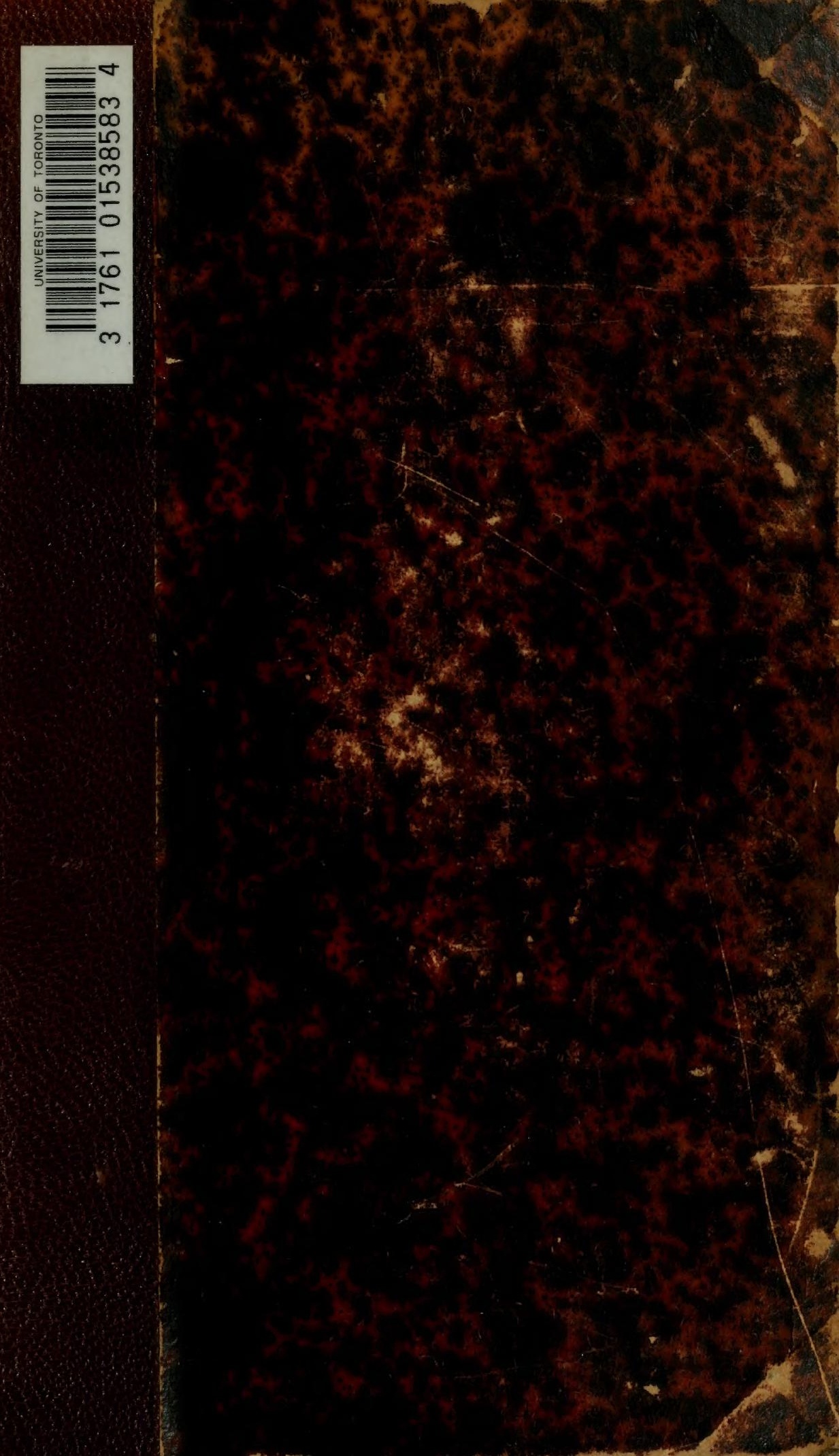




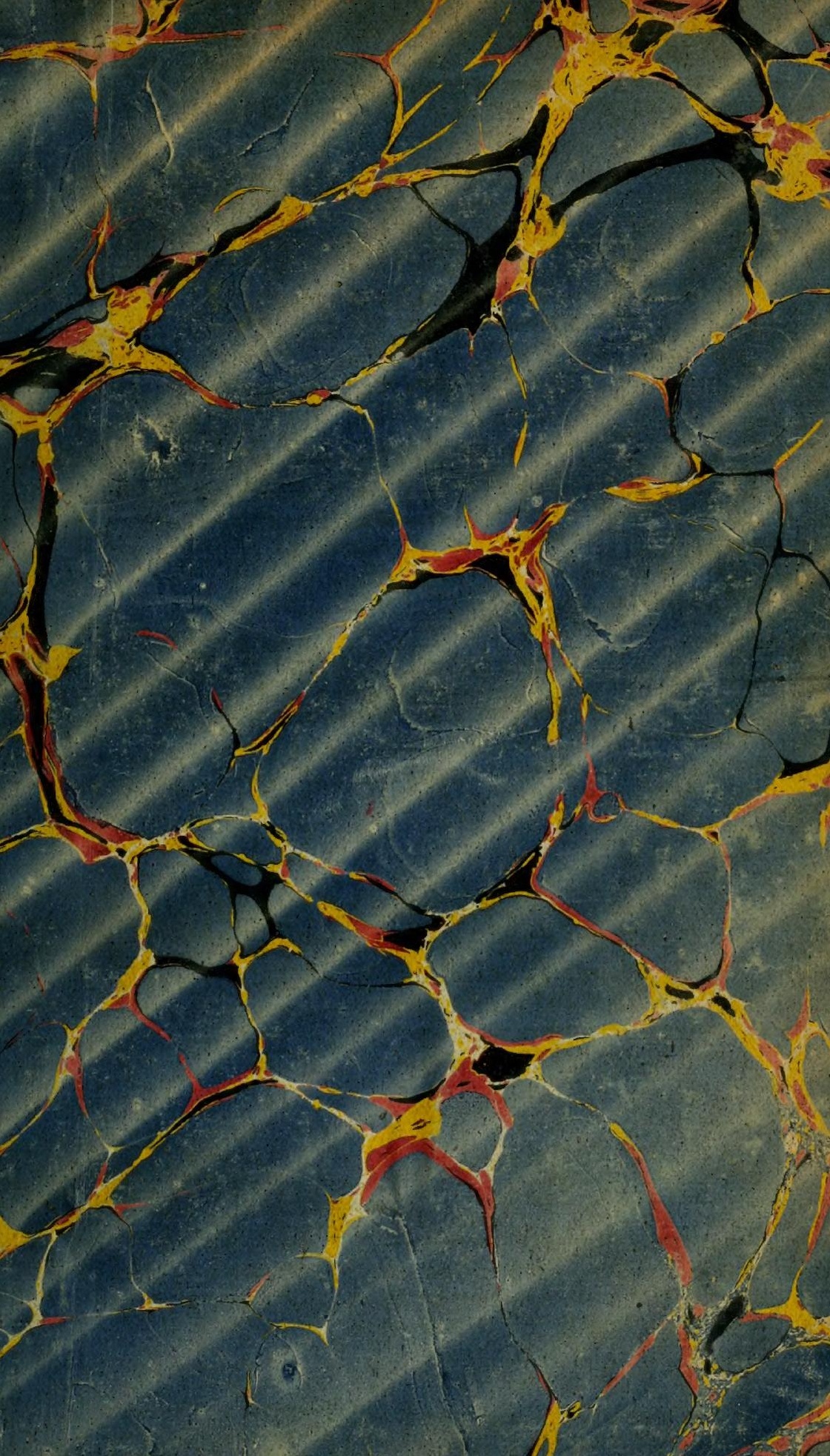




Digitized by the Internet Archive in 2010 with funding from University of Ottawa 


\section{COLRS ÉLEENTTARE}

$$
\text { DE }
$$

\section{CULTURE IDES BOIS}


POITIERS. - IMPRIMERIE GÉNÉRALE DE L'OUEST. . PARIS, 103, RUE MoNtmarthe, no 232 C. 


\section{COURS ÉLÉMENTAIRE}

I) $\mathrm{E}$

\section{CULTURE DES BOIS}

CRÉÉ A L'ÉCOLE FORESTIÈrE DE NANCY

\section{Par B. LORENTZ}

Directeur fondateur de celte école,

ancien administrateur des forêts, officier de la Légion d'Honneur

COMPLÉTÉ ET PUBLIÉ

\section{Par A. PARADE}

Conservateur des forêts, direcleur de l'école forestière

\section{SIXIEME ÉDITION}

PUBLIÉE PAR

A. LORENTZ et L. TASSY

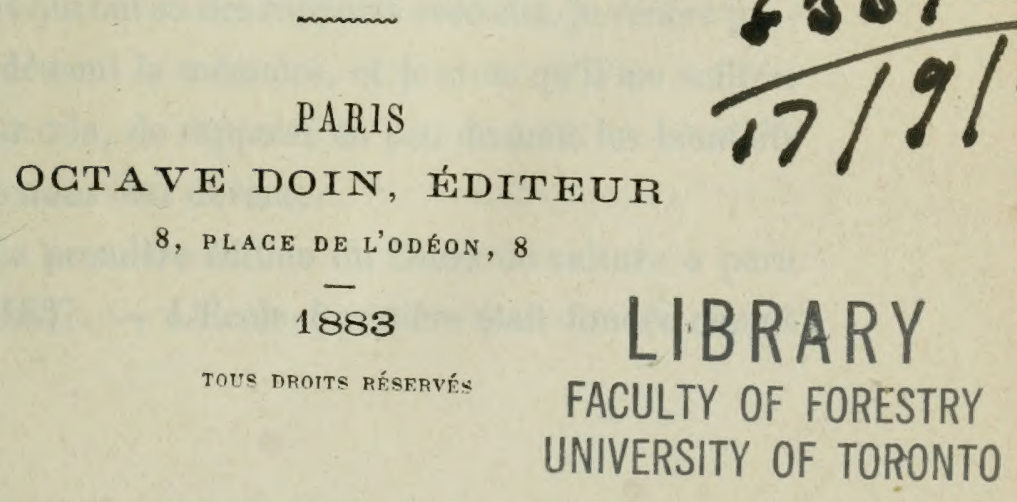




$$
\begin{aligned}
& S D \\
& 373 \\
& 167 \\
& 1883
\end{aligned}
$$

$$
\frac{25726}{2.1809}
$$




\section{PRÉFACE}

Le Cours de culture de MIM. Lorentz et Parade peut se passer de recommandation, pour continuer a faire son chemin dans le monde. La réputation qu'il s'est acquise le lui permet. Aussi, quand j'ai accepté la tàche d'écrire une préface pour l'édition qu'on en publie aujourd'hui, n'ai-je pas pris l'engagement d'en signaler tous les mérites, engagement qui d'ailleurs m'eùt mené trop loin et que je n'aurais pu remplir au gré de mes désirs. J'ai voulu seulement ne pas manquer l'occasion de rendre un nouvel hommage à deux hommes dont, comme tous ceux qui ont eu des rapports avec eux, je vénère pro- . fondément la mémoire, et je crois qu'il me suffira, pour cela, de rappeler en peu de mots les bienfaits que nous leur devons.

La première édition du Cours de culture a paru en 1837. - L'École forestière était fondée depuis 
1824. Le code forestier et l'ordonnance réglementaire avaient élé promulgués en 1827. La loi et l'administration avaient fait ce qu'il leur appartenait de faire pour la régie des forêts. Il restait à formuler et à publier les règles de culture les plus propres à féconder ce vaste domaine, règles professées par II. Lorentz, mais jusqu'alors au profit exclusif des élèves.

Je l'ai fait observer ailleurs : les matériaux nécessaires pour ce travail étaient fort incomplets ot dépourvus de liens, au moins dans notre pays. Plus nombreux en Allemagne, ils y avaient été réunis en corps de doctrine, en traités spéciaux, très estimés et très estimables; mais ces traités ne convenaient ni au climat ni à la flore de la France, ni aux mœurs de ses habitants, et j’ajouterai, ni à leur esprit, esprit logique s'il en fut et très exigeant en fait de méthode et de clarté.

MII. Lorentz et Parade connaissaient tout ce qui avait été écrit sur la matière; ils possédaient en outre un fonds précieux d'observations et d'expérience personnelles, beaucoup d'élévation dans les idées et beaucoup de précision dans l'esprit. Nul mieux qu'eux ne pouvait mener à bien la difficile 
entreprise de fixer dans un livre les principes de la science forestière. Aussi leur ouvrage obtint-il, dès la première édition, un grand el légilime succès.

Cel ouvrage a été amélioré. Il devait l'ètre. Si l'on compare la dernière édition à la première, on la trouvera beaucoup plus volumineuse. Toutefois, la différence ne porte que sur des objets secondaires. Les auteurs ont perfectionné l'œurre sans en modifier l'ordonnance, les proportions, l'harmonie, sans en altérer les théories générales, et ainsi a été réalisée leur intention, de donner aux forestiers une constitution immuable dans ses bases essentielles et néanmoins perfectible. On n’a peut-ètre pas assez remarqué cela, tant ce qui est juste, vrai, parait facile à concevoir, tant il est rare quion se rende compte de ce qu'il en coùte à un écrivain pour être méthodique. simple et clair.

Production soutenue, régénération naturelle, amélioration progressive, tel est le but de la culture des bois, disent MMI. Lorentz et Parade dans l'introduction de leur Cours; celte définition est excellente :

La production soutenue, quoique certains cas particuliers autorisent des exceptions, est une des 
conditions principales d'une exploitation rationnelle. Sans elle, il ne saurait y avoir de bonne économie domestique ou publique, et les générations futures seraient privées de toute garantie.

La régénération nuturelle est, au point de vue cultural, la fin que doivent poursuivre les forestiers. Ie Cours de culture a maintenu fermement ce précepte dans ses éditions successives, malgré les tentatives qui voulaient le lui faire abandonner. Pour moi, tout en rendant justice à l'intelligence, à la capacité et aux intentions des agents forestiers qui ont fait ces tentatives, je pense, conformément aux leçons de nos illustres maitres, que le forestier doit utiliser autant que possible l'action des agents naturels, sans chercher à y substituer la sienne, et je regarde les repeuplements artificiels, quand ils ne sont pas imposés par les circonstances, comme un vol fait à la nature, vol, - qu'on me pardonne la vivacité du mot, - qui peut avoir les plus graves conséquences.

L'amélioration progressive est un but commun à toutes les branches de l'industrie; mais elle est particulièrement recommandable en sylviculture, parce que les spéculations n’y sont bornées par 
aucune limite de temps, parce que les moindres négligences s'y traduisent sourent par des pertes irréparables, tandis que les moindres soins donnés à propos y sont susceptibles des résultats les plus avantageux. S'il est vrai de dire que les petites causes peuvent engendrer de grands effets, c'est surtout pour les forêts: personne n'ignore qu'il suffit d'un insecte gros comme la tète d'une épingle pour en compromettre l'existence. Ilais par ces mots, améTioration progressive, MII. Lorentz et Parade ont voulu tenir les agents forestiers en garde contre les témérités; ils leur conseillent de procéder avec lenteur, circonspection, comme ils l'ont fait eux-mèmes pour améliorer leur Cours. Dans ce Cours, ils ne préjugent rien; ils n'avancent que des aphorismes incontestables. Ils n'ont demandé à la science que ce qu'elle pouvait leur donner au moment où ils écrivaient; ils ne lui ont ni demandé les secrets de la vie, qu'elle ne pénétrera d'ailleurs jamais, ni emprunté des explications insuffisantes: ils n'exposent que des faits positifs, el, sous ce rapport, les moindres particularités ont été soumises à la critique la plus rigoureuse.

Maintenant, sans pousser plus loin une analyse 
dont le lecteur n'a pas besoin, je résumerai mes impressions en disant: qu'intéressant et indispen-sable pour les fonctionnaires chargés de la régie des forêts de l'État et des communes, le livre de MII. Lorentz et Parade l'est également pour les particuliers propriétaires de bois; qu’il ne néglige aucun détail de culture el qu'il laisse néanmoins en relief les hautes considérations basées sur les rapports des forèts avec l'intérèt général; qu'il fait voir enfin les liens nombreux par lesquels la sylviculture proprement dite se rattache aux autres sciences, et qu'il les fait voir avec une méthode et une netteté qui ne permettent pas de se méprendre sur leur nature et sur leur importance.

L'opinion, du reste, l'opinion mème des personnes étrangères au corps forestier, a classé depuis longtemps ce livre au rang des plus utiles.

Que l'on interroge les particuliers : ils reconnaitront qu'ils y ont trouvé tous les renseignements qui leur sont nécessaires, et que, si leur intérêt comme propriétaires n'est pas d'adopter les exploitabilités reculées, leur devoir comme citoyens est de les préconiser.

Que l'on interrnge les physiciens, les naturalistes: 
ils constateront que, dans aucun autre ouvrage, on n'a fait une application plus intelligente, plus judicieuse, plus prudente et plus exacte des sciences à la culture du sol.

Que l'on interroge les hommes d'État, les économistes, en un mot tous ceux qui songent aux moyens d'augmenter les richesses sociales : ils répondront que c'est dans ce traité qu'ils ont puisé leurs notions, leurs lumières sur les services que les forêts rendent aux sociétés.

Mais s'il en est ainsi, on ne doit point s'étonner de la popularité dont les forèts jouissent dans notre pays et de l'émotion qui s'est manifestée toutes les fois que, dans ces derniers temps, leur conservation a paru menacée. Celte popularité, ce sont MIM. Lorentz et Parade qui ont beaucoup contribué à la faire. Cette émotion, ce sont eux qui l'ont justifiée.

Voila quelques-uns des titres qui assurent à ces deux forestiers la reconnaissance de leurs concitoyens. Arrivés dans ce monde, l'un à la fin du siècle dernier (2כ̋ juin 1773), l'autre au commencement de celui-ci ( 11 février 1802), à une trentaine d'années de distance, ce fut là un fait provi- 
dentiel, s'il est permis de qualifier ainsi certains événements plutòt que d'autres. En effel, s'il fallait la hardiesse et la résolution de M. Lorentz pour tailler à l'économie forestière, dans le monument de la régénération sociale, la place à laquelle elle pouvait prétendre, il fallait la prudence, le tact et la patience de $\mathrm{M}$. Parade pour que cette place ne fùt point amoindrie par la réaction malheureuse qui fit rentrer prématurément M. Lorentz dans la vie privée; et si celui-ci, par exemple, a eu la glorieuse initiative d'affirmer hautement la supériorité du régime de la futaie sur le régime de taillis, son successeur n’a pas moins bien mérité de son pays en parvenant à maintenir ce principe, malgré tous les rfforts qui ont été faits pour l'ébranler.

Gràce aux auteurs du Cours de culture, il n'y a qu'une voix aujourd'hui sur les inconvénients du taillis, non seulement dans les bons, mais aussi, mais surtout, dans les mauvais sols. Les idées sur ce point capital ont subi un revirement complet, revirement fort heureux; carl'éducation des futaies, c'est la régénération des forces naturelles, c'est la richesse de nos descendants.

Jes àges de MM. Lorentz et Parade élaient done 
échelonnés de manière à favoriser l'accomplissement de la mission à laquelle ces forestiers s'étaient dévoués.

En exposant, ainsi que je viens de le faire, en quoi consistait cette mission et comment elle a été remplie, je suis loin cependant d'avoir montré toutes les qualités, d'avoir énuméré tous les services des deux hommes éminents que l'on a proclamés, aver raison, les fondateurs de la sylviculture française. Le deuil extraordinaire que leur mort a occasionné ne s'expliquerait pas suffisamment, si leurs actes n'avaient eu pour résultat que d'améliorer l'état de nos forêts. Il s'explique encore par des causes essentiellement morales qui leur font autant d'honneur que leurs talents comme forestiers et administrateurs :

En dehors de ce mouvement d'idées et d'affaires dont s'occupent les journaux périodiques et qui répand tant d'éclat, il y en a un autre aussi fécond, aussi intéressant, quoiqu'il attire moins l'attention, et qui fournit au plus chaud patriolisme l'occasion de s'exercer efficirement. MM. Lorentz et Parade en ont domne par leur vie un touchant témoignage. Ils ont aimé et servi la chose publique autant quion 
peut l'aimer et lit servir, et pourtint il s'est fait peu de bruit autour de leurs noms. Ah! c'est qu'ils n'ont pas cherché la célébrité. Ils se sont enfermés dans leurs attributions. C'est la meilleure règle de conduite à suivre pour celui qui veut utiliser son séjour ici-bas. Ils ont consacré à leurs fonctions, sans aucune préoccupation d'intérêt personnel, tout ce qu'ils avaient d'intelligence, de cœur et de forces physiques. Tel est le spectacle qu'ils ont offert avec une modestie parfaite, avec une foi inébranlable, et tel est l'empire, telle est la force d'attraction de la simplicité dans le vrai, dans la rertu que leurs élèves ont tous été pénétrés du mème amour et du même zèle pour la prospérité de leur pays, et se sont senlis frappés par la perte de leurs maitres, dans leurs affections les plus vives. Exemple consolant de l'heureuse influence du devoir accompli. Que chacun profite de cet exemple; que chacun s'applique à faire autour de lui tout le bien qu'il pourra, et borne son ambition à remplir, aussi complètement que possible, la tàche qui lui aura été dévolue: c'est lì la bonne morale. L'arvenir des sociétés est intéressé à ce qu'on ne s'en écarte pas.

Il me reste à faire commaitre, avant de terminer 
celle préface, que l'ouvrage, dont elle annonce au public la sixième édition, a été revu arec le plus grand soin, et qu'on a essayé d'y apporter toutes les corrections, toutes les améliorations nécessitées par les découvertes scientifiques les plus récentes. Le lecteur y retrouvera d'ailleurs le supplément important inséré par MII. Idolphe Lorentz et Henri Nanquetle dans la cinquième édition. Ces messieurs ont respeclé l'œuvre des maitres. Telle elle avait élé laissée par ses auteurs, telle elle devait en effel paraitre devant la postérité. Mais c'eùt été méconnaitre les conseils, les désirs de ММ. Lorentz et Parade que de ne pas meltre les lecteurs au courant des progrès sylvicoles survenus depuis la précédente ćdition du Cours de culture. Ainsi s'explique l'Appendice que nous reproduisons.

Cet Appendice contient, entre autres objets, deux monographies nouvelles: celles du chène chevelı el du chène occidental; une note sur l'utilité de pratiquer par contenance des coupes préparatoires à l'ensemencement dans les sapinières, et en général dans les peuplements dont l'essence dominante est susceptible de supporter longtemps le couvert; des observations sur la régénération du pin sylvestre; 
- une méthode d'aménagement pour les forcèts où le jardinage doit ètre conservé, et divers articles sur le traitement des forêts soumises au régime du taillis composé, ainsi que sur les méthodes de conversion des taillis en futaie.

Les liens de parenté et de collaboration qui unissaient MIM. Adolphe Lorentz et Henri Nanquette aux deux forestiers dont ils ont réimprimé le livre, disent assez que, dans leur Appendice, ils se sont inspirés des doctrines de nos vénérés maitres, el que ce sont encore ceux-ci qui nous parlent par leur intermédiaire.

L. TassY. 


\section{PRÉFAGE}

DE LA PREMIERE ÉDITION.

Le livre que je publie, ainsi que l'indique son titre, n'est pas de moi. M. Lorent $z$ en arait traité presque toutes les parties, lorsque, en 1830, il fut appelé au poste d'administrateur des forèts à Paris. Chargé, depuis cette époque, de professer à l'École forestière le cours de Culture des bois que M. Lorentz y avait créé, je suis devenu dépositaire de ses cahier's et de ses notes; aidé de ces dernières, j'ai continué son travail et complété l'ouvrage en lui faisant revètir une forme entièrement élémentaire. M. Lorentz l'a l'evu et m'a autorisé à le publier.

La for'me élémentaire devenait indispensable pour que notre travail atteignit son but principal, qui est d'enseig'ner la théorie d'une science de faits à des jeunes gens auxquels souvent l'idée même des forêts, qui en sont l'objet, est entièrement étrangère. Or, il est à remarquer que, sous ce rapport, aucun des auteur's forestiers français ne pouvait nous servir, quelque profonds qu'ils aient été d'ailleurs; car, pour les lire avec fruit, il faut déjà connaitre les forèts, y avoir pratiqué pendant un 
certain temps sous un guide éclairé, et, de plus, être familiarisé avec les expressions techniques, toutes choses qui manquent aux élèves lors de leur entrée à l'École.

Les ouvrages allemands sur la matière, même les plus estimés, ne laissaient guère moins à désirer quant à la forme, parce qu'il est à peu près de règle en Allemagne que, pour suivre les cours des écoles forestières, on doit avoir acquis d'abord les éléments de la science par la pratique. La traduction d'un de ces traités ne pouvait donc pas davantage satisfaire au besoin particulier de notre enseignement; et d'ailleurs, un ouvrage allemand eût toujour's présenté le double inconvénient de ne pas tenir compte de l'état actuel de nos forêts, en raison du traitement qu'elles ont subi jusqu'ici, et d'être inapplicable, sous plus d'un rapport, au sol et au climat de la France, ainsi qu'aux besoins de ses habitants et de son gouvernement.

Sans doute, on ne saurait écrire aujourd'hui sur la culture des forèts sans puiser dans les ouvrages des auteurs allemands, de mème que ceux-ci ont profité des travaux de leurs devanciers, parmi lesquels la France peut citer avec orgueil les Buffon, les Duhamel, les Réaumur, et les Varenne de Fenille. Aussi nous faisons-nous un devoir de reconnaitre publiquement ici tout le fruit que nous avons tiré de l'étude des livres allemands, surtout de ceux de Hartig et de Cotta auxquels la science forestière doit, en grande partie, le degr'é de perfection qu'elle a atteint de nos jours.

Quoique notre livre soit écrit plus particulièrement pour les élèves de l'École forestière, nous espérons cependant qu'il n'en sera pas moins d'une utilité réelle aux agents forestiers, ainsi qu'aux propriétaires de bois et aux autres per'sonnes curieuses d'acquérir quelques connaissances positives sur unẻ des parties les plus im- 
portantes de l'agronomie. Ils y rencontreront, sous une forme qu'on s'est efforcé de rendre simple et claire, les principes les plus essentiels de l'économie forestière, corroborés et éclairés par une expérience de plus de trente ans entièrement consacrée au service des forèts.

Toutefois, on se tromperait si l'on s'attendait à trouver dans cet ouvrage des règles de conduite pour tous les cas que peuvent offrir les forèts. Il n'y a pas et il n'y aura jamais de livre qui puisse dispenser le forestier d'ètre observateur attentif et intelligent de la nature. Ce que l'on peut faire dans un ouvrage élémentaire, c'est de présenter avec exactitude et netteté les principaux faits qui composent la science, de les apprécier et de les grouper avec justesse et clarté, enfin de conclure avec prudence.

Lor'sque la théorie est conçue dans un tel esprit, elle devient véritablement la base et l'utile auxiliaire de la pratique, loin d'ètre, comme on l'a quelquefois prétendu, son antagoniste. Dépourvue rle toute théorie, la pratique, en culture forestière comme en toute autre matière l'ailleurs, ne saurait être qu'une routine plus ou moins incertaine, plus ou moins obscure; de mème que, sans l'expérience et sans une certaine habitude des opérations matérielles, la théorie la mieux établie peut conduire aux plus graves méprises. C"est donc une pratique raisonnée, ou l'union intime de la pratique arec la théorie, qui constitue le forestier vraiment instruit.

Si notre livre peut contribuer à faire comprendre de tous cette utile vérité, il aura atteint le but que nous nous sommes proposé en le publiant.

PARADE.

Nancy, $1{ }^{\text {er }}$ mai 1837. 



\section{PRÉFACE}

\section{DE LA TROISIÈME ÉDITION.}

Depuis plusiem's annies déja, la seconde édition du Cours élémentaire de culture des bois est entièrement épuisée.

Des circonstances independantes de notre volontei ont. fait différer jusqu'aujourl'hui la publication d'une édition nouvelle, que nous tenions a ne pas livere au public, sans y avoir aplorte les amejiorations ef les complements dont dix-huit ammies diexperience et d'otules nous avaient successivement lémontré l'utilité.

Le lecteur, qui voudia bien comparer notres nouveau livre avec l'ancien, jugera si nous avons rempli la tache que nous nous étions imposée. Tous affirmons, du moins, que nous arons fait. dans ce but, de sérieux et constants efforts, sans, toutefois, que nous osions nous flatter dly être entièrement parrenu.

Toute science experimentale, on le sait, doit passer, en progressant, pal trois phases principales. Ians la première, les faits les plus marruants s'observent, se constatent et sont recurillis. La seconde est employe a les grouper, à les coordonner et à en déduire des rirités fonlamentales, des théories générales qui relient foutes 
les parties entre elles, les constituent en corps de doctrine et en forment $n$ érlifice dirlactique. Dans la troisième phase enfin, la throrie doit se compléter, se perfectionner saus cesse, soit au fond, soit dans la forme, en s'enrichissant d'observations et de faits nouveaux.

C'est cette troisieme phase que la sylviculture francaise nous paraít aroir atteinte lésormais, quoique, à la verité, elle ne s'y soit arancée que bien timidement jusqu'alor's et, presque, d"un pas chancelant. Ce n'est pas cepenrlant que ses nombreux adleptes, répandus aujourd'hui sur tous les points du trrritoire de l'Empire, ne fussent en mesure de foumir un riche butin, si chacun d'eux, se croyant tenu de quelque obligation enver's la science, avait à cœur d'acquitter sa lefte, ainsi quon le voit dans d'autres corps administratifs, par exemple dans ceux des mines et res ponts et chaussés. Qu'il nous soit permis de regretter que l'adninistration iles forêts n’ait jas suivi la voie trace dès longtemps par ces corpes d'élite.

On se tromperait pourtant si l'on prenait ce que nous venons de dire pour un l'eproche adressé au persomnel forestier. Cette pensée est loin de nous. Les circonsfances, nous le savons, ne lui ont pas permis de faire plus qu'il n'a fait, et nous commaissons d'ailleur's, autant que qui que ce soit, quelle est sa valeur, et ce que l'on peut. attemlie de lui. Nous n'avous voulu que constater un lait : cest que, apres aroir parcouru, depuis le milieu du siecle dernier jusqu“a nos jours, les deux premières phases de progres dont nous arous parlé, la sylviculture francaise est aujomblhui prescue stationnaire; parce que, en raison du point oir elle est par'renue, c'est surtout aux praticiens de tout ordre et le tout degré qu'il appartient de produire les matériaux qui puissent la laine avancer dans sil marche; mais, faute de certaine 
conditions favorables à leur émission, ces materiaux restent trop généralement enfouis dans la mémoire on dans les cartous de ceux qui out consacré une partie notable de leur laborieuse carrière à les rassembler.

En vain rirait-on que cest principalement aux hommes voués à l'enseignement d'une science qu'il appartient de la faire progresser. Ce serait là une idée tout à fait erronée, lorsqu'il s'agit d'une science d'application qui ne peut être assise solidement que sur le terrain de l'expérience. Les hommes d'enseignement sont évidemment moins bien placés que les praticiens proprement dits pour observer et recueillir les faits de toute nature que la culture et l'exploitation de nos forèts présentent en si graul nombre. Et d'ailleurs, ils ont une autre mission. non moins importante : celle de garder et de nourir la théorie, ce fonds commun de tous, en faisant passer au creuset de leurs meditations les travaux de la pratique. afin de ne les admettre dans l'enseinnement élémentaire qu'après les avoir soumis à l'épreure d'un raisonnement rigoureux, et, s'il y a lien, dune exprimentation satvante.

Nous le disons donc en toute sinceriti : rette troisiente edition de notre live serait et plus complete et meillemres si, pendant la longue période qui s'est écoulée depuis la publication des deux premieress, les forestiers fot par la nous entendons tous les hommes qui s'occupent de ș lviculture, qu'ils appartiennent on non a l'arministration publiqu(') avaient eu, autant (qu'il l'ent lallu, la volonti: ou la faculté de rendre compte de leurs travaux.

Espérons mieux de l'avenir. Sous un gouveruement qui veut sćrieusement le léveloppement de toutes les forces du pays et qui se glorifie, à juste titre, de féconder toutes les sources de la prospérité nationale, sous un tel régime, on ne saurait manquer d'apprécier à leur valeu. 
les précieuses richesses que recèlent encore les forêts de la France, et de rendre justice aux hommes qui sauront les faire fructifier, en même temps qu'ils s'appliqueront à perfectionner et à étendre l'art du sylviculteur.

En terminant, nous sommes heureux de pouvoir informer nos lecteur's que cette édition du Cours de culture des bois a été, comme les rrécérlentes, soumise au jugement éclairé du forestier illustre qui eut, il y a treute ans, l'insigne honneur de créer l'enseignement de la sylviculture à l'École forestière, et chez lequel les années n'ont refroidi ni le zèle ni le dévouement pour les forêts et leur prospérité.

PARADE.

Nancy, le 20 février $185 \%$.

Trentième anniversaire de l'inauguration de l'École impériale forestière. 


\section{PRÉFAGE}

DE LA QUATRIĖME ÉDITION.

Multum egerunt qui ante nos fuerunt, sed non peregerunt.

Ceux qui nous ont précédés ont beaucoup fait, mais ils n'ont pu rien parfaire.

SÉNÈ@UE.

Lorsque, il y a trente ans, l'illustre fondateur de l'École forestière, M. LoRENTz, aju's aroir créé dans cet établissement l'enseignement sylvicole, fut appelé au poste d'Arministratem des Forèts, à Paris, il me fit l'insigne honneur de me désigner au choix de l'Administration supérieure pour lui succéder dans la chaire qu’il avait occupée arec tant rl'éclat. En mème temps, il me confia les cahiers qu'il avait rédigés, me chargea de les compléter et rle mettre le tout en état d'ètre publié.

Aidé et soutenu par les conseils du maitre, je consacrai sept années à ce travail, qu'il roulut bien revoir et approuver.

Telle est l'origine du Cours élémentaire de culture des bois, dont je domne aujourd'hui la quatrième érlition.

J'ai apporté tous mes soins à améliorer cette élition; je l'ai augmentée de nombreux details sur les essences encore imparfaitement observées, de considérations nouvelles sur les particularités inhérentes aux divers modes d'exploitation. Ce's additions ne seront pas, je l'espère, sans intérèt pour le lecteur; je les dois, en partie, au concour's de plusieurs forestier's qui ont bien voulu 
répondre à l'appel que je faisais à mes confrères dans la préface de la troisième édition. Je leur adresse ici, au nom de la sylviculture, mes publics remerciements.

C'est que, en effet, ainsi que nous l'avons fait remarquer antérieurement, les sciences d'application, et la culture des bois en particulier, ne sauraient progresser sérieusement, lésormais, sans ce concours soutenu de la pratique et de la théorie : la première recueillant soigneusement tous les faits rignes d'attention; la rleuxième les rattachant, après mûr examen, au trésor commun; celle-ci se rappelant constamment le mot si profond et si vrai rapporté par DE C.LNDOLLE, que le point d'interrogation est la clef de toutes les sciences; l'autre reconnaissant, avec VARENne de Fenilde, « que l'étude des bois » est immense, et que, malgré tous les travaux dont elle » a été l'objet, l'histoire naturelle des arbres est encor'e » presque à faire. »

Ce sont donc des monographies forestières de plus en plus détaillées, de plus en plus complètes qu'il nous importe aujourd'hui d'obtenir des praticiens; elles seules feront faire à la théorie des pas rapides et assurés.

L'époque, éloignée déjà, à laquelle remonte l'apparition de notre livre, nous dispense sans doute de justifier ici, de noureau, la forme élémentaire que nous avons cru devoir lui faire revètir', tant dans l'intérêt de l'enseignement auquel il est spécialement destiné, que dans celui rle la science elle-mème, qu'il s'agissait alor's, en quelque sorte, de constituer.

Mais, si nous croyons superflu d'insister sur un point qui, d'ailleur's, ne semble avoir donné lieu à aucune objection grave, nous éplouvons, d'un autre cóté, le besoin de répondre à quelques critiques qui, pour ne pas s'ètre produites par la presse, n'en sont pas moins digues d'attention et méritent d'être examinées. 
On a dit :

"Le Cours de culture des bois devait sans doute ad»mettre dans son cadre tous les modes d'exploitation, » mais on peut reprocher à ce livre d'avoir accordé aux » méthodes du taillis et à celle de la futaie le mème soin, » les mèmes développements, et pour ainsi dire le mème » intér'êt. Par l'excellence de son but et par la perfection » des procédés qu'il emploie, ce dernier régime méritait » seul d'ètre mis en lumière par une sylviculture avan» cée. L'enseignement de l'École forestière, ne s'adres» sant d'ailleurs qu'à des fonctionnaires destinés à gérer " les forèts de l'État et des communes, devait, avec d'au» tant plus de raison, reléguer à l'arrière-plan des mé» thodes qui rappellent trop l'ignorance des temps passés » et l'enfance de l'art. »

Et d'abord, en ce qui concerne le dernier point, nous ferous remarquer que, dans toutes les spécialités (industrie, constructions, agriculture, etc.), où l'agent de l'État est placé parallèlement au particulier', l'opinion publique, en France du moins, exige du premier une instruction plus étendue et plus approfondie, parce qu'elle le considère comme étant en quelque sorte le modèle et le conseiller naturel du second.

Or, pour juger du mérite relatif des différents systèmes (qui se sont produits dans une science et pour en faire une saine application, la condition indispensable est évidemment de les avoir étudiés tous à fond et sans opinion préconçe. Cette vérité nous parait inattaquable.

En outre, bien que l'École forestière ait été instituée surtout pour former des agents de l'État, rien n'empêche cependant qu'elle ne puisse servir aussi à domner, aux particulier's propriétaires de bois, l'instruction qui leur fait défaut et dont l'utilité est mieux comprise à mesure que les produits ligneux acquièrent plus de valeur. Il y 
a lieu de peuser, au contraire, que, cédant aux réclamations qui s'élèvent de toutes parts depuis quelque temps, le Gourernement jugera bientot le moment venu de faire dans cet établissement ce qu'il a fait dans d'autres écoles de service public, et d'almettre les particulier's à venir y puiser, en qualité d'élèves libres, les comnaissances spéciales reconnues nécessaires pour gérer avec intelligence les importantes propriétés placées entre leurs mains 1 .

Dès lors, et à tous les points de vue, notre enseignement ne se trouvera-t-il pas justifié?

Un livre élémentaire, qu'on nous permette de le rappeler en terminant, n'est point un ouvrage destiné à faire triompher telle ou telle doctrine à l'exclusion des autres. Le devoir de l'auteur est de les examiner toutes avec impartialité, de faire ressortir le coté faible aussi bien que les avantages de chacune, enfin de montrer, dans un avis motivé, à laquelle appartiennent ses préférences.

Nous nous sommes efforcé de suivre cette marche dans la rédaction du Cours de culture des bois, et nous croyons n'avoir failli, en l'adoptant, ni à notre mission, ni à nos convictions.

\section{PARADE.}

Nancy, avril 1860.

1 Le sol forestier de la France a une étendue de 9,185,310 hectares, qui se décompose ainsi qu'il suit :

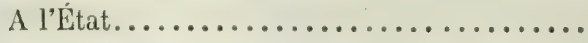

967,120 heot.

Aux communes et aux établissements publics...................... $2,058,729 \quad-$

Aux particuliers................ 6,159,461 - 


\section{COURS ÉLÉMENTAIRE}

D E

\section{CULTURE DES BOIS}

\section{INTRODUCTION.}

L'Économie forestiere comprend l'ensemble des connaissances nécessaires à l'administration la mieux entendue des forêts, eu égard aux intérèts du propriétaire en particulier et à ceux du payss en général.

Cette science est complexe. Elle emprunte aux mathématiques, à la physique et à la chimie, à l'histoire naturelle, au droit et à l'économie politique, les parties dont elle a besoin pour se constituer, ainsi 
que pour éclairer les combinaisons et les faits prattiques qui font la base du traitement des forêts.

Or, ce sont ces combinaisons, ces faits pratiques, ainsi que les différentes méthodes d'exploitation consacrécs par une expérience raisonnée, qui, réunis et coordonnés en corps de doctrine, ont reçu la dénomination de Culture des Bois ou Sylviculture, terme qui correspond à celui d'Agriculture, lorsque par ce dernier on n'entend que ce qui est relatif à la culture des champs.

Toutefois, il existe, cntre la culture der bois et celle des champs, des différences profondément tranchées, qui, sous le rapport économique du moins, détruisent en quelque sorte toute analogie entre ces deux sciences.

En effet, dans la première, la récolte ammuclle ne peut pas s'étendre, comme dans la seconde, sur la totalité du terrain mis en production; on ne peut, au contraire, exploiter chaque amée qu'une certaine partie de la superticie de ce terrain, si l'on reut retirer de la propriété (ce qui est le cas le plus grénéral) un revenu annuel et soutenu. De plus, l'exploitation des forêts n'entraine pas, comme celle des champs, la nécessité de semer ou de planter pour s'assurer une récolte; cette récolte doit, dans la plupart des 
cas, se faire de manière que la reproduction des bois en devieme une conséquence naturelle.

'Toute méthode d'exploitation des for'st-cloit done, en grénéral, satisfaire aux deux conditions fondamentales suivantes:

$1^{\circ}$ Régler la quotité des coupes ammuelles de manière à procurer un rapport soutenu;

$2^{\circ}$ Assurel, par ces coupes mêmes, la régénération naturelle.

A ces deux conditions s'en joint une troisiène, celle de tendre constamment à anciliorer et à augmenter la production, et par suite les revenus clu propriétaire.

Production soutenue, rigénérntion naturelle, amélioration prorgressice, tel est donc un réslimé le but de la Culature des Bois. 



\section{LIVRE PREMIER}

DES CLIMATS, DES SOLS, DES ESSENCES.

DÉFINITIONS ${ }^{1}$.

1. On entend par climat l'état de l'atmosphère d'un lieu donné du globe, eu égard à sa température, à son degré d'humidité et aux courants qui s'y agitent.

2. La distance d'un lieu à l'équateur, ou sa latitude, détermine son climat géographique, tandis que son climat physique ou local dépend plus particulièrement de sa situation et de son exposition.

3. Pour nous, la situation d'un lieu est caractérisée par l'élévation au-dessus du niveau de la mer et la configuration terrestre.

' Les définitions placées en tête de chaque livre sont celles des termes techniques contenus dans son texte. 
4. L'exposition est l'inclinaison d'un termin vers un point donné de l'horizon.

:. On appelle terre végétale lia couche supérieure du globe pénétrable aux racines des plantes, et terrean ou humus la partie de cette couche formée par le détritus des matières végétales.

6. Les diverses modifications que subit, dans sa composition, lit terre végétale, constituent les différents sols ou terrains.

7. On appelle arbre la plimte dont la tige est ligneuse, et gui est susceptible d'alteindre au moins la hauteur de 8 à 10 mètres ${ }^{1}$; trbrissen, la plante it tige ligneuse qui n'atteint pas la hauteur de:a mètres et se ramifie près de sa base; arbuste, la plante ligneuse dont les bourgeons ne paraissent en général qu'au printemps, et qui ne s'élìve guère au delà d'un mètre.

8. Essence est synonyme d'espèce, et s'applique aux forils. Sous le terme d'essences forestieres, on comprend toutes les espèces rl'arbres qui se rencontrent dans les forêts.

9. On qualifie d'espèce une collection de végétaux semblables entre eux, et dont les caraclères se retrouvent chez leurs ascendints el leurs descendants.

Lit variété est une légère altération de l'espèce, dont les caractères ne se perpétuent pas générale-

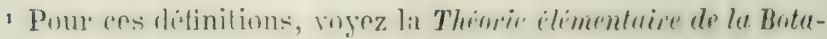
nique, par de Candolle. 
ment par la graine. Cependant, il est quelques variétés, appelées races, dont l'origine est due à des influences de sol et de climat, et dont les caractères différentiels paraissent devoir se maintenir aussi longtemps que les causes qui les ont produits.

La variation ou sous-variété est une modification de l'espèce, moins importante et encore moins durable que la variété.

10. La reproduction naturelle des arbres s'opère par les fruits. Les fruits sont produits par les fleurs.

Les fleurs sont essentiellement composées de deux sortes d'organes; les premiers, appelés étamines, représentent le sexe màle et ont pour mission de féconder les seconds, que l'on nomme pistils ou carpelles et qui constituent le sexe femelle.

La floraison des arbres se présente de plusieurs manières. Elle est : hermaphrodite, quand les deux sexes sont réunis sur la même fleur; monoïque, quand les fleurs unisexuées sont réunies sur le mème individu; diö̈que, quand les fleurs, également unisexuées, sont séparées, suivant le sexe, sur des individus différents; et enfin polygame, lorsque les fleurs mâles, femelles ou hermaphrodites se trouvent sur le mème individu ou sur des individus différents.

11. Sous le rapport de leur enracinement, on divise les essences forestières en pivotantes el traçantes, selon que leurs racines ont une tendance marquée à s'enfoncer dans la terre ou à s'étaler. 
Il est à remarquer que le pivot proprement dit, c'est-à-dire la racine centrale formant dans le sol le prolongement direct de la tige, est un organe dont le développement n'est très prononcé que dans la première jeunesse de l'arbre. Plus tard, il s'arrête dans sa croissance, alors même qu'il ne rencontre pas d'obstacle à son allongement, pour se ramifier ou pour faire place à des racines nouvelles qui sont tantôt plongeantes (pivotantes), tantôt traçantes, selon la nature des essences et la profondeur des sols.

Les ramifications extrêmes, fines et nombreuses des racines se nomment chevelu; ce sont les organes d'absorption.

12. On nomme brin de semence, ou seulement brin, l'arbre qui provient directement d'une semence; rejet, celui qui prend naissance sur une souche dont la tige a été coupée; drageon, celui qui s'élève sur une racine, et qui, séparé naturellement ou artificiellement de la souche mère, est susceptible de former un nouvel individu.

13. Un assemblage considérable de jeunes brins, de rejets ou de drageons, se nomme recru ou repenplement. Toutefois, ce dernier terme désigne plus particulièrement un assemblage de brins, tandis que l'autre s'applique de préférence aux rejets et aux drageons.

14. Les produits des forèts se rangent en diverses catégories, selon les emplois auxquels les bois sont propres. 
A. Bois de feu ou de chauffage. On le faconne de quatre manières différentes, savoir :

$1^{\circ}$ En bois de quartier, ou buches refendues;

$2 \circ$ En bois de rondin, ou bùches non fendues, moins grosses que les précédentes;

$3^{\circ}$ En fugots, ou faisceaux composés de ramilles, de branches et de quelques rondins ou quartiers ;

Et $4^{\circ}$ enfin, en bourrées, ou faisceaux renfermant exclusivement des ramilles.

Dans le commerce, on appelle plus spécialement bois de corde les bois de quartier et de rondin.

B. Bois d'ouvre. Sous cette dénomination, on comprend les bois de toute autre destination que celle du chauffage. Ils se divisent en :

$1^{\circ}$ Bois de service, qui comprend les bois de constructions civiles et navales;

$2^{\circ}$ Bois de travail ou d'ourrage, comprenant les bois employés par différents métiers, tels que lit menuiserie, l'ébénisterie, le charronnage, la tonnellerie, la fabrication des sabots, etc.

Parmi les bois de travail, on distingue les bois de fente; on nomme ainsi ceux dont l'emploi réclame le procédé de la fente. Tels sont les douves de tonneaux, de cuives, etc.; les échalas, les lattes, les cerches ou plancheltes très minces (ö millimètres et moins d'épaisseur) dont on fait les bordures des tamis, les boisseaux et autres mesures, etc. C'est encore avec des bois de fente que l'on fait des panneaux de soufflet, des pelles a four el autres, des 
attelles de collier, des bits, des arcons de selle, des rames et des gournables ou chevilles employées pour fixer les bordages à la membrure des vaisseaux.

Pour qu'un bois soit propre à la fente, il faut qu'il soit silin, d'une texture égale, que ses fibres longitudinales soient parfaitement droites, apposées régulièrement les unes contre les autres, et qu'il soit exempt de nouds.

On appelle bois merrain le bois de fente destiné plus particulièrement à la fabrication des douves.

Les bois de sciage sont ceux qu'on débite à la scie en planches de différentes dimensions.

1ว้. On nomme panage le parcours des porcs dans les forêts pour s'y nourrir de glands ou de faines.

Certaines forèts sont grevées du droit de panage à titre onéreux ou gratuit. 


\section{CIIAPITRE PREMIER.}

\section{BUT ET DIVISION DE CE. LIVRE.}

AR'TICLE PREMIER.

Des études qu'il embrasse et de leur ordre naturel.

16. Une culture raisonnée a pour base l'étude des influences auxquelles elle est soumise. Ces influences liennent aux proprietés des ohjets mèmes auxquels s'ipplique cette culture el aux propriétés des objets extérieurs. L'étude qui doit servir de base à la culture des Bois ne se borne done pas aux Bois mèmes, c'est-à-dire aux essences dont ils se composent; elle doit embrasser encore les climats sous lesquels celles-ci végètent, et les sols qui les supportent. Toutefois, les essences forment l'ohjet principal de cette étude, et ce sont elles, par conséquent, qui derraient nous occuper d'abord. Mais il est à remarquer que les essences dépendent plus des sols et des climats, que ceux-ci ne dépendent des essences. Ce sera dès lors nous conformer à l'ordre naturel que d'étudier premièrement les climals et les sols, et de passer ensuite aux essences. 
AR'TICLE II .

\section{De l'étude des Sols et des Climats.}

17. L'élude des climats et des sols, ainsi que nous venons de le faire remarquer, ne nous importe qu'en raison de l'action immédiate qu'ils exercent sur lat végétation; nous nous bornerons donc à ce qui est indispensable pour faire connaitre cette action.

18. Nous étudierons d'abord les climats en cherchant quelles sont les influences gémérales dont ils dépendent, et quelle action ils exercent à leur tour sur la régétation; puis, les divisant en climats de plaines et en climats de montiınes, nous apprendrons à connaìtre les phénomènes atmosphériques particuliers aux plaines, aux vallées, aux versants et aux plateaux, et l'influence de ces phénomènes sur la végétation. Nous serons conduits de là à prendre en considération l'exposition et les caractères propres aux quatre principaux aspects du soleil.

19. Pour les sols, nous étudierons d'abord les qualités qu'ils doivent présenter pour être favorables à la végétation, et la part qu'ils ont dans le phénomène de la nutrition. Nous examinerons ensuite leur composition, et les propriétés particulières à leurs principaux composants. Du sol lui-mème, nous descendrons au gisement, qui en est la base minérilogique; puis, nous nous occuperons de l'inclinaison. 
Enfin, nous terminerons par l'énumération des principales catégories de terrains, fondées sur lit composition, ou sur le degré d'humidité, et nous ferons connaître leurs propriétés relatives à la régélation des bois.

\section{ARTICLE III.}

\section{De l'étude des Essences.}

20. L'élude des essences étant notre objet principal, nous examinerons, pour chatcune en particulier. toutes les propriétés qui doivent aroir quelque influence sur la culture.

Ainsi, nous décrirons chaque essence en commenerant par faire connaitre ses exigences sous le ripport des climats el du sol; puis, pour embrasser le végélal dans toutes les phases de son développement, nous nous occuperons d'abord de lit floriaison el de la fructification, puis du jeune plant, enfin de l'arbre dont nous considérerons successivement le feuillage, la lige et les racines, la croissance et la durée; enfin, nous compléterons chacune de ces études par l'appréciation des qualités du bois sous le rapport des ressources quil présente à l'industrie.

21. On ne doit pas s'attendre à trourer dans nos descriptions d'arbres l'énumération de tous les caractères botaniques qui disting'uent chaque espèce 1.

1 Cescunnaissances, (qui, diailleur's, ne sont pas indispensables pour l'intelligence des règles de la Culture des bois, sont ensei- 
Nous indiquerons principalement les propriétés des arbres qui ont une influence réelle sur la culture.

2.2. Nous passerons sous silence les bois qui sont sans importance sous le rapport de leurs produits matériels, tels que les arbrisseaux el les arbusles, et nous ne parlerons pas davantige de ceux qui ne se rencontrent que très rarement dans les forêts. Parmi les essences exoliques acclimatées, quelques-unes seulement nous ont paru mériter d'ètre mentionnées, lint à cause de leur belle végétation que par rapport aux qualités de leurs bois.

23. Quant au classement des essences, nous n'entrerons encore, à cel égardrd, dans aucun détail de cliassification botanique. Nous nous contenterons de ranger les essences forestières en deux groupes: Bois feuillus et Bois résineux. Celte division nous convient principalement en ce qu'elle se fonde sur des carrictires bien tranchés tirés des phénomènes de liı végétation et de la nature même des bois.

Les arbres qui composent le premier groupe por-

grnées aux élèves de l'École forestière dans un cours de botanique appliquere, w les forestiers praticiens les possèrlent en gémiral à un legris sulfisant, soit qü̈ils les aient acquises par une longue habitude, soit quails les doivent ì une ctude applefondic. Cenx de nos lecteurs, au surplus, qui seraient entièrement étranger's à cel objet, pourront se l'approprier dans des traités spéciaux, tels que : Le nouveau Duhamel, publié par

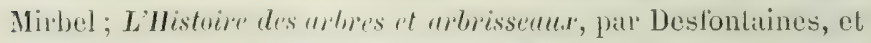
surtout la Flore forestire, publin par .I. A. Mathieu, professeur d'histoire naturelle à l'École forestière. 
tent des feuilles à limbe plus ou moins déreloppé, qui meurent et tombent à chaque automne et sont renouvelées au printemps suivant, à l'exception cependant de quelques espèces, le chêne liège et le chêne yeuse entre autres, qui conservent leurs feuilles vertes pendant plus d'une amnée. Des bourgeons, dits axillaires, se trouvent it l'aisselle de chacune de ces feuilles, d'où résulte une ramification abondante plus ou moins diffuse. Les sues qui circulent dims leurs tissus ne sont jamais résineux, et ces arbres ont tous, plus ou moins, la propriété de produire des rejets de souches et de racines, lorsque lit tige est coupée par le pied. Leur bois se compose essentiellement de fibres, de vaisseaux et de rayons ${ }^{1}$.

Les bois résineux, aussi appelés itrbres rerts, ont des fenilles linéaires, acuminées, raides, qui persistent pendint plusieurs années; le mélè̀e seul fait exception quant à la nature de ses fenilles : quoique linéaires et acuminées, elles sont tendres et tombent

' Les fibres sont des organes élémentaires qui, dans leur réunion, forment lit masse principale et lit plus compacte du bois, et qui paraît pleine à l'uil nu. - Les ruisstums sont des tubes qui se trouvent disséminés, soit solitaires, soit par faisceaux, au milieu du tissu fibrenx. Leur diametre, plus grand que celui des filmres, les fait reconnitître aisíment sur une section transversale, où ils apparaissent sous forme de petits trous.

- Les rayons sont des lames de tissu cellulaire qui varieut, selon les essences, en épaisseur, en longucur et en hauteur, et dont la direction est toujours perpendiculaire aux couches qu'clles traversent. (Voir Mathieu, Flore forestiere.) 
à chaque automne comme celles des bois feuillus. Les arbres de ce groupe n'ont pas de bourgeons à - l'aisselle de charque feuille; ils n’ont généralement que des bourgeons terminaux entourés d'un seul verticille de bourgeons axillaires, de sorte que leur ramification n'est pas diffuse comme celles des essences du groupe précédent; elle est, au contraire, plus ou moins régulire, et lia tige est susceptible d'atteindre à une plus grande hauteur.

Les bois résineux ne possident point la faculté de se reproduire par rejets 1 de souches ou de racines; leurs sues sont résineux, et leurs semences se trouvent renfermées dans un fruit ligneux composé d'écailles superposées les unes aux autres autour d'un axe commun. Ce fruit a reçu le nom de strobile ou cône.

Le bois des coniferes ne contient que des tibres et des rayons, jamais de vaisseaux.

1 Nous no ignoruns pats que plusicur's pins d Anérique, notamment le pinus trdn et le pinus rigidu, se reproduisent par rejets, el que ceitte propriété a mème été remurquée, en France comme en Allemagne, sur de très jeunes sujets de pin sylvestre qui venaient d'ètre détruils par un ineendie. Mais, d'une part, la liaculté dont il saghit ne paraît que faiblement caractérisée dans les deux espèces exotiques que nous, venons de nommer; de l'intre, lo fait signalé pour lo pin sỵlvestre est dû à une cause trop anomale pmur "qu'il puisse en ètre tenu compte autrement quau point de vue physiologique. La non-reproduction par rejuts des buis résinetux nen demeure done pas moins vraie pour nous, sous le rapport cultural. 


\section{CHAPITRE DEUXIÈME.}

\section{DES CLIMATS.}

ARTICLE PREMIER.

Du Climat en général.

24. Nous arons établi lit distinction du climit géographique et du climat physique ou local. C'est le climat local qu'il importe surtout au forestier d'étudier, comme agissant principalement sur la végétation des bois.

En général, on peut admettre que, dans les pays chauds, la végétation est non seulement plus précoce mais encore plus active que dans les pays froids, à condition que, comme sur le littoril, l'humidité atmosphérique soit abondante. Dans ces régions, les bois sont plus durs, plus pesants et plus durables que dans les pays froids. Un climat doux hàte la croissance et la maturité des bois, un climat rigoureux les retarde.

23̈. Lil situation, d’où dépend principalement le climat local, influe par l'élération au-dessus du 
niveau de lit mer, el surtout par la configuration terrestre.

26. L'élévation an-dessus du niveiu de la mer se manileste, de mème que l'éloignement de l'équateur, par une plus grande sécheresse de l'air et par un abaissement dans la température dû à un rayonnement plus actil' et à la moindre capacité de l'air raréfié pour la chaleur ${ }^{1}$; mais, tout en étant ordinairement plus raréfié et plus sec, l'air des couches supérieures de l'atmosphère est pirfois chargé, par des causes accidentelles, de grindes quantités d'humidité. En outre, les courants sont plus fréquents et ont plus de violence dans les situations élevées, sans doute parce qu'ils y rencontrent moins d'obstacles que dans les régrions basses.

Mais c'est la configuration terrestre qui a la plus grande part dans les influences qui font varier le climat local, et elle donne lieu à la distinction des climats de plaine et des climats de montagne.

\section{AR'TICLE II.}

\section{Des Climats de plaine.}

27. Les climals des plitines sont généralement plus doux et moins variables que les climats des mon-

- Dans la plus grande partie de la France, 200 mètres d'elévation au-dessus du niveau de la mer íquivalent ì jeu près à un degré de latitude. (Voir Cour's complet de Météorologic, par II. Kæmtz, traduit par M. Ch. Martins.) 
tagnes; déterminés principalement par la latitude, ils sont aussi modifiés par la nature du terrain, l'état de sa superficie, et par la proximité plus ou moins grande des mers et des montagnes.

28. Les eaux à la superficie terrestre, les lacs, les étangs, les rivières, en répandant de l'humidité dans l'atmosphère, diminuent l'intensité des chaleurs. Les masses de forèts produisent des effets analogues, par les exhalaisons aqueuses des arbres et par leur couvert qui empèche le sol de se dessécher; elles rendent encore la température plus constante, en mettant obstacle au rayonnement de la terre, en favorisant l'infiltration des eaux dans le sol que leurs racines pénètrent et divisent en tous sens, enfin en arrètant l'action des vents. Au contraire, l'ibsence totale d'eaux augmente la sécheresse et la chaleur de l'atmosphère en été; le manque de forêts ou autres plantations se fait sentir de mème, et, en hiver, il ajoute encore à l'intensité du froid.

Il doit donc être de la plus haute importince, pour l'état climatérique d'un pays, que les forèts y soient réparties d'une manière convenable.

29. Un terrain léger et profond favorise l'infiltralion des eaux et tend à adoucir le climal; un sol compacte et humide ajoute à sa rudesse.

30. Vers les còtes, les froids de l'hiver sont moins vifs, par suite du voisinage de la mer, dont la température varie peu; el les eaux, pendant l'été, rafraichissent l'air et empèchent les trop fortes chaleurs. 
Une atmosphere tris humide, la violence et la fréquence des rents caractérisent le climat de ces localités.

ARTICLL: III.

Des Climats de montagne.

31. Les climats de montagne se modifient par les mèmes causes que les climats de plaine, mais c'est surtout l'influence de la configuration terrestre qui s'y fait le plus vivement sentir. On y remarque, comme caractère général, des variations brusques et fréquentes dans la température et dinns la quantité d'humidité répandue dans l'atmosphère.

32. Trois siluations sont à distinguer dans les pays de montagnes:

Les vallées,

Les versants,

Les plateaux.

$1^{\circ}$ Dans les villées profondes, les chaleurs sont fortes pendint le jour, l'humidité atmosphérique est abondinte. L'influence des grands vents qui règnent sur les hauteurs y est ordinairement atténuée, mais l'air y est agité presque constamment par de légers courants.

Ces diverses circonstances sont toutes tris finvinrables à la végétation, qui, d'un autre còté cependinnt, se trouve exposée, dans les vallées, à l’influence nui- 
sible de plusieurs météores 1. Ce sont d'abord des brouillards épais et fréquents qui, en obscurcissant l'atmosphère, empêchent l'action bienfaisante de lir lumière, et c'est ensuite le passage trop brusque de la température de la nuit à celle du jour, d'où proviennent les gelées tardives du printemps, si funestes à la végétation.

2 Dans les régions plus hautes, les variations de température sont les mèmes que dans les rallées, mais la chaleur et l'humidité de l'atmosphère y sont moins fortes, quoique susceptibles, par moments, de s'élever à un trìs haut degrí. Des rents impétueux y règnent souvent dans une constante direction, déterminée par celles des montagnes voisines. L’action de la lumière y est vive, ot l'atmosphère chargée d'électricité.

Dans ces régions, lo régétation est moins précoce et moins active que dans les vallées.

$3^{\circ}$ Sur les plateaux des grandes hauteurs, le climat est déterminé surtout par l'élévation au-dessus du niveau de la mer, et participe d'ailleurs, en grande partie, de celui des régions immédiatement inférieures. On y remarque d'abondantes rosées, et fréquemment des pluies et des nuages qui, pendant une grande partie de l'amnée, sont transformés en neige et en givre.

' On sait que ce mot désigne généralement les phénomènes atmosphériques. 
Dans ces localités, la croissance des bois est lente et peu vigoureuse.

33. Parmi les circonstances qui modifient notablement les climats de montagne, il faut citer les abris. Ainsi, à hauteur égale au-dessus du niveau de la mer, des versants, des plateaux peuvent présenter des phénomènes climatériques très différents, selon qu'ils sont ou non abrités, dans certaines directions, par des montagnes voisines plus élevées, et selon que celles-ci sont boisées ou dénudées. Pour comprendre l'influence que cette dernière condition peut exercer, il suffit de se reporter à ce que nous avons dit plus haut [28] des effets produits par les forêts sur les climats de plaine, et de remarquer, en outre, que ces effets seront d'autant plus salutaires sur les grandes élévations, qu'en hiver les froids y sont plus vifs et plus prolongés, les neiges plus abondantes et plus persistantes; en été, l'air plus sec et plus chaud, les orages plus fréquents et plus violents; en toute saison enfin, les vents plus impétueux.

34. Nous ne parlerons pas de l'état climatérique des pays de coteaux. On conçoit qu'il doit tenir le milieu entre celui des montagnes et celui des plaines, et se rapprocher plus ou moins de l'un ou de l'autre, selon la configuration terrestre et l'élévation audessus du niveau de la mer. 
A RTICLE I V.

\section{De l'Exposition.}

33. Ainsi que nous l'avons dit, l'exposition est un élément essentiel du climat local; elle influe sur la croissance et la qualité des bois en raison de l'action non seulement du soleil, mais encore des divers météores, tels que les vents, lit pluie, la gelée, etc.. action qu'elle favorise plus ou moins.

A chacun des quatre principaux aspects se produisent des effets méléoriques particuliers.

36. A l'Est, la température est fraîche et assez sèche, parce que les rayons du soleil y arrivent le matin, lorsqu'ils ne donnent encore que peu de chaleur, et parce que les rents d'Est amènent ordinairement la sécheresse. Comme les bourgeons ne s'y développent qu'à une époque assez avancée du printemps, les gelées tardives de cette saison y sont peu à craindre; celles d'automne, au contraire, s'y font sentir de bonne heure, et peuvent quelquefois devenir nuisibles, lor'sque les jeunes plantes et les pousses de l'année ne sont point encore suffisamment lignifiées.

L'exposition de l'Est est très favorable à la végétation des bois; ils y acquièrent de belles dimensions et une texture ferme.

37. Au Nord, le climat est à peu près le mème qu'ì l'Est, toutes circonstances égáles d'ailleurs. Cependant la température y est plus froide, parce que le 
soleil y donne peu, et l'humidité plus abondante, parce qu'effectivement les rents du Nord sont moins secs que ceux du Levant.

Les arbres, dans les pentes au Nord, ont une croissance trìs rapide et parviennent aux plus belles dimensions; mais leur bois est moins dense et d'une fibre plus molle qu'aux autres expositions.

38. A l'exposition du Couchant, le sol est échiré par le soleil aux heures où il donne la chaleur la plus vive; aussi est-il, ainsi que l'atmosphère, sujet à se dessécher rapidement et à un très haut degré, à moins que des vents fréquents d'Ouest ou de Sud-Ouest, ordinairement chargés de beaucoup d'eau, ne viennent remédier à cet inconvénient. Mais, si ces vents peuvent produire quelque avantage sous ce rapport, ils sont, d'un autre côté, très nuisibles aux parties de forèt exposées immédiatement ì leur action, parce qu'en raison de leur extrême violence, et des grandes pluies qui les accompagnent, ils déracinent les arbres mal assis dans une lerre détrempée, ou les rompent très souvent.

Les bois, à cette exposition, acquièrent de la souplesse et une texture forte; mais les tourmentes trop habituelles des rents les rendent sujets à des déformations et les arrêtent souvent dans leur croissance.

39. L'exposition du Sud est la plus défavorable à la végétation. Comme le soleil y donne à peu près tout le jour, les premières chaleurs de l'année proroquent le prompt développement des hourgeons, et 
les jeunes pousses deriennent fréquemment rictimes des gelées printaniires. A cette exposition, le sol et l'atmosphère sont extrèmement chauds et secs; on y subit des rents violents, souvent accompagnés d'orages.

Les bois qui croissent dims les rersants au Midi deviennent très durs et coriaces; mais leur accroissement est lent, et leurs dimensions, tant en hauteur qu'en diamètre, sont faibles.

40. Il est à remarquer que l'influence de l'exposition sur la végétation s'affaiblit en raison de l'élévation au-dessus du niveau de la mer. Ainsi, par exemple, à une grande altitude, liı différence de lempérature, entre l'exposition du Nord et celle du Midi, est bien moins marquée qu’à des élévations moyennes ou dans les vallées. 


\section{CHAPITRE TROISIÈME.}

DES SOLS.

\section{ARTICLE PREMIER.}

Du sol en général et de son action nutritive.

41. La terre végétale se compose principalement de la terre proprement dite, fournie en général par la dégradation des roches sur lesquelles elle repose, et en outre du terreau, qui en est la partie lia plus utile au point de rue de lit nutrition. Dans les forêts, le terreau se produit abondamment par les feuilles qui tombent annuellement des arbres, et par les diverses plantes qui y pourrissent.

42. L'action du sol, sur la régétation des bois, peut ètre envisagée sous deux points de vue différents: $1^{\circ}$ sous le point de vue physique; $2^{\circ}$ sous le point de vue chimique.

43. Physiquement, le sol agit par sa profondeur, par sa compacité, par son hygroscopicité, et par les autres propriétés qui s'y rattachent, iptitude à se dessécher, ì se retirer, ete.; rnfin, par sa coloration. En vertu de sa profondeur, de sil compacilé, de sa 
DES CLIMATS, DES SOLS, DES ESSENCES.

ténacité ou de sa mobilité, le sol doit assurer aux arbres une assiette solide, favoriser l'extension des racines et permettre l'accès de l'air jusqu'à ces organes; les deux dernières propriétés déterminent aussi la facilité ou la difficulté de la culture. Par l'hygroscopicité, le sol joue un rôle très important à l'égard de la régétation : il retient plus ou moins d'eau, la cède plus ou moins facilement. Exagérée ou peu prononcée, cette propriété le rend trop humide ou trop sec. Dans le premier cas, il est sujet au retrail par les chaleurs, et les racines sont alors exposées ì être déchirées ou desséchées; dans le second, il s'émiette, devient poudreux et s'échauffe d'aulant plus que sa coloration est plus foncée.

44. Chimiquement, le sol concourt directement it la nutrition des régétiux, en leur cédant des substances minérales, qui, combinées avec l'humus, d'insolubles qu'elles étaient, se transforment en éléments solubles et, par conséquent, assimilables. Il concourt indirectement au mème but, en transmettant aux plantes certaines substances utiles qu'il recoit de l'air et des eaux de pluie. La nutrition régétale, ainsi alimentée en partie dans le sol par l'iction absorbante des racines, est complétée dans l'air par l'action de toutes les parties vertes.

Il n'étnit pas possible, au surplus, d'indiquer dans quelles mesures un sol doit présenter loutes les propriélés physiques et chimiques que nous venons d'énumérer pour qu'il offre le plus haut degré de 
fertilité. Ces mesures n'ont, en effet, rien d'absolu; elles se modifient aree les essences que l'on veut cultiver et dont les exigences varient, et, pour une mème essence, arec le climat et l'exposition.

ARTICLE II.

\section{Des principaux Composants du sol.}

4ว. Bien que les terrains présentent une variété infinie, ils ne sont cependant composés essentiellement que do trois éléments : l'argile, le calcaire, et le sable, auxquels viennent s'ajouter accidentellement le terreau et plusieurs substanoes minérales telles que le gypse (pierre à plàtre), les phosphates, l'alun, lir potasse, la soude, etc. Qunnt aux métaux que l'ony troure, le fer mérite d'être mentionné. C'est à l'oxydde de fer, en effet, que sont dues la couleur rouge et la couleur jaune d'un grand nombre de terrains, et l'on sait que la couleur influe sur l'absorption du calorique.

46. L'argile est une combinaison de silice et d'alumine; elle est très compacte, montre une très grande. avidité pour l'eau, qu'elle absorbe cependant lentrment, mais qu'elle cède de même, et dont elle ne se laisse plus pénétrer une fois qu'elle en est saturée. Exposée à une chaleur forte et prolongée, ou à un air vif et sec, elle produit beaucoup de retrait, acquiert une grande dureté et se crevasse profondément.

47. Le calcaire ou carbonate de chaux (pierre à 
chaux, craie, marbre, etc.), réduit en terre, constitue un sol meuble qui absorbe promptement et retient une grande quantité d'eau avec laquelle il se délaie et lorme de la boue, et qui, par les temps secs, perd rapidement toute cette eau et tombe en poussière.

48. Le sable est un composé de grátins plus ou moins fins non délayables dans l'eau. Il constitue un sol meuble, sans aucune consistance, dépourvu de la propriété de retenir l'eau, qu'il laisse infiltrer jusqu'aux couches les plus profondes.

49. Comme on le voit, aucune de ces terres n'est propre à former à elle seule un terrain fertile. L'argrie, à cause de sil compacité et de son imperméabilité à l'air comme à l'eau, ne permet pas aux ricines de pénétrer et de s'étendre; mais, divisée par la silice ou la chaux, en proportion convenable, elle devient un des meilleurs sols.

Lia terre calcinire, par l'éguale liacilité qu'elle possede d'absorber et de perdre beaucoup d'eau, offre un sol alternativement et brusquement trop humide it froid, puis trop sec et chaud; les plintes y régètent péniblement, quand l'argile ou une quantité considérable de terreau ne lui dome pas la consislance nécessaire.

Les sables purement siliceux, enfin, sont stériles, parce qu'en raison du manque total de cohésion entre les grains dont ils se composent, l'humidité s'infiltre ou s'évapore presque aussitòt qu'elle y pénètre, et les prive ainsi d'un des éléments essentiels 
de la végétation. Toutefois, lorsqque le sable est à l'état pulvérulent, il se modifie favorablement en ce que, bien qu'entièrement desséché à la surface, il retient, à peu de profondeur, assez de frichcheur pour permettre à certains végétaux ligneux d'y prospérer. Les dunes que l'on parvient à fixer à l'aide de semis de bois résineux sont un exemple de ce fait. Mais il n'est pas moins rrai, en général, que les sols siliceux ont hesoin, pour devenir fertiles, d'une grande quantité de terreau.

50 . Le terreau forme une terre dont lit ténacilé est supérieure à celle des terres calcaires ou siliceuses, quoique bien inférieure à celle des terres argileuses; il a, en outre, la propriété d'absorber plus d'humidité qu'aucune autre terre et de la céder avec facilité à la végétation. Le terreau corrige donc les propriétés défavorables des sols siliceux et calcaires, en leur donnant plus de consistance et en les rendant propres à retenir l'humidité, el celles des argiles, en diminuant leur trop grande compacité el leur résistance à céder l'eau absorbée. Linfin, c'est le terreau qui favorise l'assimilation des substances minérales nécessaires à la végétation.

Le ròle de ce terreau explique comment, dans beaucoup de nos anciennes forèts où il s'est accumulé, nous voyons une végétation riche el des arbres de la plus grande beauté sur des sols qui, découverts et réduits à leurs éléments minéralogiques, seraient presque stériles. 
ARTICLE III.

\section{De la base minéralogique et de l'inclinaison.}

51. Après avoir considéré le sol par rapport à ses principaux composants, il nous reste à examiner les modifications que peuvent lui faire subir sil base minéralogique et son inclinaison.

52. C'est aux gisements de roche ou d'argile pure formant la limite inférieure des terrains, que l'on a donné le nom de base minéralogique. Leur action sur la végétation se manifeste de plusicurs manières. Compacte et dure, la base minéralogique empèche évidemment les racines des arbres de s'enfoncer au delà de la couche de terre végétale; tendre et divisée par de nombreuses fissures, elle leur donne un plus liacile alccis. Stralifiée, c'est-i-dire composée de couches ou de leuillets parallè̉es, elle facilite ou arrête la pénétration des racines suivant que les joints sont obliques ou perpendiculaires à la surfice dı sol, ou qu'ils lui sont parallèles.

La base minéralogique intlue encore sur le degré d'humidité des terrains, selon qu'elle est imperméable ou qu'elle laisse l'eau s'infiltrer. Cette propriété dépend soit de la nature ou des proportions dẹs éléments constituants, el nous avons vu plus haut comment l'argile, le calcaire et le sable se comportent sous ce rapport; soit de la nature schisteuse ou 
compacte du gisement, de la direction horizontale, oblique ou verticale des couches.

53. L'inclinaison, lorsqu'elle est faible, a peu d'influence sur le terain; toutefois, les sols entièrement plats sont plus exposés aux inondations que ceux qui sont légèrement accidentés. Mais, dans les pentes fortement prononcées, on observe généralement plus de sécheresse dans la partie supérieure el plus de fraicheur dans l'inférieure, qu'à mi-còte; et les éléments meubles, solubles ou simplement délayables, étant entrainés vers le bas par l'action des eaux, la fertilité du sol au pied des montagnes augmente constamment aux dépens des parties plus élevées. Ces inconvénients, cependant, sont moins à redouter lorsque les pentes sont boisées, attendu que les arbres retiennent les terres par leurs racines, et conservent li fraicheur par leur couvert. Il est à remarquer, d'ailleurs, que l'inclinaison est favorable à la végéstation des arbres quand elle n'est pas trop forte, parce que, ne cessant pas de croìtre verticalement, leurs cimes, étagées les unes au-dessus des autres, participent plus largement à l'influence bienfaisante de la lumière, se couvrent d'un feuillage plus abondant, et l'on sait que la production ligneuse est proportionnelle au développement des parties foliacées; parce que, enfin, les racines ont plus d'espace pour s'étendre ${ }^{1}$.

1 Ce dernier arantage, qui est ríel dans les pentes douces ou moyemnes, cesse d'exister dans les pentes rapides, surtout 
ARTICLE IV.

Des diverses catégories de terrain.

วั4. Les dénominations les plus usitées pour les terrains se fondent, d'une part, sur leurs composants, de l'autre, sur la quantité d'humidité qu'ils renferment.

ร̌ร̆. On nomme terre forte, glaise, froide, celle ou l'argile se trouve visiblement en forte proportion. Les terres glaises se distinguent des terres fortes en ce qu'elles sont plus argileuses, et, par conséquent, plus rebelles à la végétation; leur couleur est ordinairement bleue ou verdàtre, et leur structure schisteuse, tandis que les secondes sont mieux pétries et colorées en blanc, en gris et en jaune. Les unes et les autres se nomment froides, lorsqu'en raison de leur humidité, de leur couleur ou de toute autre circonstance, elles sont lentes à s'échauffer, et que, pour ce motif, le développement de la végétation a lieu tardivement. כ̌6. Dans la catégorie des terres légères, on comprend les terres sablonneuses ou graveleuses composées de sable ou de gravier, soit siliceux, soit calcaire.

lorsque (comme c'est le cas le plus fréquent) le sol y manque de profondeur, les racines ayant une répugnance marquée à suivre une direction ascendinte. On reconnaît facilement la vérité de ce fait par la forme qu'affectent, dans ces localités, les cimes des arlıres, qui se développent surtout du côté de la pente et sont presque dépourvues de branches du côté opposé. 
On y compte aussi les autres genres de terres calcaires à l'état pulvérulent.

57. Les terres mameuses sont rangées tantòt parmi les terres fortes, tantòt parmi les terres légères. La marne, en effet, est un mélange intime de calcaire et d'argile qui se délite à l'air, et où l'un ou l'autre élément domine atternativement. Quand c'est le calcaire qui domine (marne calcaire), le sol peut ètre léger ; au contraire, quand l'argile l'emporte (ma'me argilcuse), la terre devient forte.

58. Le sable grus est un mélange d'environ deux tiers de sable avec un tiers d'argile, dans lequel tous les bois à peu près prospèrent.

59. Ce que l'on appelle terre franche n'est souvent qu'un sable gras arec une portion assez considérable de terreau; mais, lit plupart du temps, cette terre se compose, par parties à peu près égales, de silice, de calcaire et d'argile enrichis de beaucoup de terreau. De tous les sols c'est le meilleur, pour les bois comme pour presque toutes les plantes.

60. On donne aux sols qui renferment beaucoup de terreau (quels que soient leurs éléments minéralogiques) le nom de terrains gras ou substantiels, par opposition à ceux qui n'en contiennent que très peu ou point, et qu'on nomme maigres, pauvres, arides.

61. Sous le rapport du degré d'humidité, qui dépend non seulement des éléments terreux, mais encore de la situation, de l'exposition, etc., on divise les sols en plusieurs catégories. 
62. Les sols marécageux sont ceux qu'abreuvent abondamment des eaux croupissantes et sans écoulement; ils sont ordinairement situés dans les basfonds. On les distingue des terrains aquatiques ou mouilleux, qui sont aussi entierrement détrempés, mais où les eaux se renourellent constamment par l'écoulement. Les premiers ne présentent en général que des bois d'une végélation linguissante, tandis que les seconds peurent convenir à plusieurs essences.

63. Dims les sols humides, l'eau n’apparait pas à la surface sous une légère pression, comme cela a lieu dims les précédents; toutefois, ces sols ne se dessichent jamais entièrement, ce qui les rend particulièrement propices à certains bois.

64. Les terrains frais se dessiehent bien pendant les grandes chaleurs, mais d'ordinaire à li surface seulement, et jamais au delà de 16 centimìtres environ de profondeur. Cie degré d'humidite, favorable à presque toutes les essences, n'est d'ailleurs propre qu'aux meilleurs sols.

6ว. I ses terrains secs, que l'on nomme aussi chauds, sont ceux qui se dessèchent promptement à une profondeur assez considerable pour priver les racines des arbres de l'humidité nécessaire; il n'y a qu'un petit nombre d'essences qui puissent y végéler.

66. Toutes les différentes nuances de terrain que nous venons de citer exercent une influence plus ou moins marquée sur la qualité des bois. En général, 
les fonds humides et très substantiels produisent, avec une végétation riche, des bois d'un tissu làche qui ne restent pas aussi longtemps sains que ceux des terrains d'une fertilité moyenne, et qui, mis en weurre, sont de peu de durée. Au contraire, les sols maigres et chauds, où l'accroissement est lent et faible, fournissent un bois dur et coriace. Il est à remarquer, toutefois, que les propriétés d'un sol trop sec par lui-mèrne peurent se modifier sous une température humide, tout comme les effets produits sur la végétation par un fonds humide peuvent ètre atténués par un climat chaud. 


\section{CHAPITRE QUATRIËUE.}

\section{DES BOIS FEUILLUS.}

AR'TICLE PREMIER.

\section{Les chênes.}

67. Le genre des chènes comprend les essences qui, sous le rapport de la longérité, de lit force et des différents emplois auxquels leur bois est propre, occupent le premier rang parmi les végétaux forestiers. Les espèces principales indigènes sont les suivantes:

\section{A. - Chênes a feullles caduques.}

$1^{\circ}$ Chêne ronvre (quercus robur, Duhamel; q. sessiliflora, Sмпти); chêne mâle; chêne noir (dans quelques contrées); durelin; rouvre; chêne blanc (Blésois et Provence).

$2^{\circ}$ Chêne pédonculé (quercus pedunculata, DuHAMEL; q. racemosa, LAMARCK); chêne à grappes; 
chêne blane (Gironde, Landes, Picardie); chêne femelle; gravelin; chêne noir (Blésois); châtgne (France centrale).

$3^{\circ}$ Chêne tausin (quereus tozza, Bosc); chêne doux; chêne brosse (Anjou); chêne noir (Gironde et Landesi: chêne Angoumois ${ }^{1}$.

B. - Chênes a feuilles persistantes.

$4^{\circ}$ Chêne yeuse (quercus ilex, Lisné); chêne vert.

$丂^{\circ}$ Chêne liège (quercus suber, Lisxé); suro; sioure; surier; alcornoque ${ }^{2}$.

$6^{\circ}$ Chêne kermès (quercus coccifera, Lixwé); chêne à cochenille.

\section{$1^{\circ}$ Chêne rouvre.}

- 68. Ce chêne est un des plus répandus en France et compose des forêts considérables à lui seul, ou croissant en mélange, soit avec son congénère le pédonculé, soit avec d'autres essences.

69. Climat, Situation, Exposition. - Il appiriient aux climats tempérés, et ce n'est que rarement

- Chine chevehe (quercus cerris, Livive); chine de Bourgogne; chêne Lomberd (Doubs et Jura). Voir l'Appendice.

Note des éditeurs.

${ }^{2}$ Chêne accidental(quercus nccidentalis, Gax); corsier (Landes). Voir l'Appendice.

Note des editeurs. 
qu'on le rencontre dans les climats froids. Cependant, dans nos montagnes, il s'élève parfois à d'assez grandes hiuteurs, et, dims les Vosges, par exemple, on le trouve mème, par pieds isolés, à la vérité, mélangé au sapin. Ce sont les pays de collines et les parties inférieures des montrgnes qu'il habite de préférence. Il croit aussi ordinairement dans les plaines, mais associé au pédonculé.

Dans certains vallons froids et humides, où d'épais brouillards sont fréquents, il souffre des gelées printanières jusqu'à ce que la cimẹ de l'arbre ait dépassé la région dans laquelle ces brouillards se maintiennent habituellement.

Les pentes méridionales ne sont pas celles où il prospère le plus; quand le sol y est sec et chaud, il n'a qu'une chétive végétation, mais son bois y devient très dur [39]. Les autres expositions favorisent mieux sa croissance, lorsque le sol est d'ailleurs de bonne qualité.

70. Terrain. - Le chène rouvre se plait dans les sols moyemnement argileux, mais s'accommode des terrains graveleux, calcaires ou siliceux, pourvu qu'ils aient une certaine fraicheur. On le rencontre même dans des lieux assez arides; mais alors sa végétation, ses feuilles, ses fruits, ses dimensions enfin. se modifient et s'amoindrissent sensiblement. Les sables secs, les terrains marécageux et lles terriains mouilleux, en toute saison, lui sont contraires.

71. Floraison et fructification. - La floraison 
est monoïque, et amentacée 1 pour les mâles; les fleurs paraissent à lí fin d'avril ou au commencement de mai arec les feuilles. Les gelées printanières, qui se font sentir dans cette saison, les détruisent souvent, et sont une des causes de la rareté des fruits dans certaines contrées, où l'on observe desintervalles de $3,6,8$ et même 10 ans entre deux glandées consécutives. C'est surtout dans le Nord et l'Est de la France que cette rareté des années de semence se présente; dans les autres parties, les glandées abondantes sont généralement his ou trisannuelles, et, chaque année même, il se produit quelques fruits qui contribuent à la régénération des bois.

Le gland mûrit et tombe au mois d'octobre de l'année même de la floraison; il est lourd et s'écarte peu, dans sa chute, de l'arbre qui le produit.

Ce fruit, dont la grosseur et la forme sont extrêmement variables, selon les sols, se distingue cependant de celui du pédonculé, en ce que les glands de celui-ci sont suspendus, ordinairement au nombre

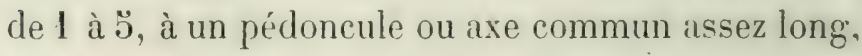
tandis que les glands du rouvre sont généralement sessiles, c'est-à-dire attachés immédiatement sur le rameau, ou du moins sur un axe très court, autour duquel ils sont groupés par bouquets de 3,4 , 与 et 6 ?

'En chatons.

2 Flore forestiere par M. Mathieu, professeur d'histoire naturelle à l'École impériale forestière. 1 vol. in- ${ }^{\circ}$. Xuncy, Grimblot, Vo Raybois et Comp., 18 8̈8. 
Le chène de brin ne devient fertile en semence que de כّ0 à 80 ans, suivant les climats et selon qu'il est plus ou moins isolé, tandis que le rejet porte fruit souvent de $2 \breve{a ̀ ~ a ̀ ~} 30$ ans. Un sol sec hàte la fructification:

72. Jeunes plants. - Les chênes sont robustes dès leur naisance, et demandent, le plus tòt possible, à être découverts. On peut mème, excepté dans les pentes au Midi, les élever sans abri; il est utile de les garantir contre les vents froids et desséchants du Nord et de l'Est.

73. Feuillage. - Le chène rouvre, selon le sol sur lequel il se trouve, a les feuilles tantòt larges, luisantes, à lobes arrondis, tantòt petites et profondément découpées, mais généralement fermes, presque coriaces et assez régulièrement distribuées sur les rameaux.

Le couvert ${ }^{1}$ de l'arbre, quoique sensiblement plus épais que celui du chêne pédonculé, est cependant toujours léger ${ }^{2}$. Cette circonstance, en permeltant aux rayons du soleil de pénétrer facilement jusqu'à la surface du sol, n'est pas avantageuse. Dans le

'Voir la définition du couvert au $4^{\mathrm{c}}$ livre.

2 Voir l'excellente étude sur les chênes, rouvre et pédonculé. publiée par MI. L. Dubois, inspecteur des forêts de Loir-et-Cher, sous le titre: Considérations multurales sur les futaies de chênes du Blésois. Blois, 18:36. Voir aussi Th. Hartig, Histoire des régitaux forestiers cultivables (texte allemand). Berlin, chez Jeanrenaud, 18 ลั2. 
terrains légers, elle facilite le desséchement, et, dans les sols fritis et de bonne qualité, surtout quand les forèts sont avancées en àge, elle favorise la végétation des herbes et des arbustes au point de compromettre souvent la reproduction de l'essence par la graine, et, par suite, sa conservation. - Les soins intelligents et continus du forestier peuvent seuls combattre une aussi fàcheuse tendance.

74. Racines. - Le chêne est généralement pivotant; ses racines s'enfoncent profondément quand l'état du sol n'y met pas obstacle; on en voit qui pénètrent jusqu’à ¿ mètres, quoique l'arbre se contente, pour prospérer, de 30 à 60 centimètres de fond.

Dans la jemesse de l'arbre, le pivot forme la racine principale, les racines latérales sont rares el peu prononcées. A un âge plus avancé, celles-ci se développent, au contraire, avec vigueur, tendant toujours plutòt à descendre qu'à s'étaler, et le pivot reste à peu près stationnaire. C'est à cette tendance des racines à s'enfoncer que le chène doit de résister, mieux que toute autre essence, aux ouragans et aux tempêtes.

Les racines du chène n'ont aucune disposition à drageonner; leur chevelu est peu abondant.

75. Croissance et durée. - La croissance du chêne est assez lente, mais assez égale jusqu'à l’àge de 180 et mème 200 ans. L'arbre vit quatre, cinq siècles, et même plus; il s'élève à 33 mètres et au delà, et parvient à une grosseur considérable. On en 
cite plusieurs qui ont atteint jusqu'ì 3 , 4, כ̆ et même 6 mètres de diamètre au pied.

76. Qualités et usages. - Le chène est l'arbre le plus estimé pour les différentes constructions. Son bois est de lit plus grande durée et résiste. mieux que tout autre, aux intempéries de l'air, lorsqu'il est parvenu à l'état parfait; l'aubier, au contraire, c'està-dire les couches les plus récemment formées, se détruit rapidement, par la vermoulure en lieu sec, par la pourriture, s'il est exposé à l'humidité. Soustraite à l'action simultanée de l'humidité, de l'air et de la chaleur, cette essence se conserve des siècles, et acquiert mème, avec le temps, une dureté presque égale à celle du fer. Elle est surtout employée dans les chantiers de la marine, et, à l'exception des màts, elle sert à peu près à toutes les parties dont un raisseau est composé. Les traverses de chemin de fer sont presque toutes en chêne, et ce n'est qu'ì défaut de pouvoir s'en procurer qu'on a recours à d'autres essences.

76 bis. Sous le rapport cultural, il est très important de connaitre les circonstances qui influent sur les qualités du bois de chêne.

Deux points sont surtout ì considérer : $1^{\circ}$ la texture ou le grain du bois; $2^{\circ}$ les vices el défauts que l'arbre est exposé à contracter en raison des conditions de sol, de climat et de traitement dans lesquelles sa croissance s'accomplit.

"La texture du bois de chène est, en général, 
" d'autant meilleure sous le rapport de la force, de

" la ténacité, de l'élasticité et de lia durée, que les

" couches amnuelles sont plus développées (de $っ$ à 15

" millimetres et au-dessus). La partie extérieure de

" chacune de ces couches, qui comprend ce qu'on

" appelle le bois d'automne, est formée d'un tissu

" serré, plein, compacte, d'apparence cornée, tandis

" que la partie intérieure ou bois de printemps n'est

" que peu développée, et ne présente qu'une zone

" étroite de vaisseaux qui apparaissent très netle-

" ment sur la tranche sous la forme de petits trous

" très rapprochés ${ }^{1 . "}$

En effet, on sait que cette zone, dite bois de printemps, composée presque exclusivement de vaisseaux, présente un tissu làche, peu résistant et trìs susceptible de décomposition, tandis que le bois d'automne, essentiellement fibreux, constitue réellement la masse solide du bois; on sait, de plus, que la zone de bois de printemps conserve à peu près la même épaisseur, quelle que soit celle de la couche annuelle entière.

Done, plus la croissance de l'arhre sera rapide, c'est-ì-dire plus le sol et le climat seront propices, le traitement bien entendu et bien suivi, plus le bois aura de qualité pour les emplois de la marine et les grandes constructions en général, et vice versa.

'Exploitation, débit et estimation des bois, par M. Nanquette, professcur d'économic forestière à l'École forestière. page 222. 
Les bois qui présentent cette forte proportion de bois d'automne sont dits nerveux; on a donné le nom de bois gras, au contraire, à ceux dont les couches innuelles sont minces et composées presque entierement de vaisseaux très ouverts.

Entre ces deux extrèmes, on comprend qu'il existe un très grand nombre de nuances.

Les vices et les défauts que le chène est exposé à contracter lorsqu'il est parvenu à de fortes dimensions, et conséquemment à un àge avancé, sont nombreux. Les bien connaitre, et savoir en apprécier la gravité dans les arbres qu'il s'agit de livrer à la consommation, est pour le forestier un sujet d'étude fort important. - Nous ne pourrions, toutefois, le traiter ici, avec les développements qu'il comporte, sans répéter, en quelque sorte, ce qui se trouve parrfiritement exposé darns l'ourrage déjà cité de M. Ninquette (pages 229 à 257), et sans sortir des limites assignées à ce Cours. Nous nous bornerons done à indiquer les défauts du chêne et les causes qui les produisent; ils sont de trois sortes:

Les uns sont dus à des accidents météoriques, tels que grands vents, grands froids, givre, verglis, variations brusques de température, coup de soleil (gélivure, roulure, lunure);

Les autres proviennent tintòt de lia sécheresse ou de l'humidité du sol, tantòt d'un état de vétusté des arbres touchant à la décrépitude (cadranure, pourriture rousse ou blanche, grisettes); 
Enfin, quelques-uns sont le résultat d'accidents provenant, directement ou indirectement, du fait de l'homme (frottures, nœuds gàtés, grisettes).

Le sylviculteur n'a que peu ou point d'action sur les causes des défauts de li première sorte; mais il peut, par un traitement intelligent et soigné de la forèt, par des mesures de police et de surveillance bien appliquées, prévenir très souvent ceux quis ont dus aux autres causes. Nous reviendrons sur ces objets dans le cours de cet ouvrage.

76 ter. Le chinne est aussi un excellent bois de fente. Il fournit le merrain le plus généralement en usage, de très bons échalas de vigne, du treillage, des cercles de futailles, et des bois propres à liı boissellerie.

Différents métiers en tirent un grand parti, mais plus particulièrement le charronnage, la tonnellerie, la menuiserie et l'ébénisterie.

"Pour ces diver's usiges, et surtout pour les deux " derniers, les chênes gras ou tendres, quand ce " défaut n'est pas exagéré, sont les plus recherchi's.

" parce que le bois est d'une plus belle teinte, mieux

„ veiné, plus ficile à traviiller, et que, mis en curre, " il n'est sujet ni à se fendre, ni à se voiler, ni à " prendre du retrait, comme le bois nerveux. - A ce " point de vue, le chène rouvre parait aroir l'avan" tage sur le pédonculé, si l'on en juge par ce qui se n passe dans les pays accidentés, où les menuisiers " et les fabricants de merrain accordent une préfé- 
" rence marquée aux chènes de montagne ou de " coteau, qui sont ordinairement de cette espèce 1."

Ainsi donc, les différentes qualités du premier de nos arbres forestiers deviennent également précieuses, selon l'usag'e auquel on le destine.

Comme bois de chauffage, le chène vieux n'est pas d'un bon usage. Il brùle sans flamme, pétille et éclate souvent, et dégage son calorique avec trop de lenteur, quoiqu'il en fournisse beaucoup; dims les apparreils à fort tirage (calorifères), à l'aide desquels on veut obtenir une température égale el soutenue, il est employé plus arantageusement. Le jeune chène (rondin) est meilleur: lorsqu'il a crù sur un terrain sec et que sal dessiccation est arancée, il brùle bien, donne beaucoup de chaleur et un beau brasier. Le charbon de chène est très emplové, surtout dans les: usines métallurgiques, et, pour cet usige, on préfère généralement le charbon de bois jeunes ou d'àge moven à celui qui provient d'arbres mùrs.

L'écorce du chène fournit le tan nécessinire à lat préparation des peaux. Le meilleur est celui qu'on retire des jeunes chènes dont l'écorce est lisse et brillante.

Le fruil du chène est pirticulièrement utile à l'engraissement des porcs.

' Ouvrage déjà eité, de M. Nanquette. 


\section{$2^{\circ}$ Chène pédonculé.}

77. Le chêne pédonculé se rencontre presque aussi fréquemment en France que le rouvre, mais il forme, plus rarement que celui-ci, l'essence dominante de nos forêts.

78. Climat, situation, exposition. - Quoique plus répandu dans les pays du Nord et moins dans ceux du Midi que son congénère, cet arbre, ainsi que nous l'avons dit, ne s'élève pas autant que celui-ci dans les montagnes et se plaît moins dans les terrains accidentés. On le trouve cependant dans les régions de coleaux, mais seulement mélangé au rouvre, landis que, dans les plaines, il prospère et domine souvent presque à l'exclusion de ce dernier.

79. Terrain. - Les terrains gras, mais surtout profonds, frais et même humides, sont ceux que le chène pédonculé recherche particulièrement. Quoique assez indifférent à la nature minérale des terres, il est cependint, de toutes nos essences forestières, celle qui s'accommode le mieux des argiles fortes. Quand il est mélangé au rouvre, on le trouve surtout en belle croissance sur le bord des eaux, dans les plis de terrain et dans toutes les places où une dépression un peu marquée du sol, en permettant à l'humidité de s'y amasser, assure à ses racines lia fraìcheur nécessiaire à leur végétation. Dans les sols 
secri vu superdiciels, ou dans les pentes prunumriss. un le voit languir et bientôt disparaître.

80 . Floraison et fructification. - Lil llopaison est la mème que celle du chène poure, si ce ne-st que les fleurs appitraissent 10 à 10 jours alvant celles de cet dernier, er qui les expose d'aulaut plus ì itre rictimes des golées du printemps et rend, pill conséquent, les glandées plus rares.

Vous alrons dit Tl les diflérences qui carracterisent les liruits de ces deux chenes. Il nous restr. seulement a faire rematrquer que, tandis que los mands du rourre passent, pour ansi dire. par loutes

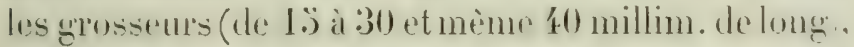
smivant lit station de l'arbore, cerse do jédoncula conservent partout it peu pres les memes dimensions 20̈ millim.), sans doute patree que, l'essence ne croissilnt que dinns les sols d'une certaine nature, les linits ne saturicut éprourer les moditications yur présenlent cenx de son congénère, beaucoup plus accommodant sous le rapport du terrain.

81. Jeunes plants. - Mème tempérament if mimes cxigences que ceux du roure, si ce nirsl. loutefois. que le pédoneule suecombe promplement sous le couvert, tandis que le roure. tout en pouvinul sen passer, le supporte cependinl assez longlemps.

82. Feuillage. - Lal rimificaltion du whe pidoncule se constilue principalement parr les bour-

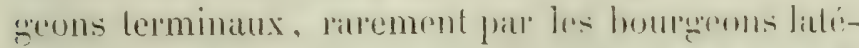

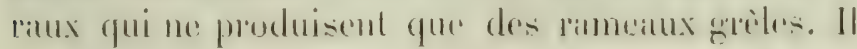


('n résulte une cime qui n’est formée que d'un pelit nombre de branches principales, plus ou moins grosses selon le volume de l'arbre, qui restent nues sur une pirlie de leur longueur, et ne portent que ver's leur extrémité un feuillage ramassé en touffes serrées, laissant dans la cime de larges ouvertures. Cette disposition rend naturellement le couvert de l'arbre tris incomplet et fort inférieur ì celui du chène rouvre ${ }^{1}$.

Les fenilles du chrine pédonculé sont plus tendres at paraissent plus lól que celles de son congénère, ce qui, en raison des lirux que l'arhore habile, les rend souvent rictimes des gelées printanières. D'un atutre còté, clles restent rertes plus longtemps en aulomne.

83. Racines. - L'enricinement du chène pédonculé ne diffère en rien de celui du rourre; toutefois, la disposition pirotinte est, chez le premier, peutètre plus prononcée encore que chez le second.

8\%. Croissance et durée. - Il n'existe pas de différence sensible, sous ce double ripport, entre les deux chènes que nous considérons. Il parait certain, néanmoins, que liı croissance du pédonculé est, en général, plus ripide que celle du rouvre, ce qui peut s'expliquer, d'abord, par la qualité des terrains qu'il exige pour prospéser, el, ensuite, par la présence plus prolongée de la leuille à l'état vert sur l'arbre.

1 Voir les ouvragés déjà cités de MMI. Th. Ilarlig, L. Dubois et A. Mathicu. 
8ə̈. Qualitès et usages.— Tout en recommaissant que les deux chines qui nous occupent participent des mèmes qualités, quoique à des degrés différents. les forestiers et les constructeurs, ainsi que les divers métiers qui travaillent ces bois, ont disculé depuis longtemps et discutent encore sur la question de saroir auxquels des deux il y a lieu d'accorder la prééminence.

Longtemps on a pensé, - el nous arons pensé el écrit nous-mème, - que le rourre, avant une plus grande force, devilil itre préféré pour les grandes constructions, le pédonculé, au contraire pour les ouvrages de fente. Nais, après aroir étudié pendant plusieurs années conséculives celte question si importante pour le sylviculteur, soulevio de nouveau, en 1856, parr la publication du remartquable mémoire de M. L. Dubois, nous devons déclitrer aujourd'hui que notre opinion s'est entièrement modifiée sur ce point.

Nous nous croyons lenu de rendre comple de ce changement.

11 a ćté établi plus haut 176 bis que le bois de chène, sans distinction d'espèce, étail d'autant plus ferme. plus résistant, plus élastique, plus nervenx enfin, que le tissu fibreux était plus abondant dans ses couches annuelles, ou, ce qui est la mème chose, que le bois d'automne était plus développé, dins une mème couche, comparativement itu bois de printemps. 
I) it vu allssi 70 of 79$]$ que le roure se plait ou résiste dans toutes sortes de terrims, depuis les ineilleurs jusqu'atux médiocres, el que le pédonculé I1 habite que les londs fortiles et frais : d'ou la conséquence que les accroissements ammuels de celui-ci seront, en général, largeses, cest-à-dire pourvus de beilucoup de bois d'aulomme, tandis que les accroissements de l'autre ne pourront présenter cet avanlage que dans les sols de bomne qualité.

De ces faits il est permis de conclure :

I' Gue le chène pédonculé, quand il aura d'ailleurs les dimensions convenables el qu'il ne sera pas vicic, fournira toujours des bois essentiellement propres aux grandes constructions;

-20ue le chène roure offrira les mèmes atvanliges, s'il a crù en bon fonds; loutefois, dins beaturoup de terrains, où sit robuste constitution el le rouvert qüil assure au sol lui permettent encore de prospérer, le grain de son bois, composé de couches imnuelles minces, manqqueril des qualités requises prour en faire un bon bois de service, mais deviendra, faur contre, d'autant plus jopre aux ouvrages de lente et de menuiserie.

\section{$3^{\circ}$ Chène tauzin.}

86 . Cet allore est très répandu dius les terrains -ablommenx de l'Ouesl de lis Frunce, depuis les P'yrémées jusqu’à Nintes, el mème jusqu’a llans. 
87. Climat, situation, exposition. - D après ce que nous venons de dire, cet arbre se plaît plus pirlticulièrement dans les climats doux et mème chauds, pourvu qu ils soient humides; toutefois, il résiste encore aux hivers des régions tempérées; il parait prchercher les plaines plutot que les montagnes, ol 111. montre pas une préférence prononcée pour un 'хуйsition plutôt que pour une autre.

88. Terrain. - Les sols légers et frais sonl will que le tauzin préfêre; on le roit rémsit dans lus dunes et ditns d'autres terrains d'mur nilure it:ar aride.

89. Floraison et fructification. - Lil $_{\text {il }}$ Mrilisun

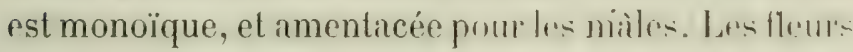

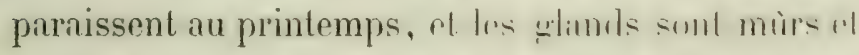

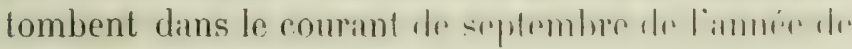
la floraison.

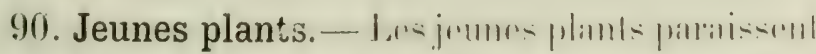

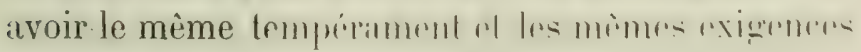
que ceux du chêne rourre.

91. Feuillage. - L s limilles du rheme timzin soml

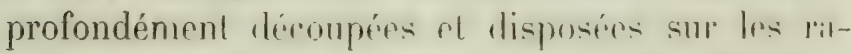
meaux. de maniepe it domner un eourept plus linene encore que celui du pédonculé.

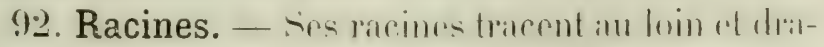
greonnent abondimment dis la jemesere. Celle diejusition to rend prerieux pour lit fixition des dumes.

93. Croissance et durée. - Lit eroissanter de col arbre est assez. rapide, mais il ne s'élère guire au 
delit de 20 à 2. moltres. el n'alloint qu'ume grosseur ordinaire. Il vit plusieurs siècles.

94. Qualités et usages. - Le chêne tauzin est peu estimé comme bois de construction; on lui reproche d'aroir trop d'aubier, d'itre noueux et tordu, de résister mal aux alternatives d'humidité et de sécheresse, et, enfin, de se tourmenter plus que les autres chênes.

Ces défauts le rendent peu propre aussi à la fente et aux autres ouvrages; mais, jeme, il fournit des cercles de futailles très recherchés, car il a la fibre coriace et beaucoup de liant.

Comme bois de feu, il est bien préférable au rouvre el au pédonculé; son charbon est très estimé. L'écoree sert an tamnage, et les glands sont particuliorement appréciés pour l'engraissement des pores.

\section{$4^{\circ}$ Chêne yeuse.}

9つ̊. Le chène yeuse ou chêne verl ne se rencontre, en France, que dans les départements méridionaux. Douns la Provence et le Languedoc, ainsi qu'en Corse, il forme de nombreuses et vastes forêts.

96. Climat, situation, exposition. - II a hesoin d'une température chaude; il se plinit sur les coteaux et sur les montignes de moyenne hauteur, of réussil ¿̀ toutes les expositions.

97. Terrain. - Cet arbre pariit préferer les sols

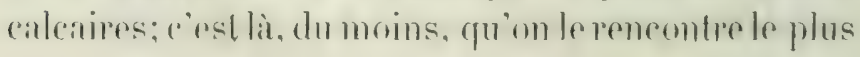


habituellement, quoiqu il croisse aussi dims d'autres terrains, mème les plus arides. A la vérité, il végète très faiblement dans ces derniers, mais arec persistance, et on peut le regarder, forestieremenl, comme le sauveur des montagnes du Midi.

98. Floraison et fructification. - La floraison est monö̈que, et amentacée pour les màles; les fleurs paraissent au printemps, quelquefois seulement en mai. Le gland mùrit en septembre, parfois mème dès l'été de l'année, et tombe avant l'hiver. Quelques chènes yeuses donnent des glands assez doux et mangeables ${ }^{1}$, sans qu'il soit possible de reconnaitre aucune différence entre les pieds qui produisent ces glands et ceux qui ne portent que des glands amers: on prélend mème que les uns et les autres se trourent parfois sur le mème arbre. II parait probable que e'est surtout dans les expositions très chatudes que le glimd devient comestible.

99. Jeunes plants. - Quoique tries robustes, el supportant bien les chaleurs, il est à conseiller cependant de leur procurer, dins les deux premières années de leur naissince, un abri contre les ardeurs du soleil.

100. Feuillage. - Les feuilles persistent pendant

1 Il existr, en Espagne et en Algérie, me variété de ìyuse appelée bullotte, dont los glands sont toujours doux el plus gros que ceux rle l'espèce type. Il serait sans doute facile de la naturaliser daus les légions de la Franee nù l’yeuse eroît spontanément. 
2 amnérs sul les lampiux, ot lombent au commeneemonl de la troisiome : elles sont brateoup plus pelites

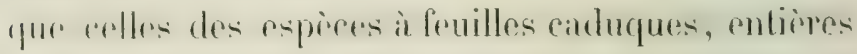

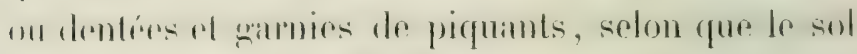

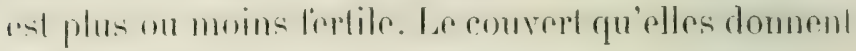
rat lrès épatis.

101. Racines. - Mans les manvats leprains oir il végèle en France, l'yeuse n’a que des racines tra-

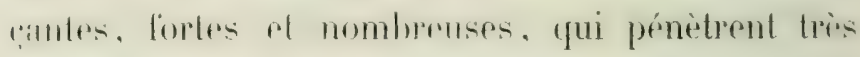
avant dius fos fissures des rochers et drageonnent ibondamment. Il est probable que, dans un hon lermin, il pirotemit davantage.

112. Croissance et durée. - La croissaner de rel

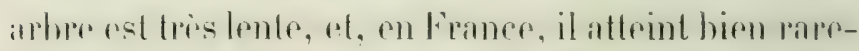
ment plus de 10 metres de haul; mais, en Italie.

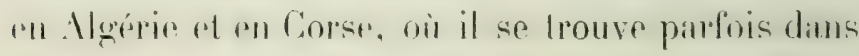
d'assez bons sols, il a de belles dimensions. Il vil plusieurs siècles.

103. Qualités et usages. - 1 o bois de l'yeuse est pemarquable jar l'homogénéité el la finesse de son grrain, sa densite el son poids. Ces qualités le rendraient éminemment propre à être employé, soil romme bois de construction, soit comme hois d'ouvarge, partout oi les pieces sont exposées à un frottement continu; anssi, Juhamel conseille-t-il déji de le prétérer, pour ces sortes d'usages, à loutes los irutres espeees do chines. On assure, de plus, que, mis en crurre, il it une tris grande durée, et prisiste longtemps à la pourifure. Nalheuleusement, on le 
roupe généralement trop jeune. ce qui fait quil ne produit guère que du chauflage. Ilais, pour ret usiage, il est très estimé. el, dans te Midi, on le troure supérieur même au hêtre.

Son écorce sert au tannage des cuirs, et on la met lort atu-dessus de relle des chines it lenilles catduques.

\section{:̊ Chinne liige.}

10'4. Le chìne liege, tris commun en kisngne. bii il se fait un grand eommerce de son écoree, of an Algérie, est cultivé arec succès dins les parties méridionales de la france. On le trouve dans les départements de Lutet-liarome, des PeréminesGrientales, du Var at de la Corse. Il mexiste deux

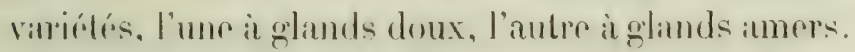
La premiere est la plus estimée pour lit qualiti de som liège.

10\%. Climat, situation, exposition. - Ciel arbre ne prospère que dans les pays à température élevée. Il réussil dans les régions moyennes des montagnes (jusqu'à 400 mètres au-dessus du niveau de la mer), iussi hien que dans les plaines. C'est dans les pentes méridionales et abritées, et partout où le soleil a beatucoup d'action, que son hois et son écoree aequierent le plus de valeur.

106. Terrain. - Le chène liège se plait particuliirement dans les sols feldspathiques. Cependant, it 
rst probable que d'iutres terres légères et divisées ne lui seraient pas contraires. Dans le département du Var, des essais de l'élever dans les terrains calcaires ont réussi, mais non sans beaucoup de soins, et il parait aussi qu'en Catalogne il croît dans des terrains de mème nature. Sa racine pivotante demande un sol profond; mais, indépendamment dn pivot, cet arbre a aussi des racines traçantes qui lui permettent de croitre dans des terrains de faible profondeur. On roit, sur les montagnes des Pyrinées-Orientales et du Var, des chènes lièges qui se contentent de très peu de fond; les racines s'implantent même dans les fissures des rochers, el donnent aux arbres une assiette tris solide. Leur écorce, dans ces situations, devient plus légère, plus fine et plus épaisse.

Les sols compactes ou humides sont ceux qui conviennent le moins à cet arbre.

107. Floraison et fructification. - La floraison estmonoïque, et amentacée pour les mâles. Les fleurs paraissent à la fin de mai ou dans le courant de juin, suivant que l'arbre est plus ou moins exposé à la chaleur et abrité des vents du Nord. Les mèmes circonstances inffuent sur liı maturité des fruits, et étitblissent à cet égard une différence de 1 à 2 mois. Les glands muirissent et tombent depuis septembre jusqu'en décembre de l'année de lit floritison. Il est probable que les glinds doux du chène liège sont dus, comme ceux de l'yeuse, à ume exposition trìs chaude. 
où la maturation s'opère d'ime manière plus complète.

Cet arbre devient fertile dès l’àge de 1 ŏ à 20 ans et porte fruit presque tous les ans.

108. Jeunes plants. - Les jeunes plants ne se montrent sensibles qu'au froid, et, particulièrement, aux gelées tardives; ils supporlent bien l'action de l'air et du soleil. Cependant, à l'exposition du midi, il convient de ne pas les laisser sans abri dans les premières années.

109. Feuillage. - Les feuilles petites, entières, plus ou moins dentées, sont nombreuses, pressées, et persistent plusieurs années. La tète de l'arbre, très rameuse, fournit un couvert épais.

110. Racines. - La racine principale pirote el s'enfonce profondément. Les racines latérales tracent au loin el sont disposées à fournir de nombreux drageons. C'est par les drageons que le chène liège s'est considérablement multiplié dans les forèts incendiées du Var.

111. Groissance et durée. - Lil croissance du chène liège est assez active, et sil durée de plusieurs siècles. Il alleint de fortes dimensions. On en cite un exemplaire qui mesurait 3 mitres $1 \ddot{\text { centimitres }}$ de tour, d'une élévation moyenne, parce qu il était isolé, mais ayant un fenillage bien nourri, des branches abondantes, fortes, et qui, par leur position verticale, annonçaient encore une végétalion vigonreuse. I in atutre chime portail 6 midres de circonfé- 
renre, et a fourni öj stires de bois liteonné, re qui pouve que lenterement de l'écorere ne lui est pats

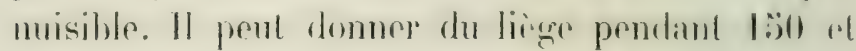
zo() ans. suivant ha fertiliti plus ou moins gramelo du lerrain.

112. Qualités et usages. - Le bois du chême liegre a beaucoup de densili. Il rat peu employer atux constructions, altendu son matrème pesanteur, ol aussi parce que, dit-on, il se pourut promptrment à l'air. Yar contre, il rest propre à la memuiserie et partienlierement estimi romme hois de chauflage.

Ciest l'enveloppe subéreuse do l'écorce qui forme le liège; livré à elle-mime, olle se crevasse profonliment el derient impropre aux usages auxquels lu lirge est destinc. Lorsquello a rití enlerée, elle est remplacée par une zone nouvelle. L '́ppoque à laquell. il convient de commeneer l'éeorement se détermine. non d'apris l’àge, mais d’apris les dimensions de l'arbre, qui doit, à ret effel. mrsurep de :30 à :30 centimètres de tour.

L'enlìvement du premier liégre ou liège mâle, qui ne donne qu'un produit grossior et presque de nulle valeur, se nomme démasclage. J fo liege femelle est celui que l'on récolte par la suite; il est éminemment propre à tous les usages du commerce. Le premier démasclage ne doil guère dépasser la moilié de la. liauteur du tronc de l'arbre; dans les écorceurmts subséquents on monte jusqu'i la naissance 
des branches, qui, en général, doivent ètre respectées, à moins (qu'elles ne soient de forle dimension. L'opération de l'écorcement se fait à la fin de juin, dans le mois de juillet, et quelquefois mème en août ${ }^{1}$.

$6^{\circ}$ Chêne kermès.

113. Le chine kermés est très abondiunt dims les départements du Midi de la Frances of. notimment, dans ceux des Bouches-du-Rhòne el du Virr, où il courre près des trois quarts du sol forestier.

114. Climat, situation, exposition. - Line température élevée lui est nécessaire ; il croit, du reste. en montagne aussi bien qu'en plaine et à tous les aspects du soleil.

11:. Terrain. - C'est dans les sols sablommeux "Lierreux qu'on le trouve le plus ordinairement. at il parait très peu exigeant sous ce rapport. Les faibles dimensions qu il acquiert doivent le faire exclure des terrains de quelque fertilité.

116. Floraison et fructification. - Lil lluraison ust monoïque, el imentacée pour les màles. Les fleurs paraissent en mai: les glands qui leur succèdent restent très petits la première année, el ne sont muirs que vers lit fin de lit seconde. Les buissons de chène:

' Voir, pour plus de détails, sur le chêne liège, la Flore furestien déjà citée de M. Mathieu, et, dans les Annules forrstirres, année 18.i8, un article fort intéressant de M. Roussel, gararle général des forêts. 
kermès portent liruit très jeunes el presque tous les ans.

117. Jeunes plants. - Cette essence étant sins importance forestière, les exigences de son jeune plint n'ont point été útudićes; mais, à en jugrer par lit grande facilité avec laquelle elle se propage, son tempérament doil itre très robuste des sa naissince, au moins pour résister aux chaleurs et à la sécheresse.

118. Feuillage. - Les feuilles sont persistimtes et épineuses, à peu près comme celles du houx; elles domnent un couvert d'autant plus épais que les buissons de chêne liermès sont très rameux el s'élèvent peu.

119. Racines. - Les racines sont nombreuses, plus traçantes, dit-on, que pivotantes, el disposées à drageonner.

120. Croissance et durée. - Ainsi qu'on vient le le dire $|11 \%|$, ce chêne ne prend que de faibles dimensions: il se ramifie dès la base, el n'atteint guère plus de 1 à 3 mètres de haut. Sa durée est probablement beaucoup moindre que celle de ses congénères; on n'a pas toutefois de notions bien certinines à ce sujet, parce qu'on l'exploite généralement très jeune.

121. Qualités et usages. - Le chène kermès, attendu ses dimensions, ne produil que du bois de bourrées et de fagots, employé en général à chauffer les tuileries, briqueteries et les four's à chaux sur place. Dims le Midi de lia France, les bois de celle 
essence sont à peu près tous lirrés au pacage. C'est sur cettc espèce que l'on trouve l'insecte appelé kiermès (liermes ilicis), qui se fixe et se nourrit sur les jeunes rameaux. On le récoltait autrefois pour en lirer une couleur rouge, estimée pour la teinture des éloffes.

L'écorce du chène liermès est, comme celle de tous les autres chènes, propre au timnitge des cuirs.

IR'TICLE: II.

\section{Le Hêtre.}

12.2. Cet inbre est l'un des plus utiles el des plus répandus de nos forèts, dont il forme fréquemment l'essence dominante. Il n'en existe qu une seule espèce indigène : le hêtre commun (fagus sylvaticia, Lixxé). On le comail aussi sous le nom de faugard, foyard ou fau.

123. Climat, situation, exposition. - Le climit tempéré est celui que cet arbre préfere; il occupe cependant, diusnos montagnes, à peu près la région du sapin; quelquefois mème il lui est supérieur. On le trouve, dims les Alpes, à $1, \ddot{\circ} 00$ mitres an-dessus du niveau de la mer, et, dans les Pyrénées, il monte jusqu'à 1,800 mètres. Le climat froid des différentes contrées de la France ne lui est donc pas essentiellement contraire.

Dins le centre, le nord, l'est el l'ouest de la 
France. on le voil prospérer inssi bien dans les plaines que dans les silualions montueuses, mais il réussit moins, dans les vallons étroits et humides, exposés aux gelées lardives, que sur les plateaux et dins les pentes abritées. Il est exch des plaines of des coteaux brùlints des départements du Midi.

L'exposition méridionale lui est contraire; il prífirre celles du Nord, du Nord-Guest et de l'Est.

124. Terrain. - Al'exception d'un sable sec. de liargile compacte et d'un l'onds marécageux, le hêtre se contente de toute espice de terrain, pourvu qu'il soil divisé. Les sols calcaires ou légèrement argileux, fortement mélangés de pierrailles, semblent lui convenir particulierement. Il n'exige qu'une prolondeur médiocre, à lit condition, toutefois, que l'épaisse couche de feuilles mortes, qu'il produit chaque année. se maintienne à la surface.

1.2̈. Floraison et fructification. - La Horaison est monoïque, et en chatons globuleux pour les males: les fleurs paraissent, avec les feuilles, dans le courant d'avril ou au commencement de mai, suivant lit température, et, comme celles du chène, elles sont souvent victimes des gelées printanieres. Ce lliost ordinairement qu'à des intervalles de plusieurs iunées que l'arbre porte semence. Le fruil, appelí faine, mùrit et tombe en octobre de l'année mème de la floraison; quoirque moins lourd que Ir gland, il me s'eloigne cependant que peu de liarbre qui l. produit. 
Le hètre ne devient fertile que vers l'àge de ä0 ans. Dans les années ou il y a fainée complète, on voil des pieds beaucoup plus jeunes chargés de graines. Iais, outre qu'elles sont raines en grande partie. cette circonstance ne peut ìtre considérie que comme tout à fait exceptionnelle.

126. Jeunes plants. - Les hêtres, dès leur naissince, sont trís délicats et demandent un abri prolongé pendant plusieurs années; ils sont cependan! moins sensibles au froid quà l'action du soleil, à laquelle ils ne peuvent résister.

127. Feuillage. - Les feuilles sont d'un tissu serré el très abondantes; elles forment un abri très épais.

128. Racines. - Daus lit premiere jeunesse, It pivot est presque aussi prononcé que celui du chène et muni de peu de racines latérales; mais celles-ci ne tardent pas à se multiplier, sans toutefois sétaler au loin, tandis que le pirot s'arrête completement. Parvenu à maturité, l'arbre présente des racines assez fortes qui tracent dans les couches supérieures du sol et ne senfoncent jamais comme celles du chêne. Pirfois elles produisent quelques drageons généralement chétifs et d'une végétation languissante.

129. Croissance et durée. - La croissance du hètre, dans les dix premières années, est fort lente: mais, aussitôt qu'il a pris un peu de consistance, il levient robuste et s'élance avec assez de rapidité. En 
sol convmable, il s'elive a plus de to metres, prend jusqu'à 1 mètre et 1 metre 30 centimetres de diamètre à la base, et prospère quelquefois pendant 300 ans.

130. Qualités et usages. - Le hêtre n'est guère propre à la charpente; son bois ne résiste ni à l'humidité ni aux variations de l'atmosphère. On n'a pu l'employer jusqu'à présent ì cet usage qu'en opérant sa dessiccation parfaite, et en le soumettant au feu jusqu'à ce que sa surface fùl un peu charbonnée. Il sert cependant à quelques pièces de construction des vaisseaux, soit celles qui sont entièrement à couvert, soit celles qui restent sous l'eau; et, depuis une dizaine d'années environ, on l'emploie à faire des traverses de chemin de fer, après l'avoir imprégné de sulfate de cuivre ou de créosote.

Du reste, son bois est d'une utilité générale. C'est un des meilleurs bois de fente : il est employé par les menuisiers, les ébénistes, les charrons, les carrossiers, les tourneurs, les layetiers el les boisseliers. On en fait des rames de bàtiments de mer, des manches de marteaux de forge et autres usines. Le sabolage qu'on en tire forme un commerce considérable. Il rist à observer qu'on ne peut fendre le hêtre yue lorsqu'il est vert, mais que, pour l'employer, il faut lui faire subir une dessiccation complete, car il prend beaucoup de retrait.

Le hêtre fournit un excellent chauffage el un charhon très recherché. 
La faine, mêlée avec le gland, sert à nourrir les porcs et à les engraisser; on en retire par ce moven un produit souvent considérable. Le panage, lorsqu'il y a suffisamment de glands et de faînes, peut être mis en adjudication; c'est une ressource très précieuse, surtout pour les pays paurres en grains. On ramasse aussi la faîne pour en extraire l'huile, qui est un très bon aliment lorsqu'elle est pressée à froid. Cette huile est meilleure à brûler et donne moins d'odeur que d'autres huiles; elle est employée avec avantage dans l'économie domestique el dans lés arts.

A RTICLE 111.

\section{Le Châtaignier.}

131. Le chataignier commun fcastanea vulgaris, Lamarck) est un arbre remarquable par son beau feuillage et son port majestueux, par sa grande utilité et par une croissance des plus rapides. On ne trouve en France, et même en Europe, qu'une seule espèce de ce genre. Quant aux variétés, qui ne sont dues qu'au climat et à la culture, elles ne se distinguent que par la grosseur et le goût du fruit.

132. Climat, situation, exposition. - On rencontre le châtaignier dins toute la France, excepté dans les départements du Nord. Un climat un peu chaud parait donner plus de solidité it son bois [24] 
plus de qualité à son fruit; ce climat lui convient aussi, parce que cet arbre, fleurissant plus tard que les autres cssences, sa fructification a besoin d'une température douce pour arriver à une maturité parfaite. Il est constant, d'ailleurs, qu'il supporte mal les froids rigoureux 1 .

Le chàtaignier se plait particulièrement sur les coteaux et surles montagnes d'une élévation moyenne. Il réussit moins dans les plaines, et l'on doit éviter de le placer sur les grandes hauleurs et dans les fonds humides.

Les pentes entièrement exposées au Midi ne lui ronviennent pas, surtout dans les localités où l'on a des gelées printanières ì craindre. Cet arbre, ayant une végétation très précoce, est souvent victime de cos gelées, lorsque, à l'entrée du printemps, le soleil hâte le développement des bourgeons. Il est done préférable de le cultiver aux expositions de l'list et du Nord-Est.

133. Terrain. - Les terres légères, siliceuses ou feldspathiques, mais substantielles et profondes. sont celles ou il prospère le mieux; cependant on le voit réussir aussi dans les sols secs et sablonneux. bans ceux qui sont légèrement humides, il a une végétation extraordinaire; mais, ayant plus souvent

- C'est, à ce qu'on prétend, l'hiver de 1709 qui a généralement detruit les chataigniers, très communs autrefois en France. Buffon (2e mémoire, Expériences sur les végétaur) rejette cette opinion comme n'aỹant aucune base sériense. 
ì soulfrir des gelées printanières, il se creuse bien plus tòt que dans les autres terrains. En général, le: terres trop compactes, humides ou marécageuse: sont contraires au chàtaignier, et il se refuse à croìtre dans les sols calcaires, ou du moins il y croìt mal.

134. Floraison et fructification. - La floraison est monoïque ou mème polygrame, car certains chat tons màles portent à leur bise des fleurs femelleou hermaphrodites. Les fleurs paraisseni dans l. mois de juin, et quelquefois en juillet seulement. Lr: fruit mûrit tard et ne se récolte, dans les climattempérés, que vers le milieu ou la fin de novembre. Sa maturité ne devance cette époque que dans les climats plus chauds, ou lorsque l'été et l'automn. ont une température élevée. Les chàtaignes sunl plus lourdes que le gland, renfermées dans une enveloppe presque ligneuse armée de pointes, qu'on appelle vulgairement hérisson; il y en a ordinairement deux et mème trois. Le marron, la plus grosse des chàtaignes, $y$ st presque toujours seul.

Le châtaignier porte fruit mème très jeune.

133.. Jeunes plants. - On peut entièrement comparer les plants de châtaignier à ceux du chène. Ils sont robustes dès leur naissance et ne prospèrent pas à l'ombre; mais ils sont, plus que les jeunes chênes, sensibles aux froids.

136. Feuillage. - Les feuilles du chàtaignier sont grandes et abondantes; elles forment un couvert assez épais. 
137. Racines. - Bien que le châtaignier ne suit pas aussi pivotant que le chêne, il l'est cependant beaucoup plus que le hètre. Ses racines sont fortes et numbreuses, elles ont foutes une tendance à s'enfoncer dans le sol, oì elles pénètrent jusqu'à un mètre et plus de profondeur. Ordinairement. le pivot cesse de s'allonger dès que l'arbre ne croit plus sensiblement en hauteur, el souvent même il meurt tout à fait. lees racines latérales drageonnent facilement.

138. Groissance et durée. - La croissance du châtaignier est très rapide dès sa jeunesse ef se soutient fort longtemps. A l'âge de 60 à 70 ans, ses dimensions sont déjà celles d'un chêne de 130 à 140 ans. Sous lo rapport de la durée, cel arbre présente les exemples les plus étonnants. Le plus gros châtaignier que l'on connaisse est celui dit des cent chevaux, qui se trouve près du mont Etna en Sicile ${ }^{1}$, et dont letrone, entièrement creux à la vérilé, a 50 mètres de circonférence ; sa naissance se perd dans les temps les plus reculés. On a mesuré d'autres châtaigniers moins vieux qui avaient des grosseurs de 10 à 15 mètres. En général, cette essence présente l'inconvénient de se creuser, sans toutefois que sa durée ait à en souffrir; mais il en résulte que les fortes pièces de châtaignier sont extrêmement rares.

- D'après M. Élisée Reclus (Revue des deux mondes, fer juillet 1865), cet arbre n'existe plus. (Note des éditeurs.) 
139. Qualités et usages. - Le chàtaignier offre un bois très propre à la charpente.Quoique plus léger, il a presque autant de force que le chêne, et l'on prétend qu'il l'égale en durée.

Dans sa jeunesse, il est très liant et propre à fair des cercles; plus tard, son bois fournit des échala: de toutes dimensions, et pour cet usage, on le préfère au chêne.

Le chàtaignier est un bon bois de fente; en Italie, on en fabrique des douves, qui, dit-on, sont d'un. qualité supérieure à celles du chème. On ne connail pas assez en France tous les usages auxquels il est propre, parce que les forèts de cette essence s'exploitent géréralement fort jeunes, et que les arbres qu'on laisse vieillir ne sont cultivés que comme fruitiers, et ne se coupent ordinairement qu'inpris leur entier dépérissement.

Le chàtaignier est moins eslimé pour le chauffage que le chène, et il est sujet à jeter des éclits. Son charbon est un peu léger et ne pourrait servir à la fonte du minerai; mais on l'emploie avec avantage à forger le fer.

La châtaigne est un fruit excellent; dans plusieurs parties de la France, elle forme la principale nourriture des habitants des campagnes.

Il parait constant que les anciennes charpentes, très bien conservées, que l'on croyait être en bois de châtaignier, sont en chêne pèdonculé (Buffon, mémoire cité plus haut). Quoi qu'il en soit, on ne peut 
rontester la végétation rapide de cet arbre, la bonte de son bois, son utilité pour la construction et l'ourrage, ainsi que l'excellence de son fruit; et, sous res divers rapports, il est digne de toute l'attention du forestier.

IRTICLE IY.

\section{L'0rme.}

140. L'Orme (ulnus, Lixxé est un des arbres les plus utiles de nos forits, mais on ne l'y rencontre que très rarement comme essence dominante. Il présente un grand nombre de viriétés ou de variations qui se rattachent les unes atux autres par une multitude de transitions. Lesprincipales espèces admises par les botanistes sont:

LOrme champêtre (ulmus campestris, Sмrт); orme it petites fenilles; ormerouge. A cette espèce appartiennent les variétés connues sous le nom d'orme tortillard et d'orme fongueux.

Lorme de montagne (ulmus montana, Surtri); orme à larges feuilles. Cette espèce est la moins estimée pour la qualité du bois, et il faut par conséquent cultiver de préférence l'orme champètre.

Il existe une troisième espèce, appelée orme diffus (ulmus effusa, Wrudevow). Elle est peu répandue et son bois n'est pas apprécié (voir la Flore forestière de M. Mathieu) 
141. Climat, situation, exposition. - Le elimat tempéré est celui où l'orme prend son plus fort développement. La grande chaleur ne lui convient pas; il s'accommode mieux du froid, et l'on rencontre sur d'assez grandes hauteurs l'orme à larges feuilles. Lorsque le terrain lui est favorable, il vient aussi bien en plaine qu'en montagne. Depuis Sully qui at itendu la culture de cet arbre en France. il a été particulièrement planté sur les grandes routes, dans le roisinage des communes, des églises, des cimetières, et sur les remparts des places fortes. Quoique rres utile, il est moins commun dans les forits.

Dans les situations blevées, il se plait aux aspects du Midi et du Couchant, tandis que, dans les régions basses, on le voit prospérer davantage aux exposilions du Nord el de l'Est.

142. Terrain. - Cet arbre no ot pas difficile sur le choix du terrain; il ne craint que les sols trop argileux, marécageux, ou arides. Dans ces delniers, on le voit dégénérer en un arbrisseau diffus (orme tortillard), à feuilles très petites, et qui ne fleurit presque jamais (M. Mathieu). Un sol frais sans ètre humide accélère sa végétation et n'ôte rien à la bonté de son bois. Il croìt avec une extrème rapidité dans les sols un peu humides, mais il y perd en qualité re qu'il gagne en accroissement.

143. Floraison et fructification. - Les fleurs de l'orme paraissent avant les feuilles, dans les premiers jours du printemps. Elles sont hermaphrodites, ot 
ordinairement très abondantes. La maturité des graines a lieu dès la fin de mai on le commencement de juin. La semence est très petite, entourée d'unr membrane circulaire fort légère ; elle est disséminér au loin par les vents, aussitòt qu'elle est mùre. Les ormes sont en général fertiles en grainst. ef rommencent très jeunes à en porter.

144. Jeunes plants. - Le temperament des jeunes plants est robuste. Cependant un premier abri leur est utile, parce qu'ils lèvent pendant les chaleurde l'été: mais ensuite on peut les abandonner à eux. mèmes.

143̈. Feuillage. - Les feuilles de l'orme sunt rudes, abondantes et donnent un couvert assez épais.

146. Racines. - Les racines, fortes et nombreuses. s'étendent au loin. Lorsque le terrain est profond. l'orme pivote autant que le chène, mais il pousse en même temps un grand nombre de racines latérales. Celte faculté de tracer lui permet de prospérer encor dans les terrains qui ont peu de fond. Les racines dr l'orme fournissent de nombreux drageons.

147. Croissance et durée. - L'orme est remarquable par la promptitude de sa croissance; il s'élève à une trìs grande hauteur et prend une grosseur considérable. Sa vie s'étend à plusieurs siècles. Unt: grande quantité d'ormes, plantés sous le ministere de Sully, existent encore et sont en bon état de croissance.

148. Qualités et usages. - Le bois d'orme est 
très dur. Un peut l'employer à la chitrpente, quoiqu'il soit, pour cet usage, inférieur au chêne et au chàtaignier. Il sert dans les chantiers de la marine. pour la carène des vaisseaux, pour les pompes ef pour toutes les parties qui sont sous l'eau.

L'orme est un des meilleurs hois de travail. Le tortillard est surtout recherché pour le charronnage: et pour tous les ouvrages qui exigent de la soliditi. L'artillerie l'emploie, de préférence à tout autre bois. pour les affùts de canon, les voitures, etc. L'ébénisterie en tire un excellent parti pour les meubles; son grain est fin, sa couleur agréablement nuancée, et il prend un beau poli. Sa fibre, très serrée et coriace, lt: rend utile à tous les ouvrages exposés au frottement, tels que les vis de pressoir, les écrous, Jes roues d'engrenage. On en fabrique aussi des arbres et des roues de moulin.

Toutes ces qualités sont celles de l'orme à petites feuilles. L'orme à larges feuilles ou de montagne ne fournit qu'un bois mou, peu résistant et peu durable; il en est de même de l'orme diffus.

Comme chauffage, l'orme est assez estimé lorsqu'il est complètement sec, mais il n'est pas de première qualité : il en est de même de son charbon.

La cendre de l'orme est une de celles des arbres forestiers qui fournissent le plus de potasse.

Les feuilles peuvent être employées comme fourrage; les bêtes à cornes et les bêtes à laine les mangent volontiers. 
A RTICLI: V.

\section{Le Frâne.}

149. Il n'y a qu'une seule espece de frène qui soit indigène dans nos forèts: c'est le frêne commun ou élevé (fraxinus excelsior, Lixxé), On le trouve ordinairement parsemé entre les autres bois, et ce n'est que par exception qu'on le voit former l'essence dominante.

1ऽ0. Climat, situation, exposition. - C'est dans les climats tempérés que le frène prend le plus bel accroissement, mais il réussit aussi dans les climats froids.

On le rencontre dans les plaines et dans les vallons, dims les pentes et sur les plateaux d'une élévation assez considérable. Toutefois, les deux premieres situations, el surtout les vallées ombreuses el fraiches, sont celles qu'il préfère.

Les expositions mépidinnales sont défavorables: ì sa croissance.

1วั1. Terrain. - Les sols profonds, fruis et assez divisés sont les plus convenables au frène; aussi, est-ce dans les prairies et sur le bord des ruisscaux yu'on le voit prendre les dimensions les plus fortes. Cependant il ne se plait pas dans les terrains marécageux. L'argile compacte et le sable pur lui sont 
contraires; on le trouve parfois en assez bon etat de croissance dans les sols secs.

152. Floraison et fructifisation. - La thoraison est polygame. Lesfleurs paraissent avant les feuilles, rers la fin d'avril et quelquefois mème plus tòt. Les fruits sont des samares munies d'une aile allongée: ils sont répandus au loin par les vents. et mûrissent rers la fin d'octobre. Leur dissémination s'opère en novembre et en décembre, et $n^{`}$ a forl souvent lieu qu'au printemps suivant.

La semence du frêne réussit abondamment presque chaque annéc, et l'arbre devient fertile de bonne heure.

133. Jeunes plants. - Quoique les jeunes frènes ne soient point délicats, ils demandent cependant à itre abrités la première année, et l’ombrage continue mêmeà leur être arantageux jusqu'à l'àge de trois ou quatre ans.

13้4. Feuillage. - La feuille du frène est composée; ses folioles sont petites. Le fruillage est léger et ne donne que peu de couvert.

1:\%.). Racines. - Les racines, menues et tric nombreuses, tracent et pirotent tout a la fois. Elles s'enfoncent jusqu ï I mètre כ̋0 centimètres et plus. et s'étendent horizontalement à 6 et à 7 mètres. Cette dernière disposition rend le frêne fort nuisible dans les champs et les prés; cependant on l'y plante fréquemment. mais on remarque que, mème it une assez 
grande distance, il mlive la nourriture aux plantes qui l'avoisinent.

Le frêne drageonne, moins abondamment toutelois que l'orme.

15้6. Croissance et durée. - Dès sa première jeunesse, le frêne a une croissance très rapide qui se soutient jusqu à 70 et 80 ans. A cet âge, l'arbre a. sourent de 30 it 33 metres de hauteur et un diamètre de 66 centimilres à sa base. Il peut atteindre des dimensions bien plus fortes encore, car il vit deux siècles et au delà.

1:\%. Qualités et usages. - Le frène peut être emplové aver arintage à la charpente, lorsqu'on le place à couvert ou entièrement sous l'eau. Mais on le voit fort rarement servir à cet usage, car, dès que les pièces sont un peu fortes, elles acquièrent une valeur très grande comme bois d'ouvrage. Le frène sert à la. menuiserie, à l'ébénisterie et à la boissellerie; il est surtout recherché pour le charronnage (particulièrement pour les timons et brancards) à cause de sa fibre souple et coriace. Les sabotiers et les tourneurs en font grand cas, on l'emploie aussi dans les manufactures d'armes. Son chauffage et son charbon sont très estimés et valent ceux du hêtre.

L'écorce peul servirau tannage; on en retire une couleur bleue.

Les cendres fournissent beaucoup de potasse; rnfin, les feuilles, soit vertes, soit sisches. sont un- 
plovées dans les pays paurres en fourrage, a la nourriture du bétail qui en est très friand.

ARTICLL VI.

\section{Les Ėrables.}

158. On rencontre communément trois especes d'érable dans nos forêts. Ce sont:

L'érable sycomore (acer pseudo-platanus, Isixí) :

L'érable plane (acer platanoides, Lisxé):

Et l'érable à petites fenilles, ou érable champètre (acer campestre, Linné).

Comme les frènes, les érables ne forment presque jannilis l'essence dominante des bois.

1399. Glimat, situation, exposition. - Sous le triple rapport du climat, de la situation et de l'exposition, les érables ont à peu près les mèmes exigences que le trine. Le plane et le sycomore supportent mieux, toutefois, les grandes élévations: dans les Alpes, on a trourí ce dernier a 1,700 mètres au-dessus du niveau de la mer.

160. Terrain.-Les sols profonds, frais et divisés sont ceux que les érables préferent; mais ils saccommodent très bien des terrains de moins bonne qualité, tels que ceux qui conviemnent, par exemple, au hètre, avec lequel ils rroissent souvent en mélange. 
L'argile compacte, les sables secs et les marais Jeur sont entièrement contraires.

161. Floraison et fructification. - Lil Horaison ust hermaphrodite. accidentellement et par avortement, polygame. Les fleurs de l'érable plane paraissent vers lia fin d'arril, et celles du sycomore et de l'érable champètre seulement dans le courant de mai. Les fruits sont des samares doubles, dont charune est munie d'une large membrane en forme d'aile; elles mùrissent on octobre of se disséminent aussitòt.

162. Jeunes plants. - Le tempérament des jeunes plants est assez robuste, mais un premier abri leur est nécessaire.

163. Feuillage. - Les f'euilles du sycomore el celles du pline sont fort grandes; celles de l'érable champêtre le sont beatucoup moins; mais le feuillage des trois est abondant et procure un couvert épais.

164. Racines. - Les racines des érables tracent et pivotent à la fois. Leur extension horizontale est beaucoup moindre que celle des racines de frène, et une profondeur de 30 it 60 centimètres leur suffit pour porter de forl beaux arbires. Il est rare qu'elles drageonnent.

163. Croissance et durée. - L'érable sycomore et le plane croissent rapidement dès leur jeunesse; le premier l'emporte cependant sur son congénère. A l'àge de 60 à 70 ans, ils ont, en sol convenable, une elévation de 20 a 25 mètres, et un diamètre de 
66 centimètres à la base. Ils peuvent atteindre des dimensions bien plus fortes encore; ils vivent en effet 150 à 200 ans, et même au delà.

L'érable champêtre, qui est tantôt arbre et tantòt arbrisseau, croìt plus lentement. Dans le premier cas, il peut s'élever de 10 à 15 mètres au plus. Il vit, comme les deux autres, 150 à 200 ans, mais sa croissance se ralentit déjà beaucoup avant qu'il ait accompli le siècle.

166. Qualités et usages. - Les érables ne servent point à la charpente, quoiqu'ils y soient propres, lorsqu'ils sont garantis des variations de l'atmosphère. Le plane et surtout le sycomore sont extrêmement recherchés pour la menuiserie et l'ébénisterie; ils prennent un beau poli, sont agréablement veinés et d'un beau jaune pâle. Les charrons, les tourneurs et plusieurs métiers encore, en font aussi très grand cas. L'érable a la propriété de ne point se tourmenter et de n'être pas attaqué par les vers.

Les qualités de ce bois pour le travail rendent nécessairement très rare son emploi comme chauffage ou comme charbon, quoiqu'il soit supérieur, pour ces usages, à la plupart des autres essences.

Les cendres des érables fournissent beaucoup de potasse.

Leurs feuilles peuvent servir à la nourriture des bètes à laine; la sève du sycomore, ainsi que celle du plane, contient du sucre, mais pas assez sans doute pour' que l'extraction en soit ivantageuse. 


\section{Le Bouleau.}

16\%. Sous le nom générique de bouleiu, on réunil, en langage forestier, deux espèces: le bouleau blanc (betula alba, Lisvé) el le boulean pubescent (betulit pubescens, Emrmandt). Les caracteres dislinctifs de ces deux espèces sont surtout botaniques; leurs qualités, leurs tempéraments diffèrent peu, el il n'est pas nécessaire, par conséquent, de les séparel l'une de l'autre dans la pratique.

Quoique très répandu dans les forèts, le bouleuu y forme rarement l'essence dominante. Ce n'est grùre que sur les grandes sommités, dérolues surtout au bouleau blanc, et quelquefois dans les marais tourbeux, que recherche particulierement le pubescent, qu'on le rencontre seul. Dans d'autres localités, s'il occupe exclusivement le terrain, ce fait est d'ordinaire le résultat d'une culture particulière, el non l'ouvrage de la nature.

168. Climat, situation, exposition. - Le bouleau supporte les climats les plus froids; en s'élevant sur les monlignes, on le voit dépasser de beaucoup la limite extrême de loutes les autres essences, et c'est, vers le póle du Nord, le dernier arbre que l'on 
rencontre. Les élés chauds et prolongés ne lui sont pis furorables, et, dans le Midi, il ne réussit que sur les grindes sommités. Dims les Pyrénées, on troure encore des bouleaux à près de 2,000 mètres audessus du niveau de la mer. Les régions tempérées n'en sont pas moins celles qu'il préfère, et où il prend le plus bel accroissement. Dans ces réggions, il prospère pirticulièrement aux expositions du Sud-list el du Sud-Ouest, quoique les autres ne lui soient point absolument contraires.

169. Terrain. - Le sol qui convient le mieux au bouleau est un sable gras; matis cel arber se contente d'ailleurs d'un terrain médiocre et d'une nalure quelconque, pourvu qu'il ne soit pas trop compacte. Le bouleau blane ne se plail pas dims les sols matrécageux, dont le pubeseent, au contraire, s'accommode très bien.

\section{0). Floraison et fructification. - Lil flolitison} est monoïque et amentacée pour les deux sexes; les fleurs paraissent en mime temps que les leuilles, vers la fin d'avril. Le fruit est un cône el mùrit diuns les derniers jour's du mois d'inoùt ou au commencement de septembre. Les semences se disséminent immédiatement el tombent avec les écaillf's qui se délachent de l'axe; elles sont très petites, munies d'une membrane légère, et sont répandues fort loin par les vents.

Le bouleau devient fertile à un àge peu arancé, et sa graine réussit presque tous les ans. 
171. Jeunes plants. - Les jeunes plants sont vigoureux dès leur naissance et résistent aux froids comme aux ardeurs du soleil.

172. Feuillage. - Les feuilles sont très abondantes, et ne donnent cependant qu'un couvert fort léger qui n'empêche point la végétation autour de l'irbre. Cette propriété, avantageuse quelquefois, est souvent un obstacle à la bonne croissance du bouleau, lorsqu'il forme l'essence dominante. En effet, les rayons du soleil, ayant action sur la surface du sol, en pompent l'humidité, empêchent que le terrain ne s'itmende par la décomposition des feuilles mortes, et, de plus, favorisent la crue des herbes et des arbustes nuisibles.

173. Racines. - Les racines du bouleau sont nombreuses, déliées et très traçantes; lorsqu'elles sont entièrement à découvert, elles produisent assez fréquemment des drageons.

174. Croissance et durée. - La croissance du bouleau est rapide, mais elle se ralentit considérablement vers la $60^{\circ}$ année. Sa durée ne dépasse pas ordinairement 80 à 90 ans.

175. Qualités et usages. - Le bouleau sert rarement à la bàtisse, et ce n'est qu'à couvert qu'il peut ètre employé à cet usage: mais il est très estimé pour le charronnage, la menuiserie, la fabrication des sabots, etc.; sa fibre est coriace, el il a, comme l'érable, la précieuse propriété de ne point se tourmenter et d'être à l'abri de la vermoulure. 
Le bouleau produit un assez bon chauffage; il brûle avec une flamme claire et égale. Son charbon est aussi fort estimé, et ses jeunes rameaux servent à faire des balais.

L'écorce est employée au tannage dans le Nord; elle contient une huile essentielle qui communique au cuir de Russie l'odeur qui le caractérise. En Pologne et en Russie, on prépare, avec la sève du bouleau, une espèce de vin et d'assez bon vinaigre.

\section{ARTICLE VIII.}

\section{Le Robinier faux Acacia.}

176. Le robinier faux acacia (robinia pseudoacacia, Lrnsé), originaire de l'Amérique septentrionale, a été introduit en France, sous Henri IV, par Robin. Il est parfaitement acclimaté et, chaque année, se propage de plus en plus. Ses précieuses qualités, la rapidité de sa croissance et la beauté de son port justifient la faveur dont il jouit parmi les arboriculteurs.

\section{Climat, situation, exposition.- Les climats} rigoureux ne conviennent pas au robinier; il demande, pour prospérer, une température douce et égale, et les grands froids le font souvent périr entièrement. Aussi est-ce dans les pays de plaines et de coteaux que sa culture est réellement avantageuse 
On doil, autant que possible, placer les hois de robiniers dins des situations abrilées des grands vents, car cet arbre se casse très facilement.

Les expositions chaudes sont celles qu'il préfère.

178. Terrain. - Le robinier prospère dans les terrains légers, mais substantiels, et principalement lans les sables gras pourvus de terreau. Les sols arides, ou monilleux, ou trop compactes lui sont contraires.

179. Floraison et fructification. - Le robinier fleurit au commencement de juin; ses fleurs sont hermaphrodites. Les semences sont petites, rondes el sans ailes; elles sont renfermées dans une gousse à laquelle elles demeurent adhérentes en tombant, ce qui donne plus de prise au vent pour les emporter. Elles mûrissent au mois d'octobre, mais la dissémination n'a lieu que pendant l'hiver ou au printemps suivant.

Le robinier devient fertile fort jeune; ses années de semence sont fréquentes.

180. Jeunes plants. - Les jeunes plants ne craignent point les ardeurs du soleil, mais ils ont hesoin, dans les premières années, d'un alıri contre les froids trop vifs.

181. Feuillage. - Les feuilles du robinier sont composées; leurs folioles sont pelites et légères, et ne donnent que peu de couvert.

18.2. Racines. - Les racines s'enfoncent jusqu'ì 33 el 66 centimetres, lorsque le sol le permel; mais 
elles tracent surtout et s'étendent fort loin. Elles sont nombreuses, munies d'un chevelu très abondant et très disposées à drageonner.

183. Groissance et durée. - La croissance du robinier est des plus rapides. A l'àge de 40 ans, il a souvent une hauteur de 12 et mème de 18 mètres sur 66 centimètres de diamètre à la base. Sa durée ne semble pas pouvoir se prolonger au delà de 80,90 et 100 ans au plus.

184. Qualités et usages. - Le bois de robinier résiste fort longtemps à la pourriture, et cette qualité, jointe à une grande dureté, le rend très propre à lit bàtisse, dinns les plices où les pièces de bois sont le plus exposées aux variations de la température et aux injures de l'atmosphère.

En Amérique, on s'en sert même pour des constructions maritimes, quand on en trouve des échantillons convenables.

Comme bois de traviil, on l'estime beaucoup; il prend un très beau poli, est agréablement nuancé, et sert à la menuiserie et à l'ébénisterie. Les échalas de vigne fournis par le robinier ont plus de durée que ceux d'aucun autre bois. En Angleterre, il est l'essence préférée pour les gournables, parce qu'il se durcit beaucoup en vieillissant.

Sa qualité, comme bois de chauffage, passe généralement pour assez médiocre; cependint, d'après les expériences de T. Harlig, il donne heaucoup de 
chaleur rayonnante, ce qui le rendrait très propre au chauffage à foyer ouvert.

Ses fenilles fournissent un fourrage excellent.

ARTICI.E IX.

Le Charme.

185. Le charme commun (carpinus betulus, LrxxÉ) est un des bois les plus utiles et les plus répandus de nos forêts. Ordinairement, on l'y trouve mélangé avec le chêne, le hêtre, le tilleul, le tremble, etc.; mais, souvent aussi, il occupe des étendues considérables comme essence dominante.

186. Climat, situation, exposition.- Le charme supporte les climats rigoureux, mais il prospère davantage dans les tempérés. Aussi est-ce dans les plaines, sur les coteaux et les montagnes de moyenne hauteur qu'on le rencontre le plus fréquemment et qu'il prend l'accroissement le plus beau. Il s'accommode à peu près de toutes les expositions; néanmoins, sa végétation est peu satisfaisante sur les pentes entièrement méridionales.

187. Terrain. - Les sols argileux, divisés par le sable ou par de petites pierrailles et riches en terrean, sont ceux que le charme préfère; mais il croît aussi dans les fonds de moindre qualité. fussent-ils même un peu humides. Les terrains secs et arides, ou trop compactes, ou marécageux lui sont contraires. 
188. Floraison et fructification. - La floraison est monoïque et en chatons cylindriques pour les deux sexes. Les fleurs paraissent en même temps que les feuilles, au commencement de mai. Le fruit, qui mùrit en octobre et se dissémine immédiatement aprìs, est un petit gland ovale, muni de còtes saillantes et renfermé dans une cupule foliacée. Cette cupule est assez grande pour favoriser la dispersion de la graine par les vents.

Dès l'àge de 30 ans, le charme devient fertile, et la semence réussit abondamment presque chaque année.

189. Jeunes plants. - Les jeunes charmes supportent mieux les froids rigoureux que les gelées printanières, et ils se trouvent bien d'ètre garantis, pendant plusieurs années, des ardeurs du soleil.

190. Feuillage. - Les feuilles sont de moyenne grandeur, d'un tissu assez serré et très abondantes; elles donnent un couvert épais, quoique moindre que celui du hêtre.

191. Racines. - On ne remarque point chez le charme un pivot bien caractérisé; mais ses racines ont toutes une tendance à s'enfoncer obliquement dans le sol, où elles pénètrent souvent jusqu'à un mètre et plus de profondeur; elles sont nombreuses, s'étendent au loin et drageonnent facilement.

192. Croissance et durée. - Sous le rapport de la croissance, le charme égale le hêtre jusqu'à l'àge de 30 à 40 ans; mais, après cette époque, il est d'or- 
dinaire dépassé par ce dernier. Cee n'est toutefois qu'à 70 ou 80 ans que son accroissement commence à se ralentir, et on le voit végéter en très bon état jusqu'à 130 et même $10 ّ 0$ ans.

193. Qualités et usages. - Le bois de charme est d'une densité très égale; il est très dur et sa fibre est coriace. On l'emploie peu à la charpente, parce qu'il résiste mal à l'action de l'humidité et aux variiltions de la température, et que, d'ailleurs, ses dimensions en grosseur et en hauteur sont rarement convenables pour cet usage. Sous le rapport de la grosseur, le charme offre, en effet, cetle particularité, que son trone n'est presque jamais rond comme celui des autres bois; il présente des cannelures nombreuses, irrégulières, souvent très profondes, qui rendent difficile, par conséquent, son équarrissage.

C'est surtout comme bois de travail qu'il est précieux. Il est employé par les charrons; on en fait des roues d'engrenage, des leviers, différents instruments aratoires, et, en général, toutes espèces de pièces exposées à un frottement continu ou à une forte pression. Son chauffage et son charbon sont de première qualité et supérieurs à ceux du hêtre.

Les cendres du charme fournissent beaucoup de potasse, et son feuillage, vert ou sec, sert ì la nourriture des bestiaux. 
ARTICLF: X.

\section{L'Alisier.}

194. Nos forîts présentent communément deux espèces d'alisier, que l'on trouve mélangées parmi les autres bois. Ce sont:

1" L'alisier blanc on allouchier (pyrus aria, EHRHARDT);

2 L'alisier torminal (pyrus torminalis, Ehrh..rot).

19\%. Climat, situation, exposition. - L'alisier torminal et surtout l'alisier blane supportent les climats froids de nos hautes montagnes, mais leur croissance y est lente; la plupart du temps, ils y dégénèrent en arbrisseaux. Ce n'est que dans les régions tempérées qu'ils ont une belle végétation, et tous deux prospèrent principalement dans les plaines et sur les coteaux.

Les expositions entièrement méridionales ralenlissent la croissance des alisiers, qui paraissent se plaire principalement à celles de l'Ouest, de l'Est of du Sud-Est.

196. Terrain. - Les ilisiers réussissent dans toutes sortes de terrains; mais ils semblent surtout préférer les sols calcaires ou argileux, assez profonds et mélangés de terreau. Ils ne supportent ni les sables secs, ni les fonds humides ou marécageux.

197. Floraison et fructification. - Les fleurs 
des alisiers sont hermaphrodites; elles paraissent dans le courant de mai, quelquefois au commencement de juin, selon la température. Les fruits qui leur succèdent sont de petites pommes rouges, presque sèches et non comestibles pour l'alisier blanc, molles et comestibles lorsque, après avoir fermenté, elles deviennent blettes, pour l'alisier torminal. Ils mùrissent au mois d'octobre, et leur dissémination a lieu dans le courant de l'hiver. Dès l'âge de 20 ans, les alisiers deviennent fertiles.

198. Jeunes plants._Les jeunes plants sont assez robustes; un léger abri semble cependant leur être favorable dans les premières années.

199. Feuillage. - Les feuilles sont grandes, épaisses, et donnent un couvert assez complet.

200. Racines. - Les racines des alisiers sont à la fois traçantes et pivotantes, lorsque le sol le permet. Elles s'accommodent cependant d'un terrain sans profondeur, et semblent avoir une disposition particulière à pénétrer dans les fentes des rochers, où elles trouvent souvent une nourriture abondante, produite par le détritus des végétaux qui y est retenu. Elles sont nombreuses et fournissent des drageons.

201. Croissance et durée. - La croissance des alisiers est lente. Leur durée peut se prolonger jusqu'à 200 ans et au delà, mais leur accroissement diminue beaucoup dès l'âge de 90 ou 100 ans. Ils s'élèvent jusqu'à 15 et 20 mètres, et prennent de 40 à 66 centimètres de diamètre à la base. L'alisier blanc 
parait moins disposé à croitre en hauteur que le torminal.

202. Qualités et usages. - Le bois des alisiers est très dur, blanc, d'un grain égal et serré; il prend un beau poli. Parmi les bois d'ouvrage, il figure au premier rang. Il est précieux pour les dents de roues, les écrous, les vis, etc.; on en fait toutes sortes de petits meubles; il sert aux sculpteurs, aux tourneurs, aux fabricants d'instruments de musique, etc.

Son chauffage el son charbon sont fort estimés. Ainsi que nous l'avons dit [197], les fruits de l'alisier torminal, que l'on nomme alises ou aloses, peuvent se manger lorsqu'on les a laissés fermenter; on en distille aussi de l'eau-de-vie, et l'on en fabrique du vinaigre.

ARTICLE XI.

\section{Le Sorbier.}

203. On trouve dans nos forèts deux espèces de sorbier :

Le sorbier desoiseleurs (pyrus aucuparia, G.ertwer);

Le sorbier cormier (pyrus sorbus, G.ertner).

Tous deux n'y existent que mêlés avec les autres essences; le premier est bien plus répandu que le second.

204. Climat, situation, exposition. - Le climat tempéré est celui où ces deux sorbiers prospèrent le mieux; mais on trouve le sorbier des oiseleurs sur 
les plus grandes hatuleurs, où il finil, à la vérilé, par dégénérer en arbrisseau, tandis que l'on voit le cormier rechercher les plaines et les vallées abritées.

Le sorbier des oiseleurs réussit à toules les expositions; mais le cormier préfère celles où le soleil est le moins ardent, et où la terre conserve, par conséquent, plus de fraîcheur.

205. Terrain. - Le sorbier des oiseleurs se contente de toute espèce de terrain, pour'ru qu'il ne soit point humide. Les sols siliceux mèlés de terreau, ainsi que les argiles divisées sont ceux qu'il préfère; le cormier, au contraire, bien plus difficile sous ce rapport que son congénère, se plitit ditvantage dims les sols calcaires et dans les terres fortes. On voit souvent le sorbier des oiseleurs prendre racine et croître dans les fentes des rocher's, el mème sur de vieilles murailles.

206. Floraison et fructification. - Les fleurs des sorbiers sont hermaphrodites; elles paraissent vers la fin de miri ou au commencement de juin. Le fruit du sorbier des oiseleurs est une petite pomme sèche, rouge et non comestible; celui du cormier est de la grosseur d'une petite poire. Lorsqu'il a subi un commencement de fermentation, il devient blet et comestible; on l'appelle sorbe. La maturité de l'un et de l'autre a lieu vers la fin de septembre ou au commencement d'octobre, et la dissémination naturelle s'opère dans le courant de l'hiver.

Le sorbier des oiseleur's devient liertile lurt jeune; 
mais il faut près de 60 ans au cormier pour qu'il porte des fruits en abondance.

207. Jeunes plants. - Les jeunes plants du sorbier des oiseleurs sont robustes dis leur naissance; un premier abri pourrait être utile à ceux du cormier.

208. Feuillage. - Le feuillage des sorbiers est délicat, et ne donne qu'un couvert fort léger.

206. Racines. - Ces deux sorbiers ont un pirot très prononcé qui pénètre jusqu'à 1 mètre 33 centimètres et plus, lorsque le sol le permet. Leurs racines tracantes sont nombreuses, s'étendent au loin et drageonnent facilement.

210. Groissance et durée. - Le sorbier des oiseleurs a une croissance peu rapide. I l’àge de 60 ou 70 ans, il atteint une hauteur de 8 à 10 mètres, sur 33 à 50 centimètres au plus de grosseur. Il vil 100 à 120 ans et au delà.

La végétation du cormier est plus lente encore; mais, vivant plus longtemps, il patrvient à des dimensions plus considérables. Il prend jusqu'à 20 mètres de hauteur sur une grosseur de près d'un mètre à la base. Sa durée varie de 150 à 200 ans.

211. Qualités et usages. - Le bois des sorbiers est dur, pesant, coriace, et prend un beau poli. Il est recherché par les menuisiers, les ébénistes, les tourneur's et les mécaniciens. Ces derniers en fabriquent des dents de roue, des vis, des écrous, des chevilles, etc. Le chauffage et le charbon en sont fort estimés; l'écorce peut servir au tannage. 
Le fruit du sorbier des oiseleurs, ainsi que l'indique son nom, sert d'appàt pour prendre les oiseaux. Celui du cormier, comme nous l'avons dit [206], est bon à manger lorsqu'on le laisse fermenter sur la paille. On en fabrique une espèce de cidre, du vinaigre et de l'eau-de-vie.

\section{AR'TICLE XII.}

\section{Le Micocoulier.}

212. Nous n'avons qu'une seule espèce de micocoulier qui soit indigène. C'est le micocoulier de Provence (celtis australis, Linxé); on l'appelle aussi fabrecoulier, falabriquier et fabréguier. Il appartient principalement (comme l'indique son nom) aux départements méridionaux.

On ne connaît aucun massif formé par le micocoutlier; mais on le cultive sur différents points, et les nombreux avantages que présente cette culture doivent faire désirer qu'elle se propage le plus possible.

213. Glimat, situation, exposition.-Ainsi que nous venons de le dire, le micocoulier appartient plus particulièrement aux climats chauds; malgré cela, il réussit fort bien dans les parties tempérées de la France, lorsqu'on a soin de le garantir, dans sa jeunesse, des froids trop vifs.

Les plaines et les élévations moyennes paraissent convenir également 'au micocoulier, et il prospère à toutes les expositions. 
214. Terrain. - Le micocoulier n'est pas difficile pour le choix des terrains; toutefois, il préfire ceux qui sont profonds, légers et un peu frais. On le trouve, dit-on, en bon état de croissince dinns des sols secs, rocailleux où d'autres essences ne croìtraient qu'avec peine.

21:. Floraison et fructification. - La fluraisun est polygame. Les fleurs paraissent en mai avec les feuilles. Le fruit est une petite drupe ronde, peu charnue, renfermant un noyau ligneux; il est mur ¿u mois de novembre et ne se dissémine ordinatrement qu'au printemps suivant.

L'arbre porte fruit à un àge peu avancé.

216. Jeunes plants. - Les jeunes plants du micucoulier résistent facilement aux ardeurs du soleil: ils ont besoin d'ètre garantis du froid dans le centre. le nord et l'est de la France. Ordinairement, on les recouvre en hiver de feuilles mortes ou de paille. jusqu'it ce qu'ils atient atteint une hatuteur de 66 centtimètres à 1 mètre, après quoi ils deviennent tris robustes.

217. Feuillage. - Les leuilles du micocoulier sont grandes; leur couvert néammoins n'est pas épais.

218. Racines. - Lorsque le sol le permet, le micocoulier pivole assez prolondément; mais il se garnit aussi de nombreuses racines tracintes, alu moyen desquelles il peut se passer de pivot dims les sols peu profonds. Il drageomne, dit-on, asser fréyuemment. 
219. Groissance et durée. - La croissince jdu micocoulier est rapide, et sa durée est de plusieurs siecles. Dims les rlimats convenables, il alleint, it l'ige dr ö0 à 60 ans, une hauteur de 12 à 16 mètres sur

Il existe, dans les environs de. Montpellier, $11 n$ mirocoulier de:3 mitres de circonférence. Celui qui in royait il y a quelques années sur une des places de la ville d'Aix était plus gros encore.

2.20. Qualités et usages. - Le bois du micoroulier est plus dur, plus coriace et plus souple que lit phupart des autres bois; aussi est-il très recherché pour le charromage et pour divers autres arts, tels que la menuiserie, lit mirrqueterie, la sculpture, etc. (1) en fait aussi d'excellents cercles de tonneaux, des fourches, des baguettes de fusil, et surtout des manches de fouets, que l'on comnait dans le commerce sous le nom de bois de Perpignan, el que l'on apprécie beaucoup à cause de leur souplesse.

Comme chauflige, il est fort estimé.

sies feuilles servent à nourrir les moutons of tos chèves.

\section{AR'TICLE XIII.}

\section{Le Cerisier.}

2.21. On troure trois especes de cerisier dins nus forêts :

$1^{\circ}$ Le cerisier merisier ou des bois (cerasus avium, de Candolte); 
- ${ }^{\circ}$ Le cerisior at yrappes (cerasus padus, ne CAxDOLLE) ;

3 Le cerisier mahaleb ou bois de Sainte-Lucie (cerasus mahaleb, Mrtene).

Lat première espece est la plus repandur, yuniqu'elle n'existe jamais conme exsentr dominantr. et. sous le ripport de ses qualités el dr sir croissimere, elle est aussi lat plus importante. Les deux aulpes, yui ne se rencontrent que plus ritrement el presgue loujour's sous forme d'atrbrisseaux, ne sont que d'un intérêt triss secondaire prom le forestier. Nons ne nous occuperons done ici que du merisier.

$2 \cdot 2.2$. Climat, situation, exposition. - Le merisier ne redoute pas les climits un peru rudes, toul en fréférint les tempérés, et on le trouve abondimment, dans les paỵs de montignes, à des situations mème isssez élevées; il prospère égálement en plaine.

Tuutes les expositions lui conviement, yuniqu"il semble se platre particulierement it celles du Midi et de l'Ouest.

2.23. Terrain. - Les terrains légers et subslamliels, quelle que suit d'ailleurs leur milure, conviennent an merisier: mats il vient aussi dans les londs médiocres of salns profondeur. poursu quil puinsey y etendre se's bacines. Les sols humides lui sont entiorement contraires, of il ne prospere pats dians Iess argiles compactes el dians less sibles seces.

2.24. Floraison et fructification. - Les fleurs du merisier sont hermaphrodites: alles paraissent 
à lat fin divril ou au commencement de mai. Le fruit, que l'on nomme cerise des bois ou merise, est une petite drupe charnue et noire qui muirit dims le mois le juin ou de juillet, et qui tombe $u n$ mois ou six semaines après.

Les merisiers portent fruit très jeunes.

-20\%. Jeunes plants. - Le tempérament des jeunes plants est tres robuste. L'ombre leur est absolument contraire.

226. Feuillage. - Quoique les feuilles de cet allore soient grandes, elles ne procurent qu'un couvert Cort léger.

2.27. Racines. - Les ricines sont nombreuses el lrarimtes; elles s'etendent fort loin et drageonnent abondamment.

2.28. Groissance et durée. - Lil croissance du merisier est très rapide, et, à l'àge de lä ans, il i déjà les dimensions d'un chène de ö). Dans une période de S̈0 à 60 ans, il prend de $20 ّ$ à 28 mètres de hauleur sur I mètre à 1 mètre 33 centimitres de grosseur au pipd. Son existence ne se prolonge que jusqu’à 70 ou 80 ans au plus.

229. Qualités et usages. - Le bois du merisier n'est point employé pour les constructions, mais il est d'autant plus recherché, comme bois de travail. par les menuisiers, les ébénistes, les tabletiers el mème les luthiers. Lorsqu'il est jeune, on l'emploir heamcoup a farre des recreles, car il est tris liant. Son 
chauffage et son charbon, sans ìtre de premiìre quitlité, sont cependant assez estimés.

Les merises servent de nourriture aux oiseaux destructeurs d'insectes, et, sous ce rapport, elles sont d'une utilité réelle, quoique indirecte, pour le forestier. On en retire, par la distillation, une liqueur forl appréciée. connue sous le nom de lirsch-u'asser, et qui, en France, se prépare principalement dans les départements des Vosgers, de la Haute-Siline, du Haut et du Bas-Rhin. Lil goomme, qui découle assez. ahondamment des merisiers sur le retour, est employée, dans beaucoup de cas, à l'ígual de la gommon arabique.

I RTICIA XIV.

\section{L'Aune.}

230. On connait dans nos forits deux espeices d'aunes: l'aune commun, aume visqueux, appelé aussi verne ou vergme (alnus glutinosa, (i.frTXerj; et l'anme blanc (alnus incana, Widdexow.) lie dernier est peu répandu.

Les aunes n'admettent point les autres essences en mélange avec eux, et, dans les parties de forèts qu'ils habitent, on les trouve presque toujours maitres exclusifs du terrain. Le frène est peut-être la seute essence qui puisse leur ètre adjointe arec arantage.

231. Climat, situation, exposition. - L'aune est un des arbres les moins difficiles sous Ir rapport du 
climal: Destomtaines dit qu'on le trouve depuis lis Litponir jusque sur les eites septentrionales de l'Llirique. Il pu est à peu prís le mìme quant à la siturlion; aur, si on le rencontre dans les plaines et dims les londs humides qu'il recherche de prélérenee, on lre retrouve cependint aussi dans les parties fort álevées des $A$ pes el des autres régions monlazneuses; la il funil, à la vérité, pall dégénérer en buisson. Ciesl surloul l'aune blane qui habile les régions froides el blevées, oi cependant son congénère le suit souvent d'assez près.

(Juant aux expositions, l’aume parait préférer los moins chatudes.

232. Terrain. - C'est principalement sur le bord de l'eau, dans les terrains humides et mime atquitliques, que l'aune prend un bel accroissement. I] prospire cependant aussi dans d'autres sols, pourvu qu'ils soient substantiels, frais et divisés. Les terres glaises lui sont absolument contraires.

l)ans les marais, dont il s'aceommode sans toutefois les préférer, l'aune est précieux, non seulement parce qu'il utilise des lerrains qui sans lui demenreraient improductifs, matis encore paree qu'il en neutralise les émanations malfaisantes.

233. Floraison et fructification. - I floraison de l'iume est monoïque et amentacén pour les deux sexes; les fleurs paraissent dans le mois de mars,

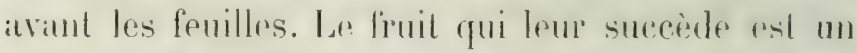

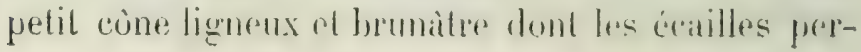


sistent sur l'axe et s'entr'ourrent simplement pour laisser échapper les graines. Celles-ci, bordées d'ume aile peu développée. sont mires en octohre et se disséminent it l'entrée de l'hirer ou au printemps. selon lat lempépiture. Les atumes deviemment fertiles dis l'âge de 12 à 1 ă ans.

234. Jeunes plants. - Les jeunes plants de l'aune n'ont pas besoin d'abri, pourru que le sol dans lecpuel ils lievent ail de la fraicheur; ils se montrent assor\% sensibles aux gelées printanières.

2:3ö. Feuillage. - I a leville de l'aume fost gramelp. el épaisse; mais le courert que son feullage procure est, malgré celir, assez ineomplet. Cette circonstaner. reunie à la fraicheur des terrains dans lesquels vónger l'aune, fail que le sol se couvre tris souvent d'herbes al de plantes diverses el n'est point meuble, comme dims d'autres foreits. Cependant, dans les sols arquitliques, que l'aune orcupe ì l'exelusion de pu'esqur loutes les autres essences, ret envahissement ne se produit pas.

236. Racines. - Les ritcines de l'allute sont nombreuses: elles pirotent peu, mais triternt an loin, surtoul guand le sol est tries humide. Tatume commun drageonue peu, landis que l'aune hlane il cette propriété au plus haut degré.

237. Croissance et durée. - L'ilccroissement de

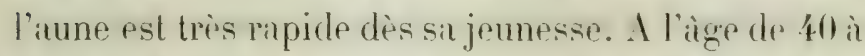
¿0 ans, il atteint somvent jusqu’a 20 ì 2\% mitres de

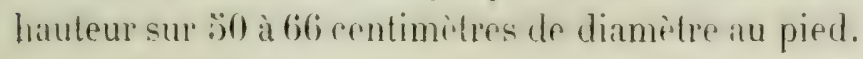


Il aequierl mime des dimensions plus fortes, car i! rit, en bon état de croissance, 80 à 90 ans .

2:38. Qualités et usages. - L'aune n'est point propre ì la charpente en plein air, ni même à courert, parer qu'il sp pique facilement; mais, employé sous raur, il est de lit plus grande durée. Iussi le recherrhe-t-on surbout pour les constructions hydriuliques. telles que corps de fontaine et de pompe. pilotis, digues, galeries dans les mines, etc.

Comme hois de travail, il est égrilement fort estimé des tourneurs, des menuisiers et des íbénistes. Il it le grain fin, égal, est agréablement reiné et prencl un beam poli. On dit que l'aune blancest préférahte, pour ces divers usagés, à l'aune commun.

Le? chauffage et le charbon de l'aune ne sont pas de première qualité; cependant il gagne considérithlement, sous ce rapport, lor'squ'nn a soin de le mettre. en lieu see, aussitot après l'abatage. Traité ainsi, il brùle d'une tlamme claire et égale, et les boulangers Ir préferent à tout autre bois pour chauffer le four. Les expériences comparatives faites sur la valeur ralorifique des deux aunes fitahlissent encore lis supériorité de l'iume blane. Celte circonstanee, jointe à la précédente, doit encourager sa culture.

Les cendres de l'aune fournissent beaucoup de potasse. Son écorce peut servir utilement au tannage: on en obtient aussi une couleur, brune ou noire, qui est employée ì teindre les cuirs et les feutres. Ifes bêtes it eornes mangent les feutlles d'uune, mais 
DES SOLS', DES ESSENCES.

s'en soucient peu; les bites i laime les refusent absolument.

\section{A IRTCI, F XV.}

\section{Le Tilleul.}

239. On connait en France deux esperes de filleuls indigènes :

Le tilleul it petites fenilles, tilleul sanvage on des bois (tilia microphylla, V Exтxat);

Le tilleul in larges fenilles ou de IInllande tilia plinlyphylla, Scopoli).

C'est le premier que l'on reneontere d'ordinaire lans les forrets, mile aux autres bois: le seronds'y trouve aussi, mais rarement.

240. Climat, situation, exposition. - Les lilleuls prospirent à peu pris dans loutr esprier de climal: on les lroure dans les plinines comme sur les monlagnes blevées. Le tilleul simvage pot celui qui supporte le mieux les climats rigoureux et les grandes himteurs, quoique sa croissance ot ses dimensions y soient bien moindres que dans les réggions tempérées.

Les expositions du Nord-Ouest el du Nord paraissent ètre celles que préfèrent les tilleuls; mais on les trouve aussien assez bon état de croissance aux autres aspects du soleil.

241. Terrain. - Les tilleuls se plaisent principaIrment dans un sol sablonneux, profond et frais; on les voit aussi prospérer diuns les terrains argileux. 
pourvu qüils soient sutfisanment rivisés; les glatises ot les marais leur sont contraires. Le tilleul des bois se contente quelquefois des terrains Jes plus ingrats. lels que les rocailles et les sables quartzeux.

24. Floraison et fructification. - Les fleurs dı lilleul sont hermaphrodiles. Celles du lilleul a larges feuilles paraissent rers lit fin de juin ou au commencement de juillet, et celles du tilleul des bois, quinz" jours plus tard.

Le fruit, qui est une petile noix globuleuse et velue. penfermant ordinairement une of quelquefois deux graines, mùit en octobre et se dissémine à l'entrés. de l'hiver.

Les tilleuls portent semence itbondamment et it un àge peu avancé.

243. Jeunes plants. - In premier abri parail favorable aux jeunes plants, qui redoulent les chileurs trop vires; ils sont, du reste. d'un tempériment robuste.

244. Feuillage. - Le feuillage des tilleuls est abondint, touffu, cl procure un couvert épais.

24:). Racines. - Ciel artore a un pivol tris prononcé qui semfonce jusqu'a I mistre el I molm ̈̈) centimilres. Les racines trateintes soml tres nonthreuses, ot setemdent à des distances tris ronsidérables; plles drageonnent assez fréquemment.

246. Groissance et durée. - Le tilleul croît avec rappidití dans sa jemmesse; à l'àge de 80 à 100 ans, il alteint une hauleur de 20 ì 30 mitres sur 66 centi- 
mètres à 1 mitre de diamètre à la base. Passé crl àge, il conlinue à croitre en grosseur. mais il commence ordinairement à se creuser dans le centre. Cette circonstance ne l'empèche pas cependant de végéter avec rigueur et de parrenir à un àge lrè avancé. Le tilleul est un des bois qui fournissent les r.semples les plus remarquables de longévité. On (emmait, Lant en Franee qu'en . Illemagne, un assez gramel nombre de ces arbres dont on porte l'àge ì 200, 300 el mème jusqu’à ö00 ans. Plusieurs d'entre eux présentent jusquà 10, 12 et 13 melpes de circonférence à la base. Tous res auberes sont de

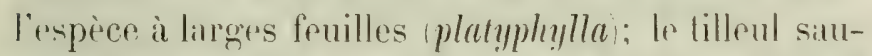
vage croil un peu moins vite ot ne dure pas alu dela de 200 à 300 ans au plus.

24.7. Qualités et usages. - Le tilleul n'est poinl propre à la charpente, mais il est tris estimr pour lit menuiserie, l'úbénisterie et mèmr pour la sculpturer. Son bois est tendre, tries blane, d'm grain égit el lin; il ne se geree et ne se fourmente point, ol nos pas sujet à la vermoulure.

Le chauffige du lilleul est peu apprécié; som

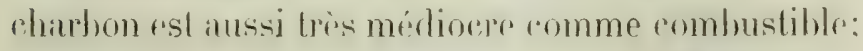
mais on s'en sert aver avantage pour la liabricalion de la poudre à tirer.

L'écorce (le liber) est employée à lis fabrication de cordes qui sont trise estimées it ratuse de leme fores el de leur souplesse. 
Les leuilles, vertesou séchées. peuvenl servirave avantage à la nourriture des bêtes à laine.

\section{AR'TICI, F XVI.}

\section{Le Peuplier.}

20.8. Le genre des perpliers est fort nombreux el mérite loute l'attention du planteur; mais une seule espece eroil spontimément dims nos bois: c'est le peupliertremble populus tremula, Irxwés. C'est done de lui seul que nous nous occuperons ici 1 .

29. Glimat, situation, exposition. - I tremble préfère les climals tempérés: il résiste cependint aussi dans les régrions froides el élevées, mais il y dégénere en arbrisseau. Les climats chauds lui paraissent contraires. Ainsi l'on remarque que, par delì la Loire, en se dirigeant vers le Midi, il devienl de plus en plus rare, et qu’il disparait mème tout ì

1 Les autres espèces de peupliers sont:

10 Le peuplier blane, appelé aussi ypréau, blano de Hollunde (populus alba, LinNé).

20 Lc peuplier grisaille (populus canescens, Sмттн).

$3^{\circ}$ Le peuplier noir (populus nigra, LiNnÉ).

Ces trois espèces cruissent spontanement dins ies îles el sur les borts du Rhin; mais, a part cette rigion, on ne les retromve guère en France que cultivées.

$4^{\circ}$ Le peuplier dlItalie ou pyramidal (populus fastigiata, PoIret).

Cette espèce, flont on connaît maintenant les pieds fomrlles, est originaire r'Orient. 
fait dans les départements les plus chauds de lia France. Les expositions du Norl el de l'Est lui conviennent particulierement. mais il prospere atussi aux autres aspects.

2ə̈0. Terrain. - Les sols légers, frais el mèmé humides sont ceux vì le tremble prend te plus bel accroissement; on le rencontre dins les terres fortes comme dans les sables purs, mais il y reste chétil. Les marais lui sont contraires.

2.ว I. Floraison et fructification. - Lil floraison du peuplier tremble est diö̈que et en chatons cylindriques pour les deux sexes; les fleurs paraissenl aviunt les fenilles, dès le mois de mars. Les fruits réussissent arec une extrème abondance charpue amnée; ce sont des capsules qui contiemnent une gramde quantité de graines, pour ansins dire microseopiques, munies d'une aigrette soyeuse, ut sourent tramsportées par les rents ì plusieurs lieues.

L'arbre devient fertile rers l'ìge de 20 it 2030 ans.

20̈2. Jeunes plants. - Les jeunes plants sont tris robustes et résistent, dés leur maissance, a loutes los influences de l'atmosphire. Ils restent tris petits lir première année; mais, à la secomle, ils prennent $\mathbf{m}$ accroissement plus rapide que celui de la plupart des autres bois. Cette circonstance, jointe ì l'alondanm.. et à la facilité avec lesquelles sa graine se répand :au loin, fitit que le tremble s'introduit dans toutes les forêls, ne litrde pas ì sy mulliplier el à régéler aux 
dépens des essences plus précieuses, si des exploilittions bien entendues n'y mettent obstacle.

2.33. Feuillage. - Le feuillige du tremble est tris léger, mobile el peu abondant; il ne donne, parr conséquent, qu'un couvert très incomplet.

254. Racines. - Le tremble est enlièrement tritrant. Ses racines sont fort nombreuses, s'étendent Iris loin el produisent des drageons en abondance.

23\%. Croissance et durée. - Linsi qu'on vient Ir Ir dire, la croissance du tremble est des plus rapides. A l'àge de 30 i a 60 anls, il aequiert une hauleur de 20 ì 30 mètres sur 66 centimitres, et mème plus, de diamètre atu pied. P'assé cel ìge, il se yourrit ordinairement à l’intérieur; cetle circonstancer at mème lieu plus tôt, lorsqu'il se trouve dans un sol humide ou très substantiel.

2006 . Qualités et usages. - Le hois du tremble est très tendre, blạme et chargé d'humidité, ce qui livil qu'il prend beancoup de retrait. Dans les parys où le sippin manque, on emploie le tremble à la char'pente en lieu sec. Il peut servir aussi, comme l'illun', it des conduites d'eau, parce gu'il résiste très longlemps à lit pourriture, surtoul dans les termains humides.

Comme bois de tratrail, il est employé à la menuiserie, à lit sculpture, à l'ébénisterie, elc. L'arbre se gaillnissant peu de branches el n'étint, par conséquent, pas noueux, on en libbrique beatcoup do roliges, ou plinches tries minces, dont on se sert 
pour l'intérieur des meubles et surtout pour les caisses d'emballage.

Son chauffage est de mauvaise qualité, mais on l'imploie volontiers pour chanfer le four, parre qu' il In'ule promptement et arec une flamme tris vire; son charbon est recherché pour lit fabrication de la poudere ì lirer.

Lécorce peut servir au lamnage, el les fenilles. virtes ou sèches, peurent s'employer comme fourlage prour les bètes à laine el mème pour les chevaux.

\section{ARTICIE XVII.}

\section{Le Saule.}

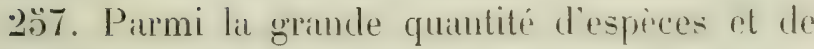
vitriétés qui composent le genre des saules, un assez friand nombre croissent spontanément ditns nos loprits. Deux espréres, cependint, méritent seules l'atIrntion du forestier, lant à callse de l'abondances ines lirpuelle elles sont répindues et de leurs stamdes dimensions, que parr rapport aux avantiegess que présente leur culture. Ce sont:

Le saule marcean (salix capræa, Lisxé);

Le saule blane (salix alba, Linvé).

Lne variété de ce dernier, it rameaux d'un jaum oringé vif au printemps, est comnue sous le nom de saule osier jaune (salix vitellina, Lixxé). Nous l'erons néanmoins remarquer que celte dénomination d'osier 
ne lui est pis spéciale el s’applirgue à tous les siunles dont les jeunes pousses flexibles sont 'muloyées par' les vanniers.

Parmi les autres espèces de saules que l'on trouve le plus communément, el qui servent à mintenir el à lixer les terres sur le bord des rivières el les ruisseaux, nous nous contenterons de nommer les suivantes:

$1^{\circ}$ Saule cendré (salix cinerea, Linvé);

2 Saule à oreillettes (salix aurita, Lixwé), particulierement commun dans les lieux marécageux et tourbeux des forêts;

3" Saule viminal salix viminalis, Lixiś, connu aussi sous le nom de saule flexible, de saule à longues fenilles;

$4^{\circ}$ Saule pourpre ou ü une étamine salix purpurea, salix monandra, dE CANdolle);

o" Saule a trois élamines ou à feuilles d'amandier. (salix triandra vel amygdalina, Linxé);

$6^{\circ}$ Saule fragile (salix fragilis, Linné).

2): Climat, situation, exposition. - Le situle mirceau prospère dims lous les climats de l'Europe. sur les montagnes les plus élerées comme dans les vallons, el a toutes les expositions; il est peut-itre, sous ce rapport, l'essence la plus remarrpuable gue nous possédions. Je siule blanc réussit aussi dans toutes les parties de la France, mais seulement dans les platines of les valloms, ol a des élévations movemes. 
259. Terrain. - Presque tous les sols conviennent au saule marceau; cependant, il se plait de préférence dans un sable gras un peu frais. C'est aussi dans un pareil sol que prospère son congénère, qui, d'ailleurs, se trouve fréquemment sur le bord des rivières et des ruisseaux, dans les prairies, el, en général, dans les lieux frais ou humides; toutefois les marais, comme les terres trop compactes, sont contraires à l'un et à l'autre.

260. Floraison et fructification. - Les saules sont dioïques. Le marceau fleurit avant l'apparition des feuilles; le saule blanc fleurit en arril, en mème temps que les feuilles poussent. Les fruits, semblables à ceux des peupliers, sont des cinsules qui contiennent des semences munies d'une aigrette. soyeuse; les vents les transportent à des distances considérables. La semence du marceau mùrit et se dissémine en mai, et celle du saule blanc à la fin de juin ou au commencement de juillet. Les stules, it peine âgés de quelques années, sont déjà fertiles, el les graines réussissent très abondamment chaque année.

261. Jeunes plants. - Ces arbres sont très robustes dès leur naissance. Comme le tremble, le marceau se répand dans toutes les forêts et y croil au détriment des autres essences, si les exploitations n'y portent remède.

262. Feuillage. - Le feuillage des saules est léger et ne donne qu'un faible couvert. 
263. Racines. - Les racines sont traçantes, nombreuses et drageonnent beaucoup.

264. Groissance et durée. - La croissance des saules est des plus rapides. Le marceau atteint, en 40 it 50 ans, une hauteur de 12 à 15 mètres sur 33 centimètres et plus de diamètre à la bise. Le saule blane prend, dans le même espace de temps, 20 ì 28 mètres de haut sur 40 à 50 centimètres de diamètre au pied. Ces arbres ne se conservent guère, en bon état de croissance, que 50 à 60 ans; passé cet àge, et sourent même plus tôt, leur trone se creuse, bien que les branches et la cime continuent à végéter avec la même activité.

26\%. Qualités et usages. - Le bois des saules est spongieux, blance el très chargé d'eau; il n'est point propre à la charpente. Comme bois de trarail, on le débite en voliges, et on en confectionne toutes sortes d'ourrages de fente; mais c'est pour la vannerie que ces arbres sont le plus précieux. On fabrique aussi des paisseaux de vigne arec le marceau, et l'on dit que, coupés en temps de sève, écorcés tout de suite et conservés à l'abri pendant un an, ils deviennent susceptibles d'une longue durée.

Le chauffige et le chimbon des saules sont de firible qualité. Le marcean est toulefois plus estimé a cet égard que le blime. On s'en sert pour chauffer le four, parce qu'il brùle avec une flamme assez vive; son charhon, qui est fort léger, est d'aillerirs employé dims la fiabrication de la poudre à canon. 
L'écorce des saules est propre au tannage des cuirs fins; leurs cendres fournissent beaucoup de politsse. et les feuilles peuvent servir de fourrage aux bètes ì laine.

\section{ARTICLE XVIII.}

\section{Le Platane.}

266. Le platane d'Occilent (platanus Occidentalis, Linné) est un arbre de première grandeur, originaire de l'Amérique du Nord et parfiitement acclimaté en France. On ne le rencontre pas en forêt, mais il mériterait d'y prendre place, à cause de ses dimensions, de la rapidité de sa croissance et de l'utilité de son bois.

267. Climat, situation, exposition. - Jusqu'it présent cet arbre n'il été planté que dans les promenades publiques, sur les routes et les canaux, dims les parcs et les jardins. Il y a lieu de croire que les régions montagneuses proprement dites ne lui conviennent pas, et qu'il se plait surtout dans les plaines ou dans les lieux abrités.

268. Terrain. - Les sols légers, frais et mème un peu humides semblent lui ìtre nécessaires. Le nom vulgaire qu'on lui donne dans l'Amérique septentrionale (waler-beech, hètre aquatique) indique que, dans ce pays, on le rencontre surtout dans les terrains mouilleux.

269. Floraison et fructification. - La floraison 
est monoïque. Les fleurs, en châtons globuleux, paraissent à la fin d'avril ou au commencement de mai. La graine, très petite et munie d'une aigrette soyeuse, mùrit et se dissémine l'automne suivant. Le platane porte fruit très jeune et presque tous les ans; mais ses graines, surtout quand elles proviennent de pieds ayant moins de 30 ia 40 ans, sont souvent vaines.

270. Jeunes plants. - Les jeunes plants paraissent réclamer un premier abri; ils lèvent et croissent rapidement.

271. Feuillage. - Les feuilles du platane, semblables, quant à la forme, à celles de nos grands érables, sont grandes, nombreuses et disposées sur les rameaux de manière à fournir un couvert aussi épais que celui du hêtre.

272 . Racines. - Les racines sont longues, fortes et traçantes.

273. Groissance et durée. - Sous le rapport de la rapidité de croissance, on a comparé le platane au peuplier noir. Il atteint les plus fortes dimensions, et vil, d'iprès ce que l'on assure, deux siècles et même au delà.

274. Qualités et usages. - Le bois du platane a beaucoup d'analogie avec celui du hètre, qu'il égale presque comme chauffage et qu'il surpasse comme bois de menuiserie et d'ébénisterie. Il est abondamment et largement maillé, agréablement nuincé et prend un beau poli. M. T. Hartig rapporte que des 
DFS SOLS, DES ESSENCES.

pièces de platane, ayant été enterrées, ont résisté pendant 14 années à la pourriture, à l'égal du chène et de l'orme, tandis que des pièces semblables en hêtre, placées dans des conditions identiques, étaient entièrement pourries au bout de 5 ans. 


\section{CHAPTTE GINOUEME.}

\section{DES BOIS RÉSINEUX.}

ARTICLE PREMIER.

\section{Le Sapin.}

273̈. Le sapin commun ahies pectinala, de CaxDOLLE) est connu aussi sous le nom de sapin aryente. blanc, ì fenilles d'if, sapin des Vosyes, de Normandie.

Il compose, à lui seul, des forits d'une grande étendue, quoiqu'on le trouve souvent mélangé avec d'autres essences, notamment aree le hitre el l'épicéa.

276. Climat, situation, exposition. - Le sapin habite les climats froids of los tempérés; il se plait davantage dans ceux-ci, et y arquirert de plus fortes dimensions. Quniqu'il ne rraigne pas les frimas, on ne le roit pas a d'aussi grandes hauteurs que l'épicéa et le mélize. En général, les montagnes lui conviennent; on le rencontre dans les Tosges, le Jura, les Alpes, les Pyrénées, les Cévennes, ot dins quelques autres parties montignneuses de la Frince. C'est 
entre $ّ 00$ et 1,000 mètres au-dessus du niveau de la mer que sa principale zone de régétation semble ìtre comprise; mais on le trouve encore à de plus grandes élévations. Dans les Pyrénées, il croit dans les régions éleries de 1,500 à 2,000 mètres; dans les Alpes, dans le Jura et dans l'Auvergne, il s'arrête à 1,0000 mètres, et dans les Vosges à 1,000 mitres.

L'exposition méridionale lui est contritre, et sil régétation y est languissinte. r'est aux exposilions du Nord et de l'Est qu'il réussit le mieux.

277. Terrain. - Le silpin demande un terrain un peu profond, frais el ficile à pénétrer. Du reste, il s'ircommode assez volontiers de toute espice de sol, sauf les terrains marécageux ou arpuatiques, dans lesquels il ne peut virre, et les sables trop légers, oi son accroissement est tris faible. Sourent on le roit en très bon état de croissance dians des terrains entièrement couverts de roches. Ses racines, ditns ce cals, s'introduisent dans les tissures et les intervalles gue présentent ces roches, of profitent de la fraicheur et du terreau qui s'y amasse abondamment.

278. Floraison et fructification. - Lit floraison est monoïque. Les fleurs paraissent fin d'avril et en mai, et sont placées vers la cime de l'arbre. Le fruit est un còne ou strobile dont la maturité a lieu au commencement d'octobre de l'année mème de la floraison. Les semences sont munies d'une aile latérale et se disséminent immédiatement apris la maturité. Cette dissémination a cela de particulier que, les 
cônes étant redressés, les écailles se détachent et tombent avec les graines, tandis que les strobiles des autres bois résineux indigènes ne font que s'entr'ouvrir pour laisser échapper la semence.

Le sapin devient complètement fertile vers l'àge de 60 à 70 ins, selon les climats, et sa semence réusșit communément tous les deux ou trois ans.

279. Jeunes plants. - Le sapin est très délicat dans sa première jeunesse, plus mème que le hêtre. Il demande un abri prolongé, surtout contre les chaleurs, auxquelles il ne peut résister.

280. Feuillage. - Les feuilles du sapin sont courtes et étroites, mais épaisses et extrèmement nombreuses, ce qui fait qu'elles donnent dans leur ensemble un couvert très complet; elles persistent ordinairement pendant trois ans sur l'arbre.

281. Racines. - Le sapin est un des bois résineux le plus fortement enracinés; ses racines latérales, ainsi que son pivot, s'enfoncent jusqu'à 1 mètre et plus, lorsque le sol le permet; mais, comme nous l'avons dit plus haut, elles s'accommodent iussi d'un terrain rocheux et s'y fixent avec vigueur.

Il arrive fréquemment que les racines d'arbres voisins s'entrelacent et s'anastomosent de telle façon que leur végétation devient solidaire. C'est à ce phénomène qu'est due la continuité de croissance de souches dont les troncs sont coupés depuis longues années. On voit alors cess souches former des couches 
annuelles de bois aussi longtemps que l'arbre auquel elles sont mariées reste sur pied.

282. Croissance et durée. - Cet arbre croît d'abord très lentement; mais, quand il a acquis un peu deforce, il s'élance avec rapidité, et parvient souvent à une hauteur de 40 à 4.5 mètres. On a coupé des sapins qui avaient atteint l'àge de 300 ans sans dépérir, et qui présentaient 6 à 9 mètres de tour à la base.

283. Qualités et usages. - Le sapin n'est pas employéà la màture, du moins dans la marine nationale '. Son bois n'a pas, comme les différentes espèces de pin, des canaux résinifères au moyen desquels le tronc se -trouve pénétré, dans toutes ses parties, d'une résine concrète qui lui donne la flexibilité et la durée nécessaires; mais il sert, du reste, dans la construction des vaisseaux, à tous les ouvrages de menuiserie qui garnissent l'intérieur du navire.

Dans les constructions civiles, cet arbre est très apprécié comme charpente, et, placé en travers, il

1 La marine du commerce se sert fréquemment, pour mature, de sapins provenant du Jura et des Alpes, et, en 1846, une commission, composée d'ingénicurs et de forestiers, a constaté, par des expériences cxécutées sous ses yeux, que les sapins tirés des forêts du département de l'Aude (Montagne-Noire) avaient plus de force, de résistance et de ténacité que les pins du Nord et du Canarla, le mélèze, lépicéa et le sapin de 'Trieste. Il serait à désirer que la pratique pût confirmer un aussì précicux résultat. 
résiste mieux et se tourmente moins que le chêne. Il est à observer que, pour cet usage ainsi que pour d'autres, contrairement à ce qui a lieu pour le chêne [76 bis], le bois de sapin (comme celui de tous les résineux, en général) est d'autant meilleur que ses couches annuelles sont plus étroites, son grain plus fin et plus serré.

On débite les sapins surtout en planches, dont on fait un commerce considérable. Ceux des Vosges el du Jura se transportent, les premiers à Paris et dans l'intérieur de la France, les autres dans le Midi et jusqu’à la Méditerranée.

Le sapin sert aux menuisiers et aux ébénistes; ceux-ci en font principalement la carcasse des meubles en marqueterie et en placage. Il est façonné en douves pour la tonnellerie, en cerches pour la boissellerie, et en planchettes minces ou bardeaux pour la couverture des maisons. Différentes pièces de charronnage se confectionnent aussi en sapin.

A l'exception de l'écorce, qui fournit un excellent combustible, son chauffage est d'une qualité médiocre, et, sous ce rapport, il est au hètre comme 11 est à $1 \%$. Son charbon, quoique inférieur aussi à celui du hètre, est cependant emploýé avec arantage à forger le fer.

Le sapin fournit une résine très liquide, dont on fabrique la térébenthine dite de Strasbourg. Elle se trouve dans les connes verts ainsi que dans la semence, mais plus particulièrement sous l'écorce vive des 
jeunes sujets, où elle existe dans de petites tumeurs ou vésicules. On la recueille avec des cornets en fer-blanc qui, au bord de la partie évasée, sont munis d'un petit bec terminé en pointe aiguë. La pointe sert à crever la tumeur, et la goutte de résine qui en sort coule jusqu'au fond du cornet. On obtient de cette térébenthine, par distillation, une huile essentielle employée dans la médecine et dians les arts. et surtout dans la composition des vernis.

Le résidu de la térébenthine distillée domne la colophane.

Le résinage du sapin ne cause aucun préjudice à l'arbre, mais il est peu productif.

Dams ruelques parties des Vosges, les habitants se servent, pour leur éclairage, de lit résine qu'ils expriment des semences du sapin.

Le sapin fournit encore le produit appelé sulim. On peut sans doute le retirer de tous les autres bois, puisqu'il n'est dù qu’aux cendres lessivées des végétaux, malis il en coute moins pour l'obtenir du sapin, un des bois qui en fournit le plus. Ordinairement on cmploie à cet effet lir sciure qui se trouve abondamment dans les scieries où le sapin se débite en planches. Dans les losges, on a l'habitude de joindre à cette sciure, en la brillint, de l'urine de hestiaux; il en résulte, à la vérité, une plus grande quantité de salin, mais il est d'une moindre qualité.

La calcination du salin produit la potasse du commerce, employée à de nombreux usages diuns les arts. 
ARTICLE II.

\section{L'Épioé\&.}

284. L'épicéa commun (picea excelsa, Link; abies picea, Mirler; abies excelsa, de Candolle) est aussi connu sous les noms de sapin épicéa, pesse, fie, sapin gentil, sapin rouge, fuve; il forme, tantôt seul, tantôt avec le sapin et le hêtre, des forêts considérables.

285. Climat, situation, exposition. - L'épicéa supporte les frimas mieux que le sapin, et réussit à de plus grandes hauteurs de montagne. On le rencontre ordinairement entre 800 et 1,800 mètres d'élévation au-dessus du niveau de la mer, et, dans les Alpes, il se trouve mème à 2,000 mètres et plus.

Quoiqu'il préfère les expositions du Nord et de l'Est, les expositions plus chaudes lui sont cependant moins contraires qu'au sapin.

286. Terrain. - Le sol propre au sapin est aussi celui qui convient à l'épicéa; mais, comme il est principalement nourri par les racines latérales, il se contente de peu de fond. Il vient mieux que le sapin dans les sols un peu humides, et s'accommode même des terrains tourbeux.

287. Floraison et fructification. - La floraison est monoïque; les fleurs paraissent en mai. Les cônes qui leur succèdent sont mûrs à la fin de l'automne qui suit lia floraison, mais la graine, sauf 
dans les années très chaudes, ne se dissémine qu'au printemps suivant. Les premières chaleurs de cette saison agissant sur les cònes, les écailles s'entr'ouvrent sans se détacher de leur axe et laissent échapper la semence qui, plus petite que celle du sapin, est aussi garnie d'une aile.

Dans quelques localités plus chaudes que celles que l'épicéa habite ordinairement, dans certaines parties des Alpes françaises par exemple, il parait que la dissémination a généralement lieu au mois d'octobre.

Vers l'àge de 50 à 60 ans, l'épicéa devient complètement fertile; il porte semence à peu près tous les deux ans.

288. Jeunes plants. - Les jeunes plants de l'épicéa sont plus robustes que ceux du sapin. Il est possible de les élever sans abri aux expositions du Nord et du Nord-Est; mais ils ne résisteraient pas de même sur les pentes méridionales, à moins que la situation ne fût très élevée [40].

289. Feuillage. - Les feuilles de l'épicéa sont plus courtes et plus étroites que celles du sapin, et, comme celles-ci, extrèmement serrées sur les rameaux, où elles persistent de 3 à š et mème, dit-on, jusqu'à 7 ans. Aussi le couvert fourni par cet arbre est-il très épais.

290. Racines. - L'épicéa n'a que très peu de pivot, et, souvent même, il en est entièrement dépourvu. 
Ses racines latérales sont plus déliees que celles du sapin; elles tracent entièrement ì la surface du sol et semblent, comme ces dernieres, aroir une disposition particulière à s'introduire dans les fissures des rochers.

291. Croissance et durée. - L'épicéa a une végétation lente dans les premieres années; mais il ne tirde pas à s'élancer, ef, dìs lors, il devance le sapin par les progrès de son accroissement. Il vit autant que cel arbre et acquiert les mimes dimensions.

292. Qualités et usages. - L'emploi de l'épicéir est généralement le mème, a le mème degré d'ulilité, que celui du sapin, tant pour les constructions que pour le trarail. Il sert en outre aux luthiers pour les tables de différents instruments de musique.

Son chauffage et son charbon sont un peu meilleurs que ceux du sapin. Dans les pays lu Nord, l'écorce s'emploie au tannage.

On obtient aussi de l'épicéa la poix jaume ou poix de Bourgogne; lia résine qui lia fournit circule principalement entre l'écorce el le bois. On fait, au printemps, des incisions à l'écorce jusqu'iux premieres couches ligneuses, ce qui procure l'écoulement du suc résineux pendant l'été, et, lorsque ce suc est coagulé, on l'enlève arec des racloirs ou limes de fer recourbées, et l'on rafraichit ainsi lit plaie. Ces incisions ne peuvent être que trìs préjudiciables anx épicéas; dans tous les cirs, elles ralentissent la végétation de l'arbre, diminuent li qualité de son bois, 
et, quand elles sont faites sans règles et sans précautions, elles occasionnent son dépérissement.

La résine obtenue, on la met, pour la faire fondre et l'épurer, dans de grandes chaudières sur un feu de flamme. Une fois liquéfiée, on la verse dans un sac de toile, puis on l'exprime au moyen de la presse; elle est ensuite reçue dans des boites ou barils pour être livrée au commerce. On peut aussi, par la distillation, obtenir la térébenthine de cette résine.

Les résidus qui sortent de la presse, ou qu'on trouve au fond des chaudières, sont conservés pour faire du noir de fumée.

ARTICLE III.

\section{Le Pin sylvestre.}

293. Le pin syluestre (pinus sy̧lvestris, Lrnvé) est connu sous une foule de dénominations diverses: pin sanvage, pin du Nord, de Riga, de IIagnenau, de Genive, Pinasse, ete. Le pin rouge ou pin d'Ecosse n'en est qu'une variété, reconnaissable à ses feuilles plus courtes, à ses cònes plus petits et réunis en verticilles, et à ses jeunes pousses rougeàtres. Le pin sylvestre forme l'essence dominante dans un grand nombre de forêts très considérables, où il se trouve fréquemment mélangé avec le chène et le bouleau.

294. Climat, situation, exposition. - Les climats tempérés sont ceux où le pin sylvestre a la végétation 
la plus active. Les paỵs froids ne lui sont cependant pas contraires, car, dans le Nord de l'Europe, en Russie surtout et en Suede, il acquiert les qualités et les dimensions les plus précieuses, el couvre, à lui seul, de vastes étendues de pays. Il habite la plaine aussi volontiers que les sols en pente; mais les grandes élérations ne lui conviennent pas. Dans ces dernières localités, la neige et le givre s'attachent en grandes masses à ses feuilles, plus qu'à celles des autres résineux des climats froids, et font ainsi rompre ses branches et, souvent mème, sa cime entière.

Le pin sylvestre réussit à toutes les expositions, et même en plein Midi. Lorsqu'il s'agit de repeupler des vides ou des parties de forêts dégradées, exposées au Midi, il est d'une grande ressource, non seulement parce qu'il se contente d'un sol maigre et sec, mais encore parce que les jeunes plants de cette essence supportent mieux que ceux des autres bois résineux les ardeurs du soleil. Nous ne parlons ici que des parties tempérées de la France; les départements du Midi ont des pins qui leur sont propres, tels que le pin maritime et le pin d'Alep; et il est probable que le sylvestre y riendrait mal, à moins qu'on ne l'y placàt à des élévations où le climat seriit moins brûlant 1.

295. Terrain. - Le pin sylvestre demande un sol profond et léger; il vient même dans des sables

' Dans les Pyrénées, on le trouve à une altitude de 1,200 mètres. 
entièrement dépourvus de liaison, et son bois y est de meilleure qualité que dans les terrains plus substantiels. Les terrains compactes lui sont entièrement contraires, et, quoiqu'il réussisse dins les calcaires, sa croissance y est cependiant beaucoup moins belle que dans les terres siliceuses.

On le troure quelquefois dans les parties humides et tourbeuses, mais sa végétation y est languissinte et il s'y présente ordinairement sous un aspect si particulier, qu'il a été pris pour une espèce différente.

\section{Floraison et fructification. - La floraison} est monö̈que; les fleurs pariissent en arril ou en mai, suivant la température.

Le strobile reste très petit la première année; au printemps suivant, il commence à grossir, et parvient à son entier développement rers la fin de l'été. Il est mûr au commencement de novembre; mais il n'entr'ouvre ses écailles pour laisser échipper la graine qu'au printemps suivant. Il lui faut done au moins dix-huit mois pour mùrir, et il fiut environ deux ans pour que la graine se dissémine; toutefois, ce qui a été dit de la dissémination de l'épicéa dans les Alpes françaises [287] s'applique aussi au pin sylvestre croissant dans les mêmes localités.

Le pin sylvestre devient complètement fertile vers la $40^{\circ}$ année. Les fruits réussissent à peu près tous les deux ou trois ans; les semences sont ailées et petites comme celles de l'épicéa. 
297. Jeunes plants. - Les pins sylvestres sont très robustes dès leur naissance, et ne prospèrent pas sous un couvert prolongé. On peut, en général, les élever sans abri; cependant, sur un terrain très sec et à une exposition entierement méridionale, il serait utile qu'ils fussent ombragés la première année.

298. Feuillage. - Les feuilles du pin sylvestre sont plus longues que celles des deux essences précédentes; mais, comme elles persistent rarement sur les rameaux plus de trois ans, il en résulte que l'arbre ne donne qu'un couvert assez léger.

299. Racines. - Les racines sont fortes et disposées à s'enfoncer. Lorsque le sol le permet, le pivot pénètre jusqu'à un mètre et plus, quoiqu'une profondeur moindre puisse suffire pour assurer ì l'arbre une assez belle végétation. Dans les terrains humides, pauvres, ou dans ceux qui manquent de fond, le pivol disparait à peu près totalement et les racines latérales prennent une direction semblable à celle des racines de l'épicéa; mais la croissance de l'arbre s'en ressent défavorablement.

300. Groissance et durée. - La végétation du pin sylvestre est très rapide dès les premières années; lorsque le sol lui convient, il s'allonge, dans sa jeunesse, quelquefois d'un mètre et au delà par an. Il vil jusqu'ì deux siècles et alteint jusqu'à 33 mètres et plus de hauteur, sur un diamètre de 1 mètre à 1 mètre 20 centimètres à la base.

301. Qualités et usages. - Le pin sylvectre est 
emploré, comme bois de construction ot de travail, aux mèmes usages que le sapin et l'épicéa. Son bois, quand il a atteint toute sa maturité, est mème considéré comme aussi solide et aussi durable que celui du chène. Dans la marine, il sert à la charpente et aux planchers mèmes des ponts, et c'est presque le seul arbre dont on fabrique les màts. La France, à cet égard, est tributaire du nord de l'Europe, ce qui doit nous faire attacher beaucoup d'importance it cultiver le pin sylvestre dans des conditions qui puissent lui permettre de remplir une aussi précieuse destination.

Or, ce n'est que dans les régions élevées de nos principales chaines de montagnes, où les accroissements sont lents et la texture ligneuse trìs serrée, que nous pouvons espérer de voir le bois du pin sylvestre acquérir le degré de souplesse et d'élisticité réclamé pour la màture. On devria donc rechercher avec soin, dans ces régions, les parties les plus abritées, où celte essence auri le moins ì souffrir des neiges et des frimas, pour y élever des massifs destinés à satisfaire à ce besoin de haute utilité publique ${ }^{1}$.

Le chauffage qu'il fournit est bien supérieur à celui du sapin et de l'épicéa; il en est de mème de son charbon, qui est recherché pour les forges.

1 Voir sur ce sujet le mímoire de MAl. Bravais et Martins, inséré dans le deuxième volume des Annales forestirips (année 18 अ, pages 369 et 361 ). 
Le pin sylvestre produit une partie du goudron dont la marine a besoin. Ce sont les souches et les racines qui en rendent le plus; on l'en retire communément en les soumettint, dans des fourneaux, à une distillation lente et graduée. Le bois, ainsi privé de résine, se réduit en un charbon que l'on emploie à divers usages. Il se fail, dinns beaucoup de pays, des adjudications trìs productives de souches de pin sylvestre, avec faculté d'établir des fours à goudron.

Dans certaines localités, les maraudeurs ont l'habitude d'entailler fortement le trone des pins sylvestres, pour en enlever quelques morceaux plus particulièrement chnrgés de résine, qu'ils reconnaissent ì une couleur jaune foncé, au poids, et à l'odeur forte qui s'en exhale. Ces morceaux, coupés en petits fragments, sont très inflammables el se vendent pour remplacer les allumeltes et pour activer le feu. II n'est pris nécessaire de dire que ce délit est des plus préjudiciables.

ARTICLE IV.

\section{Le Pin à crochets.}

302. Le pin i crochets (pinus uncinata, DE CandoLLe; p. mugho, Poiret), appelé aussi pin suffin, pinerin, suffis ', habite les régions élevées des Alpes, des

' Nous n'avons pas eu occasion d'observer le pin à crochets. Les renseignements qui nous ont permis de le dicrire sont dus, 
Pyrénées et du Jura, où il forme, tantòt seul, tantòt en mélange avec le pin sylvestre et le mélèze, des forêts assez considérables. On le trouve aussi, mais par houquets seulement, sur quelques hauts plateaux des Vosges. Outre les qualités de son bois, cette propriété de végéter en bon état dans des régions où la plupart de nos essences résineuses ne sauraient prospérer, le rend précieux pour le forestier, dont un des principaux soins doit ètre d'assurer le boisement des hautes sommités de nos chaines de montagnes 1.

303. Climat, situation, exposition. - Cet arbre, ainsi qu'on vient de le dire, appartient surtout aux climats secs et froids; dans les Alpes, il apparait à 1,500 mètres, monte jusqu'à 2,200 mètres, et se maintient même à 2,300 mètres d'altitude. Ses branches

en très grande partie, ì MIM. I) Guiny et Broilliard, qui ont administré, pendant plusieurs années, les cantonnements de. Guillestre et de Briançon.

' Le pin ehitif ou muyho (pinus pumilio ou mughus), qu'avec plusieurs botanistes nous avons considéré pendant longtemps comme une variéte du pin sylvestre, produite par l'influence simultanée d'un climat rigoureux et d'un sol tourbeux, est aujourd'hui généralement reconnu comme étant une modification du pin à crochets. Il est particulier aux tourbières et aux marais des hautes montagnes, sur lesquels sa tige et ses branches rampent souvent à une listance de plus de 10 mètres en sinntrelaçant et se coudant de manière à former des lacis impénétrables de 1 mètre äo à ? mètres de hauteur. Quvique impropre, par sa forme et ses dimensions, à tout autre usage que le chauffinge, cette variété mérite d'ètre appréciée, puisqu'elle permet de maintenir boisés des lieuxoù toute autre végétation ligneuso serait impossible. 
moins nombreuses el moins fortes, ses feuilles plus raides et plus serrées que celles du pin sylvestre, semblent être la cause pour laquelle il est moins exposé que ce dernier à souffrir de la neige et des frimas 1.

L'exposition du Sud est celle où il prospère le mieux. Dans les arrondissements de Briançon et d'Embrun, où le pin à crochets est surtout répandu, il forme l'essence dominante de presque tous les versants méridionaux.

304. Terrain. - Le pin à crochets s'accommode de toute espèce de terrain, quelles que soient sa nature et sa profondeur; on le trouve mème sur des rochers. Toutefois, les sols légers et frais sont ceux qui lui conviennent le mieux, mais il s'y carie facilement lorsqu'ils sont très substantiels.

30כ. Floraison et fructification. - La floraison est monoïque; les fleurs paraissent en juin et juillet.

Les cònes grossissent et mùrissent dans le mème espace de temps que ceux du pin sylvestre, et la semence, semblable à celle de ce dernier arbre, se dissémine à la même époque. L'arbre devient fertile à un âge peu avancé, et ses fruits sont abondants.

306. Jeunes plants. - Les jeunes plants du pin à crochets sont plus robustes que ceux du pin syl-

" Cela peut tenir aussi à ce que la neige est ordinairement fine et pulvérulente dan: le:s riviuns ćlerées qu haljite le pin à crochets.

(Nole de M. Parade.) 
restre, en ce sens que, s'ils croissent, aussi bien que ces derniers, sans abri, ils supportent, beaucoup mieux qu'eux, un couvert prolongé et résistent aux froids les plus rigoureux.

307. Feuillage. - Les feuilles sont raides et serrées sur les rameaux; elles donnent un courert épais.

308. Racines. - Les racines sont surtout tritcantes; on n'y remarque pas de pivot proprement dit. Elles pénètrent facilement et profondément dans les fentes des rochers.

309. Croissance et durée. - La croissance du pin à crochels est très lente, égale et se prolonge jusqu'à un àge fort avancé. Vers 160 à 200 ans, il atteint une circonférence au pied de 1 mètre à 1 mètre 40 sur 18 à 23 mètres de hauteur. Dans le cantonnement de Guillestre (arrondissement d'Embrum), quelques arlbres, crùs dans un sol sec et à l'exposition du Midi, mais dont on ne connait pas l'àge, ont présenté jusqu'à 3 mètres de circonférence à la base el 20 mìtres de hauteur.

310. Qualités et usages. - Le bois est estimé pour la charpente, et, à l'exception du mélèze, on le préfère, dans les Alpes, aux autres essences résineuses; il a le grain fin, il se fend et se travaille ficilement et sert à la fabrication de toutes sortes d'ustrnsilus de ménage. Comme chauffage, il est aussi très apprécié. 
La finesse et l'égalité de ses accroissements annuels [283], le petit nombre relatif de ses branches peu développées, enfin la beauté de son port, ont fait penser que le pin à crochets pourrii tdevenir une ressource précieuse pour notre mâture, si on lui laissait atteindre un âge suffisamment avancé.

Quelques échantillons fraîchement coupés de ce bois, envoyés à l'École forestière en 1856 , ont présenté cette particularité que, gorgés abondamment de térébenthine à leur arrivée, cette substance n'a pas tardé à s'évaporer pour ne laisser, dans le bois desséché, qu'une faible quantité de résine. Cette expérience peut faire craindre que, comme le sapin el l'épicéa, le pin à crochets n'ait pas, dans ses tissus, suffisamment de résine concrète pour donner à son hois l'élasticité et la force' exigées pour la màture. Cependant, il serait prématuré, à notre avis, de sc prononcer à cet égard. D'une part, en effet, les échantillons dont il s'agit n'avaient guère plus de 100 ans. ce qui est un àge peu avancé pour une essence qui, croissant aussi lentement, atteint cependant de très fortes dimensions [309] et peut vivre, par conséquent, deux à trois siècles. D'un autre còté, les échantillons ne provenaient-ils pas d'arbres crùs dans un sol frais et substantiel, tandis que ce sont certainement les terrains secs et exposés au Midi qui produisent le bois le plus incrusté de résine concrète?

Il est done nécessaire de continuer, sur les lieux, l'étude de celte importante question. On ne saurait 
trop la recommander à l'attention des forestiers en position de s'y livrer.

ARTICLE $v$.

\section{Le Pin maritime.}

311. Le pin maritime, pin de Bordeaux (pinus pinaster, Solaxd; pinus maritima, Ladiarca) constitue des parties de forèts considérables dans les Landes, en Provence, dans le Languedoc et en Corse.

\section{Climat, situation, exposition. - Quoique} cet arbre appartienne plus particulièrement aux climats chauds, on le cultive cependint avec succèdans les départements de l'Ouest; mais il y est exposé aux gelées, ne fournit pas des bois de valeur et n'atteint pas un àge avancé. Son élévation el sil grosseur y sont médiocres, et son utilité restreinte. II en est de mème dans la forèt de Fontaincbleau el dans les environs de Paris. On ne peut douter que, transporté plus au Nord, il ne soil impossible au pin maritime de s'acclimater, étant très sensible au froid.

Lorsqu'il jouit d'une température convenable, il prospère en plaine, sur les collines, el mème sur des montagnes de hauteur moyenne 1. Il est d'autiut plus utile sur les bords de li mer que ses maines

1 En Corse, on le trouve encore ì une altilude de 1,000 mètres. 
pirolantes et latérales lui donnent une assielte solide et le mettent en état de résister aux efforts des vents.

313. Terrain. - Le pin maritime se contente d'un sol médiocre, pourvu que ce sol soit profond; il réussit mème très bien dins les sables purement quartzeux, comme le sont par exemple ceux rejetés par les eaux et amoncelés sur le littoral de l'Océan. Les terrains compactes et marécageux lui sont contraires.

314. Floraison et fructification. - La floraison est monoïque. Les fleurs paraissent en mars et en avril dians le midi de la France, et au mois de mai dans les régions plus tempérées.

La semence est plus grosse que celle du pin sylvestre et munie d'une aile proportionnée à son volume; elle mùrit au bout du mème laps de temps que celle-ci, et se dissémine à la mème époque.

La fertilité de cet arbre est extraordinaire. II porte fruit presque tous les ans et dès l'àge de 12 à 10 ans, quelquefois plus jeune. Néanmoins, pour être sùr de la bonté des graines, il convient de ne les cueillir que sur des arbres plus âgés.

31ら. Jeunes plants. - Le pin maritime est trìs robuste dès sa naissance, et tout abri un peu prolongé lui est nuisible. Il n'y a que dans les sables brùlants des dunes de Griscogne et dans les expositions chaudes du midi de lit France qu'il est nécessinire de l'ombrager les premières années.

316. Feuillage. - Emoigne les fenilles du pin 
maritime soient très longues, leur ensemble cependant ne donne qu'un faible couvert; conme celles du pin sylvestre, elles tombent la troisième année.

317. Racines. - Cet arbre a une racine pirotante qui s'enfonce très avant dans le sol; il a, en outre, des racines traçantes qui, dans toute leur longueur, jettent des pivots profonds. Cette propriété, jointe à sia rapide végétation, le rend très propre à fixer les sables mobiles des dunes.

318. Croissance et durée. - La croissance du pin maritime est remarquablement prompte, et il acquiert de fortes dimensions. On roit dans une partie des dunes de Gascogne, sur un sol profond et substantiel, plusieurs arbres non résinés qui ont une élévation de 27 à 30 mètres sur 2,3 et 4 mètres de tour à 1 mètre du sol. Cette grosseur se soutient bien et donne aux arbres une forme cylindrique; leur àge est de 1300 à 170 ans, et ils ne montrent aucun signe de dépérissement. Il est done permis de penser que le pin maritime peut alteindre au moins l'àge de 200 ans diuns les terrains qui lui conviennent.

319. Qualités et usages. - Le bois du pin maritime est jugé inférieur en qualité à celui du pin sylvestre et des autres essences résineuses dont nous avons parlé. On ne s'en sert pas moins pour différentes constructions civiles. On en fait des pilotis et des étais diuns les chantiers de la marine, pour soutenir les vaisseaux pn runstruction, et on le déhite en planches el en échalas. 
Le chauffage et le charbon de ce pin sont aussi de faible qualité.

On pourrait se tromper cependant en se prononçant définitivement sur la valeur du bois du pin maritime. Pour qu'un pareil jugement pùt être porté, it faudrait que, dans le sol et dans le climat qui lui conviennent, il eùt été traité d'après le meilleur régime d'exploitation, et qu'on l'eût garanti de l'élagage el de l'extraction de la résine. Or, dans les départements des Landes et de la Gironde, cette extraction, que l'on exprime par les mots gemmage, gemmer, se pratique dans les dunes depuis plusieurs siècles '; et, dans les vastes terrains appelés landes, où le sol est généralement ingrat et où le gemmage a pris peu d'extension, on exploite le pin maritime très jeune, soit pour en tirer quelques pièces de charpente, soit pour échalasser les vignes: il en est de mème dans les départements situés plus au midi. On voit done que, dans l'un et l'autre cas, on ne saurait apprécier avec certitude les qualités de cet arbre.

Les procédés employés pour tirer de cet arbre le suc résineux donnent la mesure du préjudice qu'on lui occasionne. C'est à 23 ans ordinairement que l'on commence le gemmage, depuis le mois d'arril ou de mai jusqu'au mois de septembre. La première opé-

' Une charle du 10 octobre 1468 , octrnyie par Gaston de Foix, captal de Buch, recmnait aux habitants de la Teste la faculté de faire gemme el résine daus ses pignadas de la Teste, à charge de payer une redewance.

(Note de M. Parade.) 
ration consiste à enlever une bande d'écorce de 12 à 16 centimètres de large, depuis le pied de l'arbre, jusqu'à une hauteur de 33 à 50 centimètres, et à entailler assez profondément pour entamer l'aubier; car le suc résineux s'écoule principalement du corps ligneux et entre l'écorce et le hois. Chaque semaine le résinier rafraichit la plaie. Ces entailles se prolongent, les années suivantes, jusqu’à une hauteur de ́́ ào meitres. On commence ensuite, au pied du mème arbre, une nouvelle entaille, parallìte à lit premiìre, qui n'en est séparée que par ò à 6 centimètres d'écorce; on la conduit à la mème hauteur, et ainsi des entailles suivantes, jusqu'ì ce qu'elles fassent le tour de l'arbre. Ces entailles sont appelées quarres.

Le mode de gemmage que nous venons de ripporter est le plus modéré; on le nomme gemmage à vie. Quand on veut aller plus vite, on fait deux entailles it la fois, l'une dans le haut, l'autre dins le bas: la première dite quarpe haute, la seconde basson, at souvent on taille simultanément sur toutes les fiices de l'arbre. C'est ce qu'on appelle gemmer à mort ou à pin perdu. Jusqu’à présent, le pin maritime est principalement cultivé pour son suc résineux qui fournit des produits d'une importance incontestable, consistant en tér'benthine, brai, goudron el noir de fumée. Sous le rapport mercantile, il est hors de doute que ce mode d'exploitation est le plus profitable, el, par conséquent, il se justifie parfiritement. Mais, dans les départements de la Gironde et des Landes, on va 
plus loin, el lion prétend que le pin maritime, pour ètre d'un bon usigge, doit ètre résiné. On va jusqu'à affirmer, malgré les exemples contraires [318], que l'extraction de la résine est indispensable à cet arbre pour assurer sa prospérité et mème son existence. La dernière opinion ne nous paraît pas digne d'ètre discutée; la première, qui est fondée jusqu'ì un certain point, a besoin d'explication.

On conçoit, en effet, que le gemmage doit apporter une certaine perturbation dans la croissance du pin maritime. La nature ne lui a pas donné en vain la résine comme suc propre, et ce suc, indépendint de la sève, parait s'unir à elle pour procurer la nutrition et l'accroissement du végétal. Ce qui le prouve, c'est que des pins gemmés, et qui, étant abattus, ont pu être vérifiés, présentaient des couches annuelles très étroites, el dénotaient ainsi un accroissement ralenti, tandis que le contraire a été reconnu sur des arbres non gemmés, ayant crù d'ailleurs dans les mèmes circonstances que les premiers. Nais on a vu plus haut [283] que le bois des arbres résineux est d'autant plus ferme et plus résistant que les accroissements annuels sont plus serrés. De plus, le gemmage provoque, du centre à la circonférence de lì lige, un courant actif de térébenthine, dont la partie fluide s’écoule en laissant, dans les tissus ligneux qu'elle parcourt, une quantité notable de résine concrète ${ }^{1}$.

INathicu, Flore furestiere, page $3 \ddot{3} 3$. 
On comprend donc que le gemmage, en ralentissant prématurément la croissance du pin maritime, donne, en mème temps, à son bois, des qualités que, dans l'état de nature, il n'aurait acquises qu'à un àge plus avancé.

Reste toujours la déformation que cette pratique cause au tronc de l'arbre, lequel présente des cannelures nombreuses et profondes, devient tortueux la plupart du temps, et impropre, par conséquent, à tout usage de quelque importance.

\section{ARTICLE VI.}

\section{Le Pin laricio.}

320. Le pin laricio (pinus laricio, Porret) est aussi connu sous le nom de pin de Corse, de Calabre, parce que, dans ces deux pays, il forme de grandes forèts.

321. Climat, situation, exposition. - Ce pin se troure en Corse, ì une altitude de 1,000 it 1,500 mètres, ce qui lui donne un climat très tempéré; on le rencontre même à 1,600 el 1,700 mètres; mais, à ces hauteurs, sa régétation est lente et il est chétif. On est parvenu à l'acclimater très bien dans le nord et l'est de la France, et, quoiqu'il ne semble pas supporter les grands froids aussi bien que le pin sylvestre, il craint cependant peu nos hivers.

Comme ce dernier pin, il réussit à toutes les expositions. 
32.2. Terrain. - Les sols qu'il préfère sont les sables gras, provenant de la décomposition des roches granitiques. Il est probable qu'il ne réussirait pas, comme le pin sylvestre, dans les sables purs.

323. Floraison et fructification. - La floraison est monoïque. Les fleurs paraissent à la fin de mai, et mème au commencement de juin, sous le climat de Paris.

Ses semences, ailées et légères, sont un peu plus grosses que celles du pin sylvestre; elles demandent le mème temps que celles-ci pour la maturité et la dissémination.

324. Jeunes plants. - Le tempérament des jemes plants est aussi robuste que celui du pin sylvestre.

325. Feuillage. - Les feuilles tiennent, quant à leur longueur, le milieu entre les feuilles du pin sylvestre et celles du pin maritime; le couvert qu'elles fournissent est également fort léger.

326. Racines. - Quoique, dans la première jeunesse, le pin laricio ait un pirot prononcé, ses racines, dès qu'il a atteint un certain développement, sont entièrement traçantes, et peu étendues eu égard aux dimensions de l'arbre.

327. Croissance et durée. - La croissance du laricio est très prompte, plus prompte même que celle du pin sylvestre. Il vit plusieurs siècles et prend les plus fortes dimensions. On en roit, en Corse, qui 
ont de 33 à 40 et mème 45 mètres de haut, sur 3 et 4 mètres de circonférence à la base.

328. Qualités et usages. - Le laricio est très estimé comme bois de charpente; on l'emploie aussi dans les constructions navales. Dès 1787, on a tiré des forèts de la Corse un grand nombre de màts des plus fortes dimensions, et, de 1812 ì 1822, ces forêts ont approvisionné exclusivement le port militaire de Toulon. Cependant quelques constructeurs ont trouvé que cet arbre avait moins d'élasticité et beaucoup plus d'aubier que les pins de Riga et de Norvège. Il est permis de croire que ces observations ont été faites sur des pièces qui ne réunissaient point (probablement en raition de leur àge) toutes les qualités que l'essence est susceptible d'icquérir. Il résulte, en effet, d'un rapport fait au Ministre de la Marine, par un Ingénieur des constructions navales, envoyé en Corse vers 184\%, afin de recomnaitre les ressources que les forèts de cette ile pourraient offrir ì la marine, "que le pin laricio a le grain fin el serré. "des couches annuelles étroites, un trone parlai" tement droit et très élevé sous branches; que la ré" sine y est abondante, et, enfin, que son aubier a, ") dans certaines localités, de 20 à 30 centimìtres et, " dans d'autres, seulement de 8 à 10 centimetres d'é" paisseur sur le diamètre. " Ces faits, constatés par un juge aussi compétent, doivent nous faire atticher une haute importance aux forêts de la Corse, et nous encourager à multiplier le laricio dans toutes les 
localités de lia líance ou nous pouvons espérer de le voir réussir.

Le laricio se débite aussi en planches et en madriers; il est employé par la menuiserie et par divers autres métiers. Quand il n'est pas trop gorgé de rísine, son bois est d'un travail facile et peut mème servir à la sculpture.

Comme chauffage, il est probable qu'il ne to cide pas au pin sylvestre.

Le laricio est très résineux et susceptible de fournir les mêmes produits que les autres pins: poix. goudron, ete. Soumis au résinage, il y a une vinglime d'années, il serait à désirer qu'il pùt en ètre désormais exempt, ou du moins que le corps de cet arbre précieux ne fùt jamais gemmé. Rien llempècheritit, romme il a cite dit a l'article pin syluestre, de metlee a profit la résine qui se trouve dans la sourhe of les.s racines.

ARTICIE VII.

\section{La Pin noir.}

329. Le pin noir ou de Autriche pinus nigra, Laxwé; puns austriaca, pinus laricio austriacia. Eximathem est une espèce très voisine ou, selon plusieurs bolitnistes, une variété constinte du pin laricio. Dans less Alpes styrienues, dans les montagnes de la Dirlmatie el de la Croatie, de mème que dans une partie de la Hongrie et aux environs de Vienne. il forme des 
lurets considerables, tantiot seul, tantot en mélange aver le chène ou lo hille, le pin sylvestre ou l t melize. Ifpuis une quarmutaine d'années environ, on ¿ beaucoup propagé cet arbre dans les diverses combees de Lidlemagne, plus récemment, on it

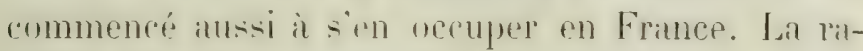
pidite de sit rovissance. Ios bomnes qualités de son

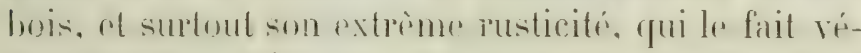
gréter en bon étal dims les sols calcaies les plus andes. justifient la litrem dont il jouit parmi les sylviculteurs.

\section{:3:). Climat, situation, exposition. - I mom-}

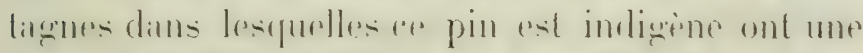

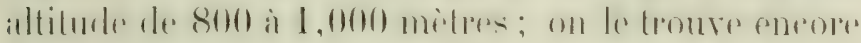

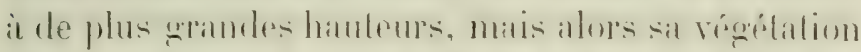

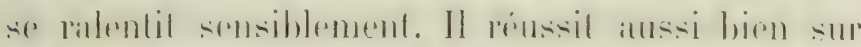

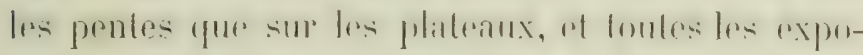
sitions semblent lui convenir!

3:31. Terrain. - lin-i yuom vient de le dire. le

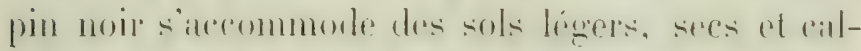
anires, quelqur paurers yüils suirnt en humus. Celle

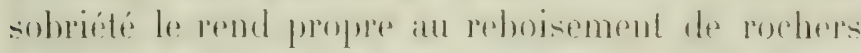

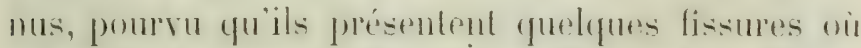
ses ranos puissent söntroduire. Il prend pied ditns les torrains provenatnl d'éboulements, lors neme qưils omt pen de fomd, ol sil régrétation n! semble

' Zoctl, conseiller' des forèts et des mines en 'Trrol, Mamuel de sylriculture des hautes montugmes. 
pas sensiblement ralentie ${ }^{1}$. Les sols argileux lui conviennent médiocrement, et, lorsqu'ils sont humides, ils lui sont absolument contraires.

332. Floraison et fructification. - Sous ce double rapport, le pin noir est entièrement semblable au pin laricio.

333. Jeunes plants. - Il est trìs robuste dès sa naissance, et ne craint ni le froid ni la chaleur.

334. Feuillage. - Les feuilles sont très serrées, d'un rert sombre, et persistent pendant 5 et 6 ans. L'arbre ayint des branches nombreuses et touffues, donne un courert épais et fournit au sol un détritus abondant.

333. Racines. - Les racines sont tracantes, robustes et s'étendent au loin; le pirot est presque nul.

336. Groissance et durée. - La régétation du pin noir est rapide et se soutient jusqu'à un àge très arancé. Quand il rroit dans les conditions qui lui conviennent, il vit 2 à 3 siècles et atteint un diamètre de 1 mètre et plus à la base, sur 30 à 33 mètres de hauteur ". Onelques auteurs ont dit que sa tige etait rarement droite. Cepentiant Zoetl, dont nous arons déjà cité l'ourrage, et qui arait longtemps administré des forèts de pin d'Autriche, ne parle pas de cette particularité et considire le pin noir comme un excellent bois pour la construction. Or, un tel

' Zoetl, même ouvrage.

"Hoess, Monographie du pin noir. 
emploi ne saurait se concilier avec un tronc de forme défectueuse.

337. Qualités et usages. - Ainsi qu'on vient de le roir, le pin roir est très estimé en Autriche comme hois de construction, et l'on assure que, sous ce rapport, sa durée égale celle du mélèze. Placé sous l'eau, il est, pour ainsi dire, incorruptible. Il donne un bon chauffage, et son charbon équivaut à peu près à celui du hêtre. Comme il est très résineux, il est soumis au gemmage, dans les pays qu'il habite. Il est probahle que la forme tortueuse du tronc, qu'on lui a reprochée, est le résultat de cette pratique, et ne provient pas d'une disposition particulière à la croissance de l'arbre [319].

\section{IR TILI, I: VIII.}

\section{Le Pin d'Alep.}

338. Le pin dlep ou de Jerusalem pinus halepensis, Muler), qui parail originaire de la Syrie et de la Barbarie, est assez commun diuns les départements méridionaux de la France.

339. Climat, situation, exposition. - Un climal chaud, ou au moins très tempéré, lui est nécessaire. C'est avec peine qu'on parrient à l'acclimater dans les environs de Paris: il souffre des froids de l'hiver, et plus au Nord il ne pourrait y résister.

Cet arbre se plait dans les plaines et sur les coteaux. 
Jans les départements du Midi, toutes les exposilions semblent lui être favorables.

340. Terrain. - Le pin d'Alep se contente d'un sol très médiocre, pourvu qu'il soit léger; en général, on remarque qu'il prospere plutiot dans les terrains calcaires que dans les siliceux.

3 1. Floraison et fructification. - La florison est monoïque; les fleurs paraissent en mai.

La maturité de la graine, qui est ailée et légère comme celle des autres pins, a lieu à la fin du second été qui suit la florison, et la dissémination ne s'opère que dans le courant du troisième.

:32. Jeunes plants. - Nime sous le ciel de lit Provence, lo jemr pin d'Dlep résiste parliailement aux ardeurs du soleil; mais, par contre, il parait craindre les grelées dans les trois ou yuiltre premieres années de son existence, lorrqu'il ast semć à découvert. Après cet âge, il devient robuste.

343. Feuillage. - Le pin d'Mep a les fenilles lris fines et assez longues; elles ne persistent guirer que 2 ans et ne foumissent, parr consérquent, qưun léger couvert.

34. Racines. - Les racines pivotent asserz profondroment, lorsque le sol le permet; mais elles s'arrommodent aussi de tracer, et l'on voil partotos dese sujets d'assez fortes dimensions, atssis sur des rochers entirrement nus dont quelques crevasses seulement permettent aux rateines de s'y eramponmes.

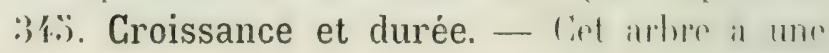


croissance tris rapide dans sa jeunesse. Situé en bon sol, il s'éleve beaucoup et est susceptible de prendre de fortes dimensions.

346. Qualités et usages. - Le pin d'- llep sert à la britisse, et surtout à la menuiserie; il a le grain fin et se travaille facilement. On extrait sa résine. qui est moins abondaute que celle du pin maritime, d'après les mèmes procédés que pour cette dernière, et l'on en labrique les mêmes produits. La résine liquide, qui découle d'abord des incisions, est conlondue dims le commeree aver lit terébenthine de Venise.

\section{ARTICE IX.}

\section{Le Pin pinier.}

39.7. I pe pin pinier ou à pignons ipinus pinea. Laxé, jin rultive, pin bon, pin de pierre, pin putrusol, ele. croit naturellement dans le midi de la France, en Italie, en Espagner ol sur les coiles de Barbarie.

En Finnce, surtout, il nest cultivi que comme arbre fruitier ou d'agrément.

34. Climat, situation, exposition. - Juoiqüil remande, pour prospérer, un climat chaud, il supprote cependant celui de l'aris el y porte fruit; mais il est douteux qu'il puisse végéter plus au Nord. Les departements maritimes de l'Ouest sont les seuls, aprìs ceñ du Midi, ou il semit possible de l'acclimator; il parait rechercher de préférence les 
plaines, les vallées, et c'est sur les bords de la mer et le long des rivières qu'il montre la plus belle croissance.

349. Terrain. - Il faut à cet arbre un terriin léger, mais profond; il se contente même d'un sol rocheux, pourvu qu'il présente des fissures qui permettent aux racines de se développer.

350. Floraison et fructification. - La floraison du pin pinier est monoïque; les fleurs paraissent à la fin de mai ou au commencement de juin. Les strobiles, qui leur succèdent, sont plus gros que ceux de tous les autres conifères, et n'atteignent leur maturité qu’à la fin de la troisième année après la floraison. A la base de chaque écaille se trouvent deux loges dont chacune renferme une amande de la grosseur d'une petite noisette, enveloppée d'une coquille ligneuse et bordée d'une aile caduque très petite.

L'arbre devient fertile à un âge peu avancé.

351 . Jeunes plants. - Les exigences du jeune plant sont peu connues parce que, les fruits étant récoltés avec soin avant la dissémination naturelle de la graine, on ne trouve guère de jeunes sujets ayant levé spontanément. Les plants que l'on emploie proviennent de pépinières où ils reçoivent les soins artificiels les plus propres à assurer et à hàter leur développement.

352. Feuillage. - Les feuilles sont longues, épaisses et assez larges; elles donnent un couvert plus complet que celles des autres pins. 
DES SOLS, DES ESSENCES.

353. Racines. - Les racines sont fortes et pivotantes.

33̈4. Croissance et durée. - Ce pin, dit-on, se distingue des autres par une cime en parasol, largement étalée et qui ne lui permet pas de croitre à l'état serré. Nous croyons qu'on se hàte trop de se prononcer à cet égard, attendu qu'il n'a encore été trouvé qu'isolé et que, dans toute la Provence qu'il habite particulièrement, ainsi qu'en Algérie où on le trouve parfois en forêt, il n'existe pas un seul massif de cette essence ${ }^{1}$.

Il parait que le pin pinier peut arriver à un grand àge et alteindre jusqu'à 30 mètres d'élération. On cite celui qui existe aux Sablettes en Provence: il it 4 mètres de circonférence; son tronc est nu jusqu'à une hauteur de 10 mètres, et, des premières branches au sommet, il a une hauteur au moins égale. La circonférence de sa tète est de 100 mètres.

$35 \%$. Qualités et usages. - Cet arbre est très propre à la charpente; on en fait des planches et des corps de pompe. En Orient, on l'emploie, dit-on, à la màture, et surtout aux bordages des vaisseaux.

- On a attribué pendant longtemps cette mème anomalie au cèdre. Or, on sait aujourd'hui que, dans les forèts de cette essence que possède l'Algérie, les cèdres à cimes coniques sont très communs et s'accommodent parfaitement de croître en massif. Tous les pins, d'ailleurs, sans en excepter le sylvestre, montrent plus ou moins de dispositinn à former une cime étalée, quand ils alteignent un åge avancé. 
II serl aussi à la menuiserie et à divers autres métiers. Son chauffage est peu apprécié.

Le pin pinier n'est pas très résineux, ce qui le g'arantil des saignées.

Sim fruil, commu sous le nom de pignon, est une amande douce, agréable à manger el donnant une boune huile; le seul inconvénient qu'elle présente, c'est d'ètre enveloppée d'un noyau dur. On trouve en Italie une variété de pin pinier qui fournit des pignons à noyiu lendre; il serait facile de l'arclimaler et de la propager ditns le midi de la France.

II TI ( LE X.

\section{Le Pin cembro.}

33̈6. Le pin cembro (pinus cembra, Lsxé, Auvier, Tinier, forme, en hussie, des forèls très étendups. En France, de mème qu'en Suisse, il est peu répandu of cooit génépalement en mélange aree l'épicéa, le mélèze et le pin à crochets '.

$33 \%$. Climat, situation, exposition. - La zonc naturelle de cet arbre se réduit en France, ainsi quion vient de le dire, aux parties les plus cilevées des

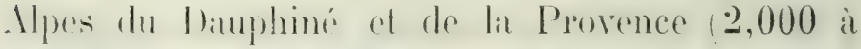
2,:00 mitres al altilude environ). Il parait que, dans

- Dans le cantonnement de Briancon, la forêt communale de Villars-Sint-Pancrace contient un massif pur de pin cembro, d'ume étendue de 200 hectures. C'est pent être le seul qui existe encore dans les . Ipes (M. Broilliard, sous-inspecteur). 
res localités, il s’accommode de foutes les exponsitions.

338. Terrain. - C'est dans les sols substantiels, frais, profonds el divisés, que le cembro se plail surtoul; mais il s’accommode aussi d'un terrain légerement humide, et ne redoute pas ceux qui sunt pierreux.

\section{$3 \% 9$. Floraison et fructification. - Les fleurs} sont monö̈ques et paraissent en mai ou au commencement de juin. Lesstrobiles renferment, sous chatpur icaille, deus amandes ì noyau dur, un peu moins grosses que celles du pinier; elles mirissent pendint

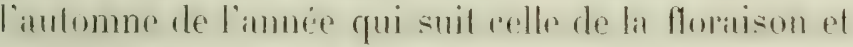
se disséminent au printemps suivant.

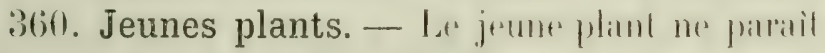
rander ni lo froid ni lesoloil; malis si, du sommonl

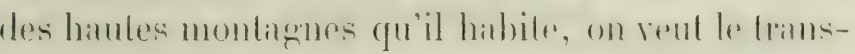

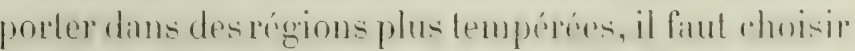

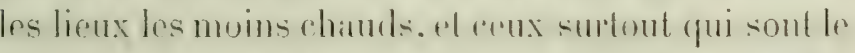
moins exposios anx gelées printmieres, anxquelles les plantes alpines sont très sensibles.

36il. Feuiliage. - I e feuillage du cermbro rit assez touffu et donne un couvert épais.

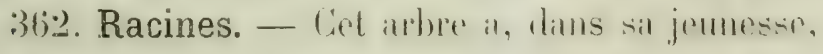
un pirot prononce, mais, plus latre, les racines laterales contionuent seules il coroitre; plles sont fortes el tracent au loin.

363. Croissance et durée. - La croissance du pin

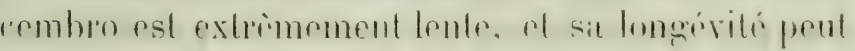


atteindre 4 à 5 siècles. Dans le Briançonnais on trouve des pieds de cette essence qui ont de 3 à 4 mètres de circonférence à la base sur 15 à 20 mètres de hauteur (M. Broilliard).

364. Qualités et usages. - Ce bois est impropre aux constructions. Il se travaille facilement et il est très recherché pour la sculpture et la menuiserie; son chauffage équivaut à peu près à celui du sapin.

Le fruit est une amande douce, bonne à manger et très nourrissante; on en exprime une huile assez agréable.

Le pin cembro contient abondamment une térébenthine très fluide; toutefois, il n'est pas soumis au résinage.

\section{AR'TICLE XI.}

Le Pin du lord Weymouth.

365̈. Le pin du lord Weymouth (pinus strobus, LinNÉ) a été importé, au commencement du dernier siècle, des Etats-Unis et du Canada en Angleterre, par le lord dont il porte le nom; mais il y a cent ans à peine qu'on a commencé à le cultiver sur le continent. Quoique exolique, on l'associe aux bois indigènes de la France, parce qu'il y est parfaitement naturalisé. Il se distingue par la beauté de son port.

366. Climat, situation, exposition. - On cultive ce pin avec succès dans toutes les parties de la France, à l'exception du Midi qui parait ne pas lui convenir; il préfère généralement les régions un peu froides. 
En Amérique, il habite surtout la plaine et les pays de coteaux.

367. Terrain. - Le pin du Lord ne se plait ni dans les sables arides, ni dans les sols trop compactes ou marécageux. C'est dans un terrain légèrement humide, profond et substantiel qu'il végète le mieux; il se contente cependant 'd'un sol maigre, pourvu qu'il soit frais, divisé, et qu'il ait du fond. Il prospère surtout le long des cours d'eau.

368. Floraison et fructification. - La floraison est monoïque; les fleurs paraissent en mai ou au commencement de juin. Les strobiles sont mûrs seize mois après la floraison, et la dissémination de la graine, qui est légère et ailée, a lieu aussitòt, c'està-dire en septembre ou en octobre de l'année qui suit celle de la floraison.

L'arbre porte semence très jeune.

369. Jeunes plants. - Les jeunes plants demandent, au moins aux expositions chaudes, de l'abri pendant les premières années; on peut assimiler leur tempérament à peu près à celui des plants d'épicéa [288].

370. Feuillage. - Le feuillage du pin du Lord est fin, léger, et donne assez peu de couvert. Ses feuilles ne persistent pas plus de deux ans.

371. Racines. - Son pivot est très prononcé; il est accompagné de racines latérales nombreuses qui s'étendent au loin.

372. Groissance et durée. - La croissance du 
pin du Lord est tris rappide; les pousses ammuelies sallongent quelqueforis, dams sa jeunesse, de 66 centimetres à 1 motre. Il atteint un àge tris avance et prend les plus fortes dimensions. En Imérique, on

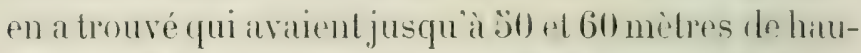
leur sur I metre 66 centimitres de diametre a la baser.

373. Qualités et usages. - Iux Ftals-Linis, le pin du lord a un hois forme; il est léger peu nourux et facile a travailler. Les Américains en tirent un grand parti pour la charpente, et mème pour lis construction des vaisseaux. Ils l'emploient aussi à lit màture; mais, quoique plus léger que le pin sylrestre. iln'est pas, pour cet effet, d'un aussi bon usagre.

Pour les constructions riviles. s'il est placé it l'abri des rariations de l'atmosphere, co bois a la durée el lit consistance de nos meilleurs pins; mais il ne faul l'employer ni sous l'eau ni dans la terre, ou il est sujet à pourrir.

Il est tris recherché par différents méliers; sit texture est fine, et il prend un beau poli.

On ne peut alfirmer que toutes res précienses quatlités se petrourent dans les pins du Lorel yue nous rultivons en France. Ceux que l'on a pu ! exploiter jusqu’à présent étaient évidemment trop jemes pour servir de sujets d'expérience; le bois en était mou, léger, peu résineux el peu resistant. Le temps seul pourra nous apprendere re que mous avons ì espéper de cette essence.

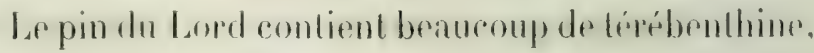


qui se rolatilise promptenent. En France, il est demeuré jusqu’à présent à l’abri du résinage; mais. dans l’Amérique du Yord, il parait qu il est soumis à cette pratique.

\section{IIT T I L L: III.}

\section{Lue Mélèze.}

374. Le méle:e (larix europara, se C.xpolde) par la beauté de son port, la durée et l'utilité deson bois. tient le premier rang parmi les bois lrísineux. Il est le seul dont les feuilles soient caduques.

Il forme des forits considerables, tantit seul, Lantòt en mélange avec l'épićá. le pin sylvestre, le pin à crochets et le cembro.

37\%. Climat, situation, exposition. - Cel ill) qui, dans le Sord de l'Europe, necupe tantrit la plaine et tantót la montagne, ne se trowse, en Frence, yue dans les . Ilpes, oi il apparait ì 1, 2011 mitres d'alti-

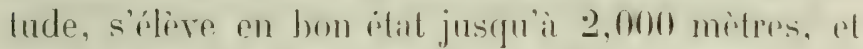
resiste mème, mais ì l'état de buisson pabougri.

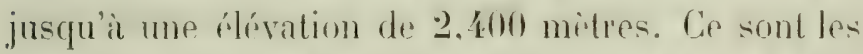
siluations où l'état habilue de l'atmosphère est a la fois sec et froid, yui semblent surtout lui convenir; il redoute les climats hrumeux et les rents humides.

Sur les montagues oi il est indigene, il prospire particulirement aux expositions du Tord et de l'Est. $A$ de grandes hauteurs, on le trouve aux aspecls du Sud et de l'Ouest, mais il n'y est pas hienremant '.

1. du Guiny. 
376. Terrain. - Le mélèze demande une terre divisée, profonde et surtout fraìche. Cette dernière condition, qu'il recherche d'une manière très marquée, le rend sourent accommodant sur les deux autres. Dans les terrains très substantiels, il est, comme le pin à crochets [304], exposé à se carier ${ }^{1}$. Les sols argileux et compactes ne lui conviennent pas plus que les sables purs et trop légers; les terrains mouillés aussi lui sont contraires.

377. Floraison et fructification. - Les fleurs sont monoïques, et paraissent en avril ou en mai, selon la température. Les graines, légères et ailées, sont mûres à la fin de l'année mème de la floraison, et se disséminent au printemps suivant; dans certaines circonstances, néanmoins, cette dissémination s'effectue, comme celle de l'épicéa [287], dès le commencement de l'automne.

Le mélèze porte semence à un àge peu avancé, surtout dans les climats tempérés, où sa trop grande fécondité, toutefois, doit être considérée comme l'indice d'un ralentissement prématuré de croissance.

378. Jeunes plants. - Dans les climats où il est indigène, le jeune plant du mélèze est robuste dès sit naissance et susceptible de résister aux froids comme aux ardeurs du soleil; mais, sous un ciel plus doux. on fera bien de l'ombrager pendant les premières années.

' M. du Guiny. 
379. Feuillage. - Les feuilles sont petites, tendres, et ne donnent qu'un couvert léger; elles fournissent cependant au sol un détritus benucoup plus abondant que celui d'aucun autre conifère (II. T. Hartig), parce qu'elles se renouvellent chaque année.

380. Racines. - Le mélèze pirote à un mètre et plus de profondeur; ses racines traçantes sont nombreuses, déliées, et s'étendent au loin.

381. Groissance et durée. - La croissance du mélèze est très prompte; il acquiert une grande hauteur et prend beaucoup de diamètre. On en a trouré sur les Alpes qui avaient de 33 à 40 mètres de hauteur sur כ̆ mètres de tour à liı base. Il peut vivre trois à quatre siècles 1 .

1 Nous devons à l'obligeance de M. Davall de Joffrey, conseiller des forèts à Vevey, canton de Viur s'suissec, la notier strivante sur un mélèze que lon vorait encore on 18.00 dans li. voisinage du hameau de Forclar, dins la villice d'ormont, sur le bord d'une forêt située sur un versant au norł cn pente rapide, à 1,400 mètres environ au-dessus du niveau de la mer.

Quoiqu'il lìt partie d'une forèt particuliere, cot arbre avai, été placé sous la surveillance spéciale de l"inspecteur forestier de la contrée, et le propriétaire avait consenti à ne pas le faire abattre. Mais la foudre le frappa et le mutila tellement, que lil hache dut l'achever.

Croissant isolé, le mélèze de Forclar était très branchu, et sa hauteur ne dépassait pas 23 mètres, mais sa souche arait, prè's du sol, un diamètre de 2 mètres. Son àge était, au plus, 27̈̈ ans, et sur la souche on comptait distinctement 237 couches de bois parfait et 13 couches seulement d'aubier; l'écorce avait une épaisseur de $0^{\mathrm{m}}\{3$.

Le premier billon, parfititement sain, d'une longueur de $y^{\text {na }} 3$, 
Dans les régions lempérées, sa végélation surpasse en rapidité celle de tous les autres bois résineux; rependant elle se ralentit déjà considérablement dès l'âge de 60 à 70 ans, et quelquefois même plus tôt. Souvent, à la vérité, il a, à cet âge, de 23 à 30 mètres de hauteur sur 25 à 33 centimètres de diamètre; mais son bois, alors, est loin de présenter les qualités (ju'on lui reconnaìl, à un àge plus avancé, dans le pays dont il est originaire.

Ces faits, qui se sont vérifiés sur différents points le l'Allemagne, et notamment dans la Forêt-Noire et dans les montagnes du Hartz, sont de nature à faire roire que le mélèze n'est point encore parfaitement icclimaté dans les situations peu élevées.

La culture en grand de cet arbre semble donc, yuant à présent, devoir se borner aux très hautes montagnes; mais on ne devra pas moins en continuer ot multiplier les essais sur nos élévations moyennes. (In sait, en effet, que ce n'est souvent qu'après plusieurs générations qu'une essence parvient à se faire entièrement au climat où elle a été importée, et à y recouvrer, au moins en partie, les propriétés qu'elle. possède dans son pays natal ${ }^{1}$.

arait au gros bout $1^{\mathrm{m} 9}$ de diamètre et $\mathbf{2 7 0}$ couches, ot au petit bout $0^{\mathrm{m}} 93 \mathrm{~s}$ et 248 couches. Il cubait done $14 \mathrm{~m}$. c. 39 . $\mathrm{d}$. c.

'Dans un grand domaine de l'Angleterre, où existaient d'infurtantes plantations de mólèzes àgés de plus d'un siècle, le propriétaire s'est décidé, il y a peu d’années, à exploiter ces arbres, dont les dimensions étaient déjà considérables. Ils 
382. Qualités et usages. - Le mélize résiste très longtemps à l'air et se conserve, dans les lieux humides et sous l'eau, aussi longtemps que le chêne. Des habitations sur les Alpes, entièrement construite: avec ce bois, dont les pièces équarries sont placées les unes sur les autres, ont une origine fort ancienne.

Dans une cabane, bâtie depuis 240 ans, on il

avaient été plantís, les uns anx expositions du Nord el de l'Es!. les autres sur des versants aux aspects du Surl et de l'Ouest.

Examinés par des ingénieurs maritimes, les jremiers furen trouvés de très bonne qualité et priyis fort cher pour ètre enployes dans la construction des vaisseaux; les autres, an contraire, parurent aux mèmes juges de rualité fort mérdiocre et tout a fait impropres aux exigences du service délite qu'il s'itgissail d'approvisionner.

Cet exemple tend, selon nous, à prouver: $1^{\circ}$ que le mélèze, transporté dans d'autres climats, y manifeste les mèmes exigences que dans son pays natal, en ce qui concerme l'expozition; $2^{\circ}$ que, quand ces exigences, jointes salls doute it cell rconcernant le sol, sunt salisfaites dans une cerlaine mesure, sun bois conserve, en grande partie, les pricieuses qualités qui le distinguent dans les régions élevées.

M. 'T. Hartig, dans son excellent ouvrage déjà cité, rapportr, de son côté, des expériences plus encourageantes encore pour prouver que les qualités si remarquables du mileze n'appartiennent pas seulement à cette essence croissant dans les climats froids.

Son illustre père avait fait disposer, pour tuteurs des arbres du jardin de l'École vétérinaire à Berlin, des perehes ou piquels de diverses essences. Ces piquets, écorcés, avaięnt un diamètre? de 3 pouces ( 1 décimètre environ). Ceux en bois de mélèze se maintinrent intacts pendant l'́x ans et ne trouvèrent de rivaux en durée que parmi les robiniers, les cembros, les thuyas et les genéviers. Line expérience analogue et simultane fit fitite avec 
trouvé les bois parfaitement sains 1. Le mélèze est également précieux pour les constructions tant civiles que navales. Si on le voit rarement dans les chantiers de la marine, la cause en est uniquement dans la difficulté de l'extraire des localités qu“il habite el dans les frais considérables de transport. Des ingénieurs de la marine le considèrent comme plus résineux, plus fort ot plus léger que le laricio, et, par conséquent, plus propre à la màture. Les bordages faits de ce bois durent plus longtemps que ceux de chène, ce qui a été reconnu dans les bìtiments employés à la navigation du lac de Genève.

Le mélèze est très propre à la menuiserie et ì d'autres métiers; il a un grain très fin et prend un beau poli. On en fail aussi du merrain, et il fournit pour la vigne de très bons échalas.

Son chauffage et son charbon ne sont pas de première qualité; cependant ils passent, dans le pars

des madriers de ' pouces qu'on enterra dans le même lieu. Ceux en mélèze, en robinier et en pin sylvestre vieux et très résineux, furent les seuls qui restèrent sains aussi longtemps que les piquets. Or, les milezes employes provenaient des entirons de Berlin.

I Il taut observer cependant que cette durée extraordinaire n'est pas uniquement due à la bonté du bois. Il n'est pas doutteux que les froids vifs et prolongés des grandes hauteurs doivent retarder la décomposition de toute espèce de bois qui : serait mis en cuvre, et que des nélèzes, employés aux constructions sur des élérations peu considérables ou dans des vallees, n'y résisteraient pas aussi longtemps, attendu les variations fréquentes que présente la température de ces localités. 
natal de l'arbre, pour ètre préférable à ceux des pins ct des sapins. Le bois de mélèze a l'inconvénient de pétiller, de lancer des éclats et de s'allumer plus difficilement que les autres bois résineux. C'est sans doute à cette dernière propriété qu'il a dù la répulation d'incombustibilité qu'on lui avait faite chez les Romains (Vitruve).

On tire du mélèze un suc résineux connu sous le nom de térébenthine de Tenise. Dans le Vilatis, on l'obtient en pratiquant dans le tronc, avec une tarière, des trous faiblement inclinés de bas en haut, mais qui ne dépassent pas l'aubier. A l'orifice de ces trous, on place des gouttières faites en bois de mélèze, par le moyen desquelles la térébenthine coule dans des auges disposées à cet effet au pied de l'arbre. Cette récolte a lieu sur les Alpes depuis la fin de mai jusqu'au commencement de septembre; elle est sans doute d'un grand produit, carr on prétend qu'un mélèze peut fournir, pendant 40 à 30 ans, de 3 à כ kilogr. de térébenthine chaque année; mais celle opération rend ordinairement le bois impropre au service.

La térébenthine distillée donne une huile essentielle qui, cependint, n'est pas aussi estimée que celle qu'on obtient de la térébenthine du sapin.

L'écorce du jeune mélèze est très propre au linnage des cuirs. 
ARTICLE XII.

\section{Le Cèdre.}

383. Le cèdre ou cèdre du Liban cedrus Libani, BArrel) est spontané en Asie, principalement sur le mont Liban et sur le mont Taurus; depuis la conquète de l'Algérie, on en a reconnu de vastes forêts dans les montagnes de l'Allis. Il peut être considéré comme le géant des conifères de ces contrées.

384. Climat, situation, exposition. - L'habitation du cèdre devrait faire présumer que cel urbre ne craint pas les climats froids ou, au moins, qu'il doit très bien réussir dins les climats tempérés de la France. En effet, sur le mont Liban, dont le sommet est couvert de neiges perpétuelles, on ne le rencontre que dans une région déjà assez élevée, et en Afrique, ce n'est qu'à 1,400 mètres au moins audessus du niveau de la mer que se trouvent les forêts de cèdre '. Cependant, dans le climat de Paris, on est forcé de lui donner beaucoup de soins dans sa jeunesse, et de l'abriter contre les gelées. Ce n'est que lorsqu'il est âgé de six à huit ans qu'on peut

' Voyez l'intéressante notice de M. Renou, inspecteur des forêts de l'Algérie, dans le $3^{e}$ vol. des Amales forestieres, année 1844, pages 1 à 7 . 
l'abandonner à lui-mème; il résiste alors aux plus: grands froids.

Les montagnes paraissent lui convenir mieux que les plaines, et la température, que lui offre son pays natal, semble exiger qu'on ne le cultive pas dans des parties trop exposées aux ardeurs du soleil.

38ə. Terrain. - Les sols graveleux, un peu secs et profonds paraissent convenir au cedre. Ainsi que les autres conifères dont nous arons parlé, il ne vient ni dans les terres trop compactes, ni dans celles qui sont marécageuses.

\section{Floraison et fructification. - La floraison} est monoïque; mais, contrairement ì ce qui existe pour les autres essences, elle n'il lieu qu'en septembre et octobre.

Des observations faites sur les védres de l'.11gérie ${ }^{\prime}$ il résulte que les graines ne muirissent, dims cette contrée, que dans le mois de juillet de lin seconde année après la floraison, c'est-ì-dire 20 mois aprìs celle-ci. La lissémination s'opere, en général. trois ou quatre mois plus tard, à l'époque des pluies d'automne, et quelquefois mème seulement à la fin de l'hiver.

Comme pour le sapin. les écailles du cone du cèdre se détachent et tombent avec la graine.

Le cèdre cultivé commence à donner des fruils vers 50 ans.

- Voỵcz la notice déjà cilée. 
387. Jeunes plants. - En Afrique, les jeunes plants sont aussi robustes que ceux du pin sylvestre 297]; mais en France, ainsi que nous l'avons déjà ،lit, ils sont plus délicats et demandent de l'abri contre le froid pendant les six à huit premières années. Cette circonstance est un obstacle majeur à la culture en grand de cet arbre, mais qui ne doit pas faire renoncer à multiplier les essais dans différentes localités et à différentes hauteurs. Il est probable qu'après plusieurs générations, le cèdre finira par s'acclimater entièrement, ou qu'au moins on reconnaitra quel est le climat qui lui convient le mieux.

388. Feuillage. - Les feuilles du cèdre sont nom_ breuses, touffues, et donnent un couvert assez épais.

389. Racines. - Le cèdre pousse, avec un pivot très fort, de nombreuses racines latérales, qui s'étendent beaucoup et le fixent fortement au sol.

390. Croissance et durée. - La croissance du riedre est lente dans le principe, mais elle devient très active après les huit à dix premieres années. 11 peut acquérir plus de dix mètres de circonférence, une hauteur considérable, et son existence se prolonge, dit-on, pendant plusieurs siècles .

391. Qualités et usages. - On attribue en général au cèdre la force et l'incorruptibilité. Cependant tous los auteurs qui en ont parlé ne sont pas du mème avis; quelques-uns lui accordent peu de qualités. 
Quant à son poids, les uns le classent au rang des bois les plus légers, les autres l'assimilent presque au chêne. Il semble difficile, dans les circonstances actuelles, de juger un arbre dont la vie est aussi longue et qui ne peut atteindre sa perfection qu'à un àge avancé. Il faudrait savoir d'où ont été tirés les cèdres sur lesquels on a fait des expériences. Les plus vieux cèdres de l'Europe datent de 1683, et le premier qui a été planté en France fut apporté par Bernard de Jussieu, en 1734. Ces arbres subsistent encore. Ceux qui en sont provenus, et qui ont été abattus, n'auraient-ils pas acquis plus de qualités si on les avait laissés sur pied plus longtemps? Avaientils crù dans le climat et dans le sol convenables? En Asie, en Afrique, le cèdre habite de grandes élévations et, jusqu'à présent, on ne lui a donné plice en Europe que dians des pares et des jardins.

L'éloge que les anciens ont fait du cèdre, les grandes constructions auxquelles il a été employé, ct le respect religieux qu'il inspiricit autrefois, pourraient déterminer notre opinion à son égard, si l'identité de cet arbre n'avait pas étémise en question, et si l'on n'avait pas prétendu qu'on donnait jadis le nom de cèdres à différentes espèces résineuses.

Espérons que nos forèts de l'Algérie pourront bientòt fournir aux travaux publies, ainsi qu'à la marine, les moyens d'expérimenter sùrement cette importante essence, et que la sylviculture sera, par là, mise à mème de décider jusqu'ì quel point les 


\section{essais d'acclimatation tentés jusqu'ici méritent d'ètre continués et étendus '.}

- D'après quelques expériences faites en Afrique par des officiers du génie, concurremment avec des agents forestiers, le cèdre serait plus léger, moins élastique et plus cassant que le sapin; il offrirait aussi une résistance horizontale moindre que ce rernier arbre, qu'il pourrait, toutefois, remplacer dans beaucoup de circonstances, mais non égaler. Comme chauffage et comme charbon, il aurait à peu près la mème valeur. Le cèdre parât plus apprécié conme bois de travail. Il prend un beau poli, est agréablement nuancé, se faconne facilement et n'est pas sujet à la vermoulure. 


\title{
LIVRE DEUXIÈME
}

\author{
PRINGIPES FONDAMENTAUX \\ DE L'EXPLOITATION DES BOIS.
}

\section{DÉFINITIONS.}

392. Le nombre d'années déterminé pour l'exploitation d'une forêt se nomme révolution.

Or, comme il est de règle de retirer d'une forèt des produits ammuels, et que la régénération du lieu où le bois a été coupé doit être, autant que possible. une conséquence immédiate de cette coupe (roir l'Introduction), il s'en suit qu'une forèt exploitée ainsi par portions annuelles présente, la plupart du temps, autant de parties de bois différant entre elles d'un an d'àge, qu'il y a d'années dans la révolution.

393. Un hois est exploitable quand il a atteint iu maximum de son accroissement moven ou de son utilité. 
Il semblerait que l'aecroissement doit être le régulateur naturel de l'exploitabilité, mais les besoins de lil société et les intérêts des propriétaires empèchent souvent qu'il n'en soit ainsi.

394. L'accroissement d'un arbre est l'augmentation de volume résultant de son grossissement et de son allongement.

L'aceroissement ammel est le volume dont il s'augmente pendant une année; l'accroissement moyen, au contraire, est la moyenne tirée de tous les accroissements annuels, depuis la naissance de l'arbre jusqu'à la dernière année de son âge.

39̋̈. Les économistes désignent ordinairement par rente la portion du produit de la terre qui excède les frais de production, en comprenant dans ces frais un certiin bénéfice qui représente la rémunération légitime de l'industrie.

Mais, dans la langue usuelle et même dans le langage scientifique, on appelle aussi rente le rapport du revenu net au prix d'achat. C'est cette dernière définition que nous adopterons en disant que la rente exprime le rapport entre le reveru net d'un bois et sa valeur capitale.

396. On entend par possibilité lit quantité de malliere quion peut retirer annuellement d'une forêt, sous la condition que cette quantité sera conslante, autant que possible; résultat que nous exprimons par le terme de rapport ou rendement soutemu.

397. Par peuplement romplet, on exprime l'étit 
d'un bois composé du plus grand nombre de tiges possible, eu égard à leur âge et à leur volume.

398. Toute étendue determinée dans une forèt pour y abattre le bois, en totalité ou avec réserve d'un certain nombre d'irbres, se nomme coupe.

399. La désignation du lieu où doit se faire une coupe s'appelle l'assictte. Ainsi, asseoir une coupe, ou fuire l'assiette d'une coupe, c'est désigner son emplacement.

400. Par vidange, on entend le transport des bois hors d'une coupe.

401. Une coupe est en usunce, lorsqu'on l'exploite; elle est usée, quand elle est exploitée et vidée.

402. Les arbres déracinés ou rompus par les vents se nomment chablis; on donne le mème nom à ceux qui sont brisés sous le poids de la neige ou du givre.

La partic d'un arbre rompu, tombée à terre, s'appelle volis; celle qui demeure debout se nomme quille, chandelier ou tronc. 


\section{CHAPITRE PREMIER.}

DE L’EXPLOIT A BILITÉ.

AR'TICLE PREMIER.

De l'exploitabilité en général.

403. L'exploitabilité d'une forèt est la base de son traitement; elle détermine l'àge auquel les bois doivent être abattus, et nous met à mème (ainsi qu'on le verra bientôt) de déterminer la possibilité.

L'exploitabilité rarie selon les considérations d'après lesquelles on l'apprécie. Les objets que l'on considere, ensemble ou séparément, pour cette appréciation, sont : la longévité des essences, la plus grande production en matière, la rente la plus élevée et les qualités les plus utiles des bois.

Arrêtée d'après ces bases, l'exploitahilité peut itre physique, absolue, relative ou composée. 
ARTICLE II.

\section{De l'exploitabilité physique.}

404. Pour réaliser l'exploitabilité physique, on laisse croître les bois jusqu'à ce qu'ils ne prennent plus qu'un très faible accroissement et qu'ils aient acquis tout le développement dont ils sont susceptibles, en raison de la nature de l'essence, du sol et du climat. Alors on ne considère les forèts que par rapport à la longévité des essences, sans s'occuper de l'intérêt du propriétaire, ni des besoins de la consommation. C'est assez dire que cette exploitabilité ne peut trouver son application qu'exceptionnellement : par exemple, lorsqu'il importe de conserver certains bois, soit à cause de leur influence manifeste sur l'état climatérique d'un pays, soit comme préservatifs contre les avalanches et les éboulements, ou bien pour des motifs d'agrément.

\section{AR'I'ICI.E III.}

\section{De l'exploitabilité absolue.}

40วั. Lin bois est parvenu à son exploitabilité $a b$ solue, lorsqu'il a atteint l'àge de son plus grand accroissement moyen. C'est cette exploitabilité qu'il faut adopter lorsque, considérant la production en matière d'une manière absolue, on ne se propose d'autre but que le maximum de cette produclion. 
406. Pour prouver que le bois coupé à l'époque de son exploitabilité absolue fournit, dans un temps donné, les plus grrands produits matériels possibles, il suffit de faire voir qu'on en obtiendrait de moindres on devançant ou en dépassint cette époque.

Or, si l'on considere la marche de la végétation d'une forêt ou d'une portion de forèt, soumise à un traitement rationnel, on observe que, dans les premières années, les accroissements ammuels sont faibles, comparés à ce qu'ils deviennent plus tard; qu'ils augmentent ensuite jusqu'à un âge plus ou moins avancé, sans toutefois suivre une marche régulièrement progressive; puis enfin, qu'ils diminuent de plus en plus ${ }^{1}$. Parallèlement à cette marche des accroissements annuels, on constate que les accroissements moyens correspondants suivent aussi d'abord une progression croissante, puis entrent dans une phase à peu près stationnaire, et enfin tendent à décroître constamment.

${ }^{1}$ Ce sont surtout nos arbres forestiers les plus importants et les plus répandus (bois durs) qui, considérés en masse, onl, dans leur première jeunesse, un accroissement très faible, comparativement à ce qu'il devient plus tard. Le plus ordinairement, cet accroissement va en augmentant d'année en annéc jusqu'à l'âg“ de $60,80,90,100$ ans, et même au delà, selon les essences, les sols et les climats, en supposant toutefois que les essences soient appropriées aux sols et aux climats. Parvenu à ce point, l'accroissement demeure sensiblement le même pendant un certain temps, puis il commence à diminuer d'une manière plus marquée et finit par devenir presque nul. 
Cela posé, admettons deux forêts parfaitement égales sous tous les rapports et soumises au mème mode de traitement: l'une, dont l'exploitation soit fixée au terme de son plus grand accroissement moyen, 100 ans, par exemple; l'autre, qui s'exploite à un terme beaucoup plus rapproché, tel que celui de 25 ans. Il est évident que la première, parcourant dans le délai de 100 ans, toute l'échelle ascendante de la végétation, fournira un produit matériel à la composition duquel auront contribué les années les plus favorables à la production; tandis que la seconde, coupée quatre fois pendant ce temps, se trouvera rejetée ainsi quatre fois dans la phase du plus faible accroissement, et ne pourra rendre, par conséquent, que des produits beaucoup moindres.

Supposons, au contraire, que l'une de ces deux forêts continue à être coupée à 100 ans et que la révolution de la seconde soit portée à 200 , il est non moins évident que cette dernière donnera dans ce délai de 200 ans moins de bois que la première, parce qu'après 100 ans, elle entrera dans une période de décroissince pendant laquelle l'autre parcourra une seconde fois l'échelle ascendante des accroissements, et atteindra de nouveau les àges moyens les plus favorables à la production.

Donc, dans les deux cas, soit que l'on devance, soit que l'on dépasse l'époque de l'exploitabilité absolue, la production est moindre que si l'on s'en tient à cette époque. 
407. De la proposition que nous venons de démontrer, ainsi que de la définition des accroissements [394\}, on déduit lacilement les corollaires suivants :

$1^{\circ}$ Tant que l'accroissement annuel augmente, l'accroissement moven augmente évidemment aussi;

$2^{\circ}$ Lorsque l'accroissement annuel diminue, l'accroissement moyen continue à augmenter aussi longtemps que l'iccroissement annuel, tout en diminuant, reste plus grand que l'accroissement moyen correspondant;

$3^{\circ}$ L'accroissement moyen atteint son point culminant lorsqu'il derient égal à l'accroissement annuel correspondant;

$4^{\circ}$ Le plus grand accroissement moyen ne se réalise nécessairement que dans la phase descendinte des accroissements annuels, d'où il suit que l'écart entre le maximum anmuel et le maximum moyen est plus ou moins grand, selon les conditions de la végétation.

408. La détermination rigoureuse de l'exploitabilité absolue d'une forêt est une question de calcul importante, qui exige une suite de recherches et d'expériences dont le détail ne saurait trouver plice ici. Au surplus, l'observation attentive de la marche de la croissance des bois a fourni à cet égard des indications assez précises pour pouvoir suppléer au calcul dans un grand nombre de cas.

les arlures sont en bon état de végétation, et leur ac- 
croissement augmente progressivement, lorsque les pousses annuelles sont fortes et allongées, le feuillage abondant et d'un vert vif et brillant, l'écorce unie, les jeunes branches souples et relevées vers le trone, l'extrémité de la cime fortement saillante.

L'accroissement a atteint son point culminant et devient stationnaire aussitôt que les pousses annuelles sont plus faibles et moins allongées que celles des années précédentes, et que la flèche de la cime est moins prononcée ${ }^{1}$.

Un arbre entre en retour ou en décroissance, lorsque la cime n'offre plus qu'une tète arrondie, et lorsqu'on voit en automne les feuilles du sommet jaunir el tomber plus tôt que celles des branches inférieures ${ }^{2}$. Ciette décroissance devient très marquée lorsque l'arbre commence à se courommer, c'est-àdire, lorsqu'il meurt quelques branches à la cime.

Le dépérissement est arrivé quand l'écorce se gerce profondément, se sépare du bois, et que par les ger-

- A ce sujet, il faut remarquer cependant qu'il serait possible quo la dernière année observée n'eût pas été favorable à la végétation à cause des froids, de la sécheresse ou des insectes. Dans co cas, la fáblesse de li pousse n'aurait qu'une cause accidentelle et momentanée, indépendante do l'ige ot do la vitalité du bois.

${ }^{2}$ Ce dernier signe, naturellement, ne peut exister chez les arbres résineux; mais le prenier, en revanche, est d'autant plus caractérisé chez cux, qu'il contraste davantage avec la forme parfaitement conique qu'affecte leur cime, lorsqu'ils sont en pleine croissance. 
çures on aperçoit des écoulements de sève; quand les mousses, les lichens, les agarics et les champignons s'attachent en grande quantité à l'écorce, et qu'on la voit marquée de taches noires et rousses.

AR'TICLE IV.

\section{De l'exploitabilité relative.}

409. Lorsqu'on se propose d'obtenir soit lit rente la plus élevée, soit les bois les plus utiles sous le rapport de leurs dimensions et de leurs qualités, l'exploitabilité est dite relative.

410. L'exploitabilité relative de première sorte trouve son application toutes les fois que, considérant une forèt comme un capital placé en fonds de terre, on ne se propose d'autre but que de faire fonctionner ce capital aux conditions les plus profitables à l'intérèt pécuniaire du propriétaire. Dans ce cas, l'exploitation d'une forêt devient une affaire de pure spéculation, et la mesure de l'avantage, que procure l'opération, se trouve exprimée par la proportion entre le revenu net de la propriété et le capital employé à le produire. Or, ce rapport entre le revenu et le capital producteur n'est autre chose que le tanx de p'acement de ce capital ou ce que nous avons appelé li rente $\lfloor 39 \check{\rfloor}\rfloor$. Et comme le taux des placements en fonds de terre se trouve établi d'une manière à peu près invariable dans thaque locialité ou province 
d'un grand pays, d'après les conditions générales auxquelles s'y font les achats et les ventes de propriétés, il s'en suit que lorsqu'on veut appliquer à une forêt l'exploitabilité relative à la rente la plus élevée, il faut constituer les peuplements et fixer la révolution de manière à obtenir le revenu maximum, sans que le rapport entre ce revenu et le capital engagé dans la propriété, s'abaisse au-dessous du taux ordinaire des placements en biens-fonds dans la localité.

410 (bis). Le capital placé dans une propriété boisée se compose, à un àge quelconque : $1^{\circ}$ de la valeur du fonds de terre; $2^{\circ}$ de la valeur sur pied du matériel ligneux; $3^{\circ}$ de la somme dont les intérêts sont égaux aux frais annupls de garde. d'entretien et d'impoit ${ }^{1}$; $4^{0}$ de ces intérèts, accumulés cux-mèmes avec intérits, depuis lit maissance du massif. - A ce capital s'ajoute chaque amnée: $1^{\circ}$ la valeur d'un accroissement anmuel ${ }^{2}$ augmentée de

- Une propriété qui s'achète $20,000 \mathrm{fr}$., et qui entraîne à $100 \mathrm{fr}$. de frais anmuels, coute en rélite 2.2,000 fr. (cu armettant le taux de $: ; \%$ ); car, pour faire fiace ì ces firais, l'acquéreur se prive de la jouissance l'un capital de 2,000 fr. De même, si la propriété est revendue $20,000 \mathrm{fl}$., lo rendeur rentre dans la jouissance du capital correspondant aux 100 fr. de frais, ce qui, de fait, porte le prix de vente à $22,000 \mathrm{fr}$.

${ }^{2}$ L'accroissement annuel du matériel ligneux est une fonction rouble du fonds de terre et des accroissements antérieurs, tout cnmme l'accroissement annuel d'une somme placée à intérèts composés est une fonction double du capital primitif et des intérêts accumulés depuis le jour où le placement a eu lieu. 
la plus-value acquise par le bois en vieillissant; $2^{\circ}$ une année de frais, plus les intérêts, pendant un an, des frais déboursés jusqu'alors.

Le revenu, au mème àge, c'est l'accumulation des valeurs des accroissements annuels que le bois a pris depuis sa naissance; ce n'est donc autre chose que la portion du capital producteur comprise dans le matériel ligneux. - Ce revenu s'accroit, chaque année, de la valeur d'un accroissement annuel augmenté de la plus-value acquise par le bois en vieillissant.

Le capital producteur, tout en s'augmentant d'une même quantité que le revenu, s'augmente done en outre d'une valeur qui croit avec la puissance des intérêts composés; et, quelle que soit la valeur progressive des accroissements annuels, il parait évident qu'elle ne peut pas être longtemps proportionnelle à cette puissance.

Il suit de là que, pour chaque année de retard apportée à l'exploitation, le rapport entre le revenu et le capital producteur, c'est-à-dire, la rente ou le taux de placement, virie avec le rapport des quantités dont ils se sont accrus, et devient égal, à un moment donné, au taux admis dans la localité.

Remarquons encore que, en exploitant un an plus tòt, le propriétaire aurait pu placer à intérêts la somme réalisée, et qu'il se serait produit un nouveau peuplement; nous pourons done conclure que: nour que le propriétaire ait intérêt à différer sa coupe l'une année, il faut que le matériel ligneux acquière 
une plus-value supérieure à l'intérêt de la somme qu'il aurait pu réaliser, un an plus tót, augmenté de la valeur des bois qui se fussent reproduits si l'exploitution avait en lieu l'année précédente.

Celte exploitabilité répond évidemment le mieux à la spéculation particulière; elle permet d'abréger les révolutions le plus possible et se réalise, généralement, avant l'exploitabilité absolue '.

411. Ouand l'exploitabilité n'est relative qu’à la qualité et aux dimensions des bois, elle peut, selon les circonstances, tantùt dépasser, tantôt devancer plus ou moins l'époque de l'exploitabilité absolue.

Le premier cas se présentes'il s'agit, par exemple, d'obtenir de fortes pièces pour les constructions naviles ou autres; le second, si la consommation ne demande que des hois de fatibles dimensions, tels qu'il en faut pour cercles de futailles ou pour paisseaux de vigne ${ }^{2}$.

- Il peut même se présenter de's cats vù un propriétaire aurait aviantage à abattre son bois avant qu'il ait atteint l'exploitabilité relative que nous considérons ici : par exemple, si des circonstances exceptionnelles et momentanéc: lui offraient à la fois l'occasion de placer' sa marchandise à des prix élevés qu'il n’espérait pas en retirer plus turd, et un emploi avantageux et sûr de ses capitaux dans un autre ordre de spéculation.

'Dans ce.dernier cas, l'exploitabilité de seconde sorte se confondrait évidemment avec celle de première sorte. 
A RTICLE V.

\section{De l'exploitabilité composée.}

412. L'exploitubilité composée trouve son application lorsqu'il s'agit d'obtenir, dans un temps donné, ou le plus grand produit matériel joint à la rente la plus élevée, ou li matière à la fois la plus considérable et la plus utile.

413. La réunion des deux premières conditions est réalisable dans les bois qui, soit à cause de la nature de l'essence, soit à cause de la mauraise qualité du fonds, entrent en décroissance à un âge peu avancé, avant lequel ils n'auraient pas de valeur commerciale assurée: Alors, en effet, lia question matérielle, tout comme la question financière, commande une rérolution de courte durée. Cette exploitabilité convient évidemment au plus haut degré à l'intérèt des particuliers, sans que l'intérèt général soit sacrifié. Au point de vue de ce dernier, il est done exact de dire que les forêts médiocres sont les seules bien placées entre les mains des particuliers.

414. Au contraire, réunir les deux autres conditions, c'est-à-dire, obtenir d'une forèt les produits les plus considérables et les plus utiles, c'estrépondre àl'intérêt général, mais au détriment du propriétaire si ce dernier est un particulier; et c'est, par conséquent, pour les forèts de l'Élit surtout, qu'on doit 
adopter l'exploitabilité dont il s'agit; on le doit notamment pour les forêts où les essences les plus importantes, les plus précieuses et les plus répandues, (chêne, hêtre, sapin, épicéa, pin sylvestre, etc.,) occupent le sol qui leur convient. L'accroissement de ces bois parcourt effectivement d'abord une longue période ascendante; puis, après avoir atteint son apogée, il se soutient pendant assez longtemps à peu prìs au même niveau. Il résulte de là qu'ils peuvent, sans décroìtre sensiblement, être conduits à un âge avancé, et acquérir, par suite, les dimensions et les qualités réclamées pour les emplois les plus intéressants. 


\title{
CHAPITRE DEUXIËME.
}

\author{
DE LA POSSIBILITÉ.
}

AR'TICLE PREMIER.

\section{De la possibilité en général.}

415. Dans une forèt telle que la théorie peut la concevoir, où les essences seraient parfaitement appropriées au sol, le peuplement complet dans toutes ses parties, et les âges des bois convenablement gradués ${ }^{1}$, dans une forêt normale, enfin, la possibilité doit être égale à l'accroissement moyen. Il est évident, en effet, que si l'on exploite chaque année un volume exactement égal à celui dont la forêt s'accroit moyennement par an, celle-ci pourra fournir le même produit annuel à perpétuité.

- Ce que l'on pourrait concevoir de plus parfait sous le rapport de la gradation des âges, ce serait une forêt qui, sur des étendues égales ou proportionnelles à la ferlilité du sol, présenterait des bois de tous les Ages, depuis l'arbre exploitable jusqu'au brin naissant. 
PRINC. FOND. DE L'EXPLOITATION DES BOIS. 187

416. Mais, comme dans la réalité les forèts sont toujours plus ou moins éloignées de l'état normal, et que d'ailleurs une foule de circonstances fortuites rendent difficile et incertaine l'exacte détermination de cet accroissement moyen, on se contente ordinairement de régler la quotité à exploiter par année. de manière qu'elle ne varie que le moins possible. C'est là ce qui reut dire: exploiter sous la condition d'un rapport soutenu.

La plupart du temps, il n'importe point, au surplus, d'arriver à une minutieuse parité entre les produits annuels et successifs, il s'agit seulement d'éviter sous ce rapport de brusques inégalités, de nature à léser à la fois et le propriétaire et le consommateur.

417. Pour déterminer lit possibilité, on peut adopter deux bases différentes: l'étendue et le volume.

AR'TICI,E II.

\section{De la possibilité par étendue.}

418. La possibilité fondée sur l'étendue s'obtient en divisant la surface totale de la forèt par le nombre d'années de la révolution, ce qui fait connaitre la contenance de la coupe annuelle. Pour procéder ainsi, on admet que les produits matériels sont entre eux comme les surfaces; d'oủ il suit qu'égaliser les unes, c'est égaliser les autres. Cette supposition 
toutefois n'est jamais rigoureusement vraie, et elle ne devient admissible que quand les essences, la. qualité du sol et la croissance du bois ne présentent généralement que peu de dissemblance. Mais il ne saurait en être ainsi dans une forèt de quelque étendue, où la situation, l'exposition et la nature du terrain apportent souvent des différences très tranchées dans l'état du bois, et où, par conséquent, sur d'égales contenances, les produits matériels peuvent ètre d'une inégalité extrême. Dans ce cas, il est évident que l'hypothèse dont il vient d'être question, cesse d'ètre admissible et que, sous peine de renoncer au rapport soutenu, il devient nécessaire de procéder autrement.

419. L’idée qui se présente d'abord pour atteindre le but, c'est de rendre les contenances des coupes proportionnelles à la fertilité du terrain. Toutefois, ce moyen n'est pas celui que l'on emploie communément, parce qu'il en existe un autre qui, plus simple en pratique, satisfait en outre à plusieurs conditions importantes.

Voici comment on procède :

420. On scinde la forêt en un certain nombre de grandes divisions, suivant les différences les plus générales du terrain et du peuplement, mais sans s'arrèter à celles qui se feraient remarquer sur de faibles étendues. Dans ces divisions, on cherche à réunir, autant que possible, des bois dontles àges soient convenablement gradués, puis on considère chacune 
d'elles comme un tout isolé qui, en présentant l'homogénéité désirable, rentre ainsi dans les conditions voulues pour permettre de baser la possibilité sur la contenance. Ces divisions ont reçu le nom de séries d'exploitation, parce que chacune d'elles est effectivement destinée à fournir, durant toute la révolution, une série de coupes successives et annuelles. Outre la facilité qu'elles donnent pour la fixation de la possibilité particulière à chacune d'elles, elles créent encore des chances de voir leurs produits annuels respectifs se compenser entre eux, ce qui tend dès lor's à assurer d'autant mieux le rapport soutenu pour la forêt considérée dans son ensemble. Enfin elles permettent d'appliquer à chaque localité, sans faire naître de confusion, la révolution et le traitement convenables et d'établir des exploitations permanentes sur les divers points de la forêt, où la consommation et l'intérêt du propriétaire peuvent les réclamer.

421. La possibilité par étendue mérite la préférence dans un grand nombre de cas, à cause de sa simplicité et de la régularité qu'elle imprime à la marche des exploitations; cependant, dans les forêts soumises au mode d'exploitation de la futaie (III livre), il devient impossible de l'adopter, en raison des exigences culturales, et on doit recourir à la possibilité fondée sur le volume. 
ARTICLE III.

\section{De la possibilité par volume}

422. Pour déterminer la possibilité par volume, on concoit qu'on pourrait procéder d'une manière analogue à celle qu'on applique à la fixation de la possibilité par étendue: Il n'y aurait qu'à substituer à la contenance de la forêt, la masse totale de bois à y abattre dans le cours de la révolution donnée, et à diviser ce volume par le nombre d'années de cette révolution; le quotient ferait connaitre la quotié de la coupe annuelle.

423. Mais pour suivre une telle marche dans la pratique, il se présente plusieurs difficultés majeures, dont la principale gìt dans l'appréciation exacte du volume à employer comme dividende. Pour le trouver, il ne suffit pas, en effet, d'estimer le matériel actuel en bois, il faut encore estimer celui qui résultera de tous les accroissements successifs pendant la révolution. Or, il est facile d'entrevoir tout ce qu'une pareille opération présente d'éventuel et de problématique, surtout lorsque la révolution est longue.

De toutes les différentes méthodes plus ou moins ingénieuses, plus ou moins compliquées, qui ont été imaginées jusqu'ici pour déterminer la possibilité en fonction des produits matériels de toute lit révolu- 
tion, aucune n'a encore conduit exactement au but, parce que la nature des choses ne permet pas d'y atteindre. Mais on peut parvenir à concilier le rapport soutenu de la forèt avec l'ordre et la régularité des exploitations, sans se baser sur une possibilité aussi rigoureusement déduite, et en procédant d'une manière simple, expéditive et cependant suffisamment sûre.

424. Après avoir, s'il y a lieu, partagé la forêt en séries [420], et avoir fixé le terme de la révolution pour chacune, on divise de même la durée de cette révolution en plusieurs époques ou périodes, afin d'embrasser plus aisément la marche des exploitations, et de pouvoir vérifier et rectifier cette marche avec plus de facilité, si, à un moment quelconque, on le jugeait nécessaire. Il convient, en général, que ces périodes soient des parties aliquotes 1 de la révolution et, de plus, égales entre elles; le plus ordinairement on les fait de 20 ou 10 années, et, dans ce dernier cas, on leur donne le nom de décennies.

425. Cela fait, on opère sur chaque série séparément, et l'on s'occupe d'y assigner à chaque période ou décennie les parties destinées à être exploitées pendant sa durée. On colloque dans la première les bois les plus vieux, et successivement dans les autres les bois moins àgés, jusqu'à la dernière, où

- Quantité contenue exactement un nombre entier de fois dans un tout. 
doivent se trouver les plus jeunes ${ }^{1}$. Dans ce travail, on examine attentivement les différences qui se présentent dans l'état du bois, selon le sol, le climat et le peuplement plus ou moins complet, circonstances d'où dépend la production. Si, sous ces divers rapports, il n'existe que des nuances, on affecte aux périodes des contenances égales parce que l'on peut considérer qu'en raison de l'étendue de ces affectations périodiques, les nuances dont il est question se compensent suffisamment pour pouvoir être négligées.

Les affectations égales en contenances doivent être. préférées autunt que possible. Mais quand les différences dans l'état du bois sont très prononcées et se présentent sur d'assez grandes surfaces pour influer d'une manière notable sur les produits matériels, et quand, surtout, l'intérêt du propriétaire réclame une égale répartition des produits par période, il devient alors nécessaire d'attribuer aux affectations des contenances proportionnelles. La pro-

' Certaines circonstances peuvent quclquefois faire classer une partie de bois dans une période à laquelle elle n'appartiendrait pas par son age : telles sont, par exemple, l'enclave au milieu d'autres parties dont il conviendrait qu'elle subît lo traitement pour satisfaire aux règles sur l'assiette des coupes, ou à d'autres principes de culture; le dépérissement prématuré; etc.

La saine application des principes dont nous venons de parler et qui seront l'objet de nos études, de même que l'inspection attentive et réfléchie des localités, fait toujours facilement découvrir et apprécier ces diverses circonstances exceptionnelles. 
portion s'établira facilement, si, rapportant chacun des différents états de production à un seul, pris pour unité, on l'exprime par le résultat de la comparaison. Le rapport des surfaces sera alor's évidemment l'inverse de celui des productions, de sorte qu'à un état de production double ou triple, par exemple, correspondra une contenance deux fois ou trois fois moins grande. Par conséquent, les affectations périodiques seront inégales en contenance, pour se trouver égales quant aux produits matériels qu'elles devront fournir.

Par ces affectations suffisamment égales quant aux produits, on assure pour toute la rérolution le rapport soutenu par période, sans néanmoins employer d'autres moyens que la simple appréciation du peuplement plus ou moins complet et de la fertilité plus ou moins grande du sol et du climit. Dégagé dès lors du soin de l'avenir, on n’a plus à s'occuper que des besoins actuels, et ce n'est que dans l'affectation de la première période que l'on cherche les éléments de la possibilité qu'il s'agit de fixer.

426. Dins ce but, on détermine d'abord le volume actuel des bois de l'affectation; en second lieu, leur accroissement probable pendint la période. Si l'on ne veut consacrer à cette double détermination que le moins de temps et le moins d'argent possible, elle peut se faire à l'aide de procédés sommaires, c'est-à-dire par de simples comptages d'arbres, 
dont on estime le volume au jugé; on ajoute ensuite quelque chose pour tenir compte de l'accroissement probable jusqu'au moment de l'abatage ${ }^{1}$. Et, comme on ne peut savoir positivement dans quelle année de la période les différentes parties de son affectation viendront en tour d'exploitation, on calcule l'accroissement de tous les bois comme s'ils devaient être abattus pour le milieu de la période. Divisant en-

'Quand les bois approchent de l'époque de leur exploitabilité absolue, ou lorsqu'ils l'atteignent, ils ont ordinairement un accroissement annuel de $2 \frac{1}{2}, 2,1 \frac{1}{2}$, ou même un pour cent de leur volume, selon les circonstances dans lesquelles ils végètent.

Lors done que l'on connaîtra le volume d'une affectation périodique, on pourra, par ce moyen, déterminer approximativement de combien il s'accroîtra jusqu'à l'abatage. Soit, par exemple, ce rolume, 10,000 stères, et que l'on estime l'accroissement annuel à 2 pour cent, l'augmentation par an sera de 200 stères.

On peut encore se contenter de l'accroissement moyen, pour en conclure l'aceroissement futur. Dans ce cas, il suffit, comme on le sait, de diviser le volume actuel par l'àge du bois. Ce moyen extrèmement simple et prompt, a l'avantage de donner généralement des résultats un peu au-dessous de la vérité et d'empêcher, par conséquent, que la possibilité ne soil dépassée.

2 On conçoit que cette manière de procéder puisse donner un résultat, sinon exact, du moins suffisamment approché. Il est évident, en effet, qu'une moitié des bois composant l'affectation sera coupée dans la première moitié de la période, et que l'autre moitié de ces bois ne sera coupée que dans la seconde moitié de la période. Done en calculant l'accroissement de tous les bois pour l'année moyenne de la période, on compense l'accroissement des arbres qui ne scront coupés qu'après cette époque par l'accroissement des arbres qui seront coupés avant. 
suite le total par le nombre d'années de la période, on obtient la possibilité cherchée.

Les procédés plus rigoureux que nous enseigne l dendrométrie 1, savoir, le cubage sur pied des arbres, au moyen de dendromètres, et la détermination de leur accroissement futur, d'après leur accroissement actuel, donnent en général un résultat plus exact, et doivent ètre préférés quand cela est possible. Mais, à quelque mode d'estimation que l'on ait recours, il sera toujours facile de rectifier les résultats obtenus qui, naturellement, ne sont pas exempts d'une certaine erreur. Il suffira, pour cela, de s'assurer, après quelques années d'exploitation, si les bois restés debout dans l'affectation de la période. pourront continuer à fournir le mème produit annuel jusqu'à la fin, et, dans le cas contraire, de faire les corrections nécessaires. En répétant cette opération plusieurs fois, si on le jugeait utile, dans le courant de la période, on finirait par approcher beaucoup de la vérité, la vérification devenant de plus en plus facile et de plus en plus exacte, au fur et à mesure qu'il reste moins de bois sur pied.

427. La première période expirée, on procédera de même pour fixer la possibilité de la seconde, et l'on examinera, en mème temps, s'il n'y a pas lieu d'apporter quelques changements aux affectations périodiques, par suite de circonstances survenues

' Dendron, arbre; metron, mesure ; dendrométrie, art de mesurer les arbres. 
dans le cours de la période écoulée, et qui influeraient notablement sur la production; et ainsi de suite de période en période, jusqu'à la fin de la révolution.

Chaque nouvelle possibilité différera sans doute, dans li plupart des cas, de la précédente, mais jamais au point de compromettre gravement le rapport soutenu. Or, c'est là le degré d'approximation auquel il s'agit d'atteindre.

428. Le mode de fixation de la possibilité par volume, tel qu'il vient d'ètre développé, doit, selon l'état des forèts que l'on rencontre, éprouver des modifications. Nous les discuterons en parlant de chaque état en particulier. 


\section{CHAPITRE TROISIENME.}

\section{DE L'ASSIETTE DES COUPES.}

ARTICIE PRMIER.

De l'assiette des coupes en général.

429. Lorsqu'il s'agit d'établir une marche rationnelle dans les exploitations d'une forèt, l'assiette des coupes est aussi importante et souvent aussi difficile que la fixation de la possibilité. Elle exerce l'influence la plus directe sur la prospérité et sur lí conservation des bois, et mérite, par conséquent, l'attention toute spéciale du forestier.

Les règles sur lesquelles elle s'appuie sont au nombre de cinq; mais il n'est pas toujours possible de se conformer à toutes à la fois, parce que souvent l'application de l'une contrarie l'application de l'autre. Quand une pareille opposition se présente, c'est au forestier à savoir, d'après les circonstances locales, à laquelle de ces règles il doit domer la préférence. 
ARTICLE II.

\section{Première règle.}

430. Dans une même série d' exploitation, les coupes doivent être assises de manière qu'elles se succèdent de proche en proche, et qu'elles aient la forme la plus régulière possible.

Quand l'assiette des coupes se fail sans suite, le jeune bois qui se trouve sur les limites de ces coupes, souffre toujours plus ou moins du couvert des arbres voisins, et, lorsque ceux-ci viennent à être exploités, ils lui causent encore un dommage notable par l'abatage, le façonnage et surtout par la vidange. Si les limites sont irrégulières et forment des sinuosités profondes, ce dommage est d'autant plus grand. Enfin, lorsque les exploitations ne se suivent point, il devient plus difficile d'exercer sur les jeunes coupes toute la surveillance qu'elles réclament, en raison des nombreux dégâts que peuvent y commettre les hommes et les animaux.

En observant la règle que nous venons d'énonicer, on évite tous ces inconvénients, et l'on se procure de plus l'avantage de juxtaposer des bois peu différents d'ìge, qui se prêtent un mutuel appui contre les vents, la neige, le givre, etc., et se poussent réciproquement dans leur croissance en hauteur.

Irais dans l'état irrégulier où se trouvent nos forèts 
actuelles, on peut rarement établir, dès à présent, une suite non interrompue de coupes; il faudrait pour cela consentir à exploiter certaines parties bien avant, et d'autres bien après l'àge d'exploitabilité, ce qui entrainerait nécessairement une perte plus ou moins considérable de produits. On doit done (du moins pour la première rérolution) se contenter d'approcher de l'ordre désirable, en disséminant les coupes le moins possible, et, dans ce but, ne pas hésiter à faire quelques sacrifices, si la trop grande inégalité d'àge des bois les rendait nécessaires.

\section{ARTICI, E III.}

\section{Deuxième règle.}

431. Les coupes doivent être disposées de manière que les bois d'une coupe en exploitation ne soient pas dans le cas d'être transportés à traver's d'autres coupes précédemment exploitées.

Cette règle a pour but de faciliter les transports, et particulièrement d'éviter les dommages des vidanges qui, ne pouvant s'exécuter la plupart du temps qu'à l'aide de nombreux charrois, sont surtout ruineuses dans les jeunes coupes. Pour s'y conformer, il suffit que chaque coupe soit indépendante des autres, et qu'elle aboutisse directement, soit sur une route ou un chemin, soit sur un ruisseau où le bois puisse se flotter, soit enfin sur les terres riveraines. 
ARTICLE IV.

\section{Troisième règle.}

432. Dans toute forêt ou série d'exploitation, les coupes doivent être assisesde manière que celles qui sont à exploiter au commencement de la révolution, se trouvent placées du côté du Nord ou de l'Est, et les dernières du. côtédu. Sud ou de l'Ouest.

Ce sont les vents soufflint de ces deux points de I'horizon, qui, en général, causent le plus de dégâts dans les forêts, parce que, étant d'ordinaire accompagnés de pluies et très souvent d'orages, ils détrempent la terre et déracinent ainsi plus facilement les arbres. Les vents du Nord et de l'Est au contraire, outre qu'ils sont ordinairement moins violents, amènent presque toujours la gelée ou la sécheresse, et, dans ce cas, les racines offrent plus de résistance.

Il est donc très essentiel que les arbres réservés en plus ou moins grand nombre dans les coupes, selon le mode d'exploitation adopté, soient garantis le plus possible des vents dangereux par la masse de la forêt.

Celte direction des coupes présente un avantage de plus dans les forêts d'essences à semences légères, parce que ces semences, qui se disséminent en grande partie par les vents chauds du Sud et de l'Ouest, sont dès lors portées, des portions demeurées intactes, 
dans celles que l'on exploite, et elles augmentent ainsi les chances de régénération.

Quoi qu'il soit à conseiller d'appliquer la troisième règle à toutes les essences, mème au chêne qui est la plus fortement enracinée, il faut observer, cependant, qu'elle est surtout d'une haute importance dans les forêts de bois résineux qui, en général, ont une tige plus élevée et moins de racines que les bois feuillus.

La situation des forêts peut seule amener des cas d'exception. On sait, par exemple, que sur les bords de la Méditerranée, ce sont trìs fréquemment les vents d'Est qui apportent les pluies, et il est également notoire que, dans les pays de montagnes, lit configuration du terrain peut être telle, que les rents, quoique soufflant du Sud et de l'Ouest, viennent cependant s'engouffrer dans les villées, où ils sont détournés de leur direction primitive. Dans de pareilles circonstances, la règle qui nous occupe doit nécessairement subir les modifications indiquées par la localité.

433. On ajoute aux arantages de la règle que nous venons d'examiner, en laissant subsister sur les lisières Ouest et Sud, lorsque les exploitations y parviennent, un rideau d'arbres en massif, d'une profondeur plus ou moins grande (de 15 à 30 mètres), selon que la situation est découverte ou abritée, l'essence traçante ou pivotante, etc. On sait que, sur les lisières, les arbres sont en général plus 
branchus, moins élancés et plus enracinés que dans l'intérieur de la forèt, et que, s'étant développés sous l'action continuelle des vents, ils résistent mieux, par suite, à leur violence.

En n'exploitant ce ridenu que 10 ou 20 ans après que la régénération des parties contiguës sera complètement assurée, on n'aura plus, alors, à se préoccuper que du faible espace sur lequel il s'étend et, au besoin, on le repeuplera artificiellement ${ }^{1}$.

ARTICLE V.

\section{Quatrième règle.}

434. Enmontagne, il faut couper d'abord les parties inférieures, et conserver les supérieures pour les demières exploitations.

Ce sont les sommités qui sont le plus exposées aux ravages des vents, et, lorsqu'elles sont boisées, elles en diminuent la violence. En commençant done l'exploitation par les plateaux, on doit craindre que les parties des pentes, immédiatement contiguës, ne soient plus suffisamment garanties et que, n'étant

' Le maintien d'un ridcau peut être utile encore pour garantir certaines essences contre les vents desicéchants ou froids, qui souvent nuisent considerablement aux jeunes plants dans les premières annes de leur existence. Dans ce cas, ce n'est pas au Nidi et à l'Ouest, mais au contraire, au Nord ou à l'Est que l'abri doit être établi. 
pas habituées ì subir l'action entière et directe des ouragans, elles ne se trouvent compromises dans leur existence et dans leur régénération. En outre, les semences tombant naturellement du haut vers le bas des montagnes, il est utile que les hauteurs restent garnies le plus longtemps possible, dans l'intérêt du réensemencement des parties inférieures.

Tels sont les motifs qui ont dicté cette règle. Mais, que l'on commence les coupes par en haut ou par en bas, il est évident que si l'on ne pouvait assurer à chaque coupe quelque moyen direct de vidange, on retomberait tôt ou tard dans l'inconvénient de faire traverser aux bois de la coupe en exploitation les coupes précédemment exploitées.

On remédie à cet inconvénient en pratiquant un chemin de voiture en lacets, de manière que toutes les coupes viennent y aboutir. Parfois on établit aussi des chemins à tranneaux ${ }^{1}$ et des lan-

- Pour établir ces chemins, on creuse dans la côte un sentier, dans une direction sinueuse et conrenablement en pente, et on le garnit de rondins ou de hois de quartier, placés parallèlement en travers du sentier, et à la distance de fä à 60 centimètres les uns des autres ; chaque bûche est maintenuc par un ou plusieur's piquets enfoncés à fleur de terre. Des deux côtés de ces loûches on adapte parfois des perches, afin d'empècher le traîneau de sortir de la voie, et l'on a soin de graisser avec du lard, du suil ou du savon les endroits des bûches sur lesquels les membrures du traineau doivent frotter, pour rendre la descente d'antant plus facile quand le traîneau est fortement chargéi. A côté de ce chemin à traîneau on pratique un autre sentier, ou du moins on 
coirs 1 . Dans certains cas, cependant, aucun de ces moyens ne peut ètre employé, soit à cause de la pente trop escarpée de la montagne, soit parce qu'elle se

établit un nombre suffisant de places sur les côtés, pour que les traineaux vides puissent être remontés sans rencontrer en chemin les traîneaux chargés.

\section{(Dictionnaire des forêts de Baudrillart.)}

1 Le lançoir ou glissoir artificiel consiste dans un canal de 66 centimètres ì 1 mètre d'ouverture, sur 50 centimètres de profondeur; il se compose de six à huit perches ou jeunes tiges d'arbres, longues, droites et unies, assemblées de manière à former un demi eylindre ereux, que l'on dispose pour que le bois qu'on y jette glisse de lui-même et se rende au bas de la montagne.

Les glissoirs qui ne sont destinés qu'an bois de chauffage ou de travail et mêne aux billes de sciage, peuvent être ririgés en ligne droite, du haut en bas de la montagne; mais quand il s'agit d'en construire pour faire glisser des pièces de longueur, on doit les diriger de manière qu'ils forment une longue courbe, afin que les pièces ne tombent pas avec trop de vitesse et n'éprouvent aucun dommage. On doit aussi diminuer la rapidité de la chute, vers l'extrémití du lanȩoir, en le rendant horizontal à cette extrémité, et l'on y pratique, lorsque cela est possible, un étang ou réservoir d'eau d'une profondeur suffisante pour amortir la violence de la chute et empêcher que les pièces ne se rompent.

Ordinairement, la surface du sol, dans les pentes de montagne, n'est pas telle que le lançoir puisse reposer immédiatement sur la terre dans tous ses points. On remédic à cet inconvénient par des cales ou baudets, qui donnent au glissoir le talus nécessaire.

Le lançoir est encore susceptible d'une amélioration importante, si, par un temps de gelée, on y répand de l'eau dont la congélation le revêt d'une croûte de glace, ou s'il tombe un peu de neige ou de verglas, ce qui lui procure également une sur- 
trouve traversée par des bancs de rochers trop considérables. Dans de telles circonstances, si, par une raison majeure on se décidait à commencer les coupes par le haut, il faudrait maintenir sur les plateaux un rideau d'une épaisseur suffisante [433] pour protéger les versants. Cet expédient devrait par exemple, ¿̀tre, employé si, à cause del'àge des bois, il était indispensable de régénérer d'abord les parties supérieures.

Quant au rideau lui-mème, mis ainsi, par destination, en dehors de toute régénération régulière, il serait traité d'après le mode jardinatoire [438] afin

face polie, et diminue le frottement des bois que l'on fitit glisser. Aussi dans les pays où l'on emploie ce moyen de vider les bois le pratique-t-on ordinairement pendant l'hiver.

La plupart des lançoirs ou glissoirs sont en bois; cependint, on en a fait en fer dans quelques pays's où l'usigge de transporter ainsi le bois est permanent. Dans le Vurtemberg, dit M. Hartig, il y en a un qui, n'aỹant que ‘z̈ centimetres de lingeur sur 27 centimètres de profondeur, est surmonté d'un toit jour que le bois ne puisse sauter en dehors.

(Dictionnaire de Baudrillart.)

Ces lançoirs sont un des movens de vidange les mieux entendus, et l'établissement en est bien moins coûteux et plus facile que celui d'un chemin à traîneaux. En y jetant les bois, on ne risque pas d'endommager les arbres voisins ni de détéliorer le sol au point de causer à la longue des ravines dangereuses, ainsi que cela arrive lorsque, conme autrefois dans les Vosges, par exemple, on lance immédiatement sur la terre. En outre, on ménage singulièrement le jeune bois et, lorsque l'opération est terminée et l'appareil enlevé, on apergoit à peine la trace du pass̀age des produits de la coupe.

(Note de l'Auteur.) 
206 PRINCIPES FONDAMENTAUX DE L'EXPLOIT. DES BOIS. que son action ne fùt jamais interrompue.

ARTICLE VI.

Cinquième règle.

435. Dans tous les cas, les coupes en montagne, autant que les localités le permettront, devront être longues et étroites et présenter leur plus petit côté aux vents dangereux.

Cette règle s'explique d'elle-même. 


\section{LIVRE TROISIËNIE}

\section{DE L'EXPLOITATION DES FUTAIES.}

\section{DÉFINITIONS.}

436. On nomme futaie la forêt destinée à produire principalement des bois de fortes dimensions et à se régénérer par la semence. En général, cette régénération doit s'opérer par les graines tombant naturellement des arbres, et ce n'est que par exception qu'elle a lieu artificiellement.

437. Une futaie est appelée régulière lorsqu'elle présente, dans toutes ses parties, un peuplement uniforme et complet, des àges convenablement gradués, et qu'elle renferme tous les éléments propres à assurer liı régénération naturelle. Le plus sûr moyen d'arriver à cette régularité est l'emploi de la méthode d'exploitation connue sous le nom de méthode du réensemencement naturel et des éclaircies, 
méthode qui sera développée dans le chapitre suivant.

438. On nomme irrégulière, au contraire, la futaie dont le peuplement est inégal et incomplet, où les àges sont mal gradués, et dans laquelle, par conséquent, ne se trouvent pas les conditions qui constituent la futaie régulière. Cet état est ordinairement dù à des exploitations routinières et vicieuses. Ainsi l'on trouve communément à l'état de futaie irrégulière, les forèts soumises au mode appelé jardinage et celles qui sont traitées à tire et aire, modes dont il sera question plus tard.

439. On exprime par clairière un lieu presque dé. garni de bois et qui ne peut se repeupler naturellement en raison du petit nombre d'arbres qui s'y trouvent.

440. Les places vides sont des espaces entièrement dégarnis de bois, ou tout au plus couverts de quelques petits arbrisseaux et arbustes.

441. On n'applique le nom de clairieres et de places viles qu'à de faibles contenances; lorsqu'elles sont considérables, elles prennent le nom de terres vaines et vagues.

442. Une partie de bois est en massif lorsque les branches des arbres se touchent sans ètre agitées par le vent.

443. Le massif est servé, quand les branches s'entrelacent; incomplet ou clairiéré, s'il existe de nombreuses clairières; entrecoupé, quand il $\mathrm{y}$ a des vides. 
44. Un massif de bois tout jeune. dont les tiges sont encore garnies de leurs branches dès la base, se nomme fourré; quand, par suite de l'état très serré de ces tiges, les branches inférieures sèchent et tombent, nous l'appelons gaulis ${ }^{1}$; enfin dès que les tiges atteignent à peu près 10 centimètres de diamètre au pied, nous qualifions le massif de perchis. Parmi les perchis, on distingue encore les hants-perchis et les bas-perchis. Ceux-ci se composent de tiges dont le diamètre ne s'éloigne pas beaucoup de 10 centimètres, tandis que les premiers peuvent présenter des tiges d'une grosseur double. Les hauts-perchis reçoivent aussi, diuns certaines localités, le nom de demi-futaies.

445. La dénomination de bois blane, impropre, mais consacrée par l'usage, indique, parmi les essences feuillues, celles de qualité médiocre et d'une contexture molle, quelle que soit d'ailleurs la couleur interne du bois : tels sontles aunes, les tilleuls, les peuplicrs et les saules. On les eùt mieux appelés bois tendres. Par opposition arec cette désignation de bois blancs ou bois tendres, on donne la dénomination générale de bois durs aux autres essences feuillues.

- Dans plusieurs départements, la dénominatien de guutis ost ronnce au peuplement que nuus appelons perrhis. Nous avons pensé que ce dernier terme convient aumitsill dont lasligesont déjà une certaine grosseur, tandis que le not yaulis, qui dérive de gaule, nous a paru s'appliquer mieux à des sujets d'un petit diamètre. 
446. On désigne par les mots morts-bois lit plupart des arbrisseaux dont la présence indique ordinairement le mauvais état des forêts: tels sont le sureau, le coudrier, le cornouiller mâle, le comouiller sanguin, le troëne, les viornes, le fusain, la bourdaine, le houx, lesépines, le genévrier, etc., etc.

4.7. Parles mots bois abromtis, on entend les jeunes bois broutés par les bestiaux ou par le gibier, et l'état de ces bois se nomme abroutissenent. Lorsque les essences sont de nature à bien repousser de souche, on remédie à cet état de détérioration par le recépage.

448. Recéper, c'est couper à fleur de terre les jeunes tiges, dans le but de les faire rejeter de sotiche. Cette opération se pratique pour régénérer non seulement les bois abroutis, mais encore ceux qui ont été dégradés par de lortes goclées, des incendies, et mème par la dent des mulots, qui souvent en pèlent presque entièrement l'écorce; on recépe dans le même but les bois qui ont langui pendant trop longtemps sous le couvert de grands arbres.

449. Mettre une forêt en défends, c'est en interdire l'entréeaux bestiaux, parce que les jeunes bois ne sont pas assez élevés pour échapper à l'abroutissement.

4วั0. On appelle forết défensable celle qu'on peut ouvrir au pàturage, parce qu'elle n'a plus rien à craindre de la dent du bétail.

4ว̈1. Lorsque, dans une coupe on laisse subsister un certain nombre d'arbres, pour un temps fet dans 
un but quelconques, ces arbres recoivent le nom de réserves et, pris dans leur ensemble, ils forment ce qu'on appelle la réserve de la coupe.

45้2. Les jeunes bois qui s'élèvent sous les réserves s'appellent le sous-bois.

453. Une coupe, exploitée sans aucune réserve. est une coupe à blanc-étoc. 


\section{CHAPITRE PREMIER.}

\section{MÉTHODE}

\section{DU RÉENSEMENGEMENT NATUREL ET DES ÉCLAIRCIES,}

ou

EXPLOITATION DES FUTAIES RÉGULIÈPES.

AR'TICLE PREMIER.

\section{Génóralités.}

4วั4. La méthode du réensemencement naturel et des éclaircies consiste à exploiter les futaies de manière à en assurer le repeuplement naturel et complet, et à favoriser le plus possible leur croissance, depuis la première jeunesse jusqu'au terme de l'exploitation.

Cette méthode repose sur des faits simples el peu nombreux observés dans la nature et, pour li plupart, expliqués par la physiologie végétale, science que l'on doit prendre pour guide dans toute méthode de culture. 
45\%. La graine ne germe que sous l'influence simultanée de l'humidité, de l'oxigène ${ }^{1}$ et d'un certain degré de chaleur. Quoique le sol ne soit point pour la germination un milieu indispensable, son action, quand la semence s'y trouve placée, n'en est est pas moins très utile. En effet, c'est par son intermédiaire que celle-ci reçoit, dans les proportions convenables, l'influence des trois agents quenous venons de nommer; il importe done que le sol ne soit ni trop léger ni trop compacte [43]. Il importe encore qu'il soil substuntiel; car dès que la radicule est développée, elle a besoin d'y trouver des sucs nourriciers qu'elle puisse s'assimiler facilement.

4วั6. La lumière n'est point nécessaive à la germination, et lorsqu'elle est très vive elle devient souvent nuisible; aussi lia nature nous montre-t-elle la plupart des graines germant à l'ombre. Mais aussitòt que la plantule a percé la terre, elle réclame la lumière qui, pour être bienfaisante, ne doit néanmoins lui être dispensée qu'arec mesure 2. L'action trop intense des rayons solaires peut tuer les plantes naissantes, et plusieurs essences forestières surtout, montrent, sous ce rapport, une grande susceptibilité [126 et 279].

Ce n'est qu'après avoir acquis un certain degré de force sous un abri protecteur, que le jeune plant

' L'un des gaz composant l'air atmosphérique.

2 Vovez de Candolle, Physiologic régétale, chap. r. 
peut, sans inconvénient, et même avec avantage, recevoir les influences atmosphériques. Cette époque varie selon le tempérament plus ou moins robuste des essences.

457. A partir du moment où les plants ont acquis assez de vigueur pour croitre librement sous l'action des météores, ils présentent, lorsqu'ils sont serrés en massif (ainsi que cela a lieu dans les forêts), une nouvelle série de phénomènes végélatifs.

Toute plante, pour croitre, exige un certain espace, aussi bien dans le sol pour y étendre ses racines, que dans l'atmosphère pour y étaler ses branches; et, àmesure de l'accroissement de la plante, l'espace qu'elle occupe devient nécessairement plus grand. Or, il est évident que, dans une jeune forêt, qui dès les premières années s'élève en massif serré, tous les plants composant ce massif ne pourront plus trouver place lorsque, par exemple, ils auront acquis un volume double. De là, la nécessité matérielle d'une diminution dans leur nombre, au fur et à mesure de leur accroissement. On remarque, en effet, dans un tel massif, qu'il s'engage une lutte entre les jeunes plants, qui tous cherchent à s'emparer, dans l'atmosphère, de la place qui leur est indispensable pour développer leurs branches et participer aux influences de la lumière. Dans cette lutte les brins les plus faibles, manquant d'espace, surmontés et dominés, ne tardent pas, ainsi que les branches inférieures des autres, à sécher et à tomber en pourriture; ef c'est 
ainsi que, d'année en année, le nombre des tiges diminue, et que celles qui persistent, parce qu'elles sont les plus élevées et les plus vigoureuses, se débarrassent de plus en plus de leurs branches basses, et prennent un füt d'une plus grande longueur.

Pendant la première jeunesse de la forêt, l'état serré ne présente pas d'inconvénients; il est certain même qu'il offre des avantages, les jeunes brins se prêtant un mutuel appui contre les intempéries et se poussant a croìtre en hauteur. Mais plus ils avancent en âgr, plus la lutte devient opiniâtre, parce que les tiges dominées, quoique privées de l'action de la lumière, sont d'autant plus longtemps à succomber qu'elles sont plus fortes, ce qui dis lors produit un ralentissement marqué dans l'accroissement de tout le massif. Néanmoins, le nombre des arbres diminue loujours insensiblement d'aprés la mème loi.

Diıns une forèt abandonnée à elle-même, cette opérition naturelle continue de la sorte, jusqu'au moment oùle peuplement s'éclaircil assez pour offrir les conditions favorables au développement des plants produits par ses semences. Alors une nouvelle générition se présente pour remplacer celle qui est parvenue à maturité.

4.58. Tel est le travail de la nature. Il faut chercher à l'imiter, non servilement, mais de manière it maintenir el ì faire naître, au besoin, toutes les circonstances propres d'une part, à assurer la régé- 
nération de la forêt, de l'autre, à en améliorer la croissunce (Voir l'Introduction).

Les divers genres de coupes qui constituent la méthode d'exploitation qui nous occupe, doivent toujours aboutir à l'un de ces deux résultals.

AR'IICLE II.

\section{Coupes de régénération.}

459. D'après ce que nous venons de voir, les conditions de la régénération du massif de futaie parvenu à son exploitabilité peuvent se résumer ainsi :

$1^{\circ}$ Sol meuble et substantiel;

$2^{\circ}$ Ensemencement complet du terrain;

$3^{\circ}$ Abri aux jeunes plants au commencement de leur existence;

$4^{\circ}$ Participation des jeunes plants aux influences atmosphériques, selon leur tempérament plus ou moins robuste.

Ces conditions, le forestier les réalise par trois coupes successives.

460. Dans la première, appelée coupe d'ensemencement, il laisse sur pied le nombre d'arbres nécessaire pour garnir de graines tout le terrain de la partie en exploitation, et pour abriter, tant contre les ardeurs du soleil que contre les gelées, les jeunes plants qui levent après la chute des semences. Si la 
graine est lourde et le jeune plant délicat, comme cela arrive pour le hêtre, par exemple, les arbres devront ètre asscz nombreux pour que les extrémités de leurs branches se touchent lorsqu'un léger vent les agite, et dans ce cas, la coupe sera dite sombre. Mais si, au contraire, les graines sont légères et les jeunes plants robustes, les arbres pourront être écartés davantage. Il est essentiel qne la réserve se compose, autant que possible, d'arbres sains et vigoureux, susceptibles de fournir abondamment de bonnes semences.

La coupe d'ensemencement satislicit évidemment aux trois premieres conditions que nous venons l'énoncer. En effet, elle conserve, par son couvert, le sol meuble ${ }^{1}$ et substantiel (lerrenu) qui s'est formé sous le massif et qui, joint au lit de feuilles, ficilite la germination de la graine et le développement du jeune plant; elleassure l'ensemencement complet; enfin elle procure à la jeune plante l'abri qui convient à son tempérament.

461. Aussitiot que les jeunes bois ont acquis un Bertain degré de force, ce dont on s'itssure surtout par la forme de leur cime el par l'ispect de leur

' Rien ne s'oppose à ce que, dans les coupes d'ensemencement, on déracine les souches des arbres abattus. Cette extraction augmentera les produits en matière et pourra, là oủ le terrain n'est pas suffisamment meuble, le préparer à recevoir utilement la semence des arbres de réserve. Il n'y a d'exception à cet égard que pour les pientes un peu raides, dans lesquelles cotte opération pourrait avoir pour suite l'ébnulement des terres. 
feuillage dans la saison de la végétation, il devient nécessaire de les faire participer darantige au bienfait de lia lumire el de l'air. In coupe qui doit remplir ce but, el qui consiste à éclaircir la réserve, c'est-à-dire à en abatlre une partie, se nomme coupe cluire on seconduire. Dans cetle opération, on enlève de préférence les arlores qui surmontent les plants les plus vigoureux et les plus élevés, en avant soin cependint d'en laisser quelques-uns afin de conserver l'ombrage encore utile. On n'en coupe aucun dans les plizes où l'ensemencement n'est pas complet, et où les jeunes brins sont encore trop faibles.

Lorsque le tempérament du jeune plint est trìs délicat, et que l'exposition el le climat rendent les chaleurs ou les gelées redoutables, on feria bien d'effectuer la coupe secondaire en plusieurs fois, c'est-àdire, déclaircir la réserve insensiblement, de manière à acclimater plus sirement le sous-hois.

462. Enfin, lorsqu'on est assuré que le sous-bois est assez fort pour se passer de tout abri, on procède a la coupe définitive en abiltant le restant de lin réserve. N'étant plus dominés d'ancune maniere, les jeunes brins sélancent dès lors et prennent une croissance rapide ${ }^{1}$.

1 C'est à tort que certains forestiers persistent, même après la coupe définitive, à maintenir des réserves sur les petites clairières qui peuvent exister, çà et là dans la coupe, par suite de

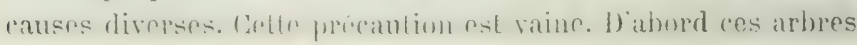


Dans certaines circonstances, on conserve dans les coupes réfinitives quelques arbres que l'on destine à parcourir une seconde rérolution, afin d'obtenir des bois de dimensions très fortes. Ces réserves doivent être placées sur le bord des chemins et sur les lisières de la forìt. Dans cette situation elles porteront moins de préjudice au sous-hois, et elles pourront d'ailleurs ètre extraites sans difficulté, si le dépérissement ou des besoins extraordinaires forçaient à les abattre.

463. Comme on le voit, la coupe secondaire et lit coupe définitive sont destinées à accomplir la quatrième et dernière condilion de la réussite du repeuplement naturel. Il est presque inutile d'iajouter que, pour assurer le succiss de ses traviux, le forestier doit prendre les mesures de police et de surveillance nécessitres, affin de gitrantir les jeunes semis de tout dommage extérieur, qui pourrail iltre causé soil par les hommes, soit par le bétril on le gibier.

464. L'exposilion of la nailure du sol doirent firire varier le nombre dos arbres qu'il eonvient de réserver dans lia coupe d'ensemencement comme dans

isolés sont la plupart du temps renversés ou brisés par les vents; en second lieu, le sol, dans ces endroits, est ordinairement ou couvert de plantes nuisibles ou durci au point qu'aucune graine ne saurail y réussir. Le meilleur moyen est rone d'abattre tous les arbress en mimn temps of de rimginerer artificiellement les places dont il sagit. Lal plantation, dans ce cas, est préférable au semis, en ce qu'elle établit plus d'égalité dans l'âge des jeunes tiges. 
la coupe secondaire. Elles peurent en mime temps faire atiancer ou retarder la coupe secondaire. Ainsi, dans les terrains qui ont une disposition prononcée ì se gazonner et à se couvrir de plantes nuisibles, de mème que sur les pentes méridionales, où le soleil frappe vivement et où la terre est sèche et légère, il est indispensible de serrer lit réserve des coupes d'ensemencement et de modérer, en les retardant, les extractions dans les coupes secondaires. Par là, on ombragera plus efficacement le sol et les jeunes plants, et l'on arrêtera la crue des mauvaises herbes '. Les mêmes précautions derront être prises en approchant de lit lisière des bois et sur les sommets où le rent disperse facilement le lit de feuilles.

En partant de ce principe, on peut laisser moins d'arbres sur pied, à mesure que les coupes s'éloignent

- Plusicurs auteurs allemands estimés, entre autres M. de Berg, enscignent que, dans les sols sces et aux expositions méridinnales, les coupes secondaires doivent être entreprises plus tôl et eclaircies plus fortement que dans les terrains frais et abrités, afin, disent-ils, de faire protiter les jeunes plants de l'influence bienfaisante des fluies douces et des rosées. Mais s'il est incontestable que ces météores sont farorables au sous-bois, il est évident aus:i, d’un autre cûté, qu'en le décourrant, comme on le conscille, on l'expose à étre suisi par les fortes chaleurs ou par les gelées printanieres. Nous pensons que mieux vaut encore risquer de relarder un peu la végétation des jeunes plants par trop de couvert, que s'exposer à les perdre par l'excès contraire; car, la riserve une fois amoindrie, il deviendrait difficile, sinon inpossille, d'obtenir une nouvelle el complète régénération naturelle des arbres restants. 
de l'exposition du Midi ou qu'elles sont moins sujettes à se gazonner. Toutefois, on ne devra jamais perdre de vue l'ensemencement que doit procurer la coupe sombre, ni l'abri que doit procurer la coupe secondaire.

ARTIGLE III.

\section{Coupes d'amélioration.}

46ว̈. Pour que la jeune forêt produite par les trois coupes successives, puisse désormais prendre tout le développement dont elle est susceptible, il importe que l'art, toujours attentif aux indications de lit nature, continue à seconder celle-ci et à hàter l'accomplissement de son œurre. Dans la plupart des cas, le besoin de cette intervention ne tarde pas à se faire sentir. En effet, quelle que soit l'attention que l'on mette à bien espacer les arbres dans la coupe d'ensemencement, et à amener la reproduction des meilleures essences, il ne s'y introduit pas moins des bois tendres, dont les graines légìres sont apportées de loin par les vents et lèvent arec une grande facilité. Ces bois tendres, auxquels se joignent les mortsbois, étint d'une végétation très prompte, ne tardent pas à dominer les plants des essences plus précieuses et à les gêner dans leur croissance. Dès qu'on s'aperçoit de cet état de choses, on doit se hâter de procéder à l'extraction des bois tendres et des mortsbois. Cette opération se nomme coupe de nettoiement. 
Ce serait une grande fiute de la retarder en vue de donner plus de valeur aux brois tendres; le dommage qui en résulterait pour les bonnes essences, serait bien loin d'ètre compensé par l'augmentation de prix qui en résulterait pour les autres.

L'époque à laquelle les coupes de nettoicment doivent se faire ne peut ètre précisée; c'est l'inspection des lieux qui seule doit en décider. Dès que les essences parasiles grènent, il faut les faire disparaitre; si elles se reproduisent et gênent encore, le nettoiement doit se répéter; et ainsi de suite, jusqu'à ce qu'enfin l'essence que l'on veut propager ait entièrement pris le dessus ${ }^{1}$.

466. Lorsque la jeune forèt se trouve débarmassée des bois tendres, et que, par suite de la lutte d'accroissement dans laquelle les jeunes plants sont engagés, elle passe de l'étut de fourré à celui de gquulis, le moment est venu d'aider la nalure dans la suppression des bois dominés. Les coupes à faire dans

' Il n'est pas toujours bon de supprimer à la fois tous les bois blanes qui se sont logés parnil les bonnes essences. Quand le repcuplement n'a pas également bien rúussi dans toutes les parties de la coupe, il devient nécessaire, dans les places incomplétement garnies, d'appuyer les tiges d'elite qui, seules, ne sauraient se soutenir et couvrir le sul. Miais, comme il importe en mème temps que ces tiges ne puissent pas itre dominées par les voisins qu'on leur aura conscrvés, on fera étêter, parmi ces derniers, ceux qui pourraient devenir nuisibles, de manière à les réduire sûrement au rôle d'auxiliaires qu'on leur destine. Aussitôt que les bonnes essences scront sulfisamment fortifiées pour former massif, les bois blancs devront etre extraits. 
ce but ont été nommées éclaircies périodiques, parce rqu'on les renourelle à des époques fixes. Pour cur comprendre l'économie, il est nécessaire d'établir les trois points suivants:

$l^{\circ}$ Pircrles d'ippres lespuelles doivent ilre exécutées les éclaircies ;

2. Ige auquel ori doit entreprendre la première;

$3^{\circ}$ Epoques auxquelles il convient de les répeiter.

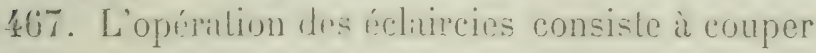
les liges les plus laibles ol les plus mal venintes. celles qui sont summóes ou pres de lietre ct rlont In végétation est languissante, enfin, les rejets de bois tendres qui se suraient peproduils ayries les nettoiements. On peut en mime temps conper quelques tiges bien renantes dinns les partios ou lo pemploment est épais, mais en y mettant une grande prudence. La principale rigle ì observer dims crenge de coupe, cest de latisser le? pruplement dins un itat convenablement serré, en un mot de no pras interrompre le massif. Dans un jeune peuplemrnt qui jusqu'alorsá crù dans un etat très serré les Jjrins::ont grêles et ont grand besoin d'appui. Une éclaircie imprudente les rendrait rictimes des orages, los frrait courber sous le poids de liz neige ou du givre, et meime sous le poids de leur propre cime. L'état de massif est tellement indispensable, qu’il faudrait, s il en était besoin, conserver même des perches mal renantes et des bois tendres qui, dans ce cas, ferient office de tuteurs, et subsisteraient jusqu'à la prochaine éclaircie. 
Il ne limt pas perdre de vue d'ailleurs, que les jeunes arbres doivent s'élancer et atteindre la hauteur dont ils sont susceptibles, cequi ne sauraitavoir lieu s'ils ne croissaient en massif.'Dansun peuplement plus àgé, les inconvénients d'une éclaircie trop forte ne seraient pas moins graves. Il en résulterait, en effet, que le sol se dessécherait et se durcirait à la surface, ce qui est défavorable à la végétation; ou qu'il se couvrirait de gazon et de plantes nuisibles, qui feraient plus tard obstacle à la régénération, ou bien, enfin, s'il se présentait une année de semence, on verrait naitre un jeune repeuplement qui, dans certains cas, ne serait pas moins gènant, la forèt n'ayant point encore atteint son exploitabiiité.

L'éclaircie, telle qu'elle vient d'être expliquée, est l'éclaircie normale ou moyemne, c'est-à-dire, celle qui, dins la plupart des cas, satisfait au but que l'on se propose. Toutefois, de mème que dans les coupes de régénération [464], le nombre des tiges à conserver dans les éclaircies périodiques doit varier suivant l'exposition, le sol, et aussi suivant l'essence: ainsi, dans un terrain de bonne qualité, exposé au Nord ou à l'Est, ou situé en plinine, une jeune forèt peut être plus largement éclaircie, pourvu toutefois que, dans la localité, les dégàts météoriques ne soient point ì redouter. Ce résultal s'obtient par l'extraction d'abord de tous les sujets dominés el, en outre, pirrmi les dominants, des brins les plus grêles, qui ont la cime étriquée, et qui empêchent les tiges 
d'ilite de derelopper leur tète circulairement. L ne lolle icharcie est dile forte; elle farorise nécessairement au plus haut degré l'aceroissoment des arbres. cn leur procurant de l'espace pour étendre à la fois leurs racines et leurs Jranches. Mais, lorsque, au contraire, les conditions de sol, d'exposition et de siluation sout délarorables, l'éclaircie doit itre fuible, c'est-a-dire qu'elle doit se bormer à supprimer les ligres mortes en cime ou tout a fait dépérissantes, conservant de la sorte non seulement toules les tiges dominantes sans exception, mais encore une parlie de celles qui sont surmuntées. Celte opéritlion, on le conçoit, a surtout en vue d'assurer au sol une formation abondante de terreilu, de le garantir des ardeur's du soleil et de mettre les massifs en ital de résister aux météores nuisibles rents. neigre. givre, ete. ). Elle sera d'une application générale en approchant de la lisiere de la forid, ou de toute autre partie très exposée aux rents, afin de prévenir li dispersion du lit de feuilles si essentiel à la rigectalion, surtout des jeunes peuplements.

468. L’àge auquel il convient d'entreprendre la première éclaircie ne peut ètre fixé: il lépend de la rapidité de l'accroissement sur lequel influent l'risence, le sol et le climat.

Le principe que l'éclaircie devient utile aussilòt que le massif passe à l'état de gaulis, est incontestable. Seulement. lorsque des accidents mitroriques sont à redouter, il ne faut la faire gut fuible. Dims 
les forìts soumises au régime forestier 1, on hésitait autrefois à entreprendre des éclaircies dans les gaulis, parce que le persomnel administratif, auquel elles pouraient être confiées, n'était ni assez nombreux, ni généralement assez instruit pour les diriger avec tout le soin et l'intelligence nécessiares. On conseillait, par suite, de n'entreprendre lia première éclaircie que quand le peuplement est ì l'état de perchis, les tiges à enlever étant alors plus faciles à distinguer, les produits quion en retire plus avantageux, et les fautes que I'on pourrait commettre moins dangereuses. Mais, de saines notions de sylviculture sont aujourd'hui répandues parmi les fonctionnaires des forêts, à tous les degrés de la hiérarchie; le nombre de ces fontionnaires a été successivement augmenté el, dans son zèle éclairé pour l'amélioration de la production forestière, l'Administration supérieure à su donner, en peu d'années, aux importantes opérations dont il s'agit, une impulsion quil'honore grandement ${ }^{2}$. On peut donc affirmer que dès à présent,

' Ce sont celles que régit l'Administration publique.

: C'est surtout en autorisant et en encourageant les exploitations dites paréronomir que l'Adninistration a assuré l'application en grand des éclaircies périodiques dans les forèts qui lui sont confiées. Cie morle, comme on sait, consiste a faire abattre et façomer les bois, non plus par des adjudicataires qui les ont achetés sur pied uprès désiguation préalable, mais par des ouvriers emplọés; soit à la journée, soit à la tàche, sous la direction spéciale des agents et préposés forestiers, après quoi seulement les produits de lexploitation sont live's at la consommation. 
il n'existe plus d'obstacles sérieux, si ce n'est dans quelques cas exceptionnels, à ce que les règles de la science forestière recoivent leur entière application, en ce qui concerne les éclaircies périodiques, dans les forêts administrées par l'État.

469. Quant aux époques auxquelles les éclaircies doivent se répéter, elles dépendent également des conditions de la végétation. Lorsque les massifs croissent rapidement, il peut être utile de les éclaircir lous les cing itns, du moins dans leur jeunesse, siul plus tard it laisser s'écouler un intervalle plus considérable. Quand la croissance est moins aclive. une éclaircie faite de dix en dix ans dans la jeunesse, el tous les ringt ans à un àge plus avancé, seria sulfisante. Entre la dernière éclaircie et la coupe d'ensemencement qui doit lui succéder, il convient de laisser un intervalle de vingt ou au moins de quinze. années, afin que les arbres puissent profiter du bienfait de l'éclaircie avant d'être abattus.

470. Les arantages qui résultent des éclaircies sont de la plus grande importance. Elles procurent une alugmentation considélable dans les produits, car tous les arbres dominés. qui naturellement auraient péri en forèt, toument entièrement au profit de la consommation et au bénéfice du propriétaire. Les brins, faibles à la vérité, que l'on coupe dìs lia première éclaircie doment du chauffage, du charbon, el peuvent mème servir à différents autres usages; ils ne sont pas à dédaigner, surtout dans les paỵs 
oii le buis a de la valeur. Dans les échirrcies subséquentes, a mesure qu'elles sont assises dans des parties plus àgées, on obtient des bois de travail et de service d'un plus grand prix. Tous ces produits, malgré leur importance, ne sont cependant qu'accessoires, et Join de nuire au produit principal (celui des coupes de régénération, ils tendent à l'augmenter. Ein effet, lorsque les arbres sont trop serrés, lorsque leurs cimes se pressent el que leur's racines sont enIrelacces, ils ne s'assimilent qu'en fiuble proportion les substances qui leur sont nécessaires, et faute d'espace pour développer leurs organes de nutrition (racines et feuilles), ils s'étiolent et contractent des tares souvent cachées, qui d'abord ralentissent leur croissince, et plus tard, entrainent prématurément te dépérissement et mème la mort. Mais, quand, en Iemps utile, on " diminué leur nombre, les arbres restants prosperent, s'élancent el grossissent, pour annsi dire, ì rue d'œil.

Les éclaircies farorisent l'accès du soleil, et permettent à l'air de circuler plus librement dans les massifs de forèts, ce qui contribue à domner aux fibres du bois la force el la souplesse exigées pour les diffirents emplois. On a reproché autrefois aux futaies de fournir un bois tendre, bien inférieur à celui des arbres crus isolément: ce défitut disparait par suite des échircies périodiques qui font participer les arbres anx influences de latmosphere el procurent it leur's tissus le degré de solidité convenable. 
Enfin. les éclaireies débarrassent les forits diune quantite de bois qui, par leur pourriture, auraient fivorisé la multiplicalion de plusieurs insectes, parliculièrement de quelques coléoptères qui se logent entre l'écorce el l'aubier, ainsi que dans l'intérieur du corps ligneux, et qui, dans les forèts résineuses surtout, itendent souvent leurs ravages de la maniere la plus désistreuse.

IHTICIS IV.

Fixation de l'exploitabilité et de la possibilité dans les futaies.

471. Lexpleitabilité d'une futare ne peut itre fixére

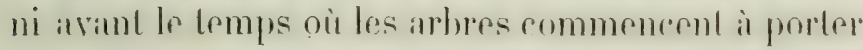
abondamment de bonnes semences, ni apris relui ou ils cessernient d'itre propres à la production d'un repeuplement complet. Du reste, cet objet doit se régler conformément aux principes que nous avons déreloppés dans le second Livre |406, 41 a d 414.].

47.2. Quant a la possibilité, elle doit itre basér sur le volume, attendu que la diversiti des coupes dont les produits sont destinés à la composer, ne salurail admeftre des exploitations it contenanees igates, ainsi guton te vertil dans lantiele suivant. 


\section{ARTICLE V.}

\section{Marche des exploitations dans une futaie régulière.}

473. Pour aroir une idée complète de la méthode du réensemencement naturel et des éclaircies, il convient de considérer la marche des exploitations dans une futaie régulière où cette méthode reçoit son application. Cet examen sera surtout utile en ce qu'il montrera ce qu'est, dans son ensemble, la futaie it l'étal normal, état auquel l'art du forestier doit chercher à ramener toutes les forêts.

Soit une telle forêt soumise à une révolution de cent ans, divisée en cinq périodes de vingt ans, à chacune desquelles est affectér une étendue déterminée [425]: on aura dans les bois de charque affectation, en se supposint placé au commencement de la révolution, les gradations d'àge suivantes:

Dans l'affect. de la $1^{\text {re }}$ pér., les bois de 100 à 81 ans.

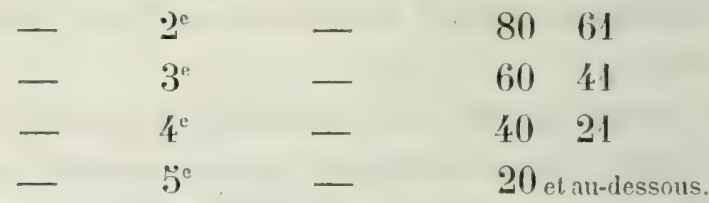

Dims l'examen qui nous occupe, nous considererons les exploitations telles qu'elles derront aroir lieu pendant lit première période: d'abord, les coupes de régémération à asseoir dans l'affectation 
de cette période, et qui fourniront les produits principanx; puis, les coupes d'amélioration qui doivent parcourir les autres affectations et dont les produits ne sont qu'accessoires. Ce que nous dirons de la marche de ces exploitations pendant la première période s'appliquera aussi aux périodes suivantes, la forêt demeurant dans les mèmes conditions.

474. Au rlébut, les coupes de régénération à entreprendre ne consistent ividemment qu'en coupes d'ensemencement, qui peuvent se succéder de proche en proche dans un ordre parliait. Ce n'est que quand les jeunes repeuplements se prísentent, que cette régularité devient impossible, parce qu'alors l'assiette et la nature des exploitations ne peurent plus être déterminées que suivint les progrìs des sousbois. Dis ce moment, e’est au forestier à apprécier chaque amnée, d'après l'élat de ces sous-bois ou d'après les apparences de réussite des graines, quelles sont les coupes qu'il convient d'effectuer.

Que, par exemple, il constate sur un point l'urgence de la coupe définitive, el que, sur un autre, il trouve la coupe secondaire non moins nécessaire, il y aura lieu de les effectuer toutes deux immédiat tement; et si les produits de ces deux coupes sont insuffisants pour parfaire la possibilite, il faudra asseoir encore une coupe d'ensemencement sur un troisième point.

Telle est la marche que doivent suivre les coupes de régénération: si on voulait leur en imprimer une 
plus récyulire, on s'exposerail à mal seconder la nature, ol'on pourrat dis lor's se priver d'une grande partie des arantages que doit prosurer la methode du réensemencement naturel.

47\%). Un a somvent tenté d'étahlir la possibilité de ces exploitations par contenanee; mais il est facile de prouver que, pour assurer au forestier la latitude dont il a hesoin, la possibilité fondée sur le volume est seule convenable.

supposons, en effet, yur l'on venille adoptep la possibilite par étendue, on pourra bien, ì la vérité. ne point rencontrer d'obstacle pendint les premieres imnées de la périnde, altendu que, n’ayant à faire alors que des coupes d'ensemencement dont learbres doivent itre espacés ì peu près partout de mime, il est dans les choses possibles que, sur d'ígailes surfaces, les produits matériels soient approximativement igaux. Mais, dis que les coupes sncondaires et définitives deviendront nécessaires, il nen sera plus ainsi, parce que, dans ces coupes, Io nombre des arbres ì ahattre varie selon les sols, les situations, les expositions, et surtout selon l'état des sous-bois, ce qui fait varier, par conséquent, les produits qu'elles fournissent sur une étendue déterminée ${ }^{\prime}$.

Si, au contraire, on base la possibilité sur te volume, on sera parfaitement lihre d'asseoir et dieffer-

2 Voir l'Appendice. 
luep les coupes en leur domnant une contenance plus ou moins gramde suivant les besoins du peuplement.

476. Les considérations d'après lesquelles doit se régler la marche des coupes d'amélioration étant différentes, la base de la possibilité relative ì ces coupes ne doit plus être la mème.

Ces coupes, en effet, n'ont point pour oljet prinripal de procurer des produits, comme les coupes de régénération : elles sont entreprises surtout en vue d'arcéléper lit renissanee des arhres destinés à partvenir au lerme de la rérolution, et de leur procurer les plus belles dimensions et les qualilés les plus précieuses. l'égalité des produits dims ces opéritlions n'est done que secondaire; pour qu'elles répundent complitement it feur hut, il importe que tes "oupes de netloiement of les échaircies du premier ige puissent s'exéruter dis l'instant ou elles sont jugées nécessaires; et, quant aux éclaircies suivantes. il est plutòt it désirer qu'elles se renouvellent à des ipoques fixes. Il serail done convenable d'imprimer it celles-ci la marche la plus riguliere possible, ol. in contraire, pour les autres, de laisser le forestier rntierement libre de les faire ruand et eomme it to jugera à propos.

Or, pour établir dans une exploitation une marche parfiritement régulière, le meilleur moyen, ineontestiblement, est de prentre pour base ha possibilite

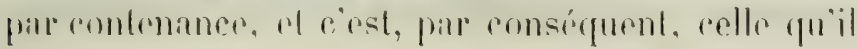


convient d'adopter pour les éclaircies périodiques, d'autant plus que rien ne s'y oppose dans la nature de ces opérations.

477. En admettant ces règles, on aura à faire annuellement, dans la futaie normale proposée pour exemple, trois genres distincts de coupes, savoir:

1Dans l'affectation de la première période, coupes de régénération, basées sur lit possibilité par volume;

$2^{\circ}$ Dans les affectations de la $2^{\circ}$, de la $3^{\mathrm{e}}$ et de la le période, éclaircies périodiques par contenances égales;

30 Dans l'affectation de la כ̈e période, nettoiement des bois blancs et premières éclaircies à exécuter ou à négliger, selon l'état du peuplement, et sims s'itstreindre à aucune possibilité.

ARTICLE VI.

Abatage, façonnage et vidange dans les futaies.

478 L'époque la plus farorable pour l'abatage des arbres dans les futaies parait itre lia fin de l'automne at l'hiver; toutefois on deva suspendre les travaux pendant les grands froids, sous l'influener desquels les arbres éclitent et se rompent facilement dans leur chute. Dans les climats tempérés, c'est ordimairement au 1:3 octubre que l'on fixe le commence- 
ment de l'abatage; dans les climats plus froids, on peut commencer dès la mi-septembre, parce que le végétation s'arrête plus tòt; et dans les pays chauds, la raison contraire pourra faire différer la coupe.

Pour les essences feuillues, il est à peu près généralement reconnu que les bois coupés dans la saison morte sont d'une plus longue durée lorsqu'ils sont mis en œuvre, et il semble certain aussi qu’employés au chauffage, ils brìlent plas facilement et donnent mème plus de chaleur que cenx qui ont été abattus dans le temps de la végétation.

Qunnt aux essences résineuses, elles ne paraissent pas éprouver les mèmes inconvénients de la coupe en temps de sive: il est au contrime assez habituel, dans les contrées que ces essences habitent, de les exploiter en éte, et heaucoup de praticiens estimés sont d'avis qu'en prenint lia préerution de les écolcer aussitòt apries l'abatage. les hois gangent mème en dureté et en solidití, outre qu'ils dreviennent plus lígers, et par suite, d'un transport plus facile 1.

Mais eu égard à lat vidange des bois, l'abalage en hiver est préférable pour toules les assences, parce qu'il permet de trinsporter les bois arant le retour de la sìve, ce qui est de lit plus grande importance diurs les coupes secondaires et dans les coupes définitives: en effet. Iors de l'ascension de la sive,

- Voyez Annales forestières, $f^{\text {er }}$ volume, page 30. 
commer parl tes grands froits, les jeunes bois sont plus caissants qu'aux autres eporpurs de lannée.

479. The mesure non moins ussontielle it prendro dans l'exploitation des coupes secondaires et des roupes définitives, surtout lorsque le sous-bois a déjit quelque élévation, e’est de faire úbrancher les arbres jusqu'à la cime, avant l'abatage '. Eu outre, on doit reiller ì er que les branchages soient immédiatement pelevés. lacomnés el sortis de lit coupe; al ensuile, it ce que l'arbre soit dirigrie, dans sa chule, de manière à fomber du ritr ou il ratusera le moins de dommage.

Sur les pentes, c'est en général rers le sommet de la montagne qu'il faudea chereher a faire tomber les arbres.

Autant qu'on le peut, on doit interdire aux roilures l'entre des coupes, et faire enlever ì dos d'homme, ou par d'autres moyens peu nuisibles, les bois de chauffage et de travail. En montagne, les chemins à tranneaux et les lancoirs doivent, le plus possible, être pratiqués: mais, dans les localités oil ces chemins ou ces limenoirs ne peurent être employés. l'époque la plus propice pour la vidange

1 Cet ébranchement doit, autant que possible, se faire en commençant paren bas: Jorsqu'on procède en sens inverse, ce qui esl plus fiecile pour linurier, les hranches sup perieures samoncelent snuvent sur les inferieures, et quand ensuite on coupe celles-ci, leur chute cause des dégàts assez eonsidérablos. 
rist celle ou la terre est couverte de neige, hors cependint le moment destrop grands froids.

Toutefois, les mesures de précaution que nous renons d'énumérer ne doivent pas ètre poussées trop loin; leur exagération purrait diminuer lis valeur des bois, en augmentant plus que de raison le prix du faronnage et de lit vidange. Ces mesures indiquent ce qu'il y a de mieux à liare pour ménager le sous-bois; c'est à l'intelligence du forestier à les modifier selon les circonstinces. Ainsi, diuns les forèts situées en plaine, et peuplées d'essences feuillues, les coupes, lorsque les repeuplements sont complets, n'exigent pas ordinairement l'enlèvement à dos d'homme des bois coupés. Les jeunes brins peurent bien ètre endommagés momentanément par le passatge des voitures, mais ils se relivent bientot, el te dommage, après un laps de temps atsez court, ne s'apereoit plus, si l'on at soin surtout de recéper immédiatement les tiges qui ont été brisées. II n'en est pais entierement de mème des essences résineuses: elles demandent plus de soins, carr, icrasi, le jeune brin se relève rarement; cassé plus bas que le dernier verticille, il ne réussil presque jamais à former une nouvelle cime; et les blessures de l'écorce, si elles pénètrent jusqu'it l'aubier, donnent lieu à des plaies chancreuses que l'arbre conserve en général pendant toute son existence. 


\section{Essences propres à la futaio.}

480. Toutes les essences peurent ètre exploitées en fulitic, puisqu'elles possident toutes lit propriété de se régénérer par lit semence. Mais il y en a un certain nombre pour lesquelles ce traitement est particulièrement avantageux. Ce sont, en dehors des essences résineuses auxquelles, comme on le sait, tout autre mode de reproduction est refusé, le chêne et le hêtre. 


\title{
CHAPITRE DEUXIËNE
}

\section{APPLICATION DE LA MÉTHODE}

\author{
DU IRÉENSEMENGEMENT NATUREL
}

ET DES ÉGLAIRGIES.

ARTIGLE PREMIER.

Exploitation du chêne en futaie.

481. Exploitabilité. - Dins quelques forèts de chêne, les révolutions ont élé portées jusqu’à $20 \% 0 \mathrm{et}$ mème 300 ans. De telles révolutions sont admissibles dans un sol profond el substantiel, mais il est certain que, dans le plus grand nombre des cas, on ne peut que perdre à reculer ainsi l'exploilation. Lin général, l'exploitabilité du chêne doit être fixée à l'époque où les arbres ont acquis les dimensions et les qualités les plus convenables à tous les genres de construction 
et de travail. Le chène, comme on sail, se plail dims des terrains substintiels. protionds el divisés, mais il croit bien aussi, quoique moins rapidement, diuns des sols assez compactes, itrgileux et froids. Fin le laissant atteindre laige de 140 a 130 ans dans les premiers terrains, et celui de 160 at 180 ans ditns les autres, on obtiendra de cel arbre toul ce qur l'on peut en attendre, au point de vue des dimensions ot de la qualité. Que si enfin, on arait à élever une futaie de chène dans un climat chaud et humide, il pourrait ètre convenable de réduire sa rérolution mème jusqu'à 120 ans .

482. Coupes de régénération. - La coupe d'ensemencement, dans une futaie de chène, doit ètre sombre, afin que le repeuplement complet du terrain soit assuré, ce qui ne saurait aroir lieu si les arbres étaient trop espacés, attendu le poids du gland. Cel état sombre a encore pour but: d'abord, d'éviter' qu'avant la chute des semences, le terrain ne se gitzomne et ne se courre de plantes nuisibles; ensuite, de conserver le lit de feuilles sèches qui, tout $\mathrm{cm}$ ajoutant à la fertilité du sol, préserve les glinds. lombés de la gelée et firrorise leur germination.

IIalgré cette précaution, c'est chose fort ordinaire de voir, dans les coupes sombres, le terrain se gazonner ou se tasser au point de devenir impropre à la reprise du gland, lorsqu'il se passe plusieurs amnées sans glandée: car les chènes. surlout quand ils ont crù en massif, ne donnent qu'un faible couvert. 
Dans ce cas, il convient, dès que lia glandée a lieu, de fitire remuer le terrain, soit à la houe, soil par les porcs, atin de mieux assurer la germination des se mences ${ }^{1}$.

Le jeune plant du chène ne demandint et ne supportant mème que très peu d'abri, il fiut se hàter, une fois l'ensemencement produit, de procéder à lit coupe secondaire. Elle doit se faire dans l'hiver qui succède à celui de la glandée, ou, au plus tard, ver's la fin de l'année suivante. Si l'on attendait plusieurs

- Vans plusieurs parties de la lirance, notamment dans le Nord et dans l'Est, et aussi dans quelques régions montasneruses du Misli et du Centre, ou les glandées ne se voient yqu ì d'assez longs intervalles [7!, les difticultis qu'eprouve lat riussite du repeuplement naturd, dans les coupes sumbles, ont rebulé eertains forestitrs au point de leur avoir suscití l'idée que le chène ne pourait se régénérer sùrement que par le semis artificiel ou lit plantation, l'un ou l'autre succédant inmédiatement à la coupe à blanc étoc des massil's de futaic. - C'est le retour à l'enfance de l'art. - Sans nous autoriser des nombreux et magnifiques exemples de régénération naturelle que présentent les futaies de chène de la Franer dans toutes les rígions où les glandées sont tant soit peu fréquentes, et sins parler des dépenses considérables qu'exigerait l'emploi du morle dont il s'igit, s'il devait se pratiquer sur une grande ischelle, il nous sulfira de faire remarquer que, dans beaucoup de localitis, par exemple dans les sols légers, siliceux ou calcaires, sur les versants un peu rapides ou aux expositions chaudes, - il y aurait la plupart du temps de sériew inconvénicnts, parlois même du danger, à déboiser complètement le terrain et à le laisser, pendant quelques annees au moins, exposé à l'influenee directe des météores. Non seulement tout le terreau formé à la surface du sol sous le couvert salutaire de la futaie se. 
années, on risquerait de voir périr les jeunes chênes sous le couvert de la coupe sombre ${ }^{1}$.

On doit, par le mème motif, ne pas retarder la coupe définitive: on y procèdera, au plus tard, dans li quatrième année après celle de l'ensemencement.

Comme le chène est un arbre extrèmement précieux, et qu'il faut, pour certaines constructions, surtoul pour les constructions natrales, des pièces de très forles dimensions, il est à conseiller de laisser quel-

rait dans ce cas desséché par le soleil et lavé par les caux, mais sutuvent la terre végútale elle-mème serait cntrainée des platteaux et des pentes dans le fond des vallées.

Heureusement rien, dans la culture des futaies de chêne, n'oblige à courir de pareils risques. - Tout en continuant à pratiquer la méthode du réensemencement naturel dans ce qu'elle a dexcellent et de supéricur, le foresticr prudent saura en modifier lapjlication, en faisant intervenir le repeuplement artificiel partout où les circonstances locales l'exigeront, cest-ì-dire toutes les fois que, soit la trop grande rareté des années de glandée, soit létat du sul à la surface, feront prevoir, pour lit réussite du repeuplement naturel, des retards qui seraient de nature à embarrasser la matrehe des exploitations et à préjudicier à la production.

Se souvenant que, pour élever des chênes de fortes dimensions, il est très avantageux de leur adjoindre en mélange certaines essences subordonnées [87], telles que le hètre et le charne par exemple, il favorisera l'introduction spontane de celles-ci, y ailera mème artificiellement au besoin, et diminuera ainsi, à la fois, et les diflicultés et les frais inhérents à la conservation de l'essence principale.

1 Cet inconvénient est très fréquent et contribue beaucoup à 
ques réserves lors de la coupe définitive 1. En les plaçant, comme nous l'avons dit plus haut, sur les bords des chemins et sur les lisières de la forèt, leurs branches auront plus d'espace pour s'étendre, et pourront ainsi former les courbes recherchées pour la

augmenter les dificultés que rencontre la régénération naturelle du chêne. En effet, si les glandées complètes sont rares, les tiers ou les quarts de glandées se voient assez souvent ef produisent des jeunes plants en bon nombre, mais qui, cependant, ne se rencontrent quépars daus la coupe et ne sauraient lurmer, plus tard, un fourré tel quon vourlrait lobtenir pour effectuer en toute sécurité la coupe secondarie et la coupe délinitive. En présence diun pareil repeuplement particl on hesite douc, la plupart du temps, à toucher à la réserré de la coupe sombre; on espère que, dans les amnexs lus plus prochaines, le semis naturel se conpletera: - bref, on altend atin de ne rien compromettre, et - précisément on compromet tout, car en mene temps que les jeunes chènes perissent sous le rouvert, celui-ci augmente d’intensiti par l'extension graduelle des cines

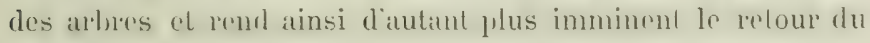
même dommage.

Mieux eût valu, - neuf' fois sur dix, - faire immédiatenent la coupe secondaire aussitôt après avoir oblenu les résultats de lit glandie particlle. Ce qui peut arriver de pis diume telle operittion, - prématurée en principe nous le reconnaissons, - c'est de permettre l'enwahissenent de bois blanes qui ctoufferaten les jounes chenres: mais lien nest plus likcile que de prérenir une telle consequence. Quelques ouvriers soigneux, places it propos dans li coupe, la débarrasseront en peu rle jours des essences parasites ot la mettront en état de recovoir fruetueusement les compléments, naturels ou artificiels, nícessailes atu premier repeuplement en chine pour tapisser entièrement le sol et pour permettre ainsi de passer à la coupe définitive.

1 De $\ddot{\text { à }} 10$ par hectare, selon les besoins de la consommatiun. 
marine. Jatns les terrains substintiels et profonds, lat Inngévilé de l'essence permettra facilement à ces réserves de parcourir une seconde révolution, surtoul si, comme on doit le supposer, elles ne sont choisies gue parmi les tiges pirlaitement bien venantes.

Il irrive presque toujours que le chènes, réservés -t isolés après l'exploitation dés coupes (les pédonculés surtoul, se garnissent de menues branches au pied et le long de la lige. La cime, dans ce cas, est moins bien nourrie, et, lorsque le fonds de terre n'est pas d'une tris bonne qualité, cette cime se couronne. ce qui entraìne peu à peu le dépérissement de l'arble.

Il est essentiel de débarrasser les chènes de cro branches gourmandes, et de renouveler cel émondirge jusqu’i ce que ha jeune forèt se soit assez éleréc pour entuurer l'arbre et emp:cher les productions dé sa tige et de son pied.

Ces branches, quand on les laisse subsister, nuisent en outre considérablement à lit croissance du suus-bois, diminuent la qualité des arbres pour lit charpente et la fente, en les rendant noueux 1, et malentissent mème l'accroissement des grosses brimches qui, senles, peurent former les courbes enployées à la construction des vaisseaux.

En meme temps quion socenperis de supprimen

' butfon a prouvé, par des expériences, que les bois noueux sont d'un quart plus laibles que les autres, seus la charge. 
les branches gourmandes des réserves, il serai tries utile dexaminer si leur cime ne présente pas dess branches sieches qui, mime sur les chènes bien renants, se forment assez souvent à l'intérieur de la ramure quand l'arbrepasse de l'état do massif à l'isolement.

Le retranchement de ces parties mortes soigneusement exécutí, avec un instrument hien tranchint et en donnant à la section une inclinaison qui assure le prompt écoulement des raux pluviales. préviendral In formation des grisettes et des nocuds gittés qui affectent trop sourent les plus belles pièers et les rendent impropres aux emplois d'élite pour lesgurls, saines, elles eussent été infiniment précieuses, soit par leur forme particuliere, soit par leurs dimensions.

483. Coupes d'amélioration. - La jeune forit obtentie par le semis naturel devia itre nettoyér des bois blancs et des morts-bois, et éclaircie périodiquement, conformément aux rìgles générales.

Comme il est tries essentiel que les chines destine's aux constructions et aux divers ourrages de fente. alcquierent une tige droite et eflilée, on feria bien, dans les deux premieres éclaircies, de serrer le malssif un peu plus qu’à l’ordinaire. Cette préciution est d'autant plus nécessaire, que les chènes ont. comme nous venons de le dire, une disposition marrquie à se garnir de branches gourmandes le long du trone, dis que entle partie se troure frappée trop 
immédiatement par la lumière. Ce n’est que lorsque les arbres ont atteint ì peu près la hauteur dont ils sont suceptibles, qu'il devient utile de les espacer davantage, afin de favoriser leur grossissement et de procurer à leur bois, essentiellement employé à la charpente, une texture plus solide.

1 la suite de ces éclaircies plus fortes, il arrive fréquemment, attendu le couvert léger du chène, que le sol se tapisse d'essences étrangères, telles que charmes, bouleaux, alisiers, bois blancs, etc., qui continuent, quoique rabougris, à végéter sous la jeune futaie. Cette végétation traînante n'offre point d'inconvénients; au contraire, elle s'oppose à l'enlèvement du lit de feuilles par les vents, empèche le sol de se durcir et de se gazonner, le maintient frais par conséquent, et produit, par ses propres détritus, plus de substance nutritive qu'elle n'en absorbe. Aussi, loin de la supprimer comme on croyait devoir le faire autrefois, faut-il la laisser subsister jusqu'au moment d'entreprendre la coupe d'ensemencement. (Voir la note page 248.)

ARTICLE II.

Exploitation du hêtre en futaie.

484. Exploitabilité. - L'époque la plus convenable pour l'exploitation d'une futaie de hêtre tombe entre 80 of 140 ans. Le plus ordinairement. 
on choisit l'àge de 120 ans, à muins que la forèt ne soit située dans un terrain très riche et dans un climat très doux. Dans ce cas, les bois acquierent plus tòt les dimensions utiles, et comme, d'un autre còté, ils sont aussi plus exposés à se carier intérieurement ', il est prudent de réduire la révolution à 100 ot mème 90 ans.

48:3. Coupes de régénération. - La coupe d'ensemencement doil présenter une réserve nombreuse, composée des arbres les plus forts et les plus sains. L'état sombre de cette coupe est doublement nécessaire dans une futaie de hitre : d'abord à cause de la semence qui, comme le ghland, est lourde, et en second lieu, parce que le jeune plant est tries délicat et a besoin d'etre protégé par un couvert épais.

Il ne faudrait pas, toutefois, conclure de lit que, sous un massif eomplet, le repeuplement réussirait. Lit fitne à la vérité y germerail et les jeunes plints liveraient, mais pour dispatrille dies la $2^{\circ}$ ou lat 3 année au plus tard, faute de lumière $\mid 4567$. Aussi lorsque, apres lia coupe sombre, la fitinée se lait trop longtemps attendre el que le massif s'est reformé par suite du développement des cimes des réserves, il devient nécessaire de remédier i cet état de choses parr quelques nouvelles extractions d'arbres, aux-

- Les essences les plus exposées à la carie intérieure, dans les sols substintiels, puraissent itro colles dont le couvert très epais maintient une grande fruteheur et lournit un terreau abondant. 
quelles il est bon, néanmoins, de ne procéder que peu arant l'époque présumée de la prochaine fainée.

Lal coupe d'ensemencement doit rester intacte jusqua'i ce que le sol soit complètement ensemencé ol mème jusqu'i ce que les plants aient acquis une cerlitine force. Il faut, à cet effet. leur laisser atteindre de 22 ì 33 centimètres de hauleur, ì peu prës; c'est alors seulement qu'il est temps de procéder ì la coupe secondaire. Cette opération doit ètre laite ave beaucoup de précaution. Dans les sols et aux expositions favorables, elle pourra supprimer à peu pris la moitié du couvert; mais si le terrain étrit sec et l'exposition chaude, il faudrait n'enlever que trìs peu d'arlores dans une mème année, et revenir ainsi a plusieurs reprises, afin d'habituer insensiblement le jeune hètre aux influences atmosphériques [464].

Lorsque, a l'aide d'une ou de plusieurs coupes secondaires, le sous-bois est parvenu à une hauteur moyenne de .50 à 66 centimètres, et même jusqu'ì un mètre, le moment est arrivé de faire la coupe définitive. En général, il n'est d'aucune utilité, lors de cette coupe, de réserver quelques arbres pour parcourir une seconde révolution, le hêtre n'étant point employé à lis charpente, et pouvant, dès l'ìge de 90 à 120 ans, convenir à tous les usages auxquels il est propre.

486. Coupes d'amélioration. - Les nettoiements de bois blanes, ainsi que les éclaireies périodiques. 
devront s'exécuter entièrement d'apris les riegles générales données ì cet ígard, en observant toutefois que le hètre supporte, plus que te chène, de croilre en massif serré,

\section{ARTICLE III.}

\section{Exploitation d'une futaie mélangée de chêne et de hêtre.}

487. Les racines du hitpe sont triesuntes, eelles du rhime, au contraire, senfoneent profondément. II en resulte que l'un at l'autre, lorequ'ils eroisent on mélange, trouvent, sins se gêner répiproquement, lis nourriture qui leur eonvient; el l'on remarque gémilalement, dans les forèts où ces essenees sont mélingées, un atcroissement plus prompl que daus celles dont le chêne est l'essence unique.

Une autre cause du meilleur aceroissement d'un pareil mélange, e’est que te hêtre, par son feuillige épais, enuserve an sol plus de fraicheur, el l'enrichit d'une couche plus athondiunte de terreau que ne ferait le chine seul. Cette silutaire influener se fait surtout remarequer dans les sols légers, siliceux on calcaires, mediocrement profonds, et elle devient particulièrement précieuse lorsqu'il s'agit d'obtenir des pièces de chène de fortes dimensions ( $\left.{ }^{1}\right)$.

' La vaste forêt du Spessart (Bavière-Rhénane) offre un exemple remarquable des avantages que présente le mélange 
488. Paprontmintí. - Comme le chìne est des deux essences li plus précieuse, il convient, s'il est dominant ou s"il forme seulement la moitié ou les deux cinquiemes du peuplement, d'adopter la rérolution qui lui est propre, el d'y subordonner celle du hêtre. Celui-ci, comme on le sait, peut sans inconvénient rester sur pied fï0 a 160 ans, lorsque le sol lui est favorable.

Ce n'est que dims le cas où la proportion du chène serait tris firible, que l'on fixernit la révolution de 120 ì 140 ans. sauf à réserver, dans les coupes défnitives, un certain nombre de chines pour croitre jusqu’à la prochaine exploitation.

480. Cocpes re régéxérATrux. - Les règles données, dims les deux articles précédents, pour espacer lit coupe d'ensemencement, devent ith suivies en observant méanmoins que, comme le chène est l'essence la plus préciense, il importe, avant toul, d'assurer sa reproduction pirl une nombreuse róserve. Par li mème raison, on derra se hâter de procéder à la coupe secomdaire aussitrit après l'ense-

dont il s'agit. Située dans un climat assez rude, sur le grès bigarré, elle produit des chênes de la plus belle venue et d'un age lrìs arancé, au moyen d'un morle particulier de traitement qui consiste principalement í iclaireir tris fortement les mas-

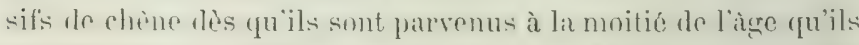
doivent atteindre (200 ans en moxemne), of it y introduire artificiellement lo hêtre, qui croît ainsi, en second ètage, sous le couvert léger des chênes, et s'y maintient en bon état jusąu'à l'exploitabilité de ceux-ci. 
mencemenl, quel que puisse d'ailleurs en ètre l'inconvénient pour le jeune hètre. De fait, cet inconvénient est souvent moins grand qu'on ne pourrait le craindre, parce qu'en raison du poids des graines, les repeuplements de charque essence existent, par taches, autour des arbres qui les ont produits; on trouve done moyen de donner du jour atux jeunes chènes tout en conservint le couverl aux hètres. I'un autre còté, les jeunes chìnes eux-mêmes qui, dans les premières amnées, ont ordinairement une croissance plus rapide que les jrumes hir, "., peuvenl (puelquefois offrir à ceux-ci un utile abrı ( $\mathbf{t}$ ).

La coupe définitive devra aussi se fitire à l'époque qui paraitra lit plus comvenable pour assurer la bomme végétation du chêne.

Souvent, malgré les précutions arre lesquellos on conduit les coupes de régénépalion, il anrive yue le hritre empiete sur le chème dans les repeuplements

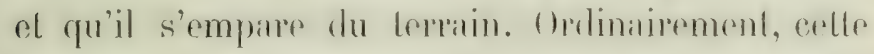
cireonstame se présenter it lis suite d'une immére qui,

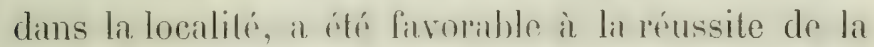
faine, alors que lo ghland y a mangué entierement ou en grande partie; olle tient encore ì ce que les fatuées partielles domment lieu à des semis naturels qui persistent sous le couvert ef envahissent aimsi

1 Dans certains terrains, notamment dans les gerrs, les deux essenees croissent souvent en rapport inverse. Dans ce cas, il laul rabattre lo hitre, sams hísiter, afin de protéger la végétation dù chênc.

(Note de M. Parade.) 
peu à peu le lerrain; tandis qu'il n'en n'est pas de meime du chène qui périt sous la futaie au bout de quelques ammés |72|. Pour remédier à cel inconvenient, et rélablir Io mélange, il faut recourir à des movens artificiels. Ces moyens consistent à se procurer, dans d'autres parties de la forêt où les chènes peuvent avoir ćté plus fertiles ${ }^{1}$ ), les glands néeessaires pour repiqure les petites places non garnies, et ì en répandre, en génépal, diums toute lit coupe. Si cette opéralion ne pouviat se faire l'année mème de la fainée, à cause d'un mangue absolu de glands. elle peurrait so remettre ì l'ammés suivante; mais. s’il fillait haisser passer ainsi plusieurs années, on frrait mieux alors de se procurer de jeunes chìnes de 3 à 4 ans, que l'on planterait parmi les hêtres, en les récépant au moment de la plantation, afin d'activer leur croissance et de leur permette d'arriver plus tôt au niveau des jeunes hêtres.

490. Colpes diaméloration. - Les netloiements et les éclaircies périorliques doivent se faire comme dinns les forits où le chine est l'essence unique. On peut, au moyen des ciclaircies, augmenter facilemenl Ie nombre relatif des chênes, en prenant toujours de prifirence les pieds à supprimer parmi les hêtres: mais, outre qu'il faut se garder de détruire le mélange, il est essentiel d'observer que, pour se servir

' Les arbres placés sur les lisières des forêts ou sur le bord des routes, sont plus fertiles que ceux qui ont crû dims l'intéricur des massife. 
de ce moyen, il litut que l'époque de l'éclaircie soit choisie très à propos, el l'opération mème exéculie irec beaucoup d'intelligence, alin de ne a las lite couper des hètres vigoureux pour linoriser peut-itre des chênes déjà maladifs.

ARTCLE IV.

\section{Exploitation du châtaignier cn futaie '.}

491. Exploitabilité. - Les révolutions comprises entre 90 et 120 ams, selon les sols et les climals, paraissent convenir au chàtaignier. Elles suftiront pour qu'on en obtienne, en raison de la promple végétilion de l'essence, tous les bois nécessitires à lit consommation, et l'on ne risquera pas de voir les arbres se creuser, ce qui arrive tris fréquemment au châtaignier, passé l'àge de 120 ans.

492. Coupes de régénération. - Sous ce ripport. le chàtiagnier pourra ètre entièrement traité comme le chène: Jeune, il est aussi robuste, et lit chàtaigne "a plus de poids encore que le gland. Quelques inbres pourront ètre réservés diuns la coupe définitive. principatement en vue d'en obtenir des fruits.

Le châtaignier ne se voit gouère en lutate qu'associé au chène et au hêtre. Il est inotoire cependant qu'il croît très bien sans mélange, et, dẻs lors, on doit admettre que les progrès de la sylviculture amèneront la création de futaies de cotte précieuse essence. 
On a observé que le chàtaignier est particulièrement incommodé par les mauvaises herbes et les arhustes. Lors done que, dins les coupes sombres, le réensemencement ne se f'era pas immédiatement après l'exploitation, et que le terrain se garnira d'herbes et d'autres plantes, il faudra, dès la première innée de semence, donner au terrain une culture entière à la houe, ou bien y pratiquer des sillons d'une largeur moyenne de 16 centimetres el espacés de 22.2 a 33 centimètres environ. Cie dernier mode, qui est bon dans toutes les situations, devra ètre exclusirement mis en usage dans les terrains en pente. Les chàtaignes s'arrèteront au fond des sillons, ou elles seront couvertes et abritées contre les gelées par les feuilles sèches.

493. Coupes d'amélioration. - Les nettoiements de bois blancs devront être particulièrement soignés dans les jeunes chàtaigneraies, attendu le tempérament du jeune plant [133̆]:

Quant aux éclaircies périodiques, il conviendra de les diriger comme dims les futaies de chène, le chàtaignier étant également un bois de construction et de fente; seulement, comme sat régétation est tris prompte, la périodicité pourra ètre plus courte. 
DES FUTAIES.

ARTICLE V.

Exploitation de l'orme en futaie !

494. Exploitabilité. - La révolution de 100 a 120 ans peut convenir pour l'exploitation de l'orme. I cel ìge, il sera propre à sittisfaire tous les besoins de la consommation, et il aurat atteint l'époque de son exploitabilité absolue.

493. Coupes de régénération. - Comme liı semence de l'orme est très petite et se discémine all loin, et que le jeme plant est robuste, on jeul se dispenser d'établir une réserve abondante dans lit coupe d'ensemencement; il suffit d'espatcer les arbres de telle manière que les branchrs soient écartées de 4 à 6 mètres. In espacement mìme plus considérable n'empècherait pas le repeuplement.

Il faut avoir égard cependant à la situation de la coupe que l'on exploite, el éviter, pour peu qu'ello soil exposée atu vent, de lrop diminuer le nombre des réserves. On conçoit que des arbres qui unt vécu 100 à 120 ans, en massif, et qui se sont élancés

1 Quoiqu'il soil très rare de rencontrer des lutaies dont lorme soit l'essence unique, limportance de cet arbre nous a c'pendant paru assez grande pour que son traitement méritat d'itre décrit avec autant de détails que eclui des essences qui doninent fréquemment dans nos bois. 
a 30 mitres de hituteur el an delà, ne peuvent ilroir une issielte bien solide, el que, sur les points les plus exposés, il serait peu prudent de leur donner tout à coup un espacement trop considérable. On pourrait craindre aussi que l'état trop clair de lis coupe d'ensemencement ne proroquàt le gazonnement du terrain. Toutefois, cet inconvénient se présentera rarement, attendu que l'orme produit des grianes en abondance, presque tous les ans, ou au moins de deux années l'une; on peut donc espérer le repeuplement immédiat de la coupe. Nais si, par extraordinaire, la crue des mauvaises herbes avait précédé l'ensemencement, on ourrirait dans lit coupe des sillons, qui auraient le double avantage de préparrer la terre à recevoir la semence et de faire nailtre une multitude de drageons.

Quant à la coupe secondaire, on devra y procéder. au plus tard, à la fin de la deuxième année qui suivra celle de l'ensemencement, et, deux ans après, on fera la coupe définitive. On pourrait mème, dans un terrain frais, ou si l'exposition n'était pas chaude, supprimer entièrement la coupe secondaire, à cause du tempérament robuste du jeune plant, et, deux ou trois ans après l'ensemencement, passer immédiatement à la coupe définitive.

496. Coupes d'amélioration. - Les nelloiements et les échincies périodiques derront ètre dirigrés d'après les règles générales; il y aura lieu cependant de ritpprocher les échaircies, d'autint plus que l'orme 
it une régétilion très rapide, et que, trargant et pivolant à la fois, il a besoin de plus d'espace pour prendre tout l'accroissement dont il est susceplible.

\section{Exploitation en futaie du fréne et des grands érables.}

497. Le frène et les grands érables peuvent, avec beaucoup d'avantage, ètre élevés en futaie, attendu que, lorsqu'ils acquitrent de fortes dimensions et qu'ils sont de bonme fente, ils deviennent d'un très grand prix pour lit menuiserie, l'ébénisterie, lia boissellerie. ete. Dordintire. ces essences se trouvent mélangées au hitpe aree lequel elles régivtent parfititement el dont elles supportent tris hien le régime, surtout lorsque lit rérolution ne dépasse pas 90 à 100 ans. Il est nécessuire aussi, dinns les coupes secondaires, de découvrir plus lìt les jeunes plants, moins délicats que ceux du hètre.

ARTICLE VII.

\section{Exploitation du charme on futaie.}

498. L Le charme n’est point une essence qui, on

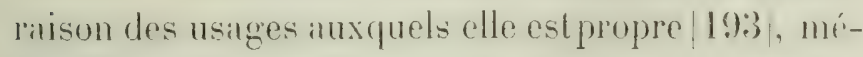
rite d'ètre élevée en futaie, maison le considere comme pourant ètre utilement mélangé, dans une futirie, a 
d'autres essences plus précieuses. C'est ainsi qu'on le trouve associé au chène et au châtaignier; et, quoiqu'il soit en général un peu dépissé par eux, il persiste assez longtemps pour leur servir d'appui jusqu'au moment où il devient possible de le faire disparaitre successivement par les éclaireies périodiques. Mêlé au chène, il produit des effets analogues à ceux du hètre, en procurant au sol une couche de terreau plns abondante, et en y maintenant la fraicheur par son couvert épais. Aussi le charme pourrait-il être très utilement introduit, en second étage (voir la note page 2.56), dins les massifs de chìne pur, situés en fonds compacts, où il se plail el réussit mieux que le hêtre.

ARTICLE VIII.

\section{Exploitation du bouleau en futaie.}

499. Le bouleau n'acquiert les qualités recherchées par les différents métiers qui l'emploient que vers l'âge de 50 à 60 ans, et il est d'ailleurs bien plus disposé à se reproduire de semence que de souche. On peut donc avec avantage l'élever en futaie, en fixant son exploitabilité à l'ìge que nous venons d'indiquer.

Quant aux coupes de régénération, l'expérience prouve qu'en les firisant à blanc étoc, ou en liaissant seulement quelques réserves éparses, on obtient des 
repeuplements tre's complets par les semences venant de la partie boisée voisine. La seule précaution à prendre, c'est d'entretenir le sol dians un état convenablement meuble, en lui donnant une légère culture à la houe, lorsqu'on s'aperçoit que l'année sera fertile en semences. Cette opération est indispensable : car sous des bouleaux, même en massif, le sol se gazonne et se couvre d'arbustes et de morts-bois, à cause du couvert trop faible de cet arbre.

Les éclaircies périodiques devront ètre très rapprochées, le bouleau demandant assez d'espace pour croître.

AR'TICLE IX.

\section{Exploitation du robinier en futaie.}

乌ั(0). Le robinier, recherché comme bois de travail, est, de plus, employé avec avantage diuns les constructions civiles et navites; il y a, par conséquent, des motifs fondés pour le traiter en futaie. Il est à observer d'ailleurs que, quand cet arbre est isolé, sa cime est très exposée à ètre brisée par les vents et que souvent lit rupture des branches principales fait écliter le tronc; sa culture en massif de futaie, en donnant le moyen d'obvier à cet inconvénient si grave, offrirait done un avantage de plus.

Quoique la végétalion extrèmement rapide du 


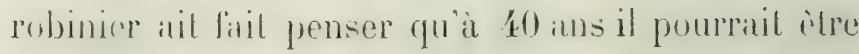
assez fort pour satisfaire à tous les besoins de lit consommation, il est à conseiller cependint de ne lixer son exploitabilité qu'à l’àge de 60 à 70 ans. De cetle minitre, on obliendra tris probablement des produits meilleur's et plus considéribles; et d'ailleurs il est à croire que sal végétation ne sera plus aussi rapide, s'il vient en missil, que lorsqu'il se trouvait isolé.

l'lus que l'orme encore, le robinier est disposé à drageonner, et sa semence, quoique non ailée, se slissimine au hoin; son jeune plant résiste aux chatlours, il croit tres rapilement el ses racines exigent hrameroup d'respatce; le mode d'exploitation preseril pour l'orme pourra done lui ètre applicable.

\section{IITICLE $\mathrm{X}$.}

\section{Exploitation d'une futaie de sapin.}

:1)1. Exploitabilité. - L'exploitabilité dı sapin tombe entre 100 et 140 ans; 120 ans est le terme le plus ordinaire et celui auquel, en général, on obtient les produits les plus utiles et les plus considérables ${ }^{1}$. Dins les sols trop humides cependant, ou lit régétition

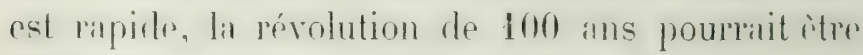
prefépible paren que. dans ces sortes de torrains, les silpins sont plus exposés encore que les hitres it la carre interpienre |84. Sur les grandes hauleurs,

' Voir l'Appendice. 
nii la ricueur du climat ralentit la croissance. Ie terme de l'exploitabilite peut itre reculi juspu it 140 ans et au-delà.

.02. Coupes de régénération. - Ainsi que nous l'avons dit dins le second livre [432], il est trì important que les exploitations d'une sapinière soient dirigées du Nord et de l'Est vers le sud et l'Ouest. Les coupes; d'ailleurs, doivent se faire à l'instar de colles des forèts de heitre. Ces deux essences se rencontrent sur un point escentiel. celui du temperirment des jeunes plants.

Dinns les trois premieres années de son existence, le sapin rešte très pelit, trìs faible, el sa tige, de mème que sa racine, s'allonge silns se ramifier. Pendint ce temps, il réclame un couvert au moins anssi épais que celui qui convient atu jeune hitre; mais, apres la troisieme année, lorequ'il al formi une of parfois deux branches laterrales, il devimut utitr de lui donner plus de lumire. Dís cette epoque, il y a done lieu de supprimer quelques arbres dims la réserve (un quart environ) ot de continuer ainsi, de deux en deux ou de trois en trois ans, jusqu'it la coupe définitive qui ne devra être fiaite que quand les brins auront atteint la hauteur do 66 centimitres à 1 mètre.

On rencontre assez souvent, dims les massifs de sapin que l'on soumet at la régénération, des repeuplements partiels qui remontent déjà à un isse\% grand nombre d'années, et régitent, languissants 
et rabougris, sous la vieille futaie. C'est à tort que certains forestiers les considèrent comme incapables de reprendre de la vigueur. Aucune essence, si ce n'est le hêtre, ne résiste autant que le sapin sous un couvert prolongé, et n'a plus que lui la propriété de se raviver dès qu'on lui donne du jour. On commet donc une faute en supprimant ces jeunes repeuplements au moment de la coupe d'ensemencement; il faut, au contraire, les conserver et, lors de cette coupe, éclaircir fortement la futaie qui les surmonte.

Il n'est pas sans intérît de réserver, dans la coupe définitive, quelques beaux sapins pour parcourir une seconde révolution. Cet arbre peut parvenir à un àge très avancé, et comme il sert beaucoup aux constructions, on obtiendra par ce moyen les pièces de fortes dimensions qui pourraient ìtre nécessaires dans certains cas. Il est entendu que de pareilles réserves ne peuvent ètre faites que dans les parties les plus abritées contre la violence des vents.

503. Coupes d'amélioration.- On se conformera sous ce rapport à ce qui a été recommandé dans les règles générales, en observant que le sapin demande. à croitre en massif très serré, surtout dans sa jeunesse. Dans les climats tempérés, l'éclaircie moyenne pourra lui être appliquée, mais, dans les localités où l'on aura à craindre les dégàls de la neige et du givre, auxquels les bois résineux sont plus exposés que les feuillus, on devra se contenter d'une éclaircie faible, afin que rerlaines tiges dominantes venant it 
être écrasées, puissent, au besoin, ìtre remplacées par celles qu'elles surmontent. Il n'est pas rare, en effet, de voir ces dernières, lorsqu'elles sont mises ì découvert, reprendre une vigueur remarquable.

ARTICLE XI.

\section{Exploitation d'une futaie mélangée de sapin et de hêtre.}

ö04. Le mélange du sapin arec le hètre est aussi favorable à la végétation que celui de cette dernière essence arec le chène. La nature nous en montre de fréquents exemples, et c'est une erreur bien funeste qui a porté certains forestiers ì détruire, dans res sortes de forèts, l'essence feuillue pour ne liaisser subsister que l'essence résineuse, alor's que tout, au contraire, semblait commander et ficiliter la conservation de ce mélange. En effet, on observe que, dans ces forèts mélangées, le sapin a une croissance remarquablement belle, et l'on reconnait, en outre, que les dégràts des vents et des insectes y sont moins à redouter que dans les forits purement résineuses. Quant à leur exploitation, lit même révolution convient aux deux essences; les jeunes plants de l'une et de l'autre ont le même tempérament, et, par conséquent, les coupes de régénération peuvent ître établies sans difficulté; dinns les différentes phases de leur croissance, les deux essences marchent à peu près du mème pas, ce qui ficilite l'exécution des 
iclaircies périodiques; et enfin, elles recherchent toutes deux, pour prospérer, mìme terrain, mème climat, mème situation et mème exposition. Elles ne different essentiellement que dans leur emploi, ce yui est un avantage évident pour lit consommation ut pour le propriétitire, d'autant plus qu'il est toujours facile de faire dominer te sapin, Iorsque sil quilité comme bois de construction doit lui mériter la préférence.

ARTICI, E XII.

Exploitation d'une futaie d'épicéa.

ö(\%). Exploitabilité. - Comme la croissance de l'épicéin est un peu plus prompte que celle du sitpin, la révolution ì laquelle il est soumis peut ètre plus courte. Ciest entre 90 et 140 ans que l'on con tixe lit durée; ordinairement, le terme de 100 à 120 ans est considéré comme le plus convenable pour obtenir de cette essence les produits les plus abondants et les plus utiles. Dans les localités où il importe de se procurer un certain nombre de pièces propres aux grandes constructions, on préfère l'ìge de 120 ans, parce que, à cause du peu de résistance que l'épicéa oppose au vent, ces pièces ne pourraient ctre fournies par les arbres qu'on réserverait dans les coupes, pour parcourir une seconde révolution. Sur les grandes hiauteurs, que souvent on le voit habiter. 
on peut laisser atteindre à l'épicéa l'ìge de 140 ans et au-delà, tandis que, dans un sol très humide, où cet arbre est, comme le sippin et le hêtre, exposé à la carrie intérieure, ce serait le cas de l'exploiter plus tòt.

\section{כั06. Coupes de régénération. — Commel'épicéa} redoute, plus que toute autre essence, les ravages des vents, et qu'il est d'ailleurs, dans sa première jeunesse, bien plus robuste et ne demande pas les mênies soins que le sippin, tous les efforts des sylviculteurs, dans l'exploitation des forets de cette essence, ont constamment tendu à préserver celles-ci du danger principal. Non-seulement on recomminde, comme extrêmement importante, la stricte observalion de la troisieme règle sur l'assiette des coupes $\mid$ 432)|, mais on a encore imaginé, toujours dans le meimr but, divers modes particulier's d'exploilition, sitvoil': $1^{\circ}$ le mode par bandes alternées; 2" le mode par bonquets; $3^{\circ}$ celui par bandes de proche en proche. Enfin, on a aussi appliqué le mode ordinaire des trois coupes de régénération.

507 . Premier mode. - Le premier mode est un de ceux qui ont eté le plus mis en pratique; il consiste à établir les coupes par bandes alternées de hois à abattre à blanc oloc, et de Jois a laisser en massil, les unes et les autres de öl it 60 mi:tres de liurgeur. L'ensemencement des bandes exploitées doit venir des bandes roisines oì tous les bois sont conservés, et, pour mieux préparer le terain des premieres à receroir la semence, on conspille d'en firire extraire 
les souches. Quelques années après l'ensemencement, on exploite les bandes qui étaient demeurées intactes. Ce retour, en effet, est indispensable, car si l'on continuait à alterner ainsi d'un bout à l'autre de la forêt, il est évident que l'on ne tarderait pas à tomber dans des massifs éloignés d'aroir atteint l'àge de l'exploitabilité, tandis qu'au point de départ, on en laisserait sur pied qui auraient dépassé cet àge:

Quoique, au premier abord, ce mode paraisse très avantageux, il présente cependant des inconvénients assez graves pour que nous n’hésitions pas à le déconseiller, comme n'ítant pas propre à conduire au but que l'on se propose:

$1^{\circ}$ L'expérience a prouvé que les bandes laissées en massif, loin de résister toujours au vent, sont souvent renversées avec une grande facilité;

$2^{\circ}$ L'ensemencement naturel des bandes mises à blanc étoc est presque toujours incomplet, les plantes nuisibles s'y jettent et rendent nécessaire un repeuplement artificiel;

$3^{\circ}$ Lorsqu'on arrive à exploiter les bandes primitirement réservées en massif que le vent a pu épargner, il est impossible d'y faire aucune réserve, et, les parties voisines étant beaucoup trop jeunes pour porter graine, il est nécessaire de recourir à un repeuplement artificiel. D'où il résulte que la moitié de lia forêt, au moins, est à repeupler artificiellement;

$4^{\circ}$ Enfin, on établit dans toute la forèt une grande inégalité d'àge entre des parties contiguës, ce qui 
entraine les inconvénients énumérés au sujet de la première règle sur l'assiette des coupes $[430]$ et, de plus, favorise l'action des vents.

วั08. Deuxième mode. - Dans le second mode, dit par houquets, au lieu de bandes alternées, on laisse çà et là, dans les parties qu'on exploite à blanc étoc. de petits bouquets ou massifs de bois, qui doivent fournir les graines nécessaires au repeuplement. Ce mode participe de tous les inconvénients du précédent, et introduit en outre une plus grande irrégularité dans les exploitations et une plus grande inégalité dans les àges des bois; on ne peut done que le rejeter.

ö09. Troisième mode. - Le troisième mode, donné par Hartig, parait plus praticable. Yoici comment cet auteur conseille de procéder.

On doit asseoir, en commenegant du còté du Nord ou du Nord-Est, une coupe longue et étroitr 1, et l'exploiter à blane f́toc. (Guand on s'apercevra que la partie voisine porte des crines et qu'on peut en espérer des semences abondantes, on extraira toutes les souches de la coupe, et l'on comblera, au moins en partie, les excavations. [lans cet état, le terrain se trouvera bien préparé à recevoir les graines poussées par les vents du sud et d'Ouest. Par pré-

- Cotta conseille de ne donner à ces coupes qu'une largeur égale à la longueur des arbres, atin de ne pas compromettre le réensemencement naturel. 
caution, on ferabien de répandre, sur ce terrain fraichement remué, 午 à : hilogrammes de semences d'ipicéa, par hectare; ce qui équiraut, à peu près, alu tiers de la quantilé néersaire pour un ensemencement complet.

Sur ee point, il faudra naturellement suspendre les exploitations, jusqu'ì ce que la coupe soit suflisimment garnie de jeunes plints. Ce n'est qu'apris le repeuploment assuré qu'on pourra asseoir, dans la partie attenante, une nouvelle coupe de mème longueur et de même largeur quo la première; el ainsi de suite.

Celte nécessité d'altendre que le repeuplement de la coupe exploitée soit assuré, pour mettre la hacho dans la partie voisine, force d'entamer lic forèt sur un autre point exploitable et mème sur trois ou quatre points différents, sur lesquels on se portr successivement, afin de laisser, dans la premiere partie exploitée, $u$ intervalle de trois ou quatre années au moins entre deux coupes consécutives, et d'assurer ainsi le repeuplement. Si, cependant, il arrivait que ces trois ou quatre années ne fussent pas suffisantes pour amener ce repeuplement, el qu'il fìl d'inilleurs impossible de suspendre les exploitations, il ne resterait plus d'autre moyen que de régénérer les coupes par un semis artificiel ou par une plitntation.

J̈10. Quatrième mode. - L'application de la méthode du rénsemencement maturel et des échircies, 
forme un quitrième mode, et c'est, sans contredit, dans lia plupart des cals, le plus recommandable.

Eu égard au tempérament du jeune plant d'ipicéa, la coupe d’ensemencement pourrait ètre plus espacée que celle du sapin, et mème que celle du hètre; ce n'est qu ì cause des faibles racines des ipicéas, qu'ilest à conseiller de multiplier les réserves autant que dans les forèts de hètre, afin qu'elles puissent se soutenir reciprorquement. Wuand le repeuplement de celte coupe sera complet et aura itteint la hauteur de 16 à 22 centim itres, on pourria faire la cuupe secondaire: et lorsque les plants seront

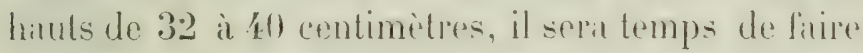
la coupe définitive.

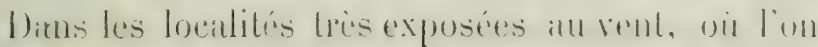
receomnait que la réserve de lit coupe secontiare ne puetracil pas résister, il est preférable de litis-er lit coupe sombre, sans y toncher, jusqua it ce que le

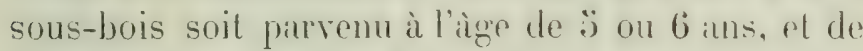
faire allors la coupe défnitive. La coupe secondaire cependant, dont l'utilité est incontestable, ne doit ètre négligée que diuns le cats d'urgente nécessité.

Ce mode, ansi que nous renons de le dire plus hatut, nous pariait le meilleur. In lemployant, on

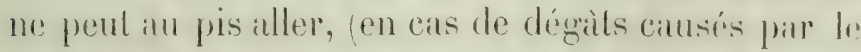
rent), qu'itre obligé de recourir sur quelques points an repenplement antificiel; mais cet inconrénient est inévitable en suivant les trois autres modes of surtout les deux premiers. On peut d'ailleurs, en 
l'appliquant, user des précautions recommandées pour le troisième: Ainsi, il est à conseiller de donner aux coupes une forme longue et étroite, et d'entamer la forèt au moins sur deux points différents, de manière à pouvoir alterner les exploitations; afin que, si la semence manque pendant quelques années sur l'un, on ne soit pas obligé d'y continuer les coupes sombres, dont lit trop grande extension finirait par donner prise aux vents.

ö11. Des quatre modes que nous renons d'exitminer, les deux premiers doivent être rejetés, ainsi que nous l'avons démontré. Le troisième a sans doute l'avantage de parer, dans beaucoup de cas, aux ravages du vent; mais, aux expositions fraîches, l'herbe et divers arbustes envahissent le terriin dès qu'il est mis à nu et étouffent les épicéas naissants ; sur les pentes chaudes et escarpées, une lumiëre trop rive el trop de chaleur les empêchent de réussir, el en général, la régénération naturelle s'opère mal, dès qu'elle n’a pas iien l'année même de l'exploitation. Enfin, Je quatrième mode, qui mérite incontestablement la préférence, présente cependant l'inconvénient de nombreux chiblis qui, dans leur chute, se rompent fréquemment ou brisent d'autres arbres restés sur pied, ce qui constilue souvent une perte considérable pour le propriétaire.

Dans les montagnes du Harz, en Sitxe, et en général dans les pilys où l'épicéa est très répandu, les difficultés d'exploitation, que présentent les forèts de 
celte essence, ont fait adopter, dans ces derniers lemps, les coupes à blanc étoc; mais au lieu de compter, comme autrefois, sur les parties roisines pour réensemencer le terrain, on a immédiatement recours à la plantation, à laquelle l'épicéa est particulièrement propre. Cette opération, dont il sera question dans le VI livre de ce Cours, réussit parfaitement et n'occasionne qu'une faible dépense, eu égard surtout aux années qu'elle fait gagner à la régétation de la jeune forèt. Aussi peut-on considérer les coupes de régénération comme presque abandonnées aujourd'hui dans les forèts d'épicéia d'outreRhin.

Tout en reconnaissant l'importance des firits que nous venons de rapporter et en admettant, ia point de vue économique, les conclusions pratiques que nos voisins en ont tirées, nous pensons que, en France, dans les localités peu nombreuses diailleurs et toujours montigneuses, où l'épicéir se rencontre comme essence dominante, on fera bien de ne recourir au repeuplement artificiel quapris avoir reconnu les inconvénients ou l'impuissance des coupes de régénération. Wécouvrir et remuer le sol en monlagne, surtoul dins les pentes rapides, nous parrait toujours une opération qui peut avoir les plus funestes conséquences. - Nous nous arrètons d'autant plus volontiers à cetavis, qu'il est à notre connaissance que, dans différentes forèts d'épicéia du Jura, des Vosges et des Alpes, l'application de la méthode du réense- 
mencement natured a produil jusqu ì ce jour des résultals satisfaisants.

312. Coupes d'amélioration. - Ce qui a eté dil sons ce ripport, au sujet des foreils de sitpin $|5033|$. est entièrement applicable à celles d'épicéa.

AR'TICLE XIII.

Exploitation d'une futaie mélangée de sapin et d'épicéa.

:3:3. Le mélange du sapin et de l'épicéa, comme colui du chène et du hètre, et par les mèmes raisons, produit les meilleurs efross sul la végrétation des deux essences. L’épicéa, à lit virité, a une croissance un peuphus prompte que le sipin, mais cette différence est hien peu sensible. Cependant, on remarque assez fréquemment, dims les torèts ainsi mèlingées, que l'épicéa tend peu à peu à empiétel sur le sapin et à le déposséder du terrain, ce qui est tìcheux en général, et surtout dans certaines localités ou, pour divers motifs, le sapin est l'essence préférée. Selon toutes les apparences, cet inconvénient tient principalement a ce que, dans les coupes de régéntération, on n'accorde pas une attention assez grande atu repeuplement dusilpin, qui réussit Jjen plus difticilement que celui de l'épictar, c'est-it-dire que, d'ahord, on ne conserve pats dans lat coupe sombre un comvert assez epalis, ef yuensuite on échircit trop fortement lit coupe secondaire. 
La précaution essentielle, pour conserver le mélange si utile des deux essences, est donc de faire les coupes d'ensemencement surtout dans les années où, pour le sapin, on peut prévoir une fructification abondante, de composer la réserve dans ces coupes du plus grand nombre possible de sapins, et enfin de régler les coupes secondaires principalement en vue des exigences de cette essence.

Il pourra bien arriver ainsi que la croissance des sapineaux gagne quelque avance sur celle des jeunes épicéas, qui exigent plus de lumière dès les premières années; mais, quoique entravés momentanément, ces derniers ne tarderont pas à reprendre leur vigueur habituelle, sitòt que le couvert sera suffisamment éclairci.

ARTICLE XIV.

Exploitation d'une futaie de pin sylvestre.

3̆14. Exploitabilité. - Dans les pays où le pin sylvestre est très commun, on ne le laisse pas atteindre le siècle. Effectivement, l'accroissement des massifs de cette essence atteint son maximum entre 60 et 80 ans; puis, après s'être maintenu pendant une vingtaine d'années, il diminue en raison de l'augmentation de l'àge. Si donc, l'intérêt principal était de fournir du chauffage, on ferait bien de fixer la révolution d'une forèt de pin sylvestre vers 80 ou 90 ans; 
encore devrait-on réserver des parties de forêt, pour les soumettre à une révolution plus longue afin d'obtenir des arbres de service de bonne qualité.

En France, où les bois de construction sont plus rares que ceux de chauffage, l'État ne peut que gagner à reculer l'exploitation du pin sylvestre. Dans le moment de sa plus forte croissance, son bois n'a pas les meilleures qualités. Ce n'est que plus tard que son grain devient serré, qu'il se charge de résine concrète et acquiert ainsi l'élasticité et la durée qui le rendent précieux pour les grandes constructions, pour la marine surtout [301]. D'après ces motifs, on fera bien de soumettre le pin sylvestre à une révolution de 120 ans dans les terrains où sa croissance est active, et de ne l'exploiter qu'entre 140 et 160 ans $^{1}$ dans les sols secs et suffisamment profonds, tels que les sables siliceux où cette essence se plaît et acquiert surtout les qualités qui la rendent propre aux grands emplois.

- Les longues révolutions ont l'inconvénient de compromettre la fertilité du sol, parce que la futaie de pin sylvestre, une fois la période d'allongement passée, ne se constitue plus en massif clos et, parvenue à un âge avancé, devient tout à fait clairiérée. Il s'ensuit que, de bonne heure, le sol se gazonne ou se couvre d'arbustes nuisibles (myrtiles, bruyères, etc.), que le vent disperse le lit de feuilles mortes et que, à la fin de la révolution, le terrain se trouve tassé, desséché, appauvri, et dans les conditions les plus favorables à la multiplication de certains lépidoptères fort nuisibles, dont les chenilles, pour hiverner, ne se terrent que dans les sols très secs. 
כ15. Coupes de régénération. - Le traitement du pin sylvestre, en ce qui concerne le réensemencement naturel, diffère sous quelques rapports de celui qu'on prescrit pour l'épicéa et le sapin; son jeune plant, comme on le sait, est plus robuste que celui des deux autres, et le couvert des arbres de réserve lui est absolument contraire dès les premières années de son existence. Aussi n'est-il pas nécessaire que la coupe d'ensemencement soit sombre, et l'on peut sans inconvénient écarter les arbres de manière que leurs branches soient distantes

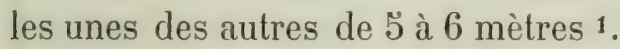

Cependant, en espaçant ainsi les réserves, on pourrait craindre les dégâts des vents, ou l'envahissement des herbes ou des plantes nuisibles. Le premier inconvénient existe beaucoup moins pour le pin sylvestre que pour l'épicéa et mème pour le sit-

Lor's done qu'on se décidera pour une révolution de 120 ans et au-dessus, on devra non-sculement conserver soigneusement tous les bois feuillus qui lèvent naturellement sous les pins, mais on pourra mème recourir arec avantage à l'expédient qui a été indiqué pour le chêne (voir la note page 250), c'est-à-dire, introduire, vers le milicu de la révolution, une essence subsidiaire destinée à protéger et à améliorer le sol. Parmi les bois feuillus, le charme, le hêtre; parni les résineux, Io sapin, seront très propres à remplir ce rôle. Ce dernier surtout, placé en second étage, donne à la végétation du pin un essor magnifique.

1 En faisant le choix des arbres de réserve, il ne faut pas préférer les pieds élancés et très élevés; il vaut mieux conserver 
pin, attendu que le pin sylvestre pivote et s'enracine plus fortement. Néanmoins, dans les parties très exposées au vent, et où l'expérience a appris que cet arbre lui résiste difficilement, on fera bien de laisser plus de réserves. Le second inconvénient est fréquent dans les pineraies et, dans certains terrains, à peu près inévitable. II peut être atténué cependant en ne faisant de coupes d'ensemencement que quand on sera assuré d'une prochaine et abondinte fructification, ce qui, dans les pins, est assez fréquent, et peut être prévu dix-huit mois à l'avance. De plus, dans l'automne ou l'hiver qui précèdera $\mathrm{I}_{\mathrm{a}}$ dissémination de la graine, on fera l'extraction des souches et l'on donnera au terrain une culture par rayons ou sillons étroits, au fond desquels les semences viendront s'arrêter el trouveront une terre meuble et fraiche, favorable à leur germination et à la réussite du jeune plant.

Dans la plupart des cas, ces travaux ne seront pas onéreux, car les souches du pin, par la résine qu'on en extrait, sont plus lucratives que celles des autres bois.

Après la coupe d'ensemencement, dès que le re-

ceux de hateur moyenne, qui sont branchus, ordinairement plus fertiles en semences, et qui d'ailleurs résistent mieux aux vents. Dans l'etat actuel de nos forèts de pin, ec choix peut avoir licu; mais, plus tard, lorsqu'clles auront été traitées régulièrement, on ne trourera plus, que sur les lisières, des arbres peu élevés et chargés de branches. 
peuplement est complet, et que les jeumes plants ont alteint à peu près la hauteur de 16 à 24 centimètres, il faut procéder à la coupe définitive. On conçoit facilement que la nature vigoureuse du jeune plant rende inutile la coupe secondaire, et qu'il faille se hàter surtout de le débarrasser du couvert qui lui est tout à fait contraire.

516. Malgré les précautions prises pour régénérer le pin sylvestre par la voie naturelle, on est forcé de reconnaître qu'il est extrêmement rare de rencontrer des repeuplements complets et bienvenants dans les forêts de cette essence. Aussi beaucoup de bons forestiers sont-ils d'avis de renoncer aux coupes d'ensemencement dans les pineraies, de couper à blane étoc et de recourir aux semis artificiels qui réussissent facilement et tries bien. A considérer la génóralité des faits, cette opinion est fondée, il fiut le dire. Que la réserve de la coupe d'ensemencement soit nombreuse ou non, que l'on hâte la coupe définitive ou qu'on la retarde, on voit fréquemment les réensemencements naturels les plus complets et les mieux venants péricliter au bout de quelques années, s'amoindrir et mème disparaìtre par places a u point de donner accès aux morts-bois et aux bois blancs. La cause du phénomène est assez difficile à indiquer. Certains auteurs l'attribuent à quelques insectes tels que le ver du hanneton, le pissode noté et l'hylobe, qui attaquent, les uns les tiges, les autres les racines des jeunes pins. M. de Berg pense, avec 
raison, selon nous, qu'il faut la chercher le plus souvent dans la position superficielle des racines des vieux pins, dont le chevelu très abondant garnit et pénètre le sol en tous sens.

En admettant cette opinion, on doit en conclure que les repeuplements naturels n'auront chance de réussir que dans les terrains assez riches et assez profonds pour que le pin, en avançant en àge, y enfonce ses racines plus bas que la couche dans laquelle les jeunes plants étendent les leurs pendant les premières années. Mais on sait que les pineraies occupent en général les sols les moins fertiles; on s'explique done aisément comment il est si rare d'y rencontrer des repeuplements naturels bien venants et complets.

N'était la considération importante de la dépense qu'occasionne le repeuplement artificiel par l'achat de la graine qui est assez chère, et par les frais de labour; n'était, de plus, la chance que l'on court de ne pas obtenir toujours de bonnes graines quand on est obligé de se les procurer par le commerce, on serait conduit, d'après ce que nous venons de dire, à poser en principe que la régénération du pin sylvestre doit s'opérer par coupes à blanc étoc suivies immédiatement de semis artificiels, et que les coupes d'ensemencement ne doivent être pratiquées qu'exceptionnellement, dans les terrains en pente, par exemple. En un mot, à nos yeux, la raison culturale conseille le premier mode, mais la raison financière 
pourrait, dans certains cas, justifier le second 1.

517. Coupes d'amélioration. Les pins sont, plus que les deux précédents conifères, exposés à se ployer ou à se rompre sous le poids de la neige et du givre, à cause de leurs branches plus étalées et de leurs feuilles plus longues ${ }^{2}$; il semblerait donc, au premier abord, qu'on ne devrait y pratiquer que l'éclaircie faible. Mais cette essence, dès qu'elle passe à l'état de gaulis, supporte mal le massif serré. Les branches qui s'entrelacent perdent leurs feuilles et ne tardent pas à périr; la tête des arbres, au lieu de se développer circulairement, devient grêle, étriquée; et quand cet état de souffrance se prolonge, les cimes, en général, se déforment, les tiges elles-mêmes contractent des maladies et le peuplement tout entier devient parfois incapable d'atteindre un âge avancé et de belles dimensions. - Pour prévenir ce dernier et grave inconvénient, sans cependant exposer la jeune pineraie à ètre brisée par la neige, le moyen le plus sûr, dont l'efficacité est aujourd'hui démontrée par l'expérience, consiste : $1^{\circ}$ à entreprendre la première éclaircie (contrairement à la théorie générale), lorsque le bois est encore à l'état de fourré, au moment où les branches les plus inférieures commencent à sécher; $2^{\circ}$ à faire cette éclaircie forte. A la vé-

\section{- Voir l'appendice.}

" Cela peut tenir aussi à ce que la neige tombe en flocons plus gros et plus lourds clans les climats habités par le pin sylvestre. 
rité, il pourra arriver ainsi que la production de terreau, si abondante surtout dans la première jeunesse des pineraies, se trouve entravée ou au moins diminuée. Mais cet inconvénient ne sera que momen-. tané, attendu que les brins restants, ayant plus d'espace pour étendre leurs branches, ne tarderont pas ì rendre au sol un épais couvert, tandis que, d'un autre côté, ils se formeront une tête bien conique, propre à assurer leur bonne croissance ultérieure ; en même temps, leur tige deviendra plus trapue et assez forte pour résister au poids de la neige.

Les éclaircies suivantes devront être plus rapprochées que dans les sapins et les épicéas, ce qui, sans doute, rendra chacune d'elles moins productive; mais, prises ensemble, elles le seront davantage et exerceront, sur la végétation des tiges destinées à croìtre jusqu'à la fin de la révolution, l'influence salutaire qui est le but principal de ce genre d'opérattion.

ARTICLE XV.

Exploitation d'une futaie de pin à crochets '.

5̈18. Les forêts de pin à crochets qui se trouvent dans les Alpes françaises n'ont pas été soumises, jusqu'ici, à une exploitation régulière et raisonnée.

' Cet article est tiré, en grande partic, d'un mémoire de $\mathrm{H}$. du Guiny sur les futaies résineuses des IIautes-Alpes. 
Ainsi que nous l'arons dit ailleurs (page 1350), ce n'est que depuis peu d'années qu'elles ont été l'objet de quelques études intelligentes qui nous ont mis ì même d'apprécier cette essence. On n'a donc pas, sur les particularités du mode de traitement à leur appliquer, de renseignements bien certains et qui soient le fruit d'une longue expérience. - Voici, toutefois, ce que l'exploration attentive des lieux, jointe aux notions acquises sur les propriétés et les exigences de l'arbre, permet d'établir à cet égard.

5ั19. Exploitabilité. L'extrême lenteur de la végétation et les retards qu'éprouve généralement le réensemencement naturel dans les hautes régions habitées par le pin à crochets, exigent qu'il ne soit exploité qu'à un àge avancé, surtout s'il était reconnu qu'il est propre à la màture. En vue de cette dernière éventualité, les révolutions qu'il semblerail convenable de lui appliquer seraient, selon l'altitude à laquelle se trouve la forêt, les suivantes:

$$
\begin{aligned}
& \text { de } 1500 \text { à } 1800^{\mathrm{m}}-160 \text { à } 180 \text { ans, } \\
& \text { de } 1800 \text { à } 3000^{\mathrm{m}}-200 \text { ans. }
\end{aligned}
$$

520. Coupes de régénération. L'abondance de lia graine du pin à crochets dans les années où elle réussit, et sa légèreté, ainsi que le tempérament robuste du jeune plant, permettront de tenir assez espacées les réserves de la coupe d'ensemencement, qu'on devra d'ailleurs choisir parmi les pieds les plus vivaces et les mieux enracinés. Toutefois, dans 
les parties exposées aux ouragans, il est à conseiller de les disposer par petits groupes ou bouquets, qui auront plus de chances de résister à l'effort du vent que des arbres isolés.

La coupe secondaire pourra se faire en une seule fois; elle sera suivie de près par la coupe définitive.

Afin de diminuer les retards de la régénération naturelle, il sera bon, partout où l'on n'aura pas à redouter les coulées de neige et l'éboulement des terres, de procéder à l'extraction des souches et même au dégazonnement partiel du sol, d'après le mode que nous arons indiqué plus haut [492 et 4953 .

Aux enseignements qui précèdent, il est nécessaire d'ajouter quelques recommandations particulières relatives, surtout, à l'assiette des coupes dans les localités exceptionnelles où croît le pin à crochets.

$1^{\circ}$ La $4^{\circ}$ règle d'assiette $[434]$ ne pouvant que rarement être appliquée dans ces hautes montagnes, faute de voies de vidange qui permettent d'éviter les inconvénients que signale la $2^{\circ}$ règle $[431]$, il est d'autant plus important d'établir, dans la partie supérieure de presque tous les massifs situés en pente rapide, un rideau tel qu'il est prescrit dans les développements de cette $4^{\circ}$ règle, mais beaucoup plus profond ( 150 à $\left.200^{\mathrm{m}}\right)$, attendu l'extrême violence des coups de vent.

De plus, la prudence exige, dans un grand nombre de cas, que l'on mette en réserve la lisière inférieure des forêts qui dominent les villages et les cul- 
tures, afin de garantir les unes et les autres contre les avalanches et contre les effets du ravinement des pentes.

$2^{\circ}$ Eu égard aux dangers qui viennent d'être signalés, il sera essentiel de ne point asseoir les coupes d'ensemencement d'un seul tenant, mais bien par coupons séparés, et d'assurer ensuite le prompt repeuplement de ces différents coupons. - Cette manière d'opérer entraînera incontestablement quelques-uns des inconvénients reprochés au mode d'exploitation appelé jardinage [438]. Toutefois, ces inconvénients seront très atténués si l'assiette des coupons, au lieu d'être abandonnée au hasard, est soumise à un certain ordre. Dès lors, les repeuplements, tout en différant d'àge, par places, ne se gêneront pas trop entre eux, el, considérés dans leur ensemble, les divers cantons composint la forêt présenteront encore une succession assez régulièrement graduée. Or, il est permis de penser que, dans les hautes régions qu'habite le pin à crochets, il serait difficile, sinon impossible, d'atteindre un résultät plus satisfaisant.

521. Coupes d'amélioration. - L'essence qui nous occupe croît, ainsi que nous l'avons dit, avec une extrême lenteur, particulièrement dans sa jeunesse, et se maintient en massif très serré. Cette double circonstance doit faire différer le commencement des éclaircies au moins jusqu'à l'âge de 40 ans; de plus, ces opérations devront ètre très faibles 
[16T], afin que le massif continue sùrement ì résister aux avalanches et aux éboulements, et qu'il procure au sol une couche abondante de terreau qui, souvent, forme la seule richesse de ce sol.

En raison, précisément, de ces éclaircies toujours faibles, leur périodicité semble pouvoir être fixée à 15 ou 20 ans. - Lors de la dernière, seulement, il sera bon de desserrer le massif plus que dans les précédentes, afin d'amener, par avance, du semis naturel el d'assurer ainsi la prompte réussite des coupes de régénération. - Le tempérament du jeune plinnt qui résiste lontemps sous le couvert $[306\rceil$ justifie cetle recommandation.

ARTICLE XVI.

Exploitation d'une futaie de pin maritime.

522. Exploitabilité. - Pour savoir positivement ce que cet arbre peut devenir sous le rapport de son accroissement, el à quelle époque il conviendrait de fixer son exploitabilité, il faudrait le cultiver sans tourmenter sa vegétation [319]. En attendant les lumières de l'expérience, on ne peut juger que par analogie.

Il est assez ordinaire que les arbres qui ont une végétation trìs rapide dans leur jeunesse, atteignent plus tôt que les autres leur maximum d'accroissement. Le pin maritime devrait être particulièrement dans ce cas. Cependiant, dans les départements méri- 
dionaux où il trouve le climat qui le fait prospérer, il paraît hors de doute que la période ascendante de son accroissement est assez longue, et, dès lors, on fera bien de retarder son exploitation jusqu'à 100 ou même 120 ans, suivant la nature du sol, afin que le bois puisse acquérir la solidité convenable pour les constructions et les autres emplois auxquels il il est propre.

Bien entendu qu'en adoptant cette exploitabilité, il faut se garder de permettre le gemmage.

523. Coupes de régénération. - Le pin marilime n'ayant point encore été soumis à un traitement régulier, on pourrait ètre incertain sur le mode à suivre; mais la nature de ses graines, le tempérament de son jeune plant et lit disposition de ses racines doivent faire conclure que cet arbre peut ètre exploité de même que le pin sylvestre. Ainsi, dans la coupe d'ensemencement, on pourra d'autant plus espacer les réserves, que le pin maritime résiste encore mieux aux coups de vent. Sa semence, à là vérité, est un peu plus grosse et un peu plus lourde que celle du pin sylvestre, mais la membrane quii lui sert d'aile est d'autant plus gramde, ce qui doit litvoriser sa dissémination au loin. La coupe secondaire deviendra inutileen raison du tempérament robuste du jeune plant; et l'extrème rapidité avec laquelle il végète fait penser que la coupe définitive devra succéder à celle d'ensemencement le plus tòt possible, c'est-à-dire, dans l'année qui suirra la naissance du 
repeuplement ou au plus tard deux années après.

524. Coupes d'amélioration. - Ce que nous avons dit à cet égard du pin sylvestre [วั17] s'applique aussi au pin maritime. La rapidité de sacroissance devra nécessairement faire avancer la première éclaircie et abréger la périodicité des éclaircies suivantes.

ว2ว้. Les règles d'exploitation que nous venons d'exposer ne s'appliquent qu'aux forêts de pin marilime qui ne doivent pas ètre soumises au gemmage. Lorsqu'on se propose au contraire de gemmer, elles doivent se modifier: d'une part, ce procédé ralentit la croissance et abrège la vie de l'arbre; de l'autre, ce n'est plus le bois, mais bien la résine qui devient le produit principal de la forêt. La première circonstance emporte avec elle des révolutions de plus courte durée, la seconde exige un mode particulier d'éclaircies très rapprochées que nous allons décrire, tel qu'il se pratique dans le midi de la France.

L'intérêt du propriétaire étant évidemment de hâter l'époque à laquelle il pourra commencer le gremmage, il importe de favoriser le développement des branches et le grossissement des tiges le plus possible. A cet effet, on éclaircit les jeunes bois pour la première fois à l'àge de 7 ans, el ensuite de 6 en 6 années, jusqu'à 25 ans, époque à laquelle ils ont ordinairement atteint la grosseur convenable. Dans ces opérations, on amène par degrés l'espacement 
des pins entre eux : les deux premières doivent conserver encore le massif, quoique clair, afin d'activer la croissance en hauteur, mais, dès la troisième, on réduit le nombre des tiges à 700 ou 800 par hectare, et, à la quatrième, on n'en laisse plus subsister que 500 ; enfin, $\breve{~ a n s ~ p l u s ~ t a r d, ~ c ' e s t-a ̀-d i r e, ~ a ̀ ~} 30$ ans, on réduit ce dernier nombre à 400 . Les 100 arbres destinés à tomber, dans la cinquième éclaircie, sont désignés dès la quatrième et gemmés à mort [319]; les autres le sont à vie à partir de la même époque.

Ces 400 pins restent ensuite sur pied de 30 à 60 ans et sont soumis au gemmage de らૅ en ら années. A 60 ans, on marque de nouveau 100 arbres pour être gemmés à mort, puis abattus, et les 300 pieds restants demeurent debout jusqu'à la coupe finale qui a lieu lantôt à 70 ou 80 ans, tantòt seulement à 100 ans, selon l'étal des bois et la qualité du sol!

526. Nous avons indiqué plus haut comment les coupes de régénération devraient être pratiquées si l'on se proposait d'y recourir; mais il est à croire que généralement on emploiera plutôt la coupe à blanc étoc suivie du semis arlificiel. En effet, dans

' Dans l'ouest et le centre de la France, où le pin maritime occupe aujourd'hui de vastes étendues, au grand avantage des propriétaires et des populations, sa végétation est beaucoup moins belle et sa durée moindre que dans le midi. II en résulte la nécessité de le couper au plus tard à 60 ans, et par conséquent de le gemmer moins longtemps. 
l'un comme dans l'autre système, un labour devient indispensable, car, par suite du grand espacement donné aux pins, le terrain ne peut manquer d'ètre couvert de toutes sortes d'arbustes et de plantes nuisibles; d'un autre côté, la graine du pin maritime coûte peu à récolter et à extraire, elle est presque toujours de très bonne qualité, el les semis qu'on en lait réussissent parfaitement; tout se réunit donc pour faire donner la préférence à la voie artificielle.

\section{ARTICLE XVII.}

\section{Exploitation d'une futaie de pin Laricio.}

527. Le laricio, comme le pin maritime, atteint assez vite son maximum d'accroissement. Ainsi, à l'àge de 80 à 100 ans, il a déjà d'assez fortes dimensions; mais, pour qu'il devienne propre à la construction et aux différents emplois qui exigent des bois de qualité supérieure, il est essentiel de le laisser vieillir bien davantage. L'àge de 160 ans pourrait être fixé pour son exploitation, et l'on fera bien de reculer ce terme jusqu'à 200 ans au moins, sur les hautes montagnes.

Le laricio a tant d'analogie avec le pin sylvestre, qu'on peut affirmer que le même traitement lui convient, tant pour les coupes de régénération que pour celles d'amélioration. 
DES FUTAIES.

AR'TICLE XVIII.

Exploitation d'une futaie de pin noir.

כ228. Les auteurs forestiers qui ont écrit surcette essence s'accordent tous pour assimiler le traitement à lui appliquer à celui du pin sylvestre. La 8 édition de la Culture des bois de Cotta, publiée en 1856 , contient, à ce sujet, les renseignements suivants :

Pour que le pin noir atteigne les dimensions et les qualités qu'il est susceptible d'acquérir, il convient de le soumettre à une rérolution de 120 à 150 ans, suivant qu'il occupe des régions plus ou moins élevées. - Dans la première jeunesse, il supporte. un peu mieux que le pin sylvestre, le couvert des grands arbres [515], mais, dès qu'il parvient à l'àge moyen, il ne se maintient pas plus que lui en massif serré, ce qui entraine la plupart du temps le gazonnement du sol et l'invasion de divers arbustes et morts-bois. - Les coupes de régénération doivent se faire dans les forìts de pin noir comme dans celles de pin sylvestre; 60 à 80 réserves suffiront, par hectare, pour composer la coupe d'ensemencement. L'essouchement des morts-bois et une culture par sillons donnée au sol, seront en général très utiles pour assurer la prompte réussite du semis naturel.

Les coupes d'amélioration, dont l'ouvrage cité ne parle pas, pourront probablement ètre effectuées 
d'après les règles que nous avons données plus haut [ڤ17] pour les forêts de pin sylvestre.

\section{ARTICLE XIX.}

\section{Exploitation d'une futaie de pin d'Alep.}

529. Le pin d'Alep, de mème que le pin maritime, atteint son plus fort accroissement dès l'àge de 60 à 70 ans ; mais, comme il est employé aux constructions, on doit croire qu'il serait préférable de le laisser arrirer au moins à l'àge de 80 ans, afin que son bois pût acquérir plus de solidité.

Pour pouvoir donner des règles sur le mode d'exploitation qui lui convient, il faudrait que cet arbre eùt été étudié en forèt, dans le climat qui lui est propre. On sait cependant qu'il n'est pas fortement enraciné, et, selon toute probabilité, son jeune plant, sous le soleil brùlant des contrées où il végète, se trouverait bien de quelque abri dans les premières années.

\section{ARTICLE XX.}

Exploitation du pin pinier, du pin cembro,et du pin du lord Weymouth.

כ330. Le pin pinier, en France du moins, n'est encore cultivé que comme fruitier. En attendant que la sylviculture s'en occupe à d'autres points de vue, ce qui est fort à désirer, il n'y a pas lieu de rechercher les particularités du mode d'ex- 
ploitation qui, un jour, pourra lui être applicable.

531. Quant au cembro dont il n'existe, dans notre pays, qu'une seule forèt, de faible étendue, à l'état pur [4ว̋6], on pourrait presque émettre la même opinion. Toutefois, cette essence se trouve, dans les Alpes, fréquemment mélangée au mélèze, au pin à crochets et à l'épicéa, et son importance, surtout au point de vue des services qu'elle est appelée à rendre pour le reboisement des hautes sommités, est assez grande pour qu'on s'attache, non seulement à la conserver là où elle existe, mais encore à la propager autant que possible. On y parviendra en réservant les pieds de cembro partout où ils se rencontreront, dans les coupes d'ensemencement aussi bien que dans les éclaircies, et en donnant aux jeunes plants tous les soins et toute l'attention qu'ils réclament pour réussir. - D'après des observations, faites tant en Suisse que dans les Alpes françaises, il paraîtrait que le traitement indiqué plus haut pour les forêts d'épicéa [弓̊l0 et 512$]$ serait celui qui conviendrait le mieux au cembro.

Non seulement les hommes, pour le commerce qu'ils en font, détournent une grande partie des graines de cembro de leur destination naturelle, mais les casse-noix (mucifraga caryocatactes) qui, à l'arrière saison, passent en troupes considérables sur les Alpes, les recherchent, dit-on, avidement et en consomment de fortes quantités '. Il semble donc

M. Broilliard. 
tout à fait à propos de ne point abandonner entièrement à la nature la propagation de cet arbre utile, mais bien de liz favoriser artificiellement, par des semis et des repiquements dont il sera question dans le $\mathrm{VI}^{\circ}$ livre de ce cours.

532. Le pin du Lord Weymouth n'est point encore un arbre des forèts d'Europe, quoique très susceptible de le devenir. A la vérité, il en existe des essais sur différents points; mais ils sont généralement faits sur une trop petite échelle, et trop récents d'ailleurs, pour qu'on puisse en déduire des règles d'exploitation. Toutefois, on connait la' légèreté de sil semence, le tempérament assez robuste du jeune plant, la nature pivotante de la racine, et la croissance rapide de l'arbre; on pourrait donc, si l'occasion s'en présentait, lui faire aisément l'application de la méthode du réensemencement naturel et des éclaircies.

ARTICLE XXI.

\section{Exploitation d'une futaie de mélèze.}

כ333. Les règles données plus haut (article $\mathrm{XY}$ du présent chap.) sur l'exploitation des forèts de pin à crochets sont entièrement applicables à celles de mélèze, croissaut dans les mèmes régions. On sait d'ailleurs que ces deux essences sont très souvent associées et il est d'observation constante, tant dans les hautes montagnes que dans les situitions mo- 
yennes où il a été introduit artificiellement, que le mélèze s'accommode parfaitement du mélange arec d'autres arbres, résineux ou feuillus, et que cet état favorise à la fois son accroissement et la régularité de forme de sa tige. II y a donc lieu de maintenir ledit état partout où il se présente naturellement et mème de chercher à le créer, lorsqu'on en a les movens.

Il est presque inutile de faire remarquer que, selon les essences irvec lesquelles le mélèze se trouvera mélangé et selon le degré de ce mélange, il pourra ètre à propos de modifier la révolution el le traitement, sur lesquels influeront nécessairement aussi, diuns une certaine mesure, la rualité du sol et le climat local.

\section{ARTICLE XXII.}

\section{Exploitation du cèdre du Liban.}

כ34́. Il serait superflu de parler de l'exploitabilité et du mode d'exploitation d'un arbre à peine connu, et qui, en France, n'existe encore que dans les plantations d'agrément. Ce sont, sans doute, les soins à donner aux jeunes plants [387] qui, jusqu'à présent, ont mis obstacle à ce que le cèdre prit place dans nos forêts. Mais, si les plantations en grand présentent beaucoup de difficultés, les forestiers peuvent se borner à quelques essais dans des situations différentes. Ils prépareront ainsi à leur's successeurs le moyen de juger cet arbre remarquable, sur lequel l'opinion encore incertaine. 


\section{CHAPITRE TROISIÈNE.}

EXPLOITATION DES FUTAIES IRRÉGULIÈRES,

QUI ONT ÉTÉ SOUMISES AU MODE

DU JARDINAGE.

ARTICLE PREMIER.

Des forêts jardinées en général.

53ว Nous n'avons traité jusqu'à présent que des futaies dont l'état régulier permet l'application de la méthode du réensemencement naturel et des éclaircies ; mais, en France, il en existe un grand nombre qui ne sont nullement dans cet état. Telles sont entre autres les forêts jardinées.

Le jardinage consiste à enlever, çà et là, les arbres les plus vieux, les arbres dépérissants, viciés ou secs, et d'autres en bon état de croissance, mais qui sont réclamés par le commerce ou la consommation 
locale. Dans ce mode d'exploitation, qui a été plus particulièrement appliqué aux essences résineuses, notamment au sapin et à l'épicéa, on a pour principe de ne jamais prendre que très peu d'arbres ì la fois sur le même point, trois à cinq au plus par hectare 1, et d'étendre autant que possible le jardinage sur toute la forêt 2 . Il résulte de cette manière d'opérer que la forèt présente, sur certains points, des sujets de tout àge confusément mêlés, depuis le jeune brin jusqu'à la vieille écorce, et que les arbres qui ont le plus de grosseur et d'élération gènent ceux qui se trouvent sous leur couvert, et en ralentissent la régétation. De plus, les arbres n'étant pas serrés entre eux, s'étendent en branches, deriennent presque toujours noueux, et n'atteignent pas la hauteur dont ils seraient susceptibles. Il en résulte encore que, s'élevant pour ainsi dire par échelons, ils ne peuvent se soutenir réciproquement et n'offrent pas assez de résistance aux coups de vent et au poids de la neige et du givre : les bois les plus faibles, arrìtés dans leur végétation par ceux qui les surmontent, contractent des germes de maladie lorsque cet état de gêne se prolonge; presque toujours ils languissent, rarement ils arrivent à un beau développement, et souvent ils meurent prématurément.

Tel est, en général, l'état des forèts jardinées. Ce-

' Dralet. Traité des bois résineux, page $15 \%$.

2 Id ., Traité de l'aménagement, page 106. 
pendant on rencontre fréquemment, dans certaines de ces forêts, de belles parties dont li prospérité est due aux soins du forestier qui a su déroger à la routine, en enlevant de préférence les arbres branchus qui surmontaient le jeune sous-bois, Celui-ci, dès-lors, débarrassé du couvert qui l'étouffait, a pu participer aux influences atmosphériques, s'est élancé et a pris une belle croissance. Des effets analogues, et sur un plus grand espace, ont souvent été opérés par des coups de vent qui ont enlevé les vieilles écorces et d'autres arbres qui dominaient de jeunes fourrés. Ainsi, l'on voit quelquefois, dans les forêts jardinées, des peuplements semblables à ceux que l'on obtient dans les futaies régulières.

5336. Le jardinage, en disséminant les exploitations sur de très grandes surfaces, rend la surveillance fort difficile, et augmente considérablement les dégâts provenant de l'abatage et de la vidange. Mais le reproche le plus grave auquel donne lieu ce mode, c'est de ne faire rendre aux forêts, dans un temps donné, que des produits matériels très inférieurs, en quantité et en qualité, à ceux que l'on obtient par la méthode du réensemencement naturel et des éclaircies. Il suffit de comparer l'influence de ces deux modes sur la végétation, pour être convaincu de cette vérité.

En effet, dans les forêts jardinées, nous voyons les sujets de toute catégoric entravés dans leur développement, pendant un temps plus ou moins long, et 
souvent jusqu'à la fin de leur existence; dans la futaie régulière, au contraire, la croissance est fitvorisée dès la première jeunesse, et activée, jusqu'au terme de la maturité, par des exploitations périodiques. Ajoutons que, dans la futaie jardinée, il n'est pas question d'enlever, comme dans la futaie régulière, les jeunes sujets dominés qui, par conséquent, sont perdus pour la consommation.

Quant à la qualité des arbres, la facilité qu'ils ont, dans la forèt jardinée, d'étendre leurs branches, les rend inférieurs, pour les constructions et la fente, à ceux qui ont crù en massif; et il est ì remarquer, en outre, que les dégaits considérables causés par l'abitage et la vidange, dans une telle forèt, y multiplient les arbres viciés, tandis que l'on n'en rencontre que peu dans les futaies régulières.

\section{ARTICLE II.}

\section{Des coupes de transformation.}

537. L'infériorité des futaies jardinées, comparativement aux futaies régulières, étant démontrée, on est nécessairement amené à conclure que la méthode du jardinage doit être supprimée et remplacée par un mode de coupes de transformation qui établisse, dans les premières de ces forêts, plus d'uniformité sous le rapport de l'àge et de la croissance des bois, et qui rende possible, par la suite, l'application de la méthode du réensemencement naturel et des 
éclaircies. Mais, pour que, dans l'exécution de ces coupes de transformation, on atteigne entièrement le but que l'on se propose, il est essentiel de les modifier d'après les divers états de peuplement qui existent dans les forêts jardinées, états qu'on peut rattacher à trois types dont chacun demande un traitement particulier.

$1^{\circ}$ Quand les vieux arbres ne sont pas très nombrenx. et que le terrain est suffisamment garni de jeunes peuplements en bon état de croissance, on doit se hâter de faire abattre tous ces vieux arbres, et mème d'autres moins âgés, si, par une tête trop rameuse, ils gênent évidemment l'ensemble du sous-bois.

Lors même que ce sous-bois serait déjà élevé, eût-il 25 ou 30 ans, il ne faudrait pas craindre de faire l'extraction dont nous venons de parler; sauf, toutefois, à prendre toutes les précautions quant à l'abatage et à la vidange, prescrites plus haut pour les futaies régulières [478 et 479]. Le dommage qu'une pareille exploitation pourra occasionner, sera, dans tous les cas, bien inférieur à celui que causeraient, par la suite, des arbres branchus s'étalant de plus en plus au-dessus de la jeune forêt.

$2{ }^{\circ}$ Quand la quantité des arbres est considérable, et qu'ils dominent un sous-bois jeune qui offre tous les signes d'une bonne végétation, sans cependant être asses vigoureux pour pouvoir être exposé tout de suite à l'air et au soleil, il convient d'effectuer d'abord une exploitation semblable à la coupe secondaire, puis 
de faire exécuter plus tard la coupe définitive, lorsque les jeunes plants paraîtront suffisamment robustes.

$3^{\circ}$ Enfin, si, sous de nombreux arbres, il existe un sous-bois entièrement rabougri par suite du couvert épais qu'il a subi trop longtemps, il faut se garder de vouloir élever une futaie avec un pareil sous-bois qui ne parviendrait jamais à un beau développement; il est préférable de le sacrifier et de préparer le terrain pour un nouveau semis naturel. Dans les forèts résineuses, les arbres existants suffiront en général pour l'ensemencement; néanmoins, il pourrait arriver que, sur quelques points, ils ne fussent pas assez nombreux pour procurer l'abri nécessaire à des plants délicats, tels que ceux du sapin, par exemple; dans ce cas, il conviendra de suppléer à ce manque d'abri au moyen du sous-bois rabougri lui-même, qu'on ne coupera point, ou qu'on ne coupera qu'en partie, selon le tempérament de l'essence, afin de laisser aux nouveaux brins le temps de se fortifier assez pour résister aux influences atmosphériques. Plus tard, on extraira le sous-bois rabougri par forme de nettoiement 1 .

- Lorsque le sapin est l'essence dominante, bon nombre de ces sous-bois rabougris, une fois débarrassés d'un couvert trop épais, se ravivent très souvent et s'élancent avec une vigueur qui semble d'autant plus grande que leur végétalion a été plus longtemps comprimée. Aussi, est-ce le cas de les conserver, toutes les fois qu'ils forment des bouquets ou petits massif's pouvant se relier avec les parties nouvellement réensemen- 
Dans les bois feuillus, le moyen de régénération seria plus prompt et plus facile, toutes les fois que les brins mal renants garniront entierement le terrain, et auront de faibles dimensions; en effet, il suffira d'aroir recours it une recépage 1 en faisant abattre en mème temps tous les vieux arbres. Ce recépage, effectué sur des brins d'un faible diamètre, fera naitre de beaux rejets qui, dans peu d'années, auront pris une assiette et une régétation presque entièrement semblables à celles de brins de semence. Mais, si les sujets rabougris étaient peu nombreux et déjà forts, il vaudrait mieux prendre le parti de les déraciner,

cées [302], à moins qüils ne soient entièrement dépérissants. Les épicéas résistent moins longtemps sous le couvert que les sapins et, une fois rabougris, sc récupèrent plus difficilement; toutefois, en bon fonds, ils se rétiblissent entièrement, pourvu ru'on ait soin de ne pas les faire passer trop brusquement de l'etat couvert à l'extrème opposé. Quant au pin, que l'on voit rarement, au surplus, à l'état de forèt jardinée, on sait qu'il ne supporte pas d'ètre surmonté, et que, une fois rabougri sous cette influence, il ne se rétablit plus. C'est ainsi que l'on trouve, dins certaines pineraies irrégulièręs (forèt de Haguenau), des gaulis ot des perchis incomplets et chétifs qui, décourerts trop tard, (quoique depuis assez longtemps, demeurent languissants et stationnaires. Évilemment c'est le pire des états, et micux vau' dritit la perte entière, car de tels peuplements resteront toujours misérables et tiennent la place d'autres qui eussent été infiniment plus productifs.

' Il est essentiel que ce recépage se fasse tout à fait à fleur de terre, afin de dommer aux rejets une assiette solide, et d'éviter qu'il ne s'en présente un trop grand nombre sur la même souche. 
afin d'obtenir un repeuplement nouveau et complet, et d'empècher que les rejets qui résulteraient du recépage ne vinssent à gèner ou à étouffer les jeunes brins de semence.

Lorsque l'essence sera le hètre, on fera mieux, en général, de viser à une régénération par la graine, cet arbre étant peu disposé à se reproduire de souche. La nature du sol, ainsi que le climat, exercent à cet égard une grande influence. C'est au forestier à apprécier ces causes locales et à s'assurer, par des expériences faites en petit, de la ficulté reproductive des souches. S'il existe le moindre doute sous ce rapport, il ne tentera pas le recépage, et cherchera, au contraire, à amener un nouvel ensemencement. Il pourra aussi, si le sous-bois n'est pas rabougri an dernier degré, se contenter de le débarrasser des arbres qui le dominent, et le laisser croitre tel qu'il est. Cet expédient se fonde sur un firit bien constint : c'est que le hètre possède, comme le sapin et plus que lui peut-ètre [ö02]. lit propriété de regagner de la vigueur, lors mème qu'il a langui pendant fort longtemps sous le couvert; et il n'est pas rare de le voir prendre une belle croissance dis que les arbres qui l'offusquaient ont disparu 1.

1 Nous pouvons citer un exemple à l'appui de cette assertion.

Ln jeune bois de hètre, dans une situation blevée des Vosges, en très maurais etat de croissance par suite d'abroutjssement, et surmonté d'un assez graud nombre de vieux arbres, paraissait réclamer le recépage. Incertain sur le suceès de cette opé- 
538. Tel est le traitement à appliquer aux principaux états de peuplement qu'on rencontre dans les forêts jardinées; on y en rencontre sans doute encore beaucoup d'autres, mais qui ne diffèrent (soit sous le rapport de l'àge, soit sous le rapport de la consislance) que par des nuances plus ou moins tranchées, de ceux que nous venons de décrire, et dont le traitement doit, par conséquent, se rapprocher plus ou moins des règles que nous avons données.

Ce qui rend surtout difficiles les coupes de transformation, c'est que ces différents peuplements se trouvent pêle-mêle sur une étendue souvent très peu considérable, et qu'il faut alors changer de mode de traitement presque à chaque pas. Pour réussir dans ces opérations, l'essentiel est d'avoir toujours bien en vue le but que l'on veut atteindre. Ce but est d'obtenir des massifs qui, bien que différents d'àge par place, puissent être régénérés à la mème époque sans qu'il en résulte une trop grande perte d'accroissement.

ration, et craignant le déboisement de cette partic de forêt, si les hêtres ne repoussaient pas, nous nous contentâmes de les mettre en défends et de les abandonner à eux-mêmes, après les avoir débarrassés des arbres qui les dominaient. Ils n'ont pas tardé à végéter avec assez de force et à gagner en hauteur; il y a trente-six ans maintenant que les bestiaux ont été éloignés de cette jeune forêt et qu'on a fait disparaitre les vieux arbres, et sa végétation continue à être des plus satisfaisantes. Le bas des liges sculement a une forme défectueuse, mais qui ne peut être d'aucun inconvénient pour le hètre, puisqu'il n'est employé qu'au chauffage et au travail. 
Les règles qu'on vient d'exposer et qui sont fondamentales du traitement des forêts jardinées, exigent qu'on se défende constamment, dans l'application, contre la tendance bien naturelle à tout forestier de chercher à élever des peuplements parfaitement réguliers. Souvent, par exemple, on serait tenté de faire disparaitre certains bouquets de perchis assez àgés déjà, parce qu'ils sont environnés de fourrés et qu'il serait possible de provoquer un nouveau repeuplement qui se raccorderait mieux avec ceux-ci. Une telle opération serait cependant une faute, car, souvent répétée, elle entrainerait immédiatement des pertes considérables d'accroissement, et, dans les situations où les semis naturels ou artificiels réussissent difficilement, elle peut mème offrir des dangers. Il est donc très important de se bien consulter avint que d'abattre, dans les coupes de transformation, d'autres bois que ceux qui, par leur àge, leurs dimensions ou leur position isolée, ne peuvent évidemment faire partie du massif quasi-régulier qu'il s'agit de constituer. C'est ce massif qui procurera un jour le peuplement normal que l'on a en vue; mais il faut savoir attendre ce résultat et ne pas lui faire plus de sacrifices que de raison. 
ARTIGLE III.

\section{Marche des coupes de transformation.}

539. Lorsqu'une forèt jardinée doit, par des coupes de transformation, être ramenée à l'état régulier, li marche de ces coupes peut ètre réglée d'une manière analogue à celle que nous arons fait connaitre pour les futaies régulières [473]. Il est à observer, toutefois, que le jardinage ne saurait ètre supprimé immédiatement dans une telle forèt, en raison de l'état assez uniforme qu'elle présente dans son irrégularité. En effet, le plus souvent, il existe, sur tous les points, des arbres exploitables entremèlés avec d'autres de l'àge moyen et du premier àge; or, si l'on se contentait d'établir une suite de coupes de transformation qui se succéderaient de proche en proche, il est érident qu'un grand nombre des arbres, qui dès à présent sont mùrs ou sur le retour, périraient arant que les coupes rinssent les atteindre. Il faut done, de toute nécessité, que, dans le nouveau mode d'exploitation, ces arbres soient enlevés à temps, et c'est dans ce but que le jardinage doit être continué.

Ainsi, pour ramener une forèt jardinée à l'état régulier, on doit établir deux exploitations distinctes: d'une part les coupes de tranformation, de l'autre, les 
coupes de jardinage portant sur les arbres mùrs et dépérissunts. Yous rerrons, dans ce qui va suivre, comment ces deux exploitations se combinent pour conduire au but proposé.

540. La révolution à adopter pour opérer lir I':unsition de l'état jardiné à l'état régulier, révolution que nous nommerons transitoire, doit itre ibrégée autant que possible; toutefois, il faut qu'elle soit assez longue pour qu'à son expiration, la forèt présente des peuplements parvenus à leur exploitabilité, ou du moins qui en approchent. Lit durée de lit révolution transitoire dépendra done principatlement de l'àge qu'auront les jeunes massil's crécis par les coupes de transformation qui auront été failes les premieres. Cette rérolution, de mème que celle d'une futaie régulière, devra ètre diviséc en périodes, dont chacune aura son affectation sur le terrain.

Mais, en déterminant ces affectations, on cherchera principalement à favoriser l'amélioration future de la forèt et, par conséquent, à observer les règles d'assietle, afin d'assurer pour l'avenir une succession aussi régulière que possible dans les coupes. De tels résultats, au cas particulier surtout, sont bien plus importants à obtenir que l'égalité des produits périodiques. Cette égalité est d’ailleurs d'inutint plus difficile à atteindre en général, que l'irrégularité de la forèt est plus grande, que les arbres présentent moins de similitude sous le rapport de leurs formes et de leurs dimensions, et enfin que 
les influences sous lesquelles ils végètent sont plus diverses. Pour parvenir à réaliser le rapport soutenu dans une forèt jardinée qu'on veut transformer, nous pensons que le meilleur moyen, peut-être, et en même temps le plus simple, est d'y multiplier les séries d'exploitation, d'après les principales différences de lertilité et de peuplement, puis, de donner aux affectittions des périodes, dans chitcune de ces séries, des contenances égales ${ }^{1}$.

Supposons que l'on adopte les principes qui viemnent d'ètre posés, et que la révolution transitoire

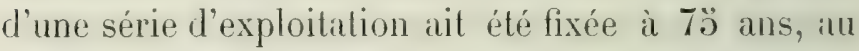
lieu de 100 qui serail la durée ordinaire, puis partitgée en périodes de 220 ans chacune; et examinons

- En effet, dans la forêt jardinée, il n'existe pas, comme dans lil futaie régulière, des peuplements dàges graduís; tous les iges au contraire s'y trouvent confusément mèlés. Sous ce rapport done, les mèmes difficultís comme les nèmes facilités se rencontreront dans une même série, qu'elle soit grande ou petite. Il suit de là que, pour constituer chacune d'elles, il serait superflu de se préoceuper de la gradation des ìges; la qualité et la configuration du sol rlevront seules être considérées, ce yui, le plus souvent, donnera la facilité de ne comprendre dins une mème série que des parties ayant à peu près la même lintilité. Quant à la formation des affectations, comme les différences de consistance entre des peuplements aussi complétement irréguliers que ceux de la forèt jardinée ne sauraicnt ividemment se déterminer aree la précision nécessaire, le mieux, dans lat plupart des cas, sera de n'en pas tenir compte. 
quelle sera la marche des exploitations, pendant toute cette révolution, de période en période.

\section{Exploitation de la $1^{\text {re }}$ période. - C'est dans} l'affectation de cette période que commenceront les coupes de transformation: on les effectuera d'après les règles données dans l'article précédent, et l'on fera bien, en outre, afin d'aider autant que possible à la végétation du sous-bois sur toute l'étendue de l'affectation, de les accompagner d'ébranchements de gros arbres, dans les parties qui ne viendront en tour d'exploitation que dans la dernière moitié de la période.

Parallelement à ces coupes de transtormation, marcheront dans les affectations des périodes 2 et 3 , les coupes jardinatoires dont nous arons parlé plus haut. Ces dernières coupes, comme nous l'avons dit, sont particulièrement destinées à faire disparaitre les bois que l'on ne pourrait laisser sur pied jusqu'à ce que les coupes de trinsformation rinssent les atteindre. On peut joindre encore à ce but, celui de préparer insensiblement lia forèt à l'état plus régulier auquel elle doit, plus tard, ètre amenée définitivement, et, dans cette vue, faire porter le jardinage, autant que possible, sur les arbres dont li présence nuit à de nombreux sous-bois, en mème temps qu'on enlèvera, par forme d'éclaircie, les tiges dominées dans les perchis. Dans l'affectation de la seconde période, le jardinage devra se borner aux arbres entièrement dépérissants; mais, dans celle de 
la troisieme, il devra porter aussi sur les arbres en retour.

I'ar ce moyen, les produits, pendant la première période, pourront atteindre le chiffre ou ils étaient atrant que lit transtormation ne commençàt, el dans certains cas même le dépasser.

:3 42. Détermination de la possibilité. - La possibilite des coupes de transformation doit ètre basée sur le volume et se déterminer à l'entrée de chaque période [4:26 el 427], comme celle des coupes de régrenération daus une futaie régulière. Ces exploiLations ne sont, en effet, que des coupes de régénération, modifiées en raison d'un état particulier de pruplement, et nous avons vu ailleurs [470̈] les motils qui empèchent que celles-ci ne soient établies par contenances égales. L'irrégularité du peuplement seule, au surplus, serait un molif suftisant pour l'tenoncer à la possibilité par contenance.

On doit s'attendre à ce que la détermination de la possibilité, dans la forèt jardinée, soit affectée d'une erreur bien plus considérable que dans la fulaie régulière. Aussi est-il indispensible de réritier le trivail d'estimation plusieurs fois dans le cours de lit période. Il est inutile de faire observer que, dins rette estimation, on ne devril comprendre que les arbres que l'on jugera devoir tomber dans les coupes de transformation.

วั43. Le point le plus difticile à régler dans les ex- 
ploitations qui nous oreupent, c'est le rolume des produits que doivent fournir annuellement los coupes de jardinage.

Dans l'affectation de la deuxième période, aronsnous dit, ees coupes devont se bormer aux arbeses dépérissants, et, dans l'affectation de lir troisiomo période, elles derront atteindre, en outre, des arbres en retour. Si done on roulait procéder rationnelloment, pour réterminer le volume à exploiter par an dans l'une et l'autre affectation, il faudrait avoir l’inrentiure des arbres déprérissants el de ceux qui puurront le devenir avant que les compes de tramaformattion n'arrivent dans les parties ou ils se trourent; al pour ce qui concerne l'affertation de la troisiome puriode en particulier, il fautrait commatre eneore lis quantité d'arbres en retnur qui derroml tomber yir suite du jardinage.

Mais, outre qu'un pareil inventaire exigerait de fort longs comptiges d'arbres, il présenterait eneorer dans l'exécution une multitude de cas tris embarrassants, en ce sens, quil serait souvent fort difficile de décider d'avance, si lel arbere dera être compris ou non dans le jardinage. Ensuite, il est evident que si, pour fixer la possibilité des coupes de transformation, on est sujet à commoltre de graves erreurs, on l'est à plus forte raison pour fixer celle des coupes de jardinage. Et cependant,ici, toute vérification dans le cours de la périodle est à peu près impossible, à moins d'imprimer aux arbres destinés ì itre alleints 
par le jardinage un signe distinctif, ce qui, en vérité, n'est guère proposable, et dans benucoup de cas, ne serait même pas efficace.

Baser la possibilité des coupes de jardinage sur le volume nous semble done tout à fait impraticable, parce que l'opération, longue et difficile à exécuter, ne pourrail ahoutir qu'à des résultats extrèmement vigues, el susceptibles de donner naissance à des erreurs pires peut-ètre que celles qu'engendrerait un entier arbitraire.

Toutefois, il faut le reconnaitre, une base quelconque est nécessitire pour ces exploitations. Peutitre la trouverait-on en se reportant ì l'ancien jardinage, dont la possibilité, comme on sait, se fixail en déterminant le nombre d'arbres ì rouper annuellement par hectare [335]. Supposons, par exemple, que ce nombre ait été $\dddot{3}$; on le réduirait à 1 ou à 2 dans l'affectation de la seconde période, où il ne s'ingit d'abattre que des arbres dépérissants, et dans l'affectation de la $3^{\circ}$ période, nù il faut faire tomber' en outre des arbres en retour, on porterait ce nombre à 3 ou à 4. Puis, si l'on croyait utile d'exprimer approximativement ce nombre d'arbres en mesure de solidité, il suffirait de déterminer, dans chaque affectation, par quelques expériences, le volume d'un arbre moyen de la catégorie que le jardinage doit atteindre; multipliant ensuite, par ce chiffre, le nombre des arbres à couper annuellement. om aurait la possibilité cherchée. 
Cette manière de procéder est sans doute peu satisfaisante, en ce que, bien évidemment, elle ne saurait conduire au degré d'approximation désirable. Mais, du moins, elle a le mérite d'ètre expéditive et de n'occasionner ni frais ni perte de temps dans des recherches auxquelles l'état des choses interdit le succès. On pourra d'ailleurs, chaque fois que l'on rérifiera la possibilité des coupes de transformation. soumettre aussi à un nouvel examen la base des coupes de jardinage et leur's résultats. Sans entrer dans des opérations de détail, le forestier entendu saura bien juger si le nombre des arbres, que tes coupes jardinatoires ont encore à atteindre, est plus petit ou plus grand qu'il ne derrait l'ètre, et s'il convient, par conséquent, d'abaisser ou d'élever le chiffipe des produits que doivent fournir ces coupes '.

344. En terminant cette discussion, nous rappellerons ce que nous avons dit plus haut:

Dans l'exploitation d'une forèt jardinée, la chose principale est la transformation, parce que d'elle doit résulter un état infiniment supérieur à ce qui existe, et dont la conséquence sera l'augmentation de la production. La possibilité n'est que secondaire: c'est en vain que l'on tenterait de la déterminer arec la mème approximation que dans une futaie régulière: l'état du peuplement y met des obstacles insurmontables. Aussi les moyens les plus simples, les plus

' Voir l'appendice. 
larges el les plus expéditifs pour en fixer le chiffre nous paraissent-ils les meilleurs.

\%ั. Exploitations des périodes 2 et 3. - Dans laffectation de la deuxirme période, les coupes de trinsformation trouveront une plus grande quantité de bois a abittre que dans celle de la premiere, les coupes de jardinage y ayant été rérluites, depuis ringl-cing ans, aux arbres dépérissants; la possibilite de ces coupes de transformation sera done augmentée. Quant aux produits que le jardinage fournissait pendant la première période, ils seront compensés d'une part par ceux qu'il continuera de fournir dans l'affectation de la troisième période, et de l'autre par les produits des éclaircies périodiques qu'il y aura lieu d'entreprendre dans l'affectation de la première.

Enfin, à la troisième période, les coupes de transformation troureront la forèt peuplée, en très grande partie, de bois mûrs, qui tous derront être coupés, et dont les produits seront renforcés encore par ceux des éclaircies périodiques à faire dans les affectations des deux périodes précédentes.

\%46. Les inconvénients du jardinage ayant été reconnus en France depuis une trentaine d'années, ce mode d'exploitation a presque généralement (du moins dans les forêts soumises au régime forestier), fait place à des coupes dans lesquelles on s'est appliqué à débarrasser d'un courert nuisible les peuplements jeunes ou d'ige moyen, el à supprimer 
partout les hois morts ou dépérissants que beaucoup de forits présentaient en abondance. Ln mème temps, on a pratiqué des éclaircies périodiques dans les jeunes massifs suffisamment réguliers qui se rencontraient cì et là. C"est, on le roit, une véritable transformation que l'on a opérée ainsi. Seulement on s'est borné, le plus souvent, à améliorer le traitement sans régler en mæ̀me temps la marche des exploitations diaprès les principes que nous renons de donner. On trouve done aujourd'hui bon nombre de lorêts qui, régularisées en partie, ne présentent plus l'état jardiné que dans quelques canlons, le surplus se composint de massifs plus ou moins réguliers, fourrés, perchis ou futaie exploitable ou à peu près.

Lorsque ces différents états de peuplement se trouveront convenablement groupés, on concoil qu'il sera assez facile d'en composer une ou plusiems séries d'exploitation dans lesquelles les parties jardinées formeront, autant que possible, l'affectation de la première période, les massifs exploitables, celle de la seconde, et ainsi de suite. La rérolution de 100 à 120 ans pourra mème sourent, dans ce cas, être immédiatement admise, afin d'établir, pour les révolutions suivantes, une gradation d'àge aussi normale que possible; et, guant au rapport soutenu, si l'on ne réussit pas toujours à l'assurer pour une même série, on y parviendra, du moins dans les masses importantes, en considérant comme liées entre olles un certain nombre de séries dont les 
produits pourront se compenser à travers les différentes périodes de la révolution [420].

Wais le cas que nous renons de considérer est le plus rare. En général, les peuplements qu’il s'agil de réunir en une série, sont dins des conditions qui rendent cette réunion fort difficile: tantril les parties jardinées out trop d'étrndue pour ne composer qu'une senle affectation, ou trop peu pour en fournir deux, el les massifs exploitables, que l'on pourrail y rattacher, à titre de complément, font défaut; Limtòl ce sont les bois d'àge moyen ou les jeunes bois qui ne sont pas représentés ou qui ne le sont pas suffisamment; tantòt enfin les peuplements de chaque catégorie sont dispersés, ce qui met obstacle à requion en compose une mème affectation.

Appliquer à de telles forèts, immédiatement, un plan normal d'exploitation, nest évidemment pas possible. Ce que l'on doit chercher ici, c'est d'achever de régulariser les différents peuplements, surtoul les parties jardinées, dans le cours d'une révolution préparaloire dont la durée dépendra de deux circonstances: $1^{\circ}$ le plus ou moins d'urgence qu’il y aura d'extraire les hois destinés ì disparaitre pour la régulatrisition du peuplement, et 20 l'àge artuel des massifs yui devront venir en tour d'exploitation apres la lransformation terminée, cest-ì-dire, formor lis premiere affectation de la rérolution qui

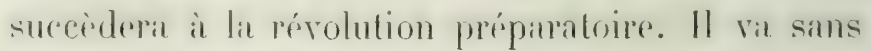
dire d'ailleurs que romme dans le premier eas, el 
bien plus encore, il sera souvent opportun et mème nécessaire, pour assurer le rapport soutenu, d'admettre la compensation de série à série pour une ou plusieurs périodes.

:47. Malgré les longs développements dans lesquels nous sommes entrés au sujet des forèts jardinées, on trouvera toujours encore, dans la pratique, des cas nombreux qui n'auront point été prérus.

Dans toutes les forèts irrégulières, el surtout dans les forêts jardinées, il est impossible de prévoir tous les états qui peurent se présenter; la théorie doil done se borner aux cas les plus généruux, et laisser le reste au coup d'ail el à l'expérience du praticien.

C'est en présence de pareilles forèts que l'instruction technique est de la plus grande nécessité, non l'instruction qui prétend tout réğler d'itrance, mais celle qui, aidée de la réflexion et du jugement, sait modifier l'application des principes en mison des circonstances.

ARTICLE IV.

Des cas où le jardinage doit être conservé.

:38. Quelque incontestable que soil l'infériorité du mode jardinatoire comparé à la méthode du rensemencement naturel et des éclaircies, il est néanmoins quelques cas où il convient de faire usage de ce mode. 
linsi, dims certaines siluations très élevées, oì le rlimat extremement rude est sorerent un obstacle ì la ríussite des repeuplements, le jardinnge peut itre avintageux, paree qußil n’éclaircit jamais la forêt que par petites plices, dims lesquelles les repeuplements resistent mieux aux rigueurs du climat que sur des surfaces plus grandes.

Le jarlinitge trouve aussi son application dans les régions où les hois sont conservés particulièrement pour abriter une localité, soit contre des rents dimgereus, soit contre des avilanches ou des éhou-

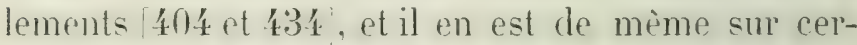
lains versints abrupts, couverts de rochers entre lesquels les bois ne peurent eloitre que çà et là, par houquets, et oi le peu de terre régétale, qui a réussi ¿ sy former. a d'ailleurs le plus grand besoin de courert pour se maintenir. En pareil cas. l'objel principal de la culture n'est plus la production du bois, mais bien la conserration de l'abri reconnu nécessaire; il faut donc, dans les exploitations, tendre ¿ maintenir la forit dans un état de consistance qui varie le moins possible, et s'appliquer, par conséquent. à n'amener que des repeuplements partiels, au fur ot à mesure de la roupe des arbres tout à fait dépérissants.

Enfin, ce mode peut encore conrenir à quelques proprictaires, communes, établissements publics ou particuliers, qui, ne possédant que des bois d'une liable itendue, veulent cependant trourer à y exploi- 
ler chilque année des pièces de dimensions diverses.

349 . Mais lorsque le jardinage derra itre maintenu, au lieu de le pratiquer comme antrefois et tel que nous l'avons décrit plus haut [כ̋3ว̋], on s'appliquera à en atténuer les inconvénients cl à le concilier avec un certain ordre dans la marche des exploitalions. Il est difficile de tracer des righles pour un traitement aussi essentiellement irrégulier que le jardinage; toutefois, on peut poser quelques principes généraux, dont on ne s’écartera que le moins possible, suivant les exigences du propriétaire el d'après les circonstances particulieres ì chatpue forêt.

Pour élablir un jardinage rationnel, il convient l'abord de diviser la forèt en parcelles, circonscrites. aulant que farire se pourra, par des limites nalurelles. cribles de montagnes, fonds de vallées, ruissealux, ot surtout pall des chemins pouvant, lors des exploitalions, servir it vider les prouluits sans dommage pour les parties voisines. Les patrcelles derront itre assery nombreuses pour you le jardinage amnuel puisse se renfermer dans l'une d'elles ou toul au plus dans deux.

Le parcellaire élabli, on déterninera l'ordre diuns lequel les parcelles derront ètre parcourues parr lo jardinage, en ayant égard à leur peuplement tant en jeunes qu'en rieux bois et en tenant compte des r'egles d'assiette; puis enfin, on fixera la périodicité des coupes de jardinage, c'est-it-dire, le nombre 
d'années à employer pour parcourir toutes les parcelles d'une série.

Pour régler ce dernier point, il sera nécessaire de déterminer : $1^{\circ}$ (quel àge et quelle grosseur les arbres doivent avoir atteints pour ètre réputés exploitubles; 20 Quel est le nombre de ces arbores; 30 Combien d'amnées devront s'écouler pour qu'un mìme nombre d'arbres non encore exploitables puissent le devenir. Ce litps de temps, toutefois, ne pourrat être qu'une moyenne qu'il sera prudent d'allonger plutót que de trop raccourcir, afin de tenir compte de l'impréru dans lia végélation; sa durée fera évidemment connaitre liı périodicité cherchée.

Quant à la possibilité, on conçoit qu'elle ne pourra ètre basée que sur le rolume; soit qu'on calcule celui-ci comme pour les coupes de transformation โว̆42], en estimant le volume actuel et le volume futur de tous les bois réputés exploitables; soit qu'on se contente de fixer le nombre de pieds d'arbres à exploiter amnuellement, ce qui suppose toujours qu'on se sera rendu comple, avec plus ou moins de précision, de leur nombre total et du rolume de l'arbre moyen.

Le jardinage lui-mème se fait d'après les règles suivantes:

$1^{\circ}$ L'exploitation portera en premiere ligne sur les arbres branchus de fortes dimensions qui surmontent, soit des perchis, soit des fourrés ou des gaulis. 
Si, dims ces derniers, il se trouve çà el là des perches isolées qui ne pourront jamais faire massif arec l'ensemble, et entravent par conséquent sa croissance, elles devront également ètre extraites quel que soit leur âge.

$2^{\circ}$ Au lieu de n'enlever, à la fois, qu'un seul arbre exploitable sur une mème place, ainsi que le voulait l'ancien jardinage, il faudra, au contraire, en abatlre plusieurs, de manière à favoriser l'accís de la lumière sur le sol autant qu'il sera nécessaire pour issurer la venue du jeune plant et son développement pendant un certain nombre d'années. On trouvera la juste mesure à cel égard, en se pénétrant bien de ce principe : que si l'ombrage des arbres est presque toujours avantageux aux sous-bois, leur couvert produit généralement l'effet contraire ${ }^{1}$.

$3^{\circ}$ Outre les bois exploitables proprement dits qui formerontle produit principal de l'exploitation innuelle, les coupes de jardinage derront fitire disparaitre encore, dans lit parcelle ou elles seront effecluées, tous les bois rabougris et dominés sur lesquels porteraient les éclaircies périodiques dins les fulaies régulières.

$4^{\circ}$ Les chablis el les arbres morts seront exploités chaque année dans toule la série, et leur produit sera précompté sur la possibilité. Dans les parcelles qui ne

1 Voir, dans le IV ${ }^{\circ}$ livre, les définitions de l'ombrage et du couvert. 
devront pas atre prochainement alteintes par les coupes jardinatoires, les vieux arbres qui causent un notid)le dommage par leur courert devront ètre élagués à une hauteur convenable.

“" Enfin, loutes les précautions recommandées pour l'abaliage el la vidange des bois, dams les futaies régulieres [48], devront ibre observées avec le plus grand soin dans les forêts jardinées!

Soir l'appendice. 


\section{CHAPITRE QUATRIENIE}

EXPLOITATION DES FUTAIES IRRÉGULIÈRES

QUI ONT ÉTÉ SOUMISES

AU MODE DIT A TIRE ET AIRE.

\section{ARTICLE PREMIER.}

\section{De l'état de ces forêts en général.}

כ็๊o. Outre les forêts jardinées, il existe encore. en France, d'autres futaies irrégulières qui, le plus souvent, ont pour essences dominantes le chène et le hêtre. Leur' état est d'ordinaire peu satisfaisint, et provient, en général, du mode d'exploitation dit à tire et aire, auquel ces forèts étaient soumises en vertu d'anciennes ordonnances.

Ce mode, qui semble avoir été conçu dans le but de remédier aux nombreux abus nés du jardinage [こ̋วّ], consistait surtout à asseoir les coupes par 
contenances égales, de proche en proche ct sans rien laisser en arrière'. Quant aux arbres de réserve, ils étaient peu nombreux dans les coupes, et celles-ci, une fois vidées, restaient abandonnées pendant tout le cours de la révolution, sans qu'on y fït aucune exploitation, ni pour assurer les conditions du repeuplement naturel [40̈9], ni pour faroriser la croissance des jeunes bois $[463 \mid$. L'ordonnance des eaux et forèts de 1669, par exemple, qui a généralisé l'application du mode à tire el aire et dont les dispositions sont demeurées en vigueur jusqqu'à la promulgation du Code forestier ${ }^{2}$, portait: que la réserve dans les coupes de futair serail de dix arbres par arpent (20 par hectare); et du reste elle ne permetlait point, ainsi que nous renons de le dire, que dims une même révolution, ces coupes fussent soumises à plusieurs exploitations.

Il résultail d'un tel mode, que les peuplements croissaient en massif trop serré pour fournir des pièces de belles proportions et d'une texture forte [470]; puis, les arbres réservés dans les coupes étaient en trop petit nombre pour assurer le repeuplement du terrain. Ils pouvaient d'autant moins l'assurer que beaucoup, parmi eux, étaient la proie des vents ou séchaient apprès peu d'années, par suite de la Iransition trop brusque de l'état serré à l'entier isole-

1 Voyez Dictionnaire des Forets par Baudrillart, page 913.

2 for août 1827 . 
ment. Enfin, l'exploitation à tire et aire, en dénudant le sol, donnait accès aux herbes et aux ronces, aux arbustes, aux morts-bois et aux bois blancs, loutes plantes qui s'opposaient à la propagation et à la croissance des essences d'élite.

วัว̆L. Toutefois, quand peu d'années arant la coupe, les graines de ces essences araient été abondantes, on voyait paraitre un repeuplement considérable, mais qui, selon le tempérament des jeunes plants, souffrait plus ou moins. Si c'étaient des chênes, ils ne tardaient pas à languir, et la plupart finissaient par succomber ; si c'étaient des hètres au contraire, ils résistaient mieux et sourent assez longtemps pour parvenir en définitive à lutler avec avantage contre les bois blancs et les morts-bois, lorsque ceux-ci, à cause de leur àge, commençaient à croìtre avec moins de force et mème à périr ${ }^{1}$. Les arbres de réserve que l'on était forcé de laisser subsister jusqu'au retour de la coupe, étendant librement leurs branches, contribuaient encore à ralentir la végétation des bonnes essences.

Mais de tels résultats, évidemment les plus heu-

I C'est ainsi que l'on peut expliquer comment, dans nos futaies d'aujourd'hui, le chêne cst rille presque généralement, tandis que le hètre s'y est conservé assez bien, malgré les entraves apjuortées à la végétation dans les vingt ou trente premières années de son existence et le retard considérable qui en résultail pour son accroissement. (Voyez Dralet, Traité du hêtre, page 79.) 
reux que pùt produire le mode à tire et aire, élaient bien plutòt l'exception que la règle. Le plus souvent ce mode arait des conséquences beaucoup plus fàcheuses, ainsi que nous l'arons dit d'abord, et que le prouvent d'ailleurs les vastes terrains couverts de bois tendres ou mème entièrement dépeuplés, que l'on rencontre en si grand nombre dans nos futaies d'aujourd'hui.

¡ö2. Comme on le roit, les futaies traitées à tire el aire offrent, en général, des massil's très diversement irréguliers, presque tous incomplets et viciés, et qui réclament un prompt remède pour ne pas le devenir davantage. Sauver les bonnes essences en faisant disparaître les causes qui entravent leur végétation, leur rendre la totalité du terrain et régénérer ainsi les sources de la production, hâter enfin le moment qui rendra possible l'introduction complète de la méthode du réensemencement naturel et des éclaircies, tel doit être le but du traitement actuel de ces forêts.

A R T ICLE II.

\section{Des coupes de transformation.}

ว้อ̌3. Pour réaliser la transformalion qui vient d’ètre indiquée, il est nécessaire de bien se rendre compte de l'état des divers peuplements que présentent d'ordinaire les forêts dont il s'agit. On peut classer ces peuplements en quatre catégories principales: 
$1{ }^{\circ}$ Les restes de vieilles futaies;

$2^{\circ}$ Les perchis;

$3^{\circ}$ Les gaulis et les fourrés;

$4^{\circ}$ Les parties totalement ruinées.

:3)4. Bien que les vieilles futaies soient fréquenment clair-plantées, le nombre d'arbres y est cependant, en général, suffisant pour procurer le repeuplement naturel, à l'aide des coupes de régénération, et parfois mème on y troure des massifs complets qui sont dans un trìs bel état de végétation. Leur traitement rentre done dans l'application pure ol simple de la méthode du réensemencement naturel et ne présente aucune difficulté.

ơ). Dans les perchis, on rencontreordinairement les anciens arbres de réserve qui ont gîné et gênent encore la végétation des jeunes bois; on y trouve ensuite, en plus ou moins grande quantité, des bois blancs dont la présence est également nuisible.

Nous parlerons d'abord des anciennes réserves.

Lorsque ces réserves sont en très petit nombre, et que l'on croit pouvoir les extraire sans causer trop de dégàts, on doit le faire, quel que soit l'àge du perchis, en apportant, dans l'abatage et dans la vidange, toutes les précautions possibles. Mais lorsque les réserves sont nombreuses ou qu'elles sont rassemblées par places, comme cela se voit dans certaines forêts où l'ordonnance n'a pas toujours été strictement observée, ce serait une faute de les abaltre toutes. Dans ce cas, en effel, le perchis qui se 
trouve parmi les réserves a crû dans une gène extrème, causée par leurs ramures volumineuses; les liges qui le composent se sont élancées, mais n'ayant recu qu'incomplètement l'action de la lumière et ayiunt manqué d'espace pour prendre du corps, elles ont un diamètre beaucoup trop faible, proportionnellement à leur hauteur, et nese souliennent contre les intempéries, que par l'appui que leur prètent los branches des arbres entre lesquels elles se sont élevées. Si donc cet appui leur était enlevé subitement. elles périraient inévitablement victimes des rents, de la neige ou du girre. Comment améliorer cet état de choses sans courir le danger que nous signalons?

On y parviendra, en se bornant à couper les réserves entièrement dépérissantes, et en faisant élaguer 1 les autres, de manière à donner insensiblement plus d'air au perchis et à favoriser sa croissance en grossemr, sans cependant le priver de l'appui qui lui est indispensable. Par ce moyen. les jeunes tiges finiront par prendre de la consislance, et le peuplement, dans son ensemble, pourril stre conduit, dans un état convenable. jusqu'au lerme de son exploitation.

1 Dans cot elagage, les branches sorvant dapuni an perchis ne devront pas être coupées entièrement; il convient, au contraire, de se bolner à les raccourcir, afin que, lout en donnant anx probes plus dirspare, alles continuent rependant encole a les appuyer. 
Cependant, si sur un point on trouvait de nombreuses réserves, et qu'elles fussent toutes dépérissantes, il faudrait se décider, malgré la perte momentanée qu'on en éprouverait, à laisser sur pied celles que l'on jugerait indispensables pour soutenir le perchis, dùt-on courir la chance de les voir périr lout à fait. Il est évident, en effet, que mieux raudrait, dans er cas, sacrifier une partie des produits actuels, que de compromettre l'existence du perehitout entier.

(Guant aux bois blanes que l'on rencontre dans les perehis, en plus ou moins grand nombre, il convient de les extraire en mème temps que los anciennes réserves; mais, comme pour celles-ci, il fitut avoir égard à l'effet que cette extraction pourra produire, et la modifier selon qu'il en sera besoin.

linsi, lorsque les bois blanes seront mélangés aux bonnesessences en faible proportion, ils devont itre coupés sans exception; au contraire, quand ils formeront une partie assez notable du peuplement pour qu'on ne puisse les enlever sans détruire le massif. il faudra en réserver le nombre indispensable à la conservation des tiges de bonnes essences. Plus tard, il y aura lieu de faire disparaitre ces bois hlanes par un second nettoiement, si les honnes essences sont alors assez nombreuses pour pouvoir former le massif it elles seules; lans le cas contraire, on devra les laisser sur pied, mème jusqu'à leur entier dépérissement, tant qu'il ne sera pas nécessaire de les enlever. 
afin d'aider and développement des perches de bonnes essences.

Il est sans doute inutile de dire que, quand l'état du perchis indiquera la nécessité d'une éclaircie soit partielle, soil générale, elle devril s'exécuter en inème temps que les deux opérations dont il vient d'être parlé.

g:66. Wans les gaulis et dans les fourrés on retrouve, de mème que dans les perchis, les anciennes réserves et les hois blanes.

Cies derniers, associés aux morts-hois el à divers antres arbustes nuisibles, sont ordinairement on trìs grand nombre, dans les fourrés surtout; leur extraction immédiate, qui doit être accompagnée de celle des vieux arbres, est de la plus grande importince. En effet, le tort qu'ils sont susceptibles d'occasionner n'est point encore consommé, et en se hàtant de les enlever, on parvient souvent à sauver des semis naturels de hêtre, et même de chêne, et à restaurer ainsi tout de suite le repeuplement.

Ces coupes de nettoiement devront se répéter autant de fois qu'on le jugera nécessaire pour assurer la prospérité des bonnes essences ; en outre, il sera ronvenable, dans les places où ces essences manqueront ou seront rares, de compléter le massif par quelques plantations.

8.57. Les parties entièrement ruinées, où les plantes parasites ont enviahi la totalité du terrain, ne peuvent évidemment être remises en bon état qu'à l'aide de 
repeuplements artificiels. Nous renroyons done, pour la manière de cultiver ces terrains, au sixième livre de ce cours; nous ferons observer seulement que, selon le tempérament des essences qu'on reut introduire, on devra ou détruire tout de suite les bois blanes, les morts-bois, etc., ou les conserver encore pendant quelque temps. Tans le premier cas, il ne suffira pas de les couper, il faudra extirper leurs souches ot leurs racines le mieux possible, afin d'empicher leur reproduction; dims le second cas, on les enlevera par forme de nettojement dès que leur couvert ne sera plus d'aucune utilité au jeune repenplement artificiel.

On abattra les vieux arbres arant de commencer les semis ou les plantations.

A R TICI,E III.

Marche des coupes de transformation.

\%48. Dans les futaies de l'espèce qui nous occupe, les anciennes exploitations, bien que faites sans principes de culture, ont cependant été assises avec un certain ordre et il existe presque toujours, dans les forêts de quelque étendue, un nombre déterminé de séries. Toutes les fois qu'il en sera ainsi, on fera bien de conserver ces anciennes séries pour régler la marche des coupes de transformation. Dans les forèts où cette distribution en séries n'exis- 
tera pas, on devra commencer par y procéder en groupant dans une mème série, autant que possible, des vieilles futaies, des perchis, des gaulis et des lourrés.

¡39. Cette distribution opérée, la marche des coupes de transformation pourrait ètre réglée à peu pris comme celle des exploitations dans ta iutaie régulière [47]. Supposons que la révolution jugúe convenable pour une série soit de 120 ans, on la parLigerail en quatre périodes de:30 ans, on affecterait i la premiè les vieilles futaies, à la seconde el à la troisième les perchis, enfin à la quatrième les gaulis et les lourrés. Cela fait, on aurait pendant la première période les exploitations suivantes:

$I^{\circ}$ Jans la première affectation : coupes de régínération réglées par volume.

2 Dans les trois autres affectations : extractions de vieilles réserves, de bois blanes, et, s’il y a lieu. de liges dominées, réglées par contenance.

Quant aux parties entièrement ruinées, il serait ronvenable d'en prescrire le repeuplement artificiel. de manière que les peuplements ainsi créés pussent itre exploités aux mèmes ípoques que les peuplements environnants '.

'Lorsque les vides dont il s'agit ici peurent, par leur situation, se rattacher à l'affectation des gaulis et fourrés, il y a licu de les repeupler immédiatement en chêne et hêtre. Ceux, au contraire, qui se trouveront enclavés dans les perchis pour-

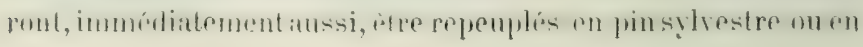


Cette marche des coupes de transformation semble, au premier abord, la plus simple et la meilleure, puisqu'elle a pour résultat de régulariser l'état de la forêt dès la première période. Cependant, selon nous, elle présente un inconvénient grave, celui de ne pas maintenir le rapport soutenu. Il est clair, en effet, que, pendant la première période. les produits seront bien plus élevés qu'ils ne l'étaient auparavant et qu'ils ne le seront dans les périodes suivantes; car ils se composeront non-seulement de coupes de régénération dans la vieille futaie, mais encore d'une quantité considérable d'anciennes réserves répandues dans toute la forêt. sans compter les hois blancs et les perches dominées qui, sur heaucoup de points, seront très productifs.

Adopter une telle marche, ce serait évidemment transgresser un des principes fondamentaux de l'exploitation des bois; re serail, en outre, dans beaucoup de localités, jeter dians la consommation plus de matiere qu'elle n’en réclimerait, et, par conséquent, en faire tomber le prix.

כ00. En général, il sera facrile d'obvier ì ce double inconvénient, à moins qu'une cirronstance. telle

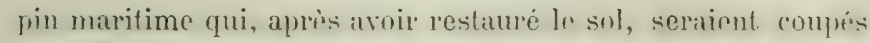
en même temps que les perchis parvenus au terme de leur exploitabilité. - Dans ce sol ainsi restauré, on introduira facilement, les essenees feuillues d'élite qui se confondront dès lors entièrement avec les parties voisines régénérées, 
que le dípérissement total des restes de vieille futaie n'y mette ohstacle; or, cette circonstance est très rare.

Cies restes de vieille futaie, in effet, quoique parvenus a maturité, sont cependant, d'ordinaire, loin de depérir. et peurent demeurer sur pied 20 at :30 ans de plus. On peut donc, au lieu de procéder immédiatement à leur régénération. les mettre an contraire en réserve, pour ne les exploiter que lorsque le peuplement du surplus de la série aura été régularisé par les coupes de transformation.

thes que l'on aura pris ce parti, la marche de ces coupes pourra ètre réglée d'une manière très simple. Car ici, de mème que dans les transformations de forèts jardinées, il faut ne pas attacher trop d'importance à la fixation de la possibilité, qui n'est qu'un objet secondaire; le principal est d'assurer l'amélioration de la forèt par des moyens prompts, faciles et sûrs.

On s'occupera done d'abord d'établir une révolution préparatoire, qui se hornera au nombre d'années nécessaire pour assurer au bois des débouchés convenables $(10,20,30$ ans par exemplei; celi lail, on séparera, sur le terrain, la vieille futaie it mettre en réserve d'avec les perchis, et ceux-ci d'avec les gaulis et les fourrés; enfin on considèrera ces deux derniers d'une part, et les perchis de l'autre, romme des séries ou sous-séries provisoires, dans chacune desquelles les coupes de transformation 
s'effectueront ammuellement par contenances égales el d’après les règles qui ont été posŕes plus hatul. ()uant aux parties ruinées, on les repeuplera, comme nous l'avons dit, de manière à pouvoir les rattacher par la suite aux massifs environnants ' .

Il est à peu près certain que ces coupes, tout en ne portant point sur la vieille futaie, satisferont cependant largement aux besoins ordinaires de la consommation. D'une part, les anciennes réserves donneront en assez grande quantité des bois de service et de travail; de l'autre, les perches dominées, el surtout les bois blanes, fourniront des ressources au chauffage, tout en donnant encore quelques pièces d'industrie.

La révolution préparatoire terminée, le peuplement de la forèt sera suffisamment régulier pour que nos successeurs soient mis à mème de conduire la futaie de plus en plus vers l'état normal. C'est à eux qu'il appartiendra de fixer la durée de la révolution définitive, la périodicité des éclaircies, etc.. parce que, mieux que nous, ils jugeront de ce qu'il

1 Lotsquion établit dos reglements de coupers dans des forete comme celles dont il s'agit, il est nécessaire de se réserver li laculté d'entreprendre, dans le cours de la révolution préparatoire, outre les exploitations régulières, toutes celles qui suraicut jugees indispensables pour atteindre entièement le but cultural, tels que nettoiements répétés daus les gaulis el fourés, ébranchements des ancieunes réserves maintenues dans les perehis, etc. 
sera convenable de faire sous ces divers rapporls ${ }^{1}$.

כ̆61. Dans les forèts irrégulières dont traile le présent chapitré, de même que dans celles qui ont été jardinées, on rencontrera des cas nombreux que nous n'avons point prévus. C'est au forestier à les apprécier el à mudifier les généralités du traitement que nous avons tracé, selon le degré d'importance qu'il accordera à l'exception².

' Voir l'appendice.

2 Voici, par exemple', une exception qui se présenle très liéquenment, surtunt dans les lorets de chène. Un trouve dans ces lorêts des parties de futaie qui, bien que peu avancées en ige, el quoique assises sur un bon sol, sont néanmoins sur le retour ou même dépérissantes, parce qu'elles proviennent de souches déjà fort vieilles. Dans ce eas, il est érident qu'il laut, avant tout, hâter lia régénération du peuplement existant, et dès lors la marche des coupes de transformation, telle que nous l'avons proposée, ne peut plus être adoptée. 


\section{LIVRE QUATRIËME}

\section{DE LEXPLOITATION DES TAILLIS}

\section{DÉFINITIONS.}

3022 . On appelle taillis, les forèts deslinées à se reproduire principalement par les rejets des souches et des racines.

Ce mode de régénération résulte de lis propriété que possèdent loutes les essences feuillues, à un degré plus ou moins élevé, de domer naissance à des rejets et à des drageons, lorsque l'arbre est coupé à fleur de terre ou à une certaine élévation au-dessus du sol [23].

Les rejets et les drageons sont, en général. d'autant plus abondants, que l'arbre a été coupé plus près de terre et que son écorce est plus spongieuse. La production des drageons est, en outre, farorisée par la disposition traçante des racines. 
ö63. Lorsque, dans l'exploitation en taillis, on réserve un certain nombre d'arbres pour rester sur pied pendant trois révolutions et plus, la forèt ainsi traitée, est appelée futaic sur taillis, el mieux taillis sous futaie ou taillis composé.

Le taillis est simple, lorsque les arbres réservés ne sunt pas maintenus au delà de deux révolutions, ou lorsque les coupes s'exploitent sins réserve.

כ̋4. Les arbres réservés dans les taillis se nomment baliveaux. On les distingue en baliveanx de l'îge, baliveaux modernes et baliveaux anciens.

Au moment de la coupe, les premiers sont âgés d'une révolution; les seconds de deux, et les autres de trois et au delà.

C'est ainsi que le Code forestier désigne les arbres de réserve; mais, dans l'usage général le mot de baliveaux seul indique ceux de l'àge, la dénomination de modernes, ceux de deux révolutions, et celle d'anciens, les autres. On a aussi adopté une distinction entre les anciens: ceux qui sont parvenus à un ìge très arancé, ont reçu souvent le nom de vieilles écorces.

Ces deux dénominations d'anciens et de vieilles ćcorces, laissent à désirer en ce qu'elles désignent des arbres d'un nombre indéterminé de révolutions.

Or, pour lit classification des réserves d'un taillis sous futaie, il importe d'indiquer l'àge des arbres; il serait donc convenable que chaque désignation fùt précise à cet égard. 
Voici comment nous arons cru pouvoir régler cet objet:

Baliveaux....... I révolution.

Modernes. . . . . . . 2 révolutions.

Anciens de $2^{\mathrm{c}}$ classe. . . . 3 révolutions.

Anciens de $1^{\mathrm{rc}}$ classe. . . . 4 révolutions.

Vieilles écorces. . . . . . 5̆ révolutions.

On pourrait mème diviser les vieilles écorces, comme les anciens, en plusieurs classes.

563 . Les arbres de réserve influent sur le tiillis, de deux manières distinctes: par le couvert et par l'ombrage.

Il importe de ne pas confondre ces denx choses.

Le couvert exerce son action sur l'espace de terrain que les branches de l'arbre surmontent; il nuit à la végétation en affaiblissant les effets de lit lumière et de la pluie, et en empèchant la formation de la rosée.

L'ombrage, au contraire, promène son influence sur un certain espace, suivant les différentes positions du soleil pendant le jour. Il est presque toujours salutaire à la croissance des bois, en ce qu'il maintient une certaine fruicheur au sol et aux plantes, sans priver celles-ci de l'action bienfaisinte de l'atmosphère et de la lumière.

Ces deux mots: couvert et ombrage, indiquent aussi la surface mème qui est couverte ou ombrigée. C'est ainsi qu'on exprime le convert des réserves en 
mètres carrés, pour établir le rapport qui doit exister entre elles et le taillis.

ว̆66. Ravaler, c'est couper à fleur de terre des souches qui, dans les exploitations précédentes, avaient été laissées trop élevées.

与67. Couper en pivot, c'est couper de manière à former un creux dans le milieu de la souche; couper en talus, c'est, au contraire, donner à la souche une inclinaison qui farorise l'écoulement des eaux pluviales.

568. On appelle cépéc ou trochée l'ensemble des rejets provenant d'une même souche.

与̆69. Ramiers: on nomme ainsi les perches du taillis et les branchages, lorsqu'ils ne sont point encore façonnés. 


\title{
CHAPITRE PREMIER.
}

\author{
- \\ IÉTHODE DU TAILLIS SIMPLE
}

ARTICLE PREMIER.

\section{Généralités.}

วั70. Dans la méthode áu taillis simple, les rejets et les drageons sont considérés comme le produit principal. On évite avec soin tout ce qui peut nuire à leur accroissement et, à cet effet, on n'étiblit que peu et souvent mème point de réserves dans les coupes. Lorsqu'on choisit quelques baliveaux, ce n'est que dans le but de procurer un peu d'ombrage aux expositions chaudes et d'obtenir les semences nécessaires pour régénérer le taillis. Ces baliveaux ne doivent jamais devenir des arbres anciens, et leur nombre doit être réglé de manière qu'en aucun cas its ne recourrent au delà du vingtième au sei ième de la surface totale. 
Toutefois, dins les forèts de l'Etat, il sera souvent convenable de faire une exception à cette règle, atin de procurer des courbes et des courbants pour la marine. Lorsque le sol et l'essence le permettront, on pourril, sur les lisières des bois et sur les bords des routes, élever des arbres bordiers destinés à atteindre un àge avancé.

571 . Les taillis ne peuvent avoir de durée indéfinie que si les souches, que l'àge ou une maladie fait périr, sont remplacées par de nouveaux pieds. Or, il est certain que ces souches ne vivent point aussi longtemps que l'arbre dont on n'aurait pas entravé la croissance naturelle; car les exploitations répétées fatiguent et altèrent les racines 1.

1 Voici l'explication physiologique de ce lait:

La relation intine ou l'équilibre qui existe entre les organes aúriens (branches) et les organes souterrains (racines) détermine, comme on le sait, la vie végétale.

Les racines, en pompant la séve ascendante, produisent le diveloppement des feuilles, et celles-ci, à leur tour, par le travail qu'elles accompliseent dans l'atmosphère, fournissent la sève descendante qui assure l'extension des racines.

Or, quand on coupe la cépée d'une souche, on détruit évidemment cet équilibre. A la vérité, de nouveaux rejets apparaissent, mais ils sont minimes, comparés aux tiges qu'ils remplacent, et ne peuvent envover, par consécquent, qu'une nourriture tout ì fait insulfisante aux racines dont une partic par suite, ne fonctionne plus, et ne tarde pas à pourrir.

Cependant, les racines étant dimintées, et les rejets s'aceroissant chaque année, l'équilibre se rétablit au bout d’un certain 
Pour que l'existence des taillis soit assurée, il fiut donc qu'il s'y opère une régénération graduelle, soit par des graines provenant des arbres réservés, soit par des drageons, ou par des rejets qui, nés très près de terre, s'enracinent et forment des pieds indépendants, alors que le centre de la souche pourrit, soit enfin par des semis artificiels ou par des plantations. En général, on doit admettre que plus une essence a de propension à drageonner, mieux elle convient au régime du taillis.

\section{ARTICLE II.}

\section{Essences propres aux taillis.}

572. Tous les arbres feuillus peurent être traités en taillis.' Le hêtre est le seul auquel ce régime semble ne point convenir. On a observé que si, dans quelques régions, les souches produisent des rejets, cette faculté leur est enlevée dès la seconde, ou au plus tard à la troisième rérolution, à moins qu'on ne

temps. Mais bientôt une nouvelle exploitation survient, les mêmes phénomènes se reproduisent, et la souche, troublée périodiquement ainsi dans ses fonctions, finit par contracter des tares, la pourriture se loge dans celles de ses parties où lit séve cesse de pénétrer, sa vitalité s’altère de plus en plus et s'éteint, en détinitive, bien plus tôt que cela ne fût arrivé si l'arbre eut obéi, dans son réreloppement, aux lois naturnlles de la végétation. 
coupe au-dessus du nœud de l'exploitation précédente; et, dans d'autres régions, on a reconnu que les souches ne repoussent pas, mème dans l'àge le plus tendre.

Parmi les arbrisseaux, il en est quelques-uns qui méritent l'attention du forestier, quoique à un moindre degré que les arbres, ce sont:

Le coudrier, les petits saules, le merisier à grappes, le comouiller mâle, les viomes, etc.

Les taillis, ordinairement, se composent d'un mélange de diverses essences. L'art du forestier consiste à favoriser les plus importantes, à assurer leur durée, leur bonne croissance et à en obtenir les produits les plus utiles.

\section{A RTICLE III .}

\section{Fixation de l'exploitabilité dans les taillis.}

วั73. L'expérience a prouvé que, pour fournir d'abondants rejets, il ne faut pas que les bois soient coupés à un âge trop avancé. Toutes les essences, en général, sont moins disposées à ce genre de reproduction, lorsqu'elles ont dépassé la période où elles croissent surtout en hauteur, pour entrer plus particulierement dins celle du grossissement, et il en est même qui alors...' s'y refusent ${ }^{1}$. D'un autre còté,

- Ce refus tient à la fois à la dureté de l'écorce et aux causes qui viennent dietre indiquées plus haul (Note de la page 340). 
il est incontestable qu'en coupant à des époques trop rapprochées, on affaiblit les souches 1 et on diminue considérablement les produits en matière [406].

D'après les observations des meilleurs forestiers, il est convenable de ne pas prolonger les révolutions de nos principales essences au delà de $\mathbf{4 0}$ ans, et de ne les fixer qu'exceptionnellement au-dessous de 15 ans. C'est entre ces deux limites qu'il convient généralement de renfermer la fixation de l'exploitabilité des taillis simples, afin d'assurer le mieux possible leur reproduction.

Paur les forèts de l'Étit el des communes, l'exploitabilité absolue, renfermée dans les limites que nous venons de poser, devra, la plupart du lemps, être préférée. Elle dépend, comme on le sait, des circonstances plus ou moins favorables à la régétition, c'est-à-dire du climat, de la situation, de l'exposition et du sol. Ainsi, tândis que, dans les meilleures conditions, on pourrait n'exploiter le chène gu'à 40 ans, on devra le couper dìs l'àge de 20 ans, et même plus tòt, lorsque ces conditions seront défitvorables. Il convient, au surplus, de ne fixer la durée de la révolution qu'après s'itre assuré, par quelques observations, de l'àge au delà duquel les essences. dans une localité donnée, ne repoussent plus de souche ou repoussent mal.

1 Voir la même note, page 340 . 
כ̈74́. Lorsqu'on a adoptél'exploitabilité absolue, ainsi entendue, pour déterminer la révolution des taillis, les données suivantes pourront être considérées, sinon comme règles, du moins à titre d'indications utiles.

Dans un bon fonds, le chêne, le hêtre. l'orme, le frêne, les grands érables et le charme pourront s'exploiter de 30 à 40 ans. Les révolutions plus longues ne sont que des exceptions rares.

La révolution de 20 à 20 ans conviendra aux mèmes essences, quand les conditions seront moins fitrorables, et clle pourra être considérée comme le plus long terme pour l'aune, le tilleul, le bouleau, le petit érable, les alisier's, les sorbiers, le merisier, et lé micocoulier, à raison surtout des usages auxquels ces essences sont propres.

Toutes ces essences pourront même s'exploiter de 10 à 20 ans, dans certains cas. Cet àge devra être préféré pour les trembles et les grands saules ainsi que pour le chutaignier qui, par exception, n'est jamais conduit jusqu'à son exploitabilité absolue, parce que, ítant employé exclusivement à faire des cercles et des chchalas, il atteint son maximum d'utilité bien avant cette époque. Le tremble, toutefois, ne se coupe, dans certaines contrées, qu'à $2 \breve{~ o u ~} 30$ ans, quand on l'emploie à la menue charpente.

Les rérolutions de $\breve{a}$ à 10 ans doivent être réservées pour les petits saules, les coudriers et autres arbrisseaux. Le robinier, cependant, peut s'exploiter 
vers 10 ans, pirce qu'à cet àge déjà, sa végétation rapide et la dureté précoce de son bois le rendent propre à fournir de bons échalas pour la vigne. Comme cette essence drageonne plus que toute autre, les courtes révolulions présentent moins d'inconvénients $!$

AR'TICLE IV.

Fixation de la possibilité dans les taillis.

575. La possibilité dans les taillis doit ètre réglée par contenance $[418]$. Cette base, ainsi que nous l'avons dit, est préférable en général à cause de sa simplicité et de la régularité qu'elle imprime à la marche des coupes. Or, cette régulitrité, c'est surtout dans les bois exploités en taillis qu'on peut la réa-

- Les besoins de erreles pour futailles et de petits paisseaux de vigne, ont, dius divers departements, fait soumettre les taillis des essenees les plus importantes à des révolutions aussi courtes. Un tel régime est fxidemment vicieux. Sans diminuer ainsi la révolution, on peut satisfaire à ces hesoins par des éclaircies, possibles à tout âge, qui produiront rles bois de quelque petite dimension qu'on les demande. C'est ainsi qu'on délivre, aux adjudicataires de coupes, des milliers de harts, qui servent aux bûcherons pour lier des fagots et aux flotteurs pour rassembler les trains. Ils sont extraits, sans aucun dommage, des taillis rle l'âge de 6 ans et au-dessous; mais ces extractions peuventavoirlicu de mêmedans des peuplements plus àgés, et fournir aux différents besoins de laconsommation. Les forêts traitées en futaie offrent la même ressource. 
liser, parce que, en raison des courtes révolutions auxquelles ces forits sont soumises, il devient plus facile d'y établir une gradition convenable dans l'àge des bois et d'asseoir les coupes de proche en proche.

Différents auteurs ont prescrit de rendre les contenances des coupes, dans les taillis, inversement proportionnelles au bon état du peuplement et à la fertilité du sol. Il est incontestable que cette mesure, bien exécutée, rendrait lesproduits annuels moins variables; mais, comme on réussit ordinairement à créer un rapport soutenu par les moyens plus simples que nous connaissons [420], on a généralement accordé peu d'attention à cette idée, et, en France, particulièrement, tous les taillis sont partagés en coupes d'égale contenance.

ARTICLE V.

Saison la plus convenable pour la coupe des taillis.

376. Il est généralement à désirer que les taillis ne soient coupés ni en automne ni en hiver, parer que l'intensité du froid, dans cette dernière saison, peut altérer les souches. L'écorce quelquefois même s'en dítiche, lorsque, gonflée par les pluies, elle íprouve l'effet d'une forte gelée. Dans ce cas, toute production de rejets devient impossible, et on ne peut plus compter que sur celle des drageons.

II ne convient pas non plus de couper les taillis en 
temps de séve; les souches s'affaiblissent, dit-on, par l'écoulement trop abondant du suc séveux. Un inconvénient plus réel, c'est la perte de la première et plus forte repousse du taillis qui est due à la séve du printemps, tandis que, par la séve d'été, on n'obtient que des rejets qui sont toujours moins vigoureux et ont plus de peine à se défendre contre les froids de l'hiver.

La coupe en temps de séve ne doit être tolérée que pour les taillis dont l'écorce est indispensable aux tanneries.

377. Les mois de férrier, mars, et quelquefois le commencement d'avril, sont, dans les cas ordinaires, et dans le climat d'une grande partie de la France, les époques les plus favorables à la coupe des taillis. D'autres époques pourront mieux convenir pour les départements méridionaux où la végétation est très précoce. La température doit endécider. En règle générale, on évitera de couper arant et pendant les grands froids et au moment de la séve.

Quelques parties du Midi de la France et celles qui sont voisines de l'Océan, n’ont guire à craindre les fortes gelées d'hiver; dins ces régions il serit préférable de commencer à couper aussitiot après la chute des feuilles, et, pour les chènes ì feuilles persistantes, aussitôt que le mouvement de la deuxième séve sera arrêté. 
ARTICLE VI,

Mode d'abatage des taillis.

:378. On doit procéder à l'abatage des taillis avec des instruments bien tranchants, afin de ne pas faire écliter la souche et l'écorce qui la recouvre. Les perches avant $0^{\mathrm{m}}, 0 \Xi$ de diamètre et au-dessus doivent être coupées à la hache; pour les brins plus faibles, il est préférable d'emplover la serpe, afin d'éviter l'ébranlement et souvent la rupture des racines que le choc de la hache occasionne aisément. On peut mème se servir, pour les tiges les plus minces, de la scie, en prenant la précaution de faire la section oblique ì l'horizon; pratiquée sur des rejets aussi petits, cette opération n'a aucun inconvénient.

En général, la souche, quelle que soit sa dimension, doit recevoir par l'abatage une forme telle que les eaux pluviales ne puissent y séjourner; de plus, la coupe doit se faire le plus près possible de terre, afin que les rejets, naissant à fleur du sol, soient rapprochés des racines et se trouvent ainsi plus solidement assis pour résister aux intempéries. Cependant, lorsque le taillis provient de souches déjà très vieilles, ou lorsque l'essence est peu disposée à se reproduire par rejets (comme le hêtre, par exemple, et mème le charme dans certains terrains froids et humides, on fera bien de couper dans le jeune bois, 
immédiatement au-dessus du noud de la précédente exploitation, parce que les pousses nourelles seront plus abondantes et se développeront arec plus de facilité que si elles avaient à traverser l'écorce épaisse et dure de la vieille souche 1. Enfin, l'abatage à une certaine hauteur de terre sera convenable dians les sols marécageux ou sujets aux inondations, afin d'éviter que l'eau, pénétrant entre l'écorce et le bois, puis s'y congelant, ne les sépare l'un de l'autre el n'empèche ainsi les bourgeons de se former'.

Quand les essences ont la proprićté de drageonner, le moyen à employer, pour assurer la perpétuité du taillis, est très simple. Lorsqu'on s'aperçoit que la faculté reproductive des souches commence à diminuer, on les coupe entre deux terres, c'est-à-dire, au-dessous du collet de la racine. En détruisant ainsi le centre vers lequel se portait la séve, celle-ci agit plus énergiquement sur les racines el donne maissince à une grande quantité de drageons qui remplacent les souches surannées.

\section{ARTICLE: VII.}

\section{Façonnage ot vidange dans les taillis.}

รั79. Pour favoriser la reproduction des litillis, il

- Ĺne précaution très utile aussi, en parcil cas, consiste à laisser subsister sur la souche, comme tirants de séve, quelques-uns des rejets dont la conservation a déjà été recommandée à d'autres tilres [วั71]. 
convient, en général, que les bois soient fiçonnés et entièrement vidés arant que les rejets ne paraissent.

Très souvent on voit l'aire d'une coupe couverte de stìres et de fagots, au milieu desquels les rejets s'élèrent et dépassent quelquefois les piles de bois. C'est ce qui arrive dans les taillis situés en sol très fertile ou humide, et dans ceux peuplés d'essences à végétation rapide. On concoit que, dans de pareilles coupes, le séjour de bois façonnés et surtout le retard de la vidange ne peuvent manquer d'occasionner du dommage.

Il y en a moins à craindre pour les taillis situés en fonds maigre et aride et dans ceux que peuplent des essences d'une végétation lente, parce que les rejets de la première année sont ordinairement peu élevés.

580. La vidange avant le temps de la séve pourra presque toujours avoir lieu dans les coupes dont on commencera l'exploitation en automne. Elle sera possible encore dans les coupes de peu d'étendue, lors même qu'on attendrait la fin de l'hiver pour y mettre la cognée. Il n'est pas nécessaire, au surplus, que les bois soient transportés hors de l'enceinte de la forìt; l'essentiel est que la coupe en soit débarrassée, et il est rare qu'on ne trouve pas de places où le produit de l'exploitation puisse ètre momentanément déposé. Par le moyen de ces places de dépôt, on pourra hàter la ridange des coupes,

1 Quind les coupes, ainsi que cela doit ètre, aboutissent 
quand mème on n'aurait commencé à les exploiter qu'après les fortes gelées.

Ce qui favorise le plus la célérité des vidanges, c'est le bon état des chemins. Il est telle forèt où la sortie des bois n'est possible que lors des grands froids ou des chaleurs de l'été, et où la difficulté des transports diminue considérablement la valeur des coupes. Dans ce cas, on est forcé de consentir à des prorogations de délai de vidange, quel que soit l'inconvénient qui en résulte. De bonnes routes forestières doivent ètre mises au rang des améliorations les plùs utiles, de celles, surtout, qui augmentent le revenu des forits et qui permettent d'apporter, dans les exploitations, la promptitude et l'ordre désirubles.

AR IICLE VIII.

Examen des dispositions que renferme le cahier des charges des adjudications de coupes.

5̈81. Le cahier des charges régissant les adjudications des coupes qui se font annuellement dans les forèts de l'Étal, des communes et des établisse-

Loutes sur une ou plusicurs laies sommières ou routes, les accotements de celles-ci deviennent les places de dépôt les plus naturelles et les plus avantageuses. 
ments publics, contient les dispositions suivantes 1 :

A. “L'abatage des bois sera entièrement terminé " le 15 avril qui suit la date de l'adjudication.

" Les bois à écorcer, en vertu de l'acte d'adjudi" cation, seront coupés avant le 15 mai.

B. " A moins de clauses contraires, les bois ") seront exploités à tire et aire et à la cognée, le

" plus près de terre que faire se pourra, de manière " que l'eau ne puisse séjourner sur les souches. Les ") racines devront rester entières.

G. " Les coupes seront nettoyées, savoir: en ce " qui concerne le ravalement des anciens étocs et ") l'enlèvement des épines, ronces et autres arbustes " nuisibles, avant le terme fixé pour l'abatage; en " ce qui concerne le faconnage des ramiers, avant "le $1^{\text {er }}$ juin.

"A l'égard des ramiers provenant des bois qui " auront été écorcés en vertu du procès-verbal " d'adjudication, ce dernier délai est prorogé jus-

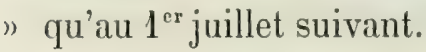

D. ") La vidange sera entièrement terminée le " 15 avril qui suit l'expiration du délai d'abatage. E. " Si des circonstances locales nécessitent d'au" tres termes que ceux fixés par l'abatage, le net-

' Your plus de facilité dans l'enseignement, nous avons classé ici les dispositions du cahier des charges, dans l'ordre où les diflérentes parlies de l'exploitation des taillis ont été traitées dans le présent chapitre. 
" toiement et la vidange des coupes, il en sera fait " une clause spéciale de l'adjudication. "

582. Par le dernier paragraphe que nous venons de rapporter, le cahier des charges a prévu que des modifications pourraient être nécessaires, et il permet de les arrêter par des clauses spéciales, qui doivent être proposées par les agents forestiers locaux et soumises à l'approbation de l'administration.

Nous allons examiner les conditions générales et en signaler les parties susceptibles d'être modifiées, lorsque d'ailleurs les circonstances rendront ces changements possibles.

\section{a. L'abatage doit être terminé au 1 J̋ avril.}

Ce terme est trop reculé pour un grand nombre de localités, eu égard aux inconvénients de la coupe en temps de séve.

D’après ce qui a été expliqué plus haut [ว̈77], il faudrait, selon les différents climats, fixer, non seulement la fin, mais aussi le commencement de l'abatage, et faire en sorte d'éviter les exploitations d'automne et d'hiver qui, hors des contrées méridionales et d'une partie de celles de l'Ouest situées dans le voisinage de la mer, ne sont pas toujours sans danger 1.

' Malheureusement, il existe souvent des obstacles presque insurmontables à une telle amélioration, par suite de l'extrème difficulté que l'un rencontre, dans-les mois de février et de mars, à se procurer des ouvriers. A cetle époque tle lannée, en 
Mais, s'il est désirable que le délai d'abatage puisse être raccourci pour les taillis en général, il est certain que celui que le cahier des charges assigne aux bois à écorcer, pourrait être prolongé avec avantage, et sans qu'il en résultât aucun inconvénient pour la reproduction.

En effet, sauf dans les régions particulièrement favorisées sous le rapport du climat, la séve n'est entièrement en mouvement que dans la seconde quinzaine d'avril, et souvent même plus tard encore. L'écorcement ne peut donc guère commencer que dans les derniers jours de ce mois ou dans les premiers jours de mai. Or, cette opération, assez longue par elle-même, pour peu que la coupe soit étendue, est sujette en outre à de fréquentes interruptions, amenées tantôt par un refroidissement subit de la température, tantôt par un vent sec, qui suffisent pour augmenter l'adhérence de l'écorce au bois et arrêter le travail.

En augmentant le délai dont il s'agit de 15 jours, on faciliterait singulièrement la récolte d'un produit de première importance pour l'une de nos principales industries, et très profitable au propriétaire,

effet, les habitants des campagnes, tous à peu près, sont occupés aux travaux agricoles, auxquels, en général, ils ne renoncent à aucun prix. En automne et en hiver, au contraire, leur temps, dans une grande partie de la France, appartient tout naturellement à l'abatage et au façonnage des bois qui deviennent ainsi une ressource précieuse pour ces pnpulations. 
sans compromettre, plus qu'on ne le fait actuellement [576], la reproduction des souches qui, en tout état de cause; ne saurait avoir lieu que sous l'action de la séve d'août.

b. Les bois seront exploités à la cognée le plus près de terre que faire se pourra.

Ce mode d'abatage, bien que généralement applicable, souffre cependant quelques exceptions, comme nous l'avons fait voir [578]. Par exemple, si, malgré le peu de disposition du hêtre à repousser de souche, on est forcé par les circonstances de le traiter en taillis, il sera prudent de le faire couper au-dessus du nœud de la précédente exploitation, car ce n'est que du jeune bois que l'on peut espérer des rejets.

C'est par ce mode que, dans l'ancienne Lorraine, on a soutenu, pendant assez longtemps, l'existence de quelques taillis de hêtre; et lorsque les agents forestiers, pour obéir aux règlements, se sont décidés à faire couper ces taillis rez-terre, les vieux bûcherons ont prédit la mort des souches et ne se sont pas trompés.

Une mesure très utile pour assurer la reproduction des taillis, et dont nous avons déjà parlé plus haut [ڤ̆71 et 578 , note], consiste à conserver les rejets traînants, soit que l'on comptesur le mouvement des voitures et des hommes dans la coupe, pour les enterrer, soit, ce qui est mieux, qu'on les fasse coucher en terre, plus soigneusement, par des ouvriers. Quoi qu'il en soit, dans l'un et l'autre cas, on peut obtenir 
par ce mojen une multitude de jeunes tiges. II serait donc à propos, en pareille circonstance, de ne pas exiger que l'exploitation se fit trop près de terre, et de recommander au contraire aux bùcherons, tout en coupant en tilus, de respecter les trainants dont il s'agit.

c. Les coupes seront nettoyées, savoir: en ce qui concerne le ravalement des anciens étocs et l'enlèvemeni des arbustes muisibles, avant le terme fixé pour l'abatage; en ce qui concerne le façonnage des ramier's, avant le $1^{\mathrm{er}}$ juin.

Les ramiers devraient expressément ètre façonnés dans le plus bref délai possible. Si le terme de cette opération n'est fixé qu'au ${ }^{\text {er }}$ juin, les adjudicataires peuvent les laisser éparpillés dans les coupes pendant tout le mois de mai et, par conséquent, dans le moment ou la séve produit déjà des rejets. D'une part, il en résultera du dommage pour le recru des souches; de l'autre, les agents et les gardes seront empêchés de visiter toutes les parties en exploiLation, ce qui pourra favoriser la fraude. Les bûcherons devraient être partagés en deux brigades: l'une chargée d'abattre et de façonner le bois de corde, l'autre de relever et de fagotter les ramiers. Celte dernière travaillerait au fur et à mesure de la chute des arbres et de l'abatage des cépées. Dans tous les temps, une coupe doit être accessible et ne présenter aucun obstacle à la surveillance journalière. Quant aux anciens étocs, c'est-à-dire, aux sou- 
ches qui sont mortes en loul ou en parlie, il viudrait mieux les extraire et leur substituer de jeunes plants bien venants, que de se contenter de les ravaler.

d. La vidange sera entièrement terminée le 1 ö avril.

Cet article des conditions générales donne aux adjudicataires une année entière, à partir de l'abatage, pour le débit et l'enlèvement des bois de leurs ventes, et doit, par conséquent, contribuer au succiss des adjudications. Il est à regretter, toutefois, que cette manière d'augmenter les revenus actuels des forêts nuise à leur conservation, et l'on ne peut qu'émettre le vœu bien formel de voir adopter des délais de vidange plus favorables à la reproduction des taillis, dans toutes les localités où le bon état des chemins et une concurrence suffisante entre les marchands de bois permettront de le faire sans un trop grand sacrifice pécuniaire. 


\section{CHAPITRE DEUXIËME.}

\section{MÉTHODE DU TAILLIS COMPOSÉ \\ OU SOUS FUTAIE.}

ARTICLE PREMIER.

\section{Gónéralités.}

583. La méthode du taillis sous futaie a pour objet caractéristique d'élever, sur les taillis, des arbres propres au service; les rejets ne sont plus alors considérés comme le produit le plus important. On veut ici obtenir, à la fois, les avantages du taillis et une partie de ceux de la futaie, c'est-à-dire régénération prompte et facile, et production de pièces de fortes dimensions. Dans cette vue, on conserve, à chaque coupe du taillis, un certain nombre d'arbres auxquels on laisse parcourir plusieurs révolutions.

584. Toutes les essences propres au taillis simple (les arbrisseaux exceptés) peuvent aussi ètre 
traitées en taillis sous futaie, et les mêmes principes d'exploitation s'appliquent à l'un et à l'autre modes. Toutefois, dans celui du taillis sous futaie, il faut considérer non seulement l'exploitabilité du sousbois, mais aussi celle de la réserve, et donner une attention particulière au choix, au nombre et ì la distribution des baliveaux, trois choses qui sont de la plus haute importance. Car, s'il est incontestable que des réserves saines, en nombre modéré et réparties avec intelligence sur le taillis, sont le plus souvent un moyen d'augmenter la quantité et l'utilité des produits en matière, il n'est pas moins vrai aussi qu'un balivage surabondant, fait sans égard aux conditions d'une bonne végétation, amène des résultats opposés. En effet, il ne faut point perdre de vue que le taillis sous futaie réunit deux éléments qui, parleur nature, s'entravent et se contrarient réciproquement: d'une part, les arbres réservés qui deviennent branchus, et s'élèvent peu, parce que chaque exploitation du taillis les isole et provoque leur végétation latérale aux dépens de leur croissance en hauteur: de l'autre, le sous-bois qui est gêné dans son développement par le couvert des réserves qui le surmontent.

Accorder ces deux éléments qui sont en opposition, en réglant le nombre et la distribution des réserves, de manière que la croissance du taillis soit entravée le moins possible, tel est le problème à résoudre dans l'exploitation du taillis sous futaie. 
A RTICI. E II.

\section{Exploitabilité des taillis sous futaie.}

o.8.. L'exploitabilité des sous-bois, dans les taillis sous futaie, doit être réglée d'après les mèmes considérations que celle des taillis simples, sauf, tontefois, que, comme il s'agit ici d'élever des arbres sur le taillis, il n'est plus possible de soumettre celui-ci à des révolutions de courte durée.

En effet, si l'on exploitait le taillis de 10 à 20 ans, la réserve d'arbres, destinés à atteindre un ìge avancé, manquerait en grande partie son but. D'abord, la plupart de ces arbres, trop grêles pour résister aux intempéries, en seraient inéritablement victimes; en second lieu, on ne peut espérer atteindre le but que l'on se propose, avec des baliveaux n'ayant que l'élévation ordinaire des taillis de cet àge, c'estì-dire, 4 à 7 mètres. On siit que, d'ordinaire, le fùt de l'arbre crû en massif, une fois isolé, ne s'allonge plus, bien que la cime continue à gagner en hauteur; la raison en est. que, dans cet état d'isolement, n’étant plus serré par des voisins qui le forȩaient à s'élever, l'arbre conserve et développe ses branches. Il est donc érident que pour obtenir, dans un taillis sous futaie, des arbres ayant la longueur de füt qu'exigent les différentes constructions. il faut, en 
général, leur permettre d'acquérir cette tongueur avant de passer à l'état de baliveaux.

D'après ces considérations, on fera bien de fixer In révolution des taillis sous futaie de 30 à 40 ans. Dès lors, on pourra faire choix de baliveaux ayant 10 à 14 mètres de hauteur et un diamètre proportionné, et qui, par conséquent, supporteront mieux les injures de l'atmosphère, en mème temps qu'ils deviendront propres à leur destination.

ว86. Plusieurs auteurs allemands, entre autres Pfeil, sont d'avis d'exploiter le sous-bois des taillis sous futaie it courte révolution. Les bois, disent-ils, qui croissent sous le couvert, perdent la faculté de repousser bien plus tôt que ceux qui végètent en plein soleil; d'où il suit que, dans les taillis composés soumis à une exploitabilité reculée, un grand nombre de souches, quoique jeunes, périssent soit immédiatement après l'exploitation, soit quelques années plus tard ' .

1 Voici en effet ce qui se passe dans ce cas: lorsqu'un brin ou une cépée vient d'être coupé et que la souche fournit des rejets, la végétation, ainsi que nous l'avons fait voir plus haut (note de la page 340 ), passe par un état transitoire, pendant lequel elle s'efforce de rétablir l'équilibre entre les tiges et les racines. Or, quand les nouveaux rejets croissent sous le couvert, ou des réserves ou des hois blanes, ils languissent, et ne. peuvent, faute de lumière, se développer de manière à rétablir promptement l'équilibre en question. Par suite, les racines, imparlaitement nourries par une séve descendante insuffisante, ne parviennent ni à se raviver elles-mêmes, ni à raviver la 
Nous reconnaissons la vérité de cette observation, sans cependant adopter le remède proposé, parce qu'il a l'inconvénient très grave aussi, que nous venons de signaler, concernant l'éducation de la réserve. Mais, par contre, il nous parait d'autant plus indispensable de ne pas négliger les travaux d'entretien (repeuplements artificiels, nettoiements, élagages), dont traite ci-après le chapitre III, et sans lesquels les produits des taillis sous futaie ne peuvent que s'amoindrir de révolution en révolution, tant en quantité qu'en qualité. Cet amoindrissement graduel, dont les exemples sont malheureusement trop fréquents en France, suit une marche d'autant plus rapide que le sol et le climat sont moins propices, el parfois mème il aboutit à la stérilité.

587. Quant ì l'exploitabilité de la réserve, on conçoit qu'elle dépendra d'abord de l'essence et de la qualité du fonds de terre, et surtout du but que le propriétaire se proposera d'atteindre. Ainsi, tandis que, dans un taillis situé en bon fonds, l'Etat et les communes peuvent avoir intérêt à prolonger le maintien de certaines réserves jusqu'au moment où elles auront acquis les dimensions et les qualités qui les rendent propres aux grandes constructions, un particulier, possédant une forêt placée dans les mêmes

sonche; la pourriture souterraine y continue ses ravages et finit juar amener la mort du pied, tantôt dans le cours de la révolution, tantôt, et le plus snuvent, à la prochaine exploitation. 
conditions de végétation, doit arrêter la vie de ses baliveaux à l'àge au-delà duquel la plus-value qu'ils acquerraient, s'il leur laissait parcourir une nouvelle révolution, cesserait de le dédommager du sacrifice pécuniaire qu'il ferait en ne les exploitant pas. En un mot:, les principes de l'exploitabilité relative, de $1^{\text {re }}$ et de $2^{\circ}$ sorte $[410$ et 411$]$, trouvent ici leur application ${ }^{1}$.

Le terme extrême de l'exploitabilité étant fixé, pour la réserve, d'après ce principe, il devient nécessairement le régulateur du balivage, en ce qui concerne du moins les catégories de baliveaux à établir sur le taillis. Ainsi, s'il s'agit, par exemple, d'élever des arbres ayant un âge quintuple de la révolution du taillis, la réserve comprendra cinq catégories de baliveaux dont le nombre variera d'après diverses considérations qui seront développées plus bas [591].

\section{ARTICLE III.}

\section{Choix des baliveaux.}

588. Les baliveaux doivent être choisis parmi les pieds les plus vifs et de la plus belle venue. En donnant la préférence aux brins de semences, qui sont

' Voir, pour plus de détails sur cette question, le Cours d'exploitation, de débit et d'estimation des bois, par M. Nanquette, page 29's et suivantes, et les Études d'aménagement de M. Tassy. 
généralement mieux venanls et plus durables que les rejets, il faut éviter de réserver desbaliveaux de l'àge trop grèles, parce qüils sont facilement ployés ou rompus par les vents, la neige et le givre.

วั89. La réserve doit se composer, en majeure partie, de chênes, comme étant l'essence la plus précieuse pour les constructions.

Iprès le chêne, on doit préférer l'orme, le frêne, les grands érables; puis le hètre et le charme. 11 est avantageux aussi de réserver quelques pieds d'alisier, de sorbier, de merisier et de bouleau. Le tremble mème pourrait, dans certains taillis, faire partie de la réserve, attendu la rapidité de sa croissance et ses belles dimensions qui le font rechercher pour diver's usages [256]. On l'en exclut cependant, et généralement avec raison, parce que l'extrême abondance de ses graines le multiplie dans les taillis, au point de compromettre la végétation des essences d'élite [252].

En général, il est utile de composer la réserve de plusieurs essences, tout en maintenant la proportion la plus forte aux plus importantes. Non seulement on assure, de celte manière, la satisfaction des différents besoins de la consommation, mais on se procure l'avantage d'élever, dans chaque canton de la forêt, sur chaque place pour ainsi dire, les arbres qui y prospèrent le mieux. On augmente donc la production générale en quantité et en qualité. A ce point de vue, on ne peut quapprouver ce qui se pratique dans 
certaines parties de l'Allemagne, où l'on a l'habitude de mêler à la réserve feuillue, un certain nombre d'arbres résineux, mélèzes ou pins sylvestres, qui réussissent parfaitement dans de telles conditions, fournissent d'excellents bois de travail et de construction et n'entravent cependant que très peu la croissance du sous-bois. Cette pratique, du reste, n'est point inconnue en France: dans différents bois particuliers de la Sologne, par exemple, on voit croitre, audessus de taillis mélangés de chêne, de chàtaignier et de bouleau, une réserve de pins sylvestres 1.

590 . On a l'habitude de ne marquer que des tiges très droites, et l'on néglige celles qui présentent quelque courbure ou dont les branches forment la fourche ${ }^{2}$, parce que, alors. ces branches sont exposées à être déchirées par les vents.

I V. le rapport de M. Ad. Brongniart, de l'Institut, à M. le Ministre de l'Agriculture et du.Commerce, sur les plantations forestières dans la Sologne.

2 La dénomination d'arbres fuurchus s'applique spécialement à ceux dont la tige se divise, à une certaine distance de la base, en deux branches qui s’élèvent verticalement et restent toujours assez rapprochées l'une de l'autre. C'est arce raison que l'on évite de comprendre des arbres ainsi conformés dans les balivages, parce qu'ils ne sont d’aucune utilité spéciale. Mais il n'en est pas de mème de ceux qui, à la naissance du houppier, portent une forte branche, laquelle attire à elle une grande partie de la séve destinée à l'alimentation de la cime, et pourra former plus tard une des courbes si précieuses pour les besoins de la marine. 'Toutefois, avant de comprendre ces arbres dans les balivages, il importe de s'assurer, par une inspection- 
Les tiges droites doivent, sans doute, être l'objet principal du balivage; mais, dans les forêts de l'Etat du moins, et pour l'essence chêne, il ne faut point perdre de vue les besoins de la marine qui sont du plus haut intérêt. Dans toutes les forêts, et surtout dans celles qui sont traitées en futaies, on n'est jamais embarrassé de trouver des pièces droites, tandis qu'on n'y rencontre pas aussi facilement les courbes et les courbants qui servent à la construction des vaisseaux. Il faut donc chercher à y pourvoir par les balivages.

AR'TICLE IV.

\section{Nombre des baliveaux.}

591. Le nombre des baliveaux à réserver doit être réglé de manière que le couvert qui en résulte ne puisse compromettre la croissance et la reproduction du taillis. C'est dire qu'il doit varier selon les essences, les sols et les expositions, et qu'il n'est pas possible de prescrire, à cet égard, des règles générales et absolues.

En effet, il est des essences, telles que le chêne, le frêne, le bouleau, etc., qui ne donnent qu'un couvert léger et n'empêchent guère la croissance du taillis, alors que le hêtre, le charme, et d'autres

attentive, que l'axe du trone et celui de la branche sont sensiblement dans un même plan, condition essentielle pour que la courbe formée par leur réunion puisse être utilisée. 
encore, peuvent étouffer ce qui végète sous leur épais feuillage.

Certaines essences aussi supportent mieux que d'autres d'être dominées. Ainsi les taillis de chêne, quels que soient le sol et l'exposition, souffrent beaucoup de la présence des arbres, tandis que l'on voit souvent les rejets de charme croitre assez bien sous le couvert et même tout près du tronc de chênes anciens.

Quand le sol sera substantiel, il y aura moins d'inconvénients, pour le taillis, à ce que les réserves soient multipliées, et qu'on les laisse parvenir à un âge avancé; d'une part, parce que la végétation du taillis est d'autant plus assurée que le terrain est meilleur; de l'autre, parce que, dans un bon fonds, les arbres prennent plus de hauteur, et que, plus ils sont hauts, moins par leur couvert ils nuisent au taillis.

Dans les terrains médiocres, peu profonds et placés à une exposition chaude, il faut chercher surtout à ombrager le taillis par de nombreuses réserves. 11 n'y aurait d'ailleurs que perte pécuniaire à réserver des arbres jusqu'à un âge avancé, dans les sols où ils ne sauraient acquérir de fortes dimensions.

En créant dans de pareils terrains un ombrage abondant, on mettra obstacle à l'évaporation trop considérable du sol et des bois eux-mêmes, sans être obligé de recourir pour cela à de vieilles réserves presque dépourvues d'utilité industrielle.

592. Nous avons dit ailleurs [587] que les catégo- 
ries de baliveiux, dont doit se composer la réserve, dépendaient du terme extrême d'exploitabilité adopté pour celle-ci, et nous venons d'indiquer quelles sont les circonstances de nature à influer sur le nombre de baliveaux à comprendre dans chaque catégorie.-On conçoit, toutefois, qu'on ne puisse établir, à cet égard, de règle fixe, mais on n'en sent pas moins le besoin de certaines données propres à servir de point de départ et de guide, lorsqu'il s'agit, dans la pratique, d'arrèter cet objet aussi important que difficile. Dans ce but, on a cherché à déterminer, par maximum et par minimum, l'étendue que peuvent recouvrir les arbres de réserve, sans compromettre le taillis; puis on a essayé de trouver l'espace moyen que recouvre un baliveau de chaque catégorie, et l'on est arrivé de cette manière à fixer le nombre d'arbres de différents àges qüil conviendrait de réserver à chaque exploitation.

Ainsi qu'il était facile de le prévoir, les expériences faites ont produit des résultats fort divers, et, par suite de ces différences, les opinions émises à ce sujet ont beaucoup varié. Nous ne rapporterons de ces opinions que celles qui se sont assez généralement vérifiées et qui sont le plus conformes à nos propres convictions. Toutefois, nous nous hâtons d'ajouter que, lorsqu'il s'agira de régler le couvert de la réserve dans une forêt ayant quelque importance, cet objet demandera toujours à ètre expérimenté particulièrement, attendu que le couvert se modifie selon les 
issences el selon la disposition des arbres à sétaler plus ou moins, disposition qui dépend à son tour du sol et du climat. Enfin, nous ferons remarquer que le couvert des vieux arbres est bien plus nuisible au sous-bois (à surface couverte égale) que celui des arbres jeunes ou d'àge moyen, parce que le feuillage les premiers est plus épais et moins pénétrable, par ronséquent, aux rayons du soleil que celui des seconds.

э93. (On admet en principe que, immédiatement avant l'exploitation, les arbres ne roivent courrir yue le tiers, au plus, du terrain, quand les circonstamers locales, quenousarons indiquées plus haul, semblent permettre une réserve abondante. Le couvert peut itre diminué, sulon los eas, juspulan sixieme de la surfice.

Le balivage normal qui vat suivere, al dont la premiere idée appartient à Cotla, nous parail applicable. it un grand nombre de nos taillis sous futaice, lorsqu'ils croissent dans un lum terrain; il ebhblit m rouvert el un ombrage molípis, ot assure dre bes-

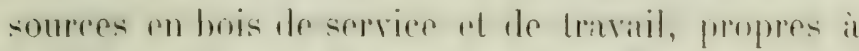
lous les besoins de la consommalion. ()u pourral litcilement, au surplus, y apporter les moditications que des circonstances particulieres rendeaient avanlageuses ou nécessaires, soit en supprimant une el mème deux catégories de réserves, soit en diminuant le nombre des pieds romposant charume des ritrigories.

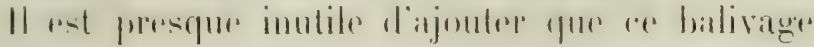


n'ist applicable quaux essences dont la consommation réclame des pièces de fortes dimensions, et qui, d'ailleurs, sont susceptibles d'atteindre un âge arancí; lelles sont, par exemple: le chène, l'orme, le frêne, l'érable.

Vous ne royons pas l'opportunité de riserver, comme on le fait souvent, des hitres el des charmes jusquà̀ 1000 ams el plus. Ces arbores, dont lr. convert est très épais, s'étulent considérablement ot écrasent le laillis, sans offrir au propriétaire, lors de lour abatage, une compensation pour les pertes yue leur présence a occasionnées. Il est rrai, foutelois, que le hitre, par ses fruits, présente quelque lídommagentent; c'est un objet à apprécier d'apris la fréquemere des années de semence dans la localité. lit vialcur de lit faine, el les difficultés de la récolte.

:94. Balivage normal. - Idmettint une révohution de 30 ans, on devait tromver, ì chaque exploitation, par hectare:

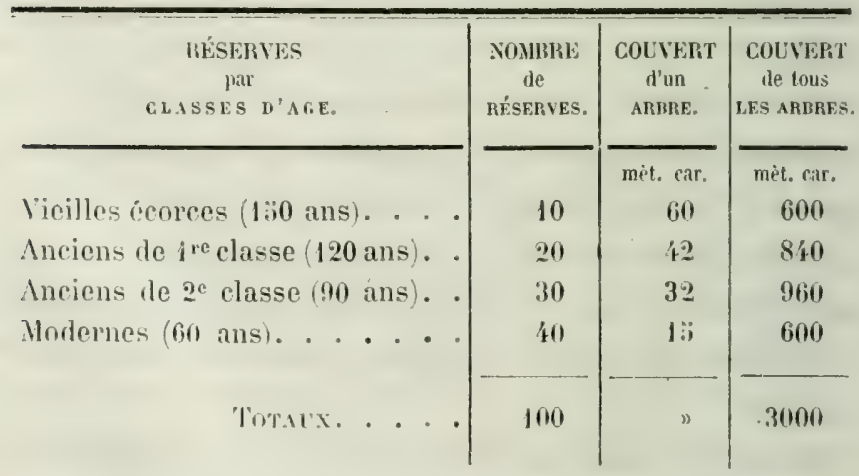


Lors de la coupe, on abattrail par hectare:

Vieilles écorces (1500 ans) . . . 10

Anciens de $1^{\text {re }}$ classe $(120$ ans $) .10$

Anciens de $2^{\circ}$ classe (90 ans) . . 10

Modernes (60 ans) . . . . 10

$$
\text { Total . . } 40 \text { arbres, }
$$

el l'on réservêrait so baliveaux de l'ìge ${ }^{1}$.

Il resterait done sur pied, après la coupe:

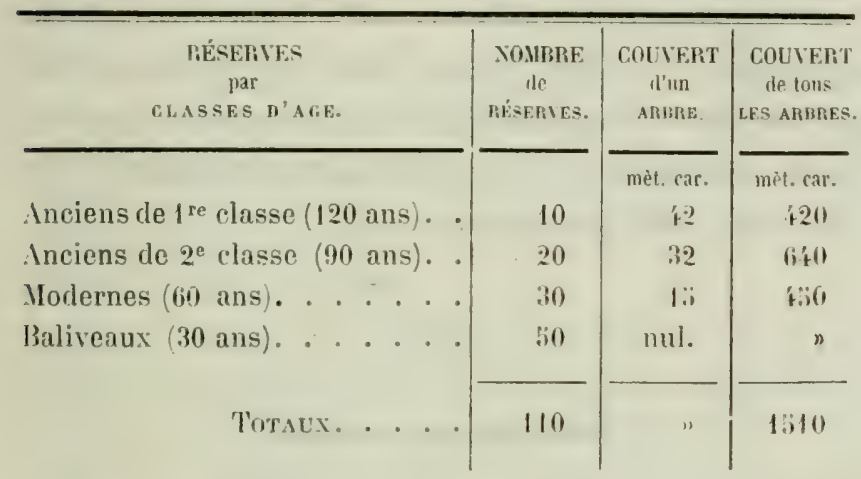

In voit que, d'apries le balivage normal que nous proposons, la réserve ne recoure, immédiatement ivint la coupe, qu'une superficie un peu au-dessous 'lu tiẹs de l'étendue totale. (1r, cest ee ripport.

- On remarfuera que lon preseril une réscrve de cinquante baliveaux de l'age, tandis qu'on ne coupe cependant que in pieds d'arbres et quion ne compte retrouver, au bout de la revolution, que quarante modernes (roir le tableau). Cette mesure a paru nécessaire pour lenil compte des nombreux accidents dont un certain momblor de baliveaux de litgesont toujours vietimes. 
mole le laillis el la fulaie, gue nous arons indigur. jlus hatul, romme nous paraissant le plus convenable pour conserver la forit en bon état, quand le sol et le rlimat sont d'ailleurs favorables à la régetation.

l'our éviter loute méprise, dans l'application, sur' lo sujet que nous venons de traiter, nous ferons re. marquer que le balirage normal ri-rlessus exposé. ur doit jas etre consideré comme un "modèle dont "m ne doive s'icarter dans aucun eas, quels que soient los conditions diune forèt el les intérêts du propritatire. Ce nost yum terme de comparaison. 111 plutrit une image de l’état de phoses en général le plus disimble, et dont il faut par ronsépguent chercher sans cesse à approcher.

IRTICLI: Y.

\section{Distribution des baliveaux.}

$.39 \%$. Lat distribution des balivenux de diffirentes matiggries présente souvent de tries gramdes difficultés. ol demande loujours lit plus serupulause altention.

-i lir coupe est siture dans un mème plinn, il fiuul cherehere it répartir l'ombrige, le plus également pussible, sur l'ensemble du terrain; si. au contritire. rlle oflre des accidents variés dans sa configuration, In balisage doit rhanger selon l'exposition de chaque partie. 
re qu'il faut surtout éviter, c'est de conserver, sur un mème point, plusieurs arbres anciens. Non seulement ils causeraient, ainsi réunis, un dommage bien plus considerable que s’ils étaient isolés: mais. suls disparaissaient ensemble à la prochaine exploitation. il en résulterail le plus souvent un vide, dans lepuel les bois blancs et les mort-bois tromeraint aeces. ou qu'il faudrait rombler par des repeuplementi itlificiels.

Toutes les fois que rela se putria. on lepa bien de réserver les arber anciens sur les lisires des bois et sur fo borl des roules et des chemins. Dams cette position, ils nuiront moins au taillis et leur propre végétation y gagnera.

.96 . Le balivage masommi dos taillis soms fulan est une operation pour l'exécution de laguelle les règles de la théorie ne sambairnt suffire, parce qu il existe une infinité de cas el de cireonstanees qu'on ne peut ni préciser ni préroir et que le coup diril cxercé du praticien est seul capable de bion appréreier. C'est assez dire que rente opération nécessile une habileté plus gramde el bien plus rare qu'on no le pense communément'.

' Les conditions dans lesquelles les agents forestiers et, avec eux, la plupart des particuliers exécutent cette importante npération du choix et de la distribution res baliveaux sont, il faut le reconnaître, peu faites pour en obtenir de buns résullat-. En effet, le mutrelage, c'est-it-dire la désignation des réserronde toutes calégories, a lieu, comme on le sait, en une seule fois, ot s'exíeule dans le printemps ou l'áté qui précesile l'explnitit- 
tion du bois. La coupe est vendue sur pied, abattue, façonnée rt videe suivant certaines conditions, imposées par le vendeur. accepties par lacquereur, et dont la principale est de représenter, au récolement, toutes les riserres frappées du marteau. 0 i, Iflumd le taillis est sur pied, enveloppant les arbres de toute: part, empêchant souvent de juger de leur port, de bien apercernir leur cine, il est, sinon impossible, du moins fort difficil. re laire toujours de bons choix. Mais ce qui est bien plus difticile encore, c'est de distribuer convenablement les diverse: catégories de réserves, de manière à répartir l'ombrage dans la mesure où il est utile, ot à atténuer, autant que possible, les inconvénients du couvert.

Nous sarons que des obstacles à peu près insurmontables sopposent à ce qu il soit rien changé à cet état de choses dans les forèts soumises au régime forestier: les règlements administratif-, la marche du service et les garanties de régulariti dont il est nécessaire de lentourer, cufin, le nombre limiti: dagents chargés des opirations dont il sagit,ne permettent pas iquant à présent du moins) d’y songer. Mais il nen est pas de mème dans les bois des particulier's, que les propriétaires exploitent eux-mèmes jour n'en vendre les produits qu'aprè: façonnage. Dans cess bois, il sera facile de modifier le procédi: actuel, en s'y prenant de la façon suivante:

On exploitera le taillis à l'époque de l'année où l'on a coutume de le faire, en laissant sur pied, d'abord, toutes les réserves de la révolution précédente et, en outre, les plus belles perches du laillis, en nombre triple ou quadruple de rolui de's baliveaux de l'ige qui devront étre maintenus défnitivement. Ces perches seront répandues sur toute la coupe, même daus les lieux où il se trouve d'anciennes réserves. Aussitôt l'abatage du taillis terminé, on procèdera au marblage de la reserve, de furon que les arbres abandonnés puissent incore itre coupés en temps utile pour la reproduction de lit souche. On conçoit aisément que celte opération pourra se latire avee promptitude et facilité, et qu elle présentera toutes les garanties désirables pour le meilleur choix des arbres nt leur espacenent le plus arantitgux. 


\title{
CHAPITIEE TROISIEMUE
}

\author{
TRAVAUX NÉGESSAIRES
}

IOH FNTIETENII IAS TAILISIS FN IOON FTAT

IRTISIE JREMIEIR.

\section{Repeuplements artificiels.}

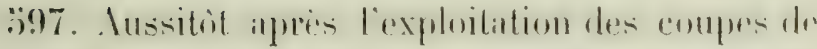
taillis, on doit s'occuper de remplacer, parr voie dr semis ou de planlation. Loutes les souches usiers. ot de regarnir de la mime mimiere les places qui ne: seraient peuplées que de bois de médiocre qualiti. Sinns cette précaution, les meilleures essences semienl souvent dépossédées par les bois blanes, et le chime surtout, la plus précieuse de toutes, finirait par disparaitre entièrement.

C'est effectirement un linit recommu, que le chine se reproduit mal de semence diuns nos taillis, mem. lorsqu'il y est dominint. Ce fait est surtout évidenl dans les taillis romposés. Nous connaissons dos 
lorèt- considéribles, ou la réserre en anciens et en vieilles écorces, formée en presque totalité de trè. beatux chines, atteste l'abondance arec laquelle cette rssence y ititit répandue autrefois, et oì l'on ne trouve plus aujourd'hui que fort peu de jeunes sujets de franc pied, propres à remplacer les arberes parvenus à maturité. \ussi est-on réduit à choisir les baliveaux parmi les perches des cépées, quoique l'on sache que les réserves de cette nature ne valent pas, ì beamoup près, celles qui proviennent de semence. En effet, un rejet n'atteint jamais l'àge auquel le brin peut parrenir; il se détériore, se creuse fréquemment pill le pied, et noffre que ritrement des ressources à la marine $\mathrm{el}$ anx grandes constructions civiles.

si nous recherchons la rause d'un inconvénient aussi grave, nous la trouvons dans le tempérament du jeune plant. On sait que les brins de chène, dès les premieres annéps de leur existence, ne supportent pas d'etre couverts 72 |. Or, dans un taillis, ils le sont en général loujours, soit par des rejets. sonil par des arbres de réserve; on comprend done qu'ilis doivent finir par périr, et que, dans une pareille siturtion, les glands, quelle que soit leur abondance, se répandent et lèvent en pure perte ${ }^{1}$

:98. Convaincu de cette vérité par l'expérience. llarlig propose de planter, par hectare, après chatqur 
exploitation, une certaine quantité de brins de chène bienvenants, pour assurer les ressources nécessaires au balivage (pour le nòtre, ce nombre serait วั0). Il reutque ces brins aient une hauteur de $\mathbf{2}$ mitres à 2 mètres 30 centimètres, qu'ils soient convenablement esparés et défendus par des tuteurs contre les coups de vent et rontre le poids de la neige et du givre.

Ce procédé n’est cependant à conseiller qu'exceptionnellement, à ciuse des difficultés et de In dípense de son exécution. En effet, non seulement lis plantation mème de tigres aussi élevées demande des soins particuliers, mais le jeune rhène, de lit hauleur indiquée par Hartig. ne devient propre à ètre lansplanté avec succìs, quaprès aroir été une première fois repiqué en pépiniere. Lors de ce repiquement. on retranche une portion de son pisot. et on fo forer ainsi à former plus de racines latérales et plus dr chevelu.

En génemal, il sera bien prefumbable de ne planter que des sujets de $\ddot{3}$ ä $̈$ ans qu'on éleve très ficilement et à fort peu de frais en pépinière croir le 6 c liver de er cours), et qu'ì défaut, quoique avec beaucoup moins d'avantage, on pourrail mème prendre directement en forèt. En les trunsplantant, on recèpe ces sujets, afin de donner à leur végétation un essor plus rapide ${ }^{\prime}$.

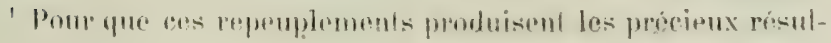


Comme nous l'arons dit plus hatul, on ne fatil res travaux que dims les patties clairiérés on les moins abrités de la rompe, el l'on arrablar, en outre, lex souches morles on déprérissutes, pour gagurer un peu de Lerraiu. Les bois bianes ef les morts-bois. s’ils peuplent seuls rertaines places do la coupe, doirent aussi itre soigneusement extirpés et remplacés par des essences meilleures ${ }^{1}$.

Outre le chine qui tient le premier rang, diuntres rssences, telles que l'orme, lo frène, les grands ératbles, etc., méritent encore d'ètre répandues, dans nos Laillis sous futaie. plus qu'elles ne le sont; elles amelioreront considérablement lir composition du taillis et pourront anssi, arre utilite, firire partic de lit réserve.

Lats qu'il est permis d'en atlendre, il est très essentiel de se souvenir des exigences de nos deux jrincipales espèces de: chêne, en ce qui concerne le terrain $\{70$ et 79$]$, atin de ne pas placer indifferemment, ainsi quion l'a fait trop souvent jusqu'ici, le rouvre dans les sols frais et humides et en plaine, le pédonculé dans les terrains accidentés, sees et peu profonds.

' Dans les taillis sous futaie, ees remplacements s'opèrent sunvent naturellement, à lit suite d'une année de semence alondante. Il niest pas rare alors de voir, dans les coupes exploitees Iepuis quelpues années, des semis très complets de chène ef d'autres essences dures, levés sous les bois blanes et les mortsbois. On conçoit qu'en coupant ceux-ci une ou plusieurs fois, jusqu'it ce que les autres soient en état de soutenir avantageusement la lutte, on fera une excellente opération [556], fort simple en elle-mène, peu coûteuse et qui, tout en épargnant inl propriétaire des frais de repeuplement bien plus considé-

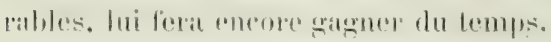


ARTILIAE II.

\section{Nottoiements ot éclaircies.}

ö99. Après aroir assuré, par les moyens que nous renons d'indiquer, la conservation des essences les plus précieuses, on ne peut point encore abandonner le jeune taillis à lui-mème; car, bien que l'on ait extirpé les bois blanes et les morts-bois dans les places où ils dominaient entièrement. il s'en troureri cependant qui, provenant soit de nouvelles semences. soit d'anciennes racines, reparititront parmi les cépées de bomnes essences, auxquelles ils ne larderont pas à nuire. Ainsi que dans les jeunes futaies, ce serit une opération des plus utiles, de faire disparaitre ces parasites, ou tout au moins d'empiecher' qu'ils ne dominent los bois durs. Par ce moven on écartera toute entrave à la régétation du taillis '.

En procédint à ce nettoiement au commencement

- En principe, il est incontestable que ces nettoiements doivent se faire suns avoir egard aux produits pécuniaires. Cependant, pour éviter une npération entièrement onéreuse et à larguelle un propriétaire aurait de la prine à se décider, ou peut ne commencel ces extractions qu’à làge de 6 à 9 ans. On en obtiendra, dès lor's, fles fagots propres au chaullage, surtout. it celui du four, et dont le prix de vente uffrira, le plus souvent, III bénéfice assez considérable. La valeur des bois dans les différentes localités fera avancer ou retarder cette opération ulitu. 
de l'automne, il est probable que lon diminueria les chances de la reproduction.

600. Lorsque le taillis, ainsi débarrassé des bois blancs, aura atteint l'àge de 12 a 1.3 ins, il sera a propos d'y faire une éclincie, d'apres les principes que nous arons déreloppés pour Ir lraitement des jeunes futaies [466]. Toutefois, on derra se borner i -nlever les perches sèches ou languissantes qui so trourent dans les cépées, et respecter soigneusement les brins de semence qui végètent, çi et là, sous lw couvert, et dont on peut espérer qu’ils vivront jusrfu'au retour de l'exploitation et augmenteront ainsi le nombre des souches propres à la reproduction. On devrit aussi litisser subsister les rejets susceptibles de s'enraciner el de devenir par la suite des pieds indépendants $[371]$. Lne seule éclaircie sera suffisante, si la forèt est exploitée de 20 à 2.3 ans ; on pourra en effectuer deux, dans le cas d'une révolution de 30 it 40 ans, surtout si l'on ne retarde pas le nettoiement dont nous venons de parler.

In propriétaire qui sera soucieux de donner des soins raisonnés et suivis à l'exploitation de son laillis, multipliera les éclaircies, favorisera par là l'accroissement des bois et obtieudra plus de produits !.

Voir l'Appendice. 
ARTICLE: III.

\section{Elagage des baliveaux.}

601. Pour compléler la série de traviux à exécuter dans les taillis, il nous reste à parter de l'èlagage ou taille des baliveanx, opriration dont on ne peut méconnaître l'utilité.

Lussitôt quion les isole, lit plupirt des arbres, et principalement le chène, se garnissentabondamment. le long du trone, de branches gourmandes qui détournerit, à leur profit, une grande partie de la séve destinée précédemment à la cime. Ces branches premant un prompt accroissement, il arrive, au bout d'un rertain nombre d'années, que lia cime n'est plus assez nourrie; elle sèche et amene le dépérissoment de l'arbre. De plus, lis lige devient tris nouruse ol moins propre, par conséquent, alu servire

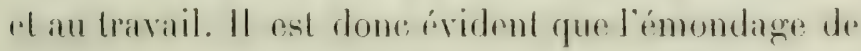
ashranchusgourmandes, pratiqur non senlement sur les réservos ancienurs el modernes. mais aussi sur les baliveaux de l'àge, est fort utile, pour que le régime du taillis sous futaic puisse atteindre son but.

Les éprques auxquelles l'émondage doit se laire me peurent ètre déterminées aree précision. Le plus urdinairement, il y a lien de le commencer trois ans atprès l'exploitation de lit coupe, et de le répéter, de trois en trois annés, jusque vers la moitir cu les 
drux liems de lat revolution: lo laillis, alors, devient assez élevé pour empêcher de nouvelles productions du trone ${ }^{1}$.

La coupe des branches se fera rez-trone, avec une serpe bien tranchante et en commencant l'entaille par le bas, afin de ne point arracher l'écorce. Quant su moyen d'exécuter cette opération avec facilití, surtoul sur de gros arbres, le meilleur pirait être de se servir d'échelles; l'ouvrier conserve ainsi les deux bras libres, et se meut plus aisément en tous sens que lorsqu'il est réduit à grimper, fìl-il mìme muni de crampons ${ }^{2}$.

La satison à choisir pour ces trivaux est le commencement de l'automne, comme étant la moins lavorable à la reproduction. Dans les pays où le bois a de la valeur, cet émondage n’est point onéreux ; les lourrées qui en résultent couvrent presque toujours les frais d'exploitation et domnent souvent même des hénéfices. Mais n'en fùt-il pas ainsi, qu'il conviendrait cependiant de tenir à son exícution: l'avantage

1 Voir l'Appendice.

- Les crampons dont se servent habituellement les élagueurs. (1) aussi les délinquants, pour grimper sur les arbres, sont très funestes aux chênes. La piqûre du crampon laisse une latee ordinairement visible à l'extéricur, el détermine à l'inléricur un épanchement de séve qui produit une tache noire, flus ou moins large, dans le bois et rlonne naissance à un vice analogue it la frutture. - Voir le Coms dexploitution, delit t

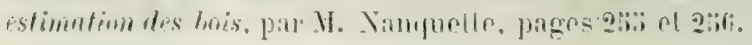


mincqué quion th obtiendra pour les arbres compensera amplement les frais.

602. Outre les branches gourmandes, l'élagage doit encore porter, quand il s'igit des baliveaux, tant anciens que modernes, sur les branches sèches qui se présenteraient, el surles branches latérales qui. sulalant trop, empèchent l'arble de gagner en hauteur derasent lo taillis en pure perte. Cette derniere opération demande du discernement de la part de relui qui la dirige, el de l'adresse dans l'exécution. Il ne faut point perdre de rue que, si lit vígétation de la jeune lige est facile a diriger par la taille, l'arbre déjà àgé peut ciprouver un grand dommage par l’enlivement total de trop fortes branches; tant parce que col enlevement interrompt l'équilibre entre la tìte et les racines, que parce que les plaies, occasionnées au frome par l'opération, ne se cicatrisent parfois qu imparfaitement et deviennent alors une cause de pourriture. C"est surtout le chene qui est plus patrticulir-

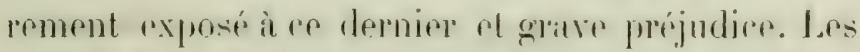
mouls giles, les griseltes, les huppes T6 his nont souvent pas d'autre origine que l'ampulation d'ume hranche fatite trop pres du trone. Il est dome it romseiller de ne jamais couper lez-trone les branrhes vives qui ont plus de 10 i 12 centimetres de diametre, et, dans ce cas, de se horner à en retrancher les extremitespour les empercherde sallonger divanlage. Mais si, par un motif particulier, lel que lis rupture aredilentolle, pare exemple, on chail forece de 
-upprimer une branche, il fiudrait, s'il se pouviat. laisser subsister un chicot de 1 mètre au moins de longueur, garni de quelques ramilles, qui y attireraient la séve et le garantiraient ainsi contre la pourriture. Il ne faut pas oublier, non plus, que l'arbre isolé a besoin, pour prospérer et pour résister aux intempéries, d'une tète plus développée que celui qui a crù en massil, et qu'il ne peut d'ailleurs jamais atteindre la hauteur de ce dernier, par celit mème qu'il a un plus graud nombre de branches it nourrir' .

603. Lélagage est un art qui, dans lia culture des bois, peut produire des effets heureux. Dans les l'orêts de l'Etat surtout, il a de l'importance, à cause des moyens qu'il offre pour faroriser lit formation des cuurbes et courbants propres aux constructions navales?

Toir l'Appendice.

"On peut consulter avec fruit, sur cet objet, le Manuel de IElayueur un de la conduite des abres forestiers, par M. Holton, Paris, chez $\mathrm{I}^{\text {me }}$ Huzard : prix : $2 \mathrm{fr}$. 


\title{
CHAPITRE QUATRIËME.
}

DU BALIVAGE DES TAILLIS

SELON L'ORDONNANCE RÉGLEMENTAIRE

\author{
RENDUE POUR L'EXÉCUTION
}

DU CODE FORESTIER.

AR'TICLL PREMIER.

Texte de l'ordonanoo.

604. Après avoir cherché à fixer les règles de l'exploitation des taillis, en nous appuyant sur l'expérience, il ne sera pas inutile de nous occuper des dispositions légales quı, en France, régissent la matière, et auxquelles les agents de l'administration des forêts sont tenus de se conformer, toutes les fois qu'il n'existe pas de règlement spécial pour les forèts qu'ils administrent. 
Ces dispositions, qui se rapportent principalement au mode de balivage, se trouvent consignées dans l'article 70 de l'ordonnance royale du $1^{\text {er }}$ août 1827 , rendue pour l'exécution du Code forestier; elles sont ainsi conçues :

"Lors de l'exploitation des taillis, il sera réservé

" 50 baliveaux de l'àge de la coupe, par hecture. Lin

" cas d'impossibilité, les causes en seront énoncées

" aux procès-verbaux de balivage et de martelage.

"Les baliveaux modernes et les anciens ne pour"ront être abattus, qu'autant qu'ils seront dépérissants " out hors d'état de prospérer jusqu'à une nouvelle 1) révolution."

ARTICI.E II.

Examen de ces dispositions et conséquences qui en découlent.

605. Cet article, calqué sur l'ordonnance de $\mathbf{1 6 6 9}$ qui a régi les forêts jusqu'en 1827, parait avoir pour but principal d'assurer à la France, qui possède peu de futaies, les ressources nécessaires en bois de construction et de travail.

On a craint, avec quelque raison sans doule, que, du silence du code et de l'ordonnance à l'égard des arbres à réserver sur les taillis, il ne résultât, en général, une anticipation sur les ressources de l'avenir, et que, pressées par des besoins toujours croissants, les générations actuelles ne vinssent à 
absorber plus que l'usufruit des richesses dont les siècles passés les ont rendues dépositaires.

En second lieu, il importait de donner aux agents forestiers un point d'appui légal qui, dans l'administration des forèts des communes el des établissements publics, leur permit de renfermer dans de justes bornes le mode de jouissance des propriétaires.

Sous ces diver's rapports, on ne peut qu'approuver la sagesse de l'article de l'ordonnance; mais il est impossible de méconnaître, d'un autre còté, les graves inconvénients de l'état de choses qu'il a consacré.

En effet, deux objets nous lrappent dans tes prescriptions qu'il renferme :

$1^{\circ}$ Un même mode de balivage est appliqué à tous les taillis;

$2^{\circ}$ Dans ce mode, on ne fixe que le nombre des baliveaux de l'âge ; celui des modernes et des anciens n'est point déterminé.

On comprend tout de suile qu'une application systématique de ce mode d'exploitation ne peut que faire commettre de nombreuses fautes. Or, au cas particulier, ces fautes seront d'autant plus graves que le balivige prescrit ne donne pas le moyen de les atténuer'; car l'ordonnance ne s'arrète que devant ls dépérissement des arbores, etn'admet, dans le nombre. des réserves, aucune modification tirée des circonstances qui influent sur li végétation et sur l'emploi 
des bois. Lintin, le traitement qu’elle ordonne, suivi à la lettre, pourrait conduire, dans un temps plus ou moins long, à la destruction de l'état de forêt auquel il doil s'appliquer (le taillis sous futaie).

C'est cette dernière assertion qu'il nous reste à prouver.

606. Pour administrer cette preuve, il nous suffira d'examiner ce que deviendrait, à la longue, un litillis sous futaie exploité d'après le régime de l'ordonnance.

I cet effet, admettons, comme plus haut [วั94], une révolution de 30 ans. Admettons, de plus, que le chène, le hêtre et la plupart des autres essences dures, peuvent, dans un sol de qualité moyenne, atteindre l'àge de 180 ans ou six révolutions, sans dépérir el sans présenter des signes évidents de mauvaise végétation, et, par conséquent, sans être dins les conditions d'exploitabilité voulues par l'ordonmance. Adoptons aussi, pour les différentes catégories de baliveaux, le mème couvert qui a servi de base à nos calculs concernant le balivage normal; rl supposons, pour les arbres de six révolutions dont il n'a pas été question dans ces calculs, un couverl de 70 mètres carrés, c'est-à-dire, supérieur de dix mètres carrés seulement, à celui des vieilles écorces de 150 ans. Enfin, fixons, comme plus haut [o:94], le déchet des baliveaux de l'àge à un cinquième.

Le lableau ci-iprès lait voir quol sera, arec ces 
DES TAILIIS.

389

données, le couvert sur un hertare, iul hout do lit sixième révolution.

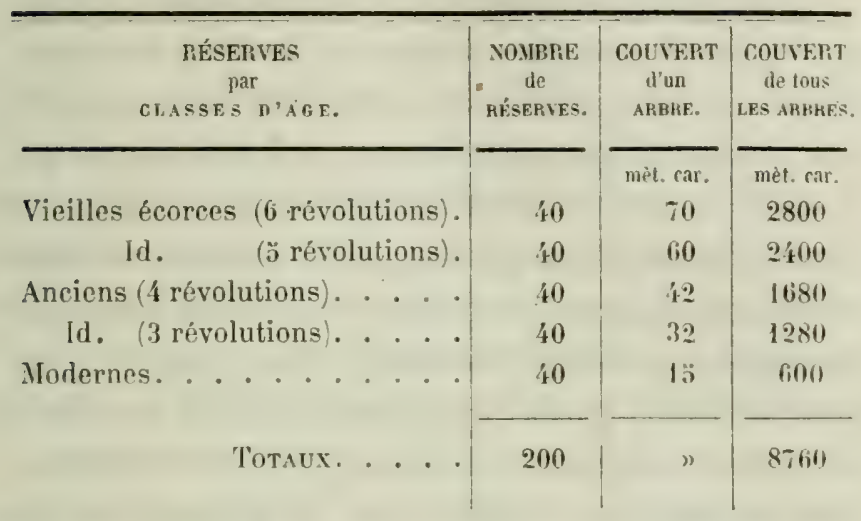

Ainsi, après six rérolutions, les 200 arbres qui existeront sur un hectare, courriront 8760 mitres carrés, et le taillis n'en orcupera plus que 1240 ru il ne sera pas surmonté ${ }^{1}$.

Autant vaudrait dire que le taillis sous futaie a disparu pour faire place à une futaie bâtarde, doul les arbres différents d’àge, branchus et inégaux hauteur, se gênent et s'entravent réciproquement.

Dans cet état de choses, si l'on vient à couper les réserves les plus àgées, il est évident qu'on ne pourri

' En réalité, dans un sol de fertilité moyenne, on verra bien rarement sur un hectare 80 vieilles ícorces parvenues sans dépérissement jusqu'à l'âge de 150 et de 180 ans. A peu prì̀ réduite par le fait aux 40 modernes et aux 80 anciens de trnis: et de quatre révolutions, la réserve ne couvrira guère plus de 4,000 mètres carrés. 
plus compler sur des rejets de souche; et les clairières, occasionnées par l'enlèvement de ces arbres, se garmissant principalement de bois blanes, ceuxci déposséderont peu à peu les bonnes essences. Toutefois, deux expédients pourraient être employés pour prévenir un aussi fàcheux résultat. L'un, que nous connaissons, serait de repeupler ces clairières par semis ou par plantation [597]; l'autre consisterait à abandomer, pendant une révolution, le mode du taillis sous futaie, et à établir des coupes de régénération avec les arbres de réserve, afin de produire, de semence, une jeune forêt susceptible d'être de nouveau exploitée en taillis. Mais, le premier de ces movens a l'inconvénient de devenir fort coûteux, en raison de la surface considérable à repeupler artificiellement chaque année (2800 mètres carrés par hectare, c'est-à-dire, plus du quart de la contenance de la coupe); le second compromet le rapport soutenu et fait succéder lit disette à l'abondance; car il oblige ¿abattre toutes les réserves dans le cours d'une même révolution, el réduit, par conséquent, les produits des révolutions suivantes au taillis seul.

A quelque point de vue qu'on le considère donc. le balivage selon l'ordonnance, exécuté à la lettre. ne saurait aboulir qu’ì de mauvais résultats. 


\section{Conclusion.}

607. Il faul le reconnaître, l'article 70 de l'ordonnance réglementaire du code forestier, quoique dicté par une sage préroyance, a maintenu dans les taillis un état de choses vicieux qu'il est urgent de remplacer par un régime d'exploitation fondé, dans chaque localité, sur les exigences culturales et sur celles de la consommation. Notre opinion n'est pas, cependant, que cet article soit abrogé immédiatement: nous croyons, au contraire, qu'il doit être maintenu quant à présent, parce que, comme nous l'avons dit au cornmencement de ce chapitre, nous reconnaissons en lui un principe conservateur, propre ì prévenir de funestes abus.

Mais, ce que nous désirons vivement, c'est son abrogation graduelle, par des dispositions d'aménagement ${ }^{1}$, basées sur un mûr examen des lieux et sur la saine application des principes d'économie forestière. Une semblable mesure n'aurait d'abord rien de contraire à la loi, et semble mème avoir été prévue par elle; car l'article $1 \ddot{3}$ du code forestier iqui s’applique aux forêts de l'litat comme à celles des com-

' On entend, par ce terme, l'opération qui consiste à régler. pour une ou plusieurs révolutions, le mode de culture d'une forêt, ainsi que la marche et la quotité de ses exploitations. 
munes et des établissements publics) dit: que les aménagements seront régléspardes ordonnances royales, Cest-ì-dire, par des actes qui ont mème force que l'ordonnance réglementaire elle-même, et qui, par conséquent, peuvent la modifier.

Trop sourent, jusqu ici, on n'a fait consister l'aménagement des taillis que dans la fixation de la révolution et dans la division du terrain en coupes d'égales contenances.

Quant au mode de balivage, on le passait sous silence, s'en référant ainsi aux dispositions de l'ordonnance réglementaire.

Yous pensons qu'au contraire ce dernier objet devrait toujours être soumis à une discussion approfondie, dans les procès-verbaux d'aménagement, et que, partout où sous ce rapport il existe une litcune dans les anciens actes, il faudrait s'empresser de la remplir, en provoquant des décrets complémentaires qui régleraient cette importante matière.

C'est ainsi que, sans s'exposer aux inconvénients d'une abrogation immédiate de l'article 70 de l'ordonnance, on réaliserait peu à peu une amélioration de la plus haute portée dans l'état de nos forêts. 


\title{
CHAPITRE CINQUIENE.
}

\author{
APPLICATION
}

DES

\section{DEUX MÉTHODES D’EXPLOITATION}

\author{
DU TAILLIS.
}

ARTICLE PREMIER.

Exploitation du chêne en taillis.

608. Le chène, l'arbre le plus intéressant pour la futaie, est en même temps l'un des plus propres à croître en taillis. Sa souche produit des rejets pendant près de deux siècles, lorsque le sol lui est favorable; elle donne des cépées abondantes et d'une croissance prompte, qui fournissent un bon bois de chauffage, de charbon et d'ouvrage.

L'écorce du chêne, qui est particulièrement recherchée pour les tanneries, est d'autant meilleure 
pour cet usige. que le bois est plus jeune el qu'il it cri plus rapidement. Celte circonstance rend les tiillis de chène très précieux el leur donne me valeur qu'aucune antre essence ne peut atteindre.

Il importe de remarquer que, sous le double rapport de la faculté de reproduction des souches et de la qualité des écorces, le chène rouvre est préférable au pédonculé. De plus, son feuillage étant plus abondant, it courre el améliore mieux le sol que ce dernier.

Si l'on veut traiter le chène en taillis sous futaie, on en ohtiendra des bois de travail et de service d'me utilité générale, et particulièrement des courbes de marine, attendu le développement que peuvent prendre, dans ce genre de forêt, les branches des arbres qui composent la réserve.

Le seul inconvénient des taillis de cette essence. déjà signalé plus haut $\lceil 597]$, est que les brins de semence y réussissent trop rarement. Lors done que les souches périssent, il devient indispensable de les remplacer au moyen du semis ou de la plantation.

609. Quand il y a lieu d'écorcer les taillis de chêne, il faut attendre que le bois soit en pleine séve. c'est-à-dire que les bourgeons commencent ì s'épitwouir, car ce n'est qu'alors qu'on peut procéder it cette opération. Le mieux est d'écorcer les arbres at les perches sur pied, aprìs que tous les menus bois non susceptibles d'écorcement auront été coupés ef enlevés.

Les bucherons chargés do l'ecorcement doivent 
d'abord faire, au pied des arbres et des perches, une entaille circulaire assez profonde pour arriver jusqu'à l'aubier. Ils fendent ensuite l'écorce, longitudinalement et par bandes, avec la pointe d'une serpe ou avec une lame quelconque, et la lèvent avee un outil en fer, en bois dur ou en os, qui a la forme d'une spatule. Cette écorce, qui se détache sans peint' lorsque la température est douce et un peu humide, s'arrache depuis la coupure circulaire au bas du tronc, jusqu'au point le plus élevé où le bùcheron puisse atteindre. II arrive souvent que les ouvriers arrachent l'écorce du haut en bas, ce qui rend d'autant plus indispensable lit coupure circulaire au pied des chènes; cette coupure empèche que lit souche el les racines ne soient dépouillées de leur écorce dont l'adhérence complète au bois est, comme on le sail, une condition indispensable de la production des rejets.

Ce premier travail fait, on coupe les chimes à fleur de terre puur en écorcer les parties supérieures qui n'avilient pu itre alteintes. On expose pendant quelque temps les écorces au soleil, pour les sécher, puis on les lie en holtes. Il faut se hàter de les meltre i couvert; car si elles étaient exposées à la pluie, elles perdraient de leur qualité.

L'écorcement diminue de quelque chose le volume du bois; on calcule cette diminution au huitième à peu près. Mais cette perte est largement compensée par la valeur de l'écorce, el il est même itrivé que le 
prix de l'écorce d'une coupe a dépassé celui du bois dont elle provenait. La perte la plus réelle est celle de la sére du printemps, qui s'écoule sans résultat et remet à la séve d'été la production de rejets moins robustes alors pour résister aux fortes gelées [马76].

La température de la France étant généralement assez douce, cet inconvénient n'est que peu à craindre dans un grand nombre de départements; et les besoins des tanneries, ainsi que les grands avantages pécuniaires que présente l'écorcement, ne permettent pas d'hésiter à pratiquer cette opération ${ }^{1}$.

L'expérience prouve d'ailleurs que l'écorcement ne compromet pas l'existence des taillis de chêne. Ce qui est trìs probable, néanmoins, c'est que les souches des arbres coupés toujours en temps de séve ne sauraient avoir la durée de celles des arbres dont la coupe ne s'effectue, au contraire, qu'en saison convenable.

ARTICLE 11 .

Exploitation du hêtre on taillis.

610. Il est incontestable que le hêtre n'est pas

' L'écorçage à la vapeur, dont M. Maître est l'inventeur, permet d'enlever et d'utiliser l'écorce des chênes abattus en toule saison: son emploi eviterait les inconvénients des procédés actuels, iau point de vue de la reproduction des taillis. 
disposé à repousser de souche; une écorce trop adhérente au bois et d'un tissu trop serré, semble s'opposer au développement facile des rejets. Quelle que soit au surplus l'organisation particulière de cet arbre, il est de fait qu'en le traitant en taillis, et surtout en le coupant à fleur de terre, on s'expose à voir mourir les souches.

Nous avons dit ailleurs โว̈78」 qu'un moyen de soutenir plus longtemps le hêtre en taillis, consiste à couper toujours au-dessus du nœud de l'exploitation précédente, afin que la séve, circulant sous une écorce plus jeune et plus tendre, ait plus de facilité à produire de nouvelles pousses. Mais, outre que ce moyen n'est pas infaillible dans certaines localités ', surtout dans les situations hautes et froides, les souches d'un taillis ainsi exploité présenteraient, au bout de quelques révolutions, des espèces de chi-

Des expériences, qui ne sunt pas encure décisives, tendent d'ailleurs à établir que l'on obtiendrait ainsi, à moins de frais, une écorce d'aussi bonne qualité que celle faite au printemps.

- De nombreuses observations ont constaté que le hêtre repoussait mieux de souche dans les terrains maigres que dans les bons fonds. Hartig avait cherché à expliquer cette particularité de la manière suivante, sans toutefois prétendre que son explication fût la vraie. "Dans un sol substantiel, dit-il, l'affluence très forte de la séve donne lieu à des bourgeons d'une grande vigueur. Mais l'écorce dure du hatre, faisant obstacle à ce qu'ils paraissent promplement à la surface extérieure, il arrive qu'ils se crispent et prennent une croissance contournée qui alors ne leur permet plus de percer. Lorsque le sol est médiocre, all contraire, la séve est peu abondante, la 
cots qui, s'élevant toujours davantage, finiraient par devenir de petits têtards, sur lesquels les rejets n'auraient plus une assiette assez solide pour résister itux coups de vent ou au poids de la neige et du givre.

611. Hartig, dont l'expérience est d'un grand poids dans toutes les questions forestières, a reconnu l'impossibilité de maintenir les taillis de hêtre, en suivant les règles ordinaires de l'exploitation des taillis; il propose, en conséquence, un mode parliculier dont voici les détails.

Supposant la forêt de hêtre soumise à une révolulion de 30 ans, il conseille de réserver, à la première exploitation, 100 baliveaux par hectare; à la deuxième, lorsque les souches auront 60 ans, il veut que l'on réserve, par hectare, 2,000 baliveaux. Trente ans plus tard, les souches ayant 90 ans, on doit interrompre le régime du taillis et exploiter d'après la méthode du réensemencement naturel. Les 100 premières réserves étant âgées de 90 ans, el les 2,000 autres de 60 ans, on peut en espérer la semence nécessaire au repeuplement du terrain. On doit alors effectuer les trois coupes de régénération, dont l'effet sera de créer une jeune forèt qui rem-

vigétation moins promple, et les bourgeons trouvent moyen de perforer lentement l'écorce. "Ce qui confirmait Hartig dans son opinion, c'est qu'il avait remarqué que, dans les bons sols, le hêtre repousse mieux lorsqu'il est coupé en temps de séve, c'est-à-dire, lorsque les souches ont perdu une partie de la séve surabondante. 
placera les anciennes souches et yui pourra de noureau ètre traitce en taillis, sauf à être régénérée comme il vient d'être dit.

Hartig ne fait mention que d'un taillis de hètre qui r’a jamais été exploité, et qui, à la première coupe, ne présente que des souches de 30 ans. Lne pareille forèt se rencontre rarement; il est plus ordinaire d'en trouver qui ont déjà été exploitées pendant ruelques révolutions et où les souches sont d'àge: très différents. Dinns ce cas, il faudra donc réserver de suite 2000 perches par hectare. Ce nombre, toutefois, ur doit pas être considéré comme invariahle; il dépend de la grosseur des perches et de la quantité d'arbres antérieurement réservés. $11 \mathrm{ne}$ s'agit, en effet, que d'établir un massif convenable, qui puisse produire le repeuplement naturel aussilòt que les perches seronl assez àgées pour porter semence.

612. En réfléchissant aux moyens jugés indispensables pour assurer la durée du hètre en taillis, on est nécessairement amené à conclure que ce mode d'exploitation ne lui convient pas et qu'on lui fait violence en l'y soumettant.

Si l'on examine avec attention les taillis de hètre, surtout ceux dont les souches sont un peu anciennes, on y trouvera toujours des clairières plus ou moins considérables, causées par la mort d'une partie de ces souches; si les clairières se remplissent, ce ser: par des essences à semences légères, le plus souvent 
par les bois blancs, à moins que des repeuplements artificiels n'y mettent obstacle. C'est ainsi que le hêtre est peu à peu dépossédé du terrain où antérieurement il dominait.

Les moyens proposés par Hartig sont sams doute suffisants pour prévenir un tel résultat; mais ils manquent de simplicité et sont peu avantageux, à cause de la suppression presque totale des produits de la deuxième révolution. En effet: couper d'abord afin d'obtenir des rejets ; puis, dès la seconde révolution, laisser sur pied li plus grande partie de ces rejets pour en former des arbres à semence; exploiter ensuite d'après la méthode du réensemencement naturel, et ne reprendre enfin le régime du taillis que lorsque la semence aura créé de nouveau une jeune forèt, tels sont ces moyens. Il suffit de les résumer pour montrer que ce n'est qu'à l'aide d'opérations compliquées et en sacrifiant le rapport soutenu, que l'on parvient à exploiter d'une manière intermittente le hêtre en taillis, tandis qu'on perpétue facilement cette essence par la méthode du réensemencement naturel, méthode dont les règles sont faciles à suivre, et par laquelle on obtient d'ailleurs, dans un temps donné, des produits en matière plus considérables et plus utiles. Toutefois, lorsque les circonstances ne permettront point de traiter les forêts de hêtre en futaie, le mode du taillis composé devra toujours ètre préféré, comme favorisant davantage la reproduction par la semence. 
613. Dans l'ancien Morvan (départements de lit Nièvre et de Saône-et-Loire) et dans plusieurs autres contrées de la France, on a adopté, très anciennement, dans les taillis de hêtre, un mode d'exploitation particulier, dont l'efficacité, en ce qui concerne du moins la reproduction des souches, est démontrée par une longue expérience. Ce mode, connu sous le nom de furetage, consiste à n'abattre, de chaque cépée, que les plus grosses perches propres à être converties en bois de corde, et à réserver soigneusement les autres. A la place des perches coupées, naissent de nouveaux rejets qui prospèrent sous le couvert des tiges conservées, jusqu'iu moment où celles-ci, ayant atteint liı grosseur qui les rend exploitables, sont coupées et remplacées à leur tour. Les souches des tiillis furetés présentent ainsi des bois de deux et mème de trois àges; jamais plles ne sont entièrement dépouillées, et c’est, à ce qu'il parait, cette dernière circonstance qui assure leur reproduction jusqu'à un àge très avancé.

Ordinairement ces taillis sont soumis à une révolution de 24 à 30 ans, et, selon que les souches portent des rejets de deux ou de trois ìges, les coupes viennent deux ou trois fois en tour d'exploitation dans la mème révolution; ainsi, par exemple, un taillis exploité à 30 ans peut ètre fureté tous les 10 ans, de manière qu'il y ait sur les souches des rejets de 10 , de 20 et de 30 ans.

Comme on n'a pas l'habitude de récerver des 
baliveaux, et que les perches du hallis sont abaltues lrop jeunes pour porter graine, les essences paritsites el les plantes nuisibles gagnent peu à jeu sur le hètre. Aussi est-il indispensable, pour entretenir ces lorêts, de remplacer les souches dépérissantes par des repeuplements artificiels.

11 poraitquin très grand inconvénient de ce mod. consiste dius les dégàts considéribles que l'abation. .t le litconnage des perches exploilibbles causent it celles qui, ne l'étant pals encore, doivent rester sur pied. Peut-être y antrit-il mọen de remédire an nit, au moins partiellement.

(Luoi quil en soit, nous pensons, d'après lous le: reuseignements que nous arons recueillis, que, si lr furetage peut se justifier dans les taillis simples d. hètre, en raison de la difficulté d’obtenir une reproduction assurée far la coupe à blane éloc, ce mode? d'exploitation ne préspute cependant pas assez d'alintages, of offre trop d'inconvénients, pour qu'on l'applique à d'autres essences.

ARTICIF: III.

Exploitation du châtaignier en taillis.

(i) 4. Lal souche du chataignier a uns longur durée, el les cépées qu'elle produit sont bien fournies al d'une rroissumer vigomense ol rapide. Cest sur-

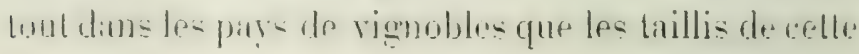


issence sont ilvantigeux, it cause des cercles de futaille et des échalas quiils fournissent el qui sont de première qualité.

Les baliveaux sont d'autimt moins à conseiller. dans les taillis de chàtaignier, que leur couvert est ipais et que les rejets supportent diffirilement d'itre Iominés. Les perches de 10 à $1 \%$ ans fournissent "lailleur's déjà des fruits; les réserves. sous le rapport du repeuplement naturel, ne sont done pas nécessitires. On peut, toutefois. en ronserver sur les lisières du taillis, affin d'obtenir quelques torte- pièceut d'augmenter lit réeolte des chataignes.

Dans les anciens départements du Rhin, où il existe heaucoup de taillis de chataignier, on a l'habitude. après l'exploitation, de cultirer In terrain, mulre les souches, pendint un ou deux ans. Opdinairement on y plante des pommes de tere. Gutre la rirolte que cette opération produil, folle is le grand avalllage d'activer considérablement la réccetation derépées en débarrassant le chàtaignier des arbu-te"l des morls-bois yui. romme on le siril, hui muisent beaucoup.

I I T I C. I, I: I I.

Exploitation de l'aune en taillis.

(i1:). Le régime du laillis est celui qui convient

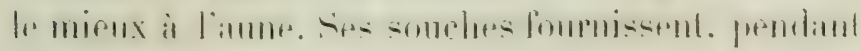
plus diun sirele, des rejets abondants of d'une 
croissance rapide, et l'aune blane drageonne mème beaucoup.

En général, les baliveaux qu'on réserve dans les aumaies, pour fournir de la semence, ne remplissent pas le but qu'on se propose.

Les aunes se trouvent d'ordinaire ou dans les mirais, ou dans les sols aquatiques, ou enfin dans des lieux très humides. Dans les deux premiers cas, la germination est entravée; dins le dernier, le terrain est tellement gazonné ou rempli de joncs et d'autres plintes nuisibles, que la graine souvent ne peut y arriver. Il ne faut done pas compter sur le semis naturel pour l'entretien du taillis; le seul moyen de repeuplement, lorsque les drageons ne réussissent pas, consiste dans la plantation, et, pour l'aune blane, dans les boutures '.

On doit cependant réserver quelques baliveaux, mais dans le seul but d'élever quelques arbres utiles aux constructions hydrauliques et à d'autres usages.

Lorsque les aunaies se trouvent placées dans des marais d'une certaine profondeur, il devient impossible de les exploiter autrement qu'en hiver, pendant les fortes gelées, quels que soient d'ailleurs les incomvénients qui peuvent en résulter pour la reproduction.

1 Voir le Vle livre de ce cours. 


\section{Exploitation du robinier faux acacia en taillis.}

616. Le robinier est une des essences les plus propres au taillis, lorsque le sol et le climat lui conviennent. En effet, non-seulement il croit aree. une rapidilé prodigieuse 1, mais il drageonne abondamment et son bois acquiert des les premières années un grain très serré.

Un inconvénient assez grave des taillis de robinier. c'est le grand nombre des épines qui garnissent les tiges; la blessure de ces épines est fort douloureuse el présente mème souyent, dit-on, quelque danger. Cette circonstance rend l'exploitation de ces taillis difficile, et beaucoup plus coùteuse, par conséquent. que celles des autres.

Ouant aux baliveaux, il n'est pas à conseiller d'en réserver. La tête du robinier est rameuse et très sujette à être déchirée par les vents; il n'est donc pas prudent de l'isoler sur le taillis.

' Dans un sol substantiel et l'rais, les rejets s'élancent souvent dès la première année à 3 et mème à 4 mètres de haut, et, à l'âge de 5 ou 6 ans, les perches mesurent jusqu'à 8 et 10 centimètres de diamètre à un mètre de terre. 
ARTILLE VI.

\section{Exploitation des taillis mélangés}

617. A quelques exceptions près, les essence: dont nous venons d'eximiner, dans les cinq articles précédents, l'exploitation en taillis, se trouvent. comme tous les autres arbres feuillus, ordinairement mélangées dans les taillis et y dominent plus ou moins, suivint que le climal et le sol conviennent davantage aux unes ou aux autres. Le chène ef lo hêtre se rencontrent rarement sitns mélange d'autres escences ol. lorsque ce mélange est établi dande justes proportions, il est loin d'ètre contraire is lit bonne croissince de nos essences d'élite. surtout de celles dont il importe de composer la réserve. 11 en est ainsi, par exemple, du chène pédonculé, lir plus précieuse de toutes au point de vue des grandmplois. Cel arbre, propre aux plaines fertiles ou humides, ne peut se maintenir à l'état pur dans lelaillic, a ruuse du couvert incomplet de sa cime 82 j: il lui conrient don que son pied soit environné d'essences secondaires 391 , qui, tout en protégeant lo -ol. ne souffrent d'ailleurs que trés peu du couvert. I.es plus belles et les meilleures pieces de chène que fournissent les taillis se remeontrent dans de telles conditions. 
Les ormes. les frènes, les érables. Les charmes of les tilleuls se reproduisent très abondanment de souche pendant 100 à 150 ans; les deux premiers ont une disposition marquée à drageonner, et lecharmes et les tilleuls, qui se garnissent de nombreux rejets au pied, se perpétuent ficilement de cette maniere, lorsque lin souche-mire vient ì mourir.

Les bouleaux. les alisier's, les sorbier's, les merisiers et les micocouliers fournissent des rejets jusqu'à 60 et 80 ans, et les saules et les trembles. dont le pied est de moindre durée. drageonnent considérnblement.

En général, le nélange des deux derniers parfoiaussi celui du tilleul avec les essences dures neat pas favorable, et, Jorsqu'il existe, il importe de la: surveiller ( 399$)$, alin d'empecher que ces essences no soient surmontées, et finalement évincépespar les boiblanes. Ce rlest que par des nelloiements frérquemment répétés que l'on peut maintenir un mélangr convenable.

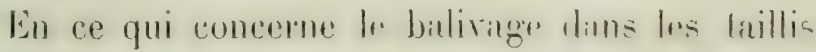
melingés, simples an composés, hules les indiralions nécessaires ont itr donnese plus hanl sond ลे ร996]. 
ARTICLE VII.

\section{Exploitation des taillis d'arbrisseaux.}

618. Il faut éviter de mêler les arbrisseaux avec les arbres. Dans les premières années, leur croissance pourrait être égale, mais, plus tard, les arbrisseaux seraient immanquablement surmontés; il ne peut être convenable d'ailleurs de soumettre les uns et les autres à la même révolution.

Ceux des arbrisseaux qui, par la qualité de leur bois et par les usages auxquels ils sont propres, méritent plus particulièrement l'attention du forestier. sont : les petits sanles, le comouiller mâle, le coudrier et le cerisier à grappes. Les deux derniers peuvent avec avantage croître en mélange. Quant au cornouiller mâle, il n'existe guère d'espèce feuillue d'une végétation aussi lente; on ne peut donc le cultiver que seul. Ordinairement on l'exclut des forêts, parce que, mêlé à d'autres essences, il en est écrasé ct, ne pouvant s'élever, il s'étale. Cependant, l'excellente qualité de son bois, sa souplesse, sa dureté devraient lui faire accorder, dans les forèts, une place petite mais exclusive, puisque sa culture ne peut s'allier à celle d'aucune autre essence.

Comme nous l'avons dit [574], c'est pour les taillis d'arbrisseaux qu'il convient d'adopter les révolu- 
tions de ö à 10 ans ; le cornouiller, toutefois, pourrait ètre coupé à un àge un peu plus avancé, vu la lenteur de sa croissance. Exploités ainsi, on les nomme menus-taillis. Il est inutile d'ajouter que ces essences ne sont traitées qu'en taillis simple et, la plupart du lemps, sans aucune réserve. 


\title{
CHAPITRE SIXIEIIE
}

\author{
DU SARTAGE
}

619. In ippelle sartange. un mode particulier dexpoitation des liallis, qui consiste it cultiver des cireales, à charque coupe, pendant un ou deux ans. après aroir brùlé, au préalable. les menus bois, hroussirilles. mort-bois et autres plantes, sur place, dan: In but de rembe le sol plus larorable à la régétation.

C"est principalement dans les Irdennes, dans les pary de Liege et de Laxembourge et sur différents points de l'Allemaque méridionate que celte pralique nis nusige. Lille est extrèmement ancienne el semblo

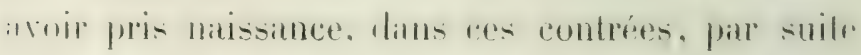

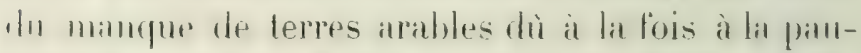
vrete dusol. à si forme accidentée et à l'àpreté du climat.

L.e cheme. particulièrement le rouve, est de loute: lis escences relle qui supporte le mieux In sartager.

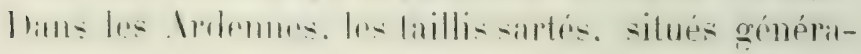


fommut en pente et sur un sol de schiste ardvisier. sont presque exclusirement composés de chène rouvre et, dans ces conditions, fournissent des bois re feu et de charhon excellents, de fort bons bois l'œuvre, quand on fait des réserves, el surtout deécorces de première qualité '. Ces écorees et les cériales formant les produits les plus importants des taillis soumis au sartage, il est de l'intérèt du propriétaire d'exploiter cenx-ci à de courtes révolutions. c'est-à-dire, de 15 à 2.5 ans.

620. Lorsque le sol est nu. dépourvu. entre les cépées, d'herbes et de gazon, l’opération du sartage a lieu de la manière suivante.

Après avoir écorcé sur pied, lor's de la séve du printemps [609], puis abaltu ol vidé le bois de lis manière ordinaire, on répand sur la surface du sol. entre les sonches ixploitées, toutes les menues hranches, brindilles, cimenux et broussailles qui n'ont point fait partie du bois de corde?. Par un

'La qualité des écor'ces est, en général, d'autant meilleure que lo liber a une épaisseur plus cousidérable, comparativement aux couches corticales of à l'ipiderme : car il paraît que lo lannin est contenu surtout dans le liber. Or, cet organe esl

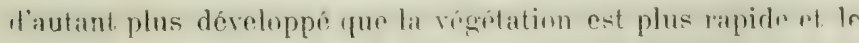
bois plus jeune; ces deux eirconstances se trouvant réunies tans les taillis sartés, on conçoit que les ecorces qu'ils fournissent doivent être de très bonne qualité.

: En général, on ne fait pas de fagots dans les taillis sartés, miis on façome en cordes, soit pour le chauflinere ordinairr. snit pour ètre carbonises (bois do charbonnette). tunt lo bois audessus de 2:i millimètres environ te diamètre. 
temps calme, on y met le feu. La flamme se propage rapidement et convertit en cendres le bois ainsi répandı. Cette mise à feu doit avoir lieu, au plus tard. dans les premiers jours de juillet ${ }^{1}$. Afin de circonscrire le feu dans la coupe en exploitation, on en pioche à la houe le pourtour sur une certaine largreur, immédiatement avant la mise à feu, et l'on dispose des hommes sur tout le périmètre, afin de combattre et de maitriser l'incendie si, par accident, il venait à atteindre les parties voisines. Dans quelques localités de l'Allemagne, on prend même la précaution, lorsque la coupe est grande, de la subdiviser en un certain nombre de parcelles ou lots dont chacune séparément est mise à feu. Les séparations s'établissent, soit en labourant le pourtour de chaque parcelle sur une certaine largeur, soit en réunissant sur ce pourtour des perches de la coupe qui. mises en tas continu, forment un petit rempart ou

Dans l'Udenwald (grand-duché de Hesse-Darmstadi) le délai (le rigueur pour la mise à feu est fixé au 10 juin; dans les Irdennes, au contraire, elle n'a lieu qu'en août et septembre. Il en résulte, évidemment, que les rejets de la première année sout détruits. C'est un retard fàcheux, et il est permis de croire que ce nouveau trouble apporté, dans la végétation des souches altère leur vitalité et compromet leur durée. Mais d'un autre côté, le recru ne se produisant qu'au printemps suivant (quel'fuefois mème dans le second printemps), les rejets naissent dans de meilleures conditions pour résister aux froids de Thiver [376 et 609], ce qui, dans un climat aussi rude, est pentit tre une circonstaner pssentielle à leur réussite (V. note, p. 3'0). 
sentier en relief. Enfin, le périmètre de la coupe entière est encore labouré sur une largeur de plusieurs mètres.

Quand le moment est venu de semer les céréales, on les répand sur le sol, et on les recourre au moyen d'un léger labour à la houe. Si cette opération est effectuée de bonne heure, on peut cultiver du sarrazin la première année et du seigle l'année suivante; mais si, au contraire, la saison est déjà avancée, il faudra se contenter d'une seule récolte de seigle'. Ces céréales devront être coupées à la faucille, en prenant toules les précautions nécessaires pour que les rejets soient respectés.

' Feu N1. de Salomon, directeur de l'Ĺcole forestière, a rapporti, en 1.833, d'une tournéc faite avec ses élères dans l'odenWalls, une céréale, appelée seigle multirunle (secrali cerente multirmele), qui est cultivée avec un très gramd succìs dians los tiillis sitrtés. Ce seigle est bisanmuel ot se seme dans les taillis avec le sarrazin. La première année, il ne s'élève qu'à environ 16 centimètres et on peut le couper, sans lui nuire, en récoltant le sarrazin. La deuxième année, ses tiges, qui sont ordinairement at vingl ou trente sur le mème pied, s'blèvent à plus de 2 metres et donnent des epis très productifs. Son emflui, en mélauge avec le surrazin, présente d'abord l'avantage de procurer deux récoltes au moyen d'une seule culture; en second lieu, on tevite les dangers de la dégradation des sols inclinés, dans lesquels, comme on le sait, les terres ameublies par la culture sont toujours exposées à ètre entrainées flar les caux pluviales. (Voir le Mémoire publié par M. de Sulomon dans le journal de la société d'agriculture de Nancy, lévrier 1836, sur le sartage des taillis de chene de l'Odenwald et sur le seigle multicaule.) 
1.r: mode de siartage que nous venons de décrire. - nomme sarlage il feu courant. Les cendres qui en résultent, ajoutent à la fertilité du sol par les sels qu'elles contiennent, el par la faculté qu'elles onl d'atlirer el de retenir l'humidité de l'air, de manière a ne lit céder que lentement aux pliniles; de plus, il tst incontestable qu'elles sont un puissant stimulant your lit végétation. On conçoit done que, à lit suite du sirtage, les céréales et le bois croissent tous deux avec beaucoup de force. Si l'atchon du feal a quelquefois pour effet de nuire à la reproduction, en détruisint les semences et les jeunes plants et en charbommint partiellement lit surface des souches, elle provoque, d'une autre part, des rejets en abondance of singulicrement vigoureux, par la lempérature élevée qu'elle communique au sol. Enfin, un dernies arantage du sartage est l’abri que los céréales prorurent aux jeunes rejels 1 .

1 C'est contre les rents froids, bien plus que contre les ardenrs du soleil, qu'il importe d'abriter les rejets, dans le. deux premières années. Dans les Ardennes, on avait coutume antrefois de réserver, aulour de chaque coupe, un cordon de lintaie qui répondait à ce besoin et offrait d'ailleurs des ressnurces d'autant plus précieuses que le sartage à fou courant ne permet guère de réserver des baliveaux dans la coupe mème. Pru à peu, ces cordons ont été exploités; on a négligé de les remplacer ot, depuis lors, on a remarqué, principalement sur les plateaux, que la reproduction des taillis élait de plus en flus compromise. On n'hésite pas à attribuer à cette circonlance, jointe aux uluus du pâturage, la dégradation considerable.

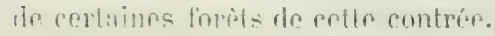


6521. Il existe encore un antre mode de sartage. dit it feu counert, anquel on a recours quand le sol est garni d'herbes, de plantes rampantes et surtout de gazon. Il consiste à peler, à la houe, liı surface du terrain couverte de gizon et d'inutres plantes, à en former un grind nombre de petits fourneaux que l'on allume et donl on répand ensuite les cendres -ur toute l'aire de la coupe. Inu reste. on procède comme il a été dit.plus haul.

Ce mode ne présente pas les avantages du premier; on en obtient, pour le bois. des rejets Inoins abondants et moins vigoureux, sims doute pitrce que, d'une part, on enlexe en certitins endroits. trop de terre avee le gazon, et que cetle terre. brîlée et calcinée, que l’on répand ensuite arec les cendres. n'est plus propre à la régétation; d'autre part, parre que les ratuines sont quelquefois roupéns ou mutilées ou trop décourertes of gue le sol liest point ígitlement échauffé sur toute sa surface '.

linfin, te sartage à leu couvert devient plus muisible dans les pentrs mapides que relui a teu courant. parce quiil ameublit la terre plus profondément et l'expose rlavinlage à s'ébouler. Il est rrai, toutefois, 'qu'il donue la ficulté de réserver. itrere plus de rhanees de suecice quelques baliveaux dans les

Le sartage ì feu couvert oftre encore un autre danger qui riside dans le repandage des cendres, tunt une pelletée seulement, jetée sur une souche, suffit pour la laire périr, quand l'incinaration du gazon tís. pas complète. 
coupes, attendu qu'on peut en éloigner le feu à volonté, ce qui ne saurait se faire en sartant à feu courant, à moins de recourir à des précautions particulières.

Quel que soit le mode de sartage que l'on emploie, il est à conseiller de le faire suivre de quelques plinliations ou semis, pour assurer la perpétuité des bonnes essences et surtout du chêne. A la vérité, ces travaux semblent moins urgents ici que dans les Laillis ordinaires, à cause de la facilité avec laquelle les rejets, dans les taillis sartés, poussent des racines qui leur sont propres et forment ainsi de nouveaux pieds indépendants de la souche mère. Ce fait, qui est incontestable, tient, sans nul doute, à ce que la terre se trouve ramenée contre les souches et soulevée au niveau de la taille d'abatage par le fait même du labour donné au sol. Toutefois, dans une coupe, il y a toujours beaucoup de places qu'il sera très utile de repeupler artificiellement.

622. Dans les Ardennes, aussitôt après la récolte des céréales, les genêts lèvent en abondance dans les coupes, surtout dans celles qui ont été sartées à feu courant. Ces arbustes étoufferaient donc les semis. En plantant des sujets de $0^{\text {" }}, 50$ à $1^{\text {m }}$ de haul, préalablement préparés en pépinière, et autour desquels on arracherait les genêts dès la première année de leur levée, le succès de l'opération serait à peu près assuré.

Lat prisence des genêts dans les jeunes coupes est 
d'ailleurs ulile aux rejels dans le premier ìge, en ce qu'elle les protège contre les intempéries. En outre, ces arbustes fournissent des produits qui peuvent s'exploiter régulièrement, quelques années après la vidange des bois, et qui sont recherchés surtout pour le chauffage du four. A cel égard, il faut remarquer, toutefois, qu'il peut y avoir du danger à laisser persister les genêts trop longtemps, dans lc but d'en tirer plus de profit, parce que, dans ce cas, les rejets de bois dur, particulierement ceux du chêne, perdent trop tôt leurs branches inférieures. ne prennent pas de corps, faute de lumière, et sont souvent exposés à languir ou à ètre écrisés par la neige, lorsque l'épais fourré de genèt vient à disparaitre. - Il importe donc de faire enlever ceux-ci soit en une fois, soit en plusieurs, 4 ou $\breve{3}$ ans, au plus tard, après la coupe.

623. La pratique du sartige, comme nous l'avons dit, n'est usitée que dans quelques localités; mais elle pourrit etre appliquée avantageusement sur différents points de la France, par les particuliers qui possident des taillis de chène, en évitant, toutefois, d'y soumettre ceux qui sont issis dans des fonds secs et légers ou en pente ripide. 


\section{CHAPITRE SEPTIËIE.}

\section{DE L'ÉTÊTEMENT ET DE L'ÉMONDAGE.}

\section{ARTICLE PREMIER.}

\section{Généralités.}

624. On fait d'un arbre un têtard, lorsqu'on abat sa tige à une certaine hauteur au-dessus du sol; on l'émonde, au contraire, en lui enlevant toutes les branches latérales jusqu'à la partie supérieure de la cime qu'on laisse intacte.

Par suite de l'une ou de l'autre opération, il se présente, au point de la coupe, des rejets que l'on exploite périodiquement à l'instar du taillis. De nouvelles branches se produisent aussi longtemps que la tige conserve sa faculté de reproduction, c'està-dire, jusqu'à ce que l'âge ou une maladie l'en aient privée.

625. Sous le rapport de la valeur des tiges, il importe peu d'en pratiquer l'étêtement à une hau- 
teur plus ou moins grande, car cette opération altère ordinairement le centre du bois, en favorisant l'infiltration des eaux pluviales, et c'est chose rare de voir des têtards vieux, dont la tige ne soit pas creuse. Il n'en est pas de même des arbres émondés; ils se conservent plus longtemps et peuvent être utilement employés, lorsqu'ils ont atteint les dimensions convenables. Cet avantage est dù à ce que, le sommet de l'arbre restant toujours garni, les eaux pluviales glissent sur la partie coupée et pénètrent moins aisément dans l'intérieur du tronc.

Mais, si l'étêtement a l'inconvénient de hàter la perle du tronc, il fournit par contre des rejets plus abondants, plus forts et plus utiles que l'émondage, dont on n'obtient que du fagotage de peu de raleur.

L'étêtement s'opère à une hauteur de 1 jusqu'à 6 et 7 mètres. On tient bas les tètards plantés sur les bords des ruisseaux, des rivières, dans les pentes, etc., pour maintenir les terres; on donne de l'élévation, au contraire, à ceux sous lesquels on cultive des céréales ou des fourrages.

En effet, c'est dans les pàturages, dans les prairies, sur le bord des champs, des routes et des chemins que les arbres émondés et les tètards trouvent plus particulièrement leur place; et l'on concoit, pour ces derniers, que plus ils seront élevés au-dessus du sol, moins ils empècheront les plantes fourragères 
ou autres, qui croissent sous leur couvert, de participer aux influences atmosphériques.

\section{Essences propres à l'étêtement et à l'émondage.}

626. Toutes les essences feuillues peurent ètre étêtées ou émondées, mais elles ne présentent pas toutes les mèmes avantages. Celles qui s'exploitent le plus ordinairement ainsi, sont : les peupliers, les grands saules, le tilleul, l'orme, l'aune, le frêne, les érables, le charme et le chêne.

A R'T ILE III.

Exploitabilité de ces bois.

627. L'exploitabilité que l'on adopte d'ordinaire pour les tètards est de 3 à 6 jusqu’à 10 ans; elle lépend de la nature des produits que l'on veut wbtenir et de l'nccroissement plus ou moins prompt des différentes essences. Ainsi, les saules et les peupliers, qui repoussent avec la plus grande activité, peuvent ètre exploités de 3 à $\breve{a}$ ans, tandis qu il linut retarder l'exploitation des autres essences. II en est absolument de mème pour les arbres soumis à l'émondage. 
APTICLE IV.

Saison la plus convenable pour l'étêtement et pour l'émondage.

628. Mars et avril sont les mois les plus convenables pour l'étêtement et pour l'émondage, sauf les modifications nécessitées par la différence desclimats. Les motifs sont les mêmes que ceux qui ont été donnés pour l'abatige des taillis [ڤ76 et $\because 7 T]$. Il est cependant des pays pauves en lourrages, bii l'on emploie le feuillage des tétards et des arbres émondés à la nourriture des bestiaux. Dans ce cas, on coupe aussitôt aprís lit séve d'atout pour pouvoü mettre encore les feuilles à profit. Celtr raison seule peut justifier la coupe à la fin de l'été.

\section{ARTICLE: V'.}

\section{Mode d'abatage.}

629. Lorsque les lètards sont jeunes encore ou d'un àge moyen, ilest avintageux de couper les rejets rez-trone; mais, lorsqu'ils vieillissent el que l'écorce devient coriace, il vaut mieux couper plus hitut, afin que lés nouvelles pousses trouvent une écorce tendre qu'elles puissent percer avec plus de filcilité. II en est de mème pour les arbres à émonder; les premiers Ahranchements se font à fleur de la lige: plus tard, 
on laisse subsister des bouts de branches pour faciliter la reproduction.

L'emploi d'instruments bien tranchants est essentiel pour cette opération, afin que la tranche soit nette et sans éclats. Pour éviter l'infiltration des pluies, la coupe des têtards doit, autant que possible, itre faite obliquement à l'horizon; celle des arbres it émonder de même, mais en commeneant l'entaille par le bas.

ARTICLE VI.

Avantages des tétards et des arbres émondés.

603. Il est des pays, par exemple dans le voisinage du Rhin, où toutes les terres vagues en parcours, tous les bords des rivières et des ruisseaux sont plantés de têtards et d'arbres émondés. Ces plantations protègent les rives contre l'envihissement des eaux et défendent les habitations et les terres cultivées contre les gliçons que le dégel et les inondations pourraient y porter; soumises à des coupes périodiques, elles fournissent en même temps un chauffage abondint. Les terrains in parcours, surtoul, ítant ainsi plantés, offrent, arec les produits an bois, de l'herbe et des feuilles pour la nourriture des bestiaux.

Les arbres émondés et les titards sont d'un trèsgramel intéprit partout oi lo bois at de lat vileur. Leur 
plantation n'enlève rien à la culture des terres; on en garnit les places improductives, les endroits marécageux, les lisières des prairies et d'autres lieux dont la charrue ne peut approcher; toutes les eaux devraient en être bordées.

Il est à regretter que des plantations aussi utiles, qui s'opèrent avec tant de facilité et qui, sans présenter aucun obstacle à l'agriculture, peuvent offrir les plus grands avantages aux propriétaires, soient encore aussi négligées dans un grand nombre de nos départements. 


\title{
LIVRE CINQUIÈVE
}

DES EXPLOITATIONS DE CONVERSION.

\section{CIIAPITRE PREMIER}

\author{
CONSIDÉRATIONS GÉNÉRALES
}

631. Les exploitations de conversion ont pour but d'introduire dans les forèts une autre méthode de culture que celle qui, jusqu'alors, y était pratiquée. A cet effet, elles doivent changer l'état du bois, et ce changement doit ètre consommé dans un temps donné.

Or, nous connaissons trois principales méthodes de culture, celle de la futrie, celle du taillis simple et celle du tirillis composé ${ }^{\text {. On }}$ peut donc concevoir les conversions suivantes :

' Nous ne comptons pas le jardinage au nombre des méthordes dexploitation rque, dans certaines circonstances, il pour- 
$1^{\circ}$ Conversion d'une fulaie en taillis simple ou en taillis composé ;

$2^{\circ}$ Conversion d'un taillis simple en taillis composé ou en futaie ;

$3^{\circ}$ Conversion d'un taillis composé en taillis simple ou en futaie.

Les règles culturales pour exécuter ces différentes opérations forment l'objet de ce livre; mais, pour les appliquer avec discernement, il faut ètre fixé sur le cas où un changement de régime devient réellement nécessaire ou utile. Cette question, qui n'est pas seulement forestière, mais qui touche aussi à l'économie politique, a été débattue à plusieurs reprises et décidée en sens divers. Il nous a semblé, toutefois, que les données nécessaires pour lit résoudre n'avaient pas toujours été bien nettement élablies, convenablement ripprochées ot discutées. Nous nous proposons done de l'examiner dans ce qui va suivre, parce que nous la considérons comme devant dominer toute la théorie des conversions.

rait être utile d'introduire, parce que ce n'est qu'un mode particulier d'exploiter certaines forèts soumises au régime de la futaie. Nous ne parlous pas non plus, sous ce rapport, du sti'tuye', de l'etitement et de l'émonduyp. Les deux derniers modes, conme on le sait, conviennent dans les terres cultivées, dans les prés et dins les pâturages, bien plutôt que dans les forèts, et l'autre, qui ne se pratique que dans quelques localités, n'est d'ailleurs qu'un taillis simple, plus la culture des céréales et l'écobuage. 


\title{
CHAPITRE DEUXIËME.
}

\author{
EXAMEN COMIPARÉ: DES TROIS PRINCIPALES \\ MÉTHODES D'EXPLOITATION.
}

ARTICLE PREMIER.

Énoncé de la question et données employées pour sa solution.

632. La question que nous venons d'indiquer dans le précédent chapitre peut se formuler ainsi :

Quelle est la méthode d'exploitation qui mérite la préférence, eu égard aux besoins de la consommation. aux intérêts du propriétaire et ì la nature des lieux?

Pour la résoudre, nous examinerons successivemont les trois méthodes d'exploitation sous les rapports suivants :

$1^{\circ}$ Quantité de produits en matière que chacune procure dans un temps donné;

$2^{\circ}$ Qualité de ces produits;

3० Rerenu qui en résulte pour Ie propriétitire: 
DES EXPLOITATIONS DE CONVERSION.

$4^{\circ}$ Enfin, influence de chaque méthode sur la ferLilité du sol ${ }^{2}$.

IRTICLE II.

De la quantité des produits en matière.

633. Dans le second livre de ce cours, nous avons démontré que, pour obtenir dans un temps donné les produits matériels les plus considérables, il faut couper le bois lorsqu'il a atteint son plus grand accroissement moyen [406]. Si done il pouvait ètre prouvé que le plus grand accroissement moyen des futaies est plus considérable que celui des taillis. il serait prouvé en mème temps que ceux-ci doirenl produire moins de matière que les autres.

Or, ce fait est établi par les expériences de tous les forestiers qui se sont occupés de recherches ì son sujet, et les auteurs francais, comme les allemands, ont fourni de nombreuses preuves à l'appui ${ }^{2}$.

IIartig, par exemple, ayant comparé entre eux (toutes itutres circonstances égales d'ailleurs) un taillis simple exploité à 30 ans et une futaie soumise it une récolution de 120 , a trouré que les produits en matière de ces deux forèts étaient dims le rapport de

1 Voir l'Appendice.

"Voyez Varenne de Fenille, de Perthuis, Coutta, Hartig et antres, 
4 à 7 . On conçoit, d'ailleurs, que ce rapport se modifie, d'abord en raison des révolutions attribuées à chaque régime et qui peuvent ètre plus ou moins favorables à la production en matière; en second lieu, selon les essences, dont les unes croissent rapidement dans leur jeunesse, mais se ralentissent de bonne heure, tandis que les autres font peu de progrès d'abord, mais ont un accroissement plus fort et plus soutenu à un àge avancé.

Sans prétendre donc exprimer, par un chiffre constant, le rapport entre les produits matériels des futaies el ceux des taillis, on peut cependant affirmer d'une manière générale: que le volume fourni par les futaies, dans un temps donné, est toujours plus grand que celui des taillis simples fourni dans le mème temps, quelque révolution que l'on adopte, pour l'une ou l'autre de ces forêts, dans les limites tracées par les exigences de la végétation et les intérêts du propriétaire.

634. La supériorité de la futaie sur le taillis simple étant établie quant à la production en volume, il sera facile de fixer la position du taillis composé à cet égard. En effet, dans ce genre de forêt, une partie du terrain est occupée par des arbres qui atteignent un développement analogue ì celui de lit futaie, l'autre partic est couverte par le taillis proprement dit; le produit matériel d'une telle combinaison doil done évidemment ètre inférieur à celui 
de lat futaie, el supérieur.au contraire au produit du taillis simple.

A RTICLE III.

De la qualité des produits matériels.

(ij).̈. Lail qualité des produits en matière est relative à l'emploi auquel on les destine. On distingue deux principaux genres d'emploi, savoir :

$1^{\circ}$ Le bois de feu (chauffage, charbon);

$2^{\circ}$ Le bois d'œurre (construction, travail).

Chacun de ces emplois exige des qualités particulières qui, la plupart du temps, ne se trouvent pas réunies dans le mème individu; ainsi le meilleur bois de construction n'est pas toujours le meilleur bois de chauffage, et vice versa.

636. Bors de FEu. - Les qualités du bois de feu doivent être de brùler facilement, d'une manière égale, sans trop de promptitude ni trop de lenteur, et de fournir, pour un volume donné, lit plus grande somme de chaleur.

Or, les expériences liaites à ce sujet, el vérifiées d'ailleurs par la pratique, tendent à établir que les bois possèdent ces différentes qualités au plus haut degré vers l'époque de leur plus grand accroissement annuel qui, comme on le sait (v. $\$ 406$, note), correspond à peu près à l'àge de $60,80,90$ ou 100 ins pour les bois durs, et à celui de 30,40 ou 20 ans pour les bois blanes. Én deça de ces époques, le 
bois a moins de puissance calorifique; au delà, il ne paraît plus gagner sous ce rapport, et il devient d'une combustion plus difficile et plus lente à mesure que les tissus s'altèrent par l'effet du dépérissement. II est à observer, toutefois, que les bois crûs sur souches atteignẻnt, plus tôt que les brins de semence, le maximum de leur puissance calorifique, el qu'ils y parviennent d'autant plus promptement que les souches sont plus àgées; en général, c'est de 25 à 30 et jusqu'à 40 ans que ce maximum se présente dans les taillis.

Lors donc qu'il ne s'agira que de bois de feu, les taillis pourront fournir des produits aussi utiles que les futaies. Il est incontestable même que le bois des taillis sera, sous ce rapport, meilleur que celui des futaies soumises à de longues révolutions; mais il est non moins vrai, d'un autre côté, que les futaies dont la révolution ne dépassera pas l'époque du plus grand accroissement moyen, ou s'arrêtera même un peu en deçà, produiront une qualité de bois sinon supérieure, au moins égale à celle des taillis *

In trouve la preuve du fait que nous avançons dans les laillis mêmes, où le bois le plus recherché pour le chauffage est cclui des buliveaux modernes, c'est-à-dire, d'arbres qui ont ordinairement de 50 à 80 ans.

Quant au charbon, il est certain (malgré l'opinion contraire répandue à cetégard) que celui que l'on obtient de brins de semence d'îge moyen, est d'une qualité au moins égale, sinon supérieure, à celle du charbon fourni par les bois taillis par- 
637. Bors d'oeuvre. - Quant à l'emploi des bois pour les constructions civiles et navales, ainsi que pour les divers ouvrages de fente, etc., il n'y a point de parallèle à établir entre les futaies et les taillis simples, ceux-ci n'étant point destinés à produire en général des bois de cette nature. Il ne reste donc qu'à comparer les arbres élevés dans les taillis composés avec ceux qui ont crû dans les futaies traitées par des éclaircies.

Les qualités que doit présenter un bois d'œuvre sont : la force et l'élasticité. Or, l'expérience prouve que ces propriétés s'acquièrent au plus haut degré sous l'influence des agents atmosphériques, à mesure que la substance ligneuse prend plus de densité, c'est-à-dire, avec l'àge. Un bois d'œuvre doil

venus à maturité. Kursten, dont les travaux sont connus et appréciés de tous les métallurgistes, donne les quantités suivantes de charbon, obtenues de 100 parties de bois (en poids), par la méthode ordinaire de carbonisation.

\begin{tabular}{|c|c|c|c|c|}
\hline Jeune chêne. & & & & \\
\hline Vieux idem. & & & & \\
\hline Jcune hêtre. & . & & & \\
\hline Vieux idem. & . & & & \\
\hline Jeune charme. & . & • & & \\
\hline Vicux idem. & . & . & 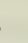 & . \\
\hline
\end{tabular}

Des maîtres de forges du Bas-Rhin et de la Meuse ont lail, sur cet objet, de nombreuses expériences en grand,et ont obtenu des résultats analogues à ceux de Karsten. Pfeil, dans son Traité de culture forestiere (1839), indique, parmi les différents genres d'utilité de la futaic, celui de fournir de très bons bois de charbon aux usines métallurgiques. 
offrir, en outre, des dimensions convenables en longueur et en grosseur, et être exempt de tous défauts ' qui le rendraient impropre à sa destination, et diminueraient beaucoup, par conséquent, sa valeur.

Nos naturalistes les plus distingués, les Réaumur. les Buffon, les Duhamel, ont successivement entrepris des recherches étendues en vue de déterminer le mérite, au point de vue des besoins en bois d'œuvre, des arbres élevés sur les taillis. Tous trois ont déclaré que les baliveaux ne répondaient guères à ces besoins, et ils ont conclu que les futaies, éclaircies périodiquement, pouvaient seules y satisfaire. Cependant, des praticiens justement appréciés, tels que de Perthuis et autres, ont combattu ces opinions, en ce qu'elles avaient de trop exclusif, et nous pensons que ce n'est pas sans raison.

On ne saurait, en effet, refuser aux baliveaux (surtout à ceux qui ont été élevés sur des taillis soumis à de longues révolutions) un degré réel d'utilité pour les constructions et l'industrie; il est mème incontestable, qu'en raison de l'état libre dans lequel ils se trouvent placés à chaque coupe de taillis, leur bois acquiert plus de densité que celui des arbres qui croitraient dans un massif trop serré. Mais il faut reconnaitre aussi que, par leur isolement et par

' Voir l'ouvrage déjà cité de II. Nanquelte, intitulé: Exploitation, débit et estimation des bois, pages 229 à 257 . 
leur extension considérable en branches, ces réserves sont exposées à une foule d'accidents météoriques qui leur causent trop souvent des défauts majeurs. D'un autre còté, elles deviennent toujours plus ou moins noueuses et perdent, par conséquent, de leur force, de leur élasticité et de leur qualité pour la fente; enfin, elles n'acquièrent jamais un fùt ni aussi élevé, ni aussi rond et droit que l'arbre venu en massif.

Si l'on compare ces bois à ceux qui ont crù dans les futaies traitées par éclaircies, il ne peut rester en conséquence aucun doute sur la supériorité des derniers. Ceux-ci, par l'étal de massif dans lequel ils sont maintenus, prennent une hauteur sous branches plus que double de celle des baliveaux, et l'appui mutuel qu'ils se prêtent contre les intempéries les exemple, presque toujours, des vices si communs aux arbres isolés. Les éclaircies périodiques ayant pour effet de distribuer l'air et la lumière dans une juste proportion, par l'espacement graduel des tiges, il en résulte un bois d'une texture plus homogène, sinon plus forte et d'une densité plus égale, sinon auksi grande.

AR'TICLE IV.

Du revenu.

638. Dańs les deux précédents articles, nous arons démontré : 
1. Que les futaies fournissent, dans un temps donné, des produits matériels plus élevés que les taillis simples et les taillis composés, et que ces derniers, sous ce rapport, sont intermédiaires entre les premiers et les seconds;

$2^{\circ}$ Que le bois des futaies est généralement d'une qualité supérieure, sauf celui qui est employé au chauffage; pour cet usage, le bois des taillis peut ètre préférable, mais dans quelques cas seulement.

Or, la valeur des produits étant nécessairement en raison de leur quantité et de leur qualité, il est évident que le revenu des futaies doit être plus grand que celui des taillis simples et des taillis composés; loutes choses étant égales d'ailleurs à l'égard du sol, du climat et des essences, et, par la mème raison, le revenu des taillis sous futaie doit, à son tour, ìtre plus élevé que celui des taillis simples.

639. Cependant, cette supériorité du revenu ne saurait être recherchée que par un propriétaire tel que l'État, par' exemple, qui ne périt point et qui, à cause de sa perpétuité même, doit considérer les forêts comme un puissant élément de prospérité publique et comme une source constante de revenus qu'il importe de rendre aussi féconde que possible. A ce dernier point de rue, la futaie convient également aux communes.

Pour le propriétaire particulier, au contraire, une forèt est un capital qu'il conservera sous sal forme actuelle, s'il lui parait convenablement placé, ou dont 
il changera l'emploi, en tout ou en partie, si cette opération doit lui procurer des bénéfices. Or, la mesure de ces bénéfices se trouve exactement donnée par le taux de placement du capital producteur; et comme la partie de ce capital comprise dans la superficie " peut être constituée très diversement, suivant l'àge auquel les bois sont exploités [ 410 bis], c'est surtout dans le rapport qui existe entre le revenu d'une forêt et la valeur de toute la superficie qu'il faut chercher la solution du problème qui nous occupe.

Ce problème peut donc se formuler ainsi :

Quel est le mode d'exploitation qui, proportionnellement au capital superficiel, produit le revenu le plus élevé?

Nous examinerons premièrement les futaies à ce

1 En langage forestier, on nomme superfiri: l'ensemble de: bois qui couvrent une etendue donnée de foret, par opposition avec le sol ou le fonds, pris abstractivement des végétaux c[u'i] nourr̈it. Lors done qu’il s'agit, comme ici, de considerer unc forêt au point de vue de lia spéculation, on est amenć à distinguer:

Le capital superficiel, ou la valeur des bois sur pied;

Le cupital funcier, ou lit valeur du fonds de terre proprement dit ;

Le cupitul prolucteur, c'est-à-rlire, la valeur réunic du fondet de la superficie, augmentée de la somme capable de fournir, en intérêts, les frais de garde, d'entretien et d'impôt.

Et enfin,

Le revenu, ou la valeur du bois parvenu au terme d'exploitabilité. 
point de vue, en recherchant si l'intérèt particulier permet de les conserver, ou si, au contraire, il ne conseille pas de les dénaturer et d'en placer la valeur d'une autre manière '. Les faits que constateri cette recherche nous serviront ensuite pour considérer, à ce mème point de vue, les taillis simples et les taillis composés.

640. Afin de rendre notre argumentation plus simple, servons-nous d'un exemple et choisissons-le tel, qu'il présenteles circonstances les plus favorables à la production d'un revenu soutenu, et, par conséquent, à l'intérêt du propriétaire.

Soit une futaie de chène, d'une contenance de 140 hectares, située sous un climat favorable, et dans un sol de qualité moyenne; soit son peuplement entièrement normal [415], et la révolution de 140 ans; soit enfin le commencement des éclaircies périodiques fixé à 20 ans, et leur marche réglée de 20 en 20 années.

Dans cette forèt, que nous pouvons nous représenter partagée en quatorze affectations décennales

- Dans le deuxième livre [ 410 bis], il a été établi que ce sont lis courtes révolutions qui profitent le plus à l'intérèt privé; il nous suffirait done de nous appuyer sur ce qui a été dit à cet égard, pour conclure que les taillis seuls peuvent être possérlés avec avantage par les particuliers. Mais comme, dans le paragraphe précité, on a dû se borner à traiter cet objet d'une manière tout à fait générale et succincte, sans entrer dans les léveloppements qu'il comporte, nous croyons qu'il ne sera pas inutile de l'examiner de nouveau ici, vu son importance. 
de 10 hectares chacune, le produit annuel se composera :

$1^{\circ}$ De la coupe d'un hectare de chènes parvenus à l'âge d'exploitabilité ${ }^{1}$;

$2^{\circ}$ De 6 hectares d'éclaircies périodiques à faire dans les bois âgés de $120,100,80,60$, 40, et 20 ans.

D'après les calculs et les expériences de la plupart des auteurs allemands, le produit des éclaircies périodiques, dans une futaie normale, est au produit principal, comme 1 est à 4 ou à 5 ; au contraire, les auteurs français ont établi que ce rapportétait comme 1 est à 2.

Bien que, selon nous, les données des forestiers allemands se rapprochent davantage de la vérité, nous admettrons néanmoins celles de nos auteurs nationaux, afin de nous placer sur le terrain le plus favorable à l'intérêt privé ?. Dans cette hypothèse, le produit matériel de la forêt proposée équivaudra donc annuellement à celui de 1 hectare 50 ares de futaie exploitable.

Or, pour apprécier ce produit annuel par rapport au capital superficiel, il est nécessaire de se rendre compte de la composition de celui-ci.

641. Le tableau ci-après fait connaître le volume

' Pour plus de facilité dans les caleuls, nous exprimons ici la possibilité des coupes de régénération par contenumre.

${ }^{2}$ Il est evident, en effet, que faire le revenu annuel plus grand, quand le capital reste le mème, c'est améliorer les conditions de placement. 
des bois existant sur l'hectare moyen de chaque affectation décennale et, par suite, le volume total de la forèt. Les données contenues dans la quatrième et la cinquième colonne, et qui ont servi à former la sixième et la septième, ont été puisées dans des tables que l'on doit à Cotta sur l'accroissement des bois dans les futaies régulières '. Nous pensons que, pour le climat de la plus grande partie de la France, ces données sont loin d'être assez élevées; cependant nous n'hésitons pas à nous en servir parce que nous les considérons principalement comme un moyen de rendre plus clairement nos idées, et que, d'ailleurs, les conclusions que nous nous proposons de tirer des faits qu'elles constatent ne seraient que plus frappantes encore, dans la supposition d'une végétation plus active.

1 Ces tables, qui sont généralement estimées en Allemagne, ont été converties en mesures françaises par feu M. de Salomon. directeur de l'École forestière, et publiées par lui à la suite de son Traité de l'aménagement des forêts. 
DE CONVERSION.

\begin{tabular}{|c|c|c|c|c|c|c|}
\hline $\begin{array}{l}\text { Affec- } \\
\text { tations } \\
\text { llécent- } \\
\text { nales. } \\
1 .\end{array}$ & $\begin{array}{c}\text { AGE DI } \\
\begin{array}{c}\text { sur l'hect. } \\
\text { le plus } \\
\text { jeune. } \\
2 .\end{array}\end{array}$ & 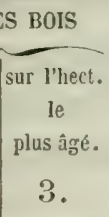 & $\frac{\text { Volunis A }}{\begin{array}{c}\text { de l'hectare } \\
\text { le } \\
\text { pius jeune. } \\
4 .\end{array}}$ & $\frac{\text { ABSOLU (1) }}{\begin{array}{c}\text { de l'hectare } \\
\text { le } \\
\text { plus âgé. } \\
5 .\end{array}}$ & \begin{tabular}{|c|} 
voluse \\
absolu \\
de \\
l'hectare \\
moyen. \\
6.
\end{tabular} & $\begin{array}{c}\text { VOLUME } \\
\text { absulu } \\
\text { de toute } \\
\text { l'affectation } \\
\text { décennale. } \\
7 .\end{array}$ \\
\hline $14^{\mathrm{e}}$ & $\begin{array}{c}\text { ans. } \\
1\end{array}$ & $\begin{array}{l}\text { ans. } \\
10\end{array}$ & $\begin{array}{l}\begin{array}{l}\text { mèt. cubl. } \\
0^{(2)}\end{array}\end{array}$ & $\begin{array}{l}\text { mèt. cub. } \\
14\end{array}$ & $\begin{array}{c}\text { mèt. cub. } \\
7\end{array}$ & $\begin{array}{r}\text { mèt. cub. } \\
70\end{array}$ \\
\hline $13^{e}$ & 11 & 20 & 16 & 28 & 22 & 220 \\
\hline $12^{\circ}$ & 21 & 30 & 30 & 54 & 42 & 420 \\
\hline $11^{e}$ & 31 & 40 & 55,5 & 74,5 & 65 & 650 \\
\hline $10^{\circ}$ & 41 & 50 & 77 & 103 & 90 & 900 \\
\hline $9^{e}$ & 51 & 60 & 105,5 & $130 . \bar{J}$ & 118 & 1180 \\
\hline 8 & 61 & 70 & 133 & 161 & 147 & 1.170 \\
\hline 7 & 71 & 80 & 163,5 & 192,5 & 178 & 1780 \\
\hline $6^{e}$ & 81 & 90 & 195,5 & 226,5 & 211 & 2110 \\
\hline $5^{\mathrm{e}}$ & 91 & 100 & 229,5 & 260,5 & 245 & 2450 \\
\hline $4 \mathrm{e}$ & 101 & 110 & 264,5 & 295,5 & 280 & 2800 \\
\hline $3^{e}$ & 111 & 120 & 299 & 329 & 314 & 3140 \\
\hline $2^{\mathrm{e}}$ & 121 & 130 & 332 & 360 & 346 & 3460 \\
\hline $1^{r c}$ & 131 & 140 & 362,5 & 389,5 & 376 & 3760 \\
\hline \multicolumn{6}{|c|}{ Volume total de la forêt. . . . } & 24410 \\
\hline
\end{tabular}

- On entend par colume clsolu ou réel, le volume plein, sans ancun interstice.

: On pourrait objecter qu'un hectare peuplé de bois d'un an 
642. On voit, par ce tableau, que le produit d'un hectare parvenu à l'àge d'exploitabilité (140 ans) est de 389,5 metres cubes; le produit annuel de notre forêt sera donc, coupes de régénération et éclaircies périodiques réunies :

$$
389^{\text {m. с. }}, 5 \times 1^{\text {h. }}, 50^{\text {a. }}=584^{\text {m. c. }}, 250
$$

On voit, de plus, que le volume en bois nécessaire pour assurer ce produit annuel d'une manière soutenue, est de 24410 mètres cubes, c'est-à-dire que le volume superficiel est environ quarante-deux fois plus grand que le revenu ou, ce qui est la même chose, que le placement en matière est fait au taux de deux et un tier's pour cent.

Ce simple rapprochement fait prévoir que, quand un propriétaire aura l'occasion de placer ses capitaux à ว̆ ou à 4 pour cent, ce qui est très admissible dans les affaires, la destruction de sa futaie lui présentera une spéculation aussi lucrative que facile à exécuter.

En effet, supposons qu'ayant l'emploi de ses fonds à 4 pour cent, il se décide à abattre sa forêt, moins les bois de 1 à 20 ans, trop jeunes pour être livrés au commerce avec avantage; il réalisera la presque totalité de la superficie, augmentera par suite ses

n'a pas zéro volume. Cela est vrai, mais ce volume est tellement faible que nous avons cru pouvoir le négliger sans commettre une erreur appréciable. 
revenus considérablement, et, outre ce bénéfice, il lui restera un bois de 20 hectares âgé de 1 à 20 ans, plus 120 hectares d'un sol susceptible de fructifier de nouveau, soit qu'on le maintienne boisé, soit qu'on y fisse d'autres cultures, si la faculté de défricher existe.

643. En présence de tels faits, il semble difficile de soutenir que l'exploitation conservatrice des futaies puisse trouver une garantie suffisante dans les exigences de l'intérêt particulier; cependant, cette opinion, qui a été défendue par des agronomes du plus éminent mérite, trouve encore aujourd'hui des partisans. Voici le raisonnement sur lequel ils se fondent :

"Tant qu'une futaie debout aura plus de valeur " pour celui qui voudra la conserver pour en atten" dre les produits, qu'elle n'en aurait actuellement " pour celui tenté d'y mettre la cognée, on peut être " assuré qu'ily a dix chances contre une pour qu'elle " ne soit pas abattue; car, s'il se rencontre un pro" priétaire pressé d'en réaliser la valeur, il se trou" vera aussi des acheteurs disposés à spéculer sur " sa conservation. Mais il est évident gu'il faut pour " cela que les bois de fort équarrissage acquièrent une "valeur qui se trouve dans un certain rapport avec " ceux de moindre dimension; c'est cette proportion " que l'on doit considérer comme le nivellement " entre les bois de service de divers genres; la con" currence seule suffira pour l'établir, et c'est de ce 
" nivellement que l'on doit attendre toute sécurité " pour les approvisionnements en bois des àges à "venir. " (De l'Industrie forestière en France, par M. de Dombasle, Annales de Roville, $8^{\mathrm{e}}$ livraison, 1832.)

644. D'aprìs le passage qu'on vient de lire, c'est une juste proportion entre les prix des bois, selon leur àge et leurs dimensions, qui assurera la conservation des futaies de la part des particuliers.

Pour vérifier l'exactitude de cette assertion, supposons un propriétaire pressé de réaliser la valeur de sa futaie, et examinons si, au moyen de prix proportionnés comme nous venons de le dire, les acheteurs les plus offrants seront plutôt disposés à spéculer sur la conservation de cette futaie que sur sa destruction. A cet effet, conservons l'exemple de la futaie normale dont nous nous sommes servi plus haut, et ajoutons au tableau des bois qui la composent, des prix gradués de la manière suivante :

Pendant les 40 premières années, le prix du mètre cube augmentera tous les dix ans de 5 francs.

Depuis l'âge de 40 ans jusqu'à celui de 100, cette augmentation sera, chaque dix ans, de $10 \mathrm{fr}$.; et enfin :

$\Lambda$ partir de 100 ans jusqu'à 140 , chaque décennie augmentera la valeur du mètre cube de 20 francs, de sorte que, dans les bois parvenus à l'àge d'exploitabilité. celte valeur sera portée au taux excessif 
DE CONVERSION.

et, pour ainsi dire, imaginaire de 160 francs 1.

Voici, avec ces données, le tableau du capital superficiel, exprimé dans la $4^{\mathrm{e}}$ colonne en mètres cubes, et dans la $6^{\mathrm{e}}$ en argent.

1 On a fait observer que ce prix de 160 fr. par mètre cube itait souvent payé, pour des pièces de choix, par les arsenaux de la marine, de la guerre et par d'autres consommateurs encore, et que, en l'adoptant comme maximum, ce n'était pas faire une hypothèse tellement exagérée qu'elle autorisât, dans tous les cas, la conclusion a furtiori que nous en tirons. On se serait dispensé de l'objection, si l'on avait réfléchi qu'il s'agit, dims notre exemple, non de bois de choix équarris à vive arête, ou tout au moins au $5^{\mathrm{e}}$ défluit, comme ceux que les arsenaux recherchent et payent fort cher, mais de la totelité du matéricl sur pied ditns une futaie exploitable, ce qui constitue we différence tout au moins du simple au double ou au triple. 


\begin{tabular}{|c|c|c|c|c|c|}
\hline $\begin{array}{c}\text { Afrec- } \\
\text { tations } \\
\text { décen- } \\
\text { nales. } \\
1 .\end{array}$ & $\begin{array}{l}\text { AGE } \\
\text { des Bois. } \\
2 \text {. }\end{array}$ & \begin{tabular}{|c|} 
voluME \\
absolu \\
de \\
l'hectare \\
moyen. \\
3.
\end{tabular} & $\begin{array}{c}\text { Volunie } \\
\text { absolu } \\
\text { de toute } \\
\text { l'affectation } \\
\text { décensale. } \\
4 .\end{array}$ & $\begin{array}{l}\text { PRIS } \\
\text { du } \\
\text { mètre } \\
\text { cube } \\
5 .\end{array}$ & $\begin{array}{l}\text { VALEUR } \\
\text { de toute } \\
\text { l'affectation } \\
\text { décennale } \\
\text { 6. }\end{array}$ \\
\hline $14^{\prime \prime}$ & 1 i 10 & $\begin{array}{c}\text { mét. cub. } \\
7\end{array}$ & $\begin{array}{r}\text { mét. cubl. } \\
70\end{array}$ & fri. & $\begin{array}{c}\text { fr. } \\
350\end{array}$ \\
\hline $13^{\circ}$ & 1120 & $2 \cdot 2$ & 220 & 10 & 2200 \\
\hline 12 & $21 \quad 30$ & 42 & 420 & 15 & 6300 \\
\hline $11^{e}$ & $31 \quad 10$ & 65 & 650 & 20 & 13000 \\
\hline $10^{\mathrm{e}}$ & 4150 & 90 & 900 & 30 & 27000 \\
\hline $9^{\mathrm{e}}$ & $51 \quad 60$ & 118 & 1180 & 40 & 47200 \\
\hline $8^{\mathrm{e}}$ & (i) & 147 & 1470 & 50 & 73500 \\
\hline 7 & $\pi$ & 178 & 1780 & 60 & 106800 \\
\hline $6^{\circ}$ & 90 & 211 & 2110 & 70 & 147700 \\
\hline $5^{e}$ & $91 \quad 100$ & 245 & 2450 & 80 & 196000 \\
\hline $4^{c}$ & $101 \quad 110$ & 280 & 2800 & 100 & 280000 \\
\hline $3^{e}$ & $111 \quad 120$ & 314 & 3140 & 120 & 376800 \\
\hline $2^{e}$ & $121 \quad 130$ & 346 & 3460 & 140 & 484400 \\
\hline $1^{\text {re }}$ & $131 \quad 140$ & 376 & 3760 & 160 & 601600 \\
\hline \multicolumn{2}{|r|}{ TотиUх... } & $\gg$ & 24410 & 》 & 2362850 \\
\hline
\end{tabular}

643. Il risulte du tableau ci-dessus que les coupes 
de régénération à faire annuellement dans la futaie produiront :

$$
389^{\mathrm{m} . c},, \breve{5} \times 160^{\mathrm{rr}}=62320^{\mathrm{rr}}
$$

Quant aux éclaircies périodiques, il est nécessaire d'entrer dans quelques détails pour en fixer la valeur.

Nous avons admis, plus haut, que le produit annuel en matière de ces opérations égalait, dans la futaie normale proposée. la moitié du produit des coupes de régénération, savoir :

\section{5}

2

Inis, comme ces 1930 mètres cubes doivent s'obtenir par six éclaircies, à faire sur six hectares, dontle peuplement le plus jeune a 20 ans et le plus àgé 120 , nous avons besoin de connaitre séparément le produit matériel de chaque éclaircie, afin d'en déter-

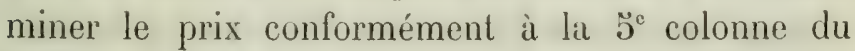
tableau, et d'obtenir ainsi la somme pour laquelle ces exploitations contribueront au revenu annicel.

Or, il est d'expérience que les produits matériels des éclaircies périodiques vont en augmentant, jusqu'à l'âge de 80 ans à peu près, pour les essences longévives, et surtout pour le chêne, et qu'ensuite ils diminuent brusquement. C'est d'après plusieurs auteurs forestiers estimés, et d'après les données pra- 
tiques que nous avons eu l'occasion de recueillir nous-même, que nous arons adopté les nombres suivants comme représentant cette échelle de production avec une exactitude suffisante pour l'exemple que nous discutons :

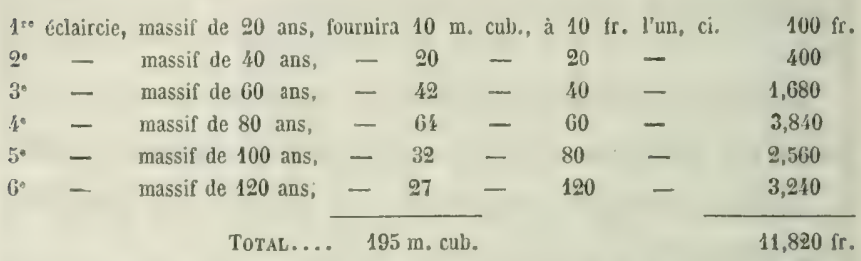
Ajuntant à cette summe la valeur des coupes de réfénération qui est de, ci.... 62,320

On trouve pour revenu de la forêt, ci.

$74,140 \mathrm{fr}$.

646. Si nous rapprochons ce revenu de 74,140 fr. de la valeur du capital superficiel, qui est de $2,362,850 \mathrm{fr}$., nous voyons que, malgré les prix excessifs attribués aux bois de fort équarrissage, on n'obtient encore qu'un placement à 3 pour cent environ.

Mais après tout, ces prix, que nous pourrions presque appeler calamiteux, nous garantiront-ils du moins que la futaie ne passera qu'entre les mains d'un acheteur disposé ì spéculer sur' sa conservation? Nullement. Cet acheleur pourra sans doute se présenter, puisque c'est au taux de 3 p. $0_{\mid} 0$ environ que se font ordinairement les placements en biensfonds; mais s'il ne se présentait pas juste au moment où le propriétaire serait pressé par un besoin 
l'argent, ou impatient de retirer plus de 3 pour cent du capital engagé dans la superficie de sa forêt, il y aurait bien des chances pour que le dit propriétaire fit abattre lui-même cette superficie.

Supposons, comme nous l'avons fait dans le premier exemple, que l'on abatte toute la futaie, moins le bois de 1 à 20 ans; on réalisera le capital suivant :

$2,362,850 \mathrm{fr} .-2,300 \mathrm{fr} .=2,360,300 \mathrm{fr} . ;$

lequel, placé à 4 pour cent seulement, rapportera par an.

$94,412 \mathrm{fr}$.

Mais le revenu, en spéculant sur la conservation, n'est que de. . . 74,140

Le bénéfice serait donc de. . . $\overline{20,272 \mathrm{fr}}$ de rente, sans compler la valeur considérable du terrain déboisé et du jeune bois de 1 à 20 ans!

Il n'y a pas beaucoup de propriétaires qui résisternient longtemps à la tentation de se procurer un tel profit.

647. Il nous semble dès lors démontré que le prix des bois de fortes dimensions, quelque élevé qu'on le suppose, ne siurait assurer la culture et la conservation des futaies de lat part des particuliers. Il est mème à croire, atu contraire, qu'avec l'augmentation de ces bois en valeur, doit croìtre aussi l'appàt de les détruire.

648. Après avoir considéré les futaies dans leurs rapports avec l'intérêt des particuliers, il nous reste 
à examiner les taillis simples et les taillis composés sous le même point de vue.

Peu de mots suffiront.

Si les futaies, surtout celles soumises à de longues révolutions, ne peuvent convenir à l'intérèt privé, la cause, comme on l'a vu, en est principalement dans le capital trop élevé, qu'il est nécessaire d'engager dans la superficie, pour se procurer un revenu soutenu; capital qui fait descendre le taux de placement bien au-dessous de celui des placements ordinaires.

Or, il n'en est pas de mème pour les taillis simples ou composés qui, s'exploitant à des époques bien plus rapprochées, n'exigent qu'un capital superficiel peu élevé comparativement au revenu qu'ils procurent 1, et offrent, par conséquent, pour le placement de ce capital, un taux convenable. Le régime du taillis convient done spécialement aux forêts possédées par les particuliers, et, sauf le cas où le sol se prêterait à un genre de culture plus lucratif, le défrichement de ces propriétés serait une fausse spéculation, dès

1 Voici quelques données à cet égard, que nous fournit un auteur allemand très estimé (Hundeshagen):

Le peuplement du taillis étant supposé normal, et le revenu en matière égal à l'unité, le capital superficiel sera: pour une révolution de 30 ans - 14 à 16.

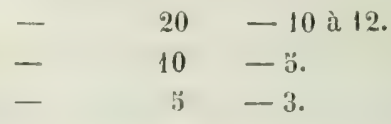


que, dans là contrée, le prix des bois est à peu près nivelé avec celui des autres denrées.

Quant à la question de savoir lequel, du taillis simple ou du taillis composé, présente le plus d'avantages à l'intérèt des particuliers, il faut distinguer:

Si le taillis est en mauvais fonds et constitué de façon à ne produire que du bois de chauffage ou de petite industrie, les particuliers n'ont aucun intérèt à y élever des réserves, et la méthode du taillis simple doit être préférée;

Si, au contraire, le sol est propre à l'éducation des essences d'élite, ils trouveront le plus ordinairement avantage à adopter des révolutions assez longues et à élever des arbres de réserve jusqu'aux dimensions requises pour la charpente. le sciage ou le merrain.

649. En résumé, la discussion du revenu des trois principales méthodes d'exploitation constate les faits suivants :

Pour l'Etat, pour les communes et pour tout propriétaire impérissable qui, par essence, n'est point apte aux spéculations commerciales, la futicie offre les plus grands avantages; le taillis composé tient le second rang, et le taillis simple occupe le dernier.

Il n'en est pas de même pour les particuliers dont la possession est précaire et qui, par suite de la division indéfinie des fortunes, peuvent être amenés à rechercher des placements à un taux élevé et des spéculations mercantiles auxquelles ne saurait résister le capital engagé dans les futaies.Pour eux, 
suivant les circonstances [648], c'est tantôt le taillis simple, tantôt le taillis composé, qui doit être mis en première ligne, et la futaie ne peut occuper que le dernier rang.

ARTICLE V.

De l'influence des différentes méthodes d'exploitation sur la fertilité du sol.

650. Les bois influent sur le sol qu'ils occupent de deux manières distinctes:

$1^{\circ}$ Par le couvert ;

$2^{\circ}$ Par l'amendement qu'ils procurent.

Le premier effet est produit par le massif plus ou moins serré et par l'épaisseur de feuillage propre à chaque essence; le second effet dépend des mêmes causes, et, en outre, des circonstances qui hâtent ou retardent la décomposition des feuilles après leur chute 1.

65̆1. Dans les futaies en général, la méthode du réensemencement naturel et des éclaircies établit un couvert constant et complet qui garantit le sol du desséchement et y maintient la fraîcheur favorable à

' On sait que les causes d'une prompte décomposition ou putréfaction sont: l'humidité, une chaleur modérée et l'air stagnant ou du moins calme. Au contraire, une forte chaleur et des courants d'air violents retardent et entravent même la putréfaction. 
la végétation des bois. Un détritus abondant, composé de feuilles, de menues branches, et qui trouve toutes les circonstances favorables à sa prompte décomposition, augmente chaque année la couche de terreau dans ces forêts, et restitue ainsi à la terre les substances minérales que s'assimilent les arbres, en même temps qu'il l'enrichit des matières organiques nécessaires à cette assimilation. Ce n'est que quand les révolutions sont très longues que ces effets cessent de se produire. Le massif alors, s'éclaircissant de plus en plus, permet l'action trop directe du soleil et de l'air sur la surface du sol; les vents dispersent le lit de feuilles; et le terreau, formé pendant de longues années, se dessèche, diminue peu à peu, el finit par perdre toutes ses qualités fertilisantes. Mais, hors ce cas qui n'est qu'une rare exception, il est incontestable que tout, dans l'exploitation des futaies régulières, concourt à conserver et à augmenter constamment la fertilité du terrain 1.

662. Par la méthode du taillis simple, le sol est à peu près complètement dénudé à chaque coupe. S'il est bon, situé en plaine ou en pente douce, les rejets s'y élèvent rapidement après la coupe et forment, dès les premières années, un nouveau fourré assez épais pour préserver le terrain des influences nuisibles de l'atmosphère. Si le sol est médiocre, peu profond, mais cependant plat, la méthode du

' Voir les notes des nos 487 et 514 . 
taillis simple, quoique ne l'amendant que faiblement, ne l'expose pas à se détériorer rapidement non plus, pourvu que le peuplement soit formé d'essences fertilisantes. Mais, quand cette méthode d'exploitation est pratiquée diıns un terrain sec, incliné et exposé aux ardeurs du soleil, il est impossible qu'elle ne produise pas les plus fàcheux résultats. En effet, à chaque coupe, les rejets nouveaux, manquant de vigueur, sont très longtemps avant de couvrir le terrain, ce qui fait que la faible couche de terreau, formée pendant la révolution écoulée, est promptement torréfiée, puis lavée par les pluies; en second lieu, l'élément minéralogique lui-même, n'étant plus recouvert, se désagrége, devient pulvérulent et est entrainé dans les bas-fonds. C'est ainsi, par exemple, que, dans les formations de grès et de calcaires qui sont entière. ment dépourvus d'argile, il suffit souvent de quelques révolutions de taillis, pour mettre le roc à nu, et exclure toute végétation autre que celle de quelques chétifs arbrisseaux et arbustes.

653. Quant à la méthode du taillis sous futaie, considérée sous le rapport de son influence sur la fertilité du sol, elle participe naturellement des deux méthodes examinées plus haut, et ses effets se rapprochent de ceux de la futaie ou de ceux du taillis simple, selon que, par le nombre et l'âge des baliveaux, le couvert est plus ou moins épais après la coupe.

63̈4. Les considérations que nous venons de pré- 
senter font voir que la méthode de la futaie ne s'applique pas exclusivement aux bons fonds, ainsi qu'on le pense communément. On peut et on doit même l'employer dans les terrains médiocres pour les améliorer, et dans les terrains secs ou pauvres [60 et 65 ], si l'on veut empêcher qu'ils ne s'appauvrissent davantage; mais, dans ce cas, il faut cultiver des essences traçantes donnant beaucoup de couvert. En ne les soumettant pas à des révolutions trop longues, les bois se maintiendront en massif serré et procureront ainsi au sol la fraîcheur et l'amendement qui, seuls, peuvent le bonifier ${ }^{1}$. L'ancienne opinion de n'exploiter' qu'en taillis les bois situés en terrain médiocre, mauvais ou dénué de profondeur, doit done être considérée comme mal fondée; car, dins l'intérêt de l'amélioration du sol et, par conséquent, de la production, dès qu'on a la faculté de choisir le régime d'exploitation, nous pensons qu'il n'y a pas à hésiter et que celui de la futaie doit être préféré.

- Pour atteindre un tel but, le hetre, parmi les essences feuillues, et l'ipiciu, parmi les résineuses, mérileraient sans doute la préférence, s’il ne devenait souvent difficile et quelquefois même impossible de les faire réussir dans les sols de mauvaise qualité dont il s'agit ici. On pourra employer avec avantage le pin syloestre et le pin d'Autriche dans les régions tempérées de la France, le pin a crochets aux plus grandes altitudes; dans les régions plus douces de l'Ouest et du Midi, le pin muritime et le pin d'Alep rendront des services analogues. Souvent ces essences pourront n'ètre cultiréss qüit titre transitrive: elles 
ARTICLE VI.

\section{Conclusion.}

655. Des faits démontrés dans le présent chapitre, on peut déduire les considérations et les principes généraux suivants, sur l'application des diverses méthodes d'exploitation dans les forêts de l'Etat, des communes et des particuliers.

65̋. La méthode de la futaie fournit les produits en matière les plus considérables et les plus utiles; elle fait rendre aux forêts les revenus les plus élevés, et elle conserve et améliore, plus que toute autre, la qualité du sol; elle répond donc, au plus haut degré, à l'intérêt général. Il suit de là, que l'Etat doit non-seulement conserver soigneusement les futaies qu'il possède, mais qu'il doit encore s'appliquer à en créer de nouvelles. Or, la conversion des taillis composés en futaie est un moyen d'atteindre ce but, d'une manière sûre et prompte, dans les forêts situées en bon fonds et peuplées de bonnes essences; elle s'impose avec plus d'urgence encore, quand il s'agit d'opérer la transformation dans des

donnent, dans la jeunesse, un couvert et des détritus abondants, à la faveur desquels les sols dégradés se restaurent suffisamment pour qu'il devienne possible d'y introduire, au bout d'une révolution, par exemple, des bois résincux ou feuillus (épicéa, sapin, hêtre, cte.) plus profitables au propriétaire. 
conditions défavorables de sol et de consistance.

657. La haute utilité d'une semblable mesure ne saurait désormais être contestée, et elle ne l'est plus, en effet, par les hommes qui comprennent que l'emploi de plus en plus répandu du combustible minéral d'une part, et de l'autre la rareté et les prix élevés des bois d'œuvre commandent logiquement d'appli. quer aux forêts nationales une culture intensive qui leur fasse rapporter, d'une manière soutenue, la plus grande quantité des produits les plus utiles.

Toutefois, pour conduire à bonne fin une entreprise de cette importance, il est essentiel de considérer qu'en administration on ne modifie sans danger ce qui est défectueux, qu'en procédant par des améliorations successives, afin d'amener, sans secousse, les intérêts existants à transiger avec ceux de l'avenir.

Dans leur état actuel, les forêts domaniales sont destinées à fournir des produits d'une haute importance, pour les principales industries du pays et pour la consommation en général ; en outre, les revenus qu'on en retire doivent subvenir dans une certaine mesure aux besoins du Trésor. Toute amélioration done, qui, dans ces forìts, ne peut se faire sentir qu'après de longues années, doit avoir pour condition de ne point froisser les intérêts actuels, tant ceux des consommateurs que ceux de l'Etat luimême. Le principe qui interdit de dépasser la possibilité des forêts, trouve ici sa réciproque ; car, s’il 
est injuste de faire tourner au profit de la génération actuelle des produits qui ne devraient écheoir qu'à ses successeurs, il le serait au moins autant de refuser satisfaction à des besoins existants, afin de préparer l'abondance dans l'avenir, à moins qu'il ne s'agisse de soustraire à la ruine les sources mêmes de la production.

658. Pour convertir en futaie un taillis composé, le problème à résoudre est donc: de conduire la forêt à l'état de futaie exploitable, sans que les produits subissent de baisse trop sensible. Or, cette solution est aujourd'hui trouvée pour tous les cas, à peu près, qui peuvent se présenter. Elle s'obtient soit par le mode de conversion même [680 à 682], soit à l'aide d'une combinaison particulière des exploitations d'une même forêt entre elles [670 et 678$]$. Nous ferons observer, cependant, que, pour apprécier l'opportunité des conversions en futaie à ce dernier point de vue, il ne faut pas toujours considérer, séparément, les forêts que l'on y destine; on doit, au contraire, les regarder comme faisant partie d'un certain ensemble de forêts, dont les produits alimentent une localité déterminée, ou s'il est permis de s'exprimer ainsi, un bassin de consommation.

Placé à ce point de vue plus élevé, on reconnaîtra souvent qu'un taillis composé peut être converti immédiatement, quoique cette opération doive amener, pour un certain temps, une baisse dans les produits particuliers de ce taillis: car, pendant le même 
temps, les produits d'une autre forêt pourront être augmentés, et ainsi, en définitive, la possibilité des forêts de la localité, tant en matière qu'en argent, ne se trouvera point altérée 1.

Mais, quelle que soit la marche adoptée, ces transformations ne pouvant s'accomplir que successivement, après de sérieuses études, il faudra, pendant un temps plus ou moins long, maintenir sous le régime du taillis composé des forêts destinées à être ultérieurement converties, et se contenter, en attendant, de préparer pour l'avenir les moyens d'exécuter cetle importante amélioration. Dans ce but, on conservera et l'on multipliera les essences précieuses, tant par le balivage que par les trivaux indiqués dans le $3^{\mathrm{e}}$ chapitre du $4^{\mathrm{c}}$ livre de ce cours.

659. Peu de mots suffiront quant à l'application que peuvent recevoir, dans les forêts domaniales, les autres genres de conversion mentionnés plus haut [631].

Il est difficile de prévoir le cas où la conversion d'une futaie en taillis pourrait être justifiée dans l'intérêt public. Quant aux autres combinaisons, elles ne se rattachent point, comme les conversions en futaie des taillis composés, à des considérations administratives d'un ordre supérieur, attendu qu'el-

' C'est, en général, de cettc manière qu'un grand propriétaire de bois, tel que l'État, devrait entendre la possibilité, qui, prise dans un sens trop étroit, devient souvent une véritable entrave à la plus grande production. 
les ne sont pas susceptibles d'acquérir le caractère de généralité dont nous avons discuté la portée. La conversion des taillis simples en futaie semble seule devoir participer, dans certains cas, de l'importance de celles des taillis composés; mais ce mode de traitement ne se rencontre que rarement, et, pour ainsi dire, par exception seulement, dans les bois de l'Etat; nous en avons indiqué la cause plus haut $[605]$.

660. Les motifs qui doivent faire préférer, en principe, la méthode de la futaie, dans les forêts de l'Etat, se reproduisent, en grande partie, au sujet des forèts communales. Les communes, en effet, sont perpétuelles comme l'Etat, et, en raison de cette perpétuité, elles doivent chercher à administrer leurs domaines dans l'intérêt du plus grand usufruit. Pour toute commune, donc. qui possède des futaies, il est de la plus grande importance de les conserver; les convertir en taillis (sauf dans quelques circonstances tout à fait exceptionnelles), ce serait consommer une véritable spoliation envers les générations futures.

Cependant, une commune doit-elle, comme l'Etat, chercher à convertir en futaie ses taillis composés? - Oui, si par quelque combinaison temporaire dans l'ensemble de l'exploitation de ses bois, elle trouve à couvrir les dépenses que pourrait entrainer la conversion.

Malheureusement, la plupart des communes en 
DE CONVERSION.

France ne possèdent que des forêts peu étendues, dont les ressources sont à peine suffisantes pour faire face, soit aux dépenses ordinaires d'utilité publique, soit aux besoins d'une population toujours croissante. On ne peut donc guère espérer de créer des futaies dans les forêts communales, tant que les conversions effectuées dans les bois domaniaux n'auront pas rendu bien manifestes les avantages et les facilités de ces transformations.

661. La majorité de ces forêts est traitée aujourd'hui en taillis composé, méthode qui, si elle est bien conduite, réunit une partie des avantages de la futaie à ceux du taillis simple [כ883]. Cette méthode d'exploitation intermédiaire semble d'ailleurs convenir assez bien à un propriétaire tel que liı commune, qui, par sa perpétuité et comme corporation, participe de l'essence de l'Etat; mais qui, à cause de ses besoins presque toujours pressants et de ses ressources bornées, est très souvent forcée, par la nécessité, de calculer comme un particulier.

Nous pensons donc, vu ces circonstances, que l'on doit, dans les forêts communales en général, se borner à conserver soigneusement les futaies existantes et maintenir du reste, la méthode du taillis composé, en s'attachant à la rendre rationnelle et à corriger son application qui, trop généralement, laisse beaucoup à désirer.

662. Quant aux forêts particulières, leur traitement, comme nous l'avons vu [639], est déterminé 
par la condition dominante d'élever le plus possible le rapport entre le revenu et le capital producteur: il est done évident que le propriétaire particulier devra préférer à la futaie, soit la méthode du taillis composé, soit celle du taillis simple, suivant les circonstances dans lesquelles la forêt se trouvera placée, et selon la valeur des produits qu'il en pourra tirer. Enfin, il est un genre de forêts, les résineuses, qui exclut absolument la méthode du taillis et commande celle de la futaie; mais alors le particulier devra nécessairement choisir la révolution la plus courte possible, parce que, comme nous l'avons prouvé ailleurs [642-646], les longues révolutions exigent un capital superficiel tellement considérable, comparativement aux intérêts qu'on en retire, que le taux du placement finit par descendre au-dessous de tous ceux que l'on trouverait dans toute autre spéculation, même la plus sûre. 


\section{CHAPITRE TROISIËIE}

\section{GONVERSION DES FUTAIES}

\section{ARTICLE PREMIER}

Conversion d'une futaie en taillis simple.

663. La conversion d'une futaip en taillis simple ne présente en général aucune difficulté. Il suffit de déterminer, d'une part, les parties de la forèt dont les souches promettent encore une reproduction certaine; de l'autre, les parties où cette circonstance n'existe pas. Dans les premieres, l'exploitation en taillis peut commencer immédiatement; dans les secondes, il est nécessaire de créer d'abord un noureau peuplement, soit par des coupes de régénération, soit par des travaux de semis ou de plantation, afin de pouvoir ensuite passer au régime du taillis.

Soit, par exemple, une futaie soumise jusqu'alors à une révolution séculaire, destinée à être convertie en un taillis de $2 \partial ̃$ ans. Si, dans la localité, on peut compter avec certitude sur la reproduction des sou- 
ches jusqu'à l'àge de 50 ans, tous les bois de cet âge et au-dessous 1 seront exploités tout de suite, en taillis, par $25^{\circ}$ de surface; le surplus de la forêt, peuplé d'une futaie de 100 à 51 ans, sera mis en coupes de régénération, de manière à produire, dans le délai de 25 ans, un repeuplement d'une gradation d'âge aussi régulière que possible, et qui, après ce temps, pourra être traité comme la partie précédente.

Ce n'est que quand une futaie a été exploitée sans ordre, que la conversion en taillis peut présenter des difficultés, parce qu'alors les parties susceptibles de se reproduire tout de suite par rejets se trouvent plus ou moins mélangées avec celles à régénérer au préalable par la semence. Dans de telles circonstances, il y aura certainement des sacrifices à faire pour rétablir l'ordre dans les exploitations.

ARTICLE II.

\section{Conversion d'une futaie on taillis composé.}

664. La marche à suivre pour opérer la conversion d'une futaie en taillis composé est absolument semblable à celle que nous venons d'indiquer dans

- Dans le cas où l'étendue de ces bois serait considérable, on les partagerait en deux séries, l'une comprenant les bois de 1 à 25 ans et l'autre ceux de 26 à 50 . 
l'article précédent, sauf les dispositions à prendre pour le balivage.

On commence donc par fixer la révolution du taillis à créer, et l'on détermine les parties de forêt qui pourront être tout de suite exploitées d'après cette méthode et celles qui, préalablement, sont à régénérer par la semence; puis on règle, d'après les principes que nous connaissons, le couvert à établir sur le taillis, et l'on arrête le nombre et les différentes catégories de baliveaux dont devra se composer la réserve [589-591].

665. Dans les parties à exploiter tout de suite en taillis, cette réserve ne pourra se composer, pour la première révolution, que de baliveaux de l'âge dont le nombre sera tel que, parvenus à la qualité de modemes, ces arbres donnent le couvert voulu. A la $2^{\mathrm{e}}$ révolution, on ne conservera de ces modernes, que la quantité fixée par le balivage adopté, et l'on complètera le couvert par des tiges de l'âge. A la $3^{\circ}$ révolution, on réservera le nombre prescrit d'anciens et de modernes, et l'on complétera encore le couvert par des baliveaux de l'âge; et ainsi de suite, de révolution en révolution, jusqu'à ce que toutes les catégories d'arbres, dont doit se composer la réserve, soient établies sur le taillis.

666. Dans les parties qu'il faut d'abord régénérer par la semence, on laissera sur pied, lors de la coupe définitive, un certain nombre d'arbres, donnant le 
couvert jugé convenable. Quand on coupera le sousbois pour la première fois, on réservera le nombre de liges de l'àge que prescrit le balivage adopté, et l'on réduira les vieux arbres dans le rapport du couvert que donneront ces jeunes baliveaux lorsqu'ils seront devenus modernes. Lit ainsi de suite, jusqu'à l'entier établissement du balivage adopté. 


\section{CHAPITRE OUATRIÈIE}

\section{GONVERSION DES TAILLIS SIMPLES}

\section{ARTICLE PREMIER.}

Conversion d'un taillis simple en taillis composé.

667. Cette opération, qui ne consiste qu'à établir successivement, sur un taillis, différentes catégories de baliveaux, est évidemment semblable, en tout, à celle qui tend à convertir en taillis composé une partie de futaie dont les souches sont encore susceptibles de reproduction; nous venons de la décrire [665 ].

\section{ARTICLE II.}

Conversion d'un taillis simple en futaie.

668. La conversion en futaie d'un taillis simple ne présente point de difficulté dans l'exécution, dès 
que les perches, dont il se compose, sont assises sur des souches assez jeunes encore.

Dans cette opération, on conserve, pour la révolution préparatoire, le terme de l'ancienne révolution du taillis, et on laisse subsister, sur chaque coupe, un massif composé des tiges les plus vigoureuses et des baliveaux ayant atteint la qualité de moderne.

On fait en sorte de supprimer, outre les perches dominées et les morts-bois, toutes les essences de qualité inférieure, à moins qu'elles ne soient indispensables pour maintenir le massif. Ces exploitations ont reçu le nom de coupes préparatoires, parce qu'elles ont, en effet, pour objet de préparer l'état de futaie, en parcourant la forêt une ou plusieurs fois, jusqu'au moment fixé pour commencer les coupes de régénération.

Soit un taillis aménagé à 30 ans, et que l'on se propose de convertir dans le même délai, on aura, au bout de ce temps, en opérant comme nous venons de le dire, une forêt qui présentera, dans la partie la plus âgée, des peuplements de 60 ans et un certain nombre d'arbres dominants de 90 , et daus la partie la plus jeune, des peuplements de 30 et des arbres dominants de 60 ans. Dès lors on pourra et on devra mème commencer l'exploitation en futaie, le massif se composant généralement de rejets. En raison de cette circonstance, on devra aussi abréger, au moins d'un tiers, la révolution qu'il eût été d'ailleurs convenable d'adopter pour la futaie. 
669. Mais cette manière de procéder, fort simple il est vrai, présente le grave inconvénient d'imposer au propriétaire une privation presque absolue depro. duits, pendant toute la révolution préparatoire où les coupesse bornent à l'extraction de quelques tiges dominées ou inutiles. Aussi n'est-elle guère admissible que dans quelques cas exceptionnels. Dans les circonstances ordinaires, on ne peut entreprendre la conversion qu'à la condition de ne pas abaisser trop sensiblement l'ancienne production. La méthode suivante a pour objet d'atteindre ce but.

670 . On détermine immédiatement la révolution qui convient à l'essence que l'on veut fiure prédominerdans les noureaux peuplements. Prenons pour exemple le même taillis que précédemment, c'est-àdire un taillis aménagé à 30 ans; supposons que celte révolution doive être fixée à 90 ans et partagíe en trois périodes de 30 ans chicune; sur le terrain, les affectations de ces périodes seront composées, savoir :

La $1^{\text {ro }}$, des peuplements de 1 à 10 ans;

Le $2^{\circ}$, des peuplements de 11 à 20 ;

La $3^{\text {e }}$, des peuplements de 21 à 30 .

Ces premières dispositions prises, on détermine le temps nécessaire pour préparer les éléments d'une régénération naturelle avant le début de la révolution de futaie.

Au cas particulier, on adoptera une révolution préparatoire de 60 ans, partagée en deux périodes 
égales, pendant lesquelles les exploitations à faire dans chacune des affectations seront ;

Première période.

$\mathrm{I}^{\text {re }}$ affectation: Coupe de nettoiement, plusieurs fois répétée, s'il est nécessaire, pendant la première moitié de la période ; coupe préparatoire, par quinzième de surface, pendant la seconde moitié.

$2^{\mathrm{e}}$ et $3^{\mathrm{e}}$ affectations réunies. Coupe de taillis, par trentième de surface, en commençant par la troisième affectation.

La coupe de taillis ne comprenant que les deux tiers de l'étendue précédemment livrée à l'exploitation, il y aura baisse de produits au commencement de la période; mais cette coupe comprendra, au début, des bois de 30 ans, puis successivement des parties plus âgées, et finalement des bois de 40 ans; il est donc permis d'espérer que, dans la deuxième quindécennie, la production, renforcée par la coupe préparatoire de la $1^{\text {re }}$ affectation, atteindra, non pas entièrement sans doute, mais à peu de chose près, celle de l'ancien taillis.

A l'expiration de la première période, les peuplements seront:

$1^{\text {re }}$ affectation: Perchis de 31 à 40 ans, avec sousbois provenant des rejets exploités dans les coupes préparatoires ; 
DE CONVERSION.

$2^{\circ}$ affectation: Taillis de 15 à 1 an;

$3^{\mathrm{e}}$ affectation: Taillis de 16 à 30 ans.

\section{Deuxième période.}

$1^{\text {re }}$ affectation: Seconde coupe préparatoire ou d'éclaircie par quinzième de surface, pendant la première et la seconde moitié de la période.

$2^{\circ}$ affectalion : Coupe de nettoiement, plusieurs fois répétée, s'il est nécessaire, pendant la première moilié de la période; première coupe préparatoire, par quinzième de surface, pendant la seconde moitié

$3^{\circ}$ affectation : Coupe de taillis par trentième de surface ${ }^{1}$.

A l'expiration de la deuxième période, les peuplements seront:

$1^{\text {re }}$ affectation: Perchis de 70 à 61 ans ;

$2^{\circ}$ affectation: Perchis de 45 à 31 ans ;

$3^{\mathrm{e}}$ affectation : Taillis de 30 à 1 an.

671. Le perchis sur souches de 61 à 70 ans étant susceptible de se régénérer par la semence, on voit que le but de la conversion se trouvera en quelque

' Si les souches cessaient d'ètre productives à $\mathbf{4} 0$ ans, il faudrait exploiter cette affectation par $2 \mathrm{r}^{\mathrm{e}}$ de surface. En pareil cas, on combinerait la marche des coupes préparatoires des $1^{\text {re }}$ et $2^{c}$ affectations, de manière à doubler leur étendue pendant les six dernières années de la période, afin de compenser la suspension des coupes de taillis. 
sorte atteint à la fin de la révolution préparatoire de 60 ans, et cela, sans que le propriétaire ait eu à s'imposer de trop fortes privations sur son revenu. De plus, la série présentera une gradation d'âge presque normale, avantage évident qui fait défaut dans la première méthode de conversion. - Enfin, le traitement des deux dernières affectations donne toute latitude d'améliorer le peuplement, en extirpant les souches usées et les essences de qualité inférieure pour y introduire les essences d'élite : 30 ans dins la deuxième affectation, et 60 ans dans la troisième sont, à coup sûr, des délais plus que suffisants pour assurer ce résultat, tout en permettant de ne faire chaque année qu'une faible dépense.

672. Que si l'on avait à convertir trois ou quatre séries contiguës qui dussent plus tard, transformées en futaie, n'en former qu'une, on pourrait procéder d'une manière tout à fait analogue. Cette réunion rendrait même l'opération plus facile. En effet, dans ce système, chaque affectation comprenant une série entière, présenterait la succession de tous les âges du taillis; on pourrait donc, dès le début et chaque année, sans interruption, pratiquer une coupe préparatoire dans la première affectation et une coupe de taillis, à l'àge de 30 ans, dans chacune des deux ou trois autres. En outre, les peuplements seraient régénérés moins prématurément, au début de la révolution de futaie.

673. Quand il s'agira de convertir des taillis ex- 
ploités à un âge moindre que 30 ans, à 20 ou 25 ans par exemple, la transformation s'effectuera d'une manière analogue; mais le nombre des coupes n'étant plus divisible par celui des périodes de la révolution de futaie, il faudra en scinder quelques-unes pour former les affectations, et l'âge des perchis en tour de régénération sera un peu moins avancé. 


\title{
CHAPITRE GINQUIẼME.
}

\author{
CONVERSION EN FUTAIE DES \\ TAILLIS COMPOSÉS ${ }^{1}$.
}

ARTICLE PREMIER.

Généralités.

674. Les taillis sous futaie soumis au régime forestier, ainsi que nous avons déjà eu l'occasion de le dire, sont loin de se trouver dans un état normal [ร86 et 597].

Faute d'une culture suffisamment soignée, les bois blancs y sont nombreux et y occupent la place d'essences plus précicuses, et, par suite du mode de

' Nuus passons sous silence la conversion d'un taillis composé en taillis simple. Il est evictent que cette opération se borne, d'une part, à la coupe de toutes les réserves, pour ne plus laisser subsister que des baliveaux de l'àge; et, de l'autre, à quelques repeuplements artificiels, afin de regarnir les clairières causécs dans le taillis par l'enlèvement des: arbres. 
balivage institué par l'ordonnance de 1669 et consacré par l'ordonnance réglementaire du code forestier, la réserve en baliveaux anciens et.en vieilles écorces y est également considérable [60כّ].

A la vérité, les dispositions de ces deux ordonnances n'ont pas toujours été rigoureuscment suivies; beaucoup d'agents forestiers ont cru pouvoir se permettre d'y déroger et sont alors tombés dans un excès contraire.

Dans les forêts de l'Etat néanmoins, on s'est plus généralement astreint à satisfaire au vœu de l'ordonnance, ce qui fait que la grande majorité des taillis composés domaniaux est encore richement pourvue d'anciennes réserves; on pourrait mème dire que bon nombre d'entre eux en sont tout à fait surchargés. Cette surabondance se remarque surtout dans les taillis qui sont situés en bon fonds et dans lesquels dominent ou dominaient jadis les essences les plus précieuses. Il importe done, lorsqu'il s'agit d'en entreprendre la conversion, de se rendre un compte exact des différents états de peuplement dus à l'ancien traitement, afin de les faire concourir le mieux possible au but qu'on se propose. Il serait difficile toutefois, et en tout cas heaucoup trop long, de donner ici une description de tous ces peuplements divers. L'arbitraire qui a régné dans la fixation du nombre et dans le choix des réserves, joint à l'absence totale ou à l'insuffisance de travaux d'entretien [597] pour régénérer le sous-bois, a produit sous ce rap- 
port la plus grande variété, non-seulement de forêt à forêt, mais souvent dans la même série d'exploitation. Cette bigarrure, lorsqu'elle se présente, est, on le comprend, une des plus sérieuses difficultés que rencontre l'opération des conversions.

Quoi qu'il en soit, on peut diviser nos taillis composés en deux grandes catégories, savoir :

$1^{\circ}$ Les taillis sous futaie régulier's, c'est-à-dire, ceux qui présentent un sous-bois bien venant, assis généralement sur des souches d'âge moyen et composé en majorité d'essences d'élite, que surmonte une réserve nombreuse d'arbres de toutes catégories, assez convenablement répartis.

$2^{\circ}$ Les taillis sous futaie irréguliers, dont les peuplements divers peuvent, tous à peu près, être ramenés aux suivants :

ฐ. Anciens et vieilles écorces, plus ou moins nombreux constituant, seuls à peu près, la réserve, et dominant un sous-bois incomplet, composé presque exclusivement de bois blancs et de morts-bois;

畹. Une réserve abondante de toutes catégories, avec sous-bois en grande partie dans un maurais état, soit à cause des essences, soit à cause de la végétation;

c. Un taillis en bon état, mais presque entièrement dépourvu de réserves, et enfin,

đII. Quelques parties régulières.

Selon que, considéré dans son ensemble, un taillis sous futaie devra être qualifié de régulier ou d'irré- 
DE CONVERSION.

gulier, il y aura lieu de lui appliquer l'une des trois méthodes de conversion dont nous allons nous occuper.

ARTICLE II.

\section{Conversion des taillis sous futaio réguliers.}

67ว. Pour ramener en futaie un taillis composé régulier, la méthode des coupes préparatoires que nous avons donnée plus haut pour la conversion des taillis simples [668] pourra être employée. Seulement, il faudra tenir compte ici d'un élément nouveau tris important; nous voulons parler des anciennes réserves qui n'existent pas dans les taillis simples, et qu'il s'agit d'associcr aux perches du taillis pour constituer la futaic à venir.

L'opportunité de conserver, à peu près généralement, dans les coupes préparaloires, les balivenux modernes d'essences longévires, est éridente. Quant aux anciens, ils devront ètre conservés aussi, toutes les fois qu'ils seront nécessaires pour maintenir le massif; mais il faudra éviter de choisir des pieds trop branchus, el surtout de vieilles écorces déjà sur le retour, à moins que leur présence ne parùt tont à fait indispensable pour empêcher qu'il n'y eût clairière, ou pour assurer, par la suite, le repeuplement en bonnes essences.

Toutes les fois que des arbres, modernes ou an- 
ciens, seront à réserver, l'élagage, tel que nous l'avons indiqué [ร๊oว et 602 ], sera de la plus grande utilité, afin d'accorder le mieux possible les cimes de ces arbres avec celles des perches environnantes, et de prévenir, en outre, le dépérissement qui pourrait les menacer.

Une dernière et importante précaution à apporter dans le choix des anciennes réserves, c'est d'examiner dans combien d'années le massif dont elles font partie reviendra en tour d'exploitation. Mais cet objet ne peut se raisonner qu'après avoir tracé la marche des coupes préparatoires, opération d'ailleurs indispensable pour assurer le succès de la conversion.

676. Marche des coupes préparatorres. Le premier point à arrêter, pour établir cette marche, est la durée de la révolution préparatoire. Cette révolution doit satisfaire à deux conditions principales:

$1^{\circ}$ Il faut qu'à son expiration, les parties les plus âgées de la forêt puissent être mises en coupe de régénération, c'est-à-dire que les arbres fournissent bonne et abondante semence ;

$2^{\circ}$ A la même époque, les parties les plus jeunes devront, eu égard à leur àge et à leur végétation, être en état de prospérer jusqu'à la fin de la révolution de futaie qui succèdera à la révolution préparatoire.

Les taillis composés, dans lesquels on trouve d'ordinaire le plus de ressources pour la conversion en 
futaie, sont ceux qui s'exploitent de 30 à 40 ans; or, pour atteindre, dans de tels taillis, le double but que nous venons d'indiquer, il suffit, en général, de donner à la révolution préparatoire une durée égale à l'ancienne révolution du taillis.

Supposons, par exemple, qu'on ait à convertir en futaie un taillis composé exploité jusqu'alors à 40 ans. Si l'on adopte ce même terme de 40 ans pour la révolution préparatoire, on trouvera, généralement, à l'expiration de cette révolution :

Dans la partie la plus âgée, un perchis de 80 ans qui sera parfaitement en élat d'ensemencer le terrain, quelle que soit l'essence, d'autant plus qu'il renfermera des réserves de 120 et 160 ans;

Dans la partie la plus jeune, des perchis de 40 ans, surmontés d'arbres de 80 et 120 ans, susceptibles de prospérer jusqu'à la fin de la révolution de futaie, attendu que cette révolution sera nécessairement abrégée, à cause de l'âge des souches.

677. La possibilité des exploitations de conversion doit, comme celle des taillis en général, être basée sur l'étendue; seulement, afin de remédier à la forte diminution que ce genre d'opération ne peut manquer d'amener dans les produits, il convient de partager la révolution préparatoire en deux périodes ou sous-révolutions, pendant chacune desquelles les coupes auront à parcourir la totalité de la forêt. Par ce moyen, la coupe annuelle s'étendant sur une contenance double de celle qu'avait la coupe ordi- 
naire en taillis sous futaie, il s'établit une chance favorable pour que les produits de la révolution préparatoire se rapprochent de ceux que procurait l'ancien mode.

Outre cet avantage, la mesure en présente encore d'autres qui ne sont pas moins importants. D'abord, elle favorise l'accroissement des bois, en rendant plus fréquentes les exploitations préparatoires; en second lieu, elle donne de grandes facilités pour le choix des réserves parmi les arbres anciens [675], surtout si l'on prend le parti, très convenable selon nous, d'asseoir et de délimiter, sur le terrain, toutes les coupes, dès le début de la révolution préparatoire. En effet, la disposition dont il s'agit permet de réserver, pendant la première sous-révolution, certains arbres anciens, utiles encore malgré leur àge et leur faible accroissement, parce qu'on sait qu'on pourra les faire abattre vingt ans plus tard, lorsque leur présence ne sera plus nécessaire. Dans la seconde sous-révolution, on trouvera non moins de facilité sous ce rapport, car on connaitra les coupes préparatoires qui, selon toutes les probabilités, seront régénérées soil au commencement, soit au milieu, soit à la fin de révolution de futaie; on sera donc à même d'apprécier si les arbres anciens ou modernes qu'on voudrait conserver, présentent des chances suffisantes de durer jusqu'au moment probable de leur abatage.

678. Mais, si favorable que soit l'expédient qui 
vient d'être indiqué, il est facile d'entrevoir cependant (et l'expérience d'ailleurs en a donné la preuve) qu'il ne suffit pas pour balancer la production du régime nouveau avec celle de l'ancien. Ce résultat ne saurait être obtenu qu'exceptionnellement et à l'aide d'une combinaison analogue à celle que nous avons exposée à propos de la conversion des taillis simples [670], et qui consiste à fiure marcher des exploitations de taillis parallèlement aux coupes préparatoires.

On déterminera donc immédiatement la révolution de futaie; on la partagera en périodes égales auxquelles on fera correspondre, sur le terrain, des affectations composées comme nous l'avons dil [670]. La différence consistera dans la présence des arbres de réserve qui concourront à la régénération avec les rejels de souches. Cette ressource qui fait défaut dans les taillis simples, permettra souvent de réduire à une seule période la durée des coupes préparatoires de chaque affectation, et ṁ̀me, dans certains cas [672], de commencer immédiatement les coupes de régénération.

La marche de ces dernières coupes se trouvant déterminée à l'avance par le plan général d'exploitation, il deviendra facile de régler le balivage des coupes de taillis sous futaie, suivant le rang des affectations où elles seront assises. C'est ainsi qu'on s'attachera à multiplier partout les baliveaux et les modernes, à supprimer un certain nombre d'anciens, et à ne 
point laisser subsister de vieilles écorces hors de l'affectation de la $2^{\mathrm{e}}$ période. On conçoil dès lors que, dans certains cas, les produits se maintiendront au niveau de ceux de l'ancien taillis sous futaie.

Entre les deux modes que nous venons d'indiquer pour la conversion des taillis sous futaie, la préférence parait devoir être donnée au dernier, parce que son application permettra d'étendre immédiatement ces transformations à de grandes surfaces, et de préparer au pays les ressources les plus précieuses, sans cependant lui imposer de grandes privations. Il n'est pas nécessaire d'ajouter que, pour se guider dans ce choix, il faudra tenir compte des considérations que nous avons développées plus haut $[658]$.

679. A l'expiration du temps consacré soit aux coupes préparatoires, soit à l'entière régénération des peuplements, nos successeurs trouveront évidemment la forêt dans un état meilleur que celui où nous l'avons prise. Il leur appartiendra d'en régler la jouissance, en fixant définitivement la révolution de la futaie, la nature et l'ordre des exploitations à y faire, etc. 
AlTICLL L 111 .

\section{Conversion des taillis sous futaie irréguliers.}

680. Le mode de conversion indiqué dans l'article précédent $[678]$ peut s’appliquer aux taillis irréguliers, sauf quelques modifications consistant principalement dans la nécessité d'effectuer ou de compléter la régénération successive des affectations, par roie de repeuplements artificiels.

Mais ces taillis se présentent quelquelois diuns des conditions particulières qui commandent dr recourir à des procédés de conversion plus expéditif:

lin effet, nous avons essatyí plus haut $|674|$ de caractériser leur état en litisint connaitre la cause do leur irrégularité, et nous arons ramené les divers peuplements qu’ils présentent à quatie types principaux, savoir:

a. Anciens et vieilles écorces plus ou moins nombreux constiluant, seuls à peu près, la réserve, ol dominant un sous-bois incomplet, composé presque rxclusivement de bois blanes ou de morts-hois;

b. Une réserve abondante de toules calégories. ivec sous-bois en gramde partie dans un maurais itilt. soit it oituse des essences, soit à cause de lit végétation ;

c. Ln taillis en bon étit, mais presque entierement dépourvu de réserves, et enfin, 
d. Quelques parties régulières.

Il suffit de considérer un tel ensemble pour reconnaître que le seul moyen de le régulariser et de le restaurer, c'est de le régénérer le plus promptement possible par la semence, de manière à y ramener les essences d'élite en profitant, pour cela, des ressources qu'offre encore la réserve.

Toutefois, il s'agit ici, comme dans les méthodes de conversion que nous avons précédemment étudiées [676 à 678], de réaliser, en les conciliant, deux conditions essentielles: il faut d'abord éviter un abaissement trop brusque des produits, et, en second lieu, constituer une gradation d'âge en rapport avec le traitement et la révolution auxquels, régénérée, la forêt doit être soumise.

681. Pour atteindre ce double but, on déterminera immédiatement la révolution de futaie. Supposons, comme plus haut [670], qu'elle soit fixée à 90 ans et partagée en trois périodes de 30 ans. Sur le terrain, on chercherait à constituer les affectations ainsi qu'il suit :

$1^{\mathrm{re}}$ affectation. Les peuplements désignés sous la lettre a ;

$2^{\mathrm{e}}$ affectation. Les peuplements désignés sous la lettre $\mathbf{b}$;

$3^{\mathrm{e}}$ affectation. Les peuplements désignés sous les lettres $\mathbf{c}$ et $\mathbf{d}^{1}$.

I On conçoit qu“il ne sera pas toujours possible d'urriver à un 
Les exploitations à faire dans chacune de ces affectations seront:

\section{Première période.}

$1^{\text {re }}$ uffectation: Coupes de régénération, en faisant intervenir le repeuplement artificiel partout où les bois existants ne suffiront pas pour assurer le repeuplementnaturel.

$2^{\mathrm{c}}$ affectution. Coupe par trentième de surface, du sous-bois, avec extraction des vieilles écorces dépérissantes et avec réserve, dans le taillis, de toutes les perches de bonnes essences susceptibles d'ètre associées à la futaie, dans l'intérêt du repeuplement futur.

$3^{\mathrm{c}}$ affectation. Coupes préparatoires, à commencer immédiatement ou à une année quelconque de la période, selon l'àge des taillis qui composeront cette affectation. Dans ces coupes, il sera procédé, à l'égard des réserves, comme il est dit plus haut [675 et $677]$.

tel résultat el qu’il faudra souvenl modifier la composition des alfectations, soit en raison de la situation respective des divers peuplements, et pour éviter le morcellement de la surface affectée à chaque période, soit parce qu'une ou plusieur's natures de peuplement (a. b), c, al, occuperont des contenances ou trop grandes ou trop petiles, comparativement aux autres. Aussi, n'entendons-nous donner ici qu'un exemple, en choisissant le cas le plus simple, atin de ne pas compliquer inutilement la démonstration, el de inieux faire comprendre la méthode. 
A l'expiration de la première période. les peuplements se composeront comme il suit :

$1^{\mathrm{re}}$ affectation. Gaulis et fourrés :

2 affectution. Futaie irrégulière, plus ou moins clair-plantée, mais susceptible d'ètre mise en coupe de régénération: - taillis dominé :

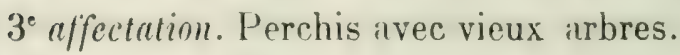

Deuxième-période.

$1^{\text {re }}$ affectation. Coupes d'amélioration.

20 affectation. Coupes de régénération, avec essouchement du tiillis, à moins qu'on ne juge utile de le conserver (hètre, par exemple), pour protéger le jeune repeuplement. Dans ce cas, il sera extrait. après lit réussite de celui-ci. par forme de nettoiement.

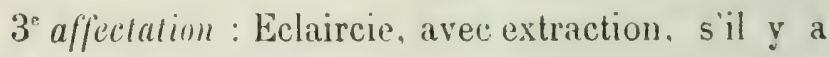
lieu, de quelques vieux bois.

A l'expiration de lit deuxième période les peuplements seront:

$1^{\text {re }}$ affectation. Perchis ;

$2^{e}$ affectation. Gaulis et fourrés ;

$3^{\circ}$ affectution. Massif de futaie sur souches.

Ainsi, dès la troisième période, la futaie sera constituée avec une gradation d'âgre convenable, et il est ívident, d'un iutre côté, que, pendantles deux premieres périodes, qui, de fitit, forment seules la révo- 
lution préparatoire, la production n'aura pas élé sensiblement abaissée.

682. Quand le mode de conversion que nous venons d'exposer devra s'appliquer à une forêt comprenant plusieurs séries de taillis composés, on trouvera la plupart du temps plus de facilité à en réunir deux ou trois pour former une même série de futaie [672], parce qu'il y aura, dins ce cas, d'autant plus de chances de rencontrer les différentes natures de peuplement que nous avons décrites, surdes surfaces assez étendues pour constiluer les affectations'.

1 Voir l'Appendice. 



\section{LIVRE SIXIÈME}

DES REPEUPLEMENTS ARTIFIGIELS.

seser

DÉFINITIONS.

683. On entend par semis l'opération par laquelle on met en terre des semences, et principalement des semences d'arbres, pour en obtenir de jeunes sujets.

684. La plantation consiste à extraire un jeune arbre du lieu où il croît, et à le replacer dans un autre lieu pour l'y faire croître.

685 . On donne le nom de bouture à unejeune branche qui, séparée de l'arbre et mise en terre, se garnit de racines et de rejets et devient ainsi un nouvel individu.

686. La marcotte est une branche que l'on couche en terre, à une certaine profondeur, sans la détacher 
de l'arbre dont elle fait partie; de lelle sorte que celui-ci lit nourrit jusqu'àce qu'elle ait pris suffisamment racine, apris quni elle pent former un individu isolé.

687. On dit que los semences s'échcuffent, lorsque, mises en tas, elles rommencentà fermenter en dégageant de la chaleur; ce qui réngit sur le germe el en détruit la vitalité.

688. Un semis est fait en plein, quand toutes les parties d'un terrilin, sans aucune exception, sont ensemencées; au contraire, le semis est partiel, si le terrain est préparé de manière qu'entre les parties ensemencées il y en ait d'autres en friche.

689. Repiquer est synonyme de planter, et s'applique aussi bien aux graines qu'aux plants. 


\section{CHAPITRE PREMIER.}

CONSIDÉRATIONS GÉNÉRALES.

690. Bien que lit culture des bois n'admette, en principe, d'autre régénération que la régénération naturelle, il se présente cependant, dans les forèts, des cas nombreux où l'action seule de lí nature ne saurait suffire pour atteindre le but que l'on se propose. et il devient indispensable de recourir à des moyens artificiels '. On doit done considérer l'art d'opérer des repeuplements de main d'homme comme l'auxiliaire plus ou moins obligé de toute méthode d'exploitation, et comme devant former. par conséquent, une partie essentielle de l'instruction du forestier.

Cette branche de la sylviculture mérite d'ailleurs

1 Ynus atons indiqué les principatux de ces cas, en traitant, dans le 2o ot te fe live, des difripentes méthorles d'exploitation el de leur applieation amx diverses essenees. (Voyez les $n^{08} 462$.

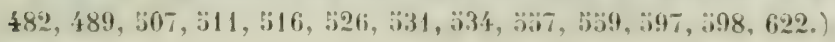


une attention plus particulière en France, où le reboisement de plusieurs contrées importantes (les Alpes, les Pyrénées, la Sologne, les Landes, etc.) est devenu une question vitale pour les habitants et préoccupe à juste titre le Gouvernement 1. En outre un grand nombre de nos forêts présentent des clairières, des vides nombreux, causés par l'abus du pâturage, par les incendies ou même par des exploitations mal entendues, et qui ne peuvent désormais être rendus à la production qu'à l'aide de repeuplements artificiels. - Enfin, ces sortes de travaux trouvent encore leur application lorsque, par suite de la dégradation soit du sol, soit du peuplement, ou par quelque autre motif, il y a lieu de substituer une essence nouvelle à celles qui composent actuellement la forêt.

691. Des quatre modes de repeuplement artificiel dont nous avons donné plus haut la définition, les plus généralement usités sont le semis et la plantation. Le semis est considéré, par beaucoup de forestiers, comme principalement applicable en grand, à cause des procédés par lesquels il s'exécute, et qui semblent à la fois plus naturels, plus simples et

1 Voir à ee sujet: Etudes sur les torrents des Hautes-Alpes, par N. Surell, ingénieur des ponts et chaussées, et le Rapport, déjà cité, sur les plantations de la Sologne, par M. A. Brongniard, de l'Institut (Amuales forestieres, tome X). V. aussi le rapport du Ministre des Finances, concernant le reboisement des montagnes (Moniteur du 3 février 1860). 
moins coûteux que ceux de la plantation. Cependant la pratique tend de plus en plus à établir la supériorité de celle-ci. Non-seulement on est parvenu à atténuer singulièrement la dépense qu'elle occasionne, en plantant des sujets très jeunes que l'on élève en pépinière, à très peu de frais; mais il est incontestable qu'une plantation bien faite présente, la plupart du temps, des chances de réussite plus assurées que le semis préparé avec le plus de soin, - parce que celui-ci a, de plus que l'autre, à lutter contre des dangers nombreux qui menacent la graine d'abord et ensuite le plant naissant. Ainsi, il vaudra toujours mieux planter que semer dans les localités exposées aux dégâts, soit du bétail, soit du gibier; dans les terrains où la crue des herbes, ou d'autres plantes nuisibles, est trop abondante; dans ceux où les jeunes plants sont exposés à être déracinés par la gelée [697]; sur les grandes sommités, et enfin, dans les lieux exposés aux inondations. Il en sera de même quand il s'agira de repeupler un terrain entièrement nu par une essence qui, dans sa première jeunesse, réclame beaucoup de couvert, comme le hêtre par exemple; ou bien lorsqu'on voudra établir le mélange de deux essences dont l'une occupe déjà le terrain [489]. Enfin, la plantation est surtout convenable pour compléter les repeuplements naturels dans les coupes de futaie [462], ainsi que pour assurer la conservation des bonnes essences et remplacer les souches manquantes dans les taillis [597]. 


\title{
CHAPITRE DEITIÊME.
}

\author{
DES SEMIS.
}

ARTICLE PPLMIER.

Des connaissanees qu'il faut posséder.

692. Outre la connaissance des climats el des sols convenables aux différentes essences, telle qu'elle at été enseignée dans le premier livre de ce Cours, il faut encore, pour opérer des semis avec succès, posséder plusieurs autres notions, ayant pour objet. savoir :

$1^{\circ}$ La maniere de récolter et de conserver les semences ;

$22^{\circ}$ Les moyens à 'mployer pour s'assurer de leur qualité ;

$3^{\circ}$ La préparation à donner au terrain;

4. La saison lit plus convenable pour semer:

$\because{ }^{\circ}$ La quantité de graine à employer ;

6. Enfin, la maniepe de sépandre el de recouvrir les semences. 
ARTICLF II,

De la récolte et de la conservation] des semenoes.

693. L'àge et l'étal de végétation des arbres sur lesquels on récolte les graines ne sont point indifférents. Les sujets qui fournissent les meilleures semences sont ceux d'àge moyen qui croissent isolés ou à peu près et présentent une cime parfaitement saine et bien développée; tandis que les arbres trop vieux ou dépérissants. de mème que les liges trop jeunes, donnent en général des graines mal condilionnées ou vianes qü il fiunt, par consécquenl, se gärder d'employer. On doit évitre rezilement de se servir desemences provenant d'atbres ribungris of dont le tempérament s'est plus ou moins abalardi en ratison des circonstances locales défiavorables dins lesquelles ils ont crì. De telles semences forment, jour ainsi dire, des variotés ou races dégénérées du type primitif el qui ny retomment, la plupart du temps. qu'iuprès avoir élé cultivées, dans de meilleures conditions, pendant plusieurs générations.

Lil récolte des semences doit, autant que possible. se lanire pitr un temps sec on qui, du moins, ne soit point pluvieux, les graines trop humides étant plus exposées à s'avarier. Pour recueillir les semences lourdes, si l'on n'aime mieux allendre leur chute na- 
turelle, on litit gauler les arbres et tendre des toiles pour les recevoir; quant aux semences légères des essences feuillues, il faut, en général, les faire cueillir et il en est de même des cònes de bois résineux.

694. Aussitôt récoltées, les graines des essences feuillues, quelle que soit leur nature, doivent être étendues, en couches minces, dans un lieu sec et aéré, et remuées souvent en vue de les laisser ressuyer et d'empêcher ainsi qu'elles ne s'altèrent. Les cônes d'arbres résineux peuvent, sans trop d'inconvénient, être entassés dicvintinge, quand les quantités récoltées ne sont pas très considérables; néanmoins, il est toujours préférable de les étendre. C'est surtoutaprès que la semence a été extraite des cônes, qu'il devient nécessaire de prendre des précautions pour lia conserver en bon état.

Selon leur nature particulière, les semences sont plus ou moins sujettes àgermer, à pourrir, à s'échauffer ou à se dessécher : il faut donc, lorsqu'il s'agit de les conserver pendant un certain temps avant de les mettre en terre, savoir les garantir contre ces différents dangers.

Les moyens à employer pour empêcher, soit une germination prématurée, soit lit pourriture, consistent d'abord à placer les graines dans un lieu frais sans être humide, tel qu'une cave, par exemple, où elles soient, autant que possible, à l'abri des variations de l'atmosphère, et ensuite à les mêler avec un corps qui puisse se charger de l'humidité qu'elles 
laissent échapper, comme du sable sec, de la paille, des feuilles sèches, etc. Le desséchement peut être prévenu par des procédés analogues, c'est-à-dire, en préservant la semence le plus possible du contact de l'air et en la mettant dans un endroit frais. Quant aux graines en danger de s'échauffer, il faut les placer dans des lieux secs et aérés, éviter de les entasser et les faire remuer souvent à la pelle.

En traitant plus bas du semis des principales essences, nous donnerons, pour chaque espèce de graine, les procédés deconservation que l'expérience a démontré être les meilleurs.

\section{ARTICLE III 。}

Des moyens de reconnaître la qualité des semencos.

695. Avant d'effectuer un semis, il est de règle de s'assurer de la qualité des graines dont on dispose, surtout lorsque c'est par lit voie du commerce qu'on se les est procurées. Cet examen ne doit jamais être négligé ; il est indispensable pour déterminer exactement la quantité de semence à employer, et l'on conçoitaisémemt l'importance d'une telle déterminittion. En effet, si la quilntité de semence est trop considérable, il en résulte un surcroit inutile de frais. Si, au contraire, la graine est trop ipargnée, le semis se trouve nécessitirement incomplet; on est forcé d'y revenir pour le compléter, et l'on aug- 
mente ainsi la dépense pécuniaire, tout en perdant du temps et en étublissint une certaine inégalité dans l'àge des jeunes bois.

Le moyen le plus surr pour vérifier la qualité des graines, est d'en prendre, au hasard, dans la provision que l'on doit éprouver, un certain nombre (plus ou moins, selon l'importance de la provision), de les semer dans une caisse, ou dans tout autre vase quelconque, rempli d'une terre substantielle et légère; puis, de placer cette caisse dans un lieu tempéré, et d'arroser souvent avec de l'eau tiède afin d'accélérer la germination. En comparant ensuite le nombre deplants levés avec le nombre de graines qui avaient été semées, on pourra apprécier la qualité de la provision entière. Si, dans cette épreuve. on reconnait que les deux tiers ou les trois quarts des graines ont levé, la semence peut ètre considérée comme étant de bonne qualité.

Le moven que nous venons d'indiquer est surtout à conseiller lorsqu'il s'agit de semis considérables ; autrement on peut se contenter de prendre quelques graines, de les ourrir, soitavec un canif, soit avec lout autre instrument, et d'examiner si l'amande et le germe sont frais et en bon étal. Cette opération peut. en général, se faire sur les petites graines comme sur les grosses, mais c'est surtout sur cellesci qu'elle se pratique aisément.

Selon l'essence, les graines doivent présenter, dans leur aspect et dans leur consistance, des carac- 
tères particuliers qui en font reconnaitre la bonté ; nous indiquerons ces caractères dans le chapitre suivant, en traitant séparément du semis de chaque essence.

696. C'est ici le cas de signaler quelques manœuvres frauduleuses des marchands de graines forestières, contre lesquelles il est utile de se précautionner.

Les semences légères, surtout les semences résineuses, étant celles qu'on achète le plus ordinairement aupoids, les marchands les humectent quelquefois au moment de la vente. On fera bien de ne consentir à la pesée des graines qu'après qu'elles auront été sorties des sacs à leur arrivée, et étendues, 'pendant une quinzaine de jours, dans un grenier ou dans une chambre aérée. Cette précaution est d'autant plus utile que, quelquefois aussi, les fournisseurs, pour en augmenter le poids, mêlent à la semence un sable très fin. Au surplus, quand même on ne peut stipuler cette condition avec les marchands, il ne faut pas moins s'empresser, à l'arrivée de la semence, de la répandre, de la remuer, et d'en opérer le desséchement; car, toute fraude à part, elle peut avoir été, pendant le transport, mouillée par la pluie ou imprégnée de l'humidité de l'atmosphère.

Une autre fraude, à laquelle les marchands ont souvent recours pour augmenter leur profit, consiste dans le mélange des graines d'épicéa aver. 
celles de pin svlvestre; le prix de la dernière étant ordinairement double du prix de l'autre. Comme la semence de pin est généralement noire, tandis que celle de l'épicéa est brun-rougeâtre, ils teignent celle-ci en noir, par un procédé qui n'altère point sa faculté germinative. On peut y ètre trompé, parce que ces deux graines ne diffërent essentiellement que par la couleur. Sans doute, en y regardant de près, on finirait par distinguer la semence de pin sylvestre d'avec celle d'épicéa: la première est plus arrondie et plus luisante; la seconde, plus allongée et plus anguleuse, prend parla teinture une couleur mate. Mais il serait toujours extrèmement difficile de reconnaitre dans quelle proportion ces deux graines sont mélangées, surtout lorsque les quantités de semences reçues sont considérables. La seule épreuve exacte est donc d'en prendre au hasard quelques pincées et les semer dans une caisse, comme nous l'avons expliqué plus haut. La levée des jeunes plants donnera la mesure de la fraude 1.

On conçoil qu'indépendamment du trop payé, ce mélange puisse entrainer de graves inconvénients. Ce sont ordinairement les pentes méridionales, entièrement dégarnies, que l'on destine à être repeuplées en pin sylvestre [294]; or, si les semences

- Les pins sylvestres levent avec une tigelle rougeâtre portant do cinq à six feuilles séminales; les épicéas naissants, au contraire, ont la tigelle jaunâtre et presque toujours neuf feuilles séminales. 
mélangées sont employées, sans examen préalable, dans de pareils terrains, on peut être assuré que tous les jeunes plants d'épicéa périront, aussitòt levés, à cause de l'ardeur trop vive du soleil. Mais quand bien même, par suite d'une exposition moins défavorable à l'épicéa, les jeunes plants de cefte essence résisteraient pendant les 2 ou 3 premières années, ils ne finiraient pas moins par ètre étouffés par le pin sylvestre qui a, dès sa naissance, une végétation beaucoup plus rapide que l'épicéa et le surmonterait complètement dès avant la dixième année. - Dans tous les cas donc le mélange des deux graines ne saurait produire qu'un semis manqué ou du moins très incomplet.

\section{AR'TICLE IV.}

\section{De la préparation du terrain.}

697. La préparation du terrain a pour but de le nettoyer, en tant que cela est nécessaire, des herbes et arbustes qui y croissent, et de le rendre assez meuble pour que la semence puisse y germer, les plants naissants y étendre leurs racines et s'y nourrir [455].

A cet égard, il est d'expérience que des labours profonds, qui auraient pour résultat de ramener à la surface des couches de terre dépourvues de principes assimilables, ne sont pas favorables aux semis. Mais 
il en est autrement d'une culture, même profonde, dont le but est d'ameublir le sol en y mélangeant le terreau. Ainsi préparée, la terre est dans les meilleures conditions pour recevoir les graines, qu'il s'agisse de semis de végétaux forestiers ou agricoles.

En effet, la couche superficielle est rendue plus perméable aux eaux pluviales; poreuse et inégale, elle condense et absorbe plus de rosée; ne faisant plus corps avec les couches inférieures, elle les protège et les garantit contre l'évaporation, comme le ferait une couverture de feuilles mortes. D'un autre côté, les racines des jeunes plants se développant davantage et s'enfonçant plus rapidement dans une terre profondément cultivée, le chevelu se trouve d'autant plus sûrement soustrait à l'action de la sécheresse. - Au contraire, dans une terre peu ou point cultivée, les racines restent grêles, superficielles, et, quand survient l'été, elles se trouvent encore dans la couche qui se durcit et se dessèche complètement; si les plants ne succombent pas toujours, ils restent du moins chétifs pendant longtemps.

La culture a donc pour résultat de maintenir et d'accroître la fraîcheur du terrain et de contribuer à fortifier le tempérament des jeunes plants.

Elle permet encore, à raison de la perméabilité du sol, d'enterrer les graines plus profondément, et de leur assurer ainsi l'humidité nécessaire à la germination. Enfin on prévient, par la culture, le déchausse- 
ment des plants, sous l'action des gelées; car il est évident qu'un plant bien enraciné y est moins exposé que celui qui l'est peu.

Dans les terres fortes ou substantielles, où l'on peut avoir à redouter I'envahissement des herbes, il sera prudent de faire précéder le semis d'une ou de plusieurs cultures agricoles.

Malgré les avantages incontestables qui résultent d'une culture complète, on ne doit pas oublier cependant que cette opération exige toujours des dépenses assez considérables. Toutes les fois done qu'il ne s'agira pas de reboiser des sols dénudés depuis longtemps, mais qu'on aura simplement à compléter des semis existants, ou à repeupler des clairières ou des vides, on ne perdra pas de vue que la végétation forestière a dû maintenir le terrain suffisamment meuble, et, dans ce cas, on pourra se borner à donner une culture légère. Nous ajouterons même que dans le cas où le sol serait, par sa composition, naturellement frais et meuble (certains terrains granitiques, par exemple), une culture légère suffira presque toujours pour assurer la réussite des semis.

698. D'après ce que nous venons de dire, on voit qu'il est de la plus haute importance de bien examiner le terrain qu'on se propose d'ensemencer, afin de lui donner la préparation convenable, tant en raison de sa nature même, que par rapport à l'essence que l'on veut y cultiver. Les modes de labour conseillés par les différents auteurs forestiers sont 
en grand nombre; toutefois nous n'en décrirons que trois, parce que jusqu'ici ils sont les seuls réellement consacrés par la pratique, et que d'ailleurs, selon nous, ils peuvent satisfaire à toutes les conditions. Ces trois modes sont :

$1^{\circ}$ Le labour entier ou le labour en plein;

$2^{\circ}$ Le labour par bandes alternes ;

$3^{\circ}$ Le labour par places, trous ou pots.

Selon la nature du sol, selon l'essence que l'on veut cultiver, il devient souvent utile de faire précéder le labour, soit de l'écobuage, soit de quelques travaux d'assainissement; nous donnerons done, sur ces deux objets aussi, les explications nécessaires 2.

699. Labour en plein. - Le labour en plein consiste à retourner la totalité du terrain à ensemencer ; il s'exécute à la charrue ou à la houe.

Le labour à la charrue est moins coûteux que le labour à la houe, et donne en outre la facilité de joindre la culture des céréales à celle du bois. Cet avantage est considérable, car souvent le produit des céréales peut couvrir entièrement les frais de culture. Toutefois, la charrue ne peut être employée

'Si nous ne parlons pas de l'épierreinent du sol, c'est qu'il est reconnu que des pierres de moyenne grosseur, lorsqu'clles ne sont pas trop nombreuses, favorisent plutôt qu'elles n'entravent la réussite des semis forestiers. D'une part elles forment un abri pour les plants qui naissent dans leur voisinage, de l'autre elles affermissent les sols trop légers, et y maintienment plus longtemps la fraîcheur. 
que dans les terrains plats ou légèrement inclinés, dans lesquels, d'ailleurs, son action n'est pas empêchée pardes pierres ou des racines trop grosses. C'est surtout dans les terres fortes et profondes, et lorsqu'il s'agit de cultiver des essences dont le pirat s'enfonce très-avint, que la charrue doit ètre préférée.

La houe (voy. fig. $\mathbf{1}^{\text {rn }}$ ) remplacerit utilement lit charrue, pour opérer des labours en plein, dans les terrains légers ou d'un accès difficile, et particulièrement dans les coupes d'ensemencement, ainsi que dans d'autres parties clair-plantées dont la régénérattion naturelle est entravée par suite du gazonnement du sol.

Il est entendu que le labour en plein, à la hove comme à la charrue, ne doit jamais être pratiqué dans les pentes rapides, où l'on pourrait craindre l'éboulement des terres.

700. Lajour par bandes alternes. - De tous les modes en usage pour préparer le terrain au semis, le mode par bandes alternes semble réunir le plus d'avantages et être le plus généralement applicable. Il consiste à ouvrir les rayons dans lesquels on sìme, et à les alterner avec des bandes qu'on laisse incultes; celles-ci ayant au moins le double de largeur des autres.

En plaine, ces rayons peuvent ètre tracés à la charrue, en prenant soin toutefois, dans les sols légers, de ne pas trop enfoncer le soc; on leur donne 
une largeur de 30 à 40 ou même 20 centimètres, et on les dirige de l'Est à l'Ouest, de manière à entasser, sur le bord méridional, le gazon et la superficie du terrain.

Én montagne, ce labour se fait à la houe, on donne aux rayons une direction horizontale, et leur largeur varie de 20 à 40 centimètres. Plus le penchant est rapide, plus le rayon doit être étroit; il faut éviter de laisser à celui-ci de la pente, dans le sens de sa largeur, et relever son bord inférieur en y entassant le gazon et les différentes plantes qu'on en extrait. Cette précaution est très essentielle afin d'empêcher que les graines ne soient entraînées par les eaux pluviales dans la bande inculte.

L'avantage d'un tel mode de labour est incontestable. En montagne, ces rayons, parallèles à l'horizon, soutenus par des bandes incultes empèchent les terres de s'ébouler; en toute situation, les semences et les jeunes plants sont abrités par les bords un peu élevés des rayons et par les herbes et les arbustes qui peuvent se trouver dans les bandes voisines. Enfin, les végétaux décomposés, les feuilles sèches s'arrêtent au fond des rayons et les eaux pluviales y sont retenues. Ainsi, l'engrais naturel, l'abri et la fraicheur assurent bien des chances de succès à un semis fait d'après ce mode. Il est inutile d'ajouter que les dépenses qu'il nécessite, tant pour le labour mème que pour l'achat des graines, sont bien moindres que celles d'un semis fait en plein. 
Le seul inconvénient auquel ce mode puisse donner lieu, est que, au moyen des bandes incultes, les herbes et autres plantes nuisibles peuvent se reproduire promptement dans les rayons cultivés et étouffer ainsi les jeunes plants ou au moins les gèner dans leur croissance. C'est au forestier à juger, selon la nature du terrain et des essences, si cet inconvénient peut réellement se présenter au point d'avoir un fàcheux résultat, et à se décider, par suite, pour le mode de préparation qu'il jugera convenable.

701. Labour par places, trous ou pots. - Ce mode de labour s'opère en formant des places ou trous carrés de 50 à 66 centimitres environ de côté, séparés les uns des autres par des intervalles de $\mathbf{6 6}$ centimètres à 1 mètre qu'on laisse en friche.

Comme dans le labour par bandes, on entasse les déblais rle ces trous sur le bord méridional, lorsqu'ils sont faits en plaine; et, si le sol est incliné, on a soin de les creuser parallèlement ì l'horizon et d'en amasser la superficie sur le bord inférieur.

Sur un terrain presque nu, on peut aligner ces trous carrés, de manière à donner au tout, à peu près l'aspect d'un damier; au contraire, on les fait sans ordre déterminé lorsque le sol présente des obstacles tels que des arbres que l'on ne voudrait point couper encore, de vieilles souches, des blocs de rochers, etc. C'est alors, surtout, que le labour par trous est avantageux, parce qu'il serait souvent impossible d'établir des rayons continus. 
Les différents modes de labour qui viennent d'ètre indiqués peuvent se pratiquer an toute saison, sauf, bien entendu, celle des fortes gelées. Pour les semis du printemps, il convient de faire préparer le terrain dans l'été ou dans l'automne précédent, que sa nature soit.d'ailleurs compacte ou légère. si le sol est léger et que, en raison des plantes parasites qui le recouvrent, il ait fallu le remuer assez profondément, il aura le temps de se raffermir suffisamment avant de recevoir la graine [697]; s'il est compacte, les gelées d'hiver, en l'attaquint et le soulevant en tous sens, le rendront plus meuble et plus propice par conséquent à la végétation. - De cette distrilıution des travaux il résultera en outre l'avantage de pouvoir se procurer plus aisément et à moins de frais les ouvriers dont on a besoin, ce qui, dans la saison du printemps où les ourrages de la campagne réclament ordinairement tous les bras, est souvent très difficile.

702. Ecobuage. - L'écobuage qui, dans la culture des champs, produit des effets forl aranlageux, peut contribuer aussi, dans beaucoup de cas, au succès des cultures forestières. Il consiste ordinairement à enlever, par tranches, à $\ddot{b}$ ou 6 centimètres de profondeur la superficie du sol couverte de plantes; à couper ces tranches en morceaux carrés pour en faire de pelits fours qu'on allume et qu'on brùle à feu étouffé; puis à répandre les cendres obtenues sur le terrain. 
Les résultats de cette opération sont: $1^{\circ}$ la destruction des mauvaises herbes et de leurs semences, ainsi que des œufs et des repaires d'animaux nuisibles; $2^{\circ}$ l'incinération des acides régétaux qui se trourent en excès dans le sol et sont contraires à la végétation des essences forestières; $3^{\circ}$ enfin, la production d'un amendement. Les deux premiers de ces résultats ne siuraient ètre qu'avantageux, quelle que soit d'ailleurs la nature du terrain; mais il n'en est pas de même du troisième, et il importe, par conséquent, de connaitre la maniére d'agir des cendres, comme amendement, afin de ne pratiquer l'écobuage que dans les sols où il peut avoir de bons effets.

" L'action des cendres sur les terrains cultivés, " dit M. de Gandolle ${ }^{2}$, est, comme la nature mème " de cette matière, complexe et variable. Les cen" dres tiennent le milieu entre les amendements et " les engrais, sous ce rapport, qu'outre les matières ") terreuses qui en constituent la masse, elles con" tiennent toujours une certaine quantité de sels et " de débris organiques. Considérées comme amen" dement, leur action est variable, selon que, four"nies par divers combustibles, elles peuvent conte") nir des quantités très diverses de matières terreuses " différentes et de sels différents. On peut dire, en " général, que : $I^{\circ}$ elles agissent mécaniquement en ") divisant les sols trop compactes, et, sous cerapport,

'Physiologie végètule, tome III, page $126 \%$. 
" plus elles sont siliceuses, plus elles ont d'action;

" $2^{\circ}$ elles ont une action hygroscopique, en absorbant

"l'humidité ; $3^{\circ}$ elles paraissent accélérer la décom-

" position du terreau ; et $4^{\circ}$ enfin, peut-être agissent-

") elles à titre d'excitants. "

" Il est donc évident, dit plus loin le mème au"teur ", et la pratique confirme cette théorie, que "l'écobuage est utile : $1^{\circ}$ dans les terrains trop ar" gileux, pour les diviser et les rendre moins hy"groscopiques; $2^{\circ}$ dims les terrains très chargés de " mauraises herbes et en mème temps très humides; " $3^{\circ}$ dans les climats où l'humidité de l'air est très " continue; $4^{\circ}$ dans les terrains marécageux, tour" beux ou froids, couverts de mousses, de joncs, de "lichens, etc., pour les exciter par les molécules " alcalines des cendres, el accélérer leur décompo" sition. "

703. Dans les cultures forestières, l'écobuage ne se pratique pas toujours comme nous l'arons expliqué plus haut; souvent on se borne à brûler, sur pied, les herbes et les arbustes qui recourrent le sol. Cette opération, qui se fait à peu près comme le sartage à leu courant qui a été décrit dims le quatrième livre [620], est avantageuse, d'abord, en ce qu'elle épargne les frais de main-d'œurre; en second lieu, parce qu'elle peut itre employie mème dans des terrains inclinés ou d'une nature assez légère. En

' Physiologie végétale, tome III, page $127 \%$. 
effet, le sol, dans ce cas, n'étant point remué, conserve toute sa compacité, et si, entre le moment de l'écobuage et celui du semis, on laisse s'écouler quelque fltemps ${ }^{2}$, les cendres, demeurant à lia surface, seront,en très grande partie, dispersées par les vents, de telle sorte qu'en définitive, les résultats de l'opération se réduiront, à peu de chose près, à la destruction des plantes nuisibles. Ajoutons que les jeunes plants, en grandissant, fourniront, par leur dépouille et par l'ombrage qu'ils procurent, une fraicheur et up engrais salutaires qui tendront nécessairement à neutraliser les effets des cendres.

On pourra donc écobuer ì feu courant pour détruire les fortes herbes, les bruyères, les myrtilles, etc., pourvu que le terrain ait quelque compacité, comme les sables gras, par exemple, et que la pente ne soil pas trop rapide. Au contraire, on devra s'interdire cette opération dans les versants escarpés, dans les sables mouvints, dans les pierrailles soit siliceuses, soil calcaires, dans les sols crayeux et, en général, dans tous les terrains qui, par leur peu de consistance, se dessèchent très facilement.

704. Assamissmanes. - Lorsqu'il y a lieu de procéder à l'assainissement d'un terrain, soil marécageux, soit aquatique, il fitut rechercher d'abord d'où proviennent les eaux surabondintes qu'il s'agit de faire disparaitre. Ordinairement elles sont produites

' On peut écobuel' en automne pour semer au printemps. 
par le débordement d'une rivière ou d'un ruisseau; ou bien par des sources, apparentes ou souterraines, qui se trouvent, soit à l'intérieur, soit à l'extérieur du terrain détrempé. Souvent aussi les eaux pluviales ou de neige, ne pouvant s'infiltrer, à cause d'un banc d'argile situé à peu de profondeur, sont maintenues dans la couche de terre végétale et s'itmassent dans les lieux les plus bas, au point de les submerger.

70ฎ. Quand ce sont les débordements d'une rivière qu'il s'agit de combattre, c'est au creusement de son lit, à l'endigage de ses rives qu'il faut avoir recours. Mais de tels ouvrages, d'ordinaire très considérables, difficiles d'ailleurs sous le rapport de l'art, et qui intéressent le plus souvent un grand nombre de propriétés riveraines, ne sont plus à compter au nombre des travaux de simple amélioration forestière; leur cxécution ne peut être confiée qu’à des hommes spéciaux.

Le cas qui rentre dans les attributions du forestier, c'est quand des accidents analogues sont causés par un simple ruisseau, soit qu'il emplisse ou exhausse son lit entier par les matières qu'il charrie, soit qu'il s'obstrue seulement sur quelques points, soit enfin qu'il se gonfle outre mesure à certaines époques de l'année. Redresser le cours de l'eau, en coupant les nombreuses sinuosités qu'il forme, par un fossé suffisamment profond; ouvrir, dans le terrain inondé, d'autres fossés de moindre dimension, 
qui débouchent dans le premier; enfin, s'il en était besoin, creuser des saignées ou rigoles plus petites encore, aboutissint a des fossés secondaires: tels sont ordinairement les moyens à employer.

706. Lorsque les marécages sont formés par des eaux de source sans écoulement, il importe de distinguer si elles sourdent dins le marais mème. ou si, au contraire, elles sont fournies par une source placée à l'extérieur, dans une situation plus élevée.

Dans le premier cas, on ourrira, dans le sens de la pente du sol, un fossé qui traverse lí partie li plus détrempée du marais; puis on observera, sur les parois de oe fossé, les points d'où les eaux suintent avec le plus d'abondance. Lit on creusera de nouveaux fossés que l'on poursuirra de manière à finir par mettre les sources ì découvert. Ajoulant ensuite, à ces fossés, des saignées plus petiles, le terrain sera facilement assaini.

Dans le second cas, lorsque les eaux viennent de l'extérieur', il convient d'ouvrir d'ithord, immédiatement au-dessus du marais, un fossé transversal pour recueillir les eaux; puis, on en ajoutera deux autres. sur les còtés, pour lés éconduire. si, malgré celir, le lermain à assainir conserve encore trop d'humidité. quelques fossés ouverts dans l'intérieur ne tarderont pas à procurer le résultat désiré.

C'est d'apris le même procédé que l'on pourra assainir, dans les forèts assises sur des couches d'ar- 
gile, les parties basses, périodiquement inondées par les eaux de pluie ou de neige.

On peut aussi, dans ces sortes de terrains, lorsqu'ils manquent de pente, avoir recours aux bétoires ou puits-perdus artificiels, pourvu toutefois que la couche d'argile, sur laquelle le sol végétal repose, ne soit pas d'une grande puissance; autrement les frais de l'opération, on le conçoit, deviendraient hors de proportion avec l'utilité qu'elle doit procurer.

707. Il ne faut pas trop se hater de repeupler les terrains qui viennent d'ètre assainis. Il convient, au contraire, de les laisser ressuyer et tasser complètement pendant une ou deux années.

ARTICLE V.

\section{De la saison la plus convenable au semis.}

708. Il est naturel de penser, en général, que la saison la plus convenable au semis de nos essences forestières nous est indiquée par l'époque de la dissémination naturelle de leurs graines. C'est un principe que l'on peut admettre, sauf les cas d'exception.

Pour les semis de glands, de faìnes et de châtaignes, par exemple, on préfère ordinairement le printemps à l'automne, quoique ce soit dans cette dernière saison que ces fruils tombent des aŕbres. Les motifs de cette exception sont dans les circons- 
tances particulières que les graines rencontrent dans un semis artificiel, et qui sont toutes différentes de celles qui, d'ordinaire, se présentent dans un semis naturel. En effet dans les semis naturels, les semences se répandent surabondamment et, pourvu que la moindre partie réussisse, le repeuplement se trouve complet; les arbres qui ont produit les graines, le lit de feuilles sèches, les mousses, etc., leur fournissent d'ailleurs un couvert précieux pour résister aux froids de l'hiver. Au contraire, dans les semis artificiels, il n'est pas possible de semer avec autant de profusion, et souvent même le prix de la semence oblige, sous ce rapport, à beaucoup d'économie ; en second lieu, les semis se font d'ordinaire dans des terrains entièrement nus ou du moins fort peu couverts; les graines sont donc bien plus exposées à geler ou à se gâter; enfin, plusieurs animaux, les sangliers et les mulots surtout, trouvant peu de nourriture pendant la mauvaise saison, se jettent quelquefois sur les semis artificiels et les ravagent totalement.

Les graines résineuses, quelle que soit d'ailleurs l'époque de leur dissémination naturelle, doivent aussi, en général, se semer plutòt au printemps qu'en automne; les oiseaux, principalement ceux de passage, y causent souvent des dommages considérables dans cette dernière saison. Un inconvénient non moins réel, et qui existe surtout dans les climats un peu rudes, c'est que les graines semées en automne lèvent trop tôt; au retour du printemps suivant, pour 
que les jeunes tiges, tendres et presque toujours peu abritées, ne soient pas souvent victimes des gelées tardives de cette époque de l'année.

\section{ARTICLE VI.}

\section{Des quantités de semence à employer.}

709. Ainsi que nous l'avons dit plus haut [693], il est très important de connaître les quantités de semence qu'il convient d'employer dans les semis. Ces quantités dépendent de la fertilité du sol, de sa déclivité, du climat local et des dangers qui, par suite, peuvent menacer les jeunes plants dans les premières années; mais elles varient surtout selon la grosseur de la graine, selon sa qualité et selon le mode de labour adopté.

Plus la graine sera grosse, plus il en faudra, en poids ou en volume, pour ensemencer une étendue déterminée; si l'on a des doutes sur sa qualité, si les dégâts d'animaux sont à craindre ou si, en général, les circonstances sont peu propices à sa réussite, la quantité à employer dans les cas ordinaires devra être augmentée; enfin, le labour en plein absorbera plus de semence que le labour partiel, soit celui par bandes, soit celui par pots. Nous ferons observer, toutefois, qu'avec ces deux derniers modes de labour, il est nécessaire d'employer proportionnellement plus de semence qu'ivec le labour en plein. 
On conçoit, en effet, qu'il est important, dans un emis par places, par exemple, que toutes les places oient bien peuplées; car si quelques-unes seulenent restaient vides, leur surface, ajoutée à celle les parties environnantes demeurées en friche, fornerait des espaces trop considérables, pour que, de ongtemps, le massif pût se former. Aussi emploie-on toujours, dans un semis partiel, les deux tiers peu près de la semence nécessaire pour un semis n plein, quoique, cependant, il n'y ait à ensenencer que le tiers ou la moitié tout au plus du terain.

En traitant, plus bas, du semis de chaque essence n particulier, nous indiquerons les quantités de raine que l'expérience a démontré devoir être emloyées par hectare, selon les différents modes de abour, en supposant la graine de qualité moyenne Iles circonslances extérieures ordinaires. 
ARTICLE VII.

\section{De la manière de semer.}

710. Dans l'opération du semis proprement dit, il faul porter son attention sur deux objets principaux : le premier, de répandre la graine le plus également possible; le second, de la recouvrir convenablement 1 .

711. Lorsque le terrain à ensemencer est considérable, il est bon de le diviser en parcelles d'égales contenances (de 20 ou 25 ares, par exemple), et de partager aussi la semence en un même nombre de parts égales. Sans cette précaution, il serait difficile de régler le travail des ouvriers semeurs, ou de le corriger à temps, s'il était fautif. Dans un semis en

- Lorsque la graine que l'on doit semer est vieille ou qu'elle a natureliement une enveloppe que l'humidité penètre difficilement, on fera bien de la mettre tremper dans de l'eau vingtquatre heures avant de la répandre. Un procédé plus efficace, cn usage chez beaucoup d'agriculteurs et qu'on a souvent imité avec succès en sylviculture, consiste à immerger la graine, pendant quarante-huit heures, dans du purin. Celte pratique, qui réussit pour les graines lourdes aussi bien que pour les légères, hate singulièrement leur germination et permet, par suite, dans les localités où les gelées tardives sont à craindre, de retarder l'époque du semis sans risquer de différer la levée du plant jusqu'au moment des chaleurs; il prévient aussi l'envahissement du semis par les herbes et autres plontes nuisibles. 
plein, les ouvriers peuvent semer à la volée, comme on sème les céréales; mais, lorsque le terrain est préparé par bandes alternes ou par pots, ils doivent, pour répandre la graine uniformément, imprimer au bras un mouvement de va-et-vient, et ne laisser échapper les semences, la main étant fermée, qu'entre le pouce et l'index. Plus la semence est légère, plus il faut rapprocher la main du sol.

712. Aussitôt semées, les graines doivent être recouvertes. Cette opération se fait, dans les semis en plein, avec une herse dans laquelle, s'il y a lieu, on entrelace des branchages, afin d'empêcher qu'elle ne s'enfonce trop; pour les semences qui n'ont besoin d'être enterrées que très légèrement, on peut aussi employer un fagot d'épines ou le rouleau. Dans les semis partiels, les semences lourdes se recouvrent à la houe, et les semences légères à l'aide d'un râteau en bois ou en fer (voy. fig. 2), selon que le sol est plus ou moins meuble. On se trouvera bien, pour ces dernières, surtout dans les sols légers, de raffermir la terre avec les pieds, ce que les jardiniers appellent tripler, afin de mieux unir la graine aux parcelles terreuses qui l'entourent immédiatement.

Le degré d'épaisseur dont il convient de recouvrir les graines, dépend, à la fois, de leur nature particulière et de la nature du sol. Les semences légères, ayant l'amande petite, et dont le plant, en levant, est très ténu, ne doivent être enterrées que fort peu, tandis que les fruits lourds, ou à noyau 
dur, ont besoin de l'être davantage, afin d'obtenir l'humidité qui leur est nécessaire pour germer. Dans un sol compacte, les graines doivent être peu couvertes; au contraire, dans un sol léger, prompt à se dessécher, il faut les enterrer plus profondément.

713. Il nous reste à parler d'une manière de semer, avantageuse surtout pour exécuter des semis partiels de semences lourdes, c'est du repiquement. Cette opération consiste à ouvrir, à la houe, de petits trous assez rapprochés, dans chacun desquels on place un ou plusieurs fruits, et que l'on recouvre ensuite avec le même instrument, ou simplement avec le pied, si le sol est bien meuble. Non seulement ce procédé permet d'économiser beaucoup la semence, mais encore chaque graine se trouve, en général, mieux enterrée et plus convenablement placée pour prospérer. Dans les lieux clairiérés, dans les coupes d'ensemencement dont le sol est gazonné [482], lorsqu'il s'agit d'établir un mélange d'essences [489], le repiquement est souvent préférable à tout autre mode de semis, parce qu'il peut s'exécuter sans labour préalable.

Outre la houe, on se sert encore, pour cette opération, de plusieurs autres instruments. Le plantoir ordinaire peut être employé; toutefois, dans les sols argileux, il a l'inconvénient de trop tasser la terre et de mettre obstacle, par là, au développement du jeune plant. On a inventé, en Allemagne, un plantoir qui lève cet inconvénient. Cet instrument est en 
fer, rond, d'une longueur de 15 centimètres environ, pointu par le bas, large au milieu de 3 à 6 centimètres, selon la grosseur de la graine qu'il doit servir à repiquer, et partagé par quatre nervures saillantes, le tout surmonté d'un manche (v. fig. 4). Lorsqu'on enfonce le plantoir, on tourne et retourne le fer, ce qui émiette et divise la terre; ainsi fait, le trou se remplit en partie de cette terre émiettée et conserve précisément la profondeur convenable pour y placer une graine, telle qu'un gland, par exemple.

Il est évident, comme nous venons de le dire, que ce plantoir n'est réellement avantageux que dans les terres fortes; car, dans un sol très léger, ce n'est plus à diviser la terre qu'il faut s'appliquer, mais bien à la raffermir. Pour obtenir ce résultat, on a construit, encore en Allemagne, un plantoir-massue (v. fig. כ). . Cet instrument se compose d'un cylindre en bois de chène de 33 centimètres environ de hauteur sur 16 à 18 centimètres de diamètre, cerclé en fer aux deux extrémités et surmonté d'un manche. Au centre de la base inférieure est fixé un boulon de 3 à 6 centimètres de longueur, sur 2 à 4 centimètres de large. Ces dimensions varient suivant la grosseur de la graine qu'on veut repiquer; et, afin de pouvoir modifier l'instrument selon qu'il est besoin, on a des boulons de différentes grosseurs qui se vissent au bout du cylindre en chêne.

Pour faire usage du plantoir-massue, on l'élève verticalement et on le litisse retomber de mime sur 
le sol. Il en résulte d'abord la cavité, formée par le boulon, dans laquelle on place la graine; ensuite, la terre est raffermie par le poids du cylindre et tassée au point de présenter un renfoncement de plusieurs centimètres, dans lequel l'humidité s'amasse, et dont les bords abritent le plant naissant '.

- Cotta conseille de repiquer aussi les semences légères. 11 n'existe aucun motif raisonnable, dit-il, pour ne pas le faire, si ce n'est que le maniement des petites graines est moins aisé, et que, par conséquent, la main-d'wuvre peut revenir trop cher. Mais, comme on peut employer, à cette opération, des femmes, et mème des enfunts, dont les doigts sont plus déliés et dont le salaire est moindre, cette objection n'est pas fondée. 


\section{CHAPITRE TROISIEิUE}

\section{APPLICATION DES RÈGLES GÉNÉRALES}

SEMIS DES ESSENCES LES PLUS IMPORTANTES.

ARTICLE PREMIER.

Semis du chêne.

714. Piécolte et conservation. - La manière de récolter les glands et de les soigner immédiatement après qu'ils ont été amassés, a été expliquée plus haut [693 et 694]. Nous ajouterons seulement qu'il faut éviter de faire amasser les premiers tombés, parce qu'ils sont ordinairement de mauvaise qualité et piqués des vers.

On connait, pour conserver les glands pendant l'hiver, différents moyens, plus ou moins applicables, selon les circonstances dans lesquelles on se trouve :

$1^{\circ}$ Dans un jardin, ou en tout autre lieu clos, on 
choisit une place bien sèche que l'on garnit d'un lit de feuilles, sèches aussi, de la hauteur de 33 centimètres environ. Sur ce lit de feuilles, on place les glands, par tas coniques d'un mètre de haut; on les recouvre d'une couche de feuilles mortes de 33 centimètres d'épaisseur, puis on ajoute encore 16 centimètres de mousse sèche, el 16 centimètres de paille. Sur le tout on établit une couverture en paille comme celles que l'on voit sur les meules de grain ou de foin; enfin, pour mieux garantir le sol de toute humidité, on ouvre un fossé circulaire autour de la place de dépôt.

$2^{\circ}$ On établit des silos ou fosses. S'ils ne doivent servir que peu de temps, on se contente d'en soutenir les parois par des pieux entre lesquels on tresse de la paille; s'ils doivent servir longtemps, on les construit en maconnerie. Dans le fond de la fosse, on met un lit de paille, les glands y sont répandus par couches de 33 centimètres d'épaisseur, alternant avec des couches aussi épaisses de menue paille et de feuilles sèches. Ainsi remplie, la fosse est recouverte de planches, par-dessus lesquelles on élève une butte de terre bien tassée, afin d'empêcher le froid et l'humidité d'y pénétrer.

$3^{\circ}$ On peut remplir de glands des tonneaux ou des caisses, qu'on perce de petits trous et qu'on plonge dans l'eau pour les y laisser jusqu'au printemps. Le séjour des glands dans l'eau les conserve et ne leur ôte rien de leur faculté germinative. 
$4^{\circ}$ On prend de grandes caisses qu'on élève sur des liteaux, dans une cave, de même qu'on place les tonneaux. On remplit ces caisses de couches alternes de sable et de glands. Il faut avoir soin d'employer du sable de rivière bien sec et éviter surtout un sable terreux. La couche supérieure de glands, ayant toujours plus de disposition à germer, doit être recouverte de 22 à 27 centimètres de sable. Il n'est pas nécessaire de donner d'autre couverture à la caisse.

Ces quatre modes de conservation du gland sont donnés par Hartig. D’après l'expérience qu'il en a faite, le premier lui parait préférable; nous pouvons indiquer le quatrieme comme nous ayant toujours parfaitement réussi, alors même que les glands n'étaient extraits des caisses qu'à une époque assez avancée du printemps. Le gland ne peut se conserver au delà du printemps.

715̆. Examex de la grane. - Le gland doit remplir complétement son enveloppe quand on le coupe par le milieu dans le sens de sa longueur, il doit être blanc. frais et luisant; le germe qui se trouve à la partie supérieure doit être intact. Si, au contraire, le fruitest desséché, d'une couleur bleuâtre uu noirâtre intérieurement, s'il a une odeur de moisi, ou s'il est piqué, sa faculté germinative est détruite.

Un autre moyen de juger de la bonté des glands consiste à en jeter un nombre déterminé dans un vase rempli d'eau: ceux qui tombent au fond sont 
bons pour la plupart, ceux qui surnagent sont mauvais. Enfin, on les juge aussi parr le poids : un litre de glands de bonne qualité doit peser environ de 550 à 600 grammes.

716. Exécution du sexis. - Lorsque le terrain à repeupler est en plaine, d'une nature compacte, garni seulement d'arbustes et de gazon, il convient de le préparer en y cultivant, soit des céréales, soit des pommes de terre, pendant une ou deux années selon qu'il sera plus ou moins facile de le nettoyer et de l'ameublir. Ce résultat obtenu, on donne un dernier labour à la charrue, et l'on répand les glands avec une demi-semaille de seigle, si c'est en automne, et d'avoine ou d'orge, si c'est au printemps.

Le semis ne doit ètre recouvert que de 3 ou 4 centimètres au plus. La herse est l'instrument le plus convenable pour cette opération. A la récolte des céréales, il faut avoir soin de couper les chaumes à une certaine hauteur, afin de ne pas endommager les jeunes chênes.

Dans les sols légers ou en pente, le labour partiel, soit par bandes, soit par places, doit ètre préféré, et, selon les circonstances, on répand les glands pour les recouvrir ensuite arec la houe, ou bien on les repique. Si les herbes ou les arbustes sont rares, ce dernier mode peut même être pratiqué sans aucun labour préalable.

Daus les terres légères, le gland doit être recouvert de 5 à 6 centimètres. 
Semé en automne 1 , il lève au bout de cinq à six mois, semé au printemps, au bout de quatre à six semaines. Cette dernière saison est toujours préférable. ainsi que nous l'avons dit plus haut [668]; mais, quand on a des semis très considérables à faire et qu'on ne peul, par divers motifs, garder jusqu'au printemps toute la quantité nécessaire de glands, il convient de partager l'opération entre les deux saisons, en prenant la précaution de semer en automne avec plus d'abondance, afin de faire la part des intempéries et des animaux.

Quoique le jeune chêne soit d'un tempérament très robuste et ne réclame en général aucun abri [72], on se trourera bien cependant, dans les départements de l'ouest et du midi, de lui adjoindre, dins la proportion d'un quart ou d'un cinquième, une ou plusieurs essences ayant une croissance rapide dis les premières années, telles que bouleau, pin sylvestre ou maritime, etc. bès que ces essences auront atteint les dimensions propres à faire de menus fagots, et que les chènes d'ailleurs se montreront suffisamment vigoureux, on s'empressera de débarrasser ceux-ci des essences auxiliaires, en les enlevant par forme de nettoiement.

- On fera bien de ne pas semer avant la mi-novembre, et plus tard dans les climats doux, afin d'empêcher la gernination d'automne qui rendrait le gland vietime des gelées de l'hiver. 
Les quantités de glands à employer, par hectare, sont:

Pour un semis en plein, 15 à 16 hectolitres; Pour un semis partiel, $\quad 10$ à 12 id. Pour le repiquement, $\quad 6$ à $7 \quad$ id.

ARTICLE II.

Semis du hêtre.

717. Récolte et conservation. - La faîne se récolte et se conserve comme le gland (1). Hartig indique, en outre, pour la conservation de cette semence, un procédé très simple et qu'il dit avoir souvent employé avec un entier succès.

Après avoir étendu les faînes dans un endroil aéré, et les avoir journellement retournées, pour en faire évaporer toute l'humidité [694], il suffit de les entasser, à une hauteur de 33 à 66 centimètres, sur le plancher d'une chambre close et de les recouvrir de 33 centimètres environ de paille, afin de les garantir du froid.

La faîne, à moins de précautions minutieuses et impossibles à pratiquer en grand, ne peut se garder que de l'automne au printemps.

718. Examen de la graine. - La qualité de la faine s'apprécie, en général, d'après les mèmes ca-

1 Cotta dit que la faine ne se conserve pas dans l'eau. 
ractères que celle du gland. On peut, en outre, en juger par le goût du fruit; il doit être agréable et rappeler celui de l'amande ou de la noisette. Une saveur rance indique la mauvaise qualité.

Un litre de faînes doit peser de 405 à $425 ٌ$ grammes.

719. ExÉcution du SEMis. - Le tempérament très délicat du jeune hêtre rend, en général, impraticable le semis en plein de cette essence, sur un terrain entièrement nu. Quand bien même on mélangerait une forte quantité de céréales à la faìne, on ne remédierait pas à cette difficulté, puisque l'abri est nécessaire pendant plusieurs années; aussi faut-il, pour réussir dans une opération de ce genre préparer, quelque temps à l'avance, l'abri indispensable.

Dans ce but, après que le terrain a été convenablement labouré, on le partage par bandes dont, sur deux, on en sème une d'essences ayant une végétation rapide, telles que bouleaux, ormes, pins, etc.; on peut aussi, pour plus d'économie, employer, selon le climat, le genêt ou l'ajonc. Si l'on veut gagner du temps, il faut planter ces essences au lieu de les semer; car ce n'est que quand elles auront atteint une certaine hauteur, qu'on pourra répandre la faine dans les bandes intermédiaires restées vides. L'abri créé de cette manière doit ètre conservé au jeune hêtre jusqu'à ce qu'il en éprouve quelque gêne, ou du moins jusqu'à ce qu'il soit assez fort pour résister 
aúx influences atmosphériques; alors les essences auxiliaires pourront être extraites par forme de nettoiement.

Toutefois, le mode d'opérer que nous venons d'indiquer ne saurait être suivi dans les terrains fortement inclinés, où le défrichement total du sol pourrait amener l'éboulement des terres. Dans ce cas il faudrait, comme à l'ordinaire, diviser la superficie en bandes horizontales de 66 centimètres à 1 mètre de largeur, dont l'une resterait inculte et dont l'autre serait préparée à l'ensemencement projeté. Celle-ci serait elle-même partagée en deux portions; dans la première, on planterait ou l'on sèmerait à l'avance, comme nous venons de l'expliquer, et l'autre, en temps opportun, recevrait la faîne.

Les difficultés d'exécution dont est entouré le semis de la faine en terrain découvert, les frais qu'il entraîne et les chances défavorables auxquelles il est exposé rendent cette opération peu avantageuse, et doivent, en général, faire choisir d'autres essences pour le repeuplement des terres vagues, ou, si l'on tenait expressément au hêtre, faire préférer la plantation comme plus sûre [691]. C'est plus particulièrement dans les futaies clairiérées, dans certains taillis entièrement épuisés, ou bien dans d'autres parties totalement envahies par les bois blancs ou les morts-bois que l'on pourra semer la faîne avec succès [รั57]. Dans de telles circonstances, le repiquement sera surtout convenable; on fera bien de jeter 
plusieurs semences dans le même trou, parce qu'il s'en trouve souvent qui sont vaines. Lorsque les jeunes plants de hêtre auront acquis suffisamment de force, les autres essences devront être extraites par forme de nettoiement.

La faîne ne doit être recouverte que de 15 à 30 millimètres, suivant la nature plus ou moins compacte du sol. Il faut faire en sorte que la terre soit bien divisée à sa surface; autrement, le jeune plant qui lève avec deux lobes séminaux très amples ne pourrait la percer.

La faine, semée en novembre, lève au bout de cinq à six mois; semée au printemps, qui est la saison préférable, les plants lèvent au bout de trois à six semaines.

Pour un semis en plein, il faut, par hectare. de.......... 8 à 10 hectolitres; pour un semis partiel, de. . 6 à 7 id. et pour le repiquement, de. . 3 à 4 id.

720. Cotta indique, dans son traité de culture des bois, un mode particulier de semer la faine sans donner aucun abri au jeune plant, mode qui, dit-il, lui a parfaitement réussi. Voici en quoi il consiste.

Le terrain étant partagé par bandes alternes, on creuse, dans le milieu de la bande cultivée, une rigole de 10 à 12 centimètres de largeur, sur autant de profondeur environ; c'est dans cette rigole qu'on sème la faîne. Immédiatement après la levée des plants, on comble la rigole, en y tirant les terres 
voisines, de manière à en entourer complètement les petites tiges jusqu'aux feuilles séminales. Il paraît, ajoute Cotta, que c'est surtout la tige des jeunes hêtres qui souffre des influences atmosphériques, et, que si on la préserve d'une manière quelconque, les jeunes plants peuvent se passer d'ombrage.

L'auteur que nous citons mérite la plus grande confiance, et nous sommes d'autant plus porté à admettre le procédé qu'il recommande, que nous connaissons un fait qui en confirme entièrement l'efficacité.

Dans la forèt de Compiègne, ci-devant domaine de la Couronne, on exécute, chaque année, des plantations très considérables de différentes essences et entre autres de hêtre. Ordinairement, les sujets plantés proviennent de semis faits en pépinière et ne sont définitivement mis en place qu'après avoir subi, dans la pépinière même, une première transplantation. Mais, pour le hêtre en particulier, comme on connait les difficultés de le semer en terrain découvert, on se procure les jeunes plants, qui doivent subir cette première transplantation, dans les massifs de futaie où ils lèvent en abondance. Ce sont les plants naissants, munis encore de leurs feuilles cotylédonaires, que l'on choisit de préférence; on les extrait de terre avec un couteau, puis, dans la pépinière, on les place, à ciel ouvert, dans des rigoles semblables à celles dont parle Cotta, en 
prenant aussi la précaution de les enterrer entièrement jusqu'aux feuilles séminales. Qnand l'été est très chaud, on perd néanmoins beaucoup de ces plants; mais ceux qui résistent, et en général c'est le très grand nombre, deviennent, dès lors, suffisamment robustes pour supporter les intempéries de tout genre.

Cette pratique du butlage des semis de hêtre en terrain découvert est aujourd'hui généralement admise en Allemagne, et les meilleurs auteurs la recommandent, tout en reconnaissant, cependant, que la plantation reste toujours le moyen le plus sùr et le plus facile pour établir le hètre sur un sol entièrement dénudé.

ARTICLE III.

\section{Semis du ohâtaignier.}

721. Récolte et conservation. - Les châlaignes se récoltent et se conservent comme les glands. Le moyen de conservation qui nous a toujours le mieux réussi est la stratification dans le sable, telle que nous l'avons décrite plus haut; on peut aussi les garder assez longtemps, en les laissant dans leur enveloppe extérieure appelée communément hérisson. La châtaigne ne se conserve que pendant un hiver.

722. Examen de la graine. - La qualité des châtaignes se reconnait par les mèmes caractères que 
celle des glands et des faìnes. La châtaigne doit avoir une silveur agréable, quoique légèrement acerbe. On a prétendu que la grosseur du fruit devait être prise en considération et qu'il fallait éviter de semer de petites châtaignes, parce qu'elles produisent des plants d'une faible végétation. C'est une erreur. Quelle que soit leur grosseur, les châtaignes produisent de bons plants, pourvu qu'elles proviennent d'arbres bien portants, qu'elles soient saines, fermes, bien remplies, et que le germe soit intact .

723. Exécution du semis. - Le châtaignier demande, plus que toute autre essence, un sol bien nettoyé. On doit, à cet effet, donner un labour convenable, soit à la charrue, soit à la houe; puis disposer le terrain par bandes alternes. Dans l'une, de 16 centimètres de largeur, on repique les châtaignes; dans l'autre d'une largeur d'un mètre environ, on cultive, pendant plusieurs années, des pommes de terre, des betteraves, etc., afin d'empêcher la crue des plantes nuisibles.

Le repiquement des chàtaignes se fait ordinairement assez dru, en raison des ennemis qu'elles ont à redouter et des différentes chances auxquelles elles sont exposées [708]. Si les plants levaient trop épais en certains endroits, on aurait toujours la facilité d'en extraire une partie pour garnir les places où le semis aurait moins bien réussi.

Le mode de semis dont nous venons de parler, s'emploie plus particulièrement pour élever le châ- 
taignier en massif de futaie. Quand il s'agit d'un taillis, on prépare le terrain de même, mais, au lieu de semer en rigoles, on établit communément des trous ou pots peu profonds, espacés d'un mètre à un mètre et demi, dans chacun desquels on repique deux ou trois châtaignes. Comme il suffit de laisser, dans chaque trou, une seule tige, on dispose des plants superflus au bout de deux ans. A l'âge de cinq ou six ans, et même plus tòt, selon sa végétation, on recèpe le jeune plant avec soin, après avoir donné, dans l'intervalle, au moins deux cultures à la terre.

On a prétendu qu'il était à propos d'abriter les jeunes châtaignier's par des bouleaux, par des saules marceaux, ou par d'autres essences d'une croissance rapide. Cette précaution est moins utile dans l'Est et le Nord de la France où le jeune châtaignier, s'il occupe les expositions qui lui conviennent, n'a pas besoin d'abri. Dans les régions plus chaudes, un tel mélange pourra être mieux à sa place.

La châtaigne ne s'enterre que de 3 à 6 centimètres au plus, selon la nature du sol. Quand on la sème en automne, elle lève après cinq ou six mois; si elle n'est semée qu'au printemps, le plant paraît au bout de trois à six semaines. Cette dernière saison doit en général être préférée.

Pour semer en rigoles, ainsi que nous l'avons indiqué plus haut, il ne faut que 9 ou 10 hectolitres de châtaignes, par hectare; quand on repique par 
pots ou trous, on conçoit que la quantité doit être de beaucoup inférieure ; 2 ou 3 hectolitres, au plus, doivent suffire.

\section{ARTICLE IV.}

\section{Somis de l'ormo.}

724. Récolte et conservation. - On récolte les semences d'orme en dépouillant les rameaux à la main. Aussitôt qu'elles sont cueillies, il est essentiel de les étendre, en couches minces, dans un lieu bien aéré et de les remuer souvent. Pour peu qu'on les laisse en tas, elles s'échauffent et perdent leur faculté germinative. En continuant à leur donner les mêmes soins, on peut rigoureusement les conserver jusqu'au printemps suivant; mais il est préférable, sous tous les rapports, de les semer immédiatement après leur maturité.

725. Examen de la graine. - La semence qui est placée au centre de la membrane doit être un peu élevée et ferme au toucher; en la coupant transversalement et en l'écrasant sur l'ongle, il faut qu'elle soit farineuse en même temps qu'humide; en outre, elle doit dégager une odeur fraîche, agréable el avoir une saveur oléagineuse prononcée.

Un litre de semence d'orme doit peser environ 40 grammes.

726. Exécution du semis. - Le labour par bandes 
alternes ou par trous carrés est le plus avantageux pour le semis de l'orme. Comme il est convenable de semer la graine aussitôt après sa maturité qui a lieu au commencement de juin, on fera bien de chercher à donner un premier abri aux jeunes plants, afin qu'ils résistent mieux aux chaleurs de l'été. A cet effet, si le sol est en plaine ou en pente douce, on pourra, dès le printemps, tracer les bandes destinées à la graine d'orme et ensemencer le reste du terrain en orge ou en avoine. De cette manière, l'abri sera assuré aux jeunes plants aussitôt qu'ils lèveront, et l'on obtiendra en mème temps un produit en céréales, ce qui n'aurait pu avoir lieu si on ne les avait semées qu'avec la graine d'orme. Lorsque le terrain sera fortement incliné, on pourra, ou subdiviser la bande cultivée [719], ou se contenter de mélanger la graine d'orme aux céréales, saul' à faire le sacrifice de celles-ci.

La semence d'orme doit ètre très légèrement recouverte; elle n'a même besoin de l'être que pour empêcher le vent de la disperser. Semée aussitôt après sa maturité, elle lève au bout de quinze jours ou de trois semaines 1, et, dès l'automne, les jeunes plants atteignent une hauleur de 16 à 22 centimètres.

1 Quand les ètés sont très chauds et secs, il arrive assez soulvent que le semis ne lève que particllement. Mais d'ordinaire, le succès n'est que retardé et se produit au printemps suivant. 
Pour effectuer un semis d'orme en plein, il faut employer, par hectare, de 28 à 30 kilogrammes de semence; pour le semis partiel, 18 à 22 kilogrammes peuvent suffire.

ARTICLE V.

\section{Somis du frône.}

727. Récolte et conservation. - Le meilleur moyen pour récolter la semence du frène est de la cueillir à la main; si l'on voulait emplover la gaule, il faudrait choisir, pour cette opération, un temps parfaitement calme.

Cette graine est sujette à se dessécher, et se conserve, en général, assez difficilement; il est bon de la mélanger avec du sable et de la placer dans un endroit frais. Ce qui vaut mieux, c'est d'ouvrir, dans quelque terrain, des rigoles ou fosses de 33 à 50 centimètres de profondeur, sur une longueur et une largeur proportionnées à la quantité de graines recueillies, d'y jeter cette graine en amas, puis de la couvrir de 12 à 16 centimètres de terre. Comme la semence de frène ne germe que lil seconde année, après avoir été mise en terre, on pourra, si l'on veut semer en automne, la laisser dans les rigoles pendint un an, et même jusqu'au printemps suivant, si c'est dans cette dernière saison seulement que doit s'effectuer le semis. Ce procédé a l'avantage de con- 
server la semence en très bon état et, de plus on sait, dès la première année, à quoi s'en tenir sur la réussite du semis.

728. Exanex de la graine. - Pour juger des semences de frène, on en coupe plusieurs transversalement. Si l'intérieur présente une substance d'un blanc bleuàtre et de la consistance de la cire, la graine est bonne; elle est mauvaise si cette substance est entièrement desséchée.

Le litre de cette semence dnit peser à peu près de 170 à 180 grammes.

729. Exécution nu semrs. - Le labour par bandes alternes ou par trous carrés est, en général, le meilleur pour les semis de frêne. Il faut faire en sorte que les jeunes plants soient un peu abrités, au moins la première année, et, dans ce hut, leur adjoindre des céréales, lorsque la nature du terrain le permel. On devri éviler de laisser prendre le dessus à la mauvaise herbe qui fait beaucoup de tort aux jeunes frênes.

Sous ce dernier rapport, il est très avantageux que la semence ait été préparée dans des fosses, parce qu'alors elle leve tout de suite, sans laisser aux plantes nuisibles le temps d'envahir le terrain.

Les semences de frêne ne doivent être enterrées que de 15 à 20 millimètres. Préparées comme nous venons de l'indiquer plus haut, et semées en automne, elles lèvent au commencement du printemps suivant; quand elles sont semées au printemps, les 
plants paraissent au bout de quatre à six semaines.

Les quantités de semences de frêne à employer par hectare, sont:

Pour le semis en plein, de 40 à 45 kilogrammes ; et, pour le semis partiel, de 27 à 38 id.

ARTICLE VI.

\section{Semis do l'órable.}

730. Récolte et conservation. - La récolte des semences d'érable a lieu comme celle des semences de frène. Quant à la conservation, il suffit, après le avoir laissées ressuyer dans un lieu aéré, de les mettre en las dans un local clos et de les retourner de temps en temps; on peut aussi les mélanger avec du sable. Traitées de la sorte, leur faculté germinative peut se conserver jusqu'au second printemps, mais il est toujours bien préférable de les semer dès le premier.

731. Examen de la graine. - Pour vérifier les graines d'érable, on en ouvre plusieurs. Elles sont bonnes lorsque, sous les capsules qui les enveloppent, on les trouve fraîches, flexibles, et d'une couleur verte. Ce dernier caractire seul, cependant, ne suffit pas pour décider de la qualité; il existe souvent quand déjà il y a desséchement. II faut donc qu'indépendamment de la couleur, la graine présente du moelleux et de la fraicheur. 
Un litre de semence d'érable de bonne qualité doit peser de 120 à 130 grammes.

732. Exécution du semis. - Ce que nous avons dit, à cet égard, du semis de frêne, s'applique aussi à celui de l'érable.

Le printemps est la saison la plus propice pour semer, les jeunes plants étant assez délicats et craignant les gelées printanières.

Pour ensemencer un hectare en plein, il est nécessaire d'employer de 60 à $6 \breve{~ k i l o g r a m ı n e s ~ d e ~ s e-~}$ mence d'érable; et, pour un semis partiel, il en faut de 40 à 45 kilogrammes.

ARTICLE VII.

\section{Semis du boulear.}

733. RÉcolte et conservation. - Comme la semence du bouleau se dissémine très promptement, il faut saisir à point l'époque de sa maturité pour la récolter. On cueille les chatons à la main. Un procédé plus facile, mais qui ne peut s'exécuter que sur des bouleaux destinés à ètre abattus prochainement, c'est de couper les rameaux qui portent la graine. Dans ce cas, iln'est pas nécessaire d'attendre l'entière maturité; en suspendant les rameaux coupés dans un endroit sec et aéré, la graine y achèvera de mûrir.

Lit semence de bouleitu est très difficile à conser- 
ver, et il est à conseiller d'en faire le semis l'année même de la récolte. Néanmoins, si l'on était forcé de la garder jusqu'au printemps suivant, il faudrait l'étendre, en couches minces, dans un grenier, la garantir des froids de l'hiver et la remuer très souvent.

734. Examen de LA Graine.-L'examen des graines de bouleau se fait comme celui des semences d'orme. Si l'on en ouvre quelques-unes, avec la pointe d'un canif, par exemple, la graine doit être farineuse; écrasée sur l'ongle, elle doit laisser quelques traces d'un suc laiteux.

Un litre de cette graine doit peser de 90 à 100 grammes.

733. Exécution du semis. - Le labour partiel convient plus particulièrement pour les semis de bouleau. Ce qu'il faut éviter surtout, c'est de trop enterrer la graine. On se contentera donc, pour établir les bandes ou les pots, de lever le gazon et d'extraire les arbustes; il suffira même, si le terrain n'est pas très gazonné, de racler la surface avec les dents d'un râteau en fer (voy. fig. 2).

En semant, il faut tenir la main le plus près possible de terre 1, afin d'empêcher que le vent

' Une bonne précaution pour faciliter l'opération du semis de la graine de bouleau, comme de toute graine très légère en général, consiste à ne la répandre que mêlée à une certaine proportion de terre très fine. 
n'emporte la graine. Le temps le plus favorable, pour cette opération, est un temps pluvieux, parce qu'alors on peut se dispenser d'enterrer la semence; mais si l'on sème par un temps sec, on devra faire en sorte de mêler seulement la graine à la terre, en promenant légèrement un râteau en bois dans les parties semées ou bien en les triplant [712] avec les pieds.

Semée en automne qui, comme nous l'avons dit. est la saison la plus convenable, la semence de bouleau lève dès le commencement du printemps.

Si l'on voulait faire un semis en plein, il faudrait employer, par hectare, de 36 à 40 lillogrammes de semence; pour les semis partiels, il suffira de 24 à 30 kilogrammes. En général, la graine de bouleau ne doit pas être épargnée, parce qu'elle est rarement de très bonne qualité.

ARTICLE VIII.

\section{Semis du robinier faux acacia.}

736. Récolte et conservation. - Les gousses du robinier se cueillent à la main. Étendues sur un grenier, elles s'ouvrent d'elles-mêmes pendant l'hiver ; il suffit ensuite de les remuer légèrement avec un râteau pour détacher les semences [179]. On sépare les graines des gousses, à l'aide du crible.

Cette semence peut se garder deux et même trois 
ans; on la conserve en tas, dans des greniers, en ayant soin d'aérer de temps à autre.

737. Examen de la graine. - La graine du robinier doit être brun foncé; l'intérieur doit être blanc, farineux et frais.

738. ExÉcution du semis. - Le semis partiel mérite la préférence. Un sol bien nettoyé favorise la végétation des jeunes plants.

Il convient de recouvrir la semence de 5 à 10 millimètres; et comme le plant, au moment de sa naissance, redoute les froids, on fera bien, quand ce sera possible, de garnir le sol d'une couche de mousse ou de feuilles mortes.

Cette susceptibilité du jeune plant doit faire préférer le printemps pour le semis du robinier. Mise en terre dans cette saison, la graine lève après trois ou quatre semaines; semée en automne, au contraire, le plant ne paraît qu'au bout de cinq ou six mois.

Quatorze à seize kilogrammes de semence de robinier sont nécessaires, par hectare, pour un semis par bandes ou par pots; si l'on voulait semer en plein, il faudrait employer, de 20 à 25 kilogrammes. 
ARTICLE IX.

\section{Semis du charme.}

739. Récolte et conservation. - Les semences de charme se cueillent à la main; on peut aussi, mais seulement par un temps très calme, faire gauler les arbres en tendant des toiles pour recevoir la graine. Les moyens de conservation sont absolument les mêmes que ceux que nous avons indiqués pour la semence de frêne [727]; comme celle-ci li graine de charme se conserve difficilement, et, mise en terre, elle ne germe que la seconde année.

740. Examen de ea graine. - En ouvrant la graine de charme, on doit la trouver blanche et fraiche; l'amande doit remplir complétement le noyau qui lui sert d'enveloppe. Un litre de semence ailée doit peser de 50 à 60 grammes; si la semence est désailée, la même capacité pèsera de 410 à 420 grammes.

741. Exécution du semis. - Ce qui a été dit pour l'exécution du semis de frêne [729] s'applique entièrement au semis de charme; seulement, la graine de cetle dernière essence pourra, dans les sols légers, être enterrée jusqu'à 3 centimètres de profondeur. 
Les quantités de semence de charme à employer par hectare sont :

Semence ailée,

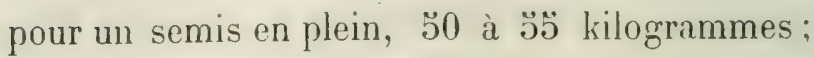
pour un semis partiel, 33 à $38 \quad$ id.

Semence désailée,

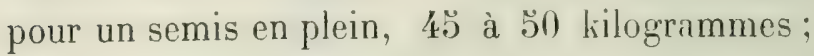
pour un semis partiel, 30 à 33 id.

Semis de l'alisier, du sorbier et du micocoulier.

742. Ces essences ne sont pas ordinairement cultivées en grand; mais il est intéressant d'en faire des semis en pépinière, pour les répandre ensuite, par la plantation, dans les forêts.

Les fruits se cueillent à l'arrière-saison; on les sème en rigoles, en évitant de trop les rapprocher, et on les recouvre de bonne terre bien émiettée. Les semences de sorbier demandent à être enterrées de 6 à 8 millimètres seulement; celles de micocoulier de 10 à 10 millimètres, et les alises de 3 à 4 centimètres. Ces deux dernières graines ne lèvent, en général, que le second printemps après avoir été semées à moins que, par des arrosements fréquents, on ne hâte leur germination.

Le micocoulier, ainsi que nous l'arons dit plus 
haut [215\%], redoute les froids dans ses premières années et, dans le Nord et dans l'Est de la France, a besoin d'être recouvert de paille, de feuilles mortes ou de mousse pendant l'hiver.

Lorsque les plants de ces trois essences ont deux ans à peu près, on leur fitit subir une première transplantation en pépinière, afin de favoriser le développement des racines; à cet effet, on les espace de 22 à 33 centimètres.

Parvenus à 66 centimètres ou à 1 mètre de haut, on les plante dans les lieux auxquels on les destine.

ARTICLE XI.

Semis de l'aune.

743. Récolte et conservation. - On cueille les petits cônes à lat main, dès qu'ils commencent à brunir; il faut se hàter, car la semence se dissémine très promptement. Cette récolte peut aussi se faire, comme celle des semences de bouleau [733], en coupant les branches qui portent graine sur les arbres destinés à être prochainement abattus; nous avons indiqué les avantages de ce procédé.

Pour obtenir la graine, il suffit d'étendre les cônes sur un plancher bien aéré et de les remuer souvent; les écailles s'entr'ouvrent peu à peu et la semence s'échappe. Mais si l'on voulait semer dès 
l'automne, il faudrait exposer les cònes ì une chaleur tempérée, afin de les faire ouvrir plus vite. Pour séparer la graine des cònes, on se sert du crible.

La semence d'aune ne se conserve que de l'automne au printemps. On la met ordinairement en tas sur un grenier; on peut aussi la plonger dans l'eau.

74́. Exanex de la grane. - La semence d'aune doit présenter ì peu près les mêmes caractires que celle d'orme ou de bouleau. Ouverte et écrasée sur l'ongle, l'amande doit ètre farineuse, légèrement humide, et dégager une odeur fraîche et agréable. La couleur de la graine est brun-marron.

Un litre de cette semence, lorsqu'elle est de bonne qualité, pèse de 320 à 340 grammes.

тй. Exícutiox nu semss. - Les sols humides, aquatiques, ou mème marécageux, dans lesquels l'aune prospère plus particulièrement, offrent des difficultés pour le semis. I Lal grande quantité d'herbes dans les premiers, une crue d'eau dans les autres, s'opposent souvent, soit à la germination de la graine, soit au développement du jeune plant. Aussi a-t-on plus généralement recours à la plantation pour établir une aunaie [691]. Cependant, comme les inconvénients que nous renons de signaler ne se présentent pas toujours, on pourra aussi, dans certains cas, employer la voie du semis.

Le labour en plein pourra ètre pratiqué, lorsque le sol le permeltra et qu'on n'aura pas besoin 
d'épargner la graine; toutefois, le labour partiel est généralement préférable. La principale précaution à prendre, pour préparer le terrain, consiste à le remuer le moins possible, tout en le débarrassant de la mauraise herbe. Souvent il sera très avantageux d'écobuer, et, dès lors, il suffira de gratter la terre, soit à la herse, si l'on veut semer en plein, soit avec le ràtenu en fer, s'il s'agit de cultiver par bandes ou par pots. Dans certains terrins, on pourra même se contenter d'introduire des bètes à cornes ou des moutons. Il n'est pas rare en effet de voir, dins les prairies, des semis naturels d'aune très bien réussis dans les pas des bestiaux ou dans les rigoles d'irrigation.

La semence ne doit, pour ainsi dire, pas ètre enterrée. Dans les semis en plein, on pourra se contenter de passer le rouleau pour la raffermir contre li terre, à moins qu'on ne redoute les dégàts des oiseaux, auquel cas il faudrait employer le firgot d'épines. Dans les semis partiels, on se servira, le plus légèrement possible, du râteau de bois. Semée en automne, qui est la saison la plus firvorable, la graine lève au mois d'avril suivant; semée au printemps, elle produit des plints au bout de cing ou six semaines.

Pour un semis en plein, il faut, par hectare, de 10 à 12 kilogrammes de semences d'aune; pour le semis partiel, 6 à 8 lilogrammes suffisent. 
ARTICLE XII.

\section{Semis du sapin.}

T'46. Récolte et coxservatjox. - On cueille les cònes à la main, dès la fin de septembre ou au commencement d'octobre. II suffit de les étendre sur un grenier et de les remuer de temps en temps au râteau, pour faire tomber les ícailles et les graines; on sépare ensuite les unes des autres au moyen du crible.

Si l'on veul dépouiller la semence de l'aile dont elle est garnie, il faut la frotter entre les mains ou dans un sac rempli au quart seulement. Quoi qu'on fasse, on ne pourra que briser une partie de l'aile; il est impossible de l'enlever entièrement parce qu'elle est très adhérente.

La graine de sapin ne se conserve guère au delà de 18 mois; si donc on ne l'emploie pas au printemps qui suit sa maturité, il faut, au plus tard, la semer un an après. Le meilleur moyen de conservation est de la mettre en lieu sec, à l'abri du froid, et de l'entasser le moins possible. D'abord il faut avoir soin de lis retourner souvent, et ensuite seulement de temps à autre, jusqu'au moment de l'employer.

747. Exhmex de la ordine. - En ouvrant les graines du silpin, on doit les trouver pleines, 
fraiches et d'une couleur blanchâtre; elles doivent dégager une odeur prononcée de térébenthine, et le germe doit être vert. La couleur extérieure est brune.

Un litre de cette semence, munie de son aile. pèse ordinairement de 200 à 21 ğ grammes; Iorsqup la graine est désailée, le poids de la même capaciti: est de 265 à 275 grammes.

748. Exécution du skMis. - Tout ce que nous avons dit plus haut [719] sur les procédés à employer pour semer le hêtre, el sur les difficultés que cette opération présente, s'ipplique entièrement au semis du sapin, à cause de l'extrême délicatesse du jeune plant. Le mode indiqué par Cotta, pour semer le hêtre sans abri [720], n'i encore réussi qu'en petit pour le sapin, et la plantation ne peut guère suppléer au semis, car cette essence résineuse, surtout en terrain découvert et aux expositions chiudes, est d'une reprise fort difficile. C'est principalement pour remettre en étal des parties de forìts ruinćes, couvertes de bois blanes, de morts-bois ou de hroussailles quelconques, que l'on peut employer le semis du sipin (1). Comme le hêtre, dans ce cas, on sèmerale

- T'outes les fois qu'on pourra abriter les semis de sapin, de. manière à préserver les jeunes plants des gelées tardives du printemps, on fera bien de répandre la graine dans l'automne même de sa maturité. Il est certain qu'unc quantité notable de semences, quelques précautions que l'on prenne pour les conserver, perd sa faculté germinative dès le premier hiver. 
sapin par places, à l'ombre de ces broussailles; ou même on repiquera la graine, si elle est rare, mais en ayant soin de ne remuer la terre que le moins possible (2).

La semence doit être recouverte, avec le râteau, à une épaisseur de 6 à 9 millimètres.

Il est difficile d'indiquer la quantité de semence nécessaire, par hectare, pour un semis fait de la sorte.

En général, il faut semer abondamment, car la graine n'est pas toujours de très bonne qualité, surtout lorsqu'elle a été conservée pendant quelque temps; et d'ailleurs les jeunes plants, fort délicats, ont bien des chances à courir. Pour un semis par bandes ou par pots, on peut employer de 40 à 45 lilogrammes de semence ailée, et de 36 à 40 kilogrammes de semence désililée, par hectare.

Ordinairement on sème depuis la fin de l'hiver jusque vers la fin du mois de mai, selon que les gelées printanières sont plus ou moins à craindre. Dans ce cas, les jeunes planls paraissent au bout de quatre à six semaines.

2 Pour repiquer des graines légères, telles que celles du sapin, ou pour les semer, par places, sous des bois blanes ou sous des broussailles, l'instrument le plus commode est la houe-râteau dont nous donnons le dessin à la fin du volume (roy. fig. 3). Les dimensions à lui donner peuvent varier selon la nature des terrains dans lesquels elle doit fonctionner. 
ARTICLE XIII.

\section{Semis de l'épicéa.}

749. Récolte et conservation. - La récolte des cônes d'épicéa peut avoir lieu depuis le mois de novembre jusqu'au mois de inars. Quand on doit faire de grinds approvisionnements, il faut s'y prendre aussitôt après la maturilé [287]; dans le cas contraire, il est préférable de ne cueillir les cônes qu'après les froids, parce que plus on rapproche cette opération de l'époque de la dissémination naturelle. plus on extrail ensuite la graine avec facilité.

L'extraction des graines a lieu, soit à l'aide de la chaleur artificielle d'un foirneau, soit à l'aide de la chaleur du soleil. Quoique ce dernier moyen mérite d'ètre préféré, en ce sens qu'on en obtient généralement des graines d'une qualité supérieure, le premier est cependant le plus employé, parce qu'il est beaucoup plus expéditif, et qu'il permet mieux de pourvoir à des approvisionnements considérables.

Hartig décrit les appareils à établir pour l'un el l'autre mode; nous donnons ci-après ces descriptions telles qu'elles sont traduites dans le Dictionnaive des Forêts de Baudrillart:

" $1^{\circ}$ On se sert d'une chambre dans la partie in" férieure d'un hàliment en maçonnerie; s'il est pos- 
" sible, on place dans cette chambre un ou plu-

" sieurs poêles pourvus de grils, afin de pouvoir

" les échauffer arec les cônes vides; ou bien on y

" établit circulairement des canaux de chaleur,

" comme dans une serre chaude, afin que le local

" puisse être échauffé dans toutes ses parties à un

" assez haut degré de température. Dans cette étuve,

" on fait construire, contre les murs et dans le mi-

" lieu de la pièce, des échafaudages sur lesquels on

" puisse placer des claies en bois ou en fil de fer de

" 1 mètre 66 centimètres à 2 mètres de longueur sur

" 82 centimètres de largeur, et formant des étages de

" 16 centimètres environ d'intervalle. Sous la der-

") nière rangée de claies, on fait pratiquer des tiroirs

" pour recevoir la graine. Ces premières disposi-

"tions prises, on charge les claies de cônes et on

" chnuffe l'éture de manière qu'un homme en puisse

") difficilement supporter la chaleur 20 à $2 \partial$ de-

") grés Réaumur; ${ }^{1}$; on entretient cette température

" jusqu'à ce que les cônes se soient ouverts. Alors

" on les remue fortement sur toutes les claies, en

" commençant par les étages supérieurs, de manière

" que les semences tombent d'élage en élage, jus-

" qu'aux tiroirs placés sous les claies inférieures ".

" Lorsque tous les cônes sont ouverts aussi com-

' Cotta dit que la température peut s'élever jusqu'à 30 et même $33^{\circ}$ Réaumur, sans que la faculté germinative de la graine ait à en soufririr.

Pour plus de facilite on a rendu les claies mobiles, en les: 
" plètement que possible, on les retire et l'on cher" che à obtenir encore la semence qui a pu y rester.

" A cet effet, on les place dans un vaisseau dont la " disposition est semblable à celle d'une baratte à " battre le beurre. Dans ce vaisseau, qui doit avoir "le fond à claire-voie serrée, afin que les semences " seules puissent y passer et être reçues dans un " vase placé au-dessous, on agite fortement les cônes ") jusqu'à ce qu'ils soient totilement dépouillés de " graines. On peut alors employer les cônes à chauf") fer les fourneaux.

" $2^{\circ}$ Pour employer la chaleur du soleil, on éta" blit des échafaudages contre le mur d'un bâtiment " exposé au midi. On y place des claies à une telle " distance les unes des autres, que le soleil puisse " donner sur les rangées les plus reculées et sur les " inférieures. Sous la dernière claie se trouve un " tiroir dont le fond est en toile grossière, afin que, " si la pluie vient à y tomber, elle la traverse faci" lement et que les graines puissent sécher. L’appa" reil entier est recouvert d'un petit toit dont li ") pente est dirigée vers le Nord.

" Lorsqu'il fait un beau soleil et une chaleur forte " on remue les cônes en commençant par les claies " des étages supérieurs et en continuant jusqu'en

faisant glisser sur roulettes dans deux coulieses. De cette manière, en poussant et en tirant lit claie. on agite aisímont tous les cônes qui s'y trouvent. 
" bas; on rassemble alors les graines tombées dans " le tiroir. Enfin, quand on juge que les cônes se ") sont ouverts autant que possible, on les enlève, et " on les place dans l'espèce de baratte dont nous " avons parlé, pour en tirer les semences qui pour" raient y être restées. "

L'appareil décrit en dernier lieu a été perfectionné en disposant sur chaque claie un couvercle mobile, qu'on ferme pendant la nuit et quand il pleut, et que l'on ouvre lorsqu'il fait beau, plus ou moins, suivant la hauteur du soleil. Afin que ce couvercle renvoie mieux les rayons calorifiques sur les cònes, on en peint la face infériemre d'une couleur blanche avec vernis.

L'extraction des semences par la chaleur artificielle a lieu encore, d'après un autre système, dans les bâtiments construits exclusivement pour cet objet. On trouvera la description d'un établissement de ce genre, avec des détails intéressants sur les manipulations auxquelles donnent lieu l'extraction, le désailement et la conservation des graines résineuses, dans les Amuales forestières, tome II, année 1843, page วั0ว. Nous renvoyons le lecteur à cel article qui est de leu M. Rich, gérant de la sécherie de graines forestières que l'Administration des forêts aviut établie à Haguenau (Bas-Rhin).

Lorsqu'on veut désailer les graines d'épicéa, on les humecte, et, après les aroir mises dans un sac yu'on ne remplit qu'au quart, on les frotte fortement 
jusqu'à ce que les ailes s'en détachent '. Ensuite, après les avoir étendues dans un lieu très aéré, afin de hâter la dessiccation, on les nettoie entièrement au moyen du van.

Le désailement de la graine est avantageux parce que le transport est rendu plus facile, par la diminution du volume et du poids, en second lieu la semence désailée se sème et se recouvre mieux, et l'on ne risque pas autant de la voir dispersée par les vents. Mais, en général, les graines qui ont subi cette opération, surtout quand on se les procure par le commerce, se conservent moins bien que celles qui sont munies de leurs ailes. Dans les établissements (principalement dans ceux d'Allemagne) où il se prépare de grandes quantités de semence pour la vente, on met quelquefois la graine en tas, après l'avoir bien humectée, et on la laisse ainsi jusqu'à ce que, en y enfonçant la main, on sente une légère chaleur; c'est alors que les iiles s'en détachent avec le plus de facilité. On conçoit aisément qu'un tel procédé prédispose les graines à la germination, et que, si le semis est retardé, il puisse s'en trouver un grand nombre qui ont perdu leur faculté germinative.

La graine d'épicéa peut, rigoureusement, se con-

- On peut éviter d'humecter la graine (ce qui vaut toujours mieux; ('n bitendant les sacs, remplis comme il est dit, sur l'are d'une grange et les frappanti àvec des flénux à battre le grain. 
server trois ou quatre ans, en lui donnant les mèmes soins qu'à celle du sapin.

7כ00. Examen de la graine. - La graine d'épicéa de bonne qualité, comme celle de sapin, est ferme et bien remplie; son odeur, en l'ouvrant, est fraîche, résineuse, et, écrasée sur l'ongle, cette graine y laisse une substance grasse, d'une apparence oléagineuse. La couleur de cette graine est brun rougeâtre.

Un litre de semence ailée d'épicéa doit peser de $12 \%$ à 140 grammes; désailée, cette graine pèse, par litre, de 400 à 430 grammes.

751. ExÉcution du semis. - Le labour par bandes alternes ou par pots convient, en général, au semis d'épicéa comme au semis de sapin. Quoique le jeune plant ne soit pas aussi délicat que celui de cette dernière essence, il a besoin, cependant, surtout aux expositions chaudes, de quelque ombrage; le couvert au contraire lui est très défavorable. Quand le terrain se trourera garni de myrtilles, de bruyères, etc., il suffira de les conserver dans les bandes ou dans les places qu'on laisse en friche; dans le cas contraire, c'est-à-dire, si le terrain était entièrement nu, il faudrait mêler une demi-semaille d'avoine ou d'orge à la graine.

Le jeune plant, restant très petit les premières années, a souvent beaucoup à souffrir des herbes qui envahissent les semis. Quand on aura à combattre cet inconvénient, on devra d'abord aug- 
menter la largeur des bandes ou des pots et ensuite faire enlever les herbes avant l'automne, soit en les arrachant, soit en les fauchant, toutes les fois qu'on le pourra sans trop de frais. Parfois la conservation du semis est à ce prix.

La graine n'a besoin d'être enterrée que de 4 à 6 millimètres; on peut même se contenter de la mèler à la terre avec un râteau en bois. Semée au printemps, qui est la saison préférable, elle lève au bout de cinq ou six semaines.

Pour le semis partiel, 13 à 15 kilogrammes de semences ailées, et 10 à 12 kilogrammes de semences désailées, suffiront par hectare. Pour un semis en plein, il fiudrait ajouter moitié en sus.

\section{ARTICLE XIV.}

\section{Semis du pin sylvestre.}

752. Récolte et conservation. - Ce qui a été dit de la récolte des cônes d'épicéa s'applique entièrement à la récolte des cônes de pin sylvestre.

L'extraction des semences se fait à l'aide des appareils qui ont été décrits dans l'article précédent; mais, ainsi que nous l'avons dit plus haut, on a aussi construit des bâtiments exclusivement destinés à cet objet, auxquels on a donné le nom de sécheries de graines forestières. L'administration des forêts pos- 
sèdait, dans lia forêt de Haguenau (Bas-Rhin), un établissement de ce genre qui lui fournissait tous les ans des semences de pin en quantités considérables.

On se sert plus particulièrement, pour la préparation des graines de pin sylvestre, des sécheries par la chaleur artificielle dont nous avons parlé plus haut, en renvoyant, pour la description de ces établissements, aux Amnales forestieres. Nous renouvelons ici ce même renvoi.

Le désailement de la graine de pin a lieu comme nous l'avons expliqué pour la graine d'épicéa.

La semence de pin sylvestre peut aussi se conserver pendant trois ou quatre ans; mais, dans ce cas, il ne faut pas la désailer. Elle se conserverait encore plus sûrement si on la laissait enfermée dans les cônes, et si on ne récoltait ceux-ci qu'à la fin de l'hiver.

753. Examex de la graine. - Les caractères qui indiquent la bonne qualité des semences sont absolument les mêmes pour le pin sylvestre que pour l'épicéa, avec cette différence, que les semences de pin sont noires ou blanches; parmi ces dernières il s'en trouve un plus grand nombre de vaines que parmi les autres. On fera donc bien, lorsqu'on achetera des graines, et que les blanches seront en majorité, de les éprouver avec d'autant plus de soin.

La semence de pin sylvestre doit peser, par litre, de 120 à 140 grammes, si elle est ailée; et de 440 à 500 grammes, si elle est dépouillée de ses ailes. 
754. Exécutiox in semis. - Le labour à donner au terrain pour le semis du pin sylvestre est le même que celui qui convient pour le semis de l'épicéa, sauf qu'il n'est pas nécessaire de ménager autant l'abri. Dans les pentes méridionales couvertes de bruyères, où souvent on sème le pin, il est essentiel de prendre une précaution particulière, en préparant le sol; c'est de creuser les bandes ou les trous jusqu'à la couche de terre inférieure au terrain noir qui se trouve à la surface, et que l'on nomme communément terre de bruyère. En répandant la graine dans ce terreau sans aucune consistance, impropre à retenir l'humidité, et qui, par sa couleur, s'échauffe à un haut degré, les semis manquent presque toujours. Souvent d'ailleurs la terre de bruyère contient un principe acide qui fait avorter complétement la germination '.

Comme les graines de l'épicéa, celles du pin sylvestre ne doivent être recouvertes que de 4 à 6 millimètres; il suffit mème de les mêler à la terre à l'aide du

- Ce principe est l'acide acétique. On a proposé, pour le neutraliser, d'employer l'écobuage qui par les cendres qu'il produit, atteindrail ce but. Ce procédé peut être suffisant pour certaines plantes qui exigent peu d'humidité et pour la culture desquelles on retourne entièrement la terre, de manière à mêler la couche de terreau à l'élément minéralogique du sol. Mais, pour les semis d'essences forestières, il ne saurait en être de même; car, tout en enlevant au terreau un principe nuisible, les cendres ajouteraient d'ailleurs à son trop de légèrelé et à son défaut d'hygroscopicité [702]. 
ràteau. Ordinairement les jeunes plants paraissent au bout de quatre à six semaines, lorsque le semis a été fait au printemps, ainsi que cela est à conseiller; mais souvent aussi, quand les graines ont été trop recouvertes ou trop échauffées dans l'extraction, elles ne germent que la seconde année.

Dans quelques parties de l'Allemagne, où les semences de pin sont très abondantes, on est dans l'usage de semer tout simplement les cônes dans les bandes ou dans les trous préparés à cet effet; puis, dès qu'au printemps lia chaleur commence à agir sur les écailles, on remue fortement les cônes avec un ràteau afin d'en faire tomber les graines et de distribuer celles-ci le plus également possible. Ce procédé a d'abord l'avantage d'économiser.les frais d'extraction et de désailement des semences; en second lieu, celles-ci sont généralement de meilleure qualité; et enfin, les cônes qui recouvrent le sol peuvent donner quelque abri aux plants naissants. Mais, d'un autre côté, on perd une partie des graines, parce qu'on ne réussit jamais à les faire sortir toutes des cônes; elles ne sont pas aussi également réparties sur le terrain, et, pour le transport, les cônes sont plus encombrants.

Les quantités de semences de pin sylvestre à employer, par hectare, pour un semis partiel sont :

Semences ailées. . . 12 à 14 kilog.
Semences désailées. . 9 à 11 id.
Cônes. . . . . . 18 à 20 hectolitres.


Semis du pin maritime.

7つ̋. Rícolte et conservation. - Ce que nous avons dit, sous ce rapport, de l'épicéa et surtout du pin sylvestre, s'applique entièrement au pin maritime.

756. Examex de la graine. - La graine de ce pin est beaucoup plus grosse que celle du précédent; sa couleur est gris ou brun mat sur une face, et d'un noir luisant sur l'autre; elle doit d'ailleurs présenter les mêmes caractères que la graine du pin sylvestre.

757. Exécution du semis. - Les différents modes de labour que nous connaissons sont tous applicables au pin maritime. On se sert assez généralement de la charrue pour la façon à donner à la terre, et de la herse pour recouvrir le semis. - La graine, d'ailleurs, étant plus grosse, a besoin d'être enterrée davantage.

La quantité de semences à employer par hectare varie. - En Sologne, par exemple (roir le rapport déjà cité de $M$. A. Brongniart), on emploie pour un semis en plein, depuis 10 jusqu'à 30 kil., sans doute, selon la qualité de la graine et aussi selon quel'on a en vue la création d'un bois plus ou moins touffu. Nous 
pensons que 1 : ij it 18 kil. de semences ailées et 12 à 14 kil. de semences désailées doivent suffire, attendu que la graine du pin maritime est généralement de très bonne qualité. Pour les semis partiels, il y aurait lieu de diminuer ces quantités d'un tiers.

La culture du pin maritime a pris, dans plusieurs contrées de l'ouest et du centre de la France (notamment dans le Maine et la Sologne), une très grande extension, par suite des produits particuliers que fournit cet arbre [319]. Mais c'est sur les bords de l'Océan et surtout dans les Landes et dans la Gironde qu'elle acquiert une importance de premier ordre. On sait que, dans ces départements, s'étend sur le littoral depuis l'embouchure de la Gironde jusqu'à celle de l'Adour, une région, appelée les dunes du golfe de Gascogne, qui occupe un espace d'environ 240 kil.

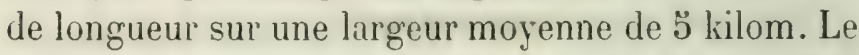
sol de cette région, exclusivement composé d'un sable quartzeux très ténu que les marées de l'Océan déposent sur la plage devient tellement mobile, dès qu'il se dessèche, que les vents le soulèvent et l'emportent au loin. Le plus léger obstacle, tel qu'un petit accident de terrain, un arbre, quelques touffes de genêt ou de gourbet (arundo arenaria), suffit souvent pour arrêter les sables dans leur marche; ils s'accumulent alors d'autant plus vite et forment des amas d'autant plus forts que les dépòts laissés par les lames sont plus considérables, et que les vents soufflent plus longtemps dans la mème direction. 
Telle est la formation des dunes, dont la hauteur et la forme diffèrent nécessairement en raison des conditions sous lesquelles elles se sont élevées; on en rencontre qui ont de 20 à 50 et même jusqu'à 100 mètres de haut.

C'est par un systeme mixte de travaux de clayonnage et de reboisement en pin maritime que l'on est parvenu à arrêter, partout à peu près, la marche des sables qui menaçait les communes roisines et leur territoire, et c'est une des gloires du corps des ponts et chaussées à qui le Gouvernement a confié au début cette grande œurre dont le service forestier est aujourd'hui chargé, d'avoir pu presque l'achever et d'avoir successivement amélioré et tellement simplifié les procédés employés, qu'ils sont aujourd'hui à la portée de toutes les personnes qui peuvent avoir à s'occuper du boisement des sables mourants.

Mais si intéressante que soit pour le forestier, comme pour l'ingénieur, l'étude approfondie de ces procédés, nous sortirions du cadre que nous nous sommes tracé pour la rédaction de ce cours, si nous entreprenions de les exposer ici en détail. Nous renvoyons donc le lecteur, curieux d'étudier cette question, aux principaux écrits où elle est traitée, savoir :

1. Mémoire sur les dunes, par Brémontier, inspecteur général des ponts et chaussées (réimprimé dans les Annales des ponts et chaussées, année 1833, page 145). 
2. Notice sur la fixation des dunes, par M. Lefort, ingénieur des ponts et chaussées (Annales des ponts et chaussées, année 1831, page 320).

3. Hémoire sur les dunes du golfe de Gascogne, par M. Laval, ingénieur en chef, directeur des ponts et chaussées (Annales des ponts et chaussées, année 1847, page 218) ${ }^{1}$.

4. Notice sur le pin maritime, par M. Lorentz, administrateur des forêts (Amales forestières, année 1842, page 57 ).

5. La dune littor'ule, par M. de Vasselot de Régné (Revue des eaux et forêts, année 1875).

ARTICLE XVI.

Semis du pin laricio et du pin d'Alep.

738. Récolte et consenvatiox. - Les règles données dans les deux précédents articles, concernant

' Ce mémoire fort remarquable, quoique moins étendu que celui de l'illustre Brémontier, fait parfaitement connaître la formation des dunes et les procédés employés pour les fixer, d'après les perfectionnements les plus récents.

Si nous passons sous silence le semis des pins à crochets rt d'Autriche, ce n'est pas (ainsi qu'on a pu s'cn convaincre par la rlescription que nous avons donnée de ces deux arbres) que nous en méconnaissions l'importance, mais bien parce que nous ne possédons aucune donnée certaine sur les particularitrs que pourrait prósenter l'opération. Il est très probable, 
ce double objet, sont applicables aux deux pins dont il s'agit.

7099. Exharex de la grane. - Les graines du laricio et celles du pin d'Alep sont à peu près d'égale grosseur et tiennent le milieu, sous ce rapport, entre les graines du pin maritime et celles du pin sylvestre. Les premières ont une couleur jaune terne et les secondes sont d'une nuance brun foncé. Ces semences doirent présenter les mêmes caractères que les précédentes.

760. ExÉcutiox de sears. - Les procédés de semis de ces deux essences sont les mèmes que ceux que nous avons indiqués pour l'épicéa et le pin sylvestre, sauf à enterrer la graine un peu plus, attendu sa grosseur. - La quantité de semence à employer par hectare ne nous est pas positivement connue par l'expérience; mais on peut à cet égard juger par analogie et en tenant compte de la qualité de la graine.

Il est probable que pour un semis partiel, il suffirait d'employer :

Semences ailéés. . . 14 à 16 kilog.

Semences désailées. . 11 à 13 id.

au surplus, que ce que nous avons dit au sujet du semis du pin sylvestre pourra s'appliquer, d’une manière générale, au semis des deux essences dont il s'agit. 


\section{ARTICLE XVII.}

\section{Semis du pin pinier ot du pin cembro.}

761. Récolte et conservation. - Les cônes de ces deux pins se cueillent à la main '. Pour les faire ourrir, il suffit de les exposer au soleil ou de les placer dans un appartement tempéré; les amandes s'en échappent facilement.

La faculté germinative de ces graines ne se conserve guère que de l'automne au printemps; elles sônt très exposées à rancir. On peut les étendre, comme les autres graines résineuses. sur des planchers secs et aérés. Si l'on roulait les garder pendant plusieurs années, il faudrait les laisser renfermées dans les cônes et, pour cela, garantir ceux-ci de la chaleur.

762. Exhmex de l. grane. - En cassant le noyau de ces graines on doit le trouver plein; l'amande doit ètre blanche, d'un goùt et d'une odeur agréables. Si elle sent le rance, la graine est gâtée.

Ln litre de semence de cembro pèse de 380 à 400 grammes.

- Dans les Alpes, on tind quelquelois res toiles sous les pins cembros pour profiter de la disseniuation naturelle : ces toiles demeurent à terre pendunt tout lautomme et jusquà l'entrée de l'hiver, car la dissémination se lait très lentement. 
763. Exécutrox ne sews - Le pin pinier et le pin cembro sont trop rares pour en faire des semis en grand. Ordinairement on repique les amandes en pépinière ou en pots, dans une terre de bonne qualité; on les recouvre de 9 à 13 millimètres et on les arrose souvent. Lorsque les plants ont un ou deux ans, on les transplante une première fois, et ce n'est que trois ou quatre ans plus tard qu'on les met définitivement en place.

Quand le repiquement de la graine se fait en automne, les plants paraissent au printemps ; lorsqu'on sème dans cette dernière saison, ils lèvent au bout de cinq ou six semaines. Souvent, néanmoins, les amandes ne germent que la seconde année.

ARTICLE XVIII.

Semis du pin du lord Weymouth.

764. Récolte et coxsenvation. - Après les avoir cueillis, il suffit d'étendre les cònes dans un lieu sec et aéré et de les retourner souvent. Les écailles s'entrouvent sins le secours de lit chaleur et laissent échapper la graine.

On n'a fail encore que peu d'essais sur la faculté qu'a cette semence de se conserver; aussi est-il it consciller de l'employer dis le printemps qui suit 
la maturité. Les moyens de conservation sont les mêmes que pour les autres graines résineuses.

765. Examex de la graine. - Ces graines doivent être d'un brun clair et présenter du reste les mêmes caractères que celles du pin sylvestre.

766. ExÉcution du semis. - Ce que nous avons dit à cet égard, dans l'article précédent, du pin pinier et du cembro, s'applique entièrement au pin du Lord. Les graines n'ont pas besoin d'être enterrées à plus de 6 à 8 millimètres.

\section{ARTICLE XIX.}

Semis du mélèze.

767. Récolte et conservatiox. - La récolte des cònes, l'extraction des graines et leur désailement se font de la manière qui a été indiquée pour le pin sylvestre et l'épicéa. Lorsqu'on le pourra, il sera préférable d'attendre le printemps pour la récolte.

Dans l'opération de l'extraction, il est essentiel de ne donner qu'une chaleur très modérée, ce qui nécessite un plus long séjour des cônes sur les claies. Une chaleur trop grande fait suinter la résine à travers les écailles, et les enduit au point qu'elles ne peuvent plus s'ouvrir.

Lorsqu'on donne à la semence de mélèze les soins que nous arons déjà indiqués pour les autres graines 
résineuses, elle peut se conserver plusieurs années, moins longtemps, cependant, que les semences de pin sylvestre et d'épicéa. Aussi est-il préférable de ne pas tarder à l'employer.

768. Exanen DE LA GRaine. - Mèmes caractères que la graine du pin sylvestre [7.3]. La semence du mélèze doit être jaunàtre et peser, par litre, 160 à 1750 grammes si elle est ailée, et 500 à 500 grammes si elle est dépouillée de ses ailes.

769. ExÉcutiox du semrs. - Les règles que nous avons données à cet égard pour le semis de l'épicéa, sont tout à fait applicables au semis du mélèze. Toutefois, comme la graine de cette dernière essence est en général fort chère, el qu'il est assez difficile de s'en procurer des quantités considérables, on préfère, la plupart du temps, semer d'abord en pépinière, et employer ensuite la plantation pour les repeuplements à exécuter en forêt. Souvent aussi on mélange la graine de mélèze avec d'autres graines de bois résineux ou feuillus, ce qui est d'autant plus à propos que le mélange, ainsi qu'on l'a vu plus haut [ð33], convient particulièrement à cette essence. La semence de mélèze est ordinairement d'assez mauvaise quitlité; si l'on voulait en faire un semis par bandes ou par trous, il ne faudrait pas l'épargner; 16 à 18 kil. de graine ailée, et 12 à 15 kil. de graines sans aile, seraient nécessaires, par hectare. Semée au printemps, cette graine lève an bout de quatre ou six semaines. 
ARTICLE XX.

\section{Semis du oèdre du Liban.}

770. Nous avons fait connaitre, dans le premier livre de ce cours [386], l'époque de la dissémination naturelle du cèdre. En cueillant les cônes à la fin d'août ou de septembre (selon que la température reste sèche ou devient pluvieuse), on pourra les conserver à volonté jusqu'au moment où l'on se proposera de semer, car la graine, renfermée dans les cônes, se maintient en parfait état pendant plusieurs années. - Pour extraire la semence, il suffit de laisser les cìnes séjourner dans l'eau pendant 24 à 36 heures. Les écailles se détachent alor's de leur axe avec une grande ficilité, et il n'y a plus qu'à en séparer la graine à l'aide d'un crible.

La semence se trourant fortement humectée par le procédé d'extraction, il est à conseiller de la semer immédiatement. Toutefois, si l'on voulait différer le semis de quelques semaines, il fiudrait faire sécher la graine, au soleil autant que possible, et l'étendre ensuite dans un lieu bien aéré en la faisant retourner fréquemment.

Les semis de cèdre ne se font qu'en pépinière ou en pots; la dernière manière est préférable, parep qu'elle permet de rentrer les jeunes plints en hiver 
[387]. Après leur aroir fait subir une première transplantation, on pourra les placer définitivement en forêt. Si on n'a pas la facilité de les garantir du froid, il faudra attendre, pour les y planter, qu'ils aient atteint leur huitième année au moins [384].

\section{ARTICLE XXI.}

Des semis mélangés.

771. Les semis mélangés deviennent utiles dans différents cas :

$1^{\circ}$ Pour créer des forêts mélangées;

$2^{\circ}$ Pour élever une essence sous l'abri d'une autre ou pour couvrir promptement le sol atin de l'empècher de se détériorer;

$3^{\circ}$ Pour économiser une semence rare et d'un prix élevé.

772. Nous sarons que les essences dont le mélange permanent est avantageux sont celles qui ont une croissance sensiblement égale, qui supportent même révolution et mème mode de traitement, et dont les racines puisent la nourriture à différentes profondeurs du sol.

Dans le troisième et dans le quatrième lirre de ce cours, nous avons indiqué les essences qu'il convient d'élever en mélange, soit pour la futaie, soit pour le tirillis; il suffira done de consulter, pour cet 
objet, les parties précitées $[\mathbf{4 8 7}, 497,498$, כ04, ร13, 533 et 617 ].

773. La propriété la plus importante des essences destinées à en abriter d'autres dans leur jeunesse ou à courrir le sol, est d'avoir une croissance très rapide dès les premières années. Celles qui, dans ce but, conviennent le mieux, sont : parmi les bois feuillus, l'orme, le bouleau, les érables, les saules et les peupliers 1; parmi les bois résineux, le pin sylvestre, le pin maritime, le pin laricio, le pin noir et le pin d'Alep. Mais. en général, les graines résineuses sont d'un prix trop élevé pour ètre employées à un tel usage, tandis qu'on se procure à très peu de frais, selon les localités, des graines de l'une ou de l'autre essence feuillue.

Ainsi que nous l'avons dit ailleurs [719], le genèt ou l'ajonc peuvent aussi être employés arec avantage.

Dès que l'essence protectricc a atteint son but, il convient de l'extraire. Dans les pays où le bois a de la valeur, le produit de cette extraction est souvent assez considérable pour faire rentrer le propriétaire, en très grande partie, dans ses arances. Aussi cette considération seule peut-elle dans beaucoup de cas, justifier le mélange des essences dans un semis.

1 Ces deux dernières ne se multiplient d'ordinaire que par boutures. 
774. Lorsque, pour économiser une graine rare ou chère, on la mélange avec une autre plus commune, la seule précaution à prendre dans le choix de cette dernière, est que les deux essences aient une régétition et des exigences à peu pris semblables pendant les trente ou quarante premières années au moins, si c'est une futaie que l'on veut créer, et pendant une durée moindre, s'il ne s'agit que d'un taillis. En s'attachant ensuite, dans les éclaircies périodiques, à extraire l'essence supplémentaire, on réussira peu à peu à faire dominer entièrement la plus précieuse.

77\%. La manière d'exécuter des semis melangés ne présente aucune particularilé, si ce n'est que, après avoir déterminé les quantités à emplover pour chaque espèce, il faut semer et recouvrir d'abord la semence qui demande à être enterrée davantage. Si, all contraire, les deux graines ont, sous ce rapport, les mèmes exigences, on les mélange nrant de les senrer, el ensuite le semis se firit comme à l'ordinaire 


\title{
CHAPITRE QUATRIËME.
}

\author{
DES PLANTATIONS.
}

ARTICLE PREMIER.

Des qualités que doivent offrir les plants.

776. Tout plant, cruel que soit son ìge, doit, pour aroir les meilleures chances de reprise, être pourvu de racines fraiches, unies, qui ne soient ni rompues, ni écorchées, ni endommagées en aucune manière; plus les racines sont nombreuses, mieux la reprise est assurée.

Si c'est un plant de haute tige (1 mètre à 1 mètre 33 centimètres et au-dessus), il est nécessaire que sa grosseur soit proportionnée à sa hauteur, afin qu'il puisse se soutenir et résister aux intempéries; il faut en outre, qu'il soit bien droit, sans aucune 
blessure, que sal lète soit suffisamment développée, et qu'il présente d'ailleurs tous les signes d'une végétation vigoureuse. Si c'est un plant de basse tige (audessous de 1 mètre), sa forme est moins importante; cependant il doit être droit et aroir surtout de belles racines, des pousses fortes et des bourgeons sains et bien formés.

77T. Les plants reprennent d'autant plus facilement qu'ils sont plus jeunes; aussi les hautes tiges ne sont-elles employées en sylviculture, que dans quelques cas exceptionnels où seules elles peurent convenir : par exemple, dans des lieux exposés aux dégâts du pâturage ou des inondations, ou bien encore, s’il s'agit dims certirins laillis composés, d'élever des sujets propres au halivage [:398]. D'ordinaire, cest toujours la plantition de bassers tiges qui est préférée; mon-seulement parce qu'elle est, comme nous venons de le dire, l'une réussite plus assurée, mais encore parce qu'elle entraine à bien moins de frais. Cette derniere considération, lorsqu'on fait des repeuplements en grand, est naturellement de beaucoup de poids.

En général, les hautes tiges ne sont propres à la transplintation que quand elles ont été élevées en pépinière; pour les basses tiges, bien que celles que l'on produit en pépinière méritent de beaucoup la préférence, on peut cependant aussi les tirer des semis naturels ou artificiels qui existent souvent, soit dans la forêt mème où il s'agit de planter, soit 
dans les forèts voisines. Si l'on prend un tel parti, il faut éviter de choisir des plants provenant d'endroils très fourrés ou très couverts; dans les uns, ils sont rabougris ou au moins très délicats et peu propres, par conséquent, à résister lorsqu'on les met en terrain découvert; dans les autres, ils manquent de racines et de branches, ce qui compromet également la réussite.

Lìge le plus convenable pour la reprise des basses tiges varie d'ailleurs selon les essences. Ainsi, on peut planter avec avantage :

De 1 à 3 ans, les pins sylvestre, maritime et laricio, l'épicéa et le mélèze, de même que les bouleaux, les aunes et les robiniers ;

De 3 à 6 ans, les sapins, les pins du lord W'eymouth, les chàtaigniers, les ormes, les frênes et les érables ;

De 4 à 8 ans, les chênes, les hètres, les charmes, les fruitiers, etc.

Il est entendu que, pour être générales, ces règles n'ont rien d'absolu, et qu'elles doivent fréquemment se modifier d'après les circonstances locales.

Quand on a des plantations considérables à exécuter, la création d'une pépinière, loin d'occasionner une dépense inutile, procure, au contraire, une grande économie; c'est aussi le moyen le plus sùr d'obtenir du plant de bonne qualité. 
AR'TICLE II.

De la culture des plants on pépinière.

778. Il faut éviter d'établir les pépinières diuns un terrain très gras ou humide ; les plants y prennent une texture làche, leurs racines y sont généralement mal conditionnées, et lorsque, plus tard, on les transplante dans une terre moins grasse ou plus sèche, ils périssent souvent dis la première année, ou bien ils sont longtemps avant de reprendre de li vigueur. Un terrain maigre et de mauvaise qualité convient moins encore pour une pépinière, parce qu'on n'y obtient que des brius limguissants, dont tous les organes, el surtout les racines, sont faibles et d'une conformation ricieuse; trimsplinntís, de tels sujets sont presque toujours victimes des circonstances plus ou moins défavorables qui sont la suite de lat transplantation. C'est dans un terrain de qualité et de compacité moyennes qu'on élèvera des plants vigoureux et qui, placés en forêt, s'accommoderont le mieux d'un sol quelconque.

Les éléments minéralogiques du terrain n'exercent qu'une très faible influence sur la réussite des cultures d'une pépinière; les labours de diverses sortes, les arrosages, les amendements au besoin, y combattent et corrigent les défauts que peut présenter le 
sol. Seulement, quand on en aura le choix, on devra donner la préférence aux sols légers sur les terres fortes, parce que celles-ci, comme on le sait, se durcissent et se crevassent profondément par la sécheresse, et, sous l'influence de labours fréquents les jeunes plants y sont facilement soulevés et déracinés par les gelées de l'hiver.

Autant que possible, on doit établir la pépinière à proximité du terrain à replanter; d'abord, afin que les plants s'habituent, dès leur naissance, au climat et au sol auxquels ils sont destinés; en second lieu, parce qu'il y a économie sur les frais de transport. Toutefois, les grandes élévations, de même que les vallées profondes, doivent être évitées; un terrain un peu abrité des grands vents, en plaine ou en pente douce, et où les plants soient le moins possible exposés aux gelées tardives du printemps, tel est l'emplacement le plus convenable. S'il se trouve, dans le roisinage, des eaux qui donnent la facilité d'arroser pendint les lemps de sécheresse, ce sera un grand avantage.

779. Le terrain destiné à une pépinière, à moins qu'il ne soit tout à fait léger, doit recevoir un labour issez complet et assez profond pour y détruire entièrement la mauvaise herbe et rendre la terre parfaitement meuble.

Ce résultat obtenu, soit par de simples labours, soit par une ou plusicurs cultures de céréales ou de pommes de terre, on divise le terrain en planches 
ou plates-bandes, en le coupant par des chemins et par des sentiers perpendiculaires les uns aux autres; enfin, si l'on a à redouter les dégâts du bétail ou du gibier, on le clôt de fossés, de palissades, de haies, etc.

Dans les plates-bandes dont il vient d'ètre parlé. et parallèlement aux sentiers qui les limitent, on ouvre des sillons ou petites rigoles, séparés entre eux par des intervalles de 20 à 30 centimètres, profonds de 25 à 30 et d'une largreur de 20 à 20 centimètres, réduite au fond à 12 ou 1 ŏ centimitres environ '. La terre qui provient de ce travail est répartie sur les intervalles demeurés libres. - L'opération terminée, on remplit les sillons d'un terreau provenant, mi-partie de feuilles morles et mi-partie de gazons décomposés et qu'il faut avoir soin, à cet effet, de préparer annuellement dans quelque place reculée de la pépinière ${ }^{2}$. On tasse ensuite légèrement ce

- Voyez Charles Heyer, Traité de sylviculture.

${ }^{2}$ Pour faire son terreau, on choisit une place ombragée, abritée du midi, mais non recouverte ou surmontée, afin que les influences atmosphériques y aient un libre accès. - On forme, d'une part, un ou plusieurs tas de feuilles mortes, de fougères et autres plantes charnues récoltées avant la maturite de leurs semences; de l'autre, des tas semblables composés de gazons, de la mauvaise herbe provenant des binages de la pépinière, du nettoyage des chemins, etc. - Ces tas doivent ètre longs, assez étroits et d'une hautcur de 1 mètre à 1 mètre 30 environ. Deux fois par an, au prinlemps et en automne, on les retourne et, dans les temps de sécheresse prolungie, on les arruse. Les gazons sont d'omdinaire entièrement 
terreau, soit avec une batte ou simplement arec la main, de façon que les intervalles entre les sillons saillissent sur ceux-ci de 3 à 4 centimètres environ. Cette précaution devient très utile lorsque, dans l'hiver qui suit la levée du semis, les jeunes plants ont été soulerés par les gelées, et qu'une partie des radicelles se trourent déchaussées. parce que, si tôt le dégel du printemps, il suffit de les recouvrix de quelques centimitres de terre pour assurer leur reprise.

780. Les semis en pépinière se font de préférenct an printemps: il faut semer tris dru de maniere que

décomposés au hout d'un an, mais il faut 3 et $f$ ans aux feuilles mortes pour être complétement réduites en terre. Les feuilles de hêtre mélangées, par parties égales, à des feuilles de bois résineux, fournissent une des meilleures qualités de terreau. Pour hâter la pourriture assez lente de ce mélinge, on fera bien d'y ajouter des fruilles qui se déconposent plus rapidement, telles que celles de frêne, érable, orme, saule, peuplier, aune, robinier, ete.

Lorsque les sillons d'une pépinière ont été, une première lois, préparés avec ce terreau, conme nous l'expliquons cidessus, il suffit d'y apporter charque année une faible quantité de terreau neuf pour entretenir la fertilité du'sol. - En prévenant ainsi, far un procérlés simple el peu cuuteux, läpuisement de la terre dans les pépinières, on évite la nécessité de les dephacer de temps à autre, à laquelle on ne saturait échapper autrement. L'intérèt qu'il y a, sous tous les rapports, à établir les pépinières à proximité des maisons de gardes et à leś y maintenir, doit faire apprécier cette méthorle de cullure et lui mériter l'aceneit des forestiers. 
les graines se touchent, pour ainsi dire, el ne recouvirir celles-ci, mème les semences lourdes, que d'une couche de terreau, tout juste suffisante pour empêcher que la pluie ne les mette à découvert. - On comprend que des semis aussi drus fournissent le plus grand nombre possible de plants, eu égard à la surface cultivée, et en second lieu, que la mauvaise herbe ne saurait les envahir. L'expérience prouve, d'ailleurs, que les plants qui en proviennent sont abondamment pourvus de racines, surtout de chevelu, et qu'ils ne s'affiment point entre eux, mème lorsqu'ils atteignent 40, 50) et jusqu'à 70 centimètres de hauteur. Lorsqu'on procède à leur extraction, les racines, quoique entrelacées, se démêlent et se séparent aisément; mais un certain nombre de tiges ayant été dominées, surtout vers le milieu du sillon, sont demeurées faibles, et ont besoin de se fortifier avant de pouvoir ître mises définitivement en place. A cet effet, on les repique dans des rigoles voisines. ou dans celles mêmes d'où elles proviennent, et on les y laisse jusqu'à ce qu'elles aient atteint les dimensions ef la vigueur désirables.

Pour blever une essence qui, comme le chine. par exemple, pousse un fort pivol des les premieres années, el diminuep l'inconvénient que cette disposilion présente lors de l'extraclion des plants, il suffit d'enfoncer, de chaque coté du sillon et obliquement rers le fond de celui-ci, le fer bien acéré d'une besche, aprosis quoi l'on referme, aver le pied. 
l'ouverture faite par l'instrument dans la terre '. Le plant ne tarde pas à remplacer le pivot, ainsi retranché, par des racines obliques qui sont moins gênantes et plus utiles lors de la transplantation. Cette opération doit se faire au commencement de l'automne qui suit la levée des plants.

Un autre procédé, pour empècher le trop grand développement du pivot, est celui que Duhamel indique sous le nom de pratique de Breatane", et dont il conseille l'emploi. Il consiste à paver en pierres plates le fond des sillons dans lesquels on sème; ces pierres, en arrètant le pivot, forcent le plant à pousser des racines latérales.

781. Quand il s'agit d'élever en pépinière des plants de haute tige, on choisit, dans les sillons de semis, les sujets les plus vigoureux et les plus élancés, et on les transplante dans une ou plusieurs plates-bandes à ce destinées, en leur donnant un espacement en tout sens de 33 à 66 centimètres. Lors de cette transplantation, on pourra retrancher le pirot du plant, qui se reproduit souvent, mème après aroir été supprimé une première fois, afin de favoriser le développement des racines latérales. On pourrait aussi, dans le même but, placer une ou plusieurs pierres plates au fond de chaque trou.

: Nous avons vu appliquer avec avantage ce procédé au hêtre qui, quoique traçant à un certain àge, pousse, dans les premières années, un pivot très rléveloppé [128].

Voyez Duhanel. Des semis et plentutions des methes, page 127. 
78.2. Les arrosements, ainsi que nous l'avons dit, seront très utiles dans les temps de grande sécheresse; mais, une fois commencés, il faut les continuer jusqu'à ce que la pluie survienne. Autrement il se forme, à la surface de la terre, une croûte qui nuit à la végétation, en empêchant l'air de parvenir aux racines. - Il est essentiel d'arroser assez abondamment pour détremper le sol jusqu'à la profondeur où plongent les racines les plus longues; on conçoit que, sans cette précaution, la peine que l'on prendrait serait sans résultat.

De tous les modes d'arrosement, celui qui offrirait le plus d'arantages, sans présenter d'inconvénient serait l'irrigation, telle qu'on la pratique dans les prairies, en faisant, dans ce cas, servir comme saignées, les sentiers qui séparent les plates-bandes. Mais les pépinières forestières sont trop rarement placées dans des situations qui rendraient applicable un parreil système; on ne peut donc en parler qu'à titre d'exception.

783. Il faut avoir un soin particulier de ne pas laisser prendre le dessus à la mauvaise herbe. Dans les sillons, il faut l'enlever à la main dès qu'elle parait, et choisir autant que possible pour cette opération un temps frais. - Si le semis a réussi convenablement, ces sarclages ne seront nécessaires que la première année. - Les intervalles entre les sillons ainsi que les chemins et sentiers, devront aussi être netloyes au moins deux fois par an, au prin- 
temps et rers la fin de l'été, des avant l'époque oir mûrissent les graines des plantes nuisibles. Les parties de la pépinière occupées par les plants de haute lige devront recovoir chaque année deux binages.

A RTICLE III.

Do la saison la plus convenable à la plantation.

78. On peut plinter depuis le temps de la chule des feuilles jusqu'au moment où les boutons commencent à s'ouvrir'; mais comme, en hiver, la terre est ordinairement ou gelée ou trop molle, on dislingue deux saisons pour planter : l'automne et le printemps.

Quand on plante en automne, les arbres, lor's de l'extraction, souffrent moins de se trouver quelque lemps hors de terre, pitrce que l'évaporation est moindre dans cette saison qu'en toute autre; en second lieu, la terre, par l'humidité dont elle s'imbibe, rl par les gelées, se tasse mieux autour des racines: enfin, il parait constant que, dans les hivers doux, les arbres poussent du chevelu; d'où il suit que, dans certains cas, des sujets plintés en automne peuvent être pourvus, dès le printemps, de nouvelles racines 1 . On peut donc, en général, considérer l'au-

Voyez Duhamel. Des semis et plantations des arlores, page lia. 
tomne comme la meilleure saison pour les plantations à faire en grand; cependant il existe aussi des cas où le printemps est préférable. Ainsi on doit choisir cette saison pour les essences qui peuvent avoir à souffrir des fortes gelées d'hiver, et, à cel égard, il faut nécessairement tenir compte du climat local; elle est encore la plus convenable pour les bois résineux qui, généralement, reprennent moins bien quand ils sont plantés en automne '. Enfin, dans les terrains trop humides, la plantation du printemps mérite souvent la préférence.

\section{ARTICLE IV:}

\section{De l'espacement à donner aux plants.}

785. Nos arbres forestiers, comme on le sait, prospèrent surtout lorsqu'ils croissent en massif;

1 Dans les régions (fuhabilent cesessenees íau moins les plus importantes et les plus rópandues), les hivers sont rigoureux. Si l'on plante des sujets très jeunes (de 1 à $3 \mathrm{ans}$ ), ce qui est à conseiller puisfu'on ne peut tailler les résineux [801], il arrive frífuenment que les fortes gelées d'hiver soulèvent les plants rt les rejettent, pour ainsi dire, hors des trous. Si l'on plante des tiges plus àgées, on les rnit souvent perdre toutes leurs feuilles pendant l'hiver el, comme ces essences vivent plus pardiculièrement par les orgunes aúrims, leur reprise, au printemps, exige, de la part des racines, un effort de végétation qu'elles ne sont que rarement en état de foumir. Le plant languit alors jusqu'en été, puis, quand surviennent les grandes chaleurs et la sécheresse, il finit par périr. 
c'est dins cet riat qu'ils prenment les plus belles proportions et qu'ils fertilisent le sol, en lui conservant de la fraìcheur et en lui procurant de l'engrais. II suit de là que, dans les plantations, il faudrait rapprocher assez les tiges, pour que, dìs les premières années, le massif pût se former. Mais, si l'on appliquait ce principe dans toute sa rigueur, il arriverait, après un très court lips de temps, que le besoin d'une éclaircie se ferait sentir, et qu'on se verrait forcé de supprimer un grand nombre de tiges, sans que, dans la plupart des cas, leur produit pût dédommager des dépenses qui auraient été faites pour les planter. Dans la pratique, il faut donc calculer l'espacement à donner aux plants de manière à concilier, autant que possible, les exigences d'une bonne végétation avec l'économie. Or, cet espacement dépend, d'abord, des dimensions des tiges, de l'essence, du sol et du climat; en second lieu, du but que le propriétaire se propose en plantant, et des sommes qu'il est en état de consacrer à cet objet.

786. Dans les plantitions de basses tiges, l'espacement varie de 66 centimètres à 1 mètre 33 centimètres; 1 mètre est la distance la plus ordinairement adoptée. Les hautes tiges s'espacent à 2, 3, 4, 5, 6 et mème jusqu'à 8 mètres.

Pour régler cet objet, l'expérience a confirmé les principes suivants :

Plus tes plants sont lorts, plus ils doivent ètre espacés. 
Certaines essences, telles que le hêtre, le sapin et, en général, les arbres dont le couvert est épais, demandent à croître très rapprochées; d'autres, au contraire, comme le bouleau, l'orme, le robinier, exigent plus d'espace.

On doit planter plus serré dins les sols secs et arides que dans les termins fertiles, dans les climats froids que dans les régions tempérées.

Lorsqu'on ne veut obtenir que du bois de feu, on peut adopter un plus grand espacement que quand il s'agit de se procurer des bois de construction ou de fente.

Quand on a des terrains très étendus à reboiser et qu'on est borné dans ses ressources pécuniaires, il faut adopter le plus grand espacement possible, afin d'arriver promptement à mettre le sol en production.

Les plantations de tètards et d'irrbres d'émondes sont celles qui admettent le plus d'espacement, parce que le sol, comme on le sait, est ordinairement utilisé, soit pour la culture, soit pour le pâturage.

787. On ne réussit à donner un égal espacement aux plants, qu'en les disposant dans un ordre régulier. On connait quatre manières de tracer les plantations: $1^{\circ}$ en allées ou files (fig. 9); $2^{\circ}$ en triangles équilatéraux (fig. 10); $3^{\circ}$ en carrés (fig. 11); et $4^{\circ}$ en triangles isociles ou quinconces ${ }^{1}$ (fig. 12).

1 Ce dernier mode est au fond le même que le troisième, si ce nest que les carrés, au lien d'etre construits, par rapporl 
C'est ordinairement avec un cordenu et des piquels que ces tracés s'exécutent; si le terrain est étendu, on peut aussi employer l'équerre d'arpenteur pour établir les alignements. Nous croyons inutile d'expliquer comment on procède à cefte opération: l'inspection des figures suffira pour le faire comprendre.

Le choix de l'une on de l'autre de ces figures n'a pis, à proprement parler, d'influence culturale, c'est plutot une affaire de goùt; cependant la plantation par files ou allées présente, dans certaines circonstance, des avantages réels. D'abord, elle permet l'ouvrir des tranchées, au lieu de trous, ce qui rend l'ouvrage plus facile et procure un sol plus meuble. dans lequel, en général, les plants prospèrent mieux, en second lieu, le tracé en est plus aisé et plus prompl, quels que soient les contours du terrain. A la vérité, les plants n'ont pas la facilité d'étendre également leurs racines de tous les còtés, puisqu'ils sont trèsrapprochés dans un sensel très espacés dans l'autre; mais on sait qu'il n'est pas nécessaire que les racines se répandent symétriquement autour d'un arbre: elles se dirigent indifféremment vers un point quelconque, pourvu qu'elles y trouvent de l'espace et rle la nourriture.

788. Le tableau ci-ippres fail connaitre la quantité

à une ligne donnée au moyen d'un système de perpendiculaires, sont formés pir un sy-teme doblipues faisant, avec la ligne donníc, un angle de fï degrés. 
we phints ì cimployer sur un hectare, quimd on adopte le tracé par triangles équilatéraux, ou celui par carrés, avec les différents espacements portés dans la première colonne.

\begin{tabular}{|c|c|c|c|}
\hline 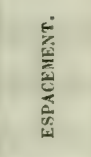 & 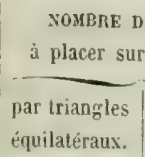 & $\begin{array}{l}\text { PI,AdTs } \\
\text { un hectare } \\
\text { par carrés. }\end{array}$ & OIISERYATIONS. \\
\hline mitres. & & & \\
\hline 0,660 & $2(0.5) 1.5$ & 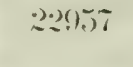 & Nous n'avons pas fitit figurer \\
\hline $1,(0)$ & 11550 & 10000 & $\begin{array}{l}\text { Sur ce tableau la plantation en } \\
\text { allées, parce que le nombre des } \\
\text { plants a cmplover, par hectare, }\end{array}$ \\
\hline $1, ; 3: 3$ & $\left(i_{0}\right) \cdot 2 ! !$ & 5653 & $\begin{array}{l}\text { dépend, a la fois, de leur espa- } \\
\text { cement sur la même file et de la }\end{array}$ \\
\hline $1,16 j ;$ & 4190 & $3(i 28$ & $\begin{array}{l}\text { distance des files entre elles } \\
\text { Mais on concoit que ce nombre } \\
\text { est tries favile à calculer, toutes }\end{array}$ \\
\hline 2,00 & 2888 & 2500 & $\begin{array}{l}\text { les fois que le terrain est un } \\
\text { carré ou qu'il peut ćtre ramené à }\end{array}$ \\
\hline 3,00 & $1: 383$ & 1111 & $\begin{array}{l}\text { celte forme; il sumtit, en effet, de } \\
\text { multiplier le nombre de plants } \\
\text { d'une tile par le nombre de toutes }\end{array}$ \\
\hline 4,00 & $7 \cdot 2.2$ & 625 & $\begin{array}{l}\text { les files. } \\
\text { Quant au tracé par trianglesiso- }\end{array}$ \\
\hline$\overline{\mathrm{C}}, 0()$ & 162 & 400 & $\begin{array}{l}\text { cèles ou quinconces, qui ne tigure } \\
\text { pas non plus ici, il est evident } \\
\text { qu'il admet lo même nombre de }\end{array}$ \\
\hline 6,00 & $3: 1$ & 278 & $\begin{array}{l}\text { tiges qua celui par carrés, pourvu, } \\
\text { toutefois, que les côtés des carrés }\end{array}$ \\
\hline 7,00 & 236 & 204 & $\begin{array}{l}\text { triangles, ainsi que nous le mon- } \\
\text { trent los figures } 11 \text { et } 12 \text {. }\end{array}$ \\
\hline 8,00 & 180 & 156 & \\
\hline
\end{tabular}


ARTICLE V.

\section{De la confection des trous.}

789. Les trous pour les plants doivent être proportionnés à la grandeur des racines, c'est-à-dire, qu'il faut que celles-ci puissent y trouver place, tout en conservant leur position et leur direction naturelles. Dans les sols très compactes, il est bon même de faire les trous plus grands, afin que les racines puissent, pendant quelques années, se fortifier et se développer dans une terre ameublie, avant de pénétrer dans des couches plus rebelles. Au contraire, dans les sols humides, on ne doit donner au trou que peu de profondeur (").

1 Si l'on avait à planter des parties aquatiques, on pourrait ne pas faire de trous du tout rt se contenter de poser le plant, bien diaplomb, sur le sol, en l'entourant d'une butte de terre assez large pour que ses racines soient entièrement couvertes, et assez élevée pour qu'il ait une assiette solide; autour de cette butte, qu'il est utile de crazonner à l'extérieur, on tracerait une petite rigole pour recevoir et éconduire les caux. Ce mode de plantation, indiqué déjà par Duhamel (roy. Truité des semis et plantations, jage 222) est rapporté par Cotta; il dit l'avoir pratiqué en grand et en avoir obtenu des résultats qui ont dépassé tout ce quon pouvait en attenrlre. Nous l'arons essayé aussi, en petit à la vérité, et l'expérience a parfaitement réussi. bans une récente publication, spécialement ennsacrón à ce mode particulier de plantation, M. le baron de Nanteuffel, 
790. Pour creuser les trous, on procède de la manière suivante: on enlève d'abord la superficie du sol, ordinairement gazonnée ou garnie de plantes quelconques, et on la place d'un côté du trou; puis on met, du côté opposé, la couche de terre végétale immédiatement inférieure, et qui est la plus riche en terreau; enfin, on entasse, sur un troisième point, les autres couches moins fertiles. Cette manière d'opérer contribue puissamment au succès des plantations, surtout de celles de hautes tiges; nous verrons plus loin quel avantage on en retire.

791. Lorsque le sol est de bonne qualité, les trous ne doivent être ouverts que peu de temps avant de planter, non-seulement pour que la terre reste plus fraîche, mais encore pour que le terreau qu'elle renferme ne perde pas ses propriétés nutritives, par l'action de l'air et de la pluie. Ce n'est que dans les sols très compactes qu'il devient nécessaire de faire les trous quelque temps à l'arance, afin que la terre se divise : ainsi, quand on veut planter au printemps, les trous peuvent être faits en automne.

\section{ARTICLF VI.}

\section{De l'extraction des plants.}

792. Quand on procède à l'extraction des plants, grand maître des forêts dans le roỵaume de Saxe, affirme l'avoir expérimenté, depuis plus de 20 ans, avec un plein succès, tant sur les essenees résineuses que sur les fienillues, et dans les sols secs aussi bien que dans les lieux humides. 
il liaul litire en surte de ménager, le plus possible, les racines et les tiges.

Les brins très petits peuvent être arrachés à la main, pour peu que la terre soit meuble et fraîche; mais, en général, il vaut mieux les extraire avec un couteau, el, dins les sols plus compactes, on fera bien de se servir d'une petite pische. l'our extraire des. liges plus fortes, si elles sont en sillons dans une pépinière, on ouvre une tranchée parallèle au premier sillon et le plus près possible des plants ; puis, avec lit bêche, on détache ceux-ci par mottes, en les soulevant un peu, et on les renverse doucement dins lit tranchée, de manière que la terre reste adhérente aux racines. On continue ainsi de sillon en sillon. Quand on arrache les plants dans les forêts, on peut aussi commencer par faire une tranchée ou un trou, puis fouiller la terre avec une pioche.

793. Lorsque des plints de basse lige doivent être transplantés en mottes, ce qui est surtout à conseiller pour les essences résineuses, on se sert, avec beaucoup d'avantage, pour les extraire, de l'une ou de l'autre des deux bêches demi-circulaires dont nous donnons le dessin à la fin du volume (voy. fig. 7 et 8). La plus grande de ces bèches convient plus particulièrement dans les terres compactes et pour des plants qui sont déjà d'une certaine force ; l'iutre, plus légère et plus facile à manier, sert dans les sols plus divisés et pour extraire des tiges plus faibles. lips bèches nt s'emploient pass seulement à l'extrac- 
tion des plants, elles servent aussi pour confectionner les trous dans lesquels les mottes doivent être placées '. On conçoit combien, par un tel procédé, la plantation est rendue facile, et quels avantages doivent en résulter pour la végétation.

794. Les hautes tiges ne peuvent être traitées par aucun des moyens que nous venons d'indiquer. Pour les extraire, il faut d'abord creuser une petite fosse autour de l'arbre, à une distance convenable pour lui laisser les racines nécessaires; puis, avec une bèche bien tranchante (roy. fig. 6), on coupe les racines latérales, et, après avoir élirgi la fosse, on finit par atteindre obliquement le pivot. Il faut se garder de pencher l'arbre avant d'en avoir détaché toutes les racines, ou de l'arracher avec effort.

ARTIGLE VII.

Du transport des plants.

795. A mesure que les ouvriers arrachent les plants de basse tige, ils doivent les mettre dans des pa-

- Pour faire les trous avec les bèches dont il est question ici, l'ouvrier enfonce l'instrument en le tournant et le retournant horizontalement à l'aide de la traverse qui se trouve adaptée à l'extrémité de la tige verticale. Il n’a pas besoin chaque fois qu'un trou vient d'être laait, de s'occuper à faire sortir la motte de terre de la bêche qui la retient. Cette opération se fait d'elle. 
niers, sans secouer la terre qüi entoure les racines, et les faire transporter tout de suite sur le terrain où l'on plante. On emploie aussi, pour le transport des plants, surtout de ceux qui sont en mottes, des brouettes de jardin ou de petits tombereaux; il n'y a que les trop fortes tiges qu'il soit nécessaire de placer sur des voitures.

Quelle que soit la dimension des plants, il faut avoir soin que les tiges ne soient point endommagées, et, à cet effet, les garnir de bouchons de mousse ou de paille, partout ou il y aurait quelque frottement à craindre pendant le transport. Les racines doivent surtout être garanties du contact de l'air ; il ne faut souvent que quelques heures de hâle, ou un coup de soleil un peu vif, pour les priver de leur vitalité.

Quand le lieu où l'on arrache les plants est éloigné du terrain à planter, les tiges doivent être réunies par bottes et les racines empaquetées dans de la mousse fraiche ou dans du foin humide. Rendues à destination, il faut les laisser ainsi en paquets jusqu'au moment de les planter ou de les mettre en jauge 1 .

même par la confection du trou suivant; la nouvelle motte chasse l'ancienne. Cette circonstance est bonne à connaître, car elle est de nature à procurer une notable économie de temps, lorsqu'il s'agit de grands travaux de repeuplement.

1 Pour mettre les plants en jauge, on ouvre une tranchée dans laquelle on les place par ballots; on recourre ensuite les racines de terre bien meuble, de minière à empècher le contact de 


\section{De la taille des plants.}

796. L'extraction des plants, si bien qu'elle soit exécutée, lèse toujours un certain nombre de racines, et, à moins que le sujet ne soit tout à fait petit, une partie de ses racines, détachée par la bêche ou la pioche, demeure en terre. Or, dans toute plante croissant librement, il existe, comme on le sait, un rapport direct entre les racines et la tige ; et ce rapport nécessaire, l'extraction l'interrompt plus ou moins comme on le voil, selon les précautions avec lesquelles on procède et selon la dimension des sujets.

La taille des plants a donc pour but: $1^{\circ}$ de restaurer les racines qui ont été lésées; $2^{\circ}$ de rétablir l'équilibre entre les racines el les branches.

797. La première de ces opérations consiste à amputer avec une serpette, toutes les parties des racines qui présentent une déchirure, une contusion ou une blessure quelconque. Il est essentiel que la

l'air. Par ce procédé, les plants peuvent être conservés assez longtemps avant de les planter, sans qu'ils aient aucun risque à courir; toutefois, si le séjour en jauge devait se prolonger beaucoup, il faudrait délier les ballots, de crainte que les racines, surtout le chevelu, ne fussent atteintes par la moisissure. On peut aussi mettre les plants dans l'eau, mais pour peu de jours seulement. 
tranche soit bien nette; elle doit être fitite en biseau, et, autant que possible, de manì̀re qu' elle pose à plat sur la terre.

Quand les plints sont arrachés déjà depuis quelque temps, et que le chevelu des racines n'a plus toute sa fraicheur, on le ravive en en coupant les extrémités; les racines trop longues, qu'on ne pourrait placer dans le trou sans les replier, doivent aussi être retranchées, ainsi que le pivot. Mais, en génériul, il fautéviter de supprimer desracines saines; un plant n'en a jamais trop.

798. La perte de racines que le plant a subie par l'extraction el par la taille dont nous venons de parler, rend nécessaire la suppression d'une partie des iranches, afin de rétablir l'équilibre détruit. Cette suppression est d'autant plus indispensable que, par la transplantation, les racines sont pendant quelque temps entravées dans leurs fonctions, et qu'elles amènent, par conséquent, moins de substances nourricières à l'arbre.

Plus le plant est fort, plus il a perdu de racines par l'extraction, plus le sol où il doit être planté est ingrat, moins il faut lui laisser de branches. Ces principes, très simples en théorie, et les seuls qui se puissent donner sur la taille des plants, laissent néanmoins, il faut l'avouer, à désirer quand on en vient à l'exécution.

Quel est, en général, le rapport des branches aux ratcines? Est-il le mème pour toutes les essences ou 
varie-t-il pour chacune, et comment? Dans quelle proportion l'ensemble des racines a-t-il été diminué par l'extraction ? est-ce du tiers, du quart, etc. ? On sait que la reprise est favorisée par les années pluvieuses, entravée au contraire par les années de sécheresse ; il faudrait donc supprimer plus de branches dins ce dernier cas ; mais sur quelle température doit-on compter? Telle est la série de questions auxquelles la taille des plants peut donner lieu, et qui, cependant, n'admettent aucune solution positive.

Aussi cette opération n'est-elle en réalité qu'un tàtonnement, dans lequel on réussit plus ou moins, selon l'expérience que l'on a acquise de la localité et des essences que l'on plante.

C'est, sans nul doute, à l'incertitude qui règne sur cet objet, qu'il faut attribuer, en grande partie, l'insuccès de tant de plantations faites dans les forêts, surtout, lorsque les plants n'avaient pu être préparés en pépinière el qu'ils étrient déjà de forte dimension.

799. Il existe, pour la plantation des hois feuillus, un procédé qui lève toutes les difficultés que nous venons de signaler. Ce procédé, c'est le recépage du plant, à 3 centimètres environ du collet de la racine, au moment mème de le mettre en terre.

Si l'on examine les conditions de végétation du plant recépé et de celui qui n'a été que taillé, on ne peut conserver aucun doute sur les avantages marqués dont jouit le premier. En effet, la grande difficulté, pour le plan taillé, c'est de rétablir l'équilibre 
entre la tige et les racines; tant que ce résultat n'est pas atteint, la végétation souffre, et trop souvent les racines s'épuisent en efforts inutiles, parce que la tige est encore trop branchue, ou parce que le sol ou la température est contraire. Aussi est-il très fréquent de voir des plantations languir pendant cinq ou six ans; après quoi elles finissent, quelquefois, par prendre leur essor, mais non sans que bon nombre de plants aient péri, en même temps que d'autres, par le dessèchement de leur cime, ont contracté des formes vicieuses. Quand, au contraire, on récèpe le plant en le mettant en terre, il se présente, sur la petite souche, un certain nombre de rejets qui, évidemment, sont le produit de la force végétative des racines; si cette force est grande, les rejets seront vigoureux, sinon, ils serontfaibles. Mais en tout cas, ils seront en rapport direct avec les racines, car ils en sont le produit; et, par conséquent, la végétation se trouvera, de prime abord, rétablie dans son état normal.

800. Sous le rapport des chances de reprise, la plantation de brins recépés est done incontestablement supérieure à celle de brins taillés. Ce fait, clairement établi en théorie, nous est d'ailleurs confirmé par une pratique qui n'admet plus aucun doute. Cependant une objection se présente: par le recépage, chaque plant pousse plusieurs rejets qui forment une petite cépée ; il semble donc que ce procédé ne puisse convenir que pour créer des taillis, et 
non des futaies, à moins que l'on ne prenne la peine au bout de quelques années, de couper, avec une serpette, tous les rejets faibles, afin de ne laisser crồtre, sur chaque souche, que le plus vigoureux '.

Si une telle opération était nécessaire, il faudrait, en effet, renoncer au recépage, pour planter des futaies en grand, ou du moins, on pourrait rencontrer des difficultés réelles d'exécution. Mais il n'en est rien ; la pratique, sur ce point, a rectifié les inductions de la théorie.

Dans la forèt de Compiègne, que nous avons déjà eu l'occasion de citer, on voit des plantations de la plus grande beauté, exécutées sur une échelle immense 2, d'année en année, depuis plus de quatre-vingts ans, et qui, toutes, ont été traitées par le procédé du recépage. Nous avons pu y étudier toutes les phases de la régétation des plants recépés.

Ces plantations se font avec des sujets de basse tige, et l'espacement ordinaire qu'on leur donne est d'un mètre. Comme nous l'avons dit, il se présente d'abord, sur chaque souche, plusicurs rejets dont l'un, cependant, est plus vigoureux que les autres. Dès que ce maitre rejet commence à former une tête, les autres, au lieu de continuer à monter, s'étalent et

' C'était l'opinion de Duhamel. Voy. Trrité des semis et pluntations des arbres, page 340 .

"Il existe plus de 3,000 hectares de plantations dans cette forêt. 
buissonnent au pied du plant; ce qui conserve aux racines plus de fraicheur et favorise visiblement le développement du maître jet. Peu d'années suffisent, dès lors, pour que le massif de toutes les tiges montantes, soit formé ; quant aux rejets traînants, ne recevant plus les rayons du soleil, ils languissent de plus en plus, s'étiolent, sèchent, et le maître rejet finit par se trouver seul sur la souche. Aussi de telles plantations, lorsqu'elles sont parvenues à l'état de gaulis, ne laissent-elles plus apercevoir la moindre trace de ces rejets traînants; toutes les tiges sont parfaitement assises, droites, nues et bien filées, sans qu'il soit possible de les distinguer, en quoi que ce soit, des brins de semence.

La plantation avec recépage peut donc convenir pour créer des futaies, aussi bien que des taillis; seulement il faut, pour les premières, se servir de basses tiges et planter assez serré, afin que le massif se forme à temps, tandis qu'au contraire, pour établir un taillis, on pourra employer avec avantage des tiges plus fortes, parce qu'on obtiendra, par là, plus promplement des cépées abondantes.

801. Le recépage, on le conçoit, ne peut être appliqué aux bois résineux; il est mème d'expérience que la taille des hranches leur est souvent plus nuisible qu'arantageuse. Ces essences, effectivement, paraissent, plus que les feuillues, puiser leur nourriture dons l'atmosphère; il semble donc nécessaire deleur ménager plus particulièrement les organes 
destinés à assurer cette nutrition. Cependant, il peut être utile, lorsqu'on plante des sujets un peu forts, de leur enlever quelques branches; mais il faut procéder avec la plus grande réserve, car les plaies des bois résineux, quand elles sont nombreuses ou d'un certain diamètre, occasionnent à l'arbre des écoulements séveux qui l'affaiblissent. Aussi est-il à conseiller de préférer, pour les plantations de bois résineux des plants de basse tige qui soient assez jeunes pour pouvoir se passer de la taille.

D'après les expériences de plusieurs planteurs distingués, notamment de M. Marsaux (v. Annales forestieres, tome $\mathrm{I}^{\text {er }}$, page 699) le plant de hêtre supporte mal le recépage. On fera donc bien de s'abstenir de cette pratique pour cette essence et de la traiter, en cequi concerne la plantation, comme les bois résineux, à moins que des essais faits dans la localité oủ l'on opère, n'äient démontré que le jeune plant de hêtre y fournit sùrement des rejets, après amputation.

ARTICLE IX.

De la mise en terre des plants ou plantation proprement dite.

802. En général, on peut poser en principe qu'un arbre doit, après la transplantation, se trouver enterré à la même profondeur qu'avant cette opération. 
Cependant, il est convenable de planter un peu plus profondément lorsqu'on peut prévoir que la terre s'affaissera, ou bien lorsque le sol est très léger et sec: d'autant plus que, dans ce dernier cas, il faut ménager, autour des tiges, un petit creux où l'eau des neiges et des pluies puisse s'amasser. Dans les sols humides, au contraire, on plante moins profondément, et, au lieu de laisser subsister un renfoncement autour de la tige, on y fait une petite butte pour faciliter l'écoulement des eaux. Les tiges recépées doivent être plantées de manière à affleurer par leur extrémité supérieure la surface du sol.

803. Pour mettre le plant en terre, on procède de la manière suivante :

On place le plant dans le milieu du trou sur une

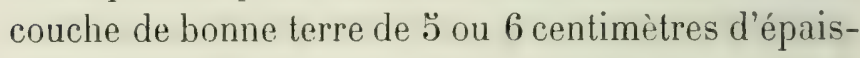
seur environ, ou bien sur les mottes de gazon provenant du trou et que l'on a pris soin de briser menu au préalable ; puis, avec la main, on étend les racines de firçon à laisser à chacune sa direction naturelle; il est essentiel qu'elles posent toutes d'aplomb et que la tige se tienne bien droite. Cela fait, on répand la bonne terre végétale, qui a été mise à part en creusant le trou [790], de manière que les racines en soient entièrement couvertes; en même temps, on remue un peu la tige en la soulevant et en la rabaissant légèrement, afin que les parcelles de terre pénétrent de toutes parts entre les racines. Enfin, pour ne négliger aucune précaution. on in- 
ARTIFICIELS.

603

troduit la main sous les racines pour remplir toutes les cavités qui pourraient encore exister. Après que la couche de bonne terre a été employée comme nous venons de l'expliquer, on achève de remplir le trou avec les couches de moindre qualité. Tout en répandant ainsi la terre sur les racines, on la raffermit de temps en temps avec la paume de la main ou avec le pied, légèrement d'abord, puis, de plus en plus fortemeni.

Quand on plante de faibles tiges, recépées ou non, on facilite l'opération sans compromettre la reprise du plant, en appuyant ce plant contre une des parois du trou par son côté le moins pourvu de racines. La paroi doit, dans ce cas, être parfaitement verticale. L'ouvrier, tenant le plant de la main gauche, conserve lia droite libre pour étendre les racines en avant du plant, les garnir de terre et remplir le trou, avec les précautions que nous venons d'indiquer.

Pour ces sortes de plantations, on emploie utilement, soit une truelle de maçon, soit une petite houe à manche très court, à l'aide de laquelle le planteur, agenouillé devant le trou pour plus de facilité dans ses mouvements, accommode celvi-ci et y ramène la terre, sans se déranger, selon que l'exige la conformation du plant dont il s'occupe.

804. Souvent, au lieu d'un seul plant, on en réunit deux, trois, et quelquefois jusqu'à cinq et six, dans le même trou. Ce mode, appelé plantation par tonffes, réussit pirfaitement pour les bois résineux, 
surtout lorsqu'ils sont très jeunes ( 1 et 2 ans); on l'a aussi appliqué avec succès au hêtre.

Les plants sont élevés en pépinière, de la façon que nous avons décrite plus haut [779], seulement les sillons sont très étroits ( 4 à 6 centimètres). Au moment de la plantation on coupe les sillons, avec une bêche, par plaques ayant à peu près la dimension d'une brique. Ces plaques sont transportées, dans des paniers, sur le terrain à planter, et là, divisées à la main, par touffes contenant le nombre de brins que nous venons d'indiquer.

Les trous se font à la houe ou avec la petite bêche demi-circulaire [793].

Les principaux avantages que présente la plantation par touffes sur celle faite avec des sujets séparés, sont :

$1^{\circ}$ Plusieurs brins réunis en une touffe permettent plus difficilement à la terre de se détacher des racines, soit dans l'extraction, soit dins le transport;

$2^{\circ}$ Les touffes couvrent plus tòt leur pied qu'un plant isolé, et la reprise d'un des brins, au moins, est à peu près assurée ;

$3^{\circ}$ Dans une mème touffe, le brin le plus vigoureux ne tarde pas à s'élever au-dessus des autres; après quelques années, ces tiges dominantes forment seules le massif de la jeune forêt et les autres tombent dans la première éclaircie.

Ce mode de plantation, que nous avons nous-mème 
pratiqué sur une assez grande échelle et avec différentes essences (pin sylvestre, épicéa, sapin), nous a toujours très bien réussi, même dans les sols et aux expositions les plus défavorables. Nous pouvons donc le recommander avec confiance, en renvoyant le lecteur, pour plus amples détails, à l'article que nous avons publié sur ce sujet dans les Annales forestières, tome IV, année 1845 , pages 329.

805̆. Lorsqu'on en aura la facilité, on fera bien d'arroser les plants immédiatement après les avoir mis en terre ; mais on conçoit qu'une telle opération n'est praticable que quandles travaux se font en petit. Ce qui l'est davantage, dans la plupart des cas, c'est de placer à droite et à gauche du plant, une ou plusieurs pierres qui maintiennent la fraìcheur et raffermissent la terre autour des racines, en mème temps qu'elles procurent à la tige, quand elle est très petite, quelque abri contre les ardeurs du soleil.

Pour les plantations en mottes, il faut avoir la précaution de remplir de bonne terre les petits interstices qui existent presque toujours entre la motte et la paroi du trou. 


\section{CHAPITRE SIXIËME.}

\section{DES BOUTURES.}

806. Toutes les essences feuillues ont, plus ou moins, la faculté de se reproduire par boutures; on a même réussi à multiplier de cette manière les bois résineux. Cependant, il n'y a que les peupliers, les saules et les platanes qui se montrent particulièrement faciles à cet égard, et qui fournissent de beaux sujets; les boutures des autres bois exigent beaucoup de soins, et les sujets qu'on en obtient sont généralement d'une faible végétation. Aussi se bornet-on, en sylviculture, à propager, de cette manière, les trois essences que nous venons de nommer, et dont il faut encore excepter toutefois, le tremble et le marceau, tous deux d'une reprise fort difficile.

Les plantations de boutures trouvent surtout leur applicition dans les terrains destinés au parcours, 
dans les prairies, sur les bords des chemins, etc.; mais elles sont aussi d'une ressource précieuse pour fixer les sables, pour maintenir les terres dans les pentes rapides et sur les bords des eaux, ainsi que pour repeupler certains lieux aquatiques dans les forêts.

On connaît deux espèces de boutures : la bouture en plançon et la bouture à bois de deux ans.

807 . Le plançon est une branche de 3 à 4 mètres de long sur 4 à 8 centimètres de diamètre, que l'on dépouille de tous ses rameaux et que l'on taille en biseau par les deux bouts. Pour le planter, on l'enfonce à une profondeur de 50 centimètres, après avoir formé, au préalable, le trou avec un pieu en fer. Toutefois, ce procédé n'est convenable que dans les lieux aquatiques; quand le sol est plus ferme, il vaut mieux ouvrir, à la bèche, un trou de 50 centimètres environ de profondeur sur 66 centimètres de côté, dans le milieu duquel on fixe le plançon et que l'on comble ensuite de bonne terre bien émiettée.

Lorsque les plançons risquent d'être endommagés, soit par le vent, soit par le bétail, etc., on leur donne des tuteurs et on les entoure, jusqu'à hauteur d'appui, d'épines ou de branchages.

C'est ordinairement avec des plançons que l'on forme les têtards. Il est à remarquer que les grands saules, tels que l'osier, le saule blanc, etc., sont les 
seuls bois qui reprennent bien de cette manière; les peupliers s'y refusent presque toujours.

808. Pour faire des boutures à bois de deux ans, un choisit des rameaux bien vigoureux, présentant, outre la pousse de l'amnée, du buis de deux ou de trois ans au plus; on leur enlève toutes les ramilles et on les réduit à 30 ou 40 centimètres de long. S'il s'agit de fixer des sables ou de maintenir des terres en pente, il est bon de leur donner même plus de longueur. La section inférieure se fait en biseau; celle du haut doit être droite, afin de ne pas blesser la main du planteur.

Lorsque le sol est bien meuble, on plante ces boutures en les enfonçant obliquement, de manière qu'elles ne dépassent la superficie que de 3 ou 4 centimètres au plus. Mais, dans une terre plus ferme, où l'on risquerait de les casser ou de déchirer l'écorce, on prépare des trous avec un plantoir un peu plus fort que les boutures; et dans les terrains tout à fait compactes, on ouvre, soit des trous, soit des tranchées avec la houe ou la bêche. Il est essentiel de bien raffermir la terre autour des boutures.

Souvent on élève des boutures en pépinière, surtout celles de peuplier et celles de platane pour les transplanter plus lard à demeure. Dans ce cas, on choisit un terrain de bonne qualité, frais, qu'on laboure convenablement et qu'on dispose par plan- 
ches. On y met les boulures en rigoles, et on les soigne de même que des plants ordinaires [781]. Des arrosements fréquents farorisent singulièrement leur reprise.

809. La saison la plus convenable pour faire des boulures est le printemp’s; néanmoins, la plupart des saules reprennent aussi de celle maniere en été. 


\title{
CIIAPITRE SEPTIËIE
}

\author{
DES MARCOTTES.
}

810. On peut appliquer le procédé du marcoltage, à toutes les essences résineuses ou feuillues; mais c'est surtout pour la propagation de ces dernières, et notamment dians les tirillis, qu'il mérite l'attention du forestier.

Lorsque les brins ou les rejets qui doivent être marcottés sont fuibles, et, par conséquent, flexibles, on peut les coucher sans difficultés dans de petites rigoles, firites à cet effet, que l'on comble ensuite de bonne terre. Mais quand ce sont des perches assez fortes déjà qu'il s'agit de fitire servir de cette maniere, il faut procéder avec plus de précaution. Pour le repeuplement des bois, ces dernières sont plus avantageuses; on en obtient des sujets plus nombreux, d'une reprise plus prompte et d'une croissance plus vigoureuse. 
Afin de réussir à ployer ces perches jusqu'à terre, on leur fait, à l'endroit où la plus grande flexion devient nécessaire, une entaille qui peut pénétrer jusqu'au centre du bois et qui doit être placée sur la face convexe de la courbure. Au moyen de cette entaille, on amène la tête de la tige sur le sol, légèrement labouré au préalable, et on l'y fixe par des crochets en bois qui la saisissent immédiatement audessous des branches inférieures et vers l'extrémité de la cime. De fortes mottes de gazon, placées sur les différentes branches principales, sont destinées à les maintenir contre terre. Cette première opération faite, on recouvre tous les rameaux de 16 à 22 centimètres de bonne terre, de manière ì n'en plus laisser passer que les extrémités, sur quitre ou cinq boutons au plus. Au moven de la terre dont on les entoure, ou bien a l'aide de molles de gazon, on donne a ces petites ramilles une position rerticale. L'entaille faite ì la perche doil, au moins pendant les premières années, itre recourerte de mottes de gazon.

Après trois ou quatre ans, il s'est formé, au-dessous de tous ces menus rameaux, des racines quileur sont propres, et qui sont suffisantes pour pourvoir a leur nutrition. Dès lors on peut done les sevrer, c'està-dire, retrancher la perche courbée qui les unissait à la souche mère ${ }^{1}$

' M. Heyer assure que les sorbiers, les irables el quelques autres arbres enenre, étant traités comme on vient de lexpli- 
Le procédé que nous venons de décrire, est rapporté par plusieurs auteurs forestiers de l'Allemagne, comme très usité dans lifférentes localités de ce pays. Dans le Hanovre, on l'emploie avec beaucoup de succès pour repeupler les clairières les taillis de hêtre. A cet effet, on réserve, lor's de l'exploitation, un certain nombre de tiges sur le lwrd de ces clairières, et l'année suivante on en opìre le marcoltage; ces tiges ont souvent 10,12 et mème $1: 3$ centimètres de diamètre à la base.

811. Lorsque les perches couchées font partie d'une cépée, il faut ériter de laisser d'autres perches debout sur la même souche. La séve ayant plus de tendance à monter droit qu'à circuler dans les branches courbées, abandonnerait celles-ci pour se porter avec affluence dans les autres, et la perte des marcottes en serait la suite. On doit donc supprimer tous les rejets, et, pour empêcher qu'il n'en repousse d'autres jusqu'à l'entière reprise des branches marcottées, on fera bien de couvrir la souche de 1 ă à 20 centimètres de terre fortement tassée en forme de pelite bulte. Dès qu'on opèrera le sevrage des marcoltes, on

quer, s'enracinent dès la première année. Il ajoute que, en général, le bois de deux ans, des branches couchées, s'enracine plus vite que les pousses de l'année, et qu'on hate d'ailleurs la production des racines, si, au moment où l'on procède au couchage d'ume branche, on lui enlève sur la face inféricure une netite plaque d'écorce jusqu'à l'aubier, avec un couteau bien thanchant. Autour de la blessure, il se forme un bourrelet sur lequel les racines ne tardent pas à se présenter. 
ARTIFICIELS.

613

pourra découvrir la souche qui ne tardera pas à fournir de nouveaux rejets.

812. Le marcotlage réussit mieux au printemps qu'en loute autre saison. 


\section{CILPITRE IIUTTIENE.}

\section{TRAYAUX DENTRETIEN A EXÉGUTER DANS LES REPEUPLEMENTS ARTIHICIELS.}

813. Par cela seul qu'un semis a bien levé et que, dans une plantation, la grande majorité des plants est restée verte ou a produit une faible pousse, la réussite de ces opérations n’est point assurée. - A vrai dire, le succès d'un repeuplement artificiel ne peut ìtre considéré comme certain qu'au bout de ä ou 6 ins, alors que les jeunes sujels, complétement enracinés, protégeant leur pied et pouvant lutter avec avintage contre les intempéries et l'envahissement des plantes nuisibles, commencent à s'élancer avec vigueur et approchent du moment où ils ront couvrir la totalité du sol par l'extension graduelle de leurs branches litérales. Jusquà cette époque, le sylviculteur ne peut abandonner son ourre; il doil, au 
contraire, sous peine de compromettre le fruit de ses travaux et de ses dépenses, la surveiller activement, écarter, autant qu'il dépend de lui, les causes qui sont de nature à entraver la végétation de la forèt naissante, et réparer, à mesure qu'ils ont lieu, les dommages que le temps a pu y causer.

814. Les travaux d'entretien consistent en sarclages et en binages. On appelle sarcler l'opération par laquelle on enlève, une à une pour ainsi dire, les mauvaises herbes qui se présentent parmi les plants composant un semis ou une plantation, tandis que biner, c'est, ainsi que le mot l'indique, donner une seconde firçon à la terre, en mème temps qu'on la débarrasse de la mauvaise herhe. Selon la nature des plantes qu'il s'igit d'extraire, selon le sol plus ou moins compacte, enfin, selon la ténuité du plant dans l'intérêt duquel l'opération a lieu, les sarclages se font ou avec un coutenu, ou avec un petit instrument appelé sarcloir et que tout le monde connait, ou bien à la main. Les binages se font toujours arec une houe légère ou avec un crochet.

813. Examinons d'abord l'application que trouvent ces travaux dans les semis.

$1^{\circ}$ Bois feuillus.

Les semis en plein sont les moins sujets à l'envahissement des mauvaises herbes, pourvu toutefois qu'ils aient bien levé. Ordinairement, comme on le sait, le sol a été préparé à la charrue et nettoyé par une ou plusieurs cultures en céréales. Si donc le 
semis forestier réussit, les jeunes plants étant répandus de toutes parts empêcheront la mauvaise herbe de s'emparer du terrain, et ne tarderont pas à la dominer et à l'étouffer. Toutefois, quelques sarclages dans les deux premières années ne sauraient être qu'avantageux.

Dans les semis partiels (par bandes et par pots) les plantes nuisibles sont beaucoup plus à redouter, parce que les parties demeurées incultes facilitent la reproduction de ces végélaux dans les parties cultivées, soit par la graine, soil par les racines. Aussi les sarclages dans les deux premières années, et les binages ensuite sont-ils, sinon indispensables, du moins extrèmement utiles, quand on ne veut pas que le semis languisse et quelquefois même que son existence soit compromise; car certaines essences feuillues, telles que le châtaignier, le frène dans ses premières années, même le chêne et quelques autres encore sont singulièrement offusquées par les hautes herbes, dans les sols où celles-ci se produisent avec abondance. On fera donc bien, dans ces sortes de semis, de ne pas se borner à sarcler ou bíner seulement les parties ensemencées, mais de donner en mème temps une légère façon aux parties demeurées primitivement incultes, pourvu que la déclivité du terrain permette de le remuer en totalité, et que l'essence ne réclame pas impérieusement un abri dans ses premieres années, comme le hêtre, par exemple. - Dans les sols frais ou dans les situations basses, 
l'opération dont il s'agit aura, outre l'arantage de débarrisser le semis de voisins incommodes et nuisibles, celui d'empicher que l'humidité atmosphérique (brouillards, rosées), en se maintenant dans les herbes, ne rende plus dangercuses pour les jeunes plants, les premières gelées de l'automne.

$2^{\circ}$ Bois résineux.

Les semis de bois résineux ne se font en général que dans des terrains assez légers, siliceux, feldspathiques ou calcaires, accidentés plutôt qu'en plaine. L'envahissement des herbes y est donc, la plupart du temps, moins à redouter que dius les terres fortes, substantielles ou humides; mais, par contre, on a à y combattre certains arbustes tels que myrtilles, bruyères, ronces, ete. - Il esl rare que l'on sème en plein et, presque toujours, c'est le semis par bandes ou par pots qui obtient la préférence. L'extrême ténuité des jeunes plants ne permet guère d'autres sarclages que ceux qui se font au couteau ou à la main. Ces opérations sont très utiles dans les deux ou trois premières années, surtout quand, pour économiser la scmence, on n’en a répandu que lit quantité striclement nécessaire el que, par conséquent, les sujets lèvent assez écirtés. Quant aux binages, ils ne sont à conseiller, ni dans les premières années, ni dans les suivantes, el il est entendu que l'on devra s'alsstenir, surtout dans les pentes, de donner une culture à la houe aux bandes intermédiaires, quand bien mème on reconnailrait que les arbustes ou les herbes 
qui les garnissent nuisent au semis en s'abattant sur les rayons cultivés. Ce qu'il y aurait de mieux à faire en pareil cas, ce serait de les faucher si ce sont des herbes, et si ce sont des arbustes, de les tondre avec

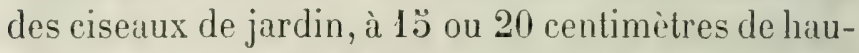
teur, comme on tond les haies dans les champs. Une forte faucille ou un croissant pourrait aussi servir à ce dernier ouvrage.

817. Dans les plantations, les sarclages, on le conçoit, seraient presque toujours insuffisants; par contre, les binages deviennent d'autant plus efficaces, tant pour éloigner des plants les herbes ou les arbustes qui pourraient les gêner, que pour procurer à leurs racines l'accès de l'air et de l'humidité. Lorsqu'on plante des essences d'élite dans de bons terrains situés en plaine, il est souvent avantageux de faire précéder la plantation, de même que le semis, d'un labour total à la charrue et mème de plusieurs cultures de céréales ou de pommes de terre. Le sol est alors parfaitement ameubli et nettoyé, et il est facile de l'entretenir dans cet état au moyen d'un ou de deux binages au plus par an. Mais, dans les repeuplements forestiers, de telles plantations ne sont guère que l'exception. Ordinairement, la qualité et la configuration du sol, autant que des motifs d'économie, font qu'on se borne à ouvrir les trous des plants, comme nous l'avons enseigné plus haut [790], sans toucher au reste du terrain. Il s'ensuit que les binages aussi ne se pratiquent qu'autour de chaque 
plant, sauf à tondre les arbustes ou à faucher les herbes dans les intervalles, s'ils devenaient nuisibles ou si l'on devait en retirer un bénéfice immédiat.

817. L'époque la plus favorable pour les sarclages comme pour les binages des semis et plantations, est celle où les plantes nuisibles poussent avec le plus de vigueur, c'est-à-dire, les mois de mai et de juin. Si, en raison de l'abondance des parasites et des inconvénients qu’elle pourrait entrainer pour les jeunes plants, dans l'automne ou l'hiver, on reconnaissait l'utilité d'un second sarclinge ou binage, il faudrait l'effectuer dans le courant de septembre.

818. Les traviux d'entretien dont nous venons de parler sont encore peu en usage parmi les forestiers qui s'occupent de repeuplements artificiels, soit que les forestiers répugnent à faire la dépense à laquelle ces opérations donnent lieu, soit qu'ils craignent de ne pouvoir suffire à la surveillance qu'elles exigent. - Nous ne méconnaissons pas les obstacles qui peuvent souvent exister sous ce double rapport, mais nous ne saurions trop insister sur l'opportunité des travaux dont il est question. Non-seulement ils assurent la réussite d'une quantité considérable de sujets qui, autrement, eussent péri ; mais ils doublent et triplent la croissance de tous dans les premières années, firvorisent le développement de leurs organes de nutrition et jettent ainsi les fondements d'une végétation prospère et vigoureuse qui, plus 
tard, dédommagera le planteur, souvent au décuple, des peines et des sacrifices pécuniaires qu'il se sera imposés.

819. Deux ou trois ans au plus tard, après qu'une plantation a été exécutée, il convient de s'occuper des remplacements ou regarnis. Pour cette opération, on emploiera de préférence des plants un peu plus forts que ne le sont ceux auxquels ils doivent ètre associés, afin qu'ils se tiennent plus facilement à leur hauteur.

Pour faire le mème travail dans un semis, il est bon d'attendre que les jeunes plants aient acquis assez de développement pour permettre de juger de la réussite du repeuplement dans son ensemble. Les regarnis se font de la sorte plus facilement et mieux; il est presque toujours préférable d'y procéder par voie de plantation.

FIN. 


\section{APPENDICE}

Renvois du $\mathrm{n}^{\circ} 67$, page 37 .

\section{CHÊNE CHEVELU}

Le chêne chevelu, commun en Orient, ne se trouve en France qu“äl'état très disséminé; il forme, dans le Doubs, l'esseuce dominante d'une partie le la for't communale de Saint-Vit. On le rencontre mélangé an l'ouvre et au pédonculé dans le Jura, la Vienue, la Lnire-Inférieure, la Provence, etc.

Climat, situation, exposition. - C'est un arbre des climats loux et tempérés; ceprndant, dans ces deruiers, il ne dépasse pas au Nord le département du Doubs. Il habite les plaines et les parties inlérieures des montagnes et recherche l'exposition de l'Est et du Sud-Est.

Terrain. - Il n'est pas difficile sous le rapjort de la nature du sol et prospère encore lá où le chène pédouculé languit. 
Floraison et fructification. - La floraison est monoïque et amentacée pour les mảles; les fleurs paraissent fin llarril ou commencement de mai, arec les feuilles, en retard d'euviron 15 jours sur le pédonculé. Le gland ne mûrit qu'à l'automne de l'année qui suit celle de la floraison et tombe aussitot après. La fructification est généralement constante.

Jeunes plants. - Le tempérament est robuste et les jeunes plants peurent se passer d'abri.

Feuillage. - Les feuilles, généralement très décou pées, ne donnent qu'un couvert léger.

Racines. - Le chène chevelu a un enracinement pivotant et profond: quand le terrain le permet, à läge de 4 ou 5 ans, le pirot mesure près d'un mètre.

Croissance et durée. - La croissance le cet arbre parait etre julus artire que celle du ronrre et du frédonculé; à ô ans, il atteint, dans le Ioubs, un mètre de circonférence; mais il y est exposé à de nombreuses gélivures qui emprechrnt de le laisser arriver à de plus grandes dianinsions. On en comnait cependant qui mesurent 2.5 m. de hauteur sur $2 \mathrm{~m}$. jo de rirconfierence. Sa longévité est prolongée.

Qualités et usages. - Le bois du rhene cherelı rappelle celui du tauzin; il est dur et tres nerveux, mais chargé d'aubier, exposé à se gercer, peu apte à la fente. Lescrélivu'es, qu'il frésentefíf́quemment dans le Doubs, le renrent inporore aux grantes constructions. 
Son chaufrage est de très bonne qualite; on l'estime à l'égal de celui du hêtre. L'écol'ce est préférée à celle du pédonculé et du rourre pour le tannage. En France, les glands, toujour's àpres, ne peuvent servir qüà l'engraissement des pores.

\section{H. N.}

\section{CHÊNE OCGIDENTAL}

Longtemps confondu avec le chène liège, le chêne occidental se rencontre peu dans la région méditerranéenne et habite particulierement less bords de l'océan depuis Bayonne jusqu'à la Gironde. Le long du golfe de Gascogne, il forme de rastes forets, seul on mélangé avec le pin maritime.

Climat, situation, exposition. - C"est un arbre des climats rloux plutot que chanis; on le trouve dans less plaines of il prospere encore jusru’a $400 \mathrm{~m}$. d'altitude. C"est surtout sur les pentes mérilionales et abritées qu’il se plaît.

'Texain. - Il recherche principalsuent les terrains de transport, siliceuxou arogilo-siliceuxdes landes.

Floraison et fructification. - La floraison est monoïque et amentacée pour les máles; les fleurs paraissent en juin; les glands qui leur succedent ne sont mûrs qu'en septembre de l'année suivante. L'arbre de- 
vient fertile ver. l'àge de 25 à 30 ans et fructifie jresque tous les ans.

Jeunes plants. - Les jeunes plants sont assez robustes, mais l'ombrarge leur est favorable et ils se trourent bien dı melange avar le pin maritime dont le couvert est très léger.

Feuillage. - Les funilles sont petites, légèrement dentées, et persistent jusquà l'entier développement le celles de l'amnée suivante. La tète de l'arbre, très rameuse, fournit un couvert assez épais.

Racines. - Le cliène occidental pivote el trace. Les racines latérales, qui s'étendent au loin, sont disposées à drageonner.

Croissance et durée. La croissance de cet arbre est assez rapinle; vel's loigre de centans, il peut atteindre denx melres de rirronférence; il ne prend qu'me hauteur de 15 a 20 mettes et ne délasse grière 4 metres de tour. Sa longérité est prolongée.

Qualités et usages. - Comme le chène liège, le

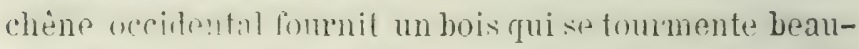
roup et smporte mal hes alternatives de sécheresse et dhumirlite: rest rom nu maurais bois rle construction ou de travail; il est an contraire très pistimé pour le chauffage et donne un excellent charbon.

Il est surtout cultive pour son écorce qui produit un liège aussi estimé que celui du chêne liège.

H. $\mathrm{N}$. 


\section{Renvoi du n ${ }^{\circ} 475$, page 232 .}

En posant le principe que la possibilité des coupes de régénération doit toujours ètre basée sur le volume, les auteurs du Cours de culture laissent entrevoir quà la rigueur les coupes d'ensemencement pourraient ètre réglées par contenance.

C'est en effet ce qui a lieu pour quelques sapinières de l'arrondissement de Remiremont, à titre d'essai seulement et dans des conditions tout à fait exceptionnelles.

Dans cette région des Vosges, située sous un rude climat, les repeuplements naturels tardent à se produire, soit que la graine jarvienne rarement à maturité, soit par suite d'accidents qui compromettent le succès des semis; au début, la croissance est d'ailleur's très lente; les jeunes plants le hètre et de sapin ont, à un degré remarquable, la faculté de supporter le couvert, dans un état de végétation latente, pendant 20,30 et mème 40 années, puis de se développer rapidement s'ils viennent à ètre dégagés arec les précautions nécessaires.

Il fallait tenir' compte de ces particularités dans l'aménagement et fixer à 40 ans la durée des périodes mesurée aux lenteurs du réensemencement naturel; mais, romme laise moyen des peuplements ne permettait pas 
d'arlopter une rérolution de plus de 1:00 ans, chaque allectation en̂t ainsi compris le tier's de la série, c'est-àdire une étendue beaucoup trop grande.

En eflet, sous le couvert rle la coupe sombre, la végétation est a peine sensib)le; les semis ne manifestent r'ellement leur existence qu'après avoir été dégagés; on comprend des lors que la grarlation des àges, rlans les nouveaux perplements, dépend de la marche que l'on imprime aux conpessecondaires et définitives. - Assises ga et lá, sans ordre, suivant les hasards du repeuplement, ces exploitations laissent sourent, après leur passagre, des bouquets de jeunes bois confusément mêlés, dont les àges extrèmes presentent un écart d'autant plus grand que la période est plus longue.

Il s’agissait l'éviter cette ir'ŕgularité le consistance, qui perpétuerait, (nu quelque sorte dans les affectations, l'aspect ot les inconvénients du jardinage, et préparerait, pour la ¿e révolution, des bois plus ou noins éloignés de l'àge d'exploitabilité.

C'est pourouoi l'on a adopté les dispositions suivantes:

La róvolution a été partagée en 6 périodes de 20 ans. La premirre afrectation doit être entièrement régénérée en :20 ans, autant que possible par voie le réźnération naturelle; mais, comme la période est trop courte, eu enard aux conditions rlimatériques, on arhnet quaprès un certain temps écoulé sans lésultats satisfaisants, le 
parter'e des coupes sombres sera repeuplé artificiellement.

Daus cette affectation, la possibilité de toutes les coupes de régénération est l'ailleurs hasée sur le volume.

Durant la mème périorle, de véritables coupe sombres, ascises de proche en proche par vingtième de surface, parcourent la totalité de la $\sim^{20}$ affectation. Si l'on jrévoit une année fertile, on prépare le sol par bandes ou sillons; mais, quel que soit l'état des semis ainsi obtenus ou de ceux qui existaient fortuitement arant le passage des coupes sombres, on rloit s'alstenir de les dégagger; c'est en deuxième période senlement que les exploitations prendront la forme de coupes claires et définitives. On espère qu'à cette éporque, il sera possible de les asseoir régulierement et de réaliser la gradation des àges, en dégageant les semis de proche en proche, sans interruption.

()n suiv'a d'ailleurs la mème marche jusqu’a la fin de la révolution, cest-i-dire que chaque afrectation sera régénérée di son tour normal d'exploitation, après avoir été traitée en coupes sombres pendant les 20 années pré cédentes.

Ln termes plus généraux, la combinaison dont il vient d'ètre parlé peut être résumée de la manière suivante :

La révolution étant de 120 ans, et la durée normale dr la période levant ètre fixée à 40 ans, on a partagé 
charne aflectation en denx parties égales con'esponrlant à deux sous-périodes de 20 ans.

Sauf la portion affectée à la première moitié de la première période et qui doit ètre régénérée par le concours des procédés naturels et artificiels, chaque affectation est régénérée en 40 ans par voie de réensemencement naturel.

On n’a donc dérogé à la méthode ordinaire qu'en deux points :

$1^{\circ}$ En réglant frar contenance la possibilite des coupes sombres:

¿0 En laissant écouler un intervalle de 20 ans entre la première coupe sombre et la première coupe secondaire, dans chaque affectation.

Contre l'application de ce mode particuliel de r'égénération dans les sapinières, on objecte:

Que le natériel des coupes sombres sera exploité prématurément;

Que les produits de la première périorle seront supérieurs à ceux du surplus de la révolution.

Ces ohjections sont plus ou moins fondées suivant les circonstances, aussi l'emploi du systeme en question ne peut-il se justifiel qu'en présence de difficultés exceptionnelles, quand les lenteur's du réensemencement naturel commandent d'adopter de très longues périodes.

Pour la grancle majorité de nos sapinières, la régle n'en subsiste pas moins de baser sur le volume la pos- 
sibilite descoupes principales scrupuleusement rirconscrites dans l'affectation qui est en tom normal le l'égénération.

\section{A. L.}

\section{Renvoi du n n $^{\circ 16}$, page 279.}

Nos plus importantes forets de pin sylvestre étaient anciennement sommises au mode a tire et aire. Ce traitement leur fut appliqué jusque vel's 1820, éporue à laquelle on lui substitua la méthode naturelle, au moins en ce qui concerne les coupes principales. Dans ces coupes, on réservait un certain nombre d'arbres dans le but dobtenir le réensenencement du terrain et, pour mieux assurer la régénération naturelle, on imposait aux adjudicataires la charge d'arracher les myrtilles, les mousses, etc., et de domner au sol une légère culture par bandes alternes.

Tel est, en peu de mots, le traitement qui était autrefois appliqué à ces forèts, dans lesquelles on trouve aujourd'hui des peuplements provenant de semis naturels, parfaitement complets et de tous les âges antérieur's à 1840 .

La faculté d"imposer des travaux sur les compes ayant été supprimée (il y a 30 ans environ), on cessa le cultiver le parterre des coupes d'ensemencement. Mais bien- 
tot on sapercut que ces coupes ne se repeuplaient pas, ou qu'elles se repeuplaient mal, ou que, tout au moins, il fallait attendre trop longtemps avant d'obtenir un semis suffisamment complet. Dès lors, on prit le parti de recourir à des repeuplements artificiels.

La facile exécution et le succès, pour ainsi dire assuré, de cette opération, d'une part; l'insuccès des coupes d'ensemencement dans les terrains non préparés, d'autre part, firent naitre, peu à peu, la conviction qu'il y avait intérèt à substituer, d'une manière générale, le repeuplement artificiel à la régénération naturelle. Mais on ne tarda pas a reconnaitre que le nouveau mode de régénération ne dommait pas des résultats meilleur's que ceux que l'on obtenait anciemement, et, comme il est beaucoul' plus couteux, on revint tout naturellement à l'anciemne pratique des coupes d'ensemencement et à la préparation du sol de ces coupes.

Une culture très légère, un simple grattage de la mousse qui recouvre les terrains secs, l'ouverture de bandes ou de sillons dans les sols trop fortement enherbés ou courerts de myrtilles, suftit jour assurer la régénération naturelle des coupes dans un bref délai.

Les repeuplements que l'on obtient ainsi ne sont pas ordinairement aussi épais, aussi drus que ceux que l'on peut avoir en semant a la main. Mais ils présentent, sur ceux-ci, plusien's arantages importants : en effet, les jeunes plants du pin sylvestre sont très robustes et 
croissent arec rapidite dans la premiere jemesse, søils sont un peu clair-semés; leurs branches basses s"étalent, leur pied devient fort et trapu, et, peu à peu, le massif se forme et se serre jusquà domner un peuplement très complet avant d'arriver à l'état de gaulis. Les semis naturels (prohablement parce qu’ils sout moins serrés) échappent presque tous a la maladie grave de la défoliation qui atteint généralement les semis artificiels, el en retardesouvent le développement pendant plusieurs années.

Enfin, malgré l'avantage qu'il pourrait y avoir à pratiquer une éclaircie forte dans les fourrés, la difficulté de procéder en grand à cette opération délicate empèche d'y recourir, et les semis artificiels, trop serrés au début, ne présentent plus quand arrive l'état de gaulis, que des tiges étiolées et souvent sans avenir.

H. N.

\section{Renvoi du no 543 , page 308.}

Dans les futaies jardinées en voie de transformation, l'usage a prévalu de régler par contenance les coupes jardinatoires destinées a faire disparaitre les bois que l'on ne pourrait laisser sur pied, jusqu'à ce que les coupes de régénération vinssent les atteindre. 
Selon que le dépérissement de ces bois est plus ou moins rapide, on parcourt deux ou trois fois, durant chaque période, les affectations qui ne sont pas en tour le régénération; les coupes sont assises de proche en proche, par contenances égales, et l’on procède en mème temps à l'enlèvement des perches dominées et sans avenir.

De cette manière, on ne réalise pas toujour's l'égalité des produits annuels; mais c'est là un point secondaire; l'essentiel est d'exploiter à propos les arbres surannés ou nuisibles, en évitant toute appréciation à long terme. Suivant les conseils du Cour's de culture [544], il s'agit, en pareil cas, de recourir aux moyens les plus simples, les plus larges et les plus expéditifs.

\section{A. I.}

\section{Renvoi du n ${ }^{\circ} 549$, page $31 \%$}

Le jardinage rationnel, tel que l'enseigne le Cours de culture [519], a rencontré dans la pratique de sérieuses difficultés en ce qui concerne la périorlicité des coupes. En effet, la marche des accroissements étant fort irrégulière dans les forèts de l'espèce, il est rarement possible de préciser le temps dans lequel les arbres d'une rertaine ratégorie de diametre parviendront a la gros- 
seur requise pour ètre réputés exploitables; et cette donnée fût-elle même acquise avec une approximation suffisante, le rapport soutenu n'en serait pas moins établi sur une base bien précaire.

Il faut donc le reconnaitre: on n'a fait et l'on ne pouvait faire que de vaines tentatives pour équilibrer les produits, a long terme, dans les forèts jardinées. C'est que la solution du problème est subordonnée à la condition de trouver, sur tonte l'étendue de la série jardinée, des loois de tous àges confusément mèlés dans des proportions constantes, c'est-à-dire un état de consistance à peu près imaginaire.

A ce point de vue, comme à celui de la quantité et de la qualité des produits, le jardinage parait devoir ètre exclu des forèts grandes ou petites, sauf les cas exceptionnels ou la situation commande d'y maintenir constamment l'état de massif, dans un intèrèt de conservation ou d'abri.

En pareil cas, le forestier intervient uniquement, soit pour enlever les arbres brisés, morts ou parrenus à un legré de maturité très avancé, soit pour favoriser l'essor des jeunes peuplements destinés à perpétuer l'état de boisement.

Or, le moyen le plus simple, le plus sùr d'obtenir ces résultats, serait de parcourir chaque année toute la série jardinée en coupant les bois parvenus au terme de l'exfloitahilité physicque. Mais cette manière de procérler 
serait fort penible si on l'appliquait à res forèts de certaine étendue; l'exploitation de produits disséminés à de grandes distances occasionnerait d'aillemrs une forte lépense; il est donc souvent néressaire de consacrer plusieurs années à ces exploitations at d'en régler la périodicité proportiomnellement aux contenances à parcourir.

'Toutefois, la période de rotation doit ètre courte pour éviter toute aprríciation lointaine sur la longévité des bois, et afin que, dans l'intervalle dedeux coupes sur un point quelconque, les produits ne puissent pas ètre dépréciés par un dépérissement imprévu.

On remplit ces conditions en fixant, par exemple, à 12 ans la périodicité des coupes jardinatoires dans les forèts de plus de 100 hrotares, et en réduisant cette périodicité jusqu’à la rendre annuelle, biemnale ou triennale, s'il s'agit d'opérer sur de faibles étendues.

Ce point établi, la marche des exploitations consiste uniquement, pour les bois le l'Ftat, a diviser l'étendue boisée en autant de parties égales qu'il y a d'années dans la période de rotation; les agents dexécution ont ensuite pleine faculté de faire, dans les limites de chaque coupe, toutes les exploitations compatibles avec la conservation des massif's.

Quant aux forets appartenant it des communes ou a les particuliers, sans poursuive le rapport soutenu a long teme, il convient cependant d'éduilibrer les pro- 
duits, dans une certaine mesure, pendant le cours de chaque période de rotation.

Les dispositions à prendre dans ce but, peuvent ètre résumées ainsi qu'il suit:

$1^{0}$ Fixer d'après la contenance de la série quelle doit ètre la périodicité des coupes:

20 Partager la série en parcelles comme il est dit au Cours de culture [549];

$3^{0}$ Déterminer le diamètre que les arbres doivent avoir atteint pour être réputés exploitables;

$4^{\circ}$ Au début de chaque période, dénombrer et cuber, séparément daus chaque parcelle, les arbres qui ont atteint on dépassé le dianitre adopté comme limite; puis divisant ce matériel par le nombre d'années de la période, réduire le volume annuel mogen que l'on peut exploiter;

50 Répartir les parcolles entre les ammés de la période, proportionmellement aux rolumes que l'on y a trouvés, en s'attachant it former chaque contingent annuel, soit d'une parcelle entière, soit d'me partie aliquote de parcelle, soit de plusieurs parcelles réunies, de telle sorte que les prorluits different le moins possible du volume annuel moyen ;

$6^{\circ} \mathrm{N}^{*}$ exploiter dans chaque coupe ammuelle que les arlures ayant atteint ou dépassé le diamètre aılopté. et mime réselver ceux rlont l'enlevement compromettrait 
l'intégrité dlu massif, faute de jeunes bois en quantité suffisante pour fermer les vides.

$7^{0}$ Exploiter en outre, quelle quesoit leur grosseur, les bois morts ou tout à fait dépérissants.

Ce règlement le possibilité, fondé sur les contenances éruivalentes, est compatible avec l'établissement d'un Inart en réserve; il suffit pour cela de formel autant, de contingents anuuels qu'il y a d'années dans la périorle, plus un tiers.

A. L.

Renvoi du n ${ }^{\circ} 560$, page 331.

Dans la pratique, on s’abstient généralement de passer par une révolution transitoire quand il s'agit de transformer les futaies précédemment soumises au mode dit à tire et aire. Limitées, pendant la premiere période, aux bois dépérissants on parvenus à 111 degré de maturité trés avancé, les extractions d'anciennes réserves, dans la plıpart des cas, n'ont pas donné d'assez grands produits pour compromettre le rapport soutenu.

On n’a recours à une période transitoire que quand la révolution adoptée étant plus longue que celle des coupes a tire et aire, il convient d'attendre que les 
hois de premiere affectation soient devenus exploitables.

A. L.

\section{Renvois des nos 597 et 600 , pages 375 et 380 .}

Quant on procede au balivage dans les taillis composés d'essences mélangées, il est bien rare de rencontrer, surtout dans les forèts situées en bon fonds, le nombre de brins de chène nécessaire à l'entretien d'une réserve convenable en sujets de cette essence.

Les auteurs de la culture attribuent cette pénurie des baliveaux de franc-pied à la difficulté que les jeunes plants de chène éprouvent à sélever au milieu des bois blancs, des morts-bois et des rejets de bois dur qui les encombrent et les dominent, bientòt après l'exploitation du taillis.

Afin d'assurer les ressources nécessaires au balivage, on prescrit de planter une quantité suffisante de jemes chênes après charue exploitation du taillis; puis on recommande de donner à ces plantations tous les soins nécessaires pour les mener à bien, notamment en pratiquant des nettoiements et des éclaircies, dans le but de les dégager du convert qui jeut nuire à leur développement. 
Si ces prescriptions étaient bien comprises et appliquées avec suite, elles produiraient le résultat que l'on poursuit. Mais elles ont, à nos yeux, un inconvénient qui n'a certainement pas échappé aux auteur's de la culture, c'est de recourir it des repeuplements artificiels, toujours fort conteux, pour assurer la régénération du chêne dans les taillis d'essences mélangées.

Nous ne songeons pas a écarter les repeuplements artificiels, d'une manière absolue; nous croyons seulement que, dans les taillis comme dans les futaies, on peut souvent évitur cette dépensè, en prenant les précantions nécessaires : $1^{0}$ pour assurer la jroduction des semis naturels en quantité suftisante; $2^{0}$ pour préselver ces semis, après l'exploitation du taillis, des dangers auxquels ils sont exposés.

Dans les taillis exploitables, où la réserve est constituée en chênes et hêtres, on trouvera presque toujours, sous le couvert des perchis, une certaine quantité de jeunes plants provenant de semis naturels. A la rigneur, cette quantité pourrait, la plupart du temps, être consilérée comme suffisante pour subvenir, ultérieurement, aux besoins du balivage. Mais on obtiendra sûrement un l'ésultat plus complet et plus satisfaisant, en pratiquant, quelques années avant la coupe principale, une éclaircie dans laquelle on anra soin de dégager le sol de tont ce qui le courre trop inmédiatement, en un mot de relryer le convert, toint en se rarlant dinterrompre 
le massif. Lorsque les taillis sont ainsi préparés, les glands et les faines se conservent parfaitement sous la feuille pendant l'hiver' (qui suit la dissémination; tandis que ces semeuces moisissent, s'échauffent et pourrissent dans les peuplements plus jeunes, plus serrés, oì l’ courert des végétaux do toute sspèce est plus rapproché du sol.

Que si, par exception et pour me cause quelconque, on n'a pas l'espoir d'obtenir des semis naturels sous le jerchis, il faudra bien planter. Mais alors, mieux vaut encore, selon nous, planter, deux ou trois ans arant l'exploitation, sous le couvert du taillis préalablement éclairci, que d'entreprentre le repenplement apres lo récolement de la compe. La plantation soms le convert du perchis, dans un sol nettoyé d'herbes et bien amenbli, est plus facile, noins conteuse et presente plus de chance de sucrés que lorsqu'on l'exécute dans un taillis nouvellement exploité. En tout cas, quand on plante, soit avant, soit après l'exploitation du taillis, il est bon d'adopter un certain ordre et de disposer les plants par bouquets ou en lignes, de facon à jouroir les retrouver plus facilement, quand il faudra leur douner les soins nécessaires pour assurer leur développement.

Ces soins sappliquent aussi bien aux brins provenant le semis naturels qu'aux sujets de plantation. Ils sont parfaitement incliqués par les auteurs cle la culture, e consistent principalement dans la pratique des nettoie- 
ments (Toir la note au bas de la page 378). Toutefois, nous croyons deroir insister sur la manière de procéder a cette opération, que l'on a trop' négligée jusqu'ici, parce qu'elle ne procure point de revenus immédiats.

Afin que l'exécution en fut bien assurée, nous voudrions que les coupes de nettoiement dans les taillis fussent assises aussi régulierement que les coupes principrales et, (qu'après son exploitation, cliaque coupe de taillis fut nécessairement et obligatoirement parcourue en nettoiement à rles éforues fixes, par exemple, tous les deux, trois ou quatre ans, suivant la rapidité de la régétation, jusqu'à l'âge fixé your la coupe l'éclaircie. Rien ne serait plus facile, nous en avons l'expérience, que de dresser les gardes à diriger ou à exécuter euxmêmes une opération qui ne demande ni déjense de force, ni beaucoup de savoir-faire. En effet, le nettoiement, comme nous l'entendons ici, ne consiste pas a extirper à la pioche les hois blancs et les morts-bois, ni même à les couper rez-terre à la serpe ou à la hache; il se réduit tout simplement, à dégager la tètedes jeunes plants dout on veut favoriser le développement, en cassant ou en coupant, a une hauteur convenable, les tiges ou lesbranches des régétaux secondaires, qui nuisent on menacent de nuire bientòt à la croissance des sujets à protéger.

H. N. 


\section{Renvois des $n^{0 *} 601$ et 603 , pages 382 et 384 .}

L'élagage, dans les forèts, ne s'applique guère qu'aux arbres qui ne croissent pas en massif. L'élagage des arbres réservés dans les taillis comprend deux opérations distinctes : l'émondagre des branches gourınandes, et la suppression ou le raccourcissement de certaines branches de la cime.

\section{I.}

Ainsi que le conseillent les auteurs de la culture, l'émondage des branches gourmandes doit toujour's avoir lieu rez-tronc. On se sert, à cet effet, l'instruments bien tranchants et de formes très variées, auxquels on domne le nom d'émondoirs. En première ligne vient la serpe qui est l'instrument préléré de tous les ouvriers, et le seul qu'ils peuvent employer commodément, quand ils sont obligés de faire usage d'échelles, pour atteindre les branches à émonder. Après la serpe ordinaire, les émondoirs dont nous donnons ici le dessin nous paraissent les meilleurs. Le premier est une serpe en forme de croissant, à laquelle on ajuste un manche de longueur variable ( 2 à 4 mètres ordinairement); le second a la forme d'une spatule et s'emmanche comme la serpe. L'un et l'autre peuvent servir indifféremment à 
642

APPENDICE

l'émondage rez-tronc des petites branches gumruandes; mais lorsqu'il s'agit de retrancher une branche un peu forte, ayant, par exemple, 4 ou j centimètres de diamètre au point d'attache, on se sert, avec plus d'avantage, de l'émondoir en spatule. Dans ce cas, on appli-
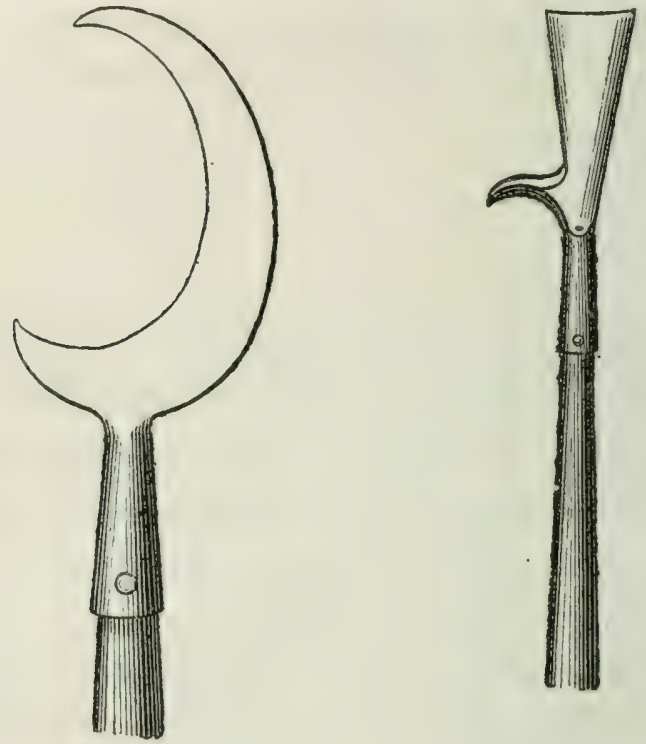

que le tranchant de l’instrument à la base de la branche, et, pendant qu'un hommesoutient l'émondoir dans cette position, un autre ouvrier lui dome l'impulsion en frappant avec un maillet contre l'extrémité inférieure du manche. On obtient ainsi des plaies bien nettes, que l'on enduit de coaltar immediatement après l'opération 
et qui se ferment avec d'autant plus de rapidité que l'arbre est plus vigoureux.

L'émondage doit se faire aussitòt que les Jranches gourmandes a!paraissent, c'est-ì-dire pendant l'été qui suit l'abatage du taillis. Si l'opríration ne peut pas ètre pratiquée dans ce délai, il sera particulièrement utile de la faire avant le déveloprement des bourgeons de l'année suivante.

Un seul émondage suffira quelquefois pour débarrasser un arbre de ses branches gourmandes, et, dans tous les cas, le premier sera le plus important. - Mais un second émonłage à 2 ans, 3 ans au plus d'intervalle, sera souvent utile ou mème nécessaire. Rarement, on sera obligré de recommencer une troisième fois l'opération.

Appliqué avec soin et en temps opportun, l'émondage des branches gourmandes suffit presque toujours pour préserver du défrérissement la cime des arbres de réserve, et dispense d'avoir recours plus tall à la suppression de grosses branches. - Nous ferons remarquer, d'ailleur's, que l'opération de l'émondage est facile à pratiquer, qu'elle n'exige pas l'emploi d'ouvrier's spéciaux difficiles à former, et (qu'on jeut en généraliser l'application dans toutes les forêts, grandes ou petites, sans aucun embarras, parce qu'elle ne demande ni beaucoup de temps, ni beancoup de frais, ni beaucoup d'habileté. Le tout est d'agir en temps utile. 
Gutre l'émondage, tonjour's nécessaire, des branches gourmandes, il pent ètre utile de retrancher ou de racconrcir certaines branches de la cime. Ainsi, les branches mortes, par exemple, devront toujours ètre supprimees en mème temps que l'on fera l'émondage des branches gourmandes. Il en sera de mème des branches cassees par le rent ou par la chute d'arbres roisins. Il est bien certain, en effet, que si on laisse subsister ces branches mortes ou rassées, la pourriture fui a envahi les premieres et qui menace d'enrahir les autres, deviendra souvent la cause de la formation de guttières; et, plus tard, de grisettes, qui atteindront le corps de l'arbre, et y produiront les ravages que l'on rencontre si souvent dans les réserves sur taillis et spérialement dans les chènes. Reste donc à savoir comment on procédera à cette amputation .

Pour les branches entièrement mortes, la section doit foujour's avoir lieu rez-tronc, tandis que l'amputation jent se faire dans la partie restée vivante le la branche, quand l'extrémité seule est morte. -De mème, onpourıa sonvent se borner ì supprimer une partie d'une branche vive et cassée en faisant la section à quelque distance au-dessous du point le fracture; mais c'est à la comblion expresse que la partie a conserver serd sus- 
ceptible de reconstituer une branche utile à l'alimentation de l'arbre. Dans tout autre cas, l'élagage devra se faire rez-tronc.

Cette opération peut se faire arec une serpe ordinaire, ou avec une scie quand il s'agit d'attaquer une glosse branche. Mais, lorsquou se servira de la scie, il faudra nécessairement raviver et polir la plaie avec la serpe et, dans tous les cas, recouvrir cefte plaie d'une couche de coaltar. Inutile de lire que l'amputation d'une branche un peu forte devra toujours commencer par une entaille assez profonde dans le còté inférieur de la section.

III.

Quant aux branches vives et intartes, nous royons. qu'en général, il y a plus de mal que de bien a attendre d'une opération, si bien laite qu'elle soit, qui amait pour objet de sommetle une partie d'entre elles a un systeme laisomed de suppression on de laccomedssement, en vue d'ameliorer la cloissance ou la forme de l'arbre qui les porte.

Pour le chène spécialement, nous partageons l'opinion que l'élagage en chicot est dangereux et qu'il vaut mieux couper rez-tronc toute branche que l'on est ilécirlé a supprimer. Yous admettons aussi qute les plaies faites au tronc peuvent se cicatriser, le plus souvent, aver 
assez de rapilite pour soustraire le corps entier de l'arbre aux dangers des gouttières, des grisettes et des nœuds gàtés; mais nous devons dire qne ces plaies, mème les mieux recouvertes, laissent ilerrière elles des taches analogues à celles qui sont connues sous le nom de frottures; que ces taches sont composées d'une ou plusieurs couches de bois mort, (mais non gàté) rui ne se ressoudent pas arec celles qui les recourrent; qu'elles se retrouvent plus tarl quand on débite l'arlspe; tt sufísent pour en déprécier la valeur ; que si, au contraire, on se fiut abstenu d'élaguer, le débit de la pièce, en planches, en charpentes ou en marine, aurait accusé, à la place de ces plaies, un nœud sain qui n’aurait altéré en rien la qualité et le prix de la marchandise. Nous pensons donc, qu'ì moins d'exceptions, il fant se dispenser d'elaguel les branches vives et intactes de la tète des réserves.

Quelles sont ces exceptions?

On comprend que, dans un taillis exploité à une courte rérolution, à 10 ou 15 ans, on puisse sommettre les jeunes réserves à loopération le la taille, et nous conseillerions volontiers aux proprietainess particuliers d'user de ce procedé pour former leur's ardnes, surtout s'ils pouraient surveiller eux-mèmes lopération, et la conlier il des ouvrier's intelligents et expérimentés.

On comprend aussi, que si un taillis est rominé par dics arhops a courert épais, comme des hètres, on puisse 
avoir intérèt, surtout quand le couvert est bas, à retrancher quelques-unes des branches inférieures, pour faroriser la croissance du sous-bois. En effet, au cas particulier du hètre, larbre ne souffrira que peu sous le rapport de sa croissance et, en raison de l'emploi que l'on en fait, il ne subira, la plupart du temps, qu'une dépréciation insignifiante.

on comprend mème que les brancha:; intërieures de la tète d'un chène, lor'squ'elles s'étalent rémesurément et horizontalement, puissent ètre raccourcies sans (langer'; car, alor's mème que, par cette opération, la vitalité de la branche serait amoinclée ou tout à fait compromise, il n'y a, pour ainsi dire, point de risque a courir au point de vue de la formation d'unegonttière, puisque, par hypothèse, la branche a une direction horizontale.

Concluons. - Larbre le plus précieux ef le plus robuste de nos forèts, le chène, est aussi celui qui est le plus facilement et le plus profondément atteint, dans la 'qualité de son bois, parles accidents de tontes sortes auxquels nos grandes essences sont exposées. Que l'on cherche à urevenir ces accidents, on a en amoindrir les conséquences daus la mesure du possible, rien de mieux; mais si l'on consulte les exploitants et tous ceux qui ont étudié le débit du chêne sous toutes les formes, tous s'écrieront, à propos de l'élagage des branches vives et intactes : ne touchez pas au chêne.

H. N. 


\section{Renvoi du n $\mathrm{n}^{\circ} 632$, page 427.}

A l'examen comparé des principales méthodes d'exploitation qu'il nous soit perınis d'ajouter encore une considération à l'avantage de la futaie:

On sait avec quelle facilité les bois blancs (tremble, saule, anne et tilleul) ainsi que le boulean, se propagent dans les taillis quand le sol est frais et fertile. - Cette invasion est dautant plus rapide que les exploitations sont plus fréquemment répétées. Les rejets de bois durs penvent lutter arec quelqu'avantage, mais les brins de semence périssent imman(ulablement étouffès, si, dès les premières années, la main du forestier ne vient pas à leur aide.

A défaut de ces soins, toujours couteux et trop souvent négligés, on a vu s'opérer dans la nature des peuplements les transformations les plus radicales; le tremble, le bonlean, les norts-bois regnent en maitres sul de rastes étendues autrefois habitées par le chêne.

Ces tristes résultats ne sont point à craindre dans les futaies; les bois blancs s'y montrent quelquefois après la coupe secondaire ou définitive, mais c'est pour disparaitre dans les éclaircies, si déjà les coupes de nettoiement n'en out pas fait justice.

A. I. 


\section{$\S 682$, page 485 .}

Pour la conversion des taillis en futaie, le cours de culture indique trois modes le conversion différents :

$1^{\text {er }}$ mode. - Dès le début et au cours d'une révolution préparatoire, supprimel les exploitations de taillis, et leur substituel des coupes d'amélioration, (nettoiements, éclaircies, extraction de réserves dépérissantes), qui parcomront toute l'ètendue de la série à convertir.

$2^{e}$ mode. - Dès le début et au cours d'une révolution préparatoire, traiter en coupes d'amélioration une partie des peuplements, et continuer parallèlement les exploitations de taillis dans le surplus de la série.

$3^{\mathrm{e}}$ mode. - Ians des conditions particulieres de peuplement et, par conséquent, à titre exceptionnel, commencer immédiatement la régénération naturelle ou artificielle l'une partie de la sirie, et traiter parallélement le surplus, soit par coupes d'amélioration, soit par coupes de taillis.

Ces trois modes de conversion ont été mis en pratique sur une assez gramle échelle, et c'est le deuxième qui a prévalu.

Cette préférence, déjà conseillée par nos maitres, s'explique aisément; on y trouve en effet diver's avantages :

Le plus essentiel, commm d'ailleurs anx denx premien's 
moles, c'est que la régénération, étant ajotirnée jusqu’à la fin d’une révolution préparatoire, s'effectuera plus facilement, parce que le taillis sera parvenu à l’àge de fertilité: parce qưil aura atteint l'élévation nécessaire pour le succès du repeuplement, et parcerque le sol se sera amélioré par suite d'un couvert prolongé.

Mais il y a aussi d'autres avantages que ne procure pas le premier mode de conversion et qui proviennent de la continuation temporaire des coupes de taillis dans une certaine portion de la série; (fu quart aux trois quarts) savoir :

$1^{0}$ Suivant la part plus ou moins grande que l'on fait à cette catégorie de coupes, on lpeut atténuer sensiblement l'inévitable diminution des revenus pendant la révolution préparatoire et même au-delà ;

$2^{\circ}$ On peut adopter, pour la première régénération de la série, une révolution transitoire différant peu de la révolution définitive qui correspond à l'exploitabilité de l'essence à faire prédominer.

Il est peutètre superflu d'ajouter que ces derniers arantages ne peuvent ètre réalisés sans inconvénient qu'a l'expresse condition de multiplier les arbres de réserve dans les coupes provisoires de taillis sous futaie, en rue d'entretenir et mème d'améliorer la fertilité du sol.

Quant au troisième mode de conver'sion, l'expérience 'qu'on en a taite n'est pas pouren recommander l'emploi : 
Quels que fussent le nombre et la fertilite des arbres de réserve, la régénération entreprise immédiatement, sans préparation, dans des taillis denviron 30 ans, a rencontré de sérieux obstacles. Pour 'assurer le succès des repeuplements naturels ou artificiels, on avait à lutter contre les rejets du taillis. Ce n'est qu'au prix d'efforts soutenus et à grauls frais qu'on parvenait à dégager ces repeuplements. Souvent, faute d'assiduité et d'argent, il y a eu des mécomptes.

On a persé mieux faire en extrayant les souches du taillis ; mais divers essais n'ont abouti qu’à montrer le danger d'un pareil expédient, surtout dans less sols maigres, dont la fertilité peut ètre compromise par tout retard dans le repeuplement.

La nécessité d’une préparation, plus ou moins longue. parait donc s'imposer en tous cas, alor's mème que les bois blancs et morts-bois domineraient dans les taillis a convertir. Eclaircir peu a peu les peuplements ainsi composés; les laisser s'élever autant que possible, mème jusqu'à l'àge voisin du lépérissement, puis les mettre en coupes d'abri, et semer ou planter les essences qui doivent tormer la jeune futaie ; telle est la marche a suive, la plus sure et aussi la moins couteuse. C'est celle que.II. Tassy a, tout le premier, recommanlée dans la deuxième édition de ses études sur l'aménagement.

A. L.

FIN. 



\section{VOCABULAIRE}

\section{DES TERMES TEGHNIQUES}

EMPLOYÉS ET DÉFINIS DANS CET GUVRAGE

t.

铛 pages

Abroutissement..........4i7 210

Accroissement annuel. . . . . . 394 172)

- moyen....... 394172

Affectation périodique. . . . . . 425191

Alise, alose. . . . . . . . . . . 20293

Aménagement. . . . . . . . 607391

Ancien. . . . . . . . . . 564 336

- de deuxième classe. . . . . . $564 \quad 337$

- de première classe. . . . . $561 \quad 337$

Arbre. . . . . . . . . . 7 6

Arbrisseau. . . . . . . . . . 7 7

Arbuste. . . . . . . . . . . 7 . 6

Assainissement. . . . . . . 704509

Assiette, asseoir une coupe. . . . . 399173 
B.

裙 pages

Balivage normal. . . . . . . . $594 \quad 370$ Balivean. . . . . . . . . . $564 \quad 336$

— de l'âge. . . . . . . . $564 \quad 336$

- moderne.......... $564 \quad 336$

- ancien.......... 564336

Bardeaux. . . . . . . . . 283122

Base minéralogique. . . . . . . $52 \quad 31$

Basse tige. . . . . . . . 776575

Bassin de consommation. . . . . . 658456

Basson. . . . . . . . . . . 319141

Bèche demi-circulaire. . . . . . . . 7939592

Biner. . . . . . . . . . 814615

Blanc étoc (coupe à) . . . . . . . . 453211

Bois abroutis. . . . . . . . 447210

- d'automne. ......... 76 bis. 4 4́

- blancs ou tendres. ...... 445 209

- de corde........... 14. 9

- défensable......... 450210

- durs............ 445209

- de fente........... 149

- feuillus. . . . . . . . . . 23 . 14

- gras............ 76 bis. 45

- merrain. ........... 14 . 10

- nerveux......... 76 bis. 45 


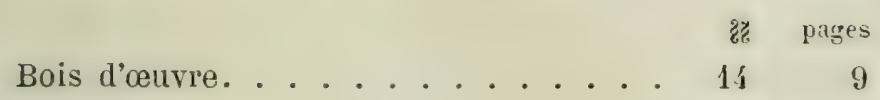

- de printemps. . . . . . . 76 bts. 4 '

- de quartier.......... 14 9

- résineux. .......... 2314

- rondin............. 149

- de service........... 149

- de sciage............ 1410

- de travail ou d'ouvrage. . . . . 14 g

Bourrée . . . . . . . . 14 9

Botiture. . . . . . . . 685 . 487

— à bois de deux ans. . . . . $808 \quad 608$

- en plançon. . . . . . . . 807607

Brin................ 128

C.

Cadranure. . . . . . . . . 76 bis. $4 \overline{0}$

Capital foncier. . . . . . . . . . 639435

- iroducteur. ............ $\begin{cases}410 \text { bis. } 181 \\ 639 & 435\end{cases}$

- superficiel. . . . . . . . 639435

Carpelles............. . . 107

Cépée. . . . . . . . . . 568338

Cerches. . . . . . . . . . 149

Chablis. . . . . . . . . . 402173

Chandelier. . . . . . . . 102173

Chevelu. ............... 11 


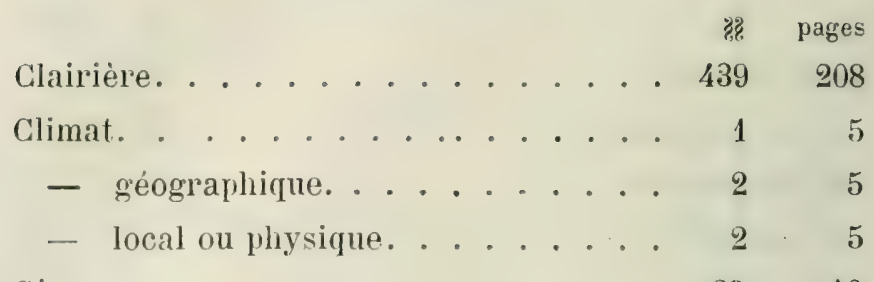
Còne. . . . . . . . . . 2316

Conversion (exploitation de). . . . 631 42. Coupe. ................. 398173

- d'anélioration. . . . . . 465 221

- par bandes alternes....... . 507265

- par bouquets. . . . . . 508267

- claire ou secondaire...... 461218

- définitive.......... 462218

- d'ensemencement. . . . . . 460 216

- de nettoiement........ 465221

- préparatoire. . . . . . $668 \quad 166$

- de régénération........ 459216

- sombre.......... 460 217

- de transformation. .... $\begin{cases}537 & 297 \\ 553 & 324\end{cases}$

Couronner (se). . . . . . . 408 179

Convert............ $565 \quad 337$

D.

Décennie........... . 424191

Défends (mettre en). . . . . . . . 449210

Dẻfensable (bois). . . . . . . . 450210 
Dendrométrie. . . . . . . . 426195

Dioïque (floraison). . . . . . 107

Drageon. . . . . . . . . 128

Dunes. . . . . . . . . $757 \quad 562$

E.

Echauffer (s'). . . . . . . . 687488

Eclaircie périodique. . . . . . . $466 \quad 223$

- faible.......... $467 \quad 225$

- forte.......... 467225

- moyenne. ........ 487224

Ecobuage. . . . . . . . 702506

- à feu courant. ..... 703509

Ecorcement. . . . . . . . . 609394

Elagage. . . . . . . . . 601381

Emondage. . . . . . . . . 624418

Espèce. . . . . . . . . . . . . 996

Essence. . . . . . . . . . 86

Etamines. . . . . . . . 107

Etêtement. . . . . . . $625 \quad 418$

Exploitabilité. . . . . . . $403 \quad 174$

- absolue. ....... 405175

- composée........ 412184

- physique........404 475

一 relative.......... 409180

Exposition. . . . . . . . 4 6 
F.

\&? pages

Fagot. . . . . . . . . 14

Feuillus (bois). . . . . . . . 2314

Floraison dioüque. . . . . . . . 107

- hermaphrodite. . . . . 107

- monoïque.......... 10 7

— polygame.......... 107

Forèt normale. . . . . . . . 415186

Fourré............. 444209

Frotture. . . . . . . 76 bis. 46

Furetage. . . . . . . . 613401

Futaie. . . . . . . . 486207

- (demi-).......... 444209

— irrégulière......... 438208

- régulière......... 437207

- sur taillis......... 563336

G.

Gaulis. .......... 444209

Gelivure........... 76 bis. 45

Gemmer, gemmage....... 319140

Glissoir. . . . . . . 434 204

Gournable. ......... 1410

Grisette. . . . . . . . 76 bis. 45 
H.

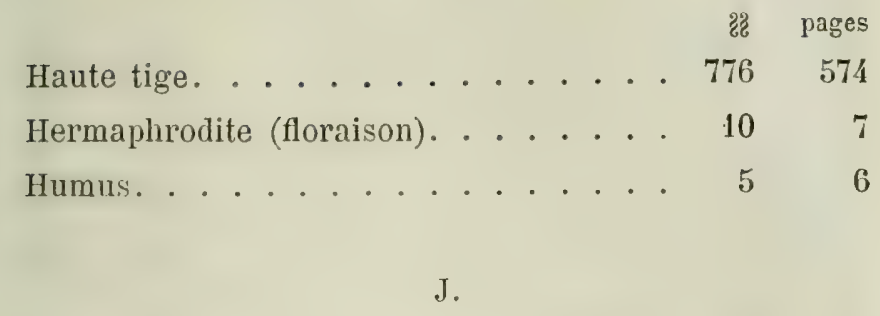

Jardinage. . . . . . . . . 535 294 Jauge (mettre en). . . . . . . $795 \quad 594$

L.

Labour en plein. . . . . . . 699502

- par bandes alternes. . . . . . 700503

- par places, trous ou pots. . . . 701505 Lancoir. . . . . . . . . 434204 Lunure. . . . . . . . . . 76 bis. 45

M.

Marcotte. . . . . . . . . 686487

Marne ........... $57 \quad 34$

Martelage. .......... $596 \quad 373$

Massif. ........... 142208

— clairiéré. . . . . . 443208

- entrecoupé......... 443208

- incomplet. ....... 443208 


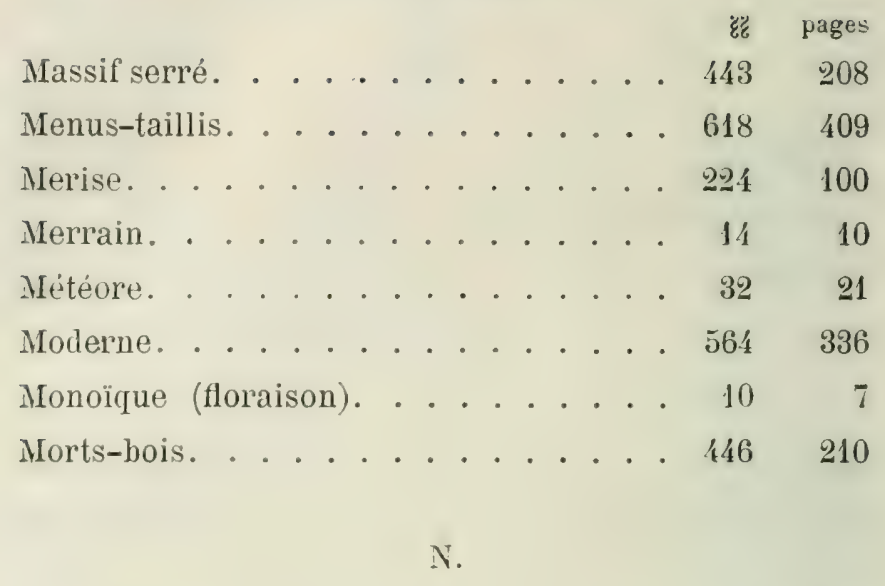

Nœuds gâtés. . . . . . . . 76 bis. 46

Ombrage............ $565 \quad 337$

P.

Panage.............. 1510

Pépinière. . . . . . . . . . . $778 \quad 577$

Perchis (haut-perchis, bas-perchis). . . 444209

Période. . . . . . . . . . 424191

Peuplement complet. . . . . . 397172

Pistil. . . . . . . . . . . 10 T

Pirot. . . . . . . . . 1148

Pivot (couper en). . . . . . . . . $567 \quad 338$

Pivotante (racine). . . . . . . . . 118 
Plancon. . . . . . . . $807 \quad 607$

Plantation. . . . . . . . . . 684. 487

- par touffes......... $804 \quad 603$

Plantoir-massue.......... $713 \quad 519$

— à nervures. . . . . a . . 713519

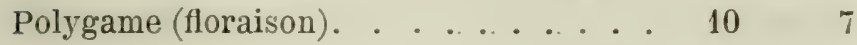

Possibilité. . . . . . . 415186

Pourriture rousse ou blanche. ....... 76 bis. 45

Produits accessoires. . . . . . 470228

- principaux......... 470228

Q.

Quarre. . . . . . . . . 319141

Quille............ 402173

R.

Race. . . . . . . . . . y 7

Racine pivotante ou plongeante.... 118

- tracante.......... 118

Ramiers. . . . . . . . . 569338

Rapport ou rendement soutenu. . . . . $396 \quad 172$

Ravaler. . . . . . . . . 566338

Recéper, recépage........ 418210

Récolement. . . . . . . . 596374

Recru. . . . . . . . . 13 
Regarnis. . . . . . . . . . 819620

Rejet............. 128

Rente. . . . . . . . . 395172

Repeuplement. . . . . . . . 13

Repiquement. . . . . . . 713518

Repiquer. . . . . . . . . . . 689488

Réserve, réserves. . . . . . . . . 451211

Résineux (bois). . . . . . . . . 2314

Retour (bois en). . . . . . . . 408179

Revenu. ............ 410 bis. 182

Révolution. . . . . . . . . 392 . 171

Roulure, bois roulé. . . . . . . . . 76 bis. 45

S.

Sable gras. . . . . . . . . 58 34

sarcler. . . . . . . . 814615

sartage. . . . . . . . . 619410

- à feu courant. ........ 620414

— à feu couvert. . . . . . . . . . 621415

Semis. . . . . . . . . 683487

- en plein. .......... 688488

- partiel........... 688488

Série d'exploitation. . . . . . . 420189

situation. .............. 35

Sol ou terrain........... 6 6 6 
Sorbe. . . . . . . . 206

Sous-bois. . . . . . . . . 452 211

Strobile. 16

Superficie. . . . . . . . 639 135

$\mathrm{T}$.

Taillis............. $562 \quad 335$

- simple.......... $563 \quad 336$

— sous futaie ou composé. . . . $563 \quad 336$

Talus (couper en). . . . . . . $567 \quad 338$

Taux de placement. . . . . . 410180

Terrain. . . . . . . . 66

Terre aquatique. . . . . . . 62 35

- chaude.......... 65035

- forte........... $55 \quad 33$

- fraîche.......... $64 \quad 35$

- franche........... $59 \quad 34$

- froide.......... 55

- glaise............. $55 \quad 33$

— humide.......... $63 \quad 35$

- légère. .......... . $56 \quad 33$

- maigre........... 60 34

- marécageuse. . . . . . . $62 \quad 35$

- substantielle........ 60 34

Terre végétale. . . . . . . . 5 . 6 
Terreau. .............. $5{ }^{\text {pages }}$ Têtard.............. 624 418

Terres vaines et vagues. . . . . . 441208 Tire et aire (exploitation à). . . . . . . $550 \quad 321$ Touffes (plantation par) . . . . . $804 \quad 603$ Tracante (racine). . . . . . . 118 Trainants. ......... 571341

Traîneaux (chemin à). . . . . 434203 Trippler. . . . . . . . . 712517 Trochée. . . . . . . . 568338

Tronc. ........... 402173

U.

Usance, coupe usée. . . . . . . . 401473

V.

Variation............ . . 9 997

Variété. . . . . . . . . . . 96

Vidange. . . . . . . . . . 400173

Vide (place vide). . . . . . . . 440208

Vieille écorce. . . . . . . . 564336

Volis. . . . . . . . . . 402173

Volume absolu ou réel. . . . . . . 641439 


\section{TABLE ANALYTIQLE DES MATIËRES}

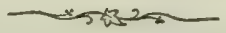

\section{INTRODUGTION}

Définition de l'économie forestière, et parties qui la constituent, page 1. - Ge qu'on entend par culture des bois ou sylviculture, 2. - Différence entre la culture des bois et celle des champs, $i b .-$ Trois conditions fondamentales de toute méthode d'exploitation: production soutenue, régénération naturelle, amélioration progressive, 3 .

\section{LIVRE PREMIER}

\section{Des climats; des sols, des essences}

Définitroxs (2? 1 à 15). Climat, page 5 . = Climat géographique, - physique ou local, $i b .=$ Situation, $i b$. = Exposition, 6. = Terre végétale $;-$ terreau; - humus $; i b .=$ Sols ou terrains, $i b .=$ Arbre $;$ - arbrisseau $;$ - arbuste $; i b$. = Essence, - essences forestiẻres, $i b .=$ Espèce $;$ - variété $;$ - variation, 7. = Floraison : monoïque; - diöque ; - hermaphrodite;polygame, $i b .=$ Racines pivotantes; - traçantes; - essences pivotantes et traçantes; - chevelues $;-8 .=$ Brin de semence ou brin; - rejet ; - drageon, $i b$. = Recru ; - repeuple- 
ment, $i b .=$ Dénomination des bois suivant l'emploi. - A. Bois de feu ou de chauffage, façonné de quatre manières : quartiers, rondins, fagots, bourrées; - B. Bois d'œuvre, divisés en bois de service et en bois de travail ou d'ouvrage; - parmi ceuxci, distinguer les bois de fente; 9. - qualités nécessaires pour la fente. 10. - Bois merrain, $i b .=$ Panage, $i b$.

\section{CHAPITRE PREMIER.}

BUT ET DIVISION DE CE LIVRE.

$A_{\mathrm{RT}}$. Ier. Des études qu'il embrasse et de leur ordre naturel (8 16). La base de la culture des bois doit être l'étude des climats, des sols, des essences; - l'étude des essences doit être précédée de celle des elimats et des sols, 11.

Art. II. De l'étude des sols et des climats (踣 17 à 19). Elle est accessoire, 12. = Marche de l'étude des elimats, $i b .=$ Marche de l'étude des sols, $i b$.

Art. III. De l'étude des essences (艮 20 à 23). C'est ici l'objet principal; marche de cette étude, 13. = On n'énumèrera pas les caractères botaniques, $i b .=$ On ne mentionnera ni arbrisseaux, ni arbustes, ni essences rares, 14. $=$ Classement des essences en deux groupes: bois feuillus et bois résincux; $i b .,-c a-$ ractères distinctifs de chaque groupe, 15.

\section{CHAPITRE DEUXIÈME.}

DES CLIMATS.

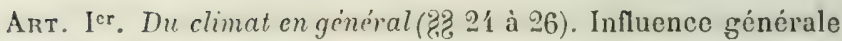
du climal physique sur la végétation des bois, 17 . = La situation influe de deux manières, $i h .=$ L'élévation se manifeste par 
plusieurs effets; - la configuration donne lieu à distinguer les climats de plaine et les climats de montagne, 18.

Art. II. Des climats de plaine (\&z 27 à 30 ). Caractères de ces climats, 19. = Influences exercées sur le climat par la présence ou par l'absence des eaux et des forèts à la superficie terrestre, $i b$. = Influence de la nature du sol sur le climat, $i b .=$ Caractères du climat des côtes, ib.

Art. III. Des climats de montagne (\&̨, 31 à 34). Caractères généraux, 20. = Trois situations : vallées, versants, plateaux ; influences climatériques favorables ou défavorables à la végétation; $1^{\circ}$ dans les vallées, $i b . ; 2^{\circ}$ sur les versants, $21 ; 3$ o sur les plateaux, $i b .=$ Influence des abris, 22. = Pays de coteaux, $i b$.

Art. IV. De l'erposition ( la végrétation, 23. = Est. Température; - influences des saisons; - caractères de la végétation, $i b .=$ Nord.Tempéralure, $i b$; - humidité; - caractères de la végétation, 24 . = Ouest. Température; - action des vents; caractères de la végrétation, $i b$. $=$ Sud. Température, $i b$. ; - gelées printanières; - orages ; - caractères de la végétation, 2̆. = L'influence de l'cxposition s'affaiblit en raison de l'élévation, $i b$.

\section{CHAPITRE TROISIÈME.}

DES SOLS.

ART. Ier. Du sol en général et de son action nutritive. (8ृ? 41 à 41). Composition de la terre végétale, 26. = L'action du sol sur la végétation des bois considérée: sous le point de vue phỹsique; - sous le point de vue chimique, $i b .=$ Physiquement, le sol agit par sa profondeur, sa cohésion, son hygroscopicité, sa coloration, $i b .=$ Chimiquement, il concourt à la nutrition, par les substances minérales, sels et oxydes qu'il r'enferme, et de plus il agit comme organe de transmission, 27.

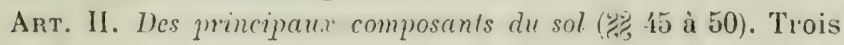


composants grénéraux : L'argile. le calcaire, le suble; - diver's sels; - oxyde de fer, 26 . = Argile; - ses propriétés, $i b .=$ Terres calcaires; - leurs propriétés, 29. = Propriétés négatives de la silice, $i b$. = Conditions nécessaires pour que chacune de ces trois terres devienne fertile, $i b$. $=$ Effets de l'abondance du terreau; - propriétés auxquelles ils sont dus, 30 .

Arт. III. De la base minéralogique et de l'inclinaison (\&马 51 à 53). Gisements qui reçoivent le nom de base minéralogique:comment leur action se manifeste suivant qu'ils sont compactes ou schisteux, verticaux ou horizontaux; - leur influence surle degré d'humidité, 31. = Influence de l'inclinaison; - les pentes douces sont favorables aux bois, et les bois maintiennent les terrains en pente, 32.

Апт. IV. Des diverses catégories de terrains (8? 54 à 66). Leurs dénominations se fondent sur les composants ou sur le degré d'humidité, 33. = Catégories tirées des composants : Terre forte, glaise, froide, $i b .=$ Terres légêres, sablonneuses ou gravelcuses, $i b .=$ Terres marneuses, marne, $34 .=$ Sable gras, $i b .=$ Terre franche, $i b .=$ Terrains gras ou substantiels, $i b$. = Terres maigres, pauvres, arides, 37. = Catégories tirées du degré d'humidité : Sols marécageux, aquatiques ou mouilleux, 35. = Sols humides, $i b .=$ Sols frais, $i b .=$ Terrains secs ou chauds, $i b .=$ Influence de ces divers sols sur la végétation des bois, $i b$.

\section{CHAPITRE QUATRIEME.}

DES B01S FEUILLUS.

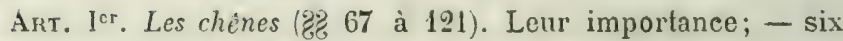
espèces principales: A. - Chènes à feuilles caduques : $1^{\circ}$ chêne rourre: 2o chène pédonculé: $3^{\circ}$ chéne tauzin; $\mathrm{B}$. - Chênes à feuilles persistantes : 1o chéne yeuse: $5^{\circ}$ chêne liége; $6^{\circ}$ chéne kermès, 37. 
10 Chène rouvre. Lieux d'habitation, 38. = C. S. E*. Climat tempéré; - il habite de préférence les pays de collines et les parties inféricures des montagnes; - se trouve dans les plaines associé au pédoncule; = craint les gelées printanières; - il prospère à toutes les expositions quand le sol lui est favorable, $\imath b .=\mathrm{T}$. Les sols moyennement argileux sont ceux qu'il préfère - il s'accommode des terrains graveleux, siliceux et calcaires, pourvu qu'ils aient de la fraîcheur; - les sables secs et les fonds marécageux lui sont contraires, 39. = F. F. Fleurs monoïques, en avril; - gelées printanières; - fruits : glands de grosseur variable, sessiles, en bouquets; - maturité, en octobre; - semence lourde; - fertilité : 50 à 80 ans pour les brins cle semence, 25 à 30 ans pour les rejets: - années de semences rares dans le Nord et l'Est de la France, $i b .=$ J. P. Tempérament robuste; - abri contre les vents desséchants, $41,=F$. Feuilles de grandeur variable, généralement fermes; - couvert léger quoique plus complet que celui du chêne pédonculé; - effets qui résultent de ce couvert léger, $b=\mathrm{l}=\mathrm{R}$. Racines pivotantes de 1 à 2 mètres de profondeur; - disposition aux différents ìges; - pas de drageons, 42. = C. D. Croissance assez lente, mais égale jusqu'à 200 ans; - longévité : 5 à 6 siècles; - très forles dimensions, $i b .=Q$. U. Dureté; - résislance aux intempéries; - durée; - il est employé aux constructions civiles et navales, $43 .=$ Cireonstances de végétation qui font varier ses qualités; - bois nerveux; - bois gr'as; vices et défauts, $i b .=$ Emploi comme bois de fente; - de chauffage; - emploi de l'écorce; - du fruit, 46.

Qo Chéne pédonculé. Lieux d'habitation, 48. = C S. E. Climat lempéré; - s'avance plus vers le Nord que le chêne rouvre: - se plait surtout dans les plaines, $i b .=T$. Sols argileux,

(') Les íniliales majuscules représententles intitulés des paragraphes du texte, de la manière suivante:

C. S. E. Climat, situation, exposition.

T. Terrain.

F. F. Floraison ef fructification.

J. P. Jeunes plants.
Q. U. Qualités et usages.

C. D. Croissance et durée.

R. Racines.

F. Feuillage. 
frais et mème humides; - redoute les sols secs ou superficiels, ainsi que les pentes prononcées, $i b .=\mathrm{F} . \mathrm{F}$. Floraison comme celle du rouvre, plus précoce de 10 à 15 jours: - glands de grosseur à peu près constante, attachés par 1, 2 ou 3 sur un pédoncule commun, assez long et grèle, 49. = J. P. Robuste; - périt promptement sous le couvert, $i b .=\mathrm{F}$. Feuilles plus tendres, plus précoces et restant plus longtemps vertes en automne que celles du rouvre; - ramification; - couvert très incomplet, $i b .=\mathrm{R}$. Plus pivotant encore que le rouvre, $50 .=$ C. D. Comme le rouvre; - croissance en général plus rapide, $i b$. $=Q$. U. Conme le rouvre, seulement il est plus particulièrement propre aux grandes constructions, et convient moins à la fente et à la menuiserie; - motifs à l'appui de cette assertion, 51 .

$3^{\circ}$ Chêne tauzin. Très répandu dans l'Ouest, 52. = C. S. E. Climat doux et même chaud; - préfère les plaines; - assez indifférent pour l'exposition, ว3. $=\mathrm{T}$. Préfère les sols légers et frais, $i b .=F$. F. Floraison monoïque, au printemps; maturité, en septembre, $i b .=J$. P. Tempérament robuste, $i b .=F$. Feuilles profondément lohées; - couvert très incomplet, $i b$. R. Racines traçantes; - drageons abondants, $i b .=$ C. D. Croissance assez rapide; - vit plusieurs siècles; - dimensions ordinaires, $i b .=Q$. U. Bois peu estimé pour les constructions, la fente et les autres ouvrages; - fournit de très bons cercles de futailles; - qualité de son chauffuge et de son charbon; - écorce; - fruit, 54.

4' Chêne yeuse. Lieux d'habitation, 54. = C \$. E. Température élevée; - coteaux, montagnes; - s'accommode de loutes les expositions, $i b .=\mathrm{T}$. Sols calcaires; - végète aussi dans les terrains sees et arides, $i b .=F$. F. Floraison monoïque, au printemps; - fruit mûr en septembre, quelquefois doux et comestible, surtout aux expositions très chaudes, $530 .=$ J. P. très robuste; - premier abri contre les ardeurs du soleil, $i b .=F$. Feuilles petites, persistantes; - couvert très épais, $i b$. R. Racines pivotantes traçantes et drageonnantes, 56. C. D. $=\mathrm{C}$. D. Croissance très lente; - vit plusieurs siècles ; - dimensions 
médiocres, $i b_{0}=Q$. U. Homogénéité, finesse du grain, densité, poids, durée; - três propre aux usages les plus précieux; - chauffage très estimé; - emploi précieux de l'écorce, ib.

$5^{\circ}$ Chêne liége. Lieux d'habitation; - deux variétés, $57 .=$ C. S. E. Température élevée; - plaines et région moyenne des montagnes; - expositions méridionales et abritées, $i b .=\mathrm{T}$. Sols granitiques; - terrains légers, calcaires; - ceux compactes ou humides lui sont contraires, 58. = F. F. Floraison monoïque, en mai ou juin; - glands parfois doux, mûrs en automne; - réussite fréquente, $\imath b=\mathrm{J}$. P. Le jeune plant n'est sensible qu'aux froids et aux gelées tardives; - premier abri aux expositions chaudes, 59. $=$ F. Feuilles petiles, entières, nombreuses, persistantes; - couvert épais, $i b .=R$. Racines pivotantes, traȩantes et drageonnantes, $i b .=\mathrm{C}$. D. Croissance assez active; - vit plusieurs siècles; - belles dimensions, $i b$. $=Q$. U. Poids, densilé ; - usage précieux; - écorce : liége mâle; - démasclage; - liège femelle; - récolte en juin, juillet, août, 60.

$6^{\circ}$ Chéne Kermès. Lieux d'habitation, 61. - C. S. E. Température élevée; - s'accommode de toutes les situations et expositions, $i b .=T$. Sols sablonneux et pierreux; - peu exigeant, $i b .=$ F. F. Floraison monoïque, en mai; - fertilité précoce et fréquente; - glands mûrs au bout de 16 mois, $i b .=$ J. P. Jeunes plants très robustes, $62 .=\mathrm{F}$. Feuilles persistantes et épineuses: - couvert très épais, $i b .=R$. Racines nombreuses traçantes, drageonnantes, $i b .=$ C. D. Croissance lente; - peu de durée; - très faibles dimensions, $i b .=Q$. U. Qualités insignifiantes; - insecte Kermès; - écorce, $i b$.

Art. II. Le hètre (๕z 172 à 130). Son importance; - une seule espèce, 63. = C. S. E. Climat qüil préfère; - hauteur où il croil; influence de la situation suivant les contrées ; - influence des expositions, $i b .=\mathrm{T}$. Ses répugnances, exigences et préférences, quant au sol, 64. = F. F. Fleurs monoïques, en avril; - sujetles aux gelées; - fruit, faîne; - semence lourde; réussite rare; - maturité, en octobre; - fertilité, à 50 ans 
$i b .=J$. P. T'empérament delieat; - abri prolongé, 65. = F. Feuilles abondantes; - couvert très épais, $i b .=$ R. Fortes; traçantes; - drageonnant rarement, $i$. = C. D. Marche de la croissance; - dimensions; - longévité, $i b .=Q$. U. Bois peu propre aux constructions, mais de bonne fente; - ses divers einplois; - dessiccation nécessaire ; - qualité de son chauffage et de son charbon; - usage de la faine, 66.

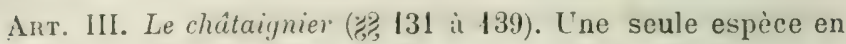
Europe, $67 .=$ C. S. E. Climats tempérés oll chauds; - siluations favorables ou contraires; - il redoute l'exposition du Sud; - gelécs printanières, $i b .=\mathrm{T}$. Sa végétation dans divers sols; - terres qui lui sont contraires; $68 .=$ F. F. Fleurs monoïques, en juin; - fruit, châtaigne; - semence lourde ; maturité, en novembre; - fertilité précoce, $69 .=$ J. P. Analogues aux jeunes chènes, $i b .=F$. Feuilles grandes; - couvert assez épais, $i b .=\mathrm{R}$. Pivot; - s'enfonce à un mètre et plus; - drageons, $70 .=$ C. D. Croissance très rapide et longtemps soutenue; - dimensions de l'arbre; - exemples de longévité, $i b .=\Omega$ U. Excellente charpente; - cercles et échalas; - merrain; - chauffage inférieur; - charbon léger; - fruit excellent, 71.

Art. 1V. L'orme (8? 140 à 4 is). Très rarement dominant; espèces ou variétés nombreuses; - celles ì petites feuilles sont plus estimées, $72=\mathrm{C}$. S. E. Influences climatériques favorables ou contraires à l'orme; - lieux où on le rencontre; - les expositions qui lui conviennent diffèrent suivant les situations, 73. = T. Sols qu'il redonte; - sa végêtation dans un sol frais et dans un sol humide, $i b .=F$. F. Fleurs hermaphrodites, en mars; - semence très petite, mùre en juin; - conformation el dissémination; - réussite fréquente; - fertilité précoce, $i$. = J. P. Tempérament robuste; - abri utile, 7\%. = F. Feuilles épaisses; - convert assez épais, $i b .=\mathrm{R}$. Double disposition; - drageons, $i b .=$ C. D. Promple croissance; - fortes dimensions; - grande longévité, ib. $=\mathrm{Q}$. U. Proprietés et usages comme bois de service; - comme bois de travail; usages précieux dus à sa fibre serrée et coriace; - qualité de 
son chauffage et de son charbon; - emploi de sa cendre; de son feuillage, $i b$.

Art. V. Le frène (Z̋ 149 à 157). Ủne seule espèce indigène, 76. = C. S. E. Climats qu'il préfère; - qu'il supporte; - sa végétation dans les situations élevées ou basses; - il craint l'exposition du Sud, $i b .=$ T. Sols favorables; - sols contraires, $i b .=F$. F. Fleurs polygames, en avril; - semence légère, ailée; - maturité en novembre; - époque de la dissémination, variable; - réussite annuelle; - fertilité précoce, 7 . $=$ J. P. Abri avantageux, $i b .=$ F. Feuilles composées; - couvert léger', $i b_{0}=\mathrm{R}$. Double disposition; -1 mètre d'enfoncement et 6 à 7 mètres d'extension horizontale; - drageons, ib. = C. C. Croissance rapide; - dimensions; - longérité, deux siècles, $78 .=$ Q. U. Emploi comme charpente; - romme bois de travail; - usages spéciaux: - chauffage et charbon estimés; - emploi de l'écorce; - des cendres; - des feuilles, $2 b$.

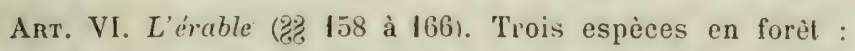
érable syeomore, érable plane, érable champêtre, $79 .=(\therefore \mathrm{S}$. E. Exigences analogues à celle du frène, $i b=T$. Sols favorables; - sols contraires, $i b=\mathrm{F}$. F. Fleurs en avril et mai; - hermaphrodites, et accidentellement polygames; - semences légères, ailées; - maturité el dissémination en octobre, 80 . $=\mathrm{J}$. P.Abri nécessaire, $i b .=$ F. Feuilles abondantes; - couvert épais, $i b .=R$. Disposition, extension et enfoncement; drageonnent rarement, $i b .=$ C. D. Erables sycomere et plane, croissance rapide; - dimensions; - longévité; - Erable champêtre, croissance plus lente; - dimensions moindres, $i b$. $=\mathrm{Q}$. U. Bois exempt de vermoulure; - emploi pour le travail; - chauffage et charbon estimés; - cendres ; - feuilles; sève, 81 .

Art. VII. Le bouleau (靽 167 à 175). Deux espèces, diffẻrant seulement par des caractères hotaniques peu essentiels, très répandues; - rarement seul et dominant, $82 .=$ C. S. E. Le bouleau supporte les climals très froids et les montagnes élevées, préfère les climats tempérés et les expositions S.-E. et $\mathrm{S} .-\mathrm{O}, i b .=\mathrm{T}$. Sols qu'il préfère et ceux dont il se contente; 
- sa végélation dans les marais, $83 .=\mathrm{F}$. F. Florason monoïque, à la fin d'avril; - semence ailée, très légère; - maturité, fin d'août; - réussite annuelle; - Eertilité précoce, $i b$. = J. P. Tempérament très robuste, $84_{0}=\mathrm{F}$. Feuilles pelites - couvert très líger; - inconvénients qui en résultent, $i b .=$ R. Traçantes; - drageons, $i b .=$ C. D. Croissance rapide; lurée de 80 à 90 ans, $i b .=Q$. U. Emploi rare dans la bàtisse; - bon bois de travail, exempt de gercures et de vermoulure - chauffage et charbon estimés; - écorce; - sève, $2 b$.

Ant. VIII. Le robinier faur acacia (\% 176 à 184). Originaire l'Amérique; - acciimaté, 8 õ. = C. . . . E. Il craint les froids ef les grands vents; - préfère les expositions chaudes, $i b .=\mathrm{T}$. Sols favorables; - suls contraires, 86. = F. F. Floriison hermaphrodite, en juin; - fruit, gousse; - semence y adhérant; - maturité, en octols'e, - dissémination, au printemps; - réussite fréquente; - fertilité précoce, $i b .=J$. P. $\Lambda$ garantir contre les froids, $i b .=F$. Feuilles petites; - peu de couvert, $i t .=R$. Disposition traçante, s'enfonçant quand le terrain le permet; - chevelu; - drageons, $86 .=$ C. D. Croissance très rapide; - dimensions; - duree de 100 ans au plus, $8 T_{.}=Q$. U. Dureté, durce très grande; - emploi comme bois de service; - comme bois de travail; - échalas; - gournables; - chauffage peu estimé ; - feuilles, $i b$.

Ant. IX. Le charme (8z 185 à 193). Souvent mélangé, quelquefois dominant, $88 .=$ C. S. E. Climats qu'il supporte; - siluation qu'il préfére: - exposition qu'ii redoute, $i b$. = T. Sols fivorables; - convenables; - contraires, $i b .=F$. F. Floraison monoïque, en mai, - semence petite, ailée, - maturité et lissémination, en octohre; - réussite annuelle; - fertilité à :30 ans, 89. = J. P. Abri contre le soleil, $i b .=\mathrm{F}$. Feuilles moyennes; - couvert très épais, $\imath b$. $=\mathrm{R}$. Direction oblique; - un mètre d'enfoncement; - drageons, $1 b$. = C. D. Marche de la croissance: - maximum à 70 ou 80 ans; - durée, 150 ans, $i b .=$ Q. U. Densité égale ; - dureté ; - fibre coriace; impropre à la charpente; - cannelures du trone; - emplo comme travail; - chauffage et charbon de première qualilé rendres: - feuillage, 90 . 


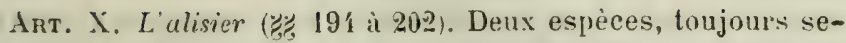
condaires : l'alisier blane, l'alisier torminal, $91 .=$ C. S. E. Ils supportent les froids; - prospèrent dans les climats tempérés dans les plaines, sur les coteaux, et aux expositions de l'Ouest, de l'Est et du Sud-Est, it. = T. Sols convenables; - favorables; - contraires, $i b .=$ F. F. Floraison hermaphrodite, en mai ; - fruit, baie; - maturité, en octobre; - dissémination, en hiver; - fertilité précoce, $i b .=$ J. P. Abri utile, $92 .=\mathrm{F}$. Feuilles grandes; - couvert assez complet, $i b .=\mathrm{R}$. double direction; - tendance particulière; - drageons, $i b .=\mathrm{C}$. D. Croissance lente; - longévité; - dimensions, $i b .=\mathrm{Q}$. U. Emploi comme bois de travail; - usages spéciaux; - chauffage et charbon estimés; - fruit, eau-de-vie, vinaigre, 93.

Ant. XI. Le sorbier (2:, 203 ì 211). Deux espèces, toules deux secondaires : sorbier des oiseleurs, sorthier cormier, 93. = C. S. E. Le premier se trouve sur les plus grandes hauteurs, et réussit it toules les expositions; - préférence du cormier, $i b .=\mathrm{T}$. Le sorbier des oiseleur's ne craint que les sols humides; - le cormier est plus difficile, 91. = F. F. F'loraison hermaphrodite, en mai; - forme des fruits; - malurite, fin de septembre; - dissémination, en hiver; - fertilité, ì âge diffèrent, $i b .=$ J. P. Abri utile seulement au cormier, 95. $=\mathbf{F}$. Couvert léger, ih. = R. Pirot; - $1^{\mathrm{m}}, 33$ d'enfoncement; - racines tracantes; - drageons, $i b .=$ (. D. D. Croissance lente; longévilé plus grande et dimensions plus fortes chez le cormier. $i b .=0$. U. Bois dur el pesant, employé par les tourneurs, menuisiers et mécaniciens; - chauffage fort estimé; - écorce; - usages différents du fruit des deux sorbiers, $i b$.

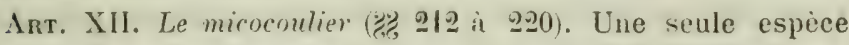
indigene; culture avantageuse, $96 .=$ C. S. E. Les climals chauds et tempérés, les plaines et les élévations moyennes, el toutes les expositions conviennent all micocoulier, $i b .=\mathrm{T}$. $\Lambda$ peu près tous les sols, $97 .=F$. F. Fleurs polygames, en mai; - fruil pelit, à noyau; - maturité, en novembre; - dissémination, au printemps; - fertilité précoce, $i b .=$ J. P. Abri contre le froid, $i b .=F$. Feuilles grandes; - couvert assez lé- 
ger, $i b .=\mathrm{R}$. Pivol et racines traçantes; - drageons, $i b .=\mathrm{C}$. D. Croissance rapide; - longue durée; - dimensions, 98. = Q. U. Dureté el souplesse; - emplois divers; - chauffage estimé; - feuilles, $\imath b$.

Ánt. XIII. Le cerisier (2马 221 à 229'. Trois espèces : cerisier commun, - à grappes, - mahaleb; - la prcmière seule est importante, $99=$ C. S. E. Il supporte les climats rudes, les situations élcvées ct toutes les expositions, 99. = T. Tous les sols, à l'exceptiun de ceux humides, lui conviennent, $i b$. F. F. Floraison hermaphrodite, en mai; - fruit, drupe, mûre en juin; - dissémination en août; - fertilité précoce, $i b .=$ I. P. Abri nuisible, 100 . = F. Feuilles grandes; - couvert léger, $i b .=$ R. Traģantes; - drageons, $i b .=\mathrm{C}$. D. Croissance très rapide; - dimensions; - durée, 80 ans, $i b .=Q$. U. ILetiers qui l'emploient : ébénistes, luthiers, etc.; - cercles; - chauffage et charbon; - merises, kirschwasser; - gomme, ib.

Art. XIV. L'aune (2\%, 230 à 238). Deux espèces : l'aune commun, l'aune blanc; - l'aune n'admet de mélange qu'avec le frêne, 101. = C. S. E. Il est assez indifferent sous ces trois rapports, $i b .=\mathrm{T}$. Il aime les terrains aquatiques; - veut un sol frais, substantiel et divisé; est avantageux dans les marais, 102. = F. F. Floraison monoïque, en mars; - fruit, cône; maturité, en octobre; - dissémination, à l'entrée de l'hiver; fertilité, â İ̈ ans, $i b .=\mathrm{J}$. P. Abıri inutile, 103. = F. Feuilles grandes et épaisses; - couvert incomplet; - effet qui en résulte, $i b .=\mathrm{R}$. Traçantes; - draģeons, $i b .=\mathrm{C}$. D. Croissance très rapide; - dimensions; - durée, 90 ans, $i b .=Q$. U. Charpente sous eau; - bon bois de travail; - chauffage recherché pour le four; - double supériorité de l'aune blanc; - cendies; - écorce; - feuilles, 104.

Art. XV. Le tilleul (2气 239 à 247). Deux espèces : tilleul des bois, tilleul de Hollande, 105. = C. S. E. Ils supportent à peu près tous les climats et les situations élevées; - expositions préférées, $i b .=T$. Ils préfèrent les terrains sablonneux, profonds et frais, $i b .=F$. F. Floraison hermaphrodite, en juin et 
juillet; - fruit, petite noix ; - maturité en octobre; - dissémination, à l'entrée de l'hiver; - fertilité précoce, 106. = J. P. Abri favorable, $i b,=F$. Feuilles abondantes; - couvert épais, $i b .=\mathrm{R}$. Pivot très prononcé $;-1$ mètre 50 centimètres d'enfoncement; - racines traçantes; - drageons, $i b .=\mathrm{C}$. D. Croissance rapide; - dimensions; - exemples de la grande longévité du tilleul de Hollande, $i b .=Q$. U. Impropre à la charpente; son emploi pour le travail; - exempt de gerçures et de vermoulure; - chauffage peu estimé; - emploi de son charbon pour la poudre; - écorce; - feuilles, 107.

Art. IVI. Le peuplier (诌, 248 à 256$)$. Le tremble est le seul peuplier de nos forêts, 108. = C. S. E. Il prospère dans les climats tempérés, et préfère les expositions du Nord et de l'Est, $i b .=\mathrm{T}$. Il est assez difficile sur le choix des sols; - ses préférences, 109. = F. F. Floraison diö̈que, en mars; - semence très-petite; - malurité el dissémination, en mai ; - fertilité à 20 ans; - réussite annuelle, $i b .=J$. P. Tempérament robuste; - causes de sa grande propagation, $i b .=F$. Feuillage très-lèger ; - couvert très incomplet, $110 .=\mathrm{R}$. Traçntes ; drageons, $i b .=$ C. D. Ciroissance très rapide; - dimensions: - pourriture hâtive à l'intérieur, $i b .=Q$. U. Charpente en lieu sec et conduites d'eau; - son emploi comme bois de travail : - chauffage du four; - emploi du charbon pour la poudre: - écorce; - feuilies, $i b$.

Art. XVII. Le saule (7?, 257 à 263). Nombreuses espèces: - le marceau el le saule blanc sont seuls très répandus en forèt. 111. = C.S. E. Le marceau prospère dans tous les climats ; les situations basses ou moyennes sont propices aux autres espèces, 112. $=\mathrm{T}$. Tousles sols conviennent au marceau $;-$ les sols frais et humides au saule blane, 113. = F. F. Fleurs dioïques, en mars et avril; - semence petite; - son organisation; - maturité et dissémination, en mai el juin; - fertilité très précoce; - réussite annuelle, $i b .=$ J. P. Tempérament robuste; - l'essence est envahissante comme la précédente, $i b .=$ F. Donne peu d'ombrage, $i b .=R$. Traçantes; - drageons. $114=$ C. D. Croissance très rapide ; - dimensions ; - passé 
60 ans, les saules se ereusent, $i b .=Q$. U. impropre a la charpente; - volige; fente; - vannerie; - mauvais chauffage, sauf pour le four; - emploi de son charbon pour la poudre; - écorce; - cendres; - feuilles, ib.

Ant. XVIII. Le platane ( $202 \%$ à $27 亿)$. Une seule espèce, originaire d'Amérique; - acelimaté ; - mérite d'ètre introduit en forêt, $115 .=$ C. S. E. Plaines, lieux frais et abrités, $i b .=T$. Sols frais, mème un peu humides, $i b .=F$. F. Fleurs monoïques, en avril et mai ; - graine aigrettée, petite; - maturité et dissémination en automue; fertilité vers 30 ans; réussite fréquente, $i l .=\mathrm{J}$. P. L'n premier abri est utile, 116. $=\mathrm{F}$. Feuilles grandes'; - couvert épais, $i b . \mathrm{R}$. = Longues, fortes et tracantes, $\mathrm{i} \eta_{\circ}=\mathrm{C}$. 1). Croissance très rapide; - durée : 200 ans, $i b .=0$. C. Egal au hèlse pour le chauffage, supérieur pour le travail; - résistance à la pourriture, ib.

\section{CHAPITRE CINQUIEME}

DES hOIS RÉSINEUX.

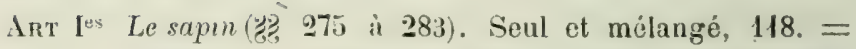
C. S. E. Il préfere les climats tempérés; - ne craint pas les frimats; sa région est entre 500 el 1000 mètres au-dessus du niveau de lamer'; - peut 'prospérer dans les plaines; - il souffre i l'exposition du Sud et se plait au Nord et à l'Est, $i b .=$ T. Il exige un sol divisé, un peu profond; - redoute les terrains aquatiques et marécageux; - eroit souvent parmı les roches, 119. = F. F. Fleurs monoïques, en mai, placees vers la eime; - fruit, cône ; - semence petite, ailée ; - maturite, en octobre; - lissémination en automne; - elle a licu d'une façon Irarliculière; - fertilité, vers 60 ans; réussite brennale, $i b=$ J. P. Très delicats, abri prolongé, $120 .=\mathrm{F}$. Courtes et étroiles; - persistance, 3 ans ; - couvert très complet, $i b .=$ R. Fortes ; - pivot ; -1 mètre d'enfoncement ; - 
les racines sont souvent anastomosées, et leur végétation solidaire, $i b .=C$. D. Croissance lente d'abord, rapide plus tard; dimensions; - longévité, $121 .=Q$. U. Très bonne charpente ; - planches ; carcasses de nieubles, etc.; - chauffage et charhon médiocres; térébenthine de Strasbourg, manière de l'obtenir ; - colophane; - éclairage; - salin, manière de l'obtenir ; - potasse, $i b$.

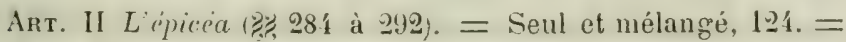
C. S. E. Il supporte les frimas et les régions supérieures à celle du sapin ; - craint, moins que celui-ci les expositions chaudes, $i b$. $=\mathrm{T}$. Mème sol que le sapin ; - il exige moins de fond; supporte un sol humide et mème tourbeux, $i b .=F$. F. Fleurs monoïques, en mai ; - fruit, cône ; - maturité, fin d'automne; - dissémination, au printemps suivant; - comment elle s'opère; fertilité, vers 50 ans; - réussite biennale, $i b .=J$. P. Abri nécessaire à l'exposition du Sud, 12.\%. = F. Feuilles plus petites que celles du sapin; - persistance de 3 à 7 ans; - Couvert tries épais, $i b .=$ R. Traçantes, $i b .=$ C. D. Croissance plus rapile, mèmes dimensions et mème durée que le sapin, 126. - Q. IT. Mèmes usages que le sapin ; - luthier's; - chauffage el charbons médiocres; - poix de Bourgogne; - mode d'extraction; - ses inconvénients, $i b$.

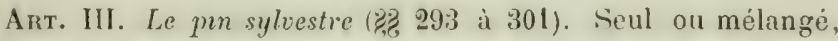
127. = C. S. E. Climats tempérés, favorables; - pays froids, convenables; - plaines et pentes favorables; - hautes montagnes, contraires; - il supporte toutes les exposilions, ib. = T. Sol profond el léger, sable pur, favorables; - terres compactes, contraires; - sa végétation dans les parties humides ou tourbeuses, 128. = F. F. Fleurs monoïques, en aviil ou mai; - fruit, cône; - semence ailée; - maturité, en novembre do la deuxième année; dissémination, au printemps suivant: fertilité, à 10 ans; - réussite biennale, 129. = J. P. Abri rarement utile, $130 .=F$. Feuilles longues ; - persistance, 3 ans; - couvert léger, $i b .=\mathrm{R}$. - Fortes; - pivot; -1 mètre d'enfoncement, $i h .=$ C. D. Croissanee rapide; dimensions ; = longévité, 2 siècles,,$b$. = Q. U. Mêmes usages que les deux 
précédents; - sa supériorité : - emploi pour la màture; chauffage et charbon : - oxtraction du goudron, carbonisation des souches, $2 b$.

ARt. IV Le pin i crochets. (隠, 302 à 310 ). Hautes régions des Alpes, des Pỹrénées et du Jura ; - tantôt seul, tantôt mélangé su pin sylvestre et an mélèze; - précieux pour reboiser les hautes montagnes, 132. = C. S. E. Climats sees et froids ; latitude : 1500 à 2500 mètres - expositions méridionales. 133. = T. Indifférent sous ce rapport, 13', = F. F. Fleur's monoïques 'en juin et juillet; - fruit, còne; - graine, comme celle du pin sylvestre ; - mêmesépoques de maturité et de dissémination ; - fertilité précoce; - fruits ajondants, $i b .=$ J. P. Très robustes; - supportent cependant un abri prolongé ; résistent aux plus grands froids, $i b=\mathrm{F}$. Feuilles roides et serrées; - couvert épais, 135. = R. Traçantes, $i b .=$ C. D. Croissance très-lente, égale, longue; dimensions, $i b .=Q$. I. Charpento estimée; - préférable aux autres résineux des Alpes, excepté le mélèze ; - très bon hois de travail ; - chauffage très estimé ; - est peut-être propre à la małlure, ib.

Art. Y. Le pin maritime ( $(2,311$ à 319). Forèts considérables, 137. = C. S. E. Il appartient aux climats chauds ; - croit dans l'Ouest, à Fontainebleau, et à Paris ; - craint les froids vifs ; - se plait dans les plaines et sur le bord de la mer, $i b .=\mathrm{T}$. Sol médiocre et mème purement quartzeux, mais profond; exclul les terrains compactes et marécageux, 138. - F. Fleurs monoïques, en avril ou mai ; - semence ailée, plus grosse que celle du sylvestre; - mêmes époques de maturité et de dissémination; fertilité à 15 ans; - réussite fréquente, $i b .=\mathrm{J} . \mathrm{P}$. Abri parfois utile, $i b .=F$. Feuilles très-longues ;-persistance, 3 ans; - couvert faible, $i b .=R$. Fortes et nombreuses; pivot; - disposition particulière, 139. = C. D. Croissance très-rapide; dimensions; - durée, 2 siècles en bon sol, et quand il n'est pas saigné, $i b .=Q$. U. Bois inférieur au pin sylvestre ; emploi pour pilotis et étais; - planches et échalas; - chauffage et charbon médiocres; - procédé pour l'extraction de la résine, $i b$. 
Arт. VI Le pinlaricio (2马, 320 à 328). Grandes forèts en Corse, 143. = C. S. E. Climat tempéré; - il réussit dans le Nord et l'Est de la France; - craint peu nos hivers; - vient à toutes les expositions, $i b .=T$. Sables gras, granitiques $144 .-F$. Floraison monoïque, fin de mai ; - semence plus grosse que celle du pin sylvestre, ailée; - mèmes époques de maturité et de dissémination, $i b .=\mathrm{J}$. P. Tempérament robuste, $i b .=\mathrm{F}$. Feuilles intermédiaires entre celles du sylvestre et du maritime; - couvert très-léger, $i b .=R$. Fortes et nombreuses, traçantes, $i b .=$ C. D. Croissance très prompte; dimensions : - longévité, plusieurs siècles, ib. - Q. U. Charpente estiméc: - bois de marine, màture ; expériences à ce sujet ; - autres emplois; - il est très-résineux, 145.

Art. VII. Le pin noir (Z尹, 329 à 337). Espèce très voisine du laricio; - seule ou mélangée - récemment introduite en France; - très rustique, 146. = C. S. E. Plaines et montagrnes jusqu'à 1000 mètres; - au-dessus, il languit ; - réussit à toutes les expositions, 147. = T. Préfère les sols léger's, sees ef calcaires; - redoute les terres argileuses, humides, ib. = F. F. Semblable en toutau laricio, 148. = J. P. Trés-robustes, $i b .=F$. Feuilles très serrées; - couvert épais, $i b .=R$. Robustes, traçantes, $i b .=$ C. D. Végétation rapide et soutenue; - dimensions; - longévité : $\mathbf{2}$ à 3 siècles, ib $=$ Q. U. Construction; - durée très longue; - incorruptible sous l'eau; chauffage et charbon estimés; - gemmage, 149.

Art. VIII. Le pin d'Alep (ZZ 338 à 316). Répandu dans le Midi de la France, 149. = C. S. E. Exige un climat chaud; - redoute les froirls ; - aime les plaines, les còteaux et l'exposition dı Sud, $i b .=\mathrm{T}$. Sol médiocre, mais léger et sec, 150. F. F. Floraison monoïque, en mai; - graine ailée; - maturité, à la fin du second été; - dissémination dans le courant du troisième, $i b .=$ J. P. Tempérament robuste dans le pays où il est indigéne, $i b=\mathrm{F}$. Feuilles longues et très fines; couvert léger, $i b .=\mathrm{R}$. Traçantes, $i b=\mathrm{C}$. D. Croissance trèsrapide; - fortes dimensions, $i b .=Q$. U. Charpente, menuiserie; - résine; s'extrait par les mêmes procédés que celle du pin maritime; - résine liquide, 151. 
Anт. IX. Le pen pinier (睠 317 à 355). Arbre de l'Europe méridionale, 151 , = C. S. E. Demande un climat chaud ; aime les plaines. les vallées, les borls de la mer et des fleures, $h .=T$. Léger et profond, sablonneux, mais frais, 152. = F. F. Floraison monoiıue, en mai et juin; - strobiles très gros; maturité, après 3 ans; - amandes grosses, comestibles, $i b=$ J. P. A étudier, $i b .=F$. Feuilles plus grandes et couvert plus épais que les autres pins, $i b .=$ R. Fortes et pivolantes, $153 .=$ C. D. Conjectures à former sur sa rroissanee et ses dimensions possibles, $i$. $=$ Q. U. Charpente, plinches, corps de pompes; - menuiserie, ete.; - fruit agréable à manger et donnart de bonne huile; - variété d'Italie à noyau plus tendre, $i b$.

Art. X. Le pin cembro (8z 356 à 36 1). Seul ou en mélange, 15\%. = C.s. E. Arbre des grandes élévations el des pays froids; - se trouve"sur les Alpes du Dauphiné et de la Provence; $y$ vient a toutes les expositions, $i b .=T .11$ se plait dans les sols substantiels, frais, profonds et divisés; - supporte un terrain légèrement humide et pierreux, 15 ö. $=\mathrm{F}$. F. Floraison monoï que en mai ou juin : - graine, amande moins grosse que celle du pinier; - maturité, après dix-huit mois, $i b$. = J. P. Doivent ètre garantis des chaleurs et surtout des gelées printanières, $i b .=\mathrm{F}$. Touffu; - couvert épais, $i b .=\mathrm{R}$. Un mètre d'enfoncement, $i b=$ C. D. Croissance très lente; - durée, plısicurs siècles; - fortes dimensions, $i b .=0$. U. Sculpture, menuiserie : - chauffage estimé ; - fruit agréable ; - bonne huile, 156.

.IRT. X1. Le pin du lord Wegmouth (8\% 365 à 373). Exolique, acclimaté en France, $156,=$ C. S. F. Réussit dans toute la France, excepté dans le Midi; - préfère les régions un peu froides, $i h .=T$. Il craint les sols arides, compactes et marécagenx; prospère dans ceux qui sont humides et profonds. 157. $=F$. F. Floraison monoï jue, fin de mai ; - malurité et dissémination des graines seize mois après; - fertilité précoce, ib. $=$ J. P. Abri utile; - analogie avec l'épicéa, $i b .=F$. Fin et léger: - peu de couvert, $i b=\mathrm{R}$ Pivot très prononcé ; nrrande extension latérale, $i h .=$ C. D. Croissance très-rapide: 
- grande durée; - dimensions très fortes, $1 b .=Q$. U. Aux Etats-Unis, son bois est ferme, léger', peu noueux ; - propre à diver's métiers ; - charpente, construetions navales et mâture; - il est sujet à pourrir ; - on ne peut ensore juger les qualités de ceux qui ont erù en France; - lérébenthine abondante: - soumis au résinage en Amérique, 158.

Arr. XII. Le mélèze (8ृ? 374 à 382 ). Son importance; est à feuilles caduques; se trouve seul on en mélange, 159. = C. S. E. Originaire des hnules montagnes et des pays froids: - il demande une atmosphère sèche et froide; - dans les règions tempérées de la France, il lui faut les expositions du Nord et de l'Est, $i b .=$ T. Il exige une terre divisée, fraîche et profonde; - craint les sols compactes ou hum des el les sables trop légers, 160. = F. F. Fleurs monoïques, en avril ou mai; graine légète el ailce; - maturite, au hout de fi mois; - disséminalion, au printemps suivant; fertilite preroce, - indice à tirer de l'abondance du fruit, $i b .=J$. P. Robustes dans leur climat; - ailleurs, abri utile, $i b .=F$. Feuilles petites; couvert tres leger, $161 .=$ R. Pivol; - un metre d'enfoncement : - racines tracantes, $i b .=(\therefore$ D. Croissance très prompte - : fortes dimensions ; - durée, plusieurs siècles; - observations sur la marche de sa végétation dans les climats temperés, $\iota_{0}=$ Q. U. Il résiste à l'air et à l'humidité ; - constructions rivilıs et navales ; - divers métiers; - merrain et échalas ; - chauffage et charbon mediveres; - térébenthine de Venise; - son extraction ; huile essentielle; - écorce, 163.

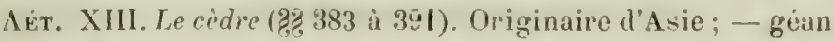
des coniferes, $166 .=$ C. S. F. Les forêts de cerdre sont situées à de grandes hauleurs sur le Liban, et at lí00 metres en dfrique; - le eedre réussit, en Enrope, dans les climats temperés; - dans le climat de Paris, il craint le froirl dans sa jennesse, $i t$. $=$ 'T'. Sols graveleux, ser's et profonds, ronvenables; - terres comprctes et marécageuses, contraires, 167. = F. F. Fleurs monoïques, en octobre; - maturité, 90 mois après; dissémination, en hiver; - s'opire, comme celle du sapin, par $I_{a}$ désarticulation des écailles, it. = J. P. En France, délicats. 
- abri peudant six ou huit ans contre les froids, 168. $=\mathrm{F}$. Feuilles nombreuses el touffues; - couvert épais, $i b .=F$. Pivot très fort; - racines latérales nombreuses, $\imath b$. = C. L. Croissance lente d'abord, très active ensuite; - dimensions énormes; - longévité considérable, $i b .=Q$. U. Incertitude et discussion à cet égard, $i b$.

\section{LIVRE DEUXIÈME}

\section{Principes fondamentaux de l'exploitation des bois.}

Définitrons (392 à 402). Révolution; - elle est figurée sur le terrain par les exploitations annuelles. 171. = Bois exploilable; - l'exploitabilité se modifie diversement, $i b .=$ Accroissement; - arnuel; - moyen, 172. = Rente, $i b .=$ Possibilité; - rapport soutenu, $i \hbar .=$ Peuplement complet, $i b .=$ Coupe. 173. = Assielte; $=$ asseoir une coupe, $i b .=$ Vidange, $i b .=$ Coupe en usance, - coupe usée, $i b$. = Chablis; - volis ; quille, chandelier ou tronc, $i b$.

\section{GHAPITRE PREMIER.}

DE L'EXPLOITABHLTTE.

Ant. Ier. De l'exploitabilité en yénéral (8 403). Elle est la base du traitement des forêts; - elle se détermine d'après diverses considérations qui dornent lieu à quatre sortes d'exploitabilité : physique, absolue, relative, composée, 174.

Int. II. De l'cxploitabilité physique (3, 104). Traitement 
qu'elle entraine; - clle ne s'applique qu'à des cas exceptionnels, 175.

Art. III. De l'exploitabilité absolue (诌 105 à 408). En quoi elle consiste; - à quoi elle répond, 173 o. = Elle donne lieu à la plus grande production matérielle possible; - démonstration de ce fait, 176. = On déduit de cette proposition quatre corollaires principaux, $178,=$ Indications nalurelles propres à détermirer l'époque de l'exploitabilité absolue; - caractères de l'accroissement progressif; - de l'accroissement stationnaire: - de la décroissance; - du dépérissement, ib.

AnT. IV. De l'exploitabilité relative deux sortes, 180. = L'exploitabilité relative à la rente la plus élevée a lieu lorsque le revenu est le plus grand possible, sans cesser d'ètre, avec le capital producleur, dans un rapport égal au taux ordinaire des placements en hiens fondsdans la localité $i b .=$ Composition du capital et du revenu à chaque àge; accroissement annuel de ces valeurs; - conséquence it en tirer pour déterminer la révolution; - cette exploitabilité convient surtout aux particulicrs; - elle tornbe avant l'époque de l'exploitabilíé absolue, 181. = L'exploitabilité relative aux produits les plus utiles varie selon cette utilité, et lantòt devance, tantòt dépasse l'exploitabilité absolue, 183.

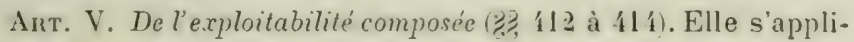
que à deux cas dont chacun réunit une double condition, 181. $=$ Combinaison du plus grand produit matériel avec la rente la plus élevée : exploitabilité convenable à l'intérêt particulier; $i b$. = Production de la matière à la fois la plus considérable et la plus utile : exploitabilité convenable à l'intérèt général; elle se réalise avec suceès dans les forèts de l'Ftat, $i b$.

\section{CHAPITRE DEUXIÈME.}

DE LA POSSIBILITÉ.

Art. Ier. De la possibilité en général. (8 415 à 417). Dans une forêt normale, elle serait égale à l'accroissement moyen, 186. 
- I Jans la réalité, il faul la régler te manière à obtenir un rapport soutenu, 187. = Elle pent se fonder sur l'étendue ou sur le volume, $i b$.

Arт. II. De la possibilié par étendue (良, 418 à 421). Comment elle sobtient; - les produits matériels sont entre eux comme les surfaces; - ce principe n'esl pas toujours admissible, IST. = Comment il doit ètre modific dans son application, 188. = Parlage de la furèt en grandes divisions, ou séries d'exploilation; - avantages qui en résultent, it). = I a possibilité par étendue c'st souvent préférée, mais n'est pas toujours applicable, 189.

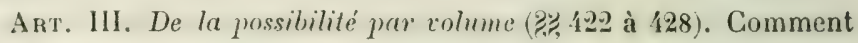
lit fixation de cette possibilité se conçoit au premier abord, 190. = Difficultés qui se présentent ; - une appréciation rigonreuse est impossible, mais n'est pas nécessaire au but rfu'on se propose, ib. = Exposition de la méthode à suivre; - périodes; - parlies aliquotes de la révolution; - décennies, 191. $=$ Répartition des exploitations dans les périodes; - circonstances à examiner; - cas où les affectations des périodes peuvent ètre de contenances égales; - cas où les contenances doivent ètre inversement proportionnelles à la production; - on calcule la possibilité sur l'affectation de la première période, 191. = Evaluation, au jugé, du volume aetuel et de l'aceroissement futur; - on estime laceroissement pour tous les bois, mais seulement pour le milieu de la période; - il existe des procédés plus rigoureux; - facilité des rectifications, 193. = On procèdera de même pour les périodes suivantes; - les inégralités, de période à période, ne seront pas assez fortes pour compromettre le rapport soulenu, $195 .=$ Ce mode peut éprouver des modifications, 196.

\section{CHAPITRE TROISIÈME.}

DE L'ASSIETtE DES COUPES

Art. Ier. De l'assiette des coupes en général (? 429). Ses dif- 
ficultés el son importance; - etle s'appuie sur cinq righles. 197.

Ant. II. Premièrc règle (尹 430). Succession et forme des coupes - inconvénients du désordre et de l'irrégularité des coupes; - avantages de la règle; - comment elle doit s'applirquer dans la pratique, 198.

Art. III. Seconde regle (\% 131). Disprosition respective des coupes; - chaque coupe doit s'appruyer sur un chemin, un ruisseau ou sur la lisière de la forèt, 199.

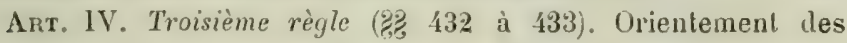
coupes; - les venis du Nord et de l'Est ne sont pas aussi dangereux que ceux du Sud et de l'Ouest: - les l'éserves doivent être garanties des vents dangereux; - avantage particulier de la règle pour les bois à semences légères; - son importance spériale pour les bois résincux; - cas d'exception lirés de la siluation des lorèts, 200. = Conservation, sur les lisières Sud et Onest, d'un rideau protecteur en massif, 201.

ART. V. Quatrième règle (\& 434). Ordre des coupes en monlagne; - danger de commencer par les plateaux; - avantages de la marehe opposée; - nécessité d'assurer ì chaqque coupe un moyen direct de vidange; - chemins de voilures; - chemins à traîneaux; - lançoirs; - marehe à suivre lor'squion ne peut assurer une vidange séparée à chaque coupe, 202.

Ant. VI. Cinquième règle (? 435). Forme à donner aux courjes en montagne, 206.

\section{LIVRE TROISIÈME.}

\section{De l'exploitation des futaies.}

Définitions (8z, 436 à 453). Futaie; = la régénération dolt ètre naturelle, 207. = Futaie régulière; - quel traitement améne cet étal, $i b .=$ Fufaie irrégulière; - causes de l'irrégularité, 
208. - Clairière, $i b$. - Places vides, $i b$. - Terres vaines et vagues, $i b .=$ Massif, $i b$. - Massif serré; - massif incomplet ou clairiérié ; - massif entrecoupé, $i b$. - Fourré ; - gaulis; - perchis, 209. = Bois blancs, mieux bois tendres; bois durs, $i b$. = Morts-bois, 210 . = Bois abroutis ; - abroulissement; le récépage y remédie, $i b$. - Recéper; - cas où cette opération se pratique, $\imath$. = Bois en défends; - bois défensables, $i b .=$ Réserves; - réserve de la coupe, $i b$. - Sous bois 211. = Coupe à blane étoe, it.

\section{CHAPITRE PREMIER.}

MÉTHODE UU RÉENSEMENCEMEXT NATUREL ET DES ÉCLAIRCIES OU EXPLOITATION DES FUTAIES RÉGULIÉRES.

Art. Ier. Généralités (靽, 454 à 458). En quoi consiste la méthode; elle repose sur des faits naturels, 212. $-=$ Action du sol sur la germination; - conditions qu'il doit réunir, 213. = La lumière, inutile à la ggermination, est nécessaire à la jeune plante; - comment son action doit s'exercer, $i b$. - Lutte nécessaire qui s'engage entre les jeunes plants serrés en massif;- diminulion du nombre des tiges et chute naturelle des branches hasses; - avantages de cet état de choses au début: - ralentıssement de croissance qui en résulte ensuite; la nature conduit ainsi une forêt jusqu'à sa régénération. 214.= Comment doit ètre imitée la marche naturelle, et conditions à réaliser, 215.

ART. II. Coupes de régénération (द⿱⺈ 459 à 461). Quatre conditions nécessaires réalisées par trois coupes, 216. = Coupe d'ensemencement; - conditions qui règlent la réserve; la coupe d'ensemencement remplitles trois premiêres conditions de la régénèration, $i b$. Coupe secondaire; - son bul; - comment on la dirige; - cas où elle se fait en plusieur's fois, 217. = Coupe définitive; - réserves qu'elle comporte ; - ces ré- 
serves ne sont point destinées à procurer un ensemencement tardif, 218. Comment l'exposition et la nature du sol doivent influer sur le nombre des réserves, dans les deux premières coupes, 219.

ArT- III. Coupes d'amélioration (8̈ 465 à 470). Les bois tendres et morts-bois s'introduisent toujours dans le repeuplement; - coupe de nettoiement ; - on ne peut en préciser l'époque d'une manière absolue, 221. - Eclaircies périodiques; - trois points à établir, 222. = Sur quels sujets portentles éclaircies; - ne jamais interrompre le massif ; - inconvénients d'une éclaircie trop forte aux différents âges; - éclaircie normale ou moyenne; - circonstances de sol, de situation et d'exposition ou l'éclaircie peut être forte; - circonstances contraires où elle doit'être faible, 223. = D'où dépend la fixation de l'éporque de la première éclaircie; - elle est utile dès que le bois est à l'état de gaulis, 295. - Fixation de la période à laisser entre les éclaircies, 227. - Avantages des éclaircies périodiques : augmentation deproduits; - ces produits accessoires ne tendent qu'à augmenter le produit principal ; les éclaircies amélior'ent la qualité du bois; - elles préservent les forêts de la multiplication des insectes, $i b$.

AnT. IV. Fixation de l'exploitabilité et de la possibilité dans les futaies (8: 471 et 472). L'exploitabilité doil-ètre fixée à un âge de fertilité complète et suivant les principes établis dans le denxième Livre, 229. = La possibilité doit se fonder sur le volume, $\imath$.

Ant. V. Marche des exploitations dans une futaie réguliëre (8: 473 à 477). Utilité de l'examen de cette marche; - hypothèse : révolution de 100 ans, périodes de $20 ;-$ embrasser les produits principaux et les produits accessoires à obtenir, dans loute la forêt, pendant la durée de la première période, $230=$ Coupes d'ensemencement pendant les premières années; - différentes coupes dans les années suivantes : - les coupes de régénération ne peuvent suivre une marche régulière, 231. = I a possibilité par étendue, applicable, à la rigueur, aux coupes d'ensemencement, ne peut plus s'admettre cians les coupes sui- 
vantes; - la possibilité par voilume permet seule d'opérer en raison des circonstances, 232. = La base de la possibilité est différente pour les coupes d'amélioration; nécessilé de leur imprimer une marche régulière ; - leur appliquer la possibilité par élendue, 233. - Trois genres de coupes à fairo annuelle. ment dans une futaie normale, 234.

Art. VI. Abatage, façonnage et vidange dans les futaies ( 8278 et 479). La meilleure saison pour l'abatage est l'hiver; - moment de le commencer; - avantages de cette saison pour la qualité du bois; - pour la vidange; - restrictions en ce qui concerne les essences résineuses, 234. = Ebranchement des arbres et autres précautions; - mode et époque de vidange; - ne point exagérer les mesures conservatuices, 236.

Art. VII. Essences propres à la futaie (: 480). Ce traitement, néressaire pour les bois résineux, n'est avantageux qne pour un certain nombre de bois feuillus, 238.

\section{GHAPITRE DEUXIÈME.}

ApPLICATION DE LA MÉTHODE DU RÉENSEMENGEMENT NATUREL ET DES ÉCLAIRCIES.

Art. I ${ }^{\text {r. }}$. Exploitation du chîne en futaie ( 28,481 à 483). E (1). Il y a perte à la reculer jusqu'à 250 et 300 ans; - l'exploitabilité peut varier, suivant les sols, de 120 à 180 ans, $239 .=$ C. R. Coupe d'ensemencement sombre; - son but; - le terrain se gazonne souvent et doit ètre remué; - ne pas retarder la coupe secondaire; - ni la coupe définitive; - quelques réserves sur celte dernière coupe; - branches gourmandes; -

1 Les intitulés des paragraphes sont représentés par les abréviations suivantes:

E. Lxploitabilité.

C. R. Coupes de rugénération.

C. A. Coupes d'amélioration. 
DES MATIERES.

émondage; - inconvénients qu'il prévient, 240. = C. A. Nettoiement conforme aux règles générales; - serrer beaucoup le massif dans les premières éclaircies; - espacer davantage dans les dernières; précautions à prendre, 245.

ArT. II. Exploitation du hêtre en futaie (2\% 484 à 486). E. Elle tornve ontre 80 et 140 ans; - cas où elle doit être abrégée, 246. = C. R. Coupe d'ensemencement sombre, pour un double motif; - fixation de l'époque de la coupe secondaire et comment elle doit s'opérer; - époque de la coupe définitive; - elle a lieu sans réserves, 247. = C. A. Conformément aux règles générales, 248.

Art. III. Exploitation d'une futcie mélangée de chènes et de hêtres ( 219. = E. On doit, en général, adopter la révolution convenable au chêne, 2 ว̃0. $=$ G. R. Réserve nombreuse en chène pour l'ensemencement; - prompte coupe secondaire nécessaire au chêne et souvent peu nuisible au hêtre; - coupe définitive en vue de la réussite du chène; empiétements du hètre; - moyens d'y remédier, $i b .=\mathrm{C}$. A. Comme dans le chène pur; - favoriser le chêne dans les éclaircies, 25 2.

Art. IV. Exploitation du chataignier en futaie (2, 491 à 493). E. Révolution de 90 à 120 ans, $253 .=$ C. R. Comme pour le chène; - labour à donner parfois dans les coupes sombres, $i b .=$ C. A Soins particuliers dans les nettoiements; - éclairvies comme pour le chêne, mais plus fréquentes, 254.

Art. V. Exploitation de l'orme en futaie (8\% 494 à 496). E. Révolution de 100 à 120 an $\div$, $255 .=$ C. R. Coupe d'ensemencement; - espacement de 4 à 6 mètres entre les branches des réserves; cas où les vents seraient à craindre; - gazonnement; - coupe secondarre : deux ans après l'ensemencement; coupe définitive : deux ans après la coupe secondaire; - cas où cette dernière peut être négligée, $i b .=\mathrm{C}$. A. Conformes aux règles générales; - éclaircies fréquentes, 256.

Ant. VI. Exploitation en futaie du frène et des grands érables $(\%, 497)$. Futaie avantageuse; - révolution de 90 à 100 ans; même traitement que pour le hêtre; - coupe secondaire plus prompte, 257. 
Art. VIl. Exploitation du charme en futaie (898). Avantages de son mélange avec d'autres essences plus précieuses, 257.

Ars. VIII. Exploitation du bouleau en futaie (8 499). Il peut se traiter avantageuscment en futaie; révolution de 50 à 60 ans; - coupes de régénération a à blanc étoc; - labour dn terram; - éclaircies très rapprochées, 258.

Art. 1X. Eryploitation du robinier en futaie (\& 500). Motifs pour le cultiver ainsi; - exploitabilité à 60 ou 70 ans; - motifs pour lui appliquer le traitement de l'orme, 259.

ArT. X. Exploitation dune futare de sapin (路501 à 503). E. Entre 100 et 140 ans; - circonstances qui la déterminent, 260. = C. P. Leur direction; - analogie avec le hêtre; - couvert de la coupe d'ensemencement plus épais; - coupe secondaire plus prompte et en plusieurs fois; - coupe définitive; - réserves, 261 . = C. A. Massif serré dans les premières années; - cas où devra être appliquée l'éclaircie moyenne; - faible. 262.

Art. XI. Exploitation d'une futaie mélangée de sapun et de

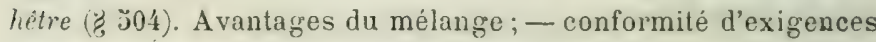
et de traitement; - diversité de produits, 263.

ArT. XII. Exploitation d'une futaie d'épicéa (28\% 505 à 512). E. Cas d'appliquer les différentes révolutions de 90 à 140 ans, $264 .=$ C. R. Danger des vents; - quatre modes particuliers d'exploitation employés pour en préserver les coupes, 265. = Premier mode: handes alternées; - ce mode présente quatre inconvénients principaux, $i b$. = Second mode : bouquets; mêmes inconvénients que le précéaent, et de plus, le désordre, 267. = Troisième mode (Hartiğ) : coupes à blanc étoc longues et étroiles, sur différents points à la fois, $i b$. = Quatrième mode : application de la méthode naturelle; - coupe sombre, serrée; - supprimer quelquefois la coupe secondaire; - combiner ce mode avec le troisième, 268. = On parait renoncer en Allemagne à la régénération naturelle des futaies d'épicéa rqui, cependant, a été tentée avec succès en France, 270. $=$ C. A. Comme pour le sapin, 272. 
ART. XIII. Exploitation d'une futaie mélangée de sapin et ípicéa (85 513). Avantages de ce mélange; - empiétement de l'épicéa; - causes de cet inconvénient; - précaution pour le prévenir, 272.

Ant. XIV. Exploitation d'une futaie de pin sylvestre (联, 514 à 317). E. Cas d'appliquer la révolution de 80 à 90 ans; - en France, et dans les forèts de lEtat, il faut, suivant les sols, exploiter entre 120 et 160 ans, $273 .=\mathrm{C}$. R. Coupe d'ensemencement : 5 à 6 mètres entre les branches des réserves; - objection relative aux dégâts des vents et à l'envahissement des plantes nuisibles; - précautions à prendre; - coupe secondaire inutile; - opportunité de la coupe définitive prompte, 275. $=$ Molifs qui font préférer la voie artificielle, 277 . $=$ C. A. Motifs qui déterminent à entreprendre la première éclaircie quand les jeunes bois sont encore à l'état de fourré, et à la pratiquer forte; - éclaircies fréquentes, 279.

Ant. XV. Exploitation d'une fulaie de pin à crochets ('), 518 à 521). Le mode de traitement est encore incertain, l'essence n'étant bien étudiée que depuis peu d'années, 280. = E. Elle semble devoir varier', suivant l'altitude, entre 160 et 200 ans, 281 . $=$ C. R. Coupe d'ensemencement assez espacée, par bouquets: - coupe secondaire en une fois, suivie de près par la coupe définitive; - hâter le repeuplement; - nécessité de conserver un rideau épais aux parties supérieures et inférieures des pentes rapides; - ne pas faire les coupes d'un seul tenant, $i b .=$ C. A. Eclaircies très faibles jusqu'à la dernière; - ne pas faire la première avant l'âge de 40 ans, 283.

ART. XVI. Exploitation d'une futaie de pin maritime ( 522 à 526). E. On ne peut la fixer que par analogie; - dans le climat du midi, on aura intérèt à la retarder jusqu'à 120 ans, 281. = C. R. Motifs pour assimiler, sous ce rapport, le pin maritime au pin sylvestre, $285 .=$ C. A. Devancer encore l'âge de la première éclaircie, et abréger la périodicité des suivantes, $2^{*} 6$. $=$ Mode de traitement et régénération des futaies, de pin maritime exploitées en vue du gemmage, $i b$. = Coupes blanc étoc avec semis artificiels, 287. 
Art. XVII. Exploitation d'une futaic de pin laricion (3, 527). Exploitabilité de 100 à 130 ans et au delà, en vue de constructions civiles et navales; - traitement comme le sylvestre, 288.

Art. XVIII. Exploitation d'une futaie de pin noir (\& 528). Tous les auleur's s'accordent pour preserire le même traitement que pour le pin sylvestre, 289.

Art. XIX. Exploitation d' une futaie de pin d'Alep (马, 529). Exploitabilité vers 80 ans; traitement présumé analogue à celui de l'épicéa, 290.

Art. XX. Erploitation du pin pinier, du pin cembro et du pin du Lord Weymouth (8\% 330 à 532). Les deux premiers n'existent qu'isolés; - les essais faits sur le troisième ne permettent pas encore d'en déduire des règles d'exploitation, 290.

Ant. XXI. Exploitation du mélèze (马े 533). Comme le pin à crochels, 292.

Art. XXII. Exploitation du cèdre du Liban (马 534$)$. Les essais d'acclimatation sont à conseiller, 293.

\section{CHAPITRF TROISIÈME.}

EXPLOITATION DES FUTAIES IRRÉGULIĖRES, QUI ONT ÉTÉ SOUMISES AU MODE DU JARDINAGE.

Авт. Iе Des forêts jardinées en général (8̨ 535 et 536). Mode du jardinage; - principe qu'on y suit; - élat que présente une forêt jardinée; - inconvénients de la confusion d'âges ; on rencontre parfois de belles parties, 294. = Difficultés de la surveillance; - infériorité des produits en quantité et en qualilé ; - la croissance, accélérée, dans la futaie régulière, est ralentie par le jardinage; - perte des produits accessoires; arbres branchus et viciés, 296.

Ant. II Des coupes de transformation (ZZ 537 et 538). But de ces coupes; - trois principaux états de peuplement à distinguer dans les forêts jardinées; - premier cas : coupe analo- 
gue à la coupe définitive; - second cas : coupe analogue à la coupe secondaire; - troisieme cas : coupe analogue à la coupe d'ensemencement; - comment le sous-bois pourra servir ; son extraction; - recépage daus les bois feuillus; - obselvations particulières au hêtre, 297. = Les divers états des folêts jardinées ne sont que des nuances de ces trois principaux; ce qui rend difficiles les coupes de transformation; - chereher toujours à égaliser la croissance, 302.

Art. III. Marche des coupes de transformation (8? 539 à 5 îi). Nécessité de continuer partiellement le jardinage; - deux exploitations dislincles, $30 \%=$ Révolution transitoire; - d'où en dépendra le terme; - péricdes et affectations; - règles à observer; - difficultés d'égaliser les produits; multiplier les séries et donner aux affectations des contenances égales; hypothèse, $30 . .3 .=$ Exploitation de la $1^{\text {re }}$ période : coupes de transformation; - ébranchements ; - jardinages dans les affectations des deux autres périodes; - comment ils s'opéreront pour chacune; - maintien des produits; $307 .=$ Détermination de la possibilité; la fonder sur le volume; - nécessité de vérifieations, 308. = Quotité de produits du jardinage ; - comment il faudrait opérer pour en déterminer la possibilité par volume ; - inconvénients et incertitude de ce mode; - se reporler à l'ancien jardinage; - avantages qu'on y trouvera, $i b .=\mathrm{La}$ transformation est la chose principale ; - la possibillté n'est ici que secondaire, $311 .=$ Exploitation des périodes, 2 et $3 ;-$ augmentation et compensalion de possibilité, 312. = Cas où le traitement a élé déjà amélioré par des exploitations intelligentes, $i$. Impossibilité de prévoir tous les cas de futaie irrigulière, 315.

Ant. IV. Des cas où le jardinage doit ctre conservé (ZZZ 348 et 549), Nécessité de maintenir le jardinage : sur les sommels élevés où le repeuplement nalurel est difficile,'afin de conserver. un abri aux parties inférieures ; - sur les versants abruptes ; dans certains bois de particuliers ou des communes, 315 . = Règles à suivre pour établir un jardinage rationnel ; - division en prarcelles ; - orrdre el périodicité du jardinage; - possibilité 
fondée sur le volume; - principes qui devront présider à la pratique du jardinage, 317.

\section{CHAPITRE QUATRIEME.}

EXPLOITATION DES FUTIIES IRRÉGULIËES QUI ONT ÉTÉ SOUMISES AU MODE DIT A TIRE ET AIRE.

Ант. Ier De l'état de ces forets en général ( 82,550 à 552). En quoi consiste le morle à tire et aire consacré par l'ordonnance de 1669 ; résultats de ce mode, 321 . = Comment il se présentait parfois un repeuplement de bonnes essences; - ce cas, le plus heureux, était le plus rare, 323. = Etat de ces futaies ; - but du traitement actuel de ces forêts, 324 .

ARt. II Des coupes de transformation (绻, 553 à 557). Quatre catégories à distinguer dans ces forèts: 10 vieilles futaies; 20 perchis; $3^{\circ}$ gaulis et fourrés; $4^{\circ}$ parties ruinées, 324. = Appliquer aux vieilles futaies le traitement des futaies régu. lières, 3 g̈ $=$ Perchis; - dans quel état on les trouve; - réserves; - cas où elles sont en petit nombre; - cas où elles sont nombreuses et rassemblées; - élagage à pratiquer parfois ; cas où il vaut mieux laisser dépérir les réserves; - bois blancs; - cas ou il faut les couper sans exception; - cas d'en réserver ; - éclaircies, ib. = Gaulis et fourrés; extraction des bois blanes et des réserves; - répéter les nettoiements, 328. = Parties ruinées; $=$ cas où les bois blanes devront être essouchés; - cas où ils devront être enlevés par nettoiement; - abatage de vieux arbres, $i b$.

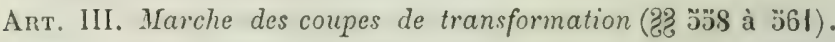
Séries; -- conserver les anciennes; - composition des séries à former, 329. = La marche des coupes pourrait se régler comme dans la futaie régulière; - exploitations qui auraient

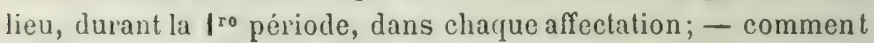
se règlerait le repeuplement des parties ruinées; - cette mar- 
che comprometl'ait le rapport soutenu; - ses inconvénients, 330. - Moyen d'y obvier; - mettre en réserve les parties les plus âgées; - révolution transitoire de courte durée; - coupes de transformation, dans les perchis et dans les gaulis et fourrés, par contenances égales; - les produits de ces coupes seront assez abondants; - ne pas fixer d'avance la révolution définitive, 331. = Ces généralités devront se modifier dans des cas nombreux, 334 .

\section{LIVRE QUATRIEME}

\section{De l'exploitation des taillis}

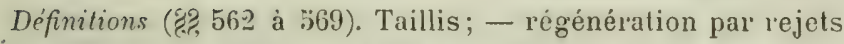
et par drageons, 335. - - Tailiis sous futaie ou composés; taillis simples, 336 . = Baliveaux; - baliveaux de l'àge; baliveaux modernes; - haliveaux anciens; - vieilles écorces; - sens précis de ces divers termes, $i b=$ Couvert et ombrage; - distinction entre res deux termes; - ils s'entendent aussi de la surface couverte ou ombragée, 337. = Ravaler, 338. = Couper en pivot; - en talus, $i b .=$ Cépe ou trochée, $i b .=$ Ramiers, $\imath b$.

\section{CHAPITRE PREMIER.}

MÉTHODE DU TAILLIS SIMPLE.

Art. Ier. Généralités (Z⿱⺈ 570 et 571). Peu ou point de réserves; - exception dans les forêts de l'Etat, 339. = Conditions de l'existence et de la durée des taillis, 340.

ART. II. Essences propres au taillis (8, 572). Les bois feuillus 
peuvent seuls être traités en taillis; - le liètre est impropre à ce mode de traitement; - quelques arbrisseaux méritent atlention; - favoriser les bonnes essences, 341.

Art. III. Fixation de l'exploitabilité dans les taillis ( 82,573 et 574). Inconvénients des révolutions trop longues ou trop courtes; - limites générales; - l'exploitabilité absolue, renfermée entre ces limiles, doit être appliquée, $342 .=$ Données sur l'exploitabilité des taillis; - cas d'appliquer, selon les sols et les essences, la révolution de 30 à 40 ans; - celle de 20 à 25 ans; - celle de 15 à 20 ans; - celle de 5 à 10 ans, 314 .

Art. IV. Fixation de la possibilité dans les taillis (8 575). I a baser sur la conlenance; - les contenances pourraient être proportionnelles; - généralement on les prend égales, 343 .

Art. V. Saison la plus convenable pour la coupe des taillis (歖 376 et 577). Inconvénients de la coupe en automne et en hiver; - en temps de sève, 316 . = Epoques à préférer selon les localités, 347 .

Art. VI. Mode d'abatage des taillis (8, 578). Instruments bien tranchants; - couper rez terre; - forme à donner aux souches; - cas de couper les jeunes bois; - procédé à appliquer aux essences drageonnantes, 348.

Art. VII. Fasonnage et vidange dans les taillis (28: 379 et 580). Les opérer avant l'apparition des rejets; - dans quels sols le retar'd est surtout à craindre, 349. = Cas où la vidange peut s'opérer avant le temps de sève; - places de dépôt; - avantages de créer de bonnes routes forestières, 330.

Art. VIII. Examen des dispositions que renferme le cahier des charges des adjudications de coupes ( 83581 et 582). Texte : $-\Lambda$. Délais d'abatage; - B. Mode d'abatage; - C. Délais de façonnage; - D. Délais de ridange; - E. Délais nécessités par les circonstances locales, 33̈1. = Ces exceptions doivent être soumises par les agents locaux à la sanction de l'Administration; - examen de ces dispositions; - $a$. Délai d'abatage trop r'eculé; - il faudrait fixer aussi je commencement de l'abatage; $-b$. Exceptions à observer pour le mode d'abatage; - $c$. Inconvévénienis d'un trop long délai pour le façonnage; - mesures 
projucs a l'abreger; $-d$. Avantages et inconvénients des délais de vidange trop reculés, 3 ว๊3.

\section{CHAPITRE DEUXIÈME.}

MÉTHODE DU TAILLIS COMPOSÉ OU SOUS FUTAIE.

Art. Ier. Généralités ( 8,383 et 384). Avantages qu'on veut réaliser par ce mode, $358 .=\mathrm{Il}$ faut considérer ici non seulement l'exploitabilité de chacun des éléments, mais encore le choix, le nombre et la distribution des baliveaux; - chercher à les concilier le micux possible, $i b$.

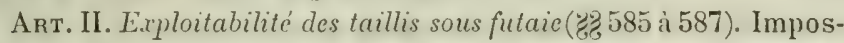
sibilité de soumettre le sous-bois à de courtes révolutions, 360 . $=$ Plusieurs auteurs allemands sont d'un avis contraire; - réfutation, 361. = L'exploitahilité de la réserve doit se déterminer d'après la qualité du propriétairo; - elle devient le régulateur du balivage, 362 .

Art. III. Choix des baliveaux (7, 588 à 390 ). Sujets à préférer sous le rapport de leur origine; - de leur végétation, $363 ;=$ de leur essence, $364 ;==$ de leur forme, 36 .

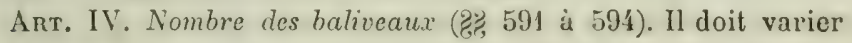
selon les essences, les sols et les expositions; - l'essence influe en raison du plus ou moins de couvert qu'elie procure, ou qu'elle supporte; - un sol fertile permet des réserves nombreuses et avancées en âge; - un sol médiocre, joint à une exposition chaude, exige que le taillis soit ombragé, mais peu couvert, 360. = Nécessité de quelques données à l'égard du balivage; - marche suivie dans les expériences sur ce sujet, 367. = Les arbres ne doivent couvrir que du tiers au sixième du terrain; - avantages du balivage normal de Colta, et comment on doit l'appliquer, 369. = Balivage normal; - il assure entre le taillis et la futaie un rapport convenable, 370 .

Arт. V. Distribution des bativeaux (ร? 395 et 596). Cas d'une 
surface plane; - d'une surface accidentée; - inconvénients de réserver plusieurs anciens sur le même point; - places où ils conviennent le mieux, 372. = Difficultés pour arriver à un balivage convenable, 373 .

\section{CHAPITRE TROISIÈME.}

TRAVAUX NÉCESSAIRES POUR ENTRETENIR LES TALLIS EN BON ETAT.

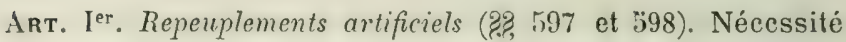
de ces opérations après l'exploitation; disparition du chêne dans les taillis, 375. = Expédient proposé par Hartig; - ses inconvénients; - préférer le semis ou la plantation avec recépage; - extraire les vieilles souches, les bois blanes et les morts-bois; - essences à introduire dans les taillis. 376 .

Art. II. Nettoiements et éclaircies (2\%, 599 et 600). Avantages des netfoiements; - époques à préférer, 379. $=\Lambda$ ge auquel il convient d'éclaircir; - sur quoi porte l'éclaircie: — une ou deux éclaircies selon la durée de la révolution; - un particulier gagnera à les multiplier, 380 .

Art. III. Elagage des baliveaux (\&̊ 601 à 603). Formation de branches gourmandes sur la tige des arbres qu'on isole; - inconvénients qui en résultent; - moment d'entreprendre l'élagage; - comment il devra se répéter ; - manière d'y procéder; - saison à choisir; - l'élagage, mème onéreux, doit être exécuté, $381=$ Suppression des branches sèches et des branches latérales nuisibles; - considérations qui doivent guider dans cet enlèvement, 383. = Importance de l'èlagage, 38.'.

\section{CHAPITRE QUATRIÊME.}

DU BALIVAGE DES TAILLIS SELON L'ORDONNANCE RÉGLEMENTAIRE DU CODE FORESTIER.

Art. Ier Texte de l'ordonnance (\& 604), Art. 70 śtablissant le nombre des baliveaux à conserver, 385 . 
Arr. II Examen de sos dispositions et conséquences qui en découlent (28 fior et 606). But de l'article; - vues utiles dans lesquelles il a été rédigé; - inconvénients rle l'état de choses qu'il consacre; - ils naissent de deux objets principaux; - le traitement prescrit doit amener la destruction du taillis sous futaie, 386. = Démonstration; hypothèse ; - calcul du convert qui s'ensuit; - deux expédients suffiront pour remédier à l'état ainsi produit; - inconvénients de l'un et de l'autre 388.

Art. III. Conchusion (\& 607). L'art. 70 ne doit pas ètre abrogé tout de suite; - il doit etpeut l'ètre par des propositions d'aménagement; importance d'une discussion du balivage dans les procès-verbaux d'aménagement, 391.

\section{GHAPITRE GINQUIÈME}

APplication des deUx méthodes d'exploitation en taIllis.

Art. I ${ }^{\mathrm{er}}$. Exploitation du chêne en taillis (ę) 608 et 609). Propriétés de la souche; de l'écorce; - utilité générale des ré serves; - nécessité des repeuplements artificiels, 393. = Ecorcement; - époque et mode; - coupure circulaire au pieddes arbres; - soins à prendre desécorces; - perte en vovolume; - elle est compensée et au delà; - perte de la première sève; - résultats qu'elle peut avoir, 394.

Ant. II. Exploitation du hêtre en taillis (\$, 610 à 613). Danger de l'exploiter selon le mode ordinaire; -ineonvénients de conper dans le jeune bois, 396. - Mode particulier proposé par Hartig; comment il s'applique aux taillis qui ont déjà été exploités, 398. = Le hêtre se perd dans le taillis ; - il est le plus souvent remplacé par les bois blanes; complication et inconvénients des mojens proposés par Hartig; - préférer le mode de la futaie; - en cas d'impossibilité, le taillis composé, 399. = Furetage adopté dans l'ancien Morvan; - en quoi il consiste; - on revient plusieurs fois sur les coupes dans une 
révolution; - nécessité des repeuplemonts artificiels; - dégàts causés par l'abatage el le façonnage; - perto re produits qui en résulte; - il n'est pas opportun de chercher à étendre co mode, 401.

Art. III. Exploitation du chataignier en taillis (\%, 61.4). Avantages de celte exploitation; - motifs pour ne réserver de baliveaux que sur les lisières; - culture du terrain après chaque exploitation, 402.

Ant. IV. Exploitation de l'aune en taillis (\& 615). Ce régime convient à l'aune; - ne pas compter, pour la régénération, sur le semis naturel; - ne réserver des baliveaux qu'en vue de leur's usages; - coupe d'hiver nécessaire dans les marais profonds, 403.

ART. V. Exploitation du robinier faux acacia en taillis (\& 616). Avantages de cette exploitation; - circonstance qui la rend difficile; - point de réserves, 405.

Ant. VI. Exploitation des taillis mélangés (\& 617). Les taillis sont presque toujours mélangés ; propriétés favorables au taillis chez différentes essences; - essences dont le voisinage est nuisible; - comment les essences doivent ètre groupées, 406.

Art. VII. Exploitation des taillis d'arbrisseaux (\& 618). Ne pas les mêler avec les arbres; - arbrisseaux quı méritent l'attention; - révolution de 5 à 10 ans; - taillis simple ; - menus-tailtis, 408.

\section{GHAPITRE SIXIÈME.}

\section{DU SARTAGE.}

(\$\$ 619 à 623). Ln quoi consiste le sartage; - localités où il est pratiqué; - courte révolution, $410 .=$ Détails de l'opération du sartage; - instruments, précautions et moyens employés - effets et avantages du sartage dit à feu courant, 4l1. $=$ Sartage à feu couverl; - en quoi il consiste; - il est moins 
avantageux que le premier; - il est nuisible dans les pentes rapides; - il permet un balivage, 415 . = Repeuplements après le sarlage, 416. = Localités propres au sartage, 417.

\section{CHAPITRE SEPTIEMME.}

DE I’ÉTÊTEMENT ET DE L'ÉMONDAfE.

ART. Ior. Généralités ( $\$ \S 624$ et 625 ). En quoi consistent ces opérations; - rejets produits et leur exploitation, 418. = L'étêtement est inférieur à l'émondage pour l'utilité de la tigre, et supérieur pour celle des rejets; - hauteur's différentes auxquelles il s'opère suivant les situations, $i b$.

Ant. II Esscnces propres à l'étêtement ct à l'émondage (\$ 626). Toutes ne sont pas avantageuses, 420 .

Ant. III. Expluitabilité de ces bois (\$627). Révolution de trois, six et dix ans, 420 .

Art. IV. Saison la plus convenable à l'ététement et à l'émondage (\$628). Mars et aviril; - coupe à la fin de l'été pour l'emploi du feuillage, 421.

ART. V. Mode d'abatage ( $(629)$. Ne plus couper rez tronc quand les sujets vieillissent; - tranche nette et oblique à l'horizon, 421.

Art. VI. Arantages des tétards et des arbres émondés (\$ 630). Soutien des rives; - produits; ces plantations n'enlèvent rien. à la culture, 422. 


\title{
LIVRE GINQUIÈME
}

\section{Des exploitations de conversion}

\author{
GHAPITRE PREMIER.
}

CONSIDÉRATIONS GÉNÉRALES.

( $\$ 631)$. But de ces exploitations; - on peut concevoir trois conversions, ou la conversion de trois sortes de forêts; question préalable à examiner, 424.

\section{CHAPITRE DEUXIÈME.}

EXAMEN COMPARÉ DES TROIS PRINCIPALES MÉTHODES D'EXPLOITATION.

Art. I ${ }^{\mathrm{er}}$. Énoncé de la question et données employées pour sa solution ( $\$ 632$ ). Comment la question peut se formuler; - quatre objets à considérer pour la résoudre, 426.

Ant. II. De la quantité des produits en matière (\&\$ 633 et 634). Les taillis produisent moins que les futaies; - expériences de Hartig; - rapport qui en résulte et ses modifications, 427. $=$ Position du taillis composé sous le rapport de la production en volume, 428.

ART. III. De la qualité des produits matériels (¿\$ 635 à 637). Elle est relative à l'emploi; - l'emploi est de deux genres : bois de feu et bois d'œurre, 429. = Bois de feu; - qualités qu'il doit réunir; - résultats des expériences sur l'âge où les bois ont acquis ces qualités; - eas où les fulaies sont supérieures ou égales aux taillis, sous ce rapport, $i b .=$ Bois d'œu- 
vre; - comment la comparaison doit s'établir; - qualités que doit présenter le bois d'œuvre; - opinions diverses des savants sur la question; - utililé réelle des baliveaux; - accidents et défauts; - supériorité des bois des futaies éclaircies, 431 .

Art. IV. Du revenu ( $\$$ g 638 à 649. A ne considérel que les produits, les futaies donneraient le plus grand revenu, $433 .=$ Cette solution ne peut covvenir qu'à un propriétaire impérissable comme l'Etal; - un particulier doit faire acception du capital superficiel; - formuler la question sous ce point de vue; - marche à suivre pour la résoudre, 43\% = Hypothèse la plus favorable possible à l'intérêt du propriétaire: - composition des produits annuels (en contenance); - choix des plus fortes données pour les produits d'éclaircies, 436 . = Composition du capital superficiel; - tableau faisant connaitre le volume de l'hectare moyen dans chaque affectation, et celui de toute la superficie, 437. = Produit annuel en volume de la forêt; - le capital superficiel est égal à quarante-deux fois le revenu, et représente un placement à environ deux et un tiers pour cent, sans tenir compte du fonds; - avantages que présente la destruction de la futaie, $4 \mathbf{4} 0 .=$ Opinion de M. de Dombasle, 411. = Vérificalion de celle assertion; - hypothèse d'une augmentation exagérée dans les prix des bois; - tableau des données et des résultals, 4 \% . = Valeur des zoupes de régénération déduite de ce tableau; - fixation de la valeur des éclaircies; -appliquer à leur's produits sé aarésl'hypothèsed'augmentation des prix; - tableau de cette échelle de production; - tolal pour le revenu de toute la forêt, $\mathbf{4 4 4}$. = Le revenu, dans celte hypothèse, n'est encore que de trois pour cent, le capital foncier négligé ; - les prix supposés ne sauraient garantir la conservation de la forèl; - calcul du bénéfice qui résulterait de sa destruction, 146. = L'augmentation de valeur du bois diminuera les chances de conservation par des particuliers, au lieu de les accroître, 447. = Examen des taillis simples et composés; les taillis simples n'exigent qu'un capital superficiel peu élevé par rapport à leur revenu; - la position du taillis composé 
est intermédiaire, $i b .=$ Conclusion de la discussion $;-$ rang qu'on doit assigner à chaque méthode d'exploitation, pour l'Etat, - pour les particuliers, 449.

Art. V. De l'influence des différentes méthodes d'erploitation sur la fertzlité du sol ( $\$ \% 650$ à 651). Les bois influent sur le sol le deux manières; - comment sont produits ces effets, 450. = Hors le cas de révolutions très longues, la futaie améliore constamment le sol, $i b .=$ Localités où le taillis ne nuit pas au sol; - localités où il le détériore, 451. = Le taillis composé participe sous ce rapport des deux autres méthodes, 452.= La méthode de la futaie ne convient pas seulement aux bons sols; - application des essences aux terrains; - cas où le taillis doit être rejeté, $i b$.

Ant. VI. Conclusion (8 655 à 662). Déductions à tirer des démonstrations précédentes, 4ö́. = Forêts de l'Etat. La futaie convient à l'intérêt général; - l'Etat doit se proposer la conversion des taillis composés, $i b .=$ Obstacles à une mesure générale; $-n$ e point froisser les intérêts actuels, $455 .=$ But 'qu'il faut atteindre dans une conversion en futaie; - de quel point de vue il faut considérer les forèts qu'on y desline; envisager la possibılité pour un bassin de consommation; cas d'opérer, ou seulement de préparer la conversion, 4วั6. = Importance secondaire des autres genres de conversion, 457. = Forêts communales. On doit y conserver les futaies, mais on ne peut espérer d'en créer immédiatement,458. = Il faut y perfectionner le taillis sous futaie, 459. = Forêts partıculières. préférer généralement le taillis simple ou composé, selon les circonstances, et, pour les bois résineux, les révolutions les plus courtes, $i b$.

\section{CHAPITRE TROISIÈME.}

\section{CONVERSION DES FUTAIES.}

ART. Ier. Conversion d'une futare en taillis simple (3,663. Deux états de peuplement à distinguer; - exemple; - cas d'une futaie irrégulièrement exploitée, 461. 
Aнт. II. Conversion d'une futare en taillis composé ( $\$ \S 664$ à 666). Fixer la révolution et les opérations à entreprendre; régler le balivage, $462 .=$ Composition de la réserve dans la partie à exploiter tout de suite en taillis, 463. = Composition de la réserve dans la partic à régénérer tout de suite par la semence, $i b$.

\section{CHAPITRE QUATRIEME.}

CONVERSION DES TAILLIS SIMPLES

Art. I ${ }^{\mathrm{er}}$. Conversion d'un taillis simple en taillis composé ( $\$ 667$ ). Opération identique à celle qu'on a décrite au $\$ 665$, 465.

ART II. Conversion d'un taillis simple en futaie (\$\$ 668 à 673 . Adopter pour la révulution transitoire le terme de l'ancienne révolution; - abréger la révolution convenable à la futaie, 46\%. = Méthode destinée à prévenir l'abaissement trop sensible de l'ancienne production, 467 . = Partage de la révolution en périodes, et de la série en affectations; - nature des exploitations durant chaque période, el état du peuplement à son expiration, $i b .=$ On parvient, par l'application de celte méthode, à une gradation convenable de l'ige des bois dans la série, et à y introduire artificiellement des essences d'élite, 469. = Traitement analogue et plus facile, si l'on avait à convertir plusieurs séries contiguës, 470. = Taillis exploités à 20 ou 25 ans, $i b$.

\section{GHAPITRE GINQUIÈME.}

CONVERSION EN FUTAIE DES TALLLIS COAPOSÉS.

Ant. Ier. Généralités (\$ 67\%). Etat que présentent les taillis composés, soumis au régime forestier; - bigarrure qu'on y re- 
marque; - abondance d'anciennes réserves dans les taillis domaniaux; - difficulté de décrire tous les peuplements divers; - division en deux grandes catégories; - taillis sous futaie réguliers; - leur état; - taillis irréguliers; - élats principaux, a, b, c, d, 472 .

AnT. II. Conversion des taillis sous futaie réguliers (\$8 675 à (79). Pralique des coupes préparatoires décrites plus haut; trailement des réserves; - conserver les modernes; - les anciens quant ils sont nécessaires; - et, par exception seulement, les vieilles écorces el les réserves branchues; - ilagage de ces dernières; - considérer l'époque où le massif dont elles font partie reviendra en four d'exploitation, 4730 . = Ifarche des conpes préparatoires. La révolution transiloire doit satisfaire a deux conditions prineipales; - elles sont, en général, remplies par une révolution transitoire égale à celle du taillis, 476 . = Possibilité par étendue; - division le la révolution transitoire en deux sous-révolutions; - avantages importants de cette mesure: - assielle el délinitation de toutes les coupes sur le terrain; - appréciation des chances de durée des anciens qu'on réserve, 4\%. = Elablissement d'exploitations de taillis, parallèlement aux coupes préparatoires; - avantages de cette mesure, 478 . $=$ Tàche à laisser à nos successeurs, 480 .

ART. III. Conversion des laillis sous futaie irréguliers (\$\$ 680 à 682). Quatre états divers à distinguer : a, b, c, d; - concilier deux condilions essentielies, $481 .=$ Détermination re la révolution transitoire el partage en périodes; - composition des affeclations correspondantes; - nature des exploitations dul'ant chaque période et dans chaque affectation; - état de ces affectations à l'expiration de chaque période, 482. = Cas où la conversion s'appliquerait à plusieurs séries de taillis composé, 485. 


\section{LIVRE SIXIÈME.}

\section{Des repeuplements artificiels.}

Définitions ( $\$ \$ 683$ à 689). Semis 487. = Plantation, $i b .=$ Bouture, $i b .-$ Marcotte, $i b$. = Semences échauffées, 488 . - Semis en plein; - semis partiel, $i b$. - Repiquer, $i b$.

\section{CHAPITRE PREMIER.}

CONSIDÉRATIONS GENÉRALES.

(\$ 690 et 691). L'emploi des moyens artificiels est souvent indispensable pour la régénération; - clairières el vides dans les forêts; - création de bois par les particuliers; - reboisement des monlagnes et des landes; - substitution d'essences, 189. - Le semis est principalement applicable en grand; cas divers où la plantation doit être préférée, 490 .

\section{CHAPITRE SECOND.}

DES SEMIS.

Art. Ier. Des connaissances qu'il faut posséder (\$ 692). Six no tions principales pour bien opérer les semis, 492.

Art. II. De la récolte el de la conservation des semences (\$\$ 693 et 69.1). P'éférer les semences movenanl d'arlores bien sains: et d'âge moyen: - temps à choisir' - manière de récolter lés semences lourles; - les semences légères, 493. $\Rightarrow$ Etaler les graines et les remuer souvent ; - moỵens d'empècher la ger- 
ninntion el lit pourriture; - le desséchement; -- l'échauffement, 494.

ART. III. Des moyens de reconnaître la qualité de la graine (\$§ 695 et 696 ). Nécessité de l'examen des semences pour déterminer la quantité à employer; - inconvénients d'un semis trop dru ou trop peu abondant; - épreuve des graines par la germination; - par l'ouverture des graines, 195. = Fraudes des marchands; - précautions contre l'humectation des semences; - mélange des graines de pin sylvestre et d'épicéa ; épreuve pour s'en assurer; - inconvénients qui résultent de ce mélange, 497.

ARt. IV. De la préparation du terrain (\$S 697 à 707). But de l'opération; - comment elle doit s'effectuer, selon les terrains, 499. = Trois modes principaux de labour, 501. = Labour en plein. Avantages du labour à la charrue; - terrain où il doit s'employer; - terrains où la houe doit ètre préférée; - le labour en plein ne peut se pratiquer dans les pentes, 502.= Labour par bandes alternées. En quoi il consiste; - comment il se pratique en plaine; - en montagne; - avantages de ce mode de labour, et inconvénients auquel il peut donner lieu, $503 .=$ Labour par places, trous ou pots. Comment il s'opère; cas où il est avantageux; - saison où doivent se pratiquer ces divers modes de travaux suivant les circonstances, $505 .=$ Ecobuage. En quoi il consiste; - ses deux principaux résultats; - quatre effets principaux des cendres, selon M. de Candolle; - quatre sortes de lerrains où l'écobuage est utile, 506. = L'écobuage praliqué comme le sarłage à feu courant; - avantages de ce mode; - terrains où il devra être pratiqué ou interdit, כ08. = Assainissements. D'où proviennent les eaux à éconduire, 509. = Débordements; - moyens d'y remédier ; - l'avaux qui rentrent dans les attributions du forestier, 510. = Marécages formés par des eaux sans écoulement; - moyens d'assainir ceux dont les eaux sourdent dans le marais mème; - cas où elles viennent d'une source plus élevée, 511. = . Ne pas repeupler tout de suite les terrains assainis, 512.

Art. V. De la saison la plus convenable aux semis (\$708). Epo 
que indiquée par la nature; - exception pour les semences lourdes; - différence entre un semis naturel et un semis artificiel; - préférer le printemps pour les essences résineuses; motifs, 512.

ART. VI. Des quantités de semence à employer (\$ 709). Circonstances qui modifient ces quautités; - proportions à observer dans les semis partiels, 514 .

ARt. VII. De la manière de seiner (\$§ 710 à 713). Deux objets principaux à observer, 516.= Procédé pour ensemencer uniformément, $i b$. = Comment les graines se recouvrent; - elles doivent s'enterrer plus ou moins selon leur nature et la nature du sol, $517 .=$ Du repiquement. En quoi il consiste; - ses avantages et dans quels lieux il s'emploie; - instruments; - plantoir; - description et manière d'employer deux plantoirs usités en Allemagne, et appliqués, l'un aux terres fortes, l'autre aux terres légères, 518 .

\section{CHAPITRE TROISIÈME.}

APPLICATION DES RÈGLES GÉNÉRALES AU SEMIS DES ESSENCES LES PLUS IMPORTANTES.

ART. Ior. Semis du chene ( $\$ 714$ à 716). R. G. (1). Ne pas ramasser les premiers glands tombés; - premier mode de conservation : tas coniques; - dellxième mode : silos ou fosses; - troisième mode : dans l'eau; - quatrième mode : dans le sable; - l'expérience est favorable au premier et quatrième mode; $321 .=E$. G. Caractères d'une bonne graine; - signes de l'altération; - épreuve par l'eau; - par le poids, 323. = E.

' Les initiales en grands caractères représentent les intitulés des paragraphes du texle, de la manière suivante :

\begin{tabular}{l|l} 
R. C. Récolte et conservation. & E. S. Exécution du semis. \\
E. G. Examen de la graine. &
\end{tabular} 
S. Marche à suivie dans un terrain en plaine, compacte et découvert; - cultures à y pratiquer; - comment la graine doit être recouverte; - mélange des céréales; - couper les chaumes à une certaine hauteur; - labour à préférer dans les sols légers ou en pente; - levée du plant ; - cas de partager le semis en deux saisons; - quantités de glands à employer par hectare, 524 .

Ant. II. Semis du hétre ( $\S 717$ à 720). R. C. Comme le gland; - procédé de conservation indiqué par Hartig, 526. = E. G. Comme pour le gland; - goût du fruit; - poids de la graine, $i b .=$ E. S. Abri indispensable; - semis ou plantation auxiliaire; - cas des pentes rapides; - repiquement de la semence ; - quantité de faînes à employer, 527. = Mode particulier indiqué par Cotta; - entourer de terre la tige des plants naissants; - plantation de hètres cotylédonaires à Compiègne, 529 .

ART. III. Semis du châtaignier (\$§ 721 à 723). R. C. Comme le gland; - préférer la stratification dans le sable, 531. = E. G. Comme le gland et la faine; - ne pas considérer la grrosseur du fruit, $i b .=E$. S. Bien nettoyer le sol; - bandes alternes et double culture; - repiquement dru; - pour créer un taillis, repiquement par trous ou pots; - l'abri est inutile; - comment le semis doit être recouvert; - levée du plant; quantité de semence à employer, 532.

Ant. IV Semis de l'orme (8.8 724 à 726). R. C. Cueillir à la main ; - ne pas laisser en tas; - semer immédiatement, 531.= E. G. Caractères que doit présenter la graine au toucher, à l'odorat et au goûl; - poids qu'elle doit avoir, $i b .=\mathrm{E}$. S Labour par bandes alternées ou par trous carrés; - premier abri à donner au plant par de l'orge ou de l'avoine; - cas d'une pente rapicle; recouvrir très légèrement ; - levée du plant ; - quantité de semenceà employer, $i b$.

Anт. V Semis du fréne (ร⿸尸, 727 à 729). Cueillir à la main ; précautions à prendre contre le desséchement; - procédé avantageux a suive dans ce but, $536 .=\mathrm{E}$. G. Couleur et consistance de l'intérieur de la graine: son poids, 537 . = E. S. Ban" 
des alternées ou trous carrés; abri par des céréales ; - précautions contre les plantes nuisibles;-comment doit se reco vrir la semence; - levée du plant; - quantité de semence à employer, $i b$

ARt. IVI Semis de l'érable (诌 730 à 732). R. C. Cueillir à la main ; conserver en tas ou dans le sable; - semer dès le premier printemps, 538 = E. G. Couleur et consistance de la graine; son poids, $i b$. E. S. Comme le frène; - semer au printemps; - quantité de semence à employer, 539.

Art. VII. Semis du bouleau (2.? 733 à 735). R. C. Cueillir à la main; cas où l'on peut couper les rameaux; semer tout de suile, 539. = E G. Amande farineuse, suc laiteux; - poids, $5 \% 0$ E. S. Labour partiel; - mode de semer; - temps à choisir et à prendre; levée du plant; - quantité de graine, ib.

Art. VIII. Semis du robinier faux acacia (8 736 à 738) Gueillir à la main ; - comment les graines se détachent et comment on les sépare des gousses; - les conserver en tas, 541. = E. G. Couleur et consistance de la graine, 542. = E. S. Semis partiel; - solbien nettoyé ; - recouvrir légèrement ; garnir le sol de mousses ou de feuilles morles; - semer au printemps; - levée du plant: - quantité nécessaire, $i b$.

Arr. IX. Semis du charme. (2ृ? 739 à 741). R. C. Récolter à la main; - avec la gaule et des toiles par un temps ealme; - conservation comme pour le frène; 5 \$3. = E. G. Amande blanche et fraiche; - noyau plein; - poids, ib. = E. S. Comme pour le frène: - enterrer davantage : - quantité à employer, ib.

Art. X. Semis de l'alisier, du sorbier et du micocoulier (o 742) Ils se sèment en pépinière ; - récolte et nianièrc de semer ; à quelle profondeur enterrer les différentes graines; - le rée tardive des plants ; - soins que réclame le micocoulier ; - re. piquement préparatoire et plantation définitive, 514 .

Art. XI. Semis de l'aune (8z 743 à 745). R. G. Cueillir de bonne heure, à la main; -- cas de couper les rameaux; comment s'obtient la graine; - la conserver en las ou dans l'eau, $54 \% .=$ F. G. Couleur, consistance et odeur de la grraine ; 
son poids, $546 .=$ E. S. Difficultés ; labour partiel - écobuer et gratter seulement la terre; - ne pas enterrer la graine; levée du plant ; - quantité nécessaire, $i b$.

Art. XII. Semus du sapin (z̨己 746 à 748). R. C. Cueillir à la main, élendre et remuer; - cribler; désailement; - entasser peu la graine, 548. = E. C. La juger à la vue, au toucher et à l'odoral; - poids, $i b .=$ E. S. Difficultés; - cas où ce semis peut s'employer; - repiquement de la semence; - comment elle doit être couverte; - quantité à employer ; - époque du semis et levée du plant, 549.

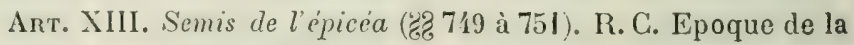
récolte; - cas de la hâter ou de la retarder; - extraction des graines; - description des appareils qui l'opèrent : $1^{\circ}$ par une chaleur artificielle; - 2. par la chaleur du soleil ; - désailement; - avantages et inconvénients, 551. = E. G. Epreuve par la vue, le toucher et l'odorat; - poids, 556.='E. S. Labour par bandes ou par pots; - ménager quelqu'abri; - enterrer à peine la graine ; levée du plant ; - quantité à employer, $i b$.

Ant. XIV. Semis du pun syleestre (28,752 à 754). R. C. Comme l'épicéa; - l'extraction de la graine s'opère par les mêmes procédés, mais plus particulièrement à ln chaleur artificielle; conservation, 557. = E. G. Comme pour l'épicéa; - infériorité des graines blanches; - poids, 558. = E. S. Labour comme pour l'épicéa; - creuser au delà de la terre de bruyère; - recouvrir comme pour l'épicéa; - levée du plant ; - mode de semer les cônes; - avartages et inconvénients ; quantité nécessaire. 559.

Ant. XV. Semis du pun maritime (马\$ 755 à 757). R. C. Comme l'épicéa et le pin sylvestre, 561 . = E. (r. Fraine plus grosse rue celle du sylvestre, colorée de gris et de brun mat, et, du reste, mêmes caractères, $i b .=E$. S. L es différents modes de culture, et, en particulier, le labour à la charrue, sont applicables à la préparation du sol; - enterrer davantage : - quantité de semence à employer; - extension et importance de la culture de celle essence en Sologne et dans les départements de l'Ouest: - mémoires et notices à consulter, ib. 
Art. XVI. Semes du pin laricio et du pin d'Alep (\$\$ 758 à 760 ). R. C. Comme pour le sylvestre et le maritime, 56\%. = E. G. Mèmes caractères que le sylvestre, - couleur et grosseur différentes, $565 .=\mathrm{E}$. S. Comme pour le sylvestre; - enterrer davantage; - quantité à employer, ib.

Art. XVII. Semis du pin pinier et du pin cembro (z̧ 761 à 763). R. C. Cueillir les cònes à la main; - étendre les graines ou les laisser dans les cônes, 566 . = E. G. Noyau plein; couleur, goût et odeur de l'amande; - poids, $i b .=\mathrm{E}$. S. Repiquement de la graine; - transplantation; - levée du plant, 567.

Art. XVIII. Semis du pin du lord Weymouth (\$\$ 764 à 766). R. C. Elendre et relourner les cònes; - employer promptement la graine, $567 .=$ F. G. Mêmes caractères que le sylvestre; - couleur, $568=\mathrm{E}$. S. Comme le pinier et le cembro; -- couverture de la graine, $i b$.

Art. XIX. Semıs du mélèze (8,\$ 767 à 769). R. C. Comme le sylvestre; - modérer la chaleur pour l'extraction des graines; - les employer promptement, 568. = F. (i. Mêmes caractères que le sylvestre; - couleur et poids, $569 .=\mathrm{E} . \mathrm{S}$. Mèmes règles que pour l'épicéa; - semer en pépinière, vu la cherté de la graine; - quanlité nécessaire; - levée du plant, ib.

Art. XX. Semis du cèdre du Llban (Z770). Cueillir les eônes en août ou septembre, selon la température; - extraire la graine au moment de l'emploỵer, en laissant séjourner les cônes dans l'eau; - sécher la graine aut soleil si on veut la conserver; - semer en pots; - transplantation préparatoire: - altendre la huitième année pour la transplantalion définilive, 570 .

ARt. XXI. Des semis mélangés ( $\$ \$ 771$ à 773). Trois cas principaux d'y avoir recours, 571. = Pour la création des forèls mélangées, se reporter aux livres précédents, $i b$. = Choix des essences destinées à en abriter d'autres; - extration de l'essence supplémertaire, 572. = Précautions et soins à prendre dans le cas du mélange de graines pour cause d'économie, et 
comment on fera dominer l'essence la plus précieuse, 573. = Cas de séparer les graines pour semer, et cas où on peut les mélanger d'abord, $i b$.

\title{
GHAPITRE QUATRIEME.
}

\author{
DES PLANTATIONS.
}

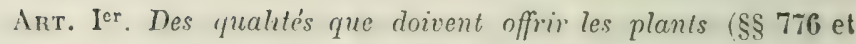
777). Etat que doivent présenter les racines; - conditions que doit réunir un plant de haute tige; - un plant de basse tige, $57 \%$. = Cas d'employer les plants de haute tige; - préférer généralement les basses tiges; - les meilleurs plants sont ceux qui ont été élevés en pépinière; - les basses tiges peuvent seules être prises en forêt; - de quels endroits il faut tirer les plants; - àge le plus eonvenable pour la reprise des hasses tiges, selon les essences; - la création d'une pépinière est une économie, 575 .

Ant. II. De la culture des plants en pépinière (28. 778 à 783). Choix du terrain; - inconvénients d'un terrain trop gras ou humide; d'un terrain maigre et de mauvaise qualité ; - choisir un terrain de fertilité moyenne; - emplacements à éviter ou à préférer, 577. = Rendre la terre parfaitement meuble: - division du terrain en plates-bandes; - cloture; - onvrir des sillons ou rigoles; - remplir ces rigoles de terreau préparé à l'avance; - nécessité đl'exhalasser les espaces intermédiaires, 578. = Semer au prirtemps dans les rigoles, très dru; - recouvrir très légèrement; - repiquement; - deux méthodes pour prévenir le développement exagéré du pivot, 580. = Soins à apporter dans la culture des plants de haute tige, 582. Arrusements; - comment on doit les pratiquer; - irrigations, 583. = Soins à prendre pour prévenir l'invasion et le développement de la mauvaise herbe, $i b$.

Ant. III. De la saison la plus convenable à la plantation 
(\% 7S1). Deux saisons : automne et printemps; - avanlages de la plantation en automne; - cas où il faut préférer le printemps, 584.

Art. IV. De l'espacement à donner aux plants (ZृZ 78ว à 798). Utilité de l'état de massif; - concilier ce principe avec l'économie; - de quelles circonstances dépend l'espacement, วั8วั. = Espacement ordinaire pour les basses tiges; - pour les haulestiges; - principes à cel égard, ¿s6. = Disposer régulièrement les plants; - quatre tracés différents; - comment on les exécule; - avantagyes de la plantation par files ou allées 587. = Tableau des quantités de plants à employer par heetare, suivant le deuxième et troisième tracé, 588.

ArT. V. De la confection des trous (88 789 à 79!). Les proportionner aux racines: - les modifier selon l'état du sol, 390 . = Cumment, on creusant les trous, on dispose les différentes couches de terre, 591. = Ouvir les trous plus ou moins long. temps d'avance, suivant le sol, $i b$.

Art. VI. De l'extraction iles plants (2,\%, 792 à 79:1). Ménager les racines et les tiges; - mole d'opérer : tranchées parallèles aux rigoles; - precautions, 591 . = Deux bêches demi circulairrs à employer pour la transplantation en mottes; - clles servent aussi à faire les trous, 592. = Extraction des hautes tiges; - manière d'y procéder, 593.

Art. VII. Du transport des plants (8, 793). Paniers ou brouetIos pour le transport des plauts en moltes; - garantir les tiges de tout froltement, et les racines du contact de l'air; - cmpaqueter les plants arrachés, 593.

Art. VIII. De la taille des plants (㺃 796 à 801). Lésion inévi table des racines par l'extraction, et interruption du rapport entre les racines et la tige; - double but de la taille des plants, 595. = Comment on ampule les racines lésées; - comment on les ravive; - en conserver autant que possible, ib. = Taille indispensable de la iigre; - principes à cat égard; - difficultés; - tâtonnement auquel on est réduit, 596. = I'rocédé du recépage; - avantages du plant recépé sur le plant taillé; - difficultés pour celui-ci de rétablir l'équilibre entre la lige et les 
racines; - cette difficulté n'existe pas pour le premier, 597 . = Objection contre le recépage quand on veut créer des fulaies, - elle est réfutée par la pratique ; - plantations dans la forêt de Compiègne; - comment il se forme un maître jet, et comment les rejets faibles ne tardent pas à périr el à disparaitre; - pour créer des fulaies, préférer les basses tiges, et les tiges plus fortes pour les taillis, 598. = La taille est nuisible aux résineux; - précautions à prendre en leur enlevant quelques branches; - préférer des basses tiges très jeunes; - s'abstenir de pratiquer le recépage des jeunes plants de hêtre, 600 .

$\Lambda \mathrm{rt}$. IX. De la muse cn terre des plants ou plantation proprement dite (8Z 802 à 805 ). - Profondeur à laquelle il faut planter : - enterrer davantage dans les sols légers et sees; moins dans les sols humides, 601. = Comment on place le plant; - répandre d'abord la bonne terre végétale; - soins à prendre pour en bien remplir toules les petites cavités; - placer ensuite les couches suivantes; - - comment on tasse la terre; - opération simplifiée pour les tiges faibles; - instruments convenables, 602 . = En quoi consiste la plantation par touffes; - éducation en pépinière; - extraction el division en touffes; - comment se font les trous; - principaux avantages de ce mode; - succès obtenus, 603. = Utilité d'un arrosement immédiat quand il est praticable; - emploi des pierres pour maintenir l'humidité, pour tasser le sol et comme abri ; - soins à prendre dans la plantation en mottes, 605 .

\section{CHAPITRE SIXIÈME.}

DES BOUTURES.

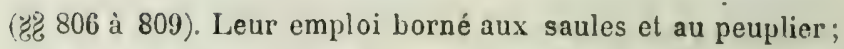
- dans quel terrain elles trouvent leur application; - deux espèces de boutures, $606 .=$ Ce que c'est qu'un plançon; comment il se plante dans les lieux aquatiques, et comment 
dans les sols plus fermes; - cas de donner des tuteurs aux plançons; - c'est avec des plançons qu'on forme les tètards; - le peuplier ne reprend pas de cette manière, 607. = En quoi consiste la bouture à bois de deux ans; - longueur à lui donner et comment s'opèrent les sections; comment on plante les boutures dans un solmeuble, et comment dans un sol ferme; - comment on traite les boutures en pépinière, 608. = Saison convenable pour faire les boutures, 609 .

\section{CHAPITRE SEPTIÈME.}

DES MARCOTTES.

(8̊ 810 à 812 ). Le marcotlage s'applique à tous les leuillus; - il est fort utile dans les taillis; - maniere d'y procéder quand les tiges sont faibles; - les perehes plus fortes sont préférables; - de quelle manière on opère le couchage de ces dernières; - à quel âge on les sèvre, 610. = Comment il faut traiter la souche dont on marcolte un certain nombre de sujets, 612. = Saison du marcottage, 613.

\section{CHAPITRE HUITIÈME.}

TRAVAUX D'ENTRETIEN A EXÉCUTER DANS LES REPEUPLEMENTS ARTIFICIELS.

(ใ⿱⺈ 813 à 819). Le succès d'un semis ou d'une plantation n'est assuré qu'après 50 ou 6 ans, et quand le jeune repeuplement va bientôt couvrir le sol; - jusque-là les travaux d'entretien sont nécessaires, 614 . = En quoi consistent ces travaux; - distinction à faire entre le sarclage et le binage, $615 .=$ Application de ces travaux dans les semis; $-1^{\circ}$ ceux de bois feuillus, 
exécutés en plein, sont moins exposés à l'envahissement de l'herbe; - utilité de quelques sarclages dans les premières années; - causes qui rendent celte invasion plus redoutable dans les semis partiels; - nécessité des sarclages et des binages fréquents, surtout pour certaines essences; - ces travaux peuvent préserver aussi les jeunes plants des premières gelées le l'automne; - $2^{\circ}$ les semis de résineux sont, à cause des sols où ils s'exéculent, moins exposés à l'envahissement des herbes; - mais d'autant plus à eelui des myrtiles, bruyères. ete.; - les sarelages seuls sont ì conseiller; - on peut fauciller les herbes et tondre les arbustes, $i b .=$ Dans les plantations, les sarclages seraient insuffisants, et il faut recourir aux binages qui facilitent l'arcès de l'air et de l'humidité aux racines, 618 . = Epoque la plus favorable pour les sarelages et les binages; - les renouveler parfois en septembre, 619.= Les travaux d'entretien sont trop rarement pratiqués, malgré l'avanlage considérable qu'ils procurent; - motifs, ib $=$ Epoque à laquelle il convient de procéder aux regarnis dans les plantalions; plants auxquels il faut accorder la préférence pour cet objet; - les regarnis se font dans les semis par voie de plantation; - on altend, pour y procéder, que le semis ait atteint quelque développement, 620 .

Table de l'Appendice, page 62\%.

Vocabulaire des termes techniques, page 653. 


\section{TABLE DE L'APPENDICE}

I3 chêne chevelu..................... (i:

Ie chène occidental.................... 1;:?

(as oủ les coupes d'ensemeneement sont réglées par contenance............................ liz.

. Cgénération naturelle du pin sylvestre..........

ièglement des coupes jardinaloires dans les forêts jardinées en conversion.................

lèglement de la possibilité dans les forèts jardinées. 19.1:2 \{évolution préparatoire dans les futaies à tire et aire en conversion..........................

'ravaux nécessaires pour maintenir le chêne dans les

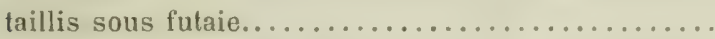
'élagage des arbres de réserve dans les taillis sous fulaie............................... nil

Ivanlages résultant du trailement en futaie........ thoix du mode de conversion des taillis sous futaic en

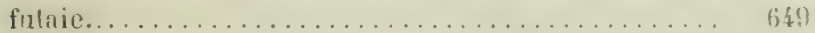





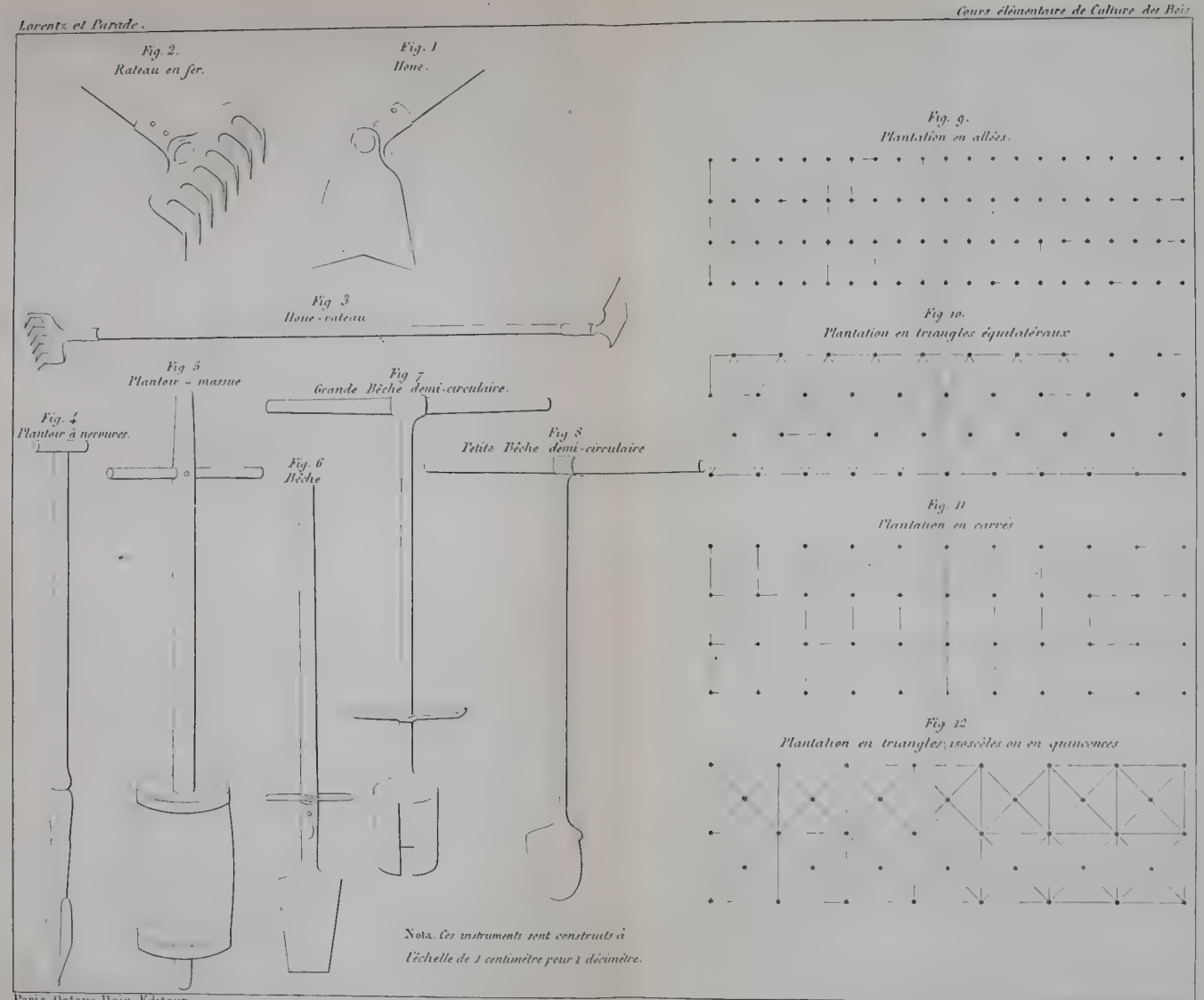








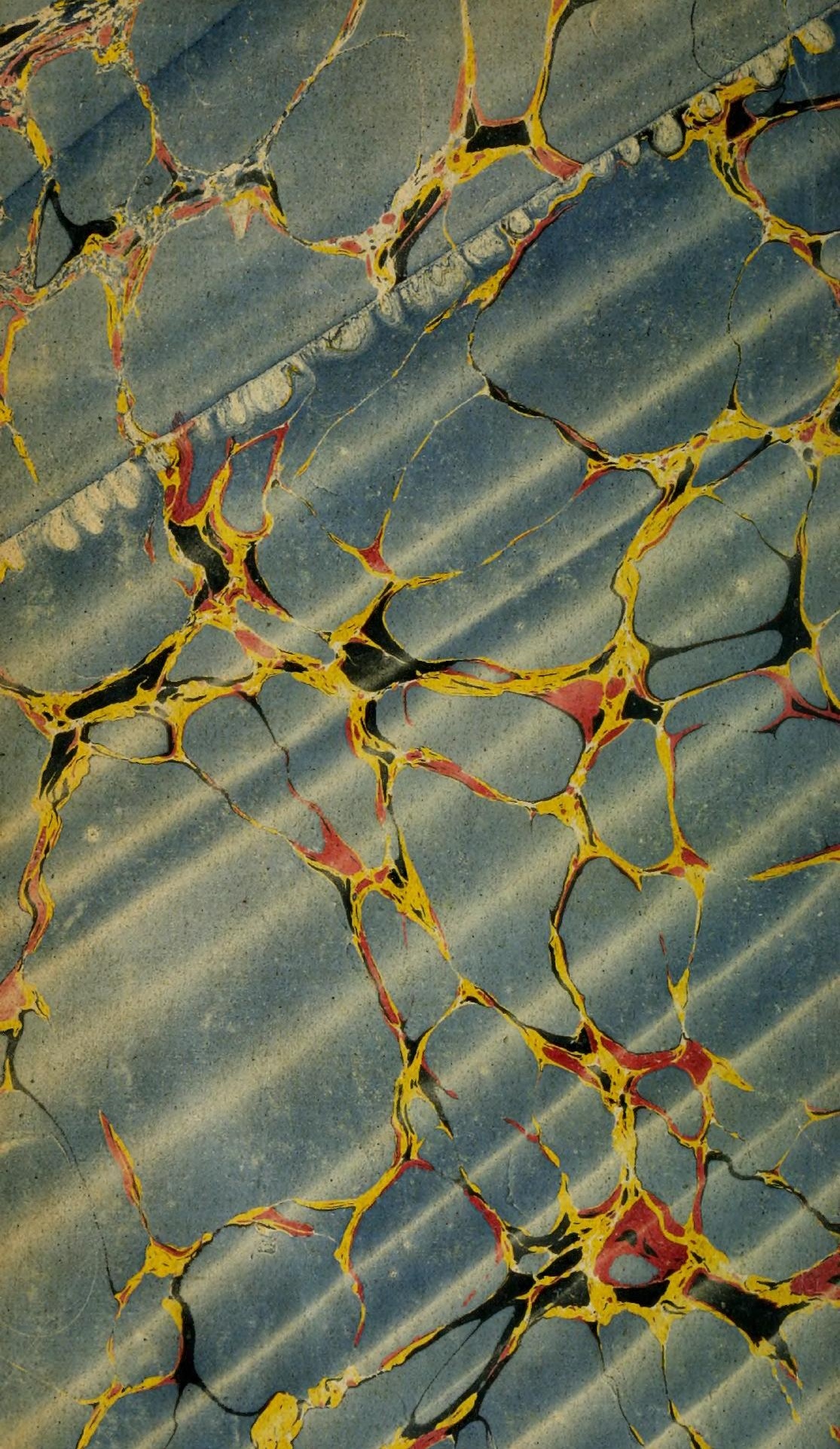




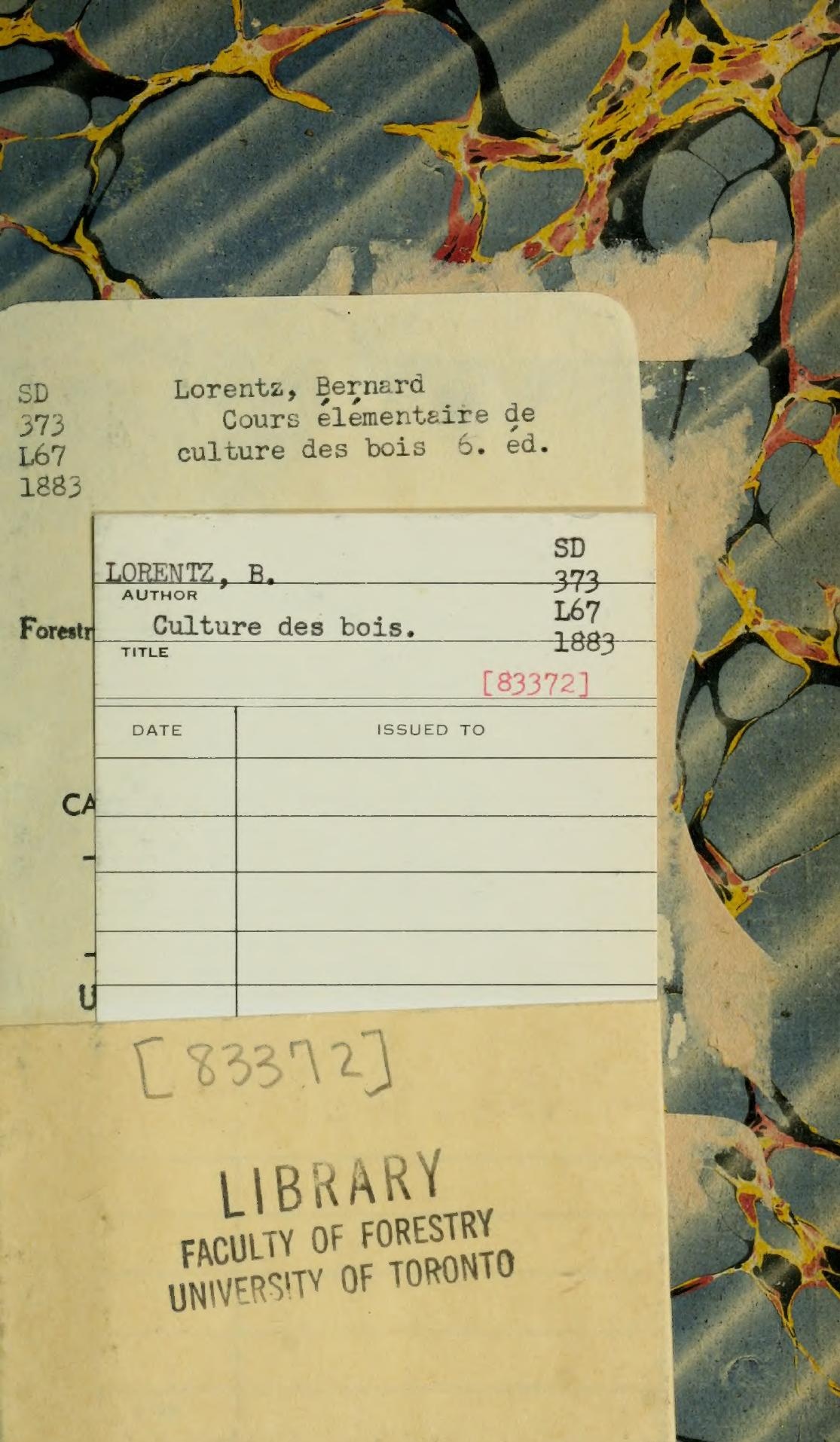


